\title{
60th Annual Conference of Indian Society of Hematology \& Blood Transfusion (ISHBT) October 2019
}

\section{Contents}

Acute Leukemia (Clinical)...

Acute Leukemia (Laboratory).......

Anemia Including Hemolyticanemia

Bleeding Disorders

Bone Marrow Failure and MDS (Clinical)

Bone Marrow Failure and MDS (Laboratory)

Chronic Leukemia (Clinical)

Chronic Leukemia (Laboratory)

Infections and support care....

Lymphoma and Myeloma (Clinical)

Lymphoma and Myeloma (Laboratory) …....................................... 110

Myeloproliferative neoplasms ................................................ 121

Stem Cell Transplantation............................................................ 128

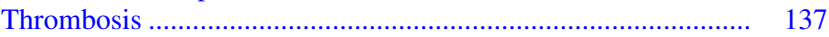

Transfusion medicine ............................................................. 143

\section{Acute Leukemia (Clinical)}

\section{Abstract 1. Reactive Plasmacytosis of Bone Marrow Imposing a Diagnostic Dilemma in Hematological Malignancies}

\section{Kanwaljeet Singh $^{* 1}$, Ankur Ahuja ${ }^{1,2}$, Tathagatta Chatterjee ${ }^{1}$ \\ ${ }^{1}$ Dept Of Pathology, AHRR, ${ }^{2}$ Army Hospital Research \& referral (AHRR), New Delhi, NEW DELHI, India}

Aims \& Objectives: This retrospective study was conducted to observe the spectra of hematological malignancies with plasmacytosis in bone marrow and to analyse the clonality of these plasma cells.

Patients/Materials \& Methods: A total 154 bone marrow aspirates that showed diagnosis of AML, ALL and MDS were screened for increased plasma cells $(>3.5 \%)$.IHC in the form of CD38, CD138, kappa/lambda and CD56 was done to evaluate the clonality of plasma cells. Further investigations including estimation of serum gamma globulins on serum Protein electrophoresis (SPEP), immunofixation and flow-cytometry were performed to rule out monoclonality
Results: Among the total of 154 patients, 84 were of AML, 58 were ALL and 12 were MDS. 5/84 (6\%) AML cases, 4/58 (6.89\%) ALL cases and 2/12 (1.67\%) MDS cases showed reactive plasmacytosis with plasma cell concentration ranging from $5 \%$ to $42 \%$. The majority of patients were in 4-5th decade in acute leukemia cases while in MDS the patients presented in 5th-6th decade

Discussion \& Conclusion: Patients with bone marrow plasmacytosis in hematological malignancies requires complete clinical evaluation, individualized investigations like SPEP, immunofixation studies and bone marrow biopsy with immunohistochemistry to arrive at a final diagnosis for patient management.

Disclosure of Interest: None Declared.

Keywords: hematological malignancies, Reactive plasmacytosis, serum Protein electrophoresis (SPEP).

\section{Abstract 2. Hyperleukocytosis in Childhood ALL: Short- Term Outcome}

Pritam Singha Roy ${ }^{* 1}$, Neelam Varma ${ }^{2}$, Richa Jain ${ }^{1}$, Man Updesh Singh Sachdeva ${ }^{2}$, Deepak Bansal ${ }^{1}$, Amita Trehan ${ }^{1}$

\section{${ }^{1}$ Pediatrics, ${ }^{2}$ Hematology, PGIMER, Chandigarh, India}

Aims \& Objectives: Fifteen to twenty percent children with ALL present with hyperleukocytosis, with short-term morbidities/mortalities secondary to respiratory \& CNS leukostasis and tumour lysis. Hyperleucocytosis is associated with poor long-term survival. The aim of this study was to evaluate the clinical, laboratory parameters, morbidity \& short-term outcomes in children with ALL presenting with hyperleukocytosis (TLC $>1,00,000 / \mu \mathrm{L}$ ).

Patients/Materials \& Methods: Analysis of children (0-12 years) with ALL presenting from January 2014 to June 2019, treated on a uniform protocol.

Results: One hundred and thirty three (17.2\%) of 759 children diagnosed with ALL had hyperleukocytosis. Fifty-three \% of T-ALL $(\mathrm{n}=94)$ patients and $12.2 \%$ of B-ALL $(\mathrm{n}=659)$ patients presented with hyperleukocytosis. The symptom diagnosis interval was 2 weeks (1-4 weeks). Median age: 6 years (IQR: $3-8), 4$ being $<1$ year. Eighty one and 50 of 133 patients with hyperleukocytosis had B-ALL \& T-ALL disease; 2 having mixed immune-phenotype. Bulky disease: $35(26 \%)$ patients, 20: T-ALL \& 15: B-ALL. Four: superior mediastinal syndrome, 3 (75\%) being T-ALL. Mean hemoglobin: 
$7.1 \mathrm{~g} / \mathrm{dl} \quad( \pm 2.3) ; \quad$ median platelet counts: $35,000 / \mu \mathrm{L}$ (IQR: 17,000-62,500). Leukostatic symptoms (CNS \& respiratory) were seen in $5 \& 6$ children. Thirteen underwent therapeutic exchange transfusions \& one had leukapheresis. Biochemical tumour lysis syndrome (TLS): 52 (39\%); 6: hyperkalemia (7.1 meq/L, IQR: 6.5-7.8), hyperuricemia: 37 (28\%)(9.9 mg/dl, IQR: 8.6-11.8). Patients received hyperhydration (1.5 to 3 times maintenance fluid) \& allopurinol. Rasburicase was used in 14 children \& 2 required hemodialyses. Cytogenetics (105 patients); 13, 6 and 3 had $\mathrm{t}(9: 22)$, $\mathrm{t}(1: 19)$ \& $\mathrm{t}(4: 11)$. One patient had CNS disease. Poor steroid response: (blast count of $>1000 / \mu \mathrm{L}$ ) on day 8 in $67(50 \%)$. End induction bone marrow was M1 in $92.5 \%(112 / 121)$ and 28/92 (30\%) patients had high MRD ( $>0.01 \%)$.

Discussion \& Conclusion: Twenty six percent cases with hyperleukocytosis had bulky disease, 39\% patients had biochemical TLS and $9 \%$ had symptomatology related to leukostasis. Poor cytogenetics was seen in $21 \%$ cases, predominantly $\mathrm{t}(9 ; 22)$. Ten percent required urgent leukoreduction (exchange/leukapheresis). Poor response to induction therapy with a high MRD was seen in $30 \%$ cases vs. $22.6 \%$ in the patients without hyperleukocytosis $(p=0.12)$. There was no mortality secondary to hyperleukocytosis. High MRD and poor cytogenetics are greater in patients with hyperleukocytosis.

Disclosure of Interest: None Declared.

Keywords: ALL, Hyperleukocytosis, Morbidity.

\section{Abstract 3. Aberrant Antigenic Expression in Acute Leukaemia: Study from A Tertiary Care Center in Southern India}

\section{Subhajit Hajra ${ }^{* 1}$, Prabhu M${ }^{1}$, Rakhee Kar $^{1}$, Debdutta Basu ${ }^{1}$ \\ ${ }^{1}$ Pathology, Jipmer, Puducherry, Puducherry, India}

Aims \& Objectives: Aberrant antigenic expression in acute leukaemia is abnormal expression of antigens which is not normally associated with acute leukaemia of specific lineage (Myeloid, B-lymphoid, T-lymphoid) and not fulfilling the criteria for diagnosis of Mixed phenotype acute leukaemia (MPAL). Some aberrant phenotype in certain leukemias may indicate presence of genetic event or may be of prognostic significance. This aberrant immunophenotype can be used as Leukemia associated immunophenotype (LAIP) for monitoring of Minimal Residual Disease (MRD). This study was done to assess the type and frequency of aberrant antigen expression in acute leukemias.

Patients/Materials \& Methods: All cases of acute leukemias diagnosed based on morphology and confirmed by immunophenotyping in the period of July 2018 to July 2019 were enrolled. Cells of interest was selected using CD45/SSC gating strategy. An immunophenotypic marker was considered positive if at least $20 \%$ and $10 \%$ cells showing expression of surface and intra-cytoplasmic markers respectively.

Results: Among 104 acute leukemias, 39 (38\%) B-Acute Lymphoblastic Leukemias (B-ALL), 13 (13\%) T-ALL, 48 (46\%) Acute Myeloid Leukemias (AML) and 4 (3\%) MPAL were found. Five (12.8\%) out of 39 B-ALLs, 3 (23\%) out of 13 of T-ALLs had aberrant antigen expression. The aberrant markers in ALLs were CD33 (50\%), CD13 (12.5\%), CD117 (12.5\%) and CD79a in T-ALL (66\% among T-ALL cases), CD5 in B-ALL (20\% among B-ALL cases). Among 48 AML, 15 (31\%) expressed aberrant antigen such as CD7 (73\%) and CD19 (27\%). Among 4 MPAL cases, one case of B-Myeloid phenotype expressed aberrant CD7.

Discussion \& Conclusion: From this study, it can be concluded that aberrant antigenic expression is common in acute leukemias (23\%). AML $(31 \%)$ more commonly expressed aberrant antigen compared to ALL (15\%). T- ALL (23\%) expressed aberrant markers more frequently compared to B-ALL (12.5\%). Most common aberrant antigen expressed by B-ALL was CD33 (50\%) and cCD79a (66\%) in T-ALL. With respect to number of antigens, ALL showed more aberrancies compared to AML (5 versus 2 aberrant markers) in this study.

Supporting Document: 13ccce08-bf34-464f-8a8d-b235cc5e5268. Disclosure of Interest: None Declared.

Keywords: Aberrant immunophenotype, Acute leukemia, Leukemia associated immunophenotype.

\section{Abstract 4. A Case Report of Down's Syndrome with Transient Abnormal Myelopoiesis (TAM)}

\author{
Khoinaijam Peepa ${ }^{* 1}$, Irom Anil², Rajkumari Banashree ${ }^{3}$, \\ Hijam Apabi ${ }^{4}$
}

${ }^{1}$ Pgt, 2nd year, MEDICINE, ${ }^{2}$ Assistant Professor, MEDICINE, ${ }^{3}$ Associate Professor, PATHOLOGY, ${ }^{4}$ Associate professor, Paediatric, Jnims, Imphal, India

Aims \& Objectives: A case report of Down's syndrome with Transient abnormal myelopoiesis (TAM).

Patients/Materials \& Methods: Down's syndrome has been known to be associated with various hematological malignancies ${ }^{1}$. They may develop a syndrome stimulating acute leukemia, commonly known as transient abnormal myelopoiesis (TAM) or myeloproliferative disease $^{2}$. We are reporting a case of Down's Syndrome with TAM.

Results: A neonate was admitted on the first day of birth with fever, jaundice and decreased feeding in NICU. On examination, he had features of Down's syndrome (Fig. 1). Complete hemogram showed $\mathrm{HB}=16 \mathrm{gm} / \mathrm{dl}, \mathrm{TLC}=1,13,000 / \mathrm{mcl}$ with blast $(50 \%)$ and platelets $=3.8 \mathrm{lakh} / \mathrm{cumm}$. The flow cytometry (peripheral blood) showed positive for CD7, CD33, CD34, CD56, CD117 \& HLADR and CD41 \& CD61 negative. The karyotyping showed 47, XY, +21 . The patient developed tumor lysis syndrome (TLS) (s. uric acid $=14.1 \mathrm{mg} / \mathrm{dl}, \mathrm{K}=6.7 \mathrm{mg} / \mathrm{dl}$, s. calcium $=8.3 \mathrm{mg} / \mathrm{dl}$, s. creatinine $=0.9 \mathrm{mg} / \mathrm{dl}$ ). Rasburicase $3 \mathrm{mg}$ was given along with hydration and other supportive measures. Patient shows improvement spontaneously with supportive management and was discharged on $20^{\text {th }}$ day. The hematological finding and TLS markers are shown in the Table 1.

\begin{tabular}{llllll}
\hline Parameters & Day1 & Day 2 & Day 5 & Day 10 & Day15 \\
\hline Hb (gm/dl) & 16 & 14.2 & 17.1 & 15.6 & 14.6 \\
TLC (/cumm) & $1,13,000$ & 74,000 & 40,930 & 36,100 & 34,760 \\
Blast (\%) & 50 & 60 & 46 & 44 & 17 \\
Platelet (lac/cumm) & 3.8 & 2.5 & 1.97 & 2.00 & 6.36 \\
Uric acid (mg/dl) & 14.1 & 6.1 & 5.0 & 4.5 & 4.0 \\
Potassium (mg/dl) & 6.7 & 5.7 & 5.1 & 5.0 & 4.3 \\
\hline
\end{tabular}

Discussion \& Conclusion: Down's syndrome with TAM usually present with leucocytosis, basophilia and thrombocytopenia \& the blast usually express immature \& myeloid antigen (CD 34, CD117 \& CD33, CD13 respectively) with at least one platelet antigen (CD36, CD41a, CD41b or CD61) and there may be aberrant expression of CD56 \& CD7. Infants who survive 4 to 8 weeks usually show complete remission ${ }^{3}$. In our case, there was absence of CD41, CD 61 unlike the classical presentation. Also basophilia and throbocytopenia were absent. He started recovering spontaneously. He was discharged on day +20 of admission in a stable condition. 
Supporting Document: ac623076-84ab-435e-889b-e342f0f43b68. Disclosure of Interest: None Declared.

Keywords: Down's syndrome, myeloproliferative disease, TAM.

\section{Abstract 5. Condensed Schedule of HiDAC in AML: Short, Safe and Effective}

\section{Shouriyo Ghosh ${ }^{* 1}$, Sambit Samanta ${ }^{1}$, Arnab Chattopadhay ${ }^{1}$, Maitreyee Bhattacharyya ${ }^{1}$}

\section{${ }^{1}$ Haematology, Ihtm Kolkata, Kolkata, India}

Aims \& Objectives: We compared the existing schedule of high dose ARA-C (HiDAC) consolidation chemotherapy (given as an IV infusion $\left(3 \mathrm{gm} / \mathrm{m}^{2}\right)$ on days 1,3 and $\left.5 \mathrm{Q} 12 \mathrm{H}\right)$ with a condensed dosing schedule, viz, on days $1-3 \mathrm{Q} 12 \mathrm{H}$ and assessed toxicity, time to hematologic recovery and quantum of supportive care required during the hospital stay.

Patients/Materials \& Methods: Institute based open labelled parallel-group clinical study was done. AML patients who achieved remission after standard induction therapy were recruited to receive HiDAC in either condensed or conventional schedule. Time to hematologic recovery, transfusion requirement, incidence of febrile neutropenia and complications arising thereof, were noted and compared between the two schedules.

Results: These are interim results of the study. A total of 26 patients were recruited ( 9 patients received HIDAC in condensed, i.e. 123 protocol and 17 patients received HiDAC in conventional i.e. 135 protocol). Time to neutrophil and platelet recovery was on an average, 4 days earlier and 7 days earlier respectively, in the condensed arm. The incidence of febrile neutropenia was also lower in patients receiving the condensed schedule. One patient died during $3{ }^{\text {rd }}$ HiDAC consolidation in the conventional schedule (135 protocol). There was marked reduction in transfusion support (PRBC and RDP/SDP) requirement in the condensed schedule. Overall, duration of hospital stay, complication rate and tolerability was better with the condensed schedule of HiDAC.

Discussion \& Conclusion: The condensed schedule of HiDAC is well tolerated and requires less supportive care. The risks of morbidity and mortality are also reduced considerably. Based on this study and another study published by the German AML study group, new guidelines may be drafted to administer HiDAC in the condensed schedule (on consecutive days 1,2 and 3) instead of the traditional administration on days 1,3 and 5 , without significantly compromising efficacy of treatment.

Disclosure of Interest: None Declared.

Keywords: AML, Condensed schedule, HiDAC.

\section{Abstract 6. Pediatric ALL Presenting with Myelofibrosis: An Unusual Presentation}

\section{Shrinza Gupta ${ }^{* 1}$, Neha Garg ${ }^{1}$, Mrinalini Kotru ${ }^{1}$, Richa Gupta ${ }^{1}$, Meera Sikka ${ }^{1}$ \\ ${ }^{1}$ UCMS and GTB hospital, Delhi, India}

Aims \& Objectives: Acute lymphoblastic leukemia (ALL) is a hematological disorder involving $\geq 20 \%$ blasts in peripheral blood and/or bone marrow. ALL commonly affects children less than 6 years of age. We present this case of pediatric ALL with myelofibrosis in view of its rarity. Only 100 cases of pediatric myelofibrosis have been reported worldwide.
Patients/Materials \& Methods: A four and a half year old girl presented to the pediatric OPD with history of fever, progressive skin pallor and weakness since 1 month. She had history of bleeding from the nose since one month and from the gums since 15 days. On examination she was severely pale and petechial rash was present over the trunk and bilateral lower limbs. Per abdominal examination revealed hepatosplenomegaly. Liver was firm in consistency and could be palpated $6 \mathrm{~cm}$ below the right costal margin. Clinically spleen was palpable $3 \mathrm{~cm}$ below the left costal margin. Investigations revealed severe anemia and thrombocytopenia for which she received 1 unit red cell concentrate and 4 unit platelet transfusion. Bone marrow aspirate and biopsy were done in view of the clinical and laboratory findings.

Results: On peripheral blood smear, it showed presence of blasts (23\%). RBCs were normocytic normochromic to microcytic hypochromic with reduced platelets. There were no tear drop cells seen on peripheral smear. On bone marrow aspirate, there was near total replacement by blasts which were of same morphology as those in peripheral smear. On bone marrow biopsy, presence of blasts along with myelofibrosis was noted. Reticulin stain suggested grade- 3 bone marrow fibrosis.

Discussion \& Conclusion: Peripheral smear, bone marrow aspiration and biopsy findings were consistent with acute lymphoblastic leukemia of B cell origin with myelofibrosis. A diagnosis of pediatric ALL with myelofibrosis is a rare presentation.

Disclosure of Interest: None Declared.

Keywords: ALL, Myelofibrosis, Pediatric acute lymphoblastic leukemia.

\section{Abstract 7. Acute Promyelocytic Leukemia Post Chemoradiation: Is It Therapy-Related or Denovo Second Malignancy?}

\author{
Aswini Prabakaran ${ }^{* 1}$, Prabhu $\mathbf{M}^{1}$, Rakhee Kar $^{1}$, Debdatta Basu ${ }^{1}$ \\ ${ }^{1}$ JIPMER, Puducherry, India
}

Aims \& Objectives: Introduction: Systemic treatment with chemotherapy and radiation have contributed to improved survival rates in patients with prior malignancy. However,the incidence of secondary malignancies are increasing following extensive exposure to chemoradiation. Of the various therapy-related myeloid neoplasms, Acute Promyelocytic Leukaemia (APML) occurring as a complication to chemo-radiotherapy is rare.Herein we report on two such cases; one with carcinoma breast and the other of cervix post chemo/radiotherapy, both of whom on follow up developed secondary APML.

Patients/Materials \& Methods: Case reports:

Case 1: 62 year-old lady was diagnosed with carcinoma right breast in Nov 2015 and was treated with right modified radical mastectomy and received 3 cycles of adjuvant chemotherapy: 5-Flurouracil, Epirubicin, Cyclophosphamide (FEC regimen) followed by 4 cycles of injection docetaxel, followed by letrozole. After 18 months of chemotherapy she presented with fever and necrotising gingivitis. Routine blood counts revealed severe pancytopenia with $\mathrm{Hb}-55 \mathrm{~g} / \mathrm{l}$, WBC- $0.74 \times 10^{9} / 1$, platelet- $10 \times 10^{9} / 1$.

Case 2: 48 year-old lady was a known case of carcinoma cervix, post radiotherapy in May 2017 and was on follow up. After 2 years she presented with bleeding per rectum for 1 month and fever for 1 week.Routine blood counts revealed severe pancytopenia with $\mathrm{Hb}$ $57 \mathrm{~g} / \mathrm{l}, \mathrm{WBC}-1.09 \times 10^{9} / \mathrm{l}$, platelet-12 $\times 10^{9} / 1$.

Results: Peripheral smear and bone marrow aspiration revealed atypical promyelocytes with characteristic Faggot cells. Based on these findings, a diagnosis of APML was made. FISH studies were positive for $\mathrm{t}(15 ; 17)$ in both. In addition, flow cytometry was also performed in case 2 and was negative for HLA-DR, CD34, positive for MPO. 
Discussion \& Conclusion: Cytotoxic agents like alkylating agents, topoisomerase inhibitors and ionising radiation are associated with risk of developing therapy-related myeloid neoplasms. Amongst which topoisomerase II inhibitors has a shorter latency period (1-5 years) and others has a longer latency period (5-10 years). Many of these cases have monoblastic or myelomonocytic morphology. However amongst all therapy related acute leukemia, therapy-related APML is rare. Whether secondary APML is therapyrelated as appears to be in the former or is a de-novo second malignancy as appears to be in the latter, it is considered the most treatable. Hence timely and accurate diagnosis of it is essential.

Supporting Document: d9df200c-483c-4f1a-bca8-c4daad38dcc9. Disclosure of Interest: None Declared.

Keywords: Acute Promyelocytic Leukaemia, secondary malignancy, Therapy-related acute leukemia.

\section{Abstract 8. Philadelphia Chromosome Positive Childhood ALL: Clinical Profile and Outcome}

Pritam Singha Roy ${ }^{* 1}$, Deepak Bansal ${ }^{1}$, Neelam Varma $^{2}$, Richa Jain ${ }^{1}$, Man Updesh Singh Sachdeva ${ }^{2}$, Amita Trehan ${ }^{1}$

\section{${ }^{1}$ Pediatrics, ${ }^{2}$ Hematology, PGIMER, Chandigarh, India}

Aims \& Objectives: The Philadelphia translocation t(9:22)(q34; q11) is one of the first cytogenetic aberration described in leukemogenesis. Contrasting to the adult ALL statistics; only around 3\% of childhood Acute Lymphoblastic Leukemia (ALL) carry this mutation. With the advent of molecularly targeted therapy (tyrosine kinase inhibitors [TKI]), the long-term survival has improved and is equivalent to a hematopoietic stem cell transplant in those who achieve remission. This study was done to evaluate the clinical profile and short term outcomes in children with $\mathrm{Ph}+\mathrm{ALL}$ treated on a uniform protocol. Patients/Materials \& Methods: Analysis of children (1-12 years) with Ph + ALL presenting from January 2014 to December 2017. All patients were managed as high risk ALL using the ICICLE protocol. Imatinib was the TKI used.

Results: Fourteen $(2.8 \%)$ of 489 children diagnosed with ALL had $\mathrm{t}(9: 22)$ or Philadelphia $(\mathrm{Ph})$ mutation positive disease. Median age: 6 years (IQR: 4-10). None had bulky/CNS positive disease at presentation. Seven $(50 \%)$ had hyperleukocytosis (TLC $>1,00,000 / \mu \mathrm{L})$. Imatinib mesylate $\left(340 \mathrm{mg} / \mathrm{m}^{2}\right)$ was added in the second/third week of induction therapy. Prophase steroid response at day 8 was good $(13 / 14)$ with 1 patient having a poor prednisolone response (blast count of $>1000 / \mu \mathrm{L})$. End induction bone marrow was M1 in $92.3 \%$ $(12 / 13)$, one patient having succumbed during induction. Fifty percent (6/12) patients had end induction high MRD (>0.01\%) [MRD not available in 1 child]. Treatment related mortality: 2 patients (Induction and consolidation phase: both sepsis) Four children relapsed (3 CNS \& 1 marrow relapse); 3 being very early relapses and one a late relapse. The event free survival is $57 \%$ (95\% CI 557-1039 days). Five patients are presently on the maintenance phase of therapy.

Discussion \& Conclusion: In our analysis $2.8 \%$ ALL patients had $\mathrm{Ph}+$ disease. A good prednisolone response was documented in the majority (13/14) as also morphological marrow remission status (12/ 13). However, $50 \%(6 / 12)$ had positive MRD at the end of induction. Two of the 4 relapsed patients had a high MRD. Though our cohort is small, the EFS at a median of 28 months is $57 \%$. Treatment related mortality $(14 \%)$ is a contributory factor towards a poor survival.

Disclosure of Interest: None Declared.

Keywords: ALL, Outcome, Philadelphia chromosome.

\section{Abstract 9. Precursor B-Cell Acute Lymphoblastic Leukemia Presenting as Hemophagocytic Lymphohistiocytosis}

\section{Anurag Gupta $^{* 1}$, Abhishek Pathre ${ }^{1}$, Neha Garg ${ }^{1}$, Sonali Dixit ${ }^{1}$, Mrinalini Kotru', Meera Sikka ${ }^{1}$}

\section{${ }^{1}$ UCMS \& GTB Hospital, Delhi, India}

Aims \& Objectives: Hemophagocytic Lymphohistiocytosis (HLH) is a life threatening hyperinflammatory disease caused by uncontrolled immune response and cytokine dysregulation accompanied by fever, cytopenia, hepatosplenomegaly and involvement of central nervous system.

Patients/Materials \& Methods: A 5-year old boy, known case of HLH with myelofibrosis presented to pediatric OPD with complaints of fever and lymphadenopathy. Patient was on chemotherapy as per HLH-2004 protocol with history of multiple blood transfusions.

Results: Complete hemogram revealed pancytopenia. Peripheral smear showed normocytic normochromic red cells with few microcytes. Few atypical cells were seen. Bone marrow aspirate smears were diluted. However, imprint smears showed presence of immature cells. Bone marrow biopsy showed replacement by monomorphic blast like cells. There was increase in reticulin grade 2 with occasional myeloid and erythroid precursors. On immunohistochemistry, the monomorphic cells were CD34(+), Tdt(+), CD10(+), PAX5(+) CD20(-), CD3(-), MPO(-) and $\mathrm{CD} 117(-)$ consistent with a diagnosis of precursor B-cell Acute lumphoblastic leukemia (B-ALL). On follow up, patient was started on BFM95 protocol for B-ALL and has achieved morphological remission.

Discussion \& Conclusion: The diagnosis of HLH requires either a molecular diagnosis consistent with primary HLH or fulfilment of 5 out of 8 diagnostic criteria. Malignancy is one of the subgroup linked to secondary HLH. Timing and severity of HLH depend not only on the infectious trigger but also on type of leukemia and clinical status of malignancy. Most commonly malignancy associated HLH has been reported in the setting of T-cell leukemias, NK-cell leukemias and lymphomas. Presentation of B-ALL as HLH is rarely reported in literature. HLH develops after a diagnosis of T-ALL with a dismal prognosis while HLH develops prior to/or concurrent with a diagnosis of B-ALL and confers a good prognosis. The present case developed HLH prior to B-ALL and has achieved morphological remission and this finding is in accordance with the previous reports. However, the possible interaction between these factors needs to studied.

Disclosure of Interest: None Declared.

Keywords: ALL, B- acute lymphoblastic leukemia, HLH.

\section{Abstract 10. BCR-ABL1-Like BCP-ALL- Cases Identified Using Customized Panel for Targeted RNA Sequencing}

Subhashree Pradhan ${ }^{* 1}$, Sweta Rajpal ${ }^{1}$, Prasanna Bhanshe ${ }^{1}$, Swapnali Joshi ${ }^{1}$, Shruti Chaudhary ${ }^{1}$, Gaurav Chatterjee ${ }^{1}$, Prashant Tembhare', P G Subramanian', ${ }^{1}$, Sumeet Gujral', Nikhil Patkar'

\section{${ }^{1}$ Hematopathology, Tata Memorial Centre Advanced Centre for Treatment, Research and Education in Cancer, Navi Mumbai, India}

Aims \& Objectives: $B C R$ - $A B L 1$-like B-cell precursor acute lymphoblastic leukemia ( $B C R-A B L 1$ like BCP-ALL) is a subtype of B-ALL characterized by a gene expression profile similar to $B C R-A B L 1$ positive 
ALL. It is associated with a high risk of relapse and poor prognosis. These patients have genetic alteration which leads to activation of cytokine receptor genes and kinase signaling pathways, which make it amenable to the ABL-type inhibitors (tyrosine kinase inhibitors) or JAK inhibitors (rearrangements of JAK2and EPOR). We designed targeted RNA sequencing panel to identify cases of $B C R-A B L 1$ like BCP-ALL.

Patients/Materials \& Methods: Cases of Precursor B-lineage ALL for whom NGS requisition was raised in Tata memorial centre from Jan 2018 to July 2019 were subjected to RNA sequencing using customized RNA sequencing panel using primers for $A B L 1, A B L 2, C S F 1 R, C R L F 2$, DUX4, EPOR, ETV6, EBF1, FGFR1, IKZF1, JAK2, KMT2A, MLLT4, MEF2D, NTRK3, NUP214, NUP98, P2RY8, PAX5, PDGFRA, PDGFRB, RUNX1, TCF3, TYK2and ZNF384 genes to detect their unknown fusion partners. Illumina Miseq (300-cycle chemistry) was used to perform the paired end sequencing. Data was analyzed using Oncofuse and Fusion Catcher. Custom scripts and bed tools-2.25 were used to determine the coverage and construct the pipeline.

Results: Seven cases of $B C R-A B L 1$ like BCP-ALLwere identified.All the 7 caseswere male. The median age of these patients was 19 years (range: 11-46 years). The median hemoglobin, total leukocyte count and platelets at the time of diagnosis were $8.7 \mathrm{gm} / \mathrm{L}, 8.5 \times 10^{9} / \mathrm{L}$ and $49 \times 10^{9} / \mathrm{L}$ respectively. The post-induction measurable residual disease by flowcytometry was positive in all 7 cases (median-2.7\%). Two patients had $E B F 1$ $P D G F R B$ fusion while there were one patient each with ETV6-ABL1, ZC $3 H A V 1-A B L 2, \quad B C R-J A K 2, \quad N U P 214-A B L$ and RANBP2/ABL1 (Table 1).

Discussion \& Conclusion: $B C R-A B L 1$ like BCP-ALL is a sub-group of ALL which needs to be identified appropriately and receive targeted therapy to improve the outcome. Conventional techniques such as FISH are not suitable as diagnostic strategies as they can neither consistently identify both partners involved in gene fusion nor they can identify the exact gene sequences for monitoring purposes. In the present study, we identified 7 cases of $B C R-A B L 1$ like BCP-ALL by using targeted gene panel designed in our laboratory for RNA sequencing.

Supporting Document: 6ccf64ad-6256-4a44-bb99-6b2e339ef525

Table 1. Clinical and laboratory parameters of BCR-ABL1-like BCP-ALL

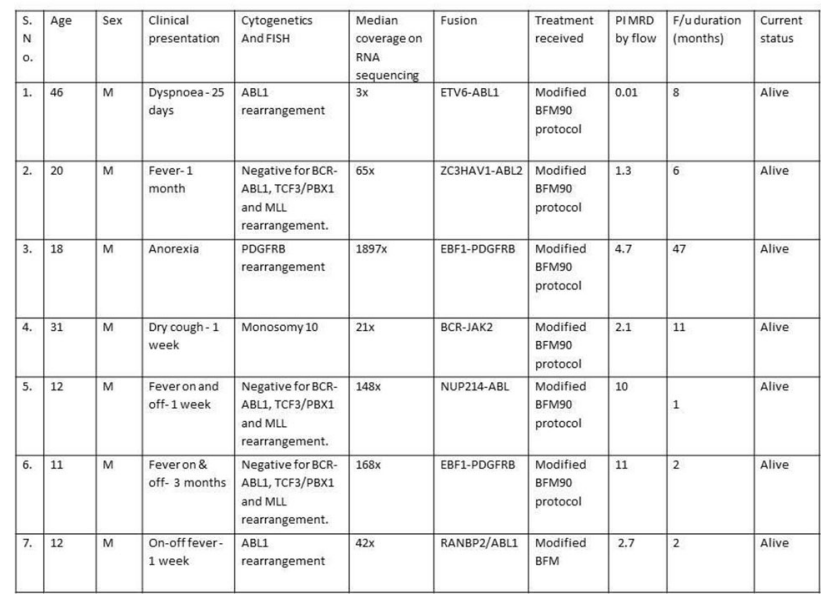

Disclosure of Interest: None Declared.

Keywords: B-ALL, NGS, Ph like ALL.
Abstract 11. Situs Inversus Totalis and Dextrocardia Associated with Acute Lymphoblastic Leukaemia

\author{
Rohit Kapoor $^{* 1}$, Anil Sharma ${ }^{1}$, Goutomi Chatterjee ${ }^{1}$, \\ Neha Rastogi ${ }^{1}$, Satya Prakash Yadav ${ }^{1}$
}

\section{${ }^{1}$ Pediatric- Hemato-Oncology and Bone Marrow Transplant, Medanta -The Medicity,near Olympus, Ch Baktawar Singh Rd, Sector 38, Gurugram, Haryana 122001, Gurgaon, India}

Aims \& Objectives: Situs inversus totalis and dextrocardia with acute leukaemia is a rare presentation and has not been reported in literature so far. We hereby report a 15 year old female with a past medical history of complex congenital heart disease, who presented with fever, syncopal episode and excessive menstrual flow subsequently diagnosed to have precursor B cell acute lymphoblastic leukaemia. We hereby report first and only case of acute lymphoblastic leukaemia with complex congenital heart anomaly with situs inversus treated on BFM -95 regimen and continues to be in complete remission till date.

Patients/Materials \& Methods: A 15 year old girl with a past medical history of pneumonia at 5 months of age and complex congenital heart disease detected at the same time (Situs Inversus and Dextrocardia-L TGA with Restrictive VSD with Moderate TR and Dilated LA/RV) presented to the emergency with history of syncopal attack, fever and excessive menses. On examination significant pallor was noted, no lymphadenopathy. Cardiac examination was significant for heart sounds, which auscultated on right side of chest; Grade 4 pan-systolic murmur present in tricuspid area, and best heard in pulmonary area. She had hepatosplenomegaly.

Results: Baseline investigation were significant for hemoglobin of $2.9 \mathrm{gm} \%$, Total leucocyte count of $6300 / \mathrm{cc}$ with polymorphs of $8.5 \%$, lymphocyte count of $83.7 \%$ and a platelet count of $40,000 / \mathrm{cc}$. Her LDH was $859 \mathrm{U} / \mathrm{l}$, reticulocyte count was $0.2 \%$. Her samples for Dengue Serology, Ns1Ag, Malaria serology, Viral markers were nonreactive. Her serum tTG $(<3)$, Beta $\mathrm{HCG}(<2.39 \mathrm{mIU} / \mathrm{ml})$, coagulation profile, ANA was negative (1:40), Troponin $(0.015 \mathrm{ng} /$ $\mathrm{ml}$ ),Serum Iron Profile (Iron levels $136 \mathrm{ugm} / \mathrm{dl}$, Total iron binding capacity $317 \mathrm{ug} / \mathrm{dl}$ ), Thyroid function test (Free T3-1.74 pg/ml, Free T4-1.58 ng/dl, TSH $-4.120 \mathrm{uIU} / \mathrm{ml}$ ) were all normal. Her ferritin levels were $2020 \mathrm{ng} / \mathrm{ml}$ and Vit B12 Levels were $<159 \mathrm{pg} / \mathrm{ml}$. Her SGOT and SGPT, were slightly elevated with $185 \mathrm{U} / \mathrm{L}$ and $96 \mathrm{IU} / \mathrm{L}$ respectively and rest LFT was normal. The BUN and S. Creatinine was $33 \mathrm{mg} / \mathrm{dl}$ and $0.80 \mathrm{mg} / \mathrm{dl}$ respectively. Supportive care in form of blood transfusion and platelet transfusion as needed to maintain hemoglobin during heavy menstrual flow were given. Ultrasound abdomen revealed liver on the left side with a span of $18.7 \mathrm{~cm}$ and her spleen was visualized in right flank with a span of $13.7 \mathrm{~cm}$ both having normal echotexture. Rest imaging showed normal adnexa, ovaries and kidneys. Bone marrow aspiration showed lymphoid blast morphology. Blasts were negative for CD34, TdT and myeloid markers however diffusely positive for CD10. Flowcytometry immunophenotypic profile was consistent with CALLA + Precursor B Cell acute lymphoblastic Leukaemia. Her karyotype was significant for $46 \mathrm{XX}, \operatorname{det}(19) \mathrm{t}(1 ; 19)(\mathrm{q} 23 ; \mathrm{p} 13)[11] / 46, \mathrm{XX}[9]$. Eventually she was started on BFM-95 induction as per protocol. Her MRD (minimal residual disease) was negative at the end of induction.Her MRD continued to be negative for day 79 bone marrow as well. She continues to be in molecular and hematological remission till date. 
Discussion \& Conclusion: The present case to best of our knowledge is first of its kind reporting an association of situs inversus totalis and dextrocardia with precursor B cell acute lymphoblastic leukaemia being successfully managed on BFM-95 protocol.

Disclosure of Interest: None Declared.

Keywords: Acute lymphoblastic Leukaemia, Dextrocardia, Situs inversus totalis.

\section{Abstract 12. Acute Lymphoblastic Leukemia with May- Hegglin Anomaly: A Case Report}

Goutomi Chatterjee ${ }^{* 1}$, Dhwanee Thakkar ${ }^{1}$, Rohit Kapoor ${ }^{1}$, Anil Sharma $^{1}$, Neha Rastogi ${ }^{1}$, Satya Prakash Yadav ${ }^{1}$

${ }^{1}$ Pediatric Hematology, Oncology and Bone marrow transplantation, Medanta, The Medicity, Gurgaon, India

Aims \& Objectives: May-Hegglin anomaly (MHA) is a rare autosomal dominant disorder characterized by thrombocytopenia, macrothrombocytes with associated sensorineural deafness, cataract and nephritis. Patients may be asymptomatic or have mild bleeding tendencies. Peripheral smear may show Dohle-like inclusion bodies in leucocytes. Clonal hematopoietic disorders like myelodysplasia and myeloid leukemia have been reported.

Patients/Materials \& Methods: We report a case of MHA developing Acute Lymphoblastic Leukemia (ALL).To our knowledge, this report is the first describing such occurrence.

Results: Fourteen year old girl presented with intermittent fever lasting for 3 months, and huge splenomegaly. Investigations revealed pancytopenia and peripheral smear showed $15 \%$ blasts, neutropenia, thrombocytopenia. Bone marrow examination showed CALLA + B cell ALL. Molecular work-up was negative for Philadelphia chromosome, ETV6-RUNX1, MLL-AF4, AF9, ENL and E2A-PBX1. Cytogenetic analysis showed normal female karyotype. She was started on treatment as per BFM-95 protocol for leukemia.

At 5-years of age, she was incidentally found to have thrombocytopenia with giant platelets and nephrotic range proteinuria during unrelated illness. Angiotensin Converting Enzyme inhibitors (ACEI) were started for proteinuria. However, there was no evidence of sensorineural deafness, cataract or major bleeds. At 10 years age, genetic confirmation of MHA was done by analysis of MYH9 gene revealing pathogenic heterozygous missense variant in exon 17, c.2152C $>$ T,p.(Arg718Trp). Child remained asymptomatic all this while with occasional epistaxis which was managed symptomatically. Mother and maternal uncle also have May-Hegglin anomaly.

Child achieved leukemia remission after Induction phase chemotherapy and continues to be in remission post Consolidation block chemotherapy. She had direct hyperbilirubinemia with transaminitis during consolidation phase, no cause could be delineated after extensive work-up and she improved clinically. She tolerated chemotherapy well without any major issues.

Discussion \& Conclusion: The occurrence of ALL in MHA maydemonstrate a potential likelihood for a genetic association and needs to be researched further. Once such an association is established, genetic screening in such cases might be prudent.

Acknowledgements: Our sincere thanks to Dr. Surjit Singh (PGI Chandigarh) and Dr. Arvind Bagga (AIIMS) who had contributed to diagnosis of May-Hegglin anomaly in this child.

Disclosure of Interest: None Declared.

Keywords: Acute lymphoblastic Leukaemia, Case report, May Hegglin anomaly.
Abstract 13. Flow Cytometric Standardization of PAX5 For the B-Cell Lineage Assignment in Acute Leukemia

Padmashree Chavan $^{* 1}$, Sitaram Ghogale ${ }^{1}$, Nilesh Deshpande ${ }^{1}$, Yajmanam Badrinath $^{1}$, Prashant Korde ${ }^{1}$, Divashree Jhurani ${ }^{1}$, Gaurav Chatterjee ${ }^{1}$, Nikhil Patkar ${ }^{1}$, Subramaniyan PG $^{1}$, Prashant Tembhare ${ }^{1}$ and Flowcytometry lab, Haematopathology Dept,TMH MUMBAI

\section{${ }^{1}$ Haematopathology Dept, Tata Memorial Centre, Navi Mumbai, India}

Aims \& Objectives: PAX5 is a nuclear protein in the paired-box (PAX) family of transcription factors. It is a pan-B-cell marker expressed from pro-B-cell stage to the early plasma cells. Expression of PAX5 by immunohistochemistry is widely used as a lineage-specific marker for B-cells. However, its expression by multicolor flow-cytometry (MFC) has not been studied in the clinical setting. On the other hand, the assignment of B-lineage on MFC can be complicated in challenging cases as the WHO criteria require multiple additional markers along with CD19. We standardized PAX5 expression by MFC and studied its utility as a B-lineage marker in the lineage-assignment of acute leukemia.

Patients/Materials \& Methods: Anti-PAX5 (PE, clone-REA140) antibody staining was standardized and studied in the blasts from acute leukemia samples using multicolor flow cytometry. We evaluated four permeabilization reagents: FACS Lyse (BD), Fix-\&-Perm (Invitrogen), Foxp3-fixation-kit (eBiosciences) and True-nuclear transcription-factor staining buffer-set (Biolegend). MFC was performed on Cytoflex flow cytometer (Beckman-Coulter) and data was analyzed using Kaluza-v2.1-software.

Results: Of the four permeabilization reagents, Foxp3-fixation-kit (eBiosciences) had shown the best results for the anti-PAX5-antibody expression with titration volume of five-microlitre. PAX5 expression was studied in 21 AML, 25 B-ALL patients and 2 mixed phenotypic acute leukemias (MPAL, B/myeloid). T-cells and normal-B-cells were taken as negative and positive controls with a median (range) MFI of PAX5 1.84 (0.18-1.4) and 5.7 (3.19-17.3), respectively. Median (range) of nMFI of PAX5 in B-ALL and AML patients were 14.7 (3.18-33.1) and -0.62 $((-2.4)-10.8)$. AML patients expressed negative PAX5 $(n M F I<3)$ except one $\mathrm{t}(8 ; 21)$ positive AML, which also showed aberrant CD19 expression. Strong expression of PAX5 (nMFI > 7) was expressed exclusively in B-ALL (17/23 samples, $p<0.001$ ). Median (SD) CV-IF of PAX5 in B-ALL and AML cases were 58.9 (41.7) and 101.5 (131.8) respectively, indicating homogenous expression in B-ALLs $(p=0.048)$. PAX5 was also expressed in two CD10-negative pro-B ALLs and two B/Myeloid MPAL patients.

Discussion \& Conclusion: We first-time standardized flow cytometric evaluation of PAX5 expression in the clinical setting. Foxp3fixation-kit (eBioscience) was the best permeabilization reagent. Flow cytometric PAX5 expression was highly specific for B-lineage ALLs and is a useful additional marker in determining B-cell lineage in acute leukemias.

Disclosure of Interest: None Declared.

Keywords: Acute leukemia, B-ALL, PAX5. 


\section{Abstract 14. MPN with T Lymphoblastic Lymphoma Associated with a Novel Cytogenetic Abnormality: A Case Report}

Dibakar Podder ${ }^{* 1}$, Nilanjan Sinha ${ }^{2}$, Sambit Samanta ${ }^{2}$, Siddhartha Sankar Roy ${ }^{2}$

${ }^{1}$ Institute of Hematology and Transfusion Medicine, Institute of Hematology and Transfusion Medicine, ${ }^{2}$ Institute of Hematology and Transfusion Medicine, Medical College and Hospital, Kolkata, India

Aims \& Objectives: To report a novel cytogenetic abnormality detected while evaluating a patient of $\mathrm{T}$ lymphoblastic lymphoma with a myeloproliferative neoplasm involving bone marrow.

Patients/Materials \& Methods: 48-year old male presented with a history of generalized skin rash involving face, trunk and lower limbs for 7 months and progressive generalized lymphadenopathy and hepatosplenomegaly for last 6 months.

Results: On evaluation complete blood count showed hemoglobin $11.5 \mathrm{~g} / \mathrm{dl}$, total WBC count 49,830/cu.mm, neutrophil 36\%, lymphocyte $3 \%$, monocyte $1 \%$, eosinophil $20 \%$, basophil $9 \%$, band and metamyelocyte $12 \%$, myelocyte $16 \%$, promyelocyte $1 \%$, blast $2 \%$, platelet $532,000 / \mathrm{cu} . \mathrm{mm}$. Bone marrow aspirate showed hypercellular particles with myeloid hyperplasia, increased eosinophils and basophils and increased megakaryocytes with dwarf forms. LAP score was low. Biopsy from skin and right inguinal node showed infiltration with atypical lymphoid cells positive for CD3, CD5, CD4, TdT, CD1a, BCL2, CD43 and CD99. The cells were negative for CD20, CD34, CD30, perforin $\mathrm{AB}, \mathrm{CD} 10, \mathrm{CD} 117$ and MPO. Ki- 67 was $85 \%$. Karyotyping done from bone marrow showed $t(13 ; 20)$ and del 14 . FISH using break apart probe for PDGFRA, PDGFRB, FGFR1 was negative. Molecular tests for bcrabl, Jak2V617F, CALR, MPL were negative.

Discussion \& Conclusion: Myeloid/lymphoid neoplasm with eosinophilia and gene rearrangement encompasses a group of diseases which present with features of myeloproliferative neoplasm and co-existing acute myeloid leukemia, $\mathrm{T}$ or $\mathrm{B}$ lymphoblastic leukemia/lymphoma associated with PDGFRA, PDGFRB, FGFR1 and PCM-JAK2 gene rearrangement. Identifying these group of disease is important as they can be targeted with tyrosine kinase inhibitors or Jak2 inhibitors. Consolidation with a allogenic stem cell transplant can only produce a long disease free survival in this group of patients This is the first case that reported the following cytogenetic abnormalities $t(13 ; 20)$ and del 14 in a case of myeloproliferative neoplasm with $\mathrm{T}$ lymphoblastic lymphoma. Disclosure of Interest: None Declared.

Keywords: Cytogenetic abnormalities, MPN, T lymphoblastic lymphoma.

\section{Abstract 15. Allogeneic Stem Cell Transplantation for Acute Lymphoblastic Leukemia: A Single Centre Experience}

Fouzia Aboobacker $^{* 1}$, Anu Korula ${ }^{1}$, Anup Devasia ${ }^{1}$, Uday Kulkarni', Aby Abraham ${ }^{1}$, Alok Srivastava', Poonkuzhali Balasubramanian ${ }^{1}$, Nancy Beryl ${ }^{1}$, Biju George ${ }^{1}$, Vikram Mathews ${ }^{1}$

\section{${ }^{1}$ Haematology, Christian Medical College, Vellore, India}

Aims \& Objectives: To find out the role of allogeneic stem cell transplantation in the management of both newly diagnosed and relapsed patients with acute lymphoblastic leukemia.

Patients/Materials \& Methods: Data on all patients with ALL who underwent allo-SCT, in the Department of Haematology, Christian
Medical College, Vellore, India from January, 2005 to December, 2018 were included in this analysis.

Results: 191 patients underwent 192 allo-SCT during this period. 145 (75.5\%) were B-cell ALL and 47 (24.5\%) T-cell ALL. Majority were males (66.7\%); median age was 20 (range: 1-62). Ph+ve ALL and ETP ALL constituted $34 \%$ of B and T-cell ALL respectively. 86 $(44.8 \%)$ patients were in CR1, while $99(51.6 \%)$ were in $\geq$ CR2 and $7(3.6 \%)$ had active disease at SCT. Among those $\geq$ CR2, $71(37 \%)$ had early relapse, and $28(14.6 \%)$ had late relapse. Indication for SCT in CR1 was $\mathrm{Ph}+\mathrm{ve}(43 \%)$, high risk disease (22\%), persistent disease/ MRD post induction (21\%) and ETP ALL (14\%). Stem cell source was PBSC in $95.8 \%$ and most had a matched related donor $(56.8 \%)$, $25.5 \%$ had a matched unrelated and $17.7 \%$ had a related haploidentical donor. Myeloablative conditioning regimens used were $\mathrm{Cy} / \mathrm{TBI}$ (76.6\%) or Busulfan based (19.3\%). $104(54.2 \%)$ developed acute GVHD $(20 \%>$ grade 3/4), and 38\% developed chronic GVHD (13.5\% extensive). The day 100 mortality was $17.7 \%$ and TRM at 1 year was 45 (23.4\%). 70 (36.5\%) patients relapsed following SCT at a median of 7 months (range: 1-66). At a median follow up of 11 months (range: $0-136$ ), the 5 year OS and EFS of the whole cohort was $37 \pm 3.8 \% \& 29.2 \pm 4 \%$ respectively. The 5 year EFS of the patients who underwent $\mathrm{SCT}$ in $\mathrm{CR} 1$ versus $\geq \mathrm{CR} 2$ versus 'refractory disease' was $49.1 \pm 6 \%$ vs $21.5 \pm 4.6 \%$ vs $0.0 \pm 0 \%(p=0.008)$ respectively. The $5 \mathrm{yr}$ EFS of early versus late relapse in the $\geq$ CR2 group was $6 \pm 3.2 \%$ vs $62.9 \pm 10.1 \%$ ( $p=0.000)$.

Discussion \& Conclusion: AlIo-SCT is an effective option for high risk disease in CR1 and in late relapses while there is limited benefit in patients with active/refractory disease and in those with early relapse of disease.

Supporting Document: 3a7ec114-287f-423d-9cb2-f95eedfd740b
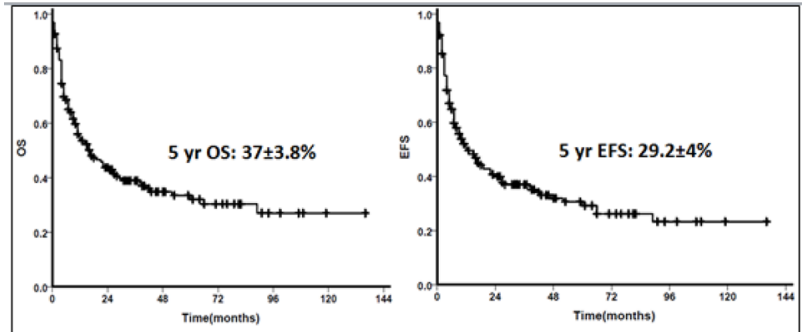

Figure 1. Kaplan-Meier estimate of the 5 year OS and EFS of the whole cohort was $37 \pm 3.8 \% \& 29.2 \pm 4 \%$ respectively, at a median follow up of 11 months (range: 0-136).

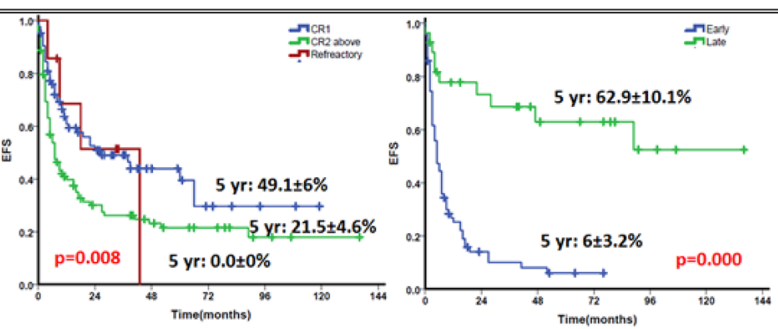

Figure 2. Kaplan-Meier estimate of the 5 year EFS of patients who underwent $S C T$ in CR1 versus $\geq C R 2$ versus 'refractory disease' was $49.1 \pm 6 \%$ vs $21.5 \pm 4.6 \%$ vs $0.0 \pm 0 \%(p=0.008)$ and the 5 year EFS of early versus late relapse in the $\geq C R 2$ group was $6 \pm 3.2 \%$ vs $62.9 \pm 10.1 \%(p=0.000)$. respectively.

Disclosure of Interest: None Declared.

Keywords: Acute lymphoblastic leukemia, allogeneic stem cell transplantation, B cell Acute lymphoblastic leukemia. 
Abstract 16. Role of Leukemic Stem Cells (LSC) in Predicting Outcome in Acute Myeloid Leukemia (AML)

\section{Chinmayee Kakirde ${ }^{* 1}$, Gaurav Chatterjee ${ }^{1}$, Manju Sengar ${ }^{2}$, Prashant Tembhare ${ }^{1}$, P G Subramanian ${ }^{1}$, Nikhil Patkar ${ }^{1}$ \\ ${ }^{1}$ ACTREC, TATA Hospital, Navi Mumbai, ${ }^{2}$ TATA Hospital, Parel, Mumbai, India}

Aims \& Objectives: Leukemic stem cells (LSC) are a largely immunophenotypically uncharacterized heterogenous population of cells which are resistant to chemotherapy and are capable of reinitiating disease in acute myeloid leukemia (AML). In this study, we sought out to establish an immunophenotypic method to characterize CD34+CD38- LSCs both at baseline and during monitoring response, and study their role in predicting outcome.

Patients/Materials \& Methods: Hundred consecutive patients of adult AMLs were evaluated at baseline and post-induction. More than one-million events were stained with a 10-color 2-tube flow cytometry (FCM) panel, acquired on Navios (BC) flow-cytometer. CD34+CD38- LSCs were gated based on CD45dim, SSClow, CD34+, CD38- and aberrant expression of markers (CD45RA, CD90, CD25, CD13, CD117, CD123, HLA-DR, CD32, CD96, CLL-1, CD44, CD47 and TIM-3). Conventional karyotyping and molecular testing were used to risk-stratify patients. The level of LSCs at diagnosis and LSC MRD positivity were analysed for their impact on overall survival (OS) and relapse-free survival (RFS) using log rank test.

Results: The median follow-up of the cohort was 23.8 months. Median LSC level at diagnosis was $0.11 \%(0.005 \%>55.9 \%)$. Based on ROC curve analysis, cases were categorized into LSC-high $(>0.1 \%, \mathrm{n}=55)$ and LSC-low $(<0.1 \%, \mathrm{n}=45)$ at diagnosis. The most useful markers aberrantly expressed in the LSCs were CLL-1 (52\%), CD45RA (48\%), CD44 (32\%), CD117 (26\%) and CD123 (24\%). LSC-high patients had significantly inferior OS and RFS as compared to LSC-low [median OS: 14.9 months (95\% CI: 12-27.53) vs not reached (NR), $P<0.0001$; median RFS: 11.3 months $(95 \%$ CI: 9.2-15.36) vs NR, $P<0.0001]$. Similarly, patients who were LSC-MRD positive at post-induction showed poorer OS and RFS compared to LSC-MRD negative patients [median OS: 15 months (95\% CI: $10.4-17.3)$ vs NR, $P=0.0004$; median RFS: 11.2 months (95\% CI: 9.2-15.5) vs NR, $P=0.0058$ ] (Fig. 1).

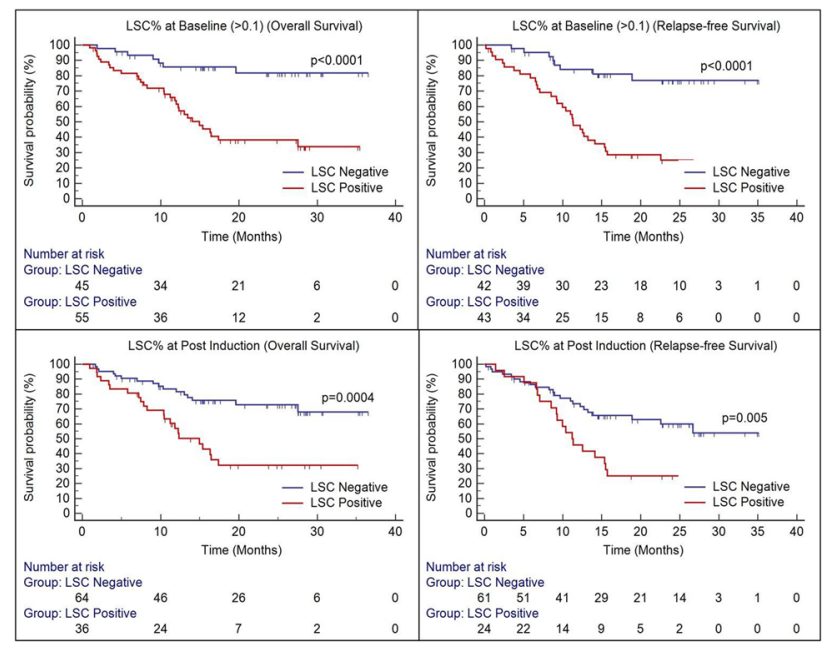

Discussion \& Conclusion: Our study shows that flow cytometric quantitation of LSCs at baseline and LSC-MRD status at post-induction are powerful predictors of outcome in adult AML patients. Integration of these parameters with currently utilized risk factors and MRD evaluation could help in better stratification of patients for proper risk-adapted therapy.

Supporting Document: 2cacd0be-fe47-4c60-928f-04a2a8d8992e. Disclosure of Interest: None Declared.

Keywords: AML, LSC, MRD.

\section{Abstract 17. Frequency of Recurrent Genetic Abnormalities by Karyotyping \& FISH in Acute Lymphoblastic Leukaemia}

\author{
Anamika Bakliwal $^{* 1}$, Rituparna Chetia ${ }^{1}$, \\ Debranjani Chattopadhya ${ }^{1}$, Sudeep Vaniyath ${ }^{1}$, \\ Ashok Kumar Rajoreya ${ }^{1}$, Uttam Kumar Nath ${ }^{1}$ and \\ Department of Clinical Haematology, AIIMS Rishikesh
}

\section{${ }^{1}$ Clinical Hematology, AIIMS Rishikesh, Rishikesh, India}

Aims \& Objectives: The objective of this study is to describe the frequency of recurrent genetic abnormalities as detected by conventional karyotyping and fluorescence-in situ hybridization (FISH) or multiplex PCR techniques, in pediatric \& adult acute lymphoblastic leukemia (ALL) patients treated in Department of Clinical Haematology at AIIMS Rishikesh.

Patients/Materials \& Methods: We analyzed data of total 47 newly diagnosed, treatment-naïve patients of acute lymphoblastic leukemia/lymphoma (ALL/LBL) treated between February 2018 \& July 2019. After confirmation of diagnosis, bone marrow cytogenetics (by G-banded karyotyping) \& peripheral blood FISH/Multiplex PCR for recurrent genetic abnormalities [t(12;21)(p13;q22); $\mathrm{t}(9 ; 22)(\mathrm{q} 34 ; \mathrm{q} 11.2) ; \mathrm{t}(4 ; 11)$ \& other KMT2A rearrangements; \& $\mathrm{t}(1 ; 19)(\mathrm{q} 23$ :p13)] were sent for risk stratification. At least RT-PCR for $B C R-A B L 1$ was sent for all patients who could not afford cytogenetics or FISH study. Pediatric \& adolescent ALL/LBL patients were treated with modified ALL IC-BFM 2009 protocol, and adult ALL/LBL patients were treated with modified Hyper-CVAD/MA protocol. All patients who were $B C R-A B L 1$ positive received Imatinib along with chemotherapy. Tests for other genetic abnormalities (e.g., IKZF1 alterations; CRLF2r \& other markers associated with Ph-like ALL, etc.) could not be done.

Results: Data of 30 pediatric/adolescent patients $(\mathrm{B}-\mathrm{ALL}=22$, $\mathrm{T}-\mathrm{ALL} / \mathrm{LBL}=8) \& 17$ adult patients $(\mathrm{B}-\mathrm{ALL}=12, \mathrm{~T}-\mathrm{ALL} / \mathrm{LBL}=$ $4, \& \mathrm{~B} /$ myeloid $\mathrm{MPAL}=1)$ were analyzed. The commonest abnormality in pediatric age group was $\mathrm{t}(12 ; 21)(\mathrm{p} 13 ; \mathrm{q} 22)$ :ETV6RUNX1 (4/30 patients; 13\%), and that in adult patients was $\mathrm{t}(9 ; 22)(\mathrm{q} 34 ; \mathrm{q} 11.2): B C R-A B L 1 \quad(6 / 17$ patients; 35\%). None of the pediatric/adolescent patients had $B C R-A B L 1$-positive disease. The complete remission rates in the study were $92 \%$ \& $83 \%$ in pediatric \& adult age groups respectively.

Discussion \& Conclusion: Both conventional karyotyping and FISH/ RT-PCR are essential for detection of recurrent genetic abnormalities in ALL/LBL patients, for the purpose of risk stratification, tailoring of treatment intensity, \& prognostication. However, in view of emerging evidence that several other molecular alterations involving $I K Z F 1$, JAK-STAT pathway, etc. may have significant impact on prognosis in ALL, these newer markers should preferably be incorporated in the molecular panel.

Supporting Document: 435fe0d1-e4c5-4feb-9f5d-4aefe4876326

Fig. 1 . 


\begin{tabular}{|c|c|c|c|}
\hline \multicolumn{4}{|c|}{ leukemia patients at AllMS Rishikesh } \\
\hline & Age \& Sex & Karyotype & FISH / Multiplex PCR \\
\hline $\begin{array}{c}\text { Pediatric \& } \\
\text { Adolescent } \\
\text { ALL } \\
(n=30)\end{array}$ & $\begin{array}{l}\text { Median: } 7.5 \text { yrs } \\
\text { (Range: } 2-18 \text { ). } \\
\text { Male }=14 \\
\text { Female }=16\end{array}$ & $\begin{array}{l}\text { Normal karyotype }=18 \\
\text { Hyperdiploidx }=1 \\
46, X Y, \text { del }(5)(q 31 ; q 35)=1 \\
\text { Hypodiploid }=0 \\
\text { Culture failure }=\mathbf{2} \\
\text { Not done }=8\end{array}$ & $\begin{array}{l}\mathrm{t}(12 ; 21)(p 13 ; q 22): E T V 6-R U N X 1=\mathbf{4} \\
\mathrm{t}(9 ; 22)(q 34 ; q 11.2): B C R-A B L 1=0 \\
K M T 2 A \text { rearrangements }[\underline{t}(4 ; 11) \text { or } \\
\text { others] }=0 \\
\mathrm{t}(1 ; 19)(q 23 ; p 13)=0 \\
\text { FISH not done }=\mathbf{6}\end{array}$ \\
\hline & Age \& Sex & Karyotype & FISH / Multiplex PCR \\
\hline $\begin{array}{l}\text { Adult ALL } \\
(n=17)\end{array}$ & $\begin{array}{l}\text { Median: } 28 \text { yrs } \\
\text { (Range: } 19-50) \\
\text { Male }=12 \\
\text { Female }=5\end{array}$ & $\begin{array}{l}t(9 ; 22)(q 34 ; q 11.2)=6 \\
\text { Complex karyotype }=1 \\
\text { Normal karyotype }=5 \\
\text { Hyperdiploidx }=0 \\
\text { Hypodjploidx }=0 \\
\text { Not done/culture failure = } 5\end{array}$ & $\begin{array}{l}\mathrm{t}(9 ; 22)(\mathrm{q} 34 ; \mathrm{q} 11.2): B C R-A B L 1=6 \\
\mathrm{t}(12 ; \underline{21)})(\mathrm{p} 13 ; \mathrm{q} 22): \text { ETV6-RUNX1 }=0 \\
K M T 2 A \text { rearrangements }[\mathrm{t}(4 ; 11) \text { or } \\
\text { others] }=0 \\
\mathrm{t}(1 ; 19)(\mathrm{q} 23 ; \mathrm{p} 13)=0\end{array}$ \\
\hline
\end{tabular}

Disclosure of Interest: None Declared.

Keywords: Acute lymphoblastic Leukaemia, FISH, Karyotype.

\section{Abstract 18. APML Management in a Resource Limited Setting: Real World Scenario}

Prakash Shekhawat ${ }^{* 1}$, Ankit Jitani ${ }^{1}$, Malini Garg ${ }^{1}$, Rajib De ${ }^{1}$, Prakas Mandal $^{1}$, Shuvraneel Baul ${ }^{1}$, Tuphan Dolai ${ }^{1}$

\section{${ }^{1}$ Haematology, NRS Medical College, Kolkata, India}

Aims \& Objectives: To study the clinicopathological profile of APML patients attending the outdoor and their response to treatment. Patients/Materials \& Methods: The data of APML patients attending the outdoor of a tertiary care hospital from October 2009 to June 2019 were analysed. They received ATRA \pm ATO \pm cytoreductive therapy in form of anthracycline depending on their risk stratification. Their presenting symptoms, clinical spectrum and response to treatment was analysed.

Results: 108 patients with suspicion of APML attended the outdoor over this period. Out of these $32.4 \%$ (35/108) patients did not turn up after initial visit due to resource constraints and bed availability. The median age of the patients was 32.1 years with male to female ratio of 1.31:1. Majority of these patients presented with anaemia $(41 \%)$ followed by fever and bleeding in $41 \%$ and $38 \%$ patients, respectively. $42 \%$ of the patients belonged to Intermediate risk, while $34 \%$ were high risk. Low risk was $8 \%$ of the total cohort. The risk stratification of $15 \%$ patients could not be done because of lack of initial records. $10.95 \%$ of patients (8/73) relapsed after completion of therapy. Out of which, $62.5 \%$ patients $(5 / 8)$ belonged to HR category. Male predilection (M: F:: 3:1) was seen in relapsed patients. In $2.7 \%$ of the patients $(2 / 73), \mathrm{t}(11 ; 17) \mathrm{ZBTB} / \mathrm{RARA}$ was detected. 1 of these patients did not respond to ATRA, hence, $3+7$ and azacitidine therapy was given, while the other directly received $3+7$ as induction therapy followed by HiDAC as consolidation. $2.7 \%$ patients $(2 / 73)$ developed extrapulmonary tuberculosis warranting treatment interruptions.

Discussion \& Conclusion: Relapse was more common in male and majority belonged to High risk category. An association of extrapulmonary tuberculosis in APML needs to be kept in mind and needs further studies.In spite ofbeing the most aggressive and curable disease, $32.4 \%$ patients didn't get treatment in view of resource constraints and bed availability.

Disclosure of Interest: None Declared.

Keywords: APML, ATO, PML RARA.
Abstract 19. Efficacy of Low Dose Rasburicase in Management of TLS in Leukaemia and Lymphoma Patients

Gopila Gupta*1, Tulika Seth1, Manoranjan Mahapatra1, Sudip Kumar Datta ${ }^{2}$, Renu Saxena ${ }^{1}$

\section{${ }^{1}$ Hematology, ${ }^{2}$ Laboratory Medicine, AIIMS, New Delhi, India}

Aims \& Objectives: Rasburicase is indicated for prevention and treatment of Tumor lysis syndrome (TLS). The recommended dose is $0.2 \mathrm{mg} / \mathrm{kg}$ for 5 days. However, the cost is prohibitive for most patients in our setting. Aim of our study was to determine the efficacy of low dose rasburicase in prevention and treatment of TLS.

Patients/Materials \& Methods: This was a prospective single arm study conducted at department of Hematology, AIIMS, New Delhi from March 2018 to June 2019. Patients of leukemia/lymphoma with lab/clinical TLS or presence of $\geq 2$ high risk factors (WBC $>50,000 /$ $\mathrm{mm}^{3}, \mathrm{LDH}>2 \mathrm{ULN}$, creatinine $>1.5 \mathrm{mg} / \mathrm{dL}$, uric acid $>7.5 \mathrm{mg} / \mathrm{dl}$ ) were included. Patients were grouped into 3 weight bands $(0.05 \mathrm{mg} / \mathrm{kg}$ dose rounded to nearest $1.5 \mathrm{mg}): 1.5 \mathrm{mg}(<30 \mathrm{~kg}), 3 \mathrm{mg}(>30-60 \mathrm{~kg})$ \& $4.5 \mathrm{mg}(>60-90 \mathrm{~kg})$. Primary end point was uric acid normalization $(<7.5 \mathrm{mg} / \mathrm{dl})$ within $24 \mathrm{~h}$ of rasburicase administration. Treatment failure was defined as inability to normalize uric acid level within $24 \mathrm{~h}$ or requirement of additional dose within $24 \mathrm{~h}$.

Results: 55 patients were recruited for this study. The median age was 20 years (3-66 years), majority were males $(72.7 \%)$, pediatric patients $(<18$ years) accounted for $43.6 \%(n=24)$ cases. Rasburicase was used prophylactically in $43(78.2 \%)$ and for treating laboratory/clinical TLS in $12(21.8 \%)$ patients. Mean serum uric acid levels at baseline and at $24 \mathrm{~h}$ were $9.19 \pm 1.81 \mathrm{mg} / \mathrm{dl}$ and $3.19 \pm 2.12 \mathrm{mg} / \mathrm{dl}$ respectively (Fig. 1). There was significant reduction in the serum uric acid and creatinine levels ( $p$ value $<0.001$ ) from baseline to $24 \mathrm{~h}$ post rasburicase administration. The response was maintained during follow-up period till $72 \mathrm{~h}$. Single dose of rasburicase as per weight bands was effective in $94.5 \%$ of patients. Failure rate was $4.6 \%$ in prophylactic group and $9.09 \%$ in treatment group. On cost analysis single low dose rasburicase was found to decrease the cost by more than 10 times.

Discussion \& Conclusion: We conclude that single dose rasburicase with frequent laboratory monitoring is effective in prevention and treatment of TLS with significant reduction in financial toxicity.

Supporting Document: b208d383-d8e6-4ac0-ab91-719634496529.

Disclosure of Interest: None Declared.

Keywords: leukemia, SERUM URIC ACID, TUMOR LYSIS SYNDROME.

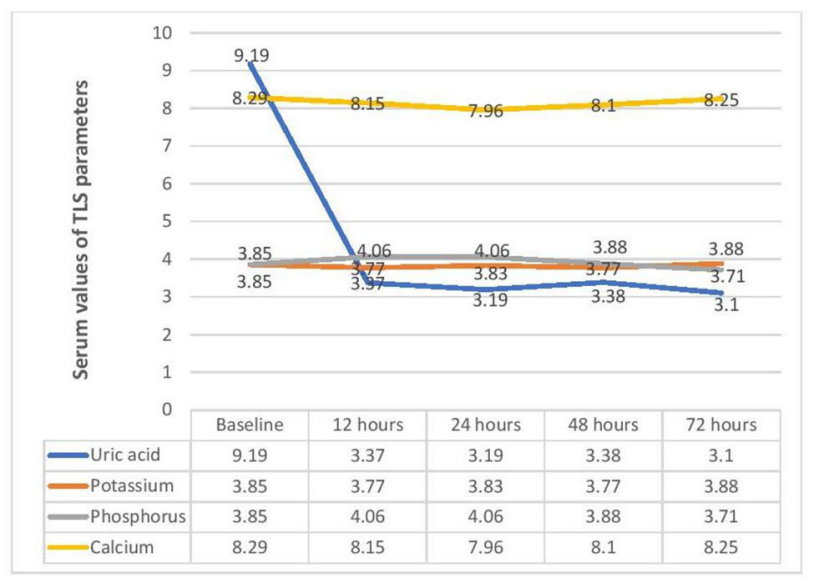

Fig. 1 Tumor lysis laboratory values from baseline to $72 \mathrm{~h}$ rasburicase administration in the study population 


\section{Abstract 20. CNS Complications in Acute Lymphoblastic Leukemia: A Tertiary Carecentre Experience from North India}

\section{Faheema Hasan ${ }^{* 1}$, Anshul Gupta ${ }^{1}$, Sanjeev Yadav ${ }^{1}$, Ruchi Gupta $^{1}$, Khaliqur Rahman ${ }^{1}$, Dinesh Chandra ${ }^{1}$, Manish SINGH ${ }^{1}$, Soniya Nityanand ${ }^{1}$ \\ ${ }^{1}$ Department of Hematology, SGPGI, Lucknow, India}

Aims \& Objectives: CNS complications during ALL treatment remain one of the most challenging issues faced by practicing hematologists. The aim of this study was to analyze the frequency, clinical characteristics and risk factors for CNS complications in ALL patients who received treated at our centre.

Patients/Materials \& Methods: It a retrospective study of all ALL patients who developed CNS complications during ALL therapy and were admitted in the Department of Haematology between 1st August 2018-31st July 2019. All patients were treated with Berlin-FrankfurtMunster (BFM) - ALL 95 chemotherapy protocol and were risk stratified accordingly. The following clinical data was retrieved from medical records: demographic profile, clinical details, baseline disease characteristics like complete blood count, immunophenotype, metabolic profile including liver \& kidney function tests along with CT scan/MRI and lumbar puncture reports.

Results: Of 123 patients who sought treatment during the study period, twenty one (17.0\%) suffered CNS complications. The most common complication in our patients was intracranial hemorrhage $(n=9 ; 42.8 \%)$, followed by posterior reversible encephalopathy syndrome $(\mathrm{n}=5$; $23.8 \%)$, CNS disease $(n=4 ; 19.04 \%)$, fungal abscess $(n=1 ; 4.76 \%)$, cortical venous thrombosis $(n=1 ; 4.76 \%)$ methotrexate induced leukoencephalopathy $(\mathrm{n}=1 ; 4.76 \%)$. Headache was the most common clinical presentation univerally present in all patients followed by seizures present in more than $90 \%$ patients. In the univariate analysis of the risk factors associated with CNS event, platelet count less than $30,000 / \mu 1$ was significantly associated with CNS bleed $(P$ value $=<0.05)$. Additionally, $\mathrm{T}$ immunophenotype as well as White Blood Cell (WBC) count $>$ $1,00,000 / \mu \mathrm{l}$ was significantly associated with CNS disease at presentation. Discussion \& Conclusion: CNS events are not an uncommon complication during ALL therapy and require a high index of suspicion for early diagnosis and management. Aggressive platelet transfusion support is needed in patients presenting with low platelet counts $(<30,000 / \mu \mathrm{l})$ at diagnosis as intracranial bleed during treatment carries a very bad prognosis. A strict collaboration between the haematologist, neurologist and neuro-radiologist is essential for optimal management of CNS complications during ALL therapy.

Disclosure of Interest: None Declared.

Keywords: Acute lymphoblastic leukemia, CNS complications, Intracranial hemorrhage.

\section{Abstract 21. RUNX1-RUNX1T1 Fusion Transcripts or $\mathbf{T}(8 ; 21)$ in Acute Myeloid Leukemia; Single Center Experience}

Avriti Baveja $^{* 1}$, Shuvraneel Baul ${ }^{1}$, T.k Dolai ${ }^{1}$, Shyamali Dutta ${ }^{1}$, R De $^{1}$, P.k Mandal ${ }^{1}$, S Bhattacharya ${ }^{1}$, I Mandal ${ }^{1}$

\section{${ }^{1}$ Hematology, NRSMCH, Kolkata, India}

Aims \& Objectives: To study the clinical and pathological profile of the patients of AML with $\mathrm{t}(8 ; 21)$.

- To study the outcome of $\mathrm{t}(8 ; 21)$ and effect of partners abnormalities on outcome.
Patients/Materials \& Methods: Study was conducted on 28 patients with newly diagnosed AML with $\mathrm{t}(8 ; 21)$ attending the haematology department of NRSMCH, Kolkata from January, 2015 to July, 2019. The patients clinical profile, immunophenotype, molecular markers and cytogenetics at baseline were collected and the outcomes with 3 plus 7 with Anthracycline and Cytarabine based induction followed by 3 cycles of cytarabine consolidation were assessed.

Results: $\mathrm{t}(8 ; 21)$ present in $11.2 \%(28)$ of all AML patients (248) attending hematology department. Amongst the patients with $\mathrm{t}(8 ; 21)$ by cytogenetics or RUNX1-RUNX1T1 fusion transcripts by RT-PCR, $64.24 \%$ (18) males, $35.7 \%$ (10) were $\leq 18$ years and $57.1 \%$ (16) between 18-60 years of age with median age of presentation 26.5 years. $35.7 \%$ (10) had myeloid sarcomas. Auer rods with large abundant granules present in $71.4 \%$ (20). Aberrant CD19 expression seen. Aberrant CD19 expression and c-Kit positively had a strong correlation ( $p$ value $<0.05$ ). Additional Cytogenetic abnormality $-\mathrm{Y},-\mathrm{X}$ and $9 \mathrm{q}$ deletion in $17.8 \%$ (5), $10.7 \%$ (3) and $3.57 \%$ (1) respectively. Those treated with intensive therapy, all patients achieved end of induction remission and among those who completed consolidation, 50\% patients faced early relapse. $t(8: 21)$ had early relapse and poor progression free survival in both c-kit positive and c-kit negative cases $(p>0.05)$.

Discussion \& Conclusion: $\mathrm{t}(8 ; 21)$ in $11.2 \%$ of AML cases with male predominance, early age of presentation. CD19 expression and c-Kit positivity, a common partner with this translocation. CD19 aberrant expression show strong association with c-Kit positivity. Early relapse and decreased progression free survival irrespective of c-kit status were observed. The way the $t(8 ; 21)$ is behaving in our study group, enhances on the importance of revision of the risk stratification for AML and for considering other therapeutic options.

Disclosure of Interest: None Declared.

Keywords: Acute myeloid leukemia, RUNX1-RUNX1T1, t(8;21).

\section{Abstract 22. 3G/M2 Versus 1.5G/M2 Cytarabine Consolidation in AML in a Tertiary Care Centre of Eastern India}

Avriti Baveja ${ }^{* 1}$, Tuphan Dolai ${ }^{1}$, Rajib De ${ }^{1}$, Prakas Mandal ${ }^{1}$, Subham Bhattacharya ${ }^{1}$, Sumit Mitra ${ }^{1}$, Shuvraneel Baul ${ }^{1}$, Shyamali Dutta ${ }^{1}$

\section{${ }^{1}$ Hematology, NRSMCH, Kolkata, India}

Aims \& Objectives: Acute myeloid leukemia (AML) has variable outcome depending upon risk stratification, performance status and therapy opted. CALGB study show $1.5 \mathrm{~g} / \mathrm{m}^{2}$ cytarabine used for consolidation has similar overall survival rates and leukaemia free survival as $3 \mathrm{~g} / \mathrm{m}^{2}$ cytarabine while achieving a better side effect profile. This study aims to assess the overall outcome in patients of Acute myeloid leukemia with cytarabine consolidation in different doses following induction therapy.

Patients/Materials \& Methods: Retrospective study on patients with newly diagnosed AML attending the haematology department of NRSMCH, Kolkata with study period January, 2017 to July, 2019. Baseline characteristics were recorded and after induction remission with $3+7$ daunorubicin and cytarabine those in remission received 3 cycles of consolidation with Cytarabine $\left(3 \mathrm{~g} / \mathrm{m}^{2} /\right.$ day)on day 1,3 and 5 for patients enrolled between January, 2017-December, 2017 and Cytarabine $\left(1.5 \mathrm{~g} / \mathrm{m}^{2} /\right.$ day $)$ on day $1,3,5$ for patients enrolled from January, 2018-July, 2019 and subsequently the data were analysed. Results: Amongst 88 patients with AML, 54 (61.34\%) were males with $14(15.9 \%)$ patients with age less than 18 years and median age of presentation was 41 years. Risk stratification revealed 10 (11.4\%), 69 (78.4\%), 9 (10.2\%) in Poor risk, Intermediate and Good risk group respectively. Figure 1, shows results in detail. $56.3 \%$ patient of patients on consolidation relapsed within one year of therapy. In all risk groups, 


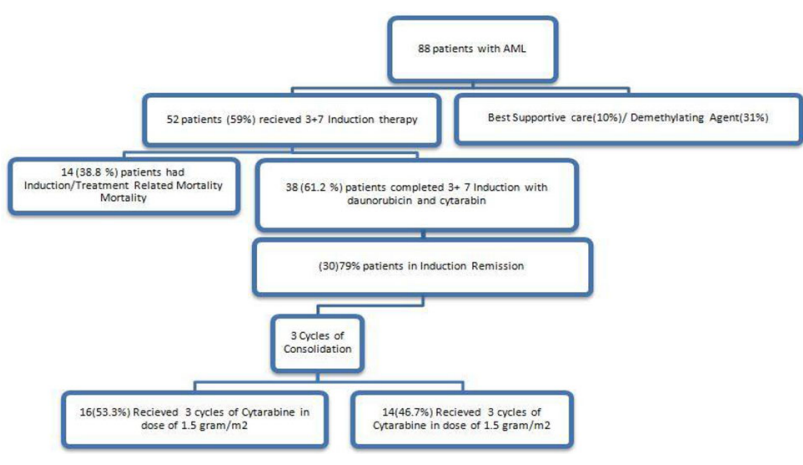

Fig. 1 Consort diagram

toxicity profile in the $3 \mathrm{~g} / \mathrm{m}^{2}$ was higher in terms of febrile neutropenia and duration of hospitalisation ( $p$ value $\leq 0.05$ ). Dose of $1.5 \mathrm{~g} / \mathrm{m}^{2}$ had decreased progression free survival $(p$ value $\leq 0.05)$ with median duration of relapse 3.5 months in comparison to $3 \mathrm{~g} / \mathrm{m}^{2}$ cohort.

Discussion \& Conclusion: In this study, high rates of early relapse and decreased progression free survival were observed in the group receiving $1.5 \mathrm{~g} / \mathrm{m}^{2} /$ day. Febrile Neutropenia and duration of hospital stay was more in the $3 \mathrm{~g} / \mathrm{m}^{2}$ (HiDAC) but with no significant difference in mortality. This study highlights on the importance of consideration of higher consolidation doses.

Supporting Document: 475fc021-ae8b-471c-9ab3-0db373ed5260.

Disclosure of Interest: None Declared.

Keywords: Acute myeloid leukemia, Consolidation, Cytarabine.

\section{Abstract 23. Outcomes in Newly Diagnosed Acute Myeloid Leukemia Patients: A Single-Center Experience}

\section{Debranjani Chattopadhyay ${ }^{* 1}$, Sudeep Vaniyath ${ }^{1}$, Ashok Rajoreya ${ }^{1}$, Rituparna Chetia ${ }^{1}$, Anamika Bakliwal ${ }^{1}$, Uttam Kumar Nath ${ }^{1}$}

\section{${ }^{1}$ Haematology, Aiims Rishikesh, Rishikesh, India}

Aims \& Objectives: Acute myeloid leukemia (AML) is the commonest subtype of acute leukemia in adults. Our study describes the overall outcomes in newly diagnosed, non-elderly AML patients of Uttarakhand \& western Uttar Pradesh, who attended Department of Clinical Haematology at AIIMS Rishikesh.

Patients/Materials \& Methods: We analyzed data of total 23 nonM3 AML patients $<60$ years of age, diagnosed at our hospital between January 2018 \& July 2019. Pediatric AML patients ( $<12$ years), elderly AML patients ( $>60$ years) and patients deemed unfit for any definitive therapy due to significant comorbidity were excluded from analysis. Diagnosis was confirmed by peripheral blood \& bone marrow morphology, and flowcytometry. All treatment-eligible patients were counselled for standard $3+7$ induction followed by high-dose cytarabine (HiDAC) consolidation. At least decitabine therapy was offered to patients who were not motivated for intensive therapy. Those who were not willing for even decitabine therapy were started on palliative hydroxyurea \& supportive care. Bone marrow was assessed for morphological complete remission (CR) after 3+7 induction or after 2 cycles of decitabine therapy.

Results: Median age of patients was 29 years (range 18-60 years). Male:female ratio was 1.3:1. In spite of repeated positive counselling, nine out of 23 patients (39\%) were not willing to undergo any definitive therapy, nine patients $(39 \%)$ opted for less intensive (decitabine) therapy \& only five patients $(22 \%)$ were motivated to start
$3+7$ induction chemotherapy followed by HiDAC consolidation. Median number of decitabine cycles administered was 4 (range 1-6 cycles). Morphological CR rates in $3+7$ arm $\&$ decitabine arm were $40 \%$ \& 50\% respectively. Two patients in $3+7$ induction died of sepsis and one patient discontinued treatment midway in induction. Two patients discontinued decitabine therapy after $1^{\text {st }}$ cycle, one died of sepsis during $1^{\text {st }}$ cycle, $\&$ another died of refractory disease after 2 cycles.

Discussion \& Conclusion: Our short-term treatment results in nonM3 AML patients of Uttarakhand \& western U.P. shows overall poor outcomes due to the high incidence of treatment non-initiation, lack of motivation for standard intensive chemotherapy due to poor socioeconomic status, poor adherence to therapy, and high treatment drop-out rates in patients undergoing definitive treatment.

Disclosure of Interest: None Declared.

Keywords: Acute myeloid leukemia, Decitabine, Induction, Consolidation.

\section{Abstract 24. Assessment of Nutritional Status in Patients with Acute Leukemia During Induction Chemotherapy}

\author{
Steve Thomas A ${ }^{* 1}$, Manoranjan Mahapatra ${ }^{1}$, Jyotsna Viveka. $P^{2}$, \\ Laksmy $\mathbf{R}^{3}$
}

${ }^{1}$ Clinical Hematology, ${ }^{2}$ Endocrinology, ${ }^{3}$ Cardiac Biochemistry, AIIMS, Delhi, India

Aims \& Objectives: Assessment of Nutritional status/nutritional parameters in patients with Acute Leukemia in the age group of 2 to 20 years during induction chemotherapy. To correlate the nutritional status with morbidity, toxicity and mortality.

Patients/Materials \& Methods: Nutritional status was assessed by BMI for age ( $\mathrm{Z}$ score, Percentile) at 0,2 and 4 weeks. BMI percentile $<5$ were classified as underweight, $5-85$ percentile as healthy weight, $85-95$ percentile as risk of overweight and $>95$ as overweight. Biochemical nutritional parameters such as Serum Albumin, Folate, Vitamin B12, Calcium, Phosphate, Serum Iron, Serum Ferritin, TIBC, UIBC and vitamin D levels were also assessed at 0,2 and 4 weeks.

Results: Out of 64 patients analyzed 45\% (29/64) of our patients were underweight at admission. BMI percentiles of patients continued to decrease at 0,2 and 4 weeks [ $45 \%$ vs $57 \%$ Vs $60 \%$ ] indicating worsening malnutrition ( $p$ value 0.0002 ). Underweight patients had significantly higher morbidities (Pneumonia, NEC, Sepsis) when compared to healthy weight patients $[65.52 \%$ Vs $39.39 \%$, p value0.043]. Similarly, underweight patients had more interruption/dose reduction of chemotherapy when compared to healthy weight $[27.9 \%$ vs $6.06 \%$, p value 0.049]. Low Albumin $(<3.5 \mathrm{gm} / \mathrm{dl})$ at admission, was seen in $20 \%$ of patients which correlated well with mortality ( $p$ 0.0001). There was increased mortality $31 \%$ in the patients with undernutrition when compared to mortality of $12 \%$ in patients with healthy weight $(p 0.140)$. There was no correlation between other nutritional parameters with toxicity, morbidity and mortality. Vitamin D insufficiency $(<30 \mathrm{ng} / \mathrm{ml})$ was seen in $95 \%$ of patients and DEXA scan done showed Osteopenia (Z score-1 to $-2 \mathrm{SD}$ ) and Osteoporosis (Z-Score $<-2.5 \mathrm{SD}$ ) in $75 \%$ of recruited patients.

Discussion \& Conclusion: Undernourished patients have a significant tendency for a greater morbidity and required more dose interruption/dose reduction of chemotherapy. However, there was no correlation between undernutrition and mortality. There is a need for complete evaluation of nutritional status for patients with acute leukemia at admission, and the role of supplemental nutrition needs to studied in larger prospective trials.

Supporting Document: 6775ea70-add7-4dd0-a6 cd-8ee84e0b905d. 


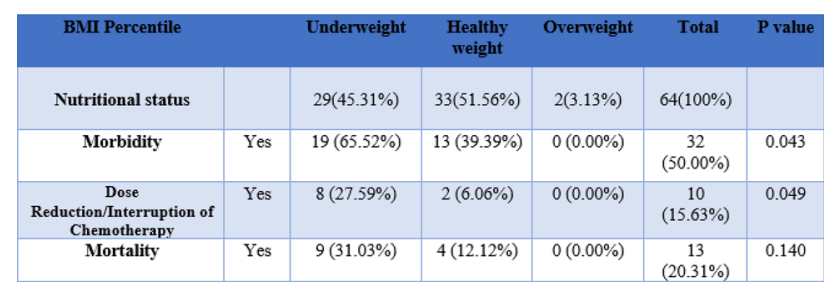

Disclosure of Interest: None Declared.

Keywords: Acute leukemia, Morbidity, undernutrition.

\section{Abstract 25. Unweaving the Tangle of AML in Pregnancy}

Neha Ganju ${ }^{* 1}$, Mukul Agarwal ${ }^{1}$, Manoranjan Mahapatra', Rishi Dhawan ${ }^{1}$

\section{${ }^{1}$ Hematology, All India Institute of Medical Sciences, Delhi, India}

Aims \& Objectives: To study maternal and fetal outcomes in pregnant women with AML.

Patients/Materials \& Methods: We analyze and present description of 7 cases of AML diagnosed during pregnancy who presented to our department from 2017 to 2019.

Results: Amongst 7 patients, two presented in first trimester, four in second trimester and 1 in third trimester. Median age of presentation was 23 years. One patient was diagnosed to have APML and rest all AML. They were risk stratified according to cytogenetics and molecular mutations. Two patients were favourable risk, three were poor risk, for one risk stratification could not be done. APML patient had low risk disease. Two patients who presented within 20 weeks of gestation underwent medical termination of pregnancy and then underwent chemotherapy. The patient with APML had a missed abortion. One patient who presented near term, underwent LSCS and delivered a healthy child. Two patients who presented between 25 and 30 weeks, received chemotherapy first followed by termination after 32 weeks. One of the patients died after few days of admission. 2 patients received decitabine based therapy while awaiting delivery, after which chemotherapy was given. None of the fetuses exposed to chemotherapy in utero have any congenital malformations at birth. Out of all 7 patients, 3 pdied and remaining 4 are in remission. za.

Discussion \& Conclusion: AML management in pregnancy requires a multi-disciplinary team comprising hematologist, obstetrician, neonatologist and genetic counsellor. AML treatment comprising chemotherapy can be associated with adverse effects on both fetus and mother, while delaying treatment till delivery can be detrimental. In India, many patients register for pregnancy only after 3-4 months of gestation, when the window of therapeutic abortion $(<20$ weeks $)$ is limited. After 28 weeks, more than $90 \%$ successful delivery rates are observed with acceptable fetal outcomes. The period between 20-28 weeks is especially challenging while making treatment decisions regarding pregnancy and chemotherapy institution and needs extensive interaction between physicians and family members.

CONCLUSION: Management of AML during pregnancy involves multi-disciplinary approach, tough decisions regarding early therapy, termination of pregnancy, fetal outcomes etc. An extensive discussion between the medical team and family members is of paramount importance.

Disclosure of Interest: None Declared.

Keywords: AML, chemotherapy, PREGNANCY.

\section{Abstract 26. Molecular Profile in Denovo/Relapsed Acute Myeloid Leukemia Using Targeted Gene Sequencing}

\section{Stitha Pragna Gudala*1, Mukul AGgARWAL ${ }^{1}$, Sahitya DSK ${ }^{2}$, Manoranjan MAHAPATRA ${ }^{2}$, Rishi DHAWAN ${ }^{2}$, Tulika Seth ${ }^{2}$ and AIIMS}

\section{${ }^{1}$ CLINICAL HEMATOLOGY, ${ }^{2}$ AIIMS, NEW DELHI, India}

Aims \& Objectives: Introduction: Acute Myeloid Leukemia (AML) is a heterogenous clonal hematopoietic progenitor malignancy. The detection of various mutations in AML has led to better risk stratification, prognostication, treatment and response assessment.

Patients/Materials \& Methods: We analyzed the genetic profile of AML patients (de novo/relapsed) attending a tertiary care center from March 2019 to August 2019. The method used for detection of mutations was targeted Next Generation Sequencing.

Results: Twenty patients were included in the study. The median age was 35.5 years. Male to female ratio is $1.2: 1$. Majority $(80 \%)$ of our patients had normal cytogenetic profile. Two patients (10\%) had complex cytogenetics, one patient $(5 \%)$ had $\mathrm{t}(8 ; 21)$ and another patient $(5 \%)$ patient had $\mathrm{t}(9 ; 11)$. The profile of genetic mutations in our study were as followed: gene encoding nucleophosmin 1 (NPM1) 35\%, tumor-suppressor genes (TP53,PHF6) 10\%,DNA-methylation-related genes (IDH2,DNMT3A) 30\%, signaling genes (FLT3-ITD/TKD,NRAS,KRAS,KIT) $\quad 40 \%, \quad$ chromatin-modifying genes (ASXL1,KDM6A,MPL) 15\%, myeloid transcription-factor genes (RUNX1,CEBPA) 10\%, cohesion-complex genes (SMC3) 5\% and spliceosomes-complex genes (STAG2) 5\%. The proportion of patients with favorable, intermediate, adverse risk were $20 \%, 45 \%$ and $35 \%$ respectively. After the molecular profiling three out of nine patients who were initially stratified into intermediate risk were re-stratified into adverse risk group. Similarly, out of seven patients who had adverse risk cytogenetics three were re-stratified into favorable risk and two into intermediate risk according to ELN risk stratification.

Discussion \& Conclusion: Molecular profiling provides important information in patients with AML particularly those with a normal cytogenetic karyotype, which forms the largest cytogenetic subset. Without the ability to further classify them, most generally fall into an intermediate risk group however these patients have variable outcomes with conventional treatment strategies which may be explained by the underlying molecular heterogenicity associated with the disease. Identification of these mutations serves as a platform for the development of novel therapeutic inhibitors and targeted therapy resulting in better outcomes compared to standard chemotherapy.

Disclosure of Interest: None Declared.

Keywords: AML, CYTOGENTICS, MUTATIONS.

\section{Abstract 27. STEMI: An Unusual Presentation of Acute Myeloid Leukemia}

Harish V. Kasarabada ${ }^{* 1}$, Kundan mishra ${ }^{2}$, Yanamandra Uday ${ }^{3}$, Suman K PRAMANIK ${ }^{3}$, Ankur AHUJA ${ }^{4}$ and ARMY HOSPITAL (RESEARCH AND REFERRAL)

\section{${ }^{1}$ Internal Medicine, ${ }^{2}$ Haematology, ${ }^{3}$ AHRR, Delhi, India, ${ }^{4}$ Haematopathology, AHRR, Delhi, India}

Aims \& Objectives: This case study is done to show a very rare and unusual presentation of Acute Myeloid Leukemia as STEMI.

Patients/Materials \& Methods: A 30-year-old previously healthy male presented with acute onset retrosternal pain of $4 \mathrm{~h}$ duration. On evaluation, ECG showed ST elevation in leads II, III, and aVF and 
troponin-I were positive. He was diagnosed as a case of Inferior wall STEMI, and he was thrombolysed with tenecteplase. He had successful thrombolysis as he became pain-free and repeated ECG showed normal ST segment. Subsequently, he had leukocytosis (WBC $28,000 / \mu \mathrm{L}$ ), and his peripheral blood smear showed $80 \%$ blasts. A bone marrow study, including flow cytometry and the cytogenetic study, confirmed the diagnosis as acute myeloid leukemia (cytogenetically normal). He was started on induction $(7+3)$ chemotherapy.

Results: We present a rare case of AML with initial presentation as STEMI. The index case emphasizes that prompt and proactive management results in patient diagnosis and saves a precious life.

Discussion \& Conclusion: AML is known to be associated with and thrombotic events, particularly the M3 and M4 variety. The mechanism is multifactorial and attributed to disseminated intravascular coagulation (DIC), hyper-leukostasis and injury to the blood vessels as well as the drugs. However, AML presenting with STEMI is very rare. There are only a handful of case reports, and it is difficult to estimate the exact prognostic significance. However, in most of the reported cases, it has been associated with poor prognosis.

Disclosure of Interest: None Declared.

Keywords: Acute leukemia, AML M3, STEMI.

\section{Abstract 28. Clinical Profile and Outcomes in FLT3 ITD Positive AML Patients in Resource Constraint Setting}

Kapil L. Barbind ${ }^{* 1}$, Kundan mishra ${ }^{2}$, Yanamandra Uday ${ }^{2}$, suman K Pramanik ${ }^{2}$, Rajan kapoor ${ }^{2}$, Ankur Ahuja ${ }^{3}$

${ }^{1}$ Dept of Medicine, ${ }^{2}$ Dept of Clinical Hematology, ${ }^{3}$ Dept of Hematopathology, Army Hospital, R \& R, Delhi, India

Aims \& Objectives: This Prospective data was carried out with an aim to look into the incidence, clinical profile, response to therapy and outcomes of FLT3 ITD positive patients of AML.

Patients/Materials \& Methods: In this prospective observational study, patients with confirmed AML were enrolled from 01 July 2018 to 30 June 2019. After history taking, a detailed clinical examination was done and recorded. These patients were also evaluated for biochemical parameters, cytogenetics, and mutations. All patients were treated with $7+3$ induction chemotherapy. The patients on follow up were also evaluated for the overall response (CR; complete remission, $\mathrm{CRi}$; complete remission without count recovery). The outcomes were recorded in terms of survival and death.

Results: Total of 49 new patients of AML were diagnosed during this period. The FLT3 ITD positive AML were 16 (32\%). Seven $(43.7 \%)$ were male, and nine (56.3) were female. The Median age of the study population was 54.5 years (34-76 years) In males median age was 53 years (36-76 years) while in females median age was 57 (34-71 years)

Only six of the sixteen $(37.5 \%)$ AML FLT3 patients were treated with $7+3$ regimen. Three of the six patients $(50 \%)$ had complete remission documented by day 30 bone marrow. The median follows up was seven months (3-12 months). The common cause of death was disease progression $(6 / 16,37.5 \%)$ and infection $(2 / 16,12.5 \%)$.

Discussion \& Conclusion: Though this study has a small number and short follow up, it highlights the poor prognosis in FLT3ITD +ve AML patients with conventional chemotherapy in resource constraints setting.

Disclosure of Interest: None Declared.

Keywords: AML, FLT3, ITD.
Acute Leukemia (Laboratory)

\section{Abstract 1. An Audit of the Flowcytometry Laboratory for Immunophenotyping in CSF and Other Body Fluids}

SubhajitBrahma $^{* 1}$, SambhunathBanerjee ${ }^{2}$, Gautam Bhagwat ${ }^{1}$, RishuVidhatri ${ }^{2}$, RakaHota ${ }^{2}$, Sekhar Krishnan ${ }^{3}$, Reena Nair ${ }^{3}$, Saurabh Bhave ${ }^{3}$, MammenChnady ${ }^{3}$, Neeraj Arora ${ }^{2}$, Deepak Mishra ${ }^{2}$

${ }^{1}$ haematopathology, TATAMEDICAL CENTER, Kolkata, ${ }^{2}$ Haematopathology, ${ }^{3}$ Clinical Haematology, Tata Medical Center, Kolkata, Kolkata, India

Aims \& Objectives: Flow cytometric immunophenotyping (FCI) is recommended in the evaluation of cerebrospinal fluid (CSF) specimens for hematologic neoplasm even when the cellularity is low.It has demonstrated to have high sensitivity and specificity for the detection of lymphoma and leukemia.

Patients/Materials \& Methods: In this study, we reviewed and audited our flowcytometry laboratory in evaluating body fluid specimens. We retrospectively analyzed immunophenotypic data from consecutive cases referred to our laboratory from January 2015 to July 2019. These cases were diagnosed based on immunophenotyping and cytomorphology as per WHO 2016 guideline. Most samples in the laboratory are collected in EDTA vials and reach the laboratory within $2 \mathrm{~h}$.

Results: Of the total 150 fluid samples received, 92 (61\%), were CSF, $43(28 \%)$, pleural fluids 13 Peritoneal fluids $(8 \%)$, two vitreous fluid (2\%) and a single Ocular fluids samples (1\%).Of the total 150 fluid samples processed abnormal cells were detected in $59 / 150(39.3 \%)$ samples processed. The included 13 BCP-ALL, 13 T-ALL, 4 AML, 19 B-CLPD, 4 Burkitt's lymphoma, one case each of ALCL, BPDCN, Multiple myeloma and Mantle cell lymphoma. In 91 (59\%) specimens normal $\mathrm{B} / \mathrm{T}$ cells were found and in $2(2 \%)$ samples no viable events could be detected. Mean WBC count of the positive specimen was 2684 cells $/ \mu 1$ however in 12 of the positive cases the counts were very low count Fluid samples $(<10$ cells $/ \mu 1)$ and the abnormality could be picked up only on flowcytometry.

Discussion \& Conclusion: Flow cytometric testing has documented value in the assessment of body fluids specimens for hematologic neoplasms. Definitive diagnostic information can be obtained from even very-low-cellularity specimens.

Disclosure of Interest: None Declared.

Keywords: Body fluid, CSF, Immunophenotyping.

\section{Abstract 2. Clinical Significance of End Induction MRD Monitoring in B Cell ALL: A Single Centre Experience}

Arun Kumar Arunachalam ${ }^{* 1}$, KotteswariKathirvel ${ }^{1}$, FaranazKamruddin $^{1}$, Anup Devasia ${ }^{1}$, Uday Kulkarni ${ }^{1}$, Fouzia NA ${ }^{1}$, Aby Abraham ${ }^{1}$, Nancy Janet ${ }^{1}$,

PoonkuzhaliBalasubramanian ${ }^{1}$, Biju George ${ }^{1}$, Vikram Mathews ${ }^{1}$

${ }^{1}$ Department of Haematology, Christian Medical College, Vellore, India

Aims \& Objectives: Assessment of minimal residual disease (MRD) has emerged as a powerful prognostic tool in both paediatric and adult acute lymphoblastic leukemia (ALL). Flow cytometry is the most widely used methodology for MRD assessment in ALL due to its wide applicability, shorter turnaround time and a sensitivity comparable to Real-Time PCR $\left(10^{-4}\right.$ to $\left.10^{-5}\right)$. In this retrospective study, 
we aimed to evaluate the prognostic relevance of MRD in B ALL patients.

Patients/Materials \& Methods: The study included 403 patients who underwent treatment for B ALL between August 2012 and March 2019 in the Department of Haematology, CMC Vellore and had their end induction MRD assessed by flow cytometry. MRD assessment was done by 4 tube- 4 colour panel (till July 2017) and 2 tube- 8 colour panel (from August 2017). MRD levels $\geq 0.01 \%$ were considered positive. Baseline demographic characteristics were collected from the patient's clinical records. Event Free Survival (EFS) and Relapse Free Survival (RFS) were calculated using Kaplan-Meier analysis and survival estimates were compared by using the log-rank test. Univariate Cox proportional hazards regression models were used to assess the association between patient characteristics and survival. Statistical analysis was done using SPSS version 16.0 software.

Results: Compared to the 4 tube- 4 colour panel, the 2 tube- 8 colour panel had wider applicability $(98.5 \%$ vs $93.2 \%)$ and showed a positive MRD in a higher percentage of patients $(43.08 \%$ vs $30.48 \%$; $p=0.046$ ). End Induction MRD had a significant impact on RFS $(34.1 \% \pm 8.5 \% \quad$ vs $83.9 \% \pm 3.4 \% ; \quad p<0.001)$ and EFS $(29.8 \% \pm 7.6 \%$ vs $81.6 \% \pm 3.5 \% ; p<0.001)$ and the survival worsened for every log increase in the MRD values. Among the other variables, older age, poor prednisolone response, presence of $B C R$ $A B L 1$ translocation and poor-risk cytogenetics were significantly associated with poor survival while $T E L-A M L 1$ translocation showed a trend towards better EFS $(p=0.097)$. On multivariate analysis MRD showed the strongest association with both RFS and EFS.

Discussion \& Conclusion: End induction MRD was the strongest prognostic indicator in B-ALL patients among the different parameters assessed in our study. End induction MRD is the ideal tool to predict treatment responses and take necessary clinical interventions including the need for a bone marrow transplant.

Disclosure of Interest: None Declared.

Keywords: ALL, Flow cytometry, MRD.

\section{Abstract 3. Leukemia Cutis in P210 Fusion Transcriptin B-ALL}

\section{Devika Gupta $^{1}$, BlessyMathews ${ }^{* 1}$, TathagatChatterjee ${ }^{1}$, Suman Pramanik ${ }^{2}$, Ankur Ahuja ${ }^{1}$}

\section{${ }^{1}$ Dept of Lab \& Molecular Medicine, ${ }^{2}$ Dept of Hematology,} Army Hospital RR, Delhi, India

Aims \& Objectives: 1 . To assess leukemia cutis in a case of B-ALL. 2. To differentiate blast crisis in CML with lymphoid blasts versus B-ALL.

Patients/Materials \& Methods: A case report study of leukemia cutis reported in our tertiary centre.

Results: 31 year old female presented with multiple erythematous papules, patches and cutaneous nodules over bilateral legs. Our patient was a diagnosed case of preB ALL (Philadelphia chromosome positive) since 05 years. At diagnosis, metaphase karyotyping by GTG banding showed a balanced translocation 46XX, t $(9 ; 22)$ and Real time polymerase chain reaction (RT-PCR)BCR/ABL translocation assay detected hybrid transcript for BCR/ABL in the leucocytes with genomic breakpoint observed at $\mathrm{b} 3 \mathrm{a} 2$ corresponding to $\mathrm{p} 210 \mathrm{kD}$ (kilodalton) protein (major fusion transcript). The immune phenotyping by flow cytometry revealed $85 \%$ dim CD45 blasts which were positive for CD19, CD22, CD10 (CALLA), CD34, HLA-DR and Tdt and negative for anti-MPO, CD56 and CD117. She was managed with second generation tyrosine kinase inhibitors (TKI) in addition to ALL chemotherapy. Patient thereafter underwent Allogenic stem cell transplant (ASCT) with brother as the HLA matched donor. She was placed on GVHD prophylaxis and her subsequent course was uneventful. After nearly 04 years of being asymptomatic, she presented with cutaneous lesions over bilateral lower extremities. On physical examination, there was no lymphadenopathy and her oral cavity was normal. There was no organomegaly or lymphadenopathy. Histopathological examination of the skin biopsy from erythematous patch lower leg was done which showed infiltration of upper and deep dermis by atypical lymphoid cells having irregular nuclei, dispersed chromatin and scant cytoplasm. There was no epidermotropism and a Grenz zone was demonstrable. Immunohistochemistry revealed that these malignant cells were immunopositive for CD10, CD20, CD19 and Tdt with Ki67 being 85\%. At this time her peripheral blood smear revealed hemoglobin-11 g/dL; WBC- 7000/cu.mm and platelet count of $1,57,000 /$ cumm. The peripheral smear and bone marrow showed absence of blasts.

Discussion \& Conclusion: LC is most frequently seen in the setting of acute myeloid leukemia with about $10-15 \%$ of the AML patients presenting as LC. The commonest subtype of AML associated beingAML M4 or 5. Other common hematological malignancies presenting with LC are chronic myeloid leukemia and myelodysplastic syndrome. Rarely, B/T ALL $(1 \%>3 \%)$ and chronic lymphocytic leukemia may also manifest as LC.

Disclosure of Interest: None Declared.

Keywords: acute lymphoblastic leukemia, Leukemia cutis, p210 fusion transcript.

\section{Abstract 4. Scatterplots from Sysmex XN-Series Analyser as Tool for Screening Acute Promyelocytic Leukaemia}

\author{
AbhirupSarkar $^{* 1}$, Aparna Ningombam ${ }^{1}$, ShreyamAcharya ${ }^{1}$ \\ ${ }^{1}$ Department of Laboratory Medicine, All India Institute of \\ Medical Sciences, New Delhi, India
}

Aims \& Objectives: To study pattern of scatterplots and Flow Cytometry Standard (FCS) data from Sysmex XN-series haematology analysers in clinically suspected patients of acute leukaemia as a tool for screening acute promyelocytic leukaemia (APML).

Patients/Materials \& Methods: We retrospectively selected 12 APML cases and 16 non-APML (other acute leukaemia, leukaemoid reaction) cases already confirmed by morphological/cytogenetic/molecular studies. Eight of the 12 APML cases were also included during the treatment and follow-up. The data of these cases from Sysmex XN-1000 haematology analyser were collected. The scatterplots of the cases were examined on the graphical user interface, and the FCS data were extracted and analysed on external flow cytometry analysis software.

Results: The 12 APML cases before treatment initiation had a typical pattern in WDF (SSC vs SFL) channel scatterplots showing a large broad-based cluster of abnormal promyelocytes with a small lymphocyte population. The eight cases later on treatment showed a growing cluster of maturing myeloid cells along with the abnormal promyelocyte cluster mentioned above in their WDF scatterplots. The 16 non-APML cases displayed various different patterns on their scatterplots, but none had such distinct clusters of the cells mentioned above and could be clearly differentiated from the APML plots. Using the FCS data, we overlaid the WDF scatterplots and generated a prototype pattern for APML.

Discussion \& Conclusion: APML is a medical emergency where treatment needs to be started as soon as diagnosis is suspected, which can reduce mortality to a large extent. Since complete blood count is usually the first investigation done in such cases, we can use the data obtained from Sysmex XN-series haematology analysers, which are now widely used, to raise early suspicion and expedite treatment of 
APML. The treatment response can also be gauged on the basis of analysis of follow-up blood samples. Hence this new tool for screening can be incredibly useful in cases of APML.

Supporting Document: b0b2eae5-546c-4cbc-a0a4-ff70f0f6c0d9.
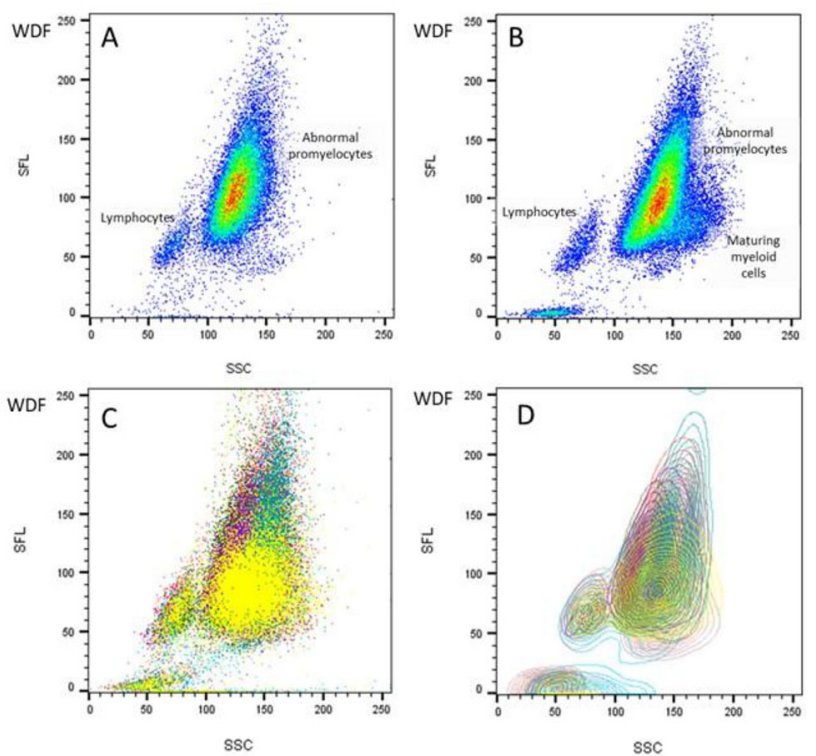

$\mathrm{SC}$

Figure: (A) WDF scatterplot of APML before start of treatment showing two distinct clusters: abnormal promyelocytes and ymphocytes. (B) WDF scatterplot of APML during treatment showing clusters of abnormal promyelocytes, lymphocytes as representing prototype of partern representing APML

Disclosure of Interest: None Declared.

Keywords: Acute Promyelocytic Leukaemia, Scatterplot, Sysmex.

\section{Abstract 5. 'SINGLE TUBE' vs 'THREE TUBE' Panel by Flow Cytometry for MRD Detection in Pediatric B-ALL}

\section{Aditi Agarwal ${ }^{* 1}$, Mrinalini Kotru ${ }^{2}$, Meera Sikka ${ }^{3}$, Pooja Dewan ${ }^{4}$ \\ ${ }^{1}$ Pathology, University College of Medical Sciences, ${ }^{2}$ University College Of Medical Sciences, ${ }^{3}$ University College of Medical Sciences, Delhi, India, ${ }^{4}$ Pediatrics, University College of Medical Sciences, Delhi, India}

Aims \& Objectives: Aim: To study the diagnostic efficacy of 'Single tube' v/s 'Three tube panel' by Flow Cytometry in detection of Minimal Residual Disease (MRD) in pediatric B-cell Acute Lymphoblastic Leukemia (B-ALL).

Objective: To determine the sensitivity of 'single tube' flow cytometry panel against 'multiple tube' panel in detection of MRD in pediatric B-ALL.
Patients/Materials \& Methods: The study was conducted in Department of Pathology and Pediatrics, University College of Medical Sciences and G.T.B. Hospital, Delhi-95 from November 2017 to March 2019. A sample size of 25 cases was taken. On Day 0 -For diagnosis $\mathrm{CBC}$ and peripheral blood smear/bone marrow aspirate was performed and baseline immunophenotyping by flow cytometry was done. On completion of induction of chemotherapy $\mathrm{CBC}$ and peripheral blood smear/bone marrow aspirate was done again along with Minimal Residual Detection by Flow Cytometry was performed using the standard 'Three tube' panel and also 'Single tube' panel (Table A).

Results: Twenty five pediatric B-ALL cases were studied. The age of the patients ranged from 2 to 18 years. The cohort included $32 \%$ females and $68 \%$ males. The $p$ value for Hemoglobin $\%$, Platelet Count and blast percentage before and after treatment were statistically significant ( $p$ value of $\mathrm{Hb}=0.002, p$ value of $\mathrm{PC}=0.0001$ and $p$ value of $\mathrm{PC}=0.0001)$. Bone marrow aspirate showed hypercellularity with near-total to total replacement by blasts. Post induction all cases showed bone marrow in morphological remission.

Immunophenotyping by flow cytometry at the time of diagnosis: All the cases of B-ALL were diagnosed on basis of positivity of CD19 along with co-positivity of any of these two markers namely CD10, CD20 and cCD79a.

MRD detection by flow cytometry using 'Three tube' panel:

MRD was found to be positive in $24 \%$ cases with cut-off for positivity taken as $>0.01 \%$ of mononuclear events. In our study $3 / 6$ cases despite being CALLA positive were MRD positive.

Percentage of MRD positivity ranged from $0.5-2.14 \%$. Cluster formation was seen in all MRD positive cases in one or more tubes.

MRD was found positive in $36 \%$ cases. Percentage of MRD positivity ranged from $0.03-64.9 \%$. Positivity was studied in three combinations of markers which were (CD38 vs CD10), (CD45 vs CD10) and (CD38 vs CD34). Two observers analysed all the cases to rule out 'observer bias'. There was $100 \%$ concordance between the two observers.

Cluster formation outside the hematogone region in any one of the marker combination was considered MRD positive.

The sensitivity of 'Single tube' panel was calculated as $100 \%$ and specificity as $84.2 \%$ taking 'Three tube' panel as the gold standard for MRD detection. The difference between the gold standard and 'Single tube' panel was not statistically significant as $p$ value $=0.25$.

Discussion \& Conclusion: To conclude MRD done on 'Single tube' panel using CD38, CD10, CD19, CD34 and CD45 is as sensitive as the 'Three tube' panel (gold standard). However, the specificity is less. CD10, CD20 and CD200 were valuable in distinguishing MRD positive from negative cases. Moreover, 'Single tube' panel had $100 \%$ concordance between the two observers suggesting the simplicity in assessment and hence, its wider applicability.

Disclosure of Interest: None Declared.

Keywords: acute lymphoblastic leukemia, B-ALL, Minimal Residual Disease.

Table A The standard 'Three tube' panel and 'Single tube' panel put for MRD detection

\begin{tabular}{|c|c|c|c|c|c|c|c|}
\hline & & FITC & $\mathrm{PE}$ & ECD & PC. 5 & PC.7 & \\
\hline \multirow[t]{3}{*}{ Three tube panel } & Tube 1 & CD $2010 \mu \mathrm{l}$ & CD $1010 \mu l$ & CD $4510 \mu \mathrm{l}$ & CD $1910 \mu \mathrm{l}$ & CD $345 \mu 1$ & $45 \mu 1$ \\
\hline & Tube 2 & CD $5820 \mu l$ & CD $1010 \mu l$ & CD $4510 \mu l$ & CD $1910 \mu \mathrm{l}$ & CD $345 \mu \mathrm{l}$ & $55 \mu l$ \\
\hline & Tube 3 & CD $3810 \mu \mathrm{l}$ & CD $2005 \mu \mathrm{l}$ & $\mathrm{CD} 4510 \mu \mathrm{l}$ & CD $1910 \mu \mathrm{l}$ & CD $345 \mu \mathrm{l}$ & $40 \mu \mathrm{l}$ (Test) \\
\hline Single tube panel & & CD $3810 \mu \mathrm{l}$ & CD $1010 \mu \mathrm{l}$ & CD $4510 \mu l$ & CD $1910 \mu \mathrm{l}$ & CD $345 \mu \mathrm{l}$ & $45 \mu 1$ \\
\hline
\end{tabular}

MRD positivity $=$ Blast events/Total mononuclear events $\times 100$ 


\section{Abstract 6. Acute Promyelocytic Leukemia at 1 Year Age: Is It the Youngest Ever Case Reported?}

\author{
Prateek Goel"1, SanjeevanSharma ${ }^{1,2}$, Venkatesan $S^{3}$, \\ Paresh Singhal $^{3}$ \\ ${ }^{1}$ Internal medicine, Afmc, ${ }^{2}$ Dept of Hematology, $\mathrm{CH}(\mathrm{SC})$, \\ ${ }^{3}$ Depatment of Pathology, Afmc, Pune, India
}

Aims \& Objectives: Acute Promyelocytic Leukemia at 1 year Age: Is it the youngest ever case reported?

We report a 1 year old infant from Pune, India born 11 Jan 2018, who presented to us in Feb 2019, with short febrile illness, acute coryza and recurrent gingival bleed of 04 days duration. Examination revealed pallor, hepatosplenomegaly, petechiae over right eyelid. Initial Blood picture showed severe anaemia (Hb-3.5 gm \%), leucocytosis (TLC$62,000 /$ cumm) and thrombocytopenia (Plt $<1.0$ lakh). PBS revealed $88 \%$ abnormal promyelocytes, $04 \%$ blast. Bone marrow aspirate showed $80 \%$ Promyelocytes with abundant granules, with Auer rods and faggots in cytoplasm (MPO +). Conventional karyotyping revealed t(15: 17), 46 XY karyotype. PML-RARA was detected in $93 \%$ cells in BM cytogenetics (FISH), and a bcr-1 transcript on RTPCR.

The patient was diagnosed as a case of high risk APL and was managed in PICU with component support, parenteral antibiotics and Induction therapy using combination of ATRA $(45 \mathrm{mg} / \mathrm{m} 2 / \mathrm{d})$, high dose Arsenic trioxide $(0.20 \mathrm{mg} / \mathrm{kg} / \mathrm{d})$ and Daunorubicin $(50 \mathrm{mg} / \mathrm{m} 2 \times 04$ doses $)$.

He responded to therapy well and had hematological remission by $\mathrm{D}+22$ of therapy and was given 2 cycles of consolidation therapy with ATRA, Arsenic, and Daunorubicin. He achieved molecular remission (RTPCR negative for PMLRARA) after the $1^{\text {st }}$ Consolidation cycle. There were no signs of differentiation syndrome during the entire treatment. His latest laboratory investigation showed $\mathrm{Hb}-10.4$, TLC- 6780/cumm, P-40/L-46, Plt- 2.56 lakh with normal LFT/RFT. Patients/Materials \& Methods: He was diagnosed as a case of high risk APL. He was regularly followed up with thrice weekly complete blood counts and Biochemical parameters. He underwent monthly PML-RARA RTPCR for response assesment and disease progression or remission.

Results: He responded to therapy well and had hematological remission by $\mathrm{D}+22$ of therapy and was given 2 cycles of consolidation therapy with ATRA, Arsenic, and Daunorubicin. He achieved molecular remission (RTPCR negative for PMLRARA) after the 1st Consolidation cycle. There were no signs of differentiation syndrome during the entire treatment. His latest laboratory investigation showed $\mathrm{Hb}-10.4$, TLC6780/cumm, P-40/L-46, Plt- 2.56 lakh with normal LFT/RFT.

Discussion \& Conclusion: Extensive literature research on APL worldwide has revealed numerous small paediatric case series of APL. Youngest case reported in literature is 18 month old. Our case is could be the first case detected as early at 12 month of age, treated with ATRA and ATO and presently in remission.

Disclosure of Interest: None Declared.

Keywords: APML, RARE, YOUNGEST.

\section{Abstract 7. Spectrum of Cytogenetic Abnormalities in Therapy Related Myeloid Neoplasms}

BexyBensega $^{* 1}$, Nancy Beryl Janet $\mathrm{A}^{\mathbf{1}}$, NivedithaRavindra ${ }^{1}$, Kulkarni Uday Prakash ${ }^{1}$, Anup J Devasia ${ }^{1}$, Fouzia N.A ${ }^{1}$, Anu Korula ${ }^{1}$, Aby Abraham ${ }^{1}$, Alok Srivastava ${ }^{1}$, Biju George ${ }^{1}$, Vikram Mathews ${ }^{1}$

\section{${ }^{1}$ Haematology, Christian Medical College, Vellore, India}

Aims \& Objectives: T oanalyze the spectrum of cytogenetic abnormalities in therapy related myeloid neoplasm.
Therapy-related myeloid neoplasms (T-MN) include therapy-related myeloproliferative neoplasms (t-MPN), myelodysplastic syndrome ( $t-$ MDS) as well as acute myeloid leukemia (t-AML). These aggressive malignancies are characterized by complex cytogenetic abnormalities and a dismal outcome with a 5-year survival of $10 \%$.

Patients/Materials \& Methods: Karyotype (GTG banding) findings in patients diagnosed with a T-MN seen in the Department of Haematology, Christian Medical College were analyzed.

Results: There were 30 patients who had T-MN with karyotyping results of whom 12 were males. The median age at diagnosis was 43 years (range $8-76$ years) and the majority were adults $(n=27)$. In total, AML or MDS were seen in 14 each and MPN in 2 patients. The prior history included carcinoma breast $(n=9)$, lymphoma $(n=3)$, adenocarcinoma, MDS as well as myeloma $(n=2$ each). Seminoma, acute lymphoblastic leukemia, carcinoma glottis, carcinoma cervix as well as osteosarcoma was the initial malignancy in one patient each and one patient developed T-MN post renal transplant. The clinical diagnosis of the earlier disease was not recorded in 6 patients. The majority underwent chemotherapy $(n=16)$ while 4 had radiation therapy while in 1 the T-MN resulted post renal transplant. The treatment details were not available in 9 .

Karyotypic abnormalities were seen in 24 patients of whom $16(66 \%)$ had complex cytogenetic abnormalities, 5 (20\%) had translocations resulting from rearrangements of the KMT2A gene (MLL) and 4 had solitary cytogenetic abnormalities. Six patients had a normal karyotype. The majority of the patients had abnormalities involving chromosomes 5 or $7(n=13,54 \%$ each); 7 of the 13 patients showed either monosomies of both 5 and 7 or partial deletions of both $5 q$ and 7q. Translocations or monosomy 17 resulting in deletion of the Tp53 gene were seen in 4 patients.

Discussion \& Conclusion: Our findings are consistent with previous reports which show T-MN to have complex karyotypes with abnormalities of chromosomes 5 and $7(42 \sim 49 \%)$ as well as rearrangements of the KMT2A gene (30\%).Sequencing of the Tp53 gene in this cohort may help to identify patients who may benefit from therapy with Decitabine as the outcome remains particularly poor for this subgroup.

Disclosure of Interest: None Declared.

Keywords: complex karyotype, Karyotype, Therapy related myeloid neoplasm.

\section{Abstract 8. Clinico-Morphological and Genomics Correlation of Acute Promyelocytic Leukemia}

RakaHota $^{{ }^{* 1}}$, Deepak K. Mishra ${ }^{1}$, Mayur Parihar ${ }^{2}$, Neeraj Arora ${ }^{3}$, Saurabh Bhave ${ }^{4}$, NiharenduGhara ${ }^{5}$, Vivek Radhakrishnan ${ }^{4}$, SubhajitBrahma ${ }^{1}$, Poonam Santra ${ }^{3}$, MammenChandy ${ }^{4}$

${ }^{1}$ Lab. Hematology, ${ }^{2}$ Dept. of Cytogenetics, ${ }^{3}$ Dept. of Molecular genetics, ${ }^{4}$ Dept of Clinical Hematology, ${ }^{5}$ Dept of PediatricHematology, Tata Medical Center, Kolkata, Kolkata, India

Aims \& Objectives: The aim of the present study is to observe the correlation between clinical presentation, cytomorphological features, cytogenetics and molecular genetics in patients of APMLs; in view of providing early diagnosis, prompt treatment to reduce the mortality. Patients/Materials \& Methods: We retrospectively analyzed 81 consecutive cases of APML based on the clinical presentation, hematological findings (PBS/BM), immunophenotypic features (CD13/CD33/cMPO positive and HLA-DR/CD34 negative), cytogenetics (karyotyping/FISH) and molecular (RT/PCR for PML-RARA) analysis from AUG 2011 to August 2019. The age range was 3 yrs to 
92 yrs. with median age at diagnosis being 47.5 years and male to female ratio was $1: 1.1$.

Results: Of the total 81 APML cases, $42(52 \%)$ patients presented with low WBC count less than $10,000 / \mu \mathrm{l}$ and $39(48 \%)$ cases had more than $10,000 / \mu \mathrm{l} \mathrm{WBC}$. Thrombocytopenia less than $40,000 / \mu \mathrm{l}$ was seen in 62 $(77 \%)$ cases and $19(23 \%)$ cases had platelet count more than $40,000 / \mu 1$. $35(43 \%)$ cases had coagulation derangements. All the cases were morphologically and immunophenotypically suggestive of APML in view of PBS/BM abnormal promyelocytes having convoluted nuclei and faggots along with cMPO/CD13/CD33/CD117 positive and CD34/HLADR negative immunophenotype. Karyotyping was performed in 52 $(64 \%)$ cases out of which $50(62 \%)$ were having $t(15,17)$ and $2(2.5 \%)$ had variant translocation $t(11,17)$. To establish the diagnosis FISH was done and data is available in $80(99 \%)$ cases of which PML-RARA fusion was found in $73(91 \%)$ cases.7 (9\%) cases were FISH negative of which 5 cases were confirmed having PML-RARA fusion by RT-PCR and 2 cases had variant $\mathrm{t}(11,17)$. RT-PCR analysis was performed in 66 (82\%) cases of which $62(94 \%)$ were PML-RARA positive and $4(6 \%)$ were negative. In 62 RT-PCR positive cases, Bcr1 (long transcript subtype) in 38 (61\%) cases, Bcr-3 (short transcription) in 22 (35\%) cases, Bcr-2 in $1(2 \%)$ case and both Bcr1/Bcr3 transcription in $1(2 \%)$ seen. Out of total 81 APML cases, $9(11 \%)$ patients were died during/before the treatment mostly due to intracranial haemorrhage and $72(89 \%)$ are alive and received treatment of ATO/ATRA along with/without anthracycline of which $14(19 \%)$ cases had episode of relapse. All the patients are under regular follow-up and monitoring.

Discussion \& Conclusion: APML with variant RARA translocations comprises $1-2 \%$ of APMLs. However, the diagnosis of these cases are challenging, as in routine practice FISH and RT-PCR targets the PML and RARA genes to detect PML/RARA fusions. We share our experience of APML from a single center. There is a subset of cases which can be missed due to cryptic/variant translocations, hence they need a comprehensive approach for the diagnosis of APMLs.

Disclosure of Interest: None Declared.

Keywords: PML-RARA, Promyelocyte, RT-PCR.

\section{Abstract 9. Post Induction Bone Marrow NK-Cell Level Predict Persistence of Residual Disease in Childhood T-ALL}

Reshma Kharat $^{* 1}$, PrathyushaGudapati ${ }^{2}$, Shripad Banavali ${ }^{1}$, Gaurav Narula $^{1}$, Sumeet Gujaral ${ }^{1}$, Prashant Tembhare ${ }^{2}$

${ }^{1}$ Tata Memorial Hospital Parel Mumbai, ${ }^{2}$ Tata Memorial Centre, Navi Mumbai, Mumbai, India

Aims \& Objectives: T-cell acute lymphoblastic leukemia (T-ALL) is known for a higher frequency of minimal residual disease (MRD) positivity and a higher risk of relapse compared to B-ALL. MRD represents the persistence of the tumor clone due to treatment resistance and immune-escape phenomenon. Thus, tumor microenvironment immune cell profile possibly contribute to MRD positivity and disease relapse. There is no data on immune cell composition of BM at diagnosis and at the time of MRD monitoring in T-ALL. Hence herein we sort out the BM immune cell profile in diagnostic and MRD samples of childhood T-ALL patients.

Patients/Materials \& Methods: We studied 40 pediatric T ALL patients (18 postinduction MRD positive, and 22 MRD negative cases) and monitored bone marrow (BM) immune cell subsets at the time of diagnosis and post-induction examination. Flow cytometry is performed using 10-color Navios flow-cytometer (Beckman Coulter) and data were analyzed using Kaluza software1.3.

Results: We studied $80 \mathrm{BM}$ samples (40 diagnostic, 40 post-induction) from 40 patients (M:F- 3:1 \& age range 1-14 years). Subsets monitored at diagnosis included $\mathrm{CD} 3+\mathrm{T}$-cells, CD4+ T-cells, CD8+ T-cells, Gamma-delta-T-cells, NK-cells, B-cells, hematogones, plasmacytoid dendritic-cells (pDC) and basophils. Median and range of different subsets at the time of diagnosis are as follows, $\mathrm{T}$ cells $2.26 \%$ $(0.18 \%>16.19 \%)$, CD4+ T-cells $5.45 \%(0.07 \%>0.89 \%), \mathrm{CD} 8+$ cells $\quad 9.36 \% \quad(0.06 \%>0.87 \%), \quad$ Gamma-delta-T-cells $\quad 0.24 \%$ $(0.01 \%>1.42 \%)$, NK cells $0.48 \%(0.05 \%>10.33 \%)$, B-cells $0.72 \%$ $(0.06 \%>13.64 \%)$, pDC $0.01 \%(0-0.62 \%)$, basophils $0.05 \%(0-0.34 \%)$. Similarly, median (SD) of T-cells, CD4+ T cells, CD8+ T cells, and NK cells subsets in post-induction marrow were $6.84 \%$ (5.69\%), $3.32 \%$ $(2.87 \%), 2.19 \%(2.70 \%)$ and $1.01 \%(1.14 \%)$ respectively. $18 / 40(46 \%)$ cases were MRD positive with median MRD level 0.6\% (range, $0.003-39.5 \%$ ). We further evaluated the difference between distribution of T/NK cell subsets in MRD(+) versus $\mathrm{MRD}(-) \mathrm{BM}$-samples using Mann-Whitney test. Interestingly, NK cell levels were significantly lower $\mathrm{MRD}(+)$ than $\mathrm{MRD}(-)$ samples $(p$ value $=0.03 \%)$. There was no difference between other T-cell subsets.

Discussion \& Conclusion: We first time studied the major immune cell subset distribution in BM samples in T-ALL in diagnostic and post-induction samples. We found that NK-cells were significantly reduced in MRD-positive post-induction BM samples highlighting the potential role of NK-cell in the pathophysiology of persistence of PIMRD in childhood T-ALL.

Disclosure of Interest: None Declared.

Keywords: Immune cell subcet, Minimal Residual Disease, T-ALL.

\section{Abstract 10. Study of T(9:22)/BCR-ABL Fusion in Pediatric ALL by fluorescence In-Situ Hybridisation}

\author{
Meera $\mathrm{M}^{* 1}$, Anita Nangia ${ }^{2}$, Jagdish Chandra ${ }^{3}$ \\ ${ }^{1}$ Pathology, Lady Hardinge Medical College, NewDelhi, \\ ${ }^{2}$ Pathology, Lady Hardinge Medical College, \\ ${ }^{3}$ Pediatrics, Kalawati Saran Children hospital, New Delhi, India
}

Aims \& Objectives: To study $\mathrm{t}(9: 22) / \mathrm{BCR}-\mathrm{ABL}$ fusion in pediatric acute lymphoblastic leukemia by fluorescence in situ hybdrisation.

Patients/Materials \& Methods: A hospital based descriptive observational study was performed in forty newly diagnosed pediatric ALL cases after bilingual written informed consent and detailed history. $1 \mathrm{ml}$ of sample (peripheral blood/bone marrow) of the cases was collected in a heparinised vial (before the commencement of induction) and was subjected to FISH analysis. Vysis LSI dual co; or dual fusion translocation probe for BCR-ABL was used and the signals were interpreted in interphase nuclei.

Results: The study found that five out of the forty ALL cases $\mathbf{( 1 2 . 5 \% )}$ showed BCR-ABL fusion signals with classic 2Y1R1G pattern in four cases and one case showed 1F1R1G fusion pattern. All the five $\mathrm{Ph}$ positive cases had hepatospenomegaly, lymphadenopathy, high total leucocyte count $(>50,000$ cells $/ \mu 1)$ and high tumor burden (blasts percentage $>50 \%$ ). Immunophenotypically, four out the five were B cell ALL and the remaining one T cell ALL. On follow up, one of the $\mathrm{Ph}$ positive case succumbed to death and another was minimal residual disease positive at the end of induction.

Discussion \& Conclusion: This study shows a higher percentage (12.5\%) of BCR-ABL fusion protein in Indian pediatric population with ALL as compared to western literatures (2-3\%). It was associated with other adverse prognostic features and poor response to treatment. This study suggest incorporating FISH as a mandatory diagnostic tool in pediatric ALL to help initiate treatment with tyrosine kinase inhibitors at the earliest in positive cases.

Disclosure of Interest: None Declared.

Keywords: BCR-ABL fusion, Fluorescence in-situ hybridisation, Pediatric acute lymphoblastic leukemia. 


\section{Abstract 11. Tumor Lysis Syndrome in Acute Leukemia and Lymphoma}

Ekta Devi $^{* 1}$, Sudhir Kumar Atri ${ }^{1}$, Mohini chinu ${ }^{1}$

\author{
${ }^{1}$ Department of Medicine and Hematology, Pt. B. D. Sharma \\ PGIMS, Rohtak, India
}

Aims \& Objectives: The tumor lysis syndrome (TLS) is the most common disease-related emergency encountered by by physicians caring for patients with hematologic cancer. This study was conducted to determine the frequency of tumor lysis syndrome (TLS) in patients of acute leukemia and lymphoma.

Patients/Materials \& Methods: This descriptive study was conducted at Pandit Bhagwat Dayal Sharma PGIMS, Rohtak and data was collected from 100 patients (age 14 years or above) of acute leukemia or lymphoma in Haematology department of medicine over a period of one year (2017-18). Serum uric acid, phosphorus, calcium and potassium levels were monitored starting from day of admission till completion of 4 weeks of induction chemotherapy for acute leukemia patients and on day 1 and day 2 of each cycle of chemotherapy in lymphoma patients. All patients received adequate hydration and allopurinol Data were analysed and following results were obtained. Results: Out of 30 patients of acute leukemia 10 fulfilled the criteria for TLS of which five patients developed spontaneous tumor lysis syndrome (STLS), 4 developed laboratory tumour lysis syndrome (LTLS) whereas only one patient developed clinical tumour lysis syndrome (CTLS). Out of 70 patients of lymphoma 9 fulfilled the criteria TLS and all of them had laboratory tumour lysis syndrome. Overall 1 patients died because of TLS. Hyperuricaemia and hyperkalemia were the most prominent findings in patients with TLS in lymphoma group. All cases of TLS occurred during first cycle of chemotherapy majority from day 0 to day 3 and cases of LTLS recovered by day 6 .

Discussion \& Conclusion: It is concluded that $28 \%$ of the patients with acute leukemia and $13 \%$ of patients with lymphoma developed TLS (including both laboratory and clinical TLS) despite all measures of prevention it can occur and result in devastating clinical effects. Disclosure of Interest: None Declared.

Keywords: Acute leukemia, LYMPHOMA, TUMOR LYSIS SYNDROME

\section{Abstract 12. Cytogenetic and Molecular Studies in a Cohort of Acute Myeloid Leukemia}

\author{
Akshay Gore ${ }^{* 1}$, Nitin Mathur ${ }^{2}$, Manorama Bhargava ${ }^{1}$, \\ Anil Yadav ${ }^{1}$ \\ ${ }^{1}$ Haematology, Cytogenetics and Molecular Haematology, \\ ${ }^{2}$ Haematology, Cytogenetic and Molecular Haematology, \\ Medanta-The Medicity, Gurugram, India
}

Aims \& Objectives: 1 . To define cytogenetic and molecular findings in AML.

2. To correlate the above findings with each other.

Patients/Materials \& Methods: 49 patients (Males-31 and Females18) diagnosed with AML in Medanta- The Medicity from April 2018 to July 2019 with an age range of 3 Years to 74 Years (Pediatrics-3 \& adults-46) were included in the study of which 35 cases were de novo AML, 5 cases AML-MRC, 7 cases APML, 2 cases therapy related AML. Karyotyping and G-banding was carried out on bone marrow aspirates/peripheral blood having more than $20 \%$ blasts using standard protocols. FISH for PML-RARA was performed on all APML cases. The karyotypes were reported as per the ISCN 16. Molecular studies were done in 35 cases of 49 cases using an RQ-PCR Panel consisting of PML- RARA, BCR-ABL, AML1-ETO, CBFB-MYH11 gene rearrangements, FLT3-ITD, FLT3-TKD (D835), NPM1, c-KIT gene mutations.

Results: On cytogenetics, 34 (69\%) patients had an abnormal and 15 $(31 \%)$ had a normal karyotype. Similar or identical abnormalities were seen in $16(47 \%)$ cases of which 6 showed molecular abnormalities as well. Distinctive abnormalities were seen in $18(53 \%)$ cases, amongst which 6 again were molecularly abnormal. Thus, a total of 12 cases showed molecular abnormalities of the 35 cases analysed (Table 1) details the karyotyping \& molecular findings in the various groups. Out of the 35 denovo AML cases 12 had normal karyotype, 2 cases showed inv(16), 2 cases had $\mathrm{t}(8: 21), 5$ cases had trisomy 8,2 cases showed monosomy 7, 3 cases showed complex karyotype and 9 cases had distinctive cytogenetic abnormalities. 2 cases of t-AML had normal karypotype out of which one had NPM1 mutation. Of the 5 cases of AML-MRC, 3 cases had complex karyotype and one had monosomy 7 , and one showed normal karyotype. Of the 7 APML cases, six showed $\mathrm{t}(15: 17)$ and one had a variant translocation $\mathrm{t}(11: 17)$.

Discussion \& Conclusion: In reported literature, normal karyotype is the single largest subset in AML comprising 40 to $50 \%$ of adult AML and $25 \%$ in Pediatric cases. We, however found a high percentage of AML cases with distinctive abnormal karyotype, some of them not as per WHO classification 2016

Supporting Document: 54c07aad-d122-4744-bdff-0b26260b0117.

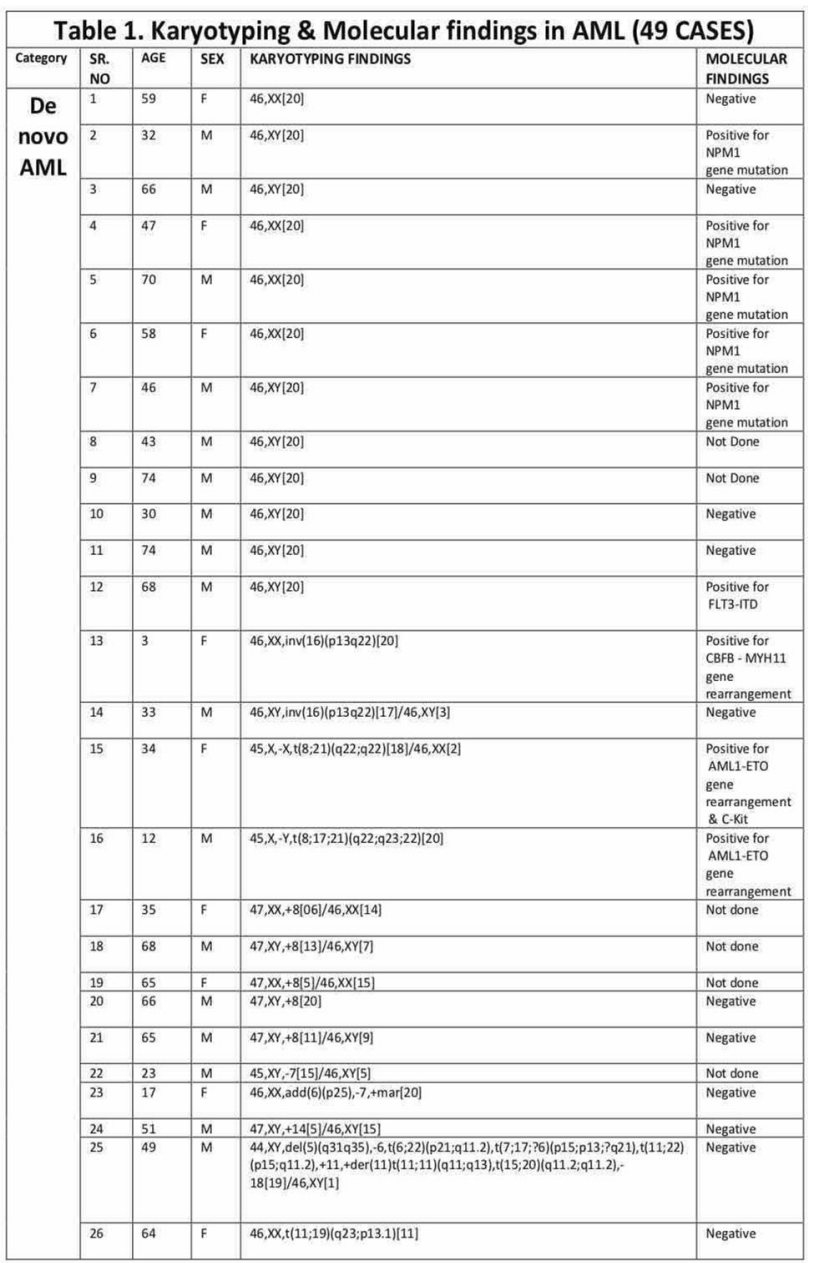

Disclosure of Interest: None Declared.

Keywords: AML, AML Genomics, Cytogenetic abnormalities. 


\section{Abstract 13. CD34 Negative B-Acute Lymphoblastic Leukemia}

\author{
Neha Garg ${ }^{* 1}$, Richa gupta ${ }^{1}$, Rajesh Pathak $^{1}$, Mrinalini kotru ${ }^{1}$ \\ ${ }^{1}$ UCMS and GTBH, Delhi, Delhi, India
}

Aims \& Objectives: CD34 antigen is expressed by early hematopoietic progenitor cells and acute leukemia cells.Its expression is associated with good prognosis in acute myeloid leukemia. Literature is sparse on its prognostic significance in B- acute lymphoblastic leukemia (B-ALL) with only few studies with differing results done in the west. No study has been published from India. The present study aimed to study the frequency and correlation of CD34 expression with other prognostic markers in B-ALL patients in India.

Patients/Materials \& Methods: Seventy B-ALL patients diagnosed by flow cytometry over a period of $3 \frac{1}{2}$ year were taken out from the records of Hematology section of department of pathology of UCMS and GTB hospital. Immunophenotyping including CD34, HLA-DR, TdT, CD117, CD10, CD19, CD20, CD 79a, cMPO, cCD3, CD3, CD5, CD7, CD13, CD 33, CD64, CD11b were performed using flow cytometry. Correlation of CD34 expression was studied with gender, age $(<1$, $1-10,>10$ years $)$, total leucocyte count $\left(<50 \times 10^{9} / \mathrm{L},>50 \times 10^{9} / \mathrm{L}\right)$ FAB morphological type (L1, L2) and immunophenotypic markers. $P$ value $<0.05$ was considered significant.

Results: CD34 antigen was expressed in 49/70 (70\%) while it was absent in 21/70 (30\%) cases. CD 13, CD33, CD5, CD7 were more frequently expressed in CD34 negative B-ALL but the results were not statistically significant. No correlation of CD34 expression was found with gender, age, total leucocyte count, FAB morphological type and other immunophenotypic markers studied.

Discussion \& Conclusion: CD34 is an immaturity marker expressed in leukemia cells. The frequency of CD34 positivity in B-ALL (70\%) was in accordance with previous Indian data reporting 68\% (753/1104) CD34 positive B-ALL cases. No significant association was found between CD34 negative or positive cases with prognostic markers or aberrant immuphenotypic expression. Our results are in contrast to studies from the west. This may be due to the different sociodemographic profile and biology of the disease in Indian patients. Larger studies need to be done in Indian setup to clearly understand the biology of CD34 negative B-ALL and thus paves the way for future research.

Disclosure of Interest: None Declared.

Keywords: B- acute lymphoblastic leukemia, ed 34, IMMUNOPHENOTYPING.

\section{Abstract 14. Therapy Related Acute Promyelocytic Leukemia in a Case of Testicular Mixed Germ Cell Tumor}

\section{ManveenKaur $^{* 1}$, RanjeevBhagat ${ }^{1}$, Sanjay Gupta $^{2}$, KislayDimri $^{3}$ \\ ${ }^{1}$ PATHOLOGY, ${ }^{2}$ SURGERY, ${ }^{3}$ RADIOTHERAPY, Gmch, Chandigarh, Chandigarh, India}

Aims \& Objectives: The current management protocol of testicular mixed germ cell tumors (GCT) is based on a combination of surgery to remove teratomatous component and chemotherapy for the rest of chemosensitive components with high cure rates. However, there is also an increased risk of developing secondary malignancies.

We report a case of Acute promyelocytic leukemia (APML) occurring two years after etoposide- based therapy for mixed GCT of the testis in a 30-year-old male.

Patients/Materials \& Methods: A 30-year-old male presented in 2016 with a swelling in the left scrotal region since last two months. The lesion measured $7.7 \times 9.1 \times 8.7 \mathrm{~cm}$. A clinical diagnosis of malignant testicular tumor was suggested and left high inguinal orchidectomy was performed. A final diagnosis of mixed germ cell tumor of left testis was made and the patient was started on chemotherapy regimen comprising of cisplatin, bleomycin and etoposide. The patient was doing well till early 2019 when he presented with fever, fatigue and pancytopenia. Bone marrow examination was performed.

Results: Bone marrow aspirate was hypercellular and showed $93 \%$ abnormal promyelocytes with numerous Auer rods and faggot cells. RT- PCR was positive for PML-RARA gene fusion. A diagnosis of APML was finally rendered and patient started on All-trans retinoic acid- based chemotherapy.

Discussion \& Conclusion: Solid cancers are more commonly encountered secondary tumors as compared to hematological malignancies such as acute myeloid leukemia (AML), acute lymphoblastic leukemia (ALL) and Chronic myeloid leukemia (CML). Risk of leukemia after treatment of testicular germ cell tumors has been estimated to be increased by $20-300$ fold by various studies. The occurrence of APML following mixed GCT of testis is a rare event with only one previously published case in the literature.

Disclosure of Interest: None Declared.

Keywords: Acute promyelocytic leukemia, mixed GCT, Testis.

\section{Abstract 15. Nodal Erythroid Sarcoma: A Diagnostic Enigma}

Surabhi Jain $^{* 1}$, SaumyaranjanMallick ${ }^{1}$, Prashant Ramteke ${ }^{1}$, Mukul Agarwal $^{2}$, Rishi Dhawan ${ }^{2}$, Renu Saxena ${ }^{2}$

${ }^{1}$ Pathology, ${ }^{2}$ Haematology, AIIMS, New delhi, India

Aims \& Objectives: Here we describe a case of erythroid sarcoma in a young female.

Patients/Materials \& Methods: A 20-year old girl presented with easy fatigability, abdominal pain and generalized lymphadenopathy. She had history of multiple transfusions. Intravenous vitaminB12 did not improve the condition. Hemogram, peripheral smear, bone marrow aspiration and excisional biopsy of the cervical lymph node was performed.

Results: Laboratory investigations showed Hb-5 mg/dl, TLC-3000. Peripheral smear showed normocytic hypochromic red blood cells (RBCs) with many nRBCs. Pancytopenia was also seen. Bone marrow aspirate was a dry tap. Sections from formalin fixed lymph nodal biopsy, on histomorphology showed partial effacement of architecture and paracortical zone expansion. There was marked proliferation of erythroid cells- erythroblasts and nucleated red blood cells in paracortical zone, sinusoidal pattern with the erythroblasts occupying almost $60 \%$ of the nodal parenchyma. The erythroblasts were immunopositive for glycophorin, E-cadherin and CD117 (focal) while were negative for CD3, CD20, MPO, SALL4, CD34, CD23. Few myeloid cell (immunopositive for MPO) and megakaryocytes (immunopositive for CD61) were also noted. A possibility of erythroid sarcoma was considered.

Discussion \& Conclusion: Erythroid sarcomas are very rare and prove to be a diagnostic dilemma for the pathologists. The main differential arises from dysplasia of erythroid precursors in megaloblastic erythroid hyperplasia. These may mimic other small round cell tumors or lymphomas too. Immunohistochemistry and flowcytometry can aid in the diagnosis with the erythroid markers. The prognosis is uncertain, with poor survival history reported more due to associated myelodysplastic syndrome, chromosomal anomalies. Accurate and early diagnosis with an integrated approach with pathologist, oncologist, genetics can improve the overall outcome. Disclosure of Interest: None Declared.

Keywords: erythroblast, erythroid sarcoma, nodal. 


\section{Abstract 16. Occurrence of IDH1 and IDH2 Mutations Among AML Patients}

SunisthaBhattacharjee ${ }^{* 1,1}$, SomashreeChakraborty ${ }^{1}$, SreeparnaPodder ${ }^{1}$, Rudra Ray ${ }^{1}$, AkshathaNayak ${ }^{1}$, Shankha Dip Ghatak ${ }^{1}$, Ankita Biswas ${ }^{1}$, MaitreyeeBhattacharyya ${ }^{1}$

${ }^{1}$ Hematology, Ihtm, Medical College, Kolkata, KOLKATA, India

Aims \& Objectives: The aim of this study was to determine the occurrence of IDH1 and IDH2 mutations among AML patients at a tertiary health centre.

Patients/Materials \& Methods: A total of 100 diagnosed AML patients attending IHTM OPD at Medical College, Kolkata during the period of July 2018 to June 2019 were analysed for the presence of IDH1 and IDH2 mutations. Peripheral blood samples and bone marrow aspirates were used for analysing the mutations in both IDH1 and IDH2 gene. ARMS PCR was conducted to identify the R140 mutation in IDH2 gene. DNA sequencing analysis was done to determine IDH1 mutations. The product size of the amplified fragment is 592 bp which spans exon 4 of the enzyme [Accession no. NG_023319.1].

Results: Analysis for IDH2 gene mutation showed that there were 9 patients with R140 mutation in IDH2 gene. Sequencing analysis revealed that amongst 100 patients, 8 patients had different types of mutations in IDH1 gene among whom 5 had mutations associated with R132 amino acid and three patient had mutation associated with silent mutation G105 amino acid of IDH1 (Table 1). Among the five patients having mutation involving R132; two was found to have a substitution from $\mathrm{R}$ to $\mathrm{H}$ (Arginine to His) which is recognized as a key and the most prevalent mutation. The other three patients with R132 mutation were detected to have $\mathrm{R}$ to $\mathrm{C}$ in IDH1. There was a high frequency of mutation at another conserved arginine residue in 109 position of the IDH1 gene fragment in patients [70/100].

Discussion \& Conclusion: The incidence of IDH mutations rounded off to $>10 \%$ which is significant for further investigation and prognosis of AML patients. The co-occurrence of IDH-TET2, IDHDNMT3A and IDH-FLT3 can be studied for further stratification of abnormalities to organize and correlate clinical symptoms and therapeutic care more efficiently. mRNA expression study and biophysical analysis may be done to investigate the impact of different mutations in IDH1 gene to understand their impact better. Supporting Document: 648ca436-7a5b-46b1-a738-7b3b4e50e570.

\begin{tabular}{|l|l|l|l|l}
\hline & $\begin{array}{l}\text { Total no. } \\
\text { of patients } \\
\text { recruited } \\
{[\mathrm{N}=100]}\end{array}$ & $\begin{array}{l}\text { IDH2 (R140) } \\
\text { mutations } \\
\text { found } \\
{[\mathrm{N}=9]}\end{array}$ & $\begin{array}{l}\text { IDH1 (R132) } \\
\text { mutations } \\
\text { found } \\
{[\mathrm{N}=5]}\end{array}$ & $\begin{array}{l}\text { IDH1 (G105) } \\
\text { mutations } \\
\text { found } \\
{[\mathrm{N}=3]}\end{array}$ \\
\hline Mean Age & 38.025 & 51.44 & 42.2 & 54 \\
\hline No. of males & 56 & 5 & 1 & 1 \\
No. of females & 44 & 4 & 4 & 2 \\
\hline Mean WBC count & 50,168 & 55,769 & 64,440 & 24600 \\
\hline Mean Blast \% & 56.33 & 56.11 & 75 & 59.66 \\
\hline \multicolumn{5}{c}{ Table 1 }
\end{tabular}

Disclosure of Interest: None Declared.

Keywords: Acute leukemia, DNA sequencing, IDH mutation.

\section{Abstract 17. A Comparative Study of Research Parameters on Sysmex XN with the Immuno-Phenotype of Blasts}

\author{
Shruti Mishra ${ }^{* 1}$, Rasheeda Mohamedali ${ }^{1}$, Debasish Jhas ${ }^{1}$, \\ Gaurav Chhabra ${ }^{1}$
}

\section{${ }^{1}$ Pathology and Lab Medicine, AIIMS, Bhubaneswar, India}

Aims \& Objectives: The aim of our pilot study was to compare the variations in positional parameters provided by Sysmex XN analyzer with the nature of blasts as determined by multi-color flowcytometry. Patients/Materials \& Methods: This study was conducted in AIIMS, Bhubaneswar. All index acute leukemia cases diagnosed at our center were included. Treated and relapsed cases were excluded from the study. Diagnosis was done either on peripheral blood or bone marrow aspirate. Same sample was processed in Sysmex XN analyzer and Flowcytometer [BD FACS Canto II]. Smears were examined by experienced hematopathologists in all cases. The positional parameters provided by WNR and WDF channels of Sysmex XN were collected. We determined the normal ranges with mean in our laboratory after analyzing 20 normal samples.

Results: 54 cases were included in this pilot study. We had 20 cases each of B-cell precursor Acute Lymphoblastic Leukemia and Acute Myeloid Leukemia. 4 cases of Acute Promyelocytic leukemia, 3 cases of AML-M5a and 7 cases of T-cell ALL out of which 3 were Early T-cell precursor ALL.

Discussion \& Conclusion: We found that NE-WY was the most reliable parameter in predicting acute leukemias. It was uniformly deranged in all types of leukemias. AML and AML with monocytic differentiation, both showed similar pattern of derangement in neutrophilic parameters, while the later showed more increased values in MO-WY. T-cell ALL cases showed lower than normal range values for MO-X while in B-cell ALL cases the value was raised. Acute Promyelocytic leukemia in comparison to AMLs and AMLs with monocytic differentiation showed deranged values for LY-WY and LY-YZ.

We, conclude in our pilot study that the positional parameters can be used as predictors of the nature of blasts and extended study can help us calculate cut-off values for the same.

Disclosure of Interest: None Declared.

Keywords: Acute leukemia, Flow cytometry, Sysmex XN.

\section{Abstract 18. A Novel Machine Learning Derived Genomics-Based Scoring System Is Predictive of Outcome in CBF-AML}

\section{AnamShaikh $^{* 1}$, ChinmayeeKakirde ${ }^{1}$, ShrinidhiNathany ${ }^{1}$, Gaurav Chatterjee ${ }^{1}$, Prashant Tembhare ${ }^{1}$, PapagudiSubramanian $^{1}$, Sumeet Gujral ${ }^{1}$, Manju Sanger ${ }^{1}$, BhausahebBagal $^{1}$, NavinKhattry ${ }^{1}$, Nikhil Patkar ${ }^{1}$ \\ ${ }^{1}$ Hematopathology, Tata Memorial Centre, ACTREC, Navi Mumbai, India}

Aims \& Objectives: Core binding factor acute myeloid leukemia (CBF-AML) is one of the commonest subtypes of AML characterized by a high frequency of somatic mutations. Here we investigated the feasibility of improving risk prediction of CBF-AML using machine learning algorithms.

Patients/Materials \& Methods: Next generation sequencing panel that targeted 50 genes implicated in the pathogenesis of myeloid malignancies was used to sequence 106 patients of CBF-AML accrued over a six year period (2012-2018). Post data analysis, we 


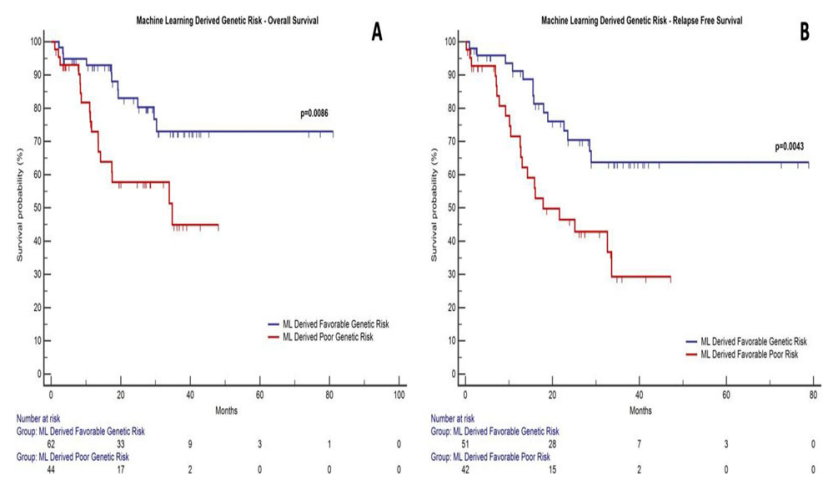

Fig. 1 .

devised supervised machine learning (ML) approach for identification of mutations most likely to predict for favorable outcome in CBFAML. A total of 11 variables were included for feature selection to predict for favorable outcome (including mutations in ASXL2, CSF3R, FLT3, KIT, NF1, NRAS, RAD21, TET2 and WT1 genes as well as mutation burden). Based on the ML results top 6 selected variables were allotted an individual score. A final score for that case was devised as a sum total of the individual scores. These sums were used to generate a genetic risk for a patient. Results of the genetic risk were analyzed for their impact on overall survival (OS) and relapse free survival (RFS) using log rank test. Multivariate analysis was performed using cox proportional hazards regression model.

Results: A total of 181 somatic mutations were identified in this subset of AML with $86.7 \%$ harbouring at least one somatic mutation. Based on ML data, a genetic score was formulated that incorporated mutations in RAD21, FLT3, KIT D816, ASXL2, NRAS genes as well as high mutation burden $(\geq 2)$ into two genetic risk classes (favorable risk and poor ML derived genetic genetic risk). Patients classified as poor genetic risk had a significantly lower OS and RFS as compared to patients with favorable genetic risk (Fig. 1). On multivariate analysis poor genetic risk was the most important independent risk factor that predicted for inferior OS.

Discussion \& Conclusion: We describe a novel ML derived genomics scoring model that provides a mechanism to risk stratify CBFAML, a seemingly homogeneous disease entity and identify CBF mutated AML patients who are at higher risk of relapse.

Supporting Document: 21e2df6f-2bec-4869-8750-ac96dd63f325. Disclosure of Interest: None Declared.

Keywords: Core Binding Factor Acute Myeloid Leukemia, Genomics based scoring sysytem, Machine learning.

\section{Abstract 19. CD304 is a Highly Useful Marker for MRD Detection in Adult B-ALL}

VisheshDudakia $^{* 1}$, PrathyushaGudapati ${ }^{1}$, Gaurav Chatterjee ${ }^{1}$, Sitaram Ghogale ${ }^{1}$, Nilesh Deshpande ${ }^{1}$, Y Badrinath ${ }^{1}$, Nikhil Patkar $^{2}$, P G Subramanian ${ }^{3}$, Prashant Tembhare ${ }^{1}$

${ }^{1}$ Hematopathology (Flow cytometry), ${ }^{2}$ Hematopathology (Molecular), ${ }^{3}$ Hematopathology, Tata Memorial Centre, Navi Mumbai, India

Aims \& Objectives: Neuropilin-1 (NRP-1)/CD304 or blood dendritic cell antigen-4 (BDCA-4) is a nontyrosine kinase transmembrane glycoprotein receptor and acts as a co- receptor for vascular endothelial growth factor (VEGF). CD304 is expressed in plasmacytoid dendritic cells, diverse human solid tumors, and a subset of B cell precursor acute lymphoblastic leukemia (BCPALL). However, information on CD304 expression-pattern in BCPALL and its utility in BCPALL minimal residual disease (MRD) testing is still limited. In this study, we sought out to study the expression pattern of CD304 and its role in the MRD detection in the BCPALL.

Patients/Materials \& Methods: We studied the CD304 (FITC, AD517F6, MB) expression in leukemic-blasts of 135 adult BCPALL samples at baseline and post-induction time-points using 13-color and 10-color flow-cytometric immunophenotyping on Cytoflex and Navios flow-cytometer respectively. Data-analysis was performed using Kaluza-V1.3-software. CD304-expression was considered as positive with $\geq 10 \%$ CD304-positivity. Mean fluorescent intensity (MFI) and coefficient-of-variation of immunofluorescence (CV-IF) of CD304 was measured on logical scale. Cytogenetic studies were performed using FISH and conventional methods.

Results: Median age of 135 patients was 27 years (range 16-57 years). CD304 was positive in 71 (52.6\%) BCPALL cases. Median MFI (range) and percentages of CD304 on blasts at diagnosis were $0.55(0.12-6.8)$ and $22.9(0.3-99.8)$ respectively. Median MFI (range) and percentages of CD304 on blasts among CD304 positive group at diagnosis were $0.77(0.06-9.3)$ and 40.6 (10.5-99.8). At post induction, among CD304 positive group 22/58 cases (38\%) had residual disease with $\geq 10 \% \mathrm{CD} 304$-positivity. At post induction, among CD304 positive group 39/71 cases (55\%) had residual disease with $\geq 10 \%$ CD304-positivity.

Discussion \& Conclusion: CD304 is expressed by leukemic-blasts in $>50 \%$ of the adult BCPALL patients and is a stable marker for MRD detection. Incidence of MRD positivity is higher in CD304 positive adult BCPALL highlighting CD304 expression as a high risk factor.

Disclosure of Interest: None Declared.

Keywords: B-ALL, CD304, MRD.

\section{Abstract 20. Cytogenetic Characterization of Prognostically Relevant Low Hypodiploid/Near Haploid in BCP-ALL}

DhanlaxmiShetty $^{* 1}$, PurviMohanty ${ }^{1}$, HemaniJain ${ }^{1}$, KrutiChaubal $^{1}$, Prashant Tembhare ${ }^{2}$, Chetan Dhamne ${ }^{3}$, Hasmukh Jain ${ }^{3}$, Gaurav Narula ${ }^{3}$, Manju Sengar ${ }^{3}$, NavinKhattry ${ }^{4}$, SripadBanavali ${ }^{3}$

${ }^{1}$ Cancer Cytogenetic Department, ${ }^{2}$ Hematopathology Laboratory, ACTREC, Tata Memorial Centre, Navi Mumbai, ${ }^{3}$ Department of Medical Oncology, Tata Memorial Hospital, Mumbai, ${ }^{4}$ Department of Medical Oncology, ACTREC, Tata Memorial Centre, Navi Mumbai, India

Aims \& Objectives: To characterize rarely identified low hypodiploid and near haploid B-Cell Precursor Acute Lymphoblastic Leukemia (BCP-ALL) patients with respect to cytogenetic abnormalities.

Patients/Materials \& Methods: A large cohort of 407 newly diagnosed BCP-ALL patients were studied by standard cytogenetics, and/ or fluorescence in situ hybridization (FISH) using comprehensive panels for detection of aneuploidies.

Results: Of the total cohort of 407 BCP-ALL patients analyzed between January-July 2019, hypodiploidy ( $<46$ chromosomes) was detected by FISH or ploidy analysis on stained metaphases in 18/407 (4.4\%) cases. Cytogenetically, the hypodiploid cases were further divided into High-hypodiploidy with chromosome number between 40-45 chromosomes in $11 / 18(61.1 \%)$ cases, low-hypodiploidy (31-39 chromosomes) in 5/18 (27.7\%), and near-haploidy (24-30 chromosomes) in $2 / 18(11.1 \%)$ cases. 
The subset of 8 patients with low hypodiploid $(\mathrm{n}=5)$ and near haploid $(n=2)$ clones were further characterized; 2 of the 5 low hypodiploid cases showed mosaicism with both low hypodiploid (31-39 chromosomes) and diploid clone while the other three cases showed unique mosaicism with the presence of hypodiploid and highhyperdiploid/near triploid (doubled) clones with chromosome number between 50 to 78 chromosomes. Similarly, the 2 cases with near haploid clones showed a near haploid clone with 24-30 chromosomes and a doubled clone arising due to duplication of the near haploid chromosome set harboring chromosomes in the high hyperdiploid range (51-67 chromosomes). These cases with masked hypodiploid clones $(\mathrm{n}=5)$ included 3 young adults and 2 paediatric patients and are currently being treated using standard protocol for high-risk ALL. These cases were confirmed to be mosaics by standard cytogenetics, DNA Index (DI) by flow cytometry, and FISH showing tri-tetraploid and monosomic clones on a comprehensive panel encompassing 23 chromosomes.

Discussion \& Conclusion: Hyperdiploidy is associated with a good prognosis in childhood acute lymphoblastic leukemia (ALL) while hypodiploidy is a recurring abnormality detected in approximately $1 \%$ of children with ALL and is associated with an extremely poor prognosis (Safavi et.al 2017). We identified 1.9\% near haploid-low hypodiploid cases which is slightly higher than the studies in the west. Conclusively, a significant proportion cases with hypodiploidy may have been overlooked in previous studies due to the presence of a doubled hypodiploid clone and the absence of hypodiploid interphase/ metaphase cells. An increased awareness among cytogeneticists and clinicians about the unique karyotypic "signature" of a doubled hypodiploid clone coupled with the coordinated use of DI, FISH and LOH studies when indicated are important for the identification of patients with masked hypodiploidy so that they can be assigned to appropriate very high-risk treatment strategies.

Disclosure of Interest: None Declared.

Keywords: acute lymphoblastic leukemia, Ploidy analysis, Risk stratification.

\section{Abstract 21. Leukemia Stem Cells: A Novel Independent Marker for Prognostication of Acute Myeloid Leukemia}

\author{
Priyanka Mishra ${ }^{* 1}$, Seema Tyagi ${ }^{1}$, H P Pati ${ }^{1}$, \\ ManoranjanMahapatra ${ }^{1}$
}

\section{${ }^{1}$ Hematology, AIIMS, Delhi, India}

Aims \& Objectives: The proportion of $\mathrm{CD} 34^{+} \mathrm{CD} 38^{-} \mathrm{CD} 123^{+}$leukemia stem cells (LSCs) at diagnosis of AML have been found to correlate with induction remission, relapse free survival and overall survival in few studies. Most AML patients have normal cytogenetics with negative routinely tested molecular markers, stratifying them as prognostically variable intermediate risk. We aimed to study correlation of proportion of LSCs at diagnosis with absolute blast count, ELN risk group, induction remission (IR), time to remission (TTR) and relapse.

Patients/Materials \& Methods: 47 AML patients were prospectively recruited and immunophenotyping for $\mathrm{CD} 34^{+} \mathrm{CD} 38^{-} \mathrm{CD} 123^{+} \mathrm{LSCs}$ as percent of blasts was done usingsequential gating. Relevant clinical and laboratory data was collected and patients were followed up for IR, TTR and relapse (early and late).

Results: Of 47 patients, 28 achieved induction remission and 19 had no remission or died prior to remission. The proportion of $\mathrm{CD} 34^{+} \mathrm{CD} 38^{-} \mathrm{CD} 123^{+}$LSCs was significantly more $(p=0.001)$ in the non- IR [mean $65.7 \%$, median $86.1 \%$, range $12.1-95.1 \%$ ) versus IR group (mean $27.5 \%$, median $18.4 \%$, range $12.1-85.6 \%$ ) with statistically significant correlation between percentage of LSCs and TTR \{Spearman's correlation coefficient $r_{\mathrm{s}}=0.432$ and $p=0.024\}$.Over mean follow up of6 months, 5 of 28 relapses had higher LSCs at baseline (range $0.8-32.4 \%$, mean 17.4\%) vs the nonrelapse group (range 3-58.7\%, mean $16.6 \%$ ), but not significant. $(p=0.596)$ possibly due to short follow up.ELN 2017 stratified adverse risk group had higher LSCs (mean 34.8\%, SD $=28.13 \%$ ) than favorable risk (mean $13.06 \%, \mathrm{SD}=5.35 \%, p=0.028$ ). In intermediate risk, patients with IR had significantly $(p=0.000)$ lower LSCs (mean 21.18\%, range 3-85.6\%.) than non- IR group (mean = $73.85 \%$, range $12.1-97.9 \%$ ).

Discussion \& Conclusion: The proportion of CD $34^{+} \mathrm{CD} 38^{-} \mathrm{CD} 123^{+}$ LSCs at diagnosis correlate with IR, TTR and risk stratification as per ELN 2017. They are thus emerging independent prognostic marker in AML, especially the patients stratified in the highly variable intermediate risk group due to normal cytogenetics and molecular studies. Disclosure of Interest: None Declared.

Keywords: AML, Leukemia Stem Cell, Prognosis.

\section{Abstract 22. Expression Of CD34, HLA-DR and TDT in Acute Leukaemias: A Single Centre Experience}

Col ProsenjitGanguli ${ }^{* 1,2}$, RehanAhmed ${ }^{3}$, Col Jyoti Kotwal ${ }^{4}$, Brig Ajay Sharma ${ }^{5}$, U D Gupta ${ }^{6}$, Y K Jaiswal ${ }^{7}$, Lt Gen VeluNair ${ }^{8}$

${ }^{1}$ Dept Of Pathology \& Lab Sciences, Command Hospital Alipore Road, ${ }^{2}$ Command Hospital Alipore Road Kolkata, kolkata, ${ }^{3} \mathrm{PhD}$ Fellow,Dept Of Haematology \& BMT, AH(RR), Delhi Cantt, ${ }^{4}$ Haematology, ${ }^{5}$ Haematology \& BMT, SGR Hosp, New Delhi, ${ }^{6}$ Exp Medicine, NJIL \&OMD, AGRA, ${ }^{7}$ School Of Studies in Biochemistry, Jiwaji University, Gwalior, ${ }^{8}$ Haematology \& BMT, Apollo Hospitals, Ahmedabas, India

Aims \& Objectives:Aim: Evaluate Immunophenotypic Expression of CD34,HLA-DR \&TdT in Acute Leukaemias of Different Lineages at Presentation.

Objectives:

1. To evaluate morphologically evidenced and flow cytometrically evaluated Acute Leukaemias.

2. To compare \& correlate the expression of CD34,HLA-DR $\&$ TdT in these cases.

Patients/Materials \& Methods: Patients: This study included n $=132$, morphologically diagnosed cases of acute leukaemia of different lineage (Acute myeloid leukaemia, Acute lymphoblastic leukaemia \& CML in blast crisis) based on evaluation of PBS/BM samples from latter half of 2013 till 2014. All the cases of acute leukaemia were diagnosed \& classified into various types of Acute Leukemias on the basis of their cell surface/cytoplasmic markers profile. The study was done utilising CE/IVD approved reagents using flow cytometry. Retrospective statistical analysis was performed to estimate distribution of expression of CD34, HLA-DR and TdT in acute leukaemia.

Material \& Methods: Immunephenotyping was performed in the Army Hospital Research \& Referral (R\&R) Delhi, on the basis of morphological analysis of PBS/BM samples. Flow cytometer (FC500, Beckman Coulter, USA) was used for diagnosis of acute leukaemia of all lineages. FCM staining, acquisition and analysis were performed to diagnose and subtyping of acute leukaemia. FCM was standardized $\&$ titre as per composed protocol for various marker profiles with subtyping like acute lymphoblastic leukaemia (B-ALL, T-ALL), acute myeloid leukaemia (AML-M0, AML-M1, AML-M2, AML-M3, AML-M4, AML-M5, AML-M6 \& AML-M7). The gating strategy were FSC Vs SSC and SSC Vs CD45 dim and the blast population gated within the dot plot during acquired cell population (minimum 50,000 Cells). 
Results: Observations \& Results: Co-expression analysis of cell surface/cytoplasmic markers, sequential gating strategy for surface markers progression and back gating strategy for mixed phenotypic acute leukaemia were very useful strategy during cell analysis through flow cytometer. Total $n=132$ cases, AML $(n=44) ; 33.33 \%$, MPAL $(\mathrm{n}=11) ; 8.33 \%$, APML $(\mathrm{n}=3) ; 2.3 \%$, MDS $(\mathrm{n}=8) ; 6 \%$, CML $(\mathrm{n}=9) ; 6.8 \%$, B-ALL $(\mathrm{n}=25) ; 18.9 \%$, T-ALL $(\mathrm{n}=13)$; $9.85 \%$, ALL $(n=6) ; 4.5 \%$ and no clear opinion $(n=13) ; 9.85 \%$. Immunophenotypic profile expressed the maximum $26 \%$ for CD34 and HLA-DR in blast cells, the second most expressed markers combination was CD34, HLA-DR and TdT was $19 \%$ followed by $11 \%$ of CD34 and other $11 \%$ of HLA-DR \&TdT. Rest of the individual or combined markers expressed $8 \%, 7 \% \& 5 \%$ respectively for TdT, HLA-DR \& CD34 \&TdT. In APML CD34 \& HLA-DR observed negative as WHO 2008 recommendation.

Discussion \& Conclusion: These specific phenotypic markers expressed individually and in combination of each other irrespective of specific lineages acute leukaemias. This analysis may help for prediction of progression \& aggression of malignant diseases of haematological origin.

Supporting Document: f924277f-2427-4af8-bb07-584c69a9c542.

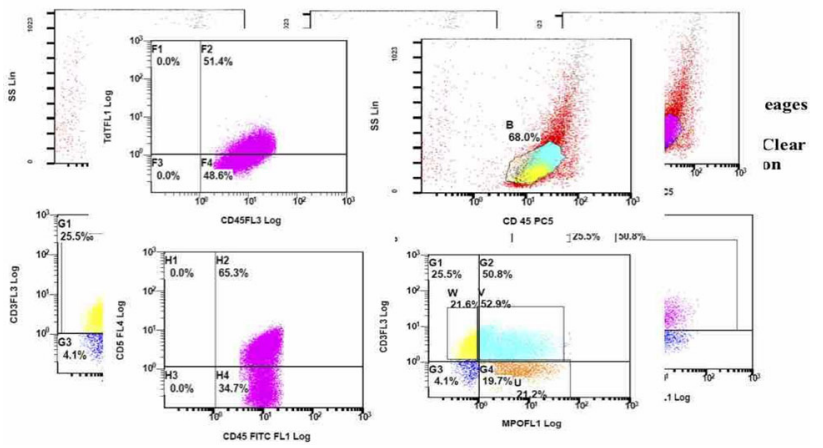

Disclosure of Interest: None Declared.

Keywords: Acute leukemia, Flow cytometry, IMMUNOPHENOTYPING.

\section{Abstract 23. Isolated Central Nervous System Relapse in Acute Lymphoblastic Leukemia, A Series of Five Cases}

\section{S Venkatesan ${ }^{* 1}$, Isha Sharma ${ }^{1}$, SanjeevanSharma ${ }^{2}$, Ajay Malik ${ }^{1}$ \\ ${ }^{1}$ Dept of Pathology, ${ }^{2}$ Dept of Hematology, Armed Forces Medical College, Pune, India}

Aims \& Objectives: We here collate the clinical and hematological parameters of known cases of ALL on therapy/follow up, presenting with isolated CNS relapse.

Patients/Materials \& Methods: 05 ALL cases presented to this hospital between 2015 and 2019 with symptoms varying from headache to fever with abdominal distension. These were known cases of ALL, who were under therapy or on follow up after therapy. All cases underwent CSF examination, which was the primary modality of diagnosis. Peripheral smear and bone marrow examination were also carried out to rule out a systemic relapse.

Results: The patients were between 07 months to 42 yrs of age. 03 were females. 02 out of 05 patients, presented with isolated CNS relapse after 3.5 years of completed therapy but during the course of illness, developed bone marrow infiltration and succumbed to their illness, while others without medullary involvement are under follow up and are clinically asymptomatic. At the time of diagnosis, karyotyping revealed $t(12,21), t(1,14), t(9,22)$ in different patients. 01 was a case of T-Cell ALL. Patients were managed with GMALL induction along with 10 cycles of maintenance.

Discussion \& Conclusion: ALL is the most common hematolymphoid malignancy in children accounting for almost $75 \%$ of childhood leukemias. $70-80 \%$ of cases achieve complete remission. These patients may also exhibit CNS involvement. Relapse in the CNS may occur alone or with accompanying bone marrow infiltration. Advances in chemotherapy and effective CNS prophylaxis have significantly decreased the incidence of CNS relapse of ALL to $5-10 \%$. A high degree of suspicion is essential to identify these cases of isolated CNS relapse, so that definitive therapy be offered at the earliest.

Disclosure of Interest: None Declared.

Keywords: Acute lymphoblastic Leukaemia, CNS disease, Relapse.

\section{Abstract 24. A Novel KMT2A Fusion in a Case of Early T-Cell Precursor Acute Lymphoblastic Leukemia}

Arusha Shetty $^{* 1}$, Shilpi More ${ }^{1}$, Prasanna Bhanshe ${ }^{1}$, DurvaRaut ${ }^{1}$, SwetaRajpal $^{1}$, Shruti Chaudhary ${ }^{1}$, SwapnaliJoshi ${ }^{1}$, Prashant Tembhare ${ }^{1}$, Sumeet Gujral ${ }^{1}$, PapagudiSubramanian ${ }^{1}$, Nikhil Patkar ${ }^{1}$

${ }^{1}$ Hematopathology, Advanced Centre For Treatment, Research, And Education In Cancer, Mumbai, India

Aims \& Objectives: Early T-cell precursor acute lymphoblastic leukemia (ETPALL) is characterized by a unique immunophenotype with limited early T-cell differentiation and poor overall survival. Mutational landscape of ETPALL has been found to be similar to that of myeloid leukemias rather than other T-cell leukaemias. A number of fusions have been described in T-ALL, detected in almost 55\% cases of T-ALL. We describe a novel fusion between KMT2A and MTMR2 in a 31-year-old male with ETPALL.

Patients/Materials \& Methods: DNA and RNA extraction were doneusing peripheral blood of the patient.For DNA sequencing, we used a 51 gene $(151.5 \mathrm{kB})$ low-cost hybrid capture based targeted sequencing myeloid panel involving single molecule molecular inversion probes implicated in myeloid malignancies. All samples were sequenced at high coverage using this assay on an Illumina Miseq. For RNA sequencing, double stranded cDNA was synthesized and then fragmented, A-tailed and ligated using half functional sequencing motifs. We designed the panel (ABL2, ABL1, CSF1R, ETV6, FGFR1, IKZF3, KMT2A, MEF2D, MLL, NTRK3, NUP98, NUP214, P2RY8, PAX5, RUNX1, TCF3, ZNF384, CRLF2, DUX4, $E B F 1, E P O R, J A K 2, P D G F R B, P D G F R A, T Y K 2)$ to detect any putative partner of a fusion gene where one of the partners is known. Illumina Miseq (300-cycle chemistry) was used to perform paired end sequencing. Data was analysed using STAR-Fusion-v1.2.0, Fusion Catcher and Fusion-Inspector. Custom scripts and bedtools-2.25 were used to determine the coverage and construct the pipeline.

Results: A 31-year-old male, presented with leucocytosis $\left(61.5 \times 1 \hat{0}^{9} /\right.$ L) with $80 \%$ blasts on peripheral smear and was diagnosed as ETPALL on flow-cytometry. FISH revealed $K M T 2 A$ translocation in $50 \%$ cells. RNA sequencing revealed $K M T 2 A-M T M R 2$ fusion with breakpoint at exon 8 of $K M T 2 A$ and exon 3 of MTMR2. Patient was started on BFM 90 protocol (high-risk group) for ALL. 
Discussion \& Conclusion: KMT2A translocations have been reported in a diverse array of pediatric and adult myeloid and lymphoid leukemias and are seen in $8 \%$ cases of T-ALL. More than 100 KMT2A fusion partner genes have been described. However literature search has not revealed any fusion with MTMR2 gene which has been described primarily in solid tumors. This novel fusion might help in refining our knowledge on the disease pathogenesis.

Disclosure of Interest: None Declared.

Keywords: ETPALL, KMT2A, MTMR2.

\section{Abstract 25. Genomic Landscape of Pediatric Acute Myeloid Leukemia}

SwapnaliJoshi $^{* 1}$, Nikhil Patkar ${ }^{1}$, Shruti Chaudhary ${ }^{1}$, SwetaRajpal $^{1}$, SumitGujral ${ }^{1}$, PgSubramanium ${ }^{1}$

\section{${ }^{1}$ Hematopathology, Tata Memorial Centre, Actrec, Kharghar, India}

Aims \& Objectives: Acute myeloid leukemia (AML) is a genetically heterogenous disease and recent advances in genetics testing have helped us to identify mutations that are therapeutically targetable. However, the genetic landscape of paediatric AML is different from adults. Hence, we aimed to study the genomic landscape of paediatric AML patients by comprehensively evaluating the mutational profile using high through-put next generation sequencing (NGS).

Patients/Materials \& Methods: This was a retrospective study. Paediatric AML cases registered in Tata memorial centre from Jan 2018 to July 2019 were enrolled in the study. Clinical and cytogenetics data were retrieved from the electronic database. DNA was extracted from blood/bone marrow samples using Qiagen Gentra Pure gene method. For mutational screening we developed a 51 gene $(151.5 \mathrm{kB})$ low-cost hybrid capture based targeted sequencing myeloid panel involving single molecule molecular inversion probes implicated in myeloid malignancies. All samples were sequenced at high coverage using this assay on an Illumina Miseq. Sequencing data analysis was done using custom pipeline.

Results: Total of 125 patients were included with median age at diagnosis was 8 years (range $0-15$ years). Among 125 cases, the most frequent mutation was found in the RAS/MAPK pathway genes (n-53, $42.4 \%)$. This was followed by mutations in the $K I T(20,25 \%), A S X L$ $(10,8 \%)$, and FLT3 $(12,9.6 \%)$ gene. Interestingly, $44.8 \%$ (n-56) of children had more than one mutation. Six $(4.8 \%)$ cases showed coexistence of NRAS and FLT3 gene mutation. Less frequently mutated gene that we found were GATA1, GATA2, JAK2, PTPN11, NPM1, RUNX1, STAG2, WT1, and U2AF. 13 (10.4\%) patients did not reveal any mutations. Among the core binding factor leukemia group $(\mathrm{n}-28,21.6 \%)$, the most frequent mutation was seen in the KIT gene (n-10, 37\%).

Discussion \& Conclusion: In our study, $89.6 \%$ of paediatric leukemia cases harbour at least one mutation. Unlike in adult AML patients who most frequently harbour mutations in the FLT3 gene, mutations in the genes involving the RAS/MAPK pathway were most frequent in our cohort. NGS in these patients helps to identity the presence of mutations that can be therapeutically targetable as well help in predicting prognosis.

Disclosure of Interest: None Declared.

Keywords: Acute myeloid leukemia, Mutational spectrum, Next generation sequencing.
Abstract 26. Scatterplot Patterns of Acute Myeloid Leukemia with Cup Shaped Blast in Sysmex XN-Series Analyser

Aparna N ${ }^{* 1,1}$, AbhirupSarkar ${ }^{1}$, ShreyamAcharya ${ }^{1}$, Vijay Antony ${ }^{1}$, Ruchi Tiwari ${ }^{1}$, Renu Saxena ${ }^{1}$

${ }^{1}$ Department of Laboratory Medicine, All India Institute Of Medical Sciences, Delhi, India

Aims \& Objectives: To analyse the scatter plot patterns of diagnosed AML with cup shaped blast in the peripheral smear.

Patients/Materials \& Methods: Scatter plots from Sysmex XN series were retrieved for four diagnosed cases of AML with cup shaped blast in peripheral smear. The WDF and WNR plots of all four cases were analysed.

Results: WDF SSC -SFL plot of all 4 cases of AML showed near identical characteristic pattern. The cells appear to arise from the lymphocyte region, slightly dips then encompasses the monocyte region, finally merging together and rising towards area of higher SFL giving the characteristic "inverted heart" shape. The right aspect of this inverted heart is more accentuated than the left aspect in all 4 cases. WNR plots were insignificant in all 4 cases.

Discussion \& Conclusion: Review of available literature revealed only studies which dealt with the mutational analysis of cases of AML demonstrating cup shaped blasts. There was no available academic precedent which deals with analysis of scatterplot pattern of such cases. Identification of the characteristic pattern in the WDF SFL-SSC pattern in scatter plot may help in early diagnosis of AML with cup shaped blast. This study hopes to provide a novel diagnostic approach to these diseases. For this purpose, future large scale scatterplot studies and statistical cum mutational analyses are required to establish this pattern as a definitive diagnostic criteria of such leukemias.

Supporting Document: 68869573-285d-4571-9ecd-ff51256e3ffb.

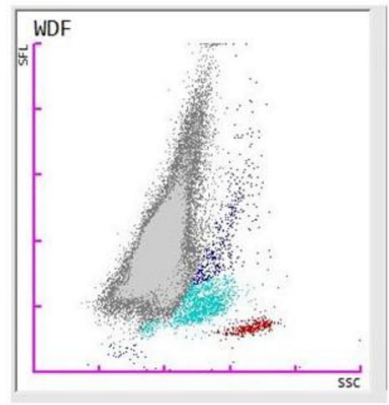

Patient 1

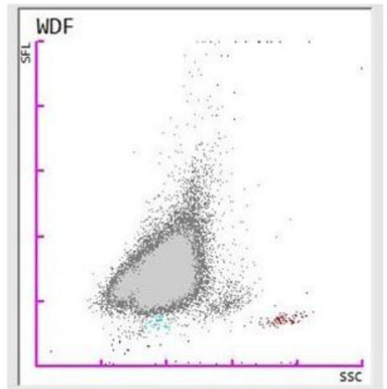

Patient 3

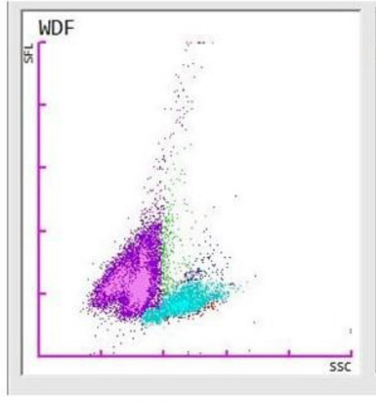

Patient 2

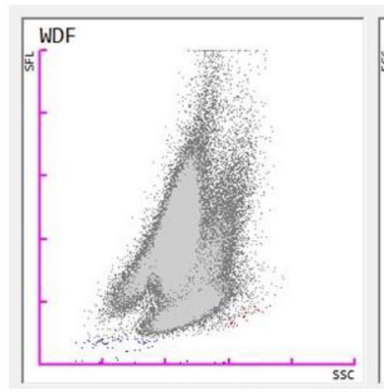

Patient 4
Disclosure of Interest: None Declared.

Keywords: AML, Cup-shaped-blast, Sysmex XN. 


\section{Abstract 27. Aberrant Antigen Expression in Acute Leukemia on Flowcytometry: A Tertiary Care Centre Experience}

\author{
LovekeshMonga $^{* 1}$, Monika Gupta ${ }^{2}$, Sunita Singh ${ }^{3}$, Rajeev Sen ${ }^{3}$ \\ ${ }^{1}$ Pathology, PT BDS, PGIMS, Rohtak, ${ }^{2}$ Pathology, PT BDS, \\ PGIMS, Rohtak, ${ }^{3}$ Pathology, PT BDS, PGIMS, Rohtak, Rohtak, \\ India
}

Aims \& Objectives: To evaluate the frequency of aberrant antigen expression in acute leukemia on immunophenotyping.

Patients/Materials \& Methods: The study included 100 patients in whom both bone marrow aspiration and flowcytometry were performed. Acute leukemia patients with blasts $>20 \%$ of all age groups were included in the study. Flowcytometric analysis (FCA) was done using the monoclonal antibody panel of acute leukemia on Peripheral blood/Bone marrow.

Results: Out of 100 cases of Acute Leukemia, 53 cases were categorised as AML, 43 cases as ALL and 4 cases MPAL. ALL were subcategorised on the basis of IPT into B-ALL and T-ALL which comprised of $88 \%$ and $12 \%$ total ALL (43\%) cases.

Discussion \& Conclusion: CD33 and CD13 were the most commonly expressed antigens in AML with CD7 being the most common aberrancy. CD19 was expressed in all cases of B-ALL followed by cCD79a, CD10, Tdt (86.8\%) with CD13 being the most common aberrancy in B-ALL. cCD3, CD7 and CD5 were expressed in all cases of T-ALL with aberrant antigen expression in $80 \%$ of cases of T-ALL.MPAL cases showed expression of B/Myeloid antigens.The presence of aberrancy helps to identify a neoplastic process. It has been demonstrated that several immunophenotypes of blast cells from cases of AL do not exhibit the features of normal cellular differentiation but show the expression of aberrant markers. To conclude aberrant immunophenotypes are quiet common in $\mathrm{AL}$ and have important implications for the diagnosis, monitoring and also establish baselines for subsequent detection of MRD.

Disclosure of Interest: None Declared.

Keywords: Aberrant antigen, Acute leukemia, flowcytometry.

\section{Abstract 28. Comparative Study Between Two Hematology Analyzers for Platelet and Its Indices in Cancer Patients}

\section{UlkaGosavi $^{* 1}$, PreetiChavan ${ }^{1}$, ManikchandraTiwari ${ }^{1}$, Sanjay KUMAR ${ }^{1}$ \\ ${ }^{1}$ Hemato-oncology, Actrec, Tata Memorial Centre, Navi Mumbai, India}

Aims \& Objectives: To compare performance of haematology cell counters BC6000-Mindray and Advia2120i-SIEMENS for Platelet parameters in an oncology centre.

Patients/Materials \& Methods: In this correlation study we compared Platelet parameter like Platelet Count (PLT), Mean Platelet Volume (MPV), Platelet Distribution Width (PDW), Large Platelets (LP) and Immature Platelet Fraction (IPF) results obtained from two different cell counters BC6000-Mindray and Advia2120i-SIEMENS and correlated them statistically. We analysed blood samples of 804 cancer patients which were received for routine analysis in the laboratory on SIEMENS cell counter-Advia 2120i. Left over samples were processed on BC6000-Mindray cell counter as part of validation exercise. Results obtained for PLT, MPV, PDW, LP/IPF parameter were compared between the two analysers.
The results of linear regression analysis

\begin{tabular}{llll}
\hline Parameters & Units & $\begin{array}{l}\text { Correlation } \\
\left(\mathrm{r}^{2}\right)\end{array}$ & $\begin{array}{l}\text { coefficient } \\
(\mathrm{y})\end{array}$ \\
\hline PLT & $\begin{array}{l}\text { Slope intercept } \\
\mathbf{4 L} \text { cells/ }\end{array}$ & $\mathbf{0 . 9 8 9 7}$ & $\mathbf{1 . 0 0 6 4} \times$ \\
$\mathrm{MPV}$ & $\mathrm{fL}$ & 0.012 & $1.2769 \times$ \\
$\mathrm{PDW}$ & $\% \mathrm{CV}$ & 0.1702 & $3.443 \times$ \\
$\mathrm{LP} / \mathrm{IPF}$ & $\times 10^{3}$ cells/ & -0.109 & $1.0246 \times$ \\
& $\mu \mathrm{L}$ & & \\
\hline
\end{tabular}

Results: Platelet and its indices results of 804 cancer patients obtained from both the cell counters were evaluated using linear regression analysis. Coefficient of correlation $\left(\mathrm{r}^{2}\right)$ more than 0.9 and a slope of 1 were considered as significant.

Discussion \& Conclusion: For cancer patients, PLT, MPV, PDW, LP are some of important parameter which helps in the course of treatment. These parameters help the clinicians to evaluate bleeding tendencies of patients, and guide the clinicians in administering chemotherapy drugs to the patients. MPV, PDW, LP gives indication for thrombopoesis and also tells us about aniso-poikilocytosis of platelets, which is very important for cancer care.

In our study, parameters like PLT showed good correlation and minimal bias among the results obtained from the two cell counters. Among the Platelet Indices parameters MPV, PDW, LP/IPF did not show any correlation.

It can be concluded that although BC6000-Mindray shows good correlation with Advia 2120i for Platelet count; however, there was no correlation for MPV, PDW, LP/IPF. Advia2120i-SEIMENS showed better comparability for MPV, PDW, LP with peripheral stained smears using microscopy.

Disclosure of Interest: None Declared.

Keywords: Cell counters, Correlation study, Platelets.

\section{Abstract 29. Precursor B- Acute Lymphoblastic Leukemia with TCF3-HLF Fusion}

Deepika Yadav $^{* 1}$, SwetaRajpal ${ }^{1}$, Prasanna Bhanshe ${ }^{1}$, Shruti Chaudhary ${ }^{1}$, SwapnaliJoshi ${ }^{1}$, Gaurav Chattarjee ${ }^{1}$, Prashant Tembhare ${ }^{1}$, Sumeet Gujral ${ }^{1}$, PapagudiSubramanian ${ }^{1}$, Nikhil Patkar ${ }^{1}$

\section{${ }^{1}$ Hematopathology, Tata Memorial Centre, ACTREC, Navi Mumbai, India}

Aims \& Objectives: TCF3 gene encodes for transcription factor responsible for B cell development and $H L F$ gene encodes for transcription factor of hematopoietic stem cell. Precursor B lineage acute lymphoblastic leukemia (B-ALL) cases with TCF3-HLF are rare and are associated with a high risk of relapse. The 5-year event free survival in patients with this fusion is 0 . Clinically these patients are known to present with features of disseminated intravascular coagulation (DIC). The patients with this fusion are known to have bright CD20 expression. These patients are found to respond to Blinatumomab and hematopoietic stem cell transplant. Here we describe four cases of B-ALL with rare TCF3-HLF fusion detected using inexpensive laboratory developedtargeted RNA sequencing.

Patients/Materials \& Methods: Double stranded cDNA was synthesized and then fragmented, A-tailed and ligatedusing half functional sequencing motifs.As a part of B-ALL workup, laboratory 
developed targeted RNA sequencing using primers for $A B L 1, A B L 2$, CSF1R, CRLF2, DUX4, EPOR, ETV6, EBF1, FGFR1, IKZF1, JAK2, KMT2A, MLLT4, MEF2D, NTRK3, NUP214, NUP98, P2RY8, PAX5, PDGFRA, PDGFRB, RUNX1, TCF3, TYK2 and ZNF384was done in Illumina Miseq (300-cycle chemistry)designed so as to detect any presumed partner of a fusion gene. Analysis of FASTQ files of fusion assay was done using OncofuseandFusionCatcher.

Results: Fourcaseswere found to haveTCF3-HLF fusion. The median age of these patients was 10 years (range: 7-41 years). Three cases were female and only one was male. Only one patient presented with features of DIC. CD20 expression was moderate in all the four cases. Two patients succumbed to their illness within 15 days of diagnosis. Third patient had not achieved remission post-induction (MRD-8.6\%) and succumbed to her illness at 12 months of diagnosis though treatment intensification was done. Fourth patient also did not achieve post-induction remission (MRD-6.5\%) and is on regular follow-up. The relevant clinical and laboratory details are summarized in Table 1.

Discussion \& Conclusion: We detected a rare fusion (TCF3-HLF) using a novel low-cost technique that can be utilized to discover unique fusions genes by using the knowledge of one partner from the fusion. Targeted NGS helps to detect clinically rare fusions as described in these cases which can guide us for appropriate treatment and patient care.

Supporting Document: 273c9fe7-010f-4f0e-b49d-d68cd793375a.

Table 1. Clinical and laboratory findings of cases of B-ALL with TCF3-HLF fusion

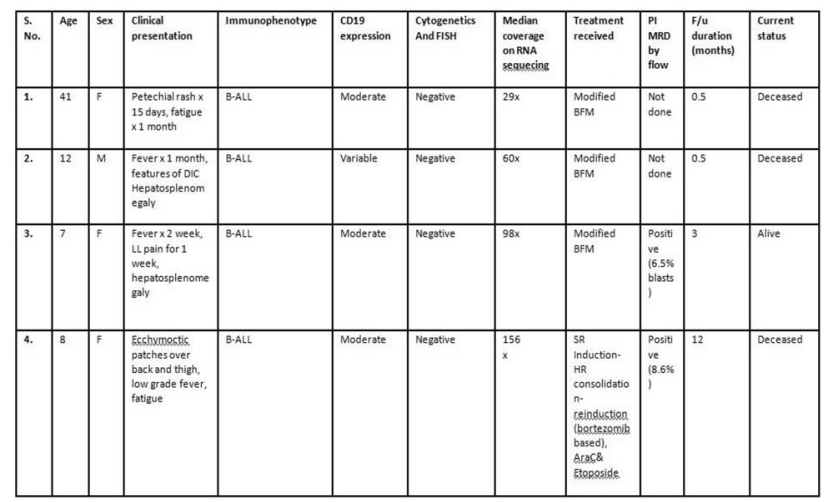

Disclosure of Interest: None Declared.

Keywords: B-ALL, RNA sequencing, TCF3-HLF.

\section{Abstract 30. To Explore the Utility of Diagnostic Immunophenotypes for Detection of MRD in B-ALL}

Nupur Das ${ }^{* 1}$, RituGupta ${ }^{1}$, Ankit Malhotra ${ }^{1}$, Sanjeev K Gupta ${ }^{1}$, Saroj Singh $^{2}$, Sandeep Rai ${ }^{2}$, Vijay K. Prajapati ${ }^{2}$, Ranjeet K. Sahoo ${ }^{3}$, Sameer Bakshi ${ }^{3}$, Lalit Kumar ${ }^{3}$

${ }^{1}$ Laboratory oncology, B.R.A. IRCH, ${ }^{2}$ Laboratory Oncology, B.R.A. IRCH, ${ }^{3}$ Medical Oncology,B.R.A. IRCH, AIIMS, New Delhi, India

Aims \& Objectives: To find out the usefulness of diagnostic immunophenotypes in assessment of measurable residual disease (MRD) in B-ALL.

Patients/Materials \& Methods: A total of 171 B-ALL patients were evaluated for MRD evaluation at post-induction time point. All the samples were collected in EDTA and processed within $24 \mathrm{~h}$ of collection using bulk-lysis protocol. Data was acquired on Gallios Flow
Cytometer and analyzed with Kaluza V2.0 software. A single tube 10-colour panel was used for evaluation at diagnosis as well as MRD assessment time points.

Results: MRD assessment was done by three independent investigators combining both difference from normal (DFN) and leukemia associated aberrant immunophenotype (LAIP) approach and final consensus on discrepancies was reached by joint evaluation. Out of 171 patients, $36.84 \%(n=63)$ were MRD positive and $63.15 \%$ $(\mathrm{n}=108)$ were negative for MRD. Baseline immunophenotype (IPT) was not required in $91.81 \%$ (157/171) of cases. In $8.18 \%(14 / 171)$ cases, review of diagnostic IPT was required. These included 10 cases with a flowcytometric pattern overlapping with stage I/II hematogones and 04 cases with under expression of CD10. The MRD assessment in cases with under expression of CD10 was achieved using CD123 and CD81. Among MRD positive cases, 35/63 (55.55\%) showed presence of $\geq 2$ LAIPs and 28/63 (44.44\%) had $\leq 2$ LAIPs but were evaluable with DFN approach.

Discussion \& Conclusion: Combining both difference from normal (DFN) and leukemia based immunophenotype (LAIP) approach, nearly $92 \%$ of cases can be evaluated. However, baseline IPT is needed for reliable MRD diagnosis in $8 \%$ of the cases. Hematogone pattern is most challenging in MRD assessment by multiparametric flow cytometry as it can lead to false negative result. Identification and validation of additional flow cytometric markers which can differentiate leukemic blasts from hematogones, is required. Till that time, careful evaluation of baseline immunophenotype is warranted in seemingly MRD negative cases with hematogone pattern.

Conclusion: Baseline IPT is not mandatory for B-ALL MRD analysis by flowcytometry as it is required in less than $10 \%$ of cases. However, it is advised to explore more and more aberrant markers in cases with hematogone pattern to make a reliable MRD assessment in B-ALL. Disclosure of Interest: None Declared.

Keywords: B-ALL, flowcytometry, MRD.

\section{Abstract 31. Validations and Implementation of Combined DNA/RNA Sequencing in Myeloid Neoplasms}

KunjalPatel $^{* 1}$, Poonam santra ${ }^{1}$, Kallolsaha ${ }^{1}$, Saurabh Bhave ${ }^{2}$, Vivek Radhakrishnan ${ }^{2}$, Reena Nair ${ }^{2}$, MammenChandy ${ }^{2}$, Mayur Parihar ${ }^{1}$, Deepak Mishra ${ }^{1}$, Neeraj Arora ${ }^{1}$

${ }^{1}$ Molecular genetics and lab Haematology, ${ }^{2}$ Clinical Haematology, Tata Medical Center Kolkata, Kolkata, India

Aims \& Objectives: The aim of this study was to evaluate and validate oncomine Myeloid Research Assay (comprising of 40 DNA target genes, 29 driver genes and a broad fusion panel) for establishing assay sensitivity, specificity and precision using Ion torrent PGM.

Patients/Materials \& Methods: Archival DNA samples $(\mathrm{n}=17)$ with known mutations in (FLT3-ITD, CEBPA, NPM1, PTPN11, cMPL1, IDH1/2,TET, EZH2, U2AF1, SETBP1, ASLX1, TP53) and RNA samples (n = 11) with FISH/RT-PCR confirmed fusions (NUP 214, RUNX1, PML RARA BCR 1, PML RARA BCR 3, MLLT3, B14A3, B19A2) were retrieved as positive controls and negative controls for the validation. The sequences were analyzed using variant calling software (Ion Reporter Annotate variants version 5.4, 5.6 and 5.10) caller to identify variants relevant to the clinical indication. After thorough optimization and validation, this panel was used for prospective analysis in patients withmyeloid disorders [AML $(n=17)$, MDS $(n=7)$, MPN $(n=3)$ ] $\operatorname{AUL}(\mathrm{n}=1)$ and anaemia $(\mathrm{n}=1)$.

Results: In the four validation runs (318 chip - 4 DNA/RNA) comprising 28 known samples, all mutations/rearrangements were 
concordant except two cases $(F L T 3-I T D>200$ bp and SETBP1, G870S $3.2 \%$ ). For the prospective myeloid disorders (27), mutations were observed in 24 genes, maximum cases having FLT3 $(\mathrm{n}=4)$ and NRAS ( $\mathrm{n}=4)$ mutations. The second most frequently mutated genes were IDH1, RUNX1, ASXL1, U2AF1, TET2, SH2B3, CEBPA. Seventeen of the 29 patients had mutations in $>1$ gene, six cases had single gene mutations and no mutation was detected in five patients. In four cases fusions were detected, BCR-ABL $(\mathrm{n}=1), M L L(\mathrm{n}=1), P M L-R A R A$ (bcr3) ( $\mathrm{n}=1)$, and $R U N X 1 T 1(\mathrm{n}=1)$. The RUNXIT1 rearranged AMLM2 and had additional mutation in NRAS. The PML-RARA (bcr3) was FISH negative and had additional mutation in $S F 3 B 1$ gene.

Discussion \& Conclusion: We demonstrate the validation and implementation of a combined targeted DNA and RNA sequencing for routine clinical use in myeloid malignancies.

Disclosure of Interest: None Declared.

Keywords: Myeloid neoplasm, Next generation sequencing, Validations.

\section{Abstract 32. Correlation Study Between Two Haematology Cell Counters for Complete Blood Count in Cancer Patients}

\author{
ManikchandraTiwari $^{* 1}$, PreetiChavan ${ }^{1}$, Sanjay KUMAR ${ }^{1}$, \\ UlkaGosavi $^{1}$ \\ ${ }^{1}$ Hemato-oncology, ACTREC, Tata Memorial Centre, Navi \\ Mumbai, India
}

Aims \& Objectives: To compare performance of hematology cell counters BC6000-Mindray and Advia2120i-SIEMENS for Complete Blood Count (CBC) test in an oncology centre.

Patients/Materials \& Methods: In this correlation study we compared CBC results obtained from two different cell counters BC6000Mindray and Advia2120i-SIEMENS and correlated them statistically. We analysed blood samples of 813 cancer patients which were received for routine analysis in the laboratory on SIEMENS cell counter-Advia 2120i. Left over samples were processed on BC6000-Mindray cell counter as part of validation exercise. Results obtained for WBC, HGB, PLT, Neutrophils, Lymphocytes, Monocytes, Eosinophils, Basophils and RDW-CV parameter were compared between the two analysers.

The results of linear regression analysis

\begin{tabular}{llll}
\hline Parameters & Units & $\begin{array}{l}\text { Correlation } \\
\text { cient }\left(\mathrm{r}^{2}\right)\end{array}$ & $\begin{array}{c}\text { coeffi- } \\
\text { Slope inter- } \\
\text { cept }(\mathrm{y})\end{array}$ \\
\hline WBC & $\begin{array}{c}\mathbf{\times 1 0}^{\mathbf{3}} \text { cells/ } / \\
\boldsymbol{\mu} \mathbf{L}\end{array}$ & $\mathbf{0 . 9 9 7}$ & $\mathbf{0 . 9 3 3 7 \times}$ \\
Neutrophil & $\%$ & $\mathbf{0 . 9 1 1 8}$ & $\mathbf{0 . 9 7 2 5 \times}$ \\
Lymphocyte & $\%$ & 0.8448 & $0.867 \times$ \\
Monocyte & $\%$ & 0.6086 & $0.762 \times$ \\
Eosinophil & $\%$ & 0.8816 & $0.9111 \times$ \\
Basophil & $\%$ & 0.7115 & $2.3848 \times$ \\
Hemoglobin & $\mathbf{g} / \mathbf{d L}$ & $\mathbf{0 . 9 9 3 6}$ & $\mathbf{0 . 9 9 1 8} \times$ \\
RDW-CV & $\%$ & 0.3989 & $0.9952 \times$ \\
Platelet & $\times \mathbf{1 0}$ cells/ & $\mathbf{0 . 9 8 5}$ & $\mathbf{1 . 0 1 3 8 \times}$ \\
& $\boldsymbol{\mu L}$ & &
\end{tabular}

Results: CBC results of 813 cancer patients obtained from both the cell counters were evaluated using linear regression analysis. Coefficient of correlation $\left(\mathrm{r}^{2}\right)$ more than 0.9 and a slope of 1 were considered as significant.

Discussion \& Conclusion: For cancer patients, White Blood Cell count, Absolute Neutrophil Count (calculated from Neutrophil), Hemoglobin and Platelet are some of important parameter in the course of treatment. These parameters help the clinicians in evaluating the cell counts, bicytopenia, pancytopenia, anemia status, and guides the clinicians in administering chemotherapy drugs to the patients.

In our study, parameters like WBC, Neutrophil, Hemoglobin and Platelet showed good correlation and minimal bias among the results obtained from the two cell counters.

Among the differential parameters Lymphocyte, Monocyte, Eosinophil and Basophil did not show good correlation. RDW-CV showed no correlation.

It can be concluded that although BC6000-Mindray shows good correlation with Advia $2120 \mathrm{i}$ for few important CBC parameters; Advia2120i-SIEMENS showed overall better performance for important parameters and also for differential counts and RDW-CV. Disclosure of Interest: None Declared.

Keywords: Cell counters, Complete Blood Count, Correlation study.

\section{Abstract 33. Spectrum of Cytogenetic Abnormalities in Paediatric \\ B- Other Acute Lymphoblastic Leukaemia}

Tanvi Gupta ${ }^{* 1}$, Arun S R ${ }^{1}$, Sipra Rani Patel ${ }^{1}$, Ashish BabuGorantla ${ }^{1}$, Neeraj Arora ${ }^{1}$, Deepak Kumar Mishra ${ }^{1}$, NiharenduGhara $^{2}$, Arpita Bhattacharya ${ }^{2}$, VaskarSaha ${ }^{2,3}$, Shekhar Krishnan ${ }^{2,3}$, Mayur Parihar ${ }^{1,3}$

${ }^{1}$ Department of Cytogenetics and Lab Haematology, ${ }^{2}$ Department of Paediatric Haematology and Oncology, ${ }^{3}$ Tata Translational Cancer Research Centre, Tata Medical Center, Kolkata, India

Aims \& Objectives: Risk stratification based on cytogenetic abnormalities is an essential aspect of modern treatment protocols in BCPALLs. Aneuploidies, ETV6-RUNX1, BCR-ABL1 fusions and KMT2A gene rearrangement designate patients into either standard risk or high risk subgroups. Patients lacking these chromosomal alterations are termed as "B-other" ALLs which is a heterogenous subgroup accounting for $30 \%$ of paediatric BCP ALLs. Associated with an intermediate risk, they have variable outcomes. We describe the spectrum of cytogenetic abnormalities in this subgroup.

Patients/Materials \& Methods: The data of newly diagnosed BCPALL patients (August 2011- July 2019) was retrospectively analyzed. Karyotyping and FISH was done on bone marrow aspirate samples as per standard cytogenetic protocols and reported in accordance with the International System for Human Cytogenetic Nomenclature (ISCN) 2009, 2013 and 2016.The B-others were defined using karyotyping and FISH as previously published.

Results: Of the total 577 paediatric BCP ALLs, 176 patients (30.5\%) were categorised as B-other ALLs. Median age was 6.5 years with a male predominance $(\mathrm{M}: \mathrm{F}=1.74: 1)$. We classified the B-others into 6 subgroups based on cytogenetic findings i.e. (a) chromosome $9 p$ abnormalities, (b) chromosome $7 \mathrm{p}$ abnormalities, (c) combined $7 \mathrm{p}$ and 9p, (d) c-MYC rearrangement, (e) other structural aberrations and f) numerical aberrations.

Successful karyotypes were obtained in 170/176 cases. Among them, $66(39 \%)$ patients revealed normal karyotype. 40 (24\%) patients had chromosome $9 p$ abnormalities while $7 p$ aberrations were noted in 2 patients $(1 \%)$. Combined $9 \mathrm{p}$ and $7 \mathrm{p}$ abnormalities constituted 
$9 \%(\mathrm{n}=15)$ of the total cohort, of which 7 had $\operatorname{dic}(7 ; 9)$ and 3 cases with $\operatorname{dic}(9 ; 12)$ translocation.

Two patients had c-MYC gene rearrangements. Other structural abnormalities were seen in 34 patients $(20 \%)$ with deletion $6 q$ being the commonest present in $12(7 \%)$ cases followed by other non recurrent translocations. Numerical abnormalities were seen in 11 (6\%) patients with isolated trisomies of chromosomes 21, 5, 8 and 22. Discussion \& Conclusion: B-other BCP ALL is a heterogeneous group that requires further refinement and sub categorisation. The cytogenetic categorisation using karyotype and altered FISH signal patterns (triple FISH) compliments further genomic studies in identification of clinically relevant novel entities based on fusions, gene expression and copy number variations.

Supporting Document: 159bb87b-481c-4d7e-929a-c79f918aa498.

Paediatric B-other-ALL $(n=170)$

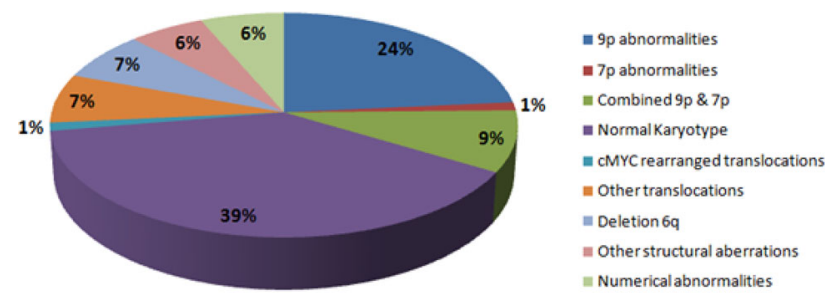

Distribution of cytogenetic abnormalities in Paediatric B other ALL

Disclosure of Interest: None Declared.

Keywords: B-other ALLs, Cytogenetic abnormalities, Karyotype.

\section{Abstract 34. Subclonal MEF2D Fusions in Precursor B Cell Acute Lymphoblastic Leukaemia}

Pranay Gurung ${ }^{* 1}$, Tanvi Gupta ${ }^{1}$, Sipra Rani Patel ${ }^{1}$, Neeraj Arora ${ }^{1}$, Deepak k Mishra ${ }^{1}$, NiharenduGhara ${ }^{2}$, Arpita Bhattacharya ${ }^{2}$, ReghuKS ${ }^{2}$, VaskarSaha ${ }^{2,3}$, Shekhar Krishnan ${ }^{2,3}$, Mayur Parihar ${ }^{1,3}$

${ }^{1}$ Department of Cytogenetics and Lab Haematology, ${ }^{2}$ Department of Paediatric Haematology and Oncology, ${ }^{3}$ Tata Translational Cancer Research Centre, Tata Medical Center, Kolkata, India

Aims \& Objectives: Precursor B Cell acute lymphoblastic leukaemia (ALL) is characterized by a constellation of chromosomal abnormalities which are prognostic and predictive of outcome. MEF2D (Myocyte enhancing factor 2D) gene rearrangement positive ALL is a novel clonal entity reported in $\sim 4 \%$ of pediatric precursor B-ALL. Here, we report a case Pro B ALL with $M E F 2 D$ fusions seen at a subclonal level.

Patients/Materials \& Methods: FISH was performed as per standard procedures.

Results: Our patient, a 9 yr old boy presented with history of fever and black tarry stools since 4 months. A complete blood count revealed bicytopenia with $34 \%$ blasts on peripheral blood examination and $90 \%$ blasts on bone marrow examination. The immunophenotyping picture was consistent with Pro-B Cell ALL. The blasts expressed CD19, cCD79a, sCD22, TdT, CD38 and HLADR and were negative for CD10 and CD5. FISH analysis performed on BMA sample was negative for ETV6/RUNX1,BCR/ABL1 fusion,
KMT2A and TCF3 genes rearrangement.ETV6 deletion was seen in $90 \%$ of cells. As the blasts were negative for CD10, FISH analysis for $M E F 2 D$ and ZNF384 genes rearrangement was performed which revealed $M E F 2 D$ gene rearrangement in $30 \%$ of cells and deletion of one copy of ZNF384 in $90 \%$ of cells.

The patient was started on ICiCLE ALL-14 protocol. In view of day 8 poor prednisolone response and positive post induction MRD $(0.93 \%)$, the treatment was intensified. Post consolidation BMA was in remission and MRD negative. During maintenance the patient developed disseminated fungal infection resistant to systemic anti fungal therapy and was lost to follow up.

Discussion \& Conclusion: We present a case of $M E F 2 D$ gene rearrangements in a subclone $(30 \%)$ of blasts, the major clone $(90 \%)$ showing deletions of ZNF384 and ETV6 genes. These patients present with CD10 negative and CD38 positive blasts and are known to repsond to HDAC inhibitor therapy.Screeneing for $M E F 2 D$ fusions is reccomended in Pro B cell ALLs that are CD 10 negative,CD38 positive and express myeloid antigens once the $K M T 2 A$ gene rearrangements have been ruled out. $M E F 2 D$ fusions have been identified on RNA sequencing and ours is a first report of these novel fusions in a subclone of the leukaemic blasts.

Disclosure of Interest: None Declared.

Keywords: B ALL, MEF2D, ZNF384.

\section{Abstract 35. Systematic Cytogenetic Strategy to Indentify Masked Hypodiploidy in BCP-ALL}

Tanvi Gupta ${ }^{* 1}$, Arun S R ${ }^{1}$, Gautam G Bhagwat ${ }^{1}$, SubhajitBrahma', Neeraj Arora ${ }^{1}$, Deepak Kumar Mishra', NiharenduGhara $^{2}$, Vivek S Radhakrishnan ${ }^{3}$, Shekhar Krishnan ${ }^{2,4}$, MammenChandy ${ }^{3}$, Mayur Parihar ${ }^{1}$

${ }^{1}$ Department of Cytogenetics and Lab Haematology, ${ }^{2}$ Department of Paediatric Haematology and Oncology, ${ }^{3}$ Department of Clinical Haematology, ${ }^{4}$ Tata Translational Cancer Research Centre, Tata Medical Center, Kolkata, India

Aims \& Objectives: Low Hypodiploidy with $<40$ chromosomes is associated with extremely poor prognosis Precursor B Acute Lymphoblastic Leukemia (BCP-ALL). In some patients, the hypodiploid clone doubles the number of chromosomes through a process called endoreduplication masking the hypodiploid clone. Karyotype reveals only metaphases that have 60 to 79 chromosomes (duplicated). We present a systematic strategy combining flow DNA index (FDI) and targeted fluorescence in situ hybridisation (FISH) approach to identify masked hypodiploidy.

Patients/Materials \& Methods: The data of newly diagnosed BCPALL patients (October2016-July2019) was retrospectively analyzed. Karyotyping, FISH analysis, FDI analysis and FDI cut offs to define ploidy levels were performed as per our previous publications. Targeted FISH to identify monosomies of 3,7,15 and 17 were performed in selected cases as per the algorithm (Fig. 1) to identify masked hypodiploidy.

Results: Of the 318 patients diagnosed as BCP ALL, 6 cases were classified as masked hypodiploidy ( 5 adults). On karyotyping, three of these showed a near triploid clone (duplicated) and three a normal karyotype.

The FDI in the 3 patients with a near triploid karyotype were: a single near triploid peak (1.43), low hypodiploid (0.73) peak and bimodal peak distribution (0.79/1.55). The patient with a single near triploid FDI peak showed monosomy 7 and single copy of ETV6, BCR, ABL1 and $K M T 2 A$ genes on FISH while patient showing low hypodiploid peak had monosmies of 3,7,15 and 17 detected by FISH. The patient with bimodal peaks on FDI showed similar monosomies. 


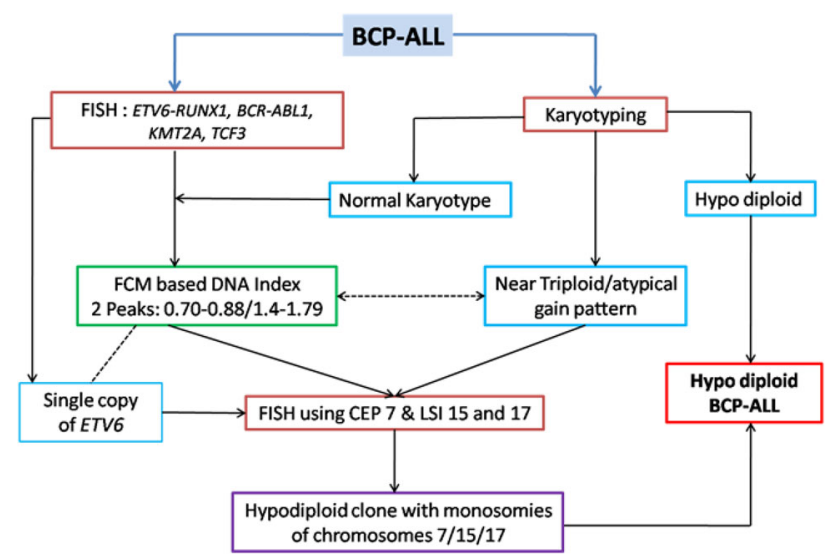

Fig. 1 Algorithm to identify masked hypo diploid BCP-ALL

All the patients with a normal karyotype showed bimodal peaks on FDI $(0.83 / 1.56,0.86 / 1.65,0.91 / 1.61)$ and monosomies of chromosome 7,15 and 17 on FISH analysis. Single allele of ETV6 and ABL1 genes were detected in one of these patients.

Discussion \& Conclusion: Masked hypodiploidy is a diagnostic challenge. Although SNP array analysis showing copy neutral LOH helps identify these patients, it is expensive and not easily available. Awareness of pattern of gains and losses of chromosomes is essential in suspecting and diagnosing these cases. Chromosomes 3, 7, 12, 15 and 17 commonly show monsomy in these patients. Complimenting this information with FDI and targeted FISH helps unmask the hidden hypodiploid clone in a sensitive specific and cost effective manner allowing optimal risk stratification.

Supporting Document: 1f499267-dabd-49f9-a7b6-99ffc293d4ab.

Disclosure of Interest: None Declared.

Keywords: BCP-ALL, Hypodiploidy, Near triploid.

\section{Abstract 36. Characterization of Chromosomal Patterns in Hypodiploid B-Cell ALL}

Shily Sipporah ${ }^{* 1}$, Nancy Beryl Janet ${ }^{1}$, BexyBensega ${ }^{1}$, Kulkarni Uday Prakash ${ }^{1}$, Anup J Devasia ${ }^{1}$, Fouzia N.A ${ }^{\mathbf{1}}$, Anu Korula ${ }^{1}$, Aby Abraham ${ }^{1}$, Alok Srivastava ${ }^{1}$, Biju George ${ }^{1}$, Vikram Mathews ${ }^{1}$

\section{${ }^{1}$ Haematology, Christian Medical College, Vellore, India}

Aims \& Objectives: To characterize the chromosomal patterns in hypodiploid B-cell acute lymphoblastic leukemia.

Near-haploid (24-31 chromosomes) and low-hypodiploid (32-39 chromosomes) B-ALL have poor outcomes and frequently present as high hyperdiploidy/triploidy confounding risk stratification.

Patients/Materials \& Methods: Cytogenetic data of 2542 B cell ALL patients with hypodiploidy seen between 2006-2018 at the Christian Medical College was reviewed.

Results: There were a total of 48 patients $(1.9 \%)$ including both adults $(n=25)$ and children $(n=23)$. The patients were categorized based on their modal chromosome number.

Near haploidy was seen only in children $(n=2)$. Low hypodiploidy was seen in $8(3.4 \%)$ children (median age 13 years) and $14(6 \%)$ adults (median age 36 years) and had a slightly higher number of males (15 males).
There were 15 children (median age 5.5 years) and 11 adults (median age 30 years) with high hypodiploidy in which structural abnormalities involving chromosomes 7,9 and 12 were the most frequent $(n=19)$.

The frequent translocations include the $t(9 ; 22)$ and the $\operatorname{dic}(7 ; 9)$ in 3 each and the $t(1 ; 19)$ in 2 .

The most commonly retained disomies in the near haploid group were 10 and 21 whereas in the low hypodiploid group it was $1,8,19$, 21 and 22 as well as $\mathrm{X}$ or $\mathrm{Y}$ in both groups.

A co-existing hyperdiploid/triploid clone was seen in 9 (18\%). Discussion \& Conclusion: Our analysis is comparable to published reports from other groups with respect to the absence of near haploidy in adults, the pattern of retained disomies and the co-existing hyperdiploid/triploid clone. It is important to analyze the pattern of chromosomal gains in hyperdiploid and triploid clones to identify a hidden hypodiploid clone.

Disclosure of Interest: None Declared.

Keywords: B-cell acute lymphoblastic leukemia, Hypodiploidy, poor risk.

\section{Abstract 37. Frequency of Common Leukemia Specific Fusion Transcripts in Pre B-Cell Acute Lymphoblastic Leukemia}

SenthamizhSelviAnandan" ${ }^{* 1}$, Sathya Mani ${ }^{1,1}$, Anu Korula ${ }^{1}$, Fouzia NA ${ }^{1}$, Kulkarni Uday Prakash ${ }^{1}$, Anup J Devasia ${ }^{1}$, Aby Abraham ${ }^{1}$, Alok srivastava ${ }^{1}$, Biju George ${ }^{1}$, Vikram Mathews', PoonkuzhaliBalasubramanian'

\section{${ }^{1}$ Haematology, Christian Medical College, Vellore, India}

Aims \& Objectives: Precursor B-cell Acute lymphoblastic leukemia (PreB-ALL) is one of the most common hematological malignancy. Testing for common leukemia specific fusion transcripts such as $B C R$ $A B L 1, T E L-A M L 1, E 2 A-P B X 1$ and $M L L-A F 4$ helps in risk stratification and ruling out Ph-like ALL. Here we looked for the presence of these fusions in patients with preB-ALL and compared the frequency among pediatric, adolescent and young adults (AYA) as well as older individuals.

Patients/Materials \& Methods: Between Jan 2008 and July 2019, all patients with bone marrow morphology and immunophenotype profile suggestive of preB-cell ALL cases were included in this study. RNA was extracted from peripheral blood/bone marrow samples at the time of diagnosis; cDNA was synthesized using High capacity cDNA synthesis kit. RT-PCR was done to test for the presence of $B C R$ $A B L 1, T E L-A M L 1, E 2 A-P B X 1$ and $M L L-A F 4$ fusion transcripts using European Biomed Protocol. The frequencies of these fusion transcripts were compared with respect to age: pediatric ( $/=40$ years). duals.

Results: There were 1855 patients with preB-ALL of which 1231 were pediatric, 444 were AYA and 180 were older adults. Of these patients, 548 patients had at least one of these fusion transcripts detected by RT-PCR (BCR-ABL1 p210: $\mathrm{n}=59 ; B C R-A B L 1$ p190: $\mathrm{n}=167 ; \quad E 2 A-P B X 1: \mathrm{n}=102 ;$ TEL-AML1: $\mathrm{n}=200 ; \quad M L L-A F 4:$ $\mathrm{n}=20$ ). The frequency of the fusion transcripts in each age group is illustrated in figure. There was significantly high incidence of $B C R$ $A B L 1$ in the older adults and AYA compared to pediatric patients (32 vs. 24 vs. $4.9 \%$ respectively; $p<0.0001)$. $B C R-A B L 1$ p 210 transcript was more prevalent in the older adults compared to the other 2 groups where p190 was more common (BCR-ABL1 p210: $23 \& 20 \%$ in pediatric and AYA vs. $41 \%$ in older adults; $p=0.008$ ). On the other hand, TEL-AML1 was more prevalent in the pediatric group compared to the other 2 groups ( 15.5 in pediatric vs. 2 and $0.6 \%$ respectively in AYA and older adults; $p<0.0001$ ). 
Discussion \& Conclusion: These frequencies are similar to previous reports from the West. These common fusion transcripts are seen in only $20-30 \%$ of preB-ALL while the genetic abnormalities in the remaining patients need to be evaluated to better risk stratify this group of patients.

Supporting Document: a71d9bb5-81b8-47e0-b8b3-e37a295b08a3.

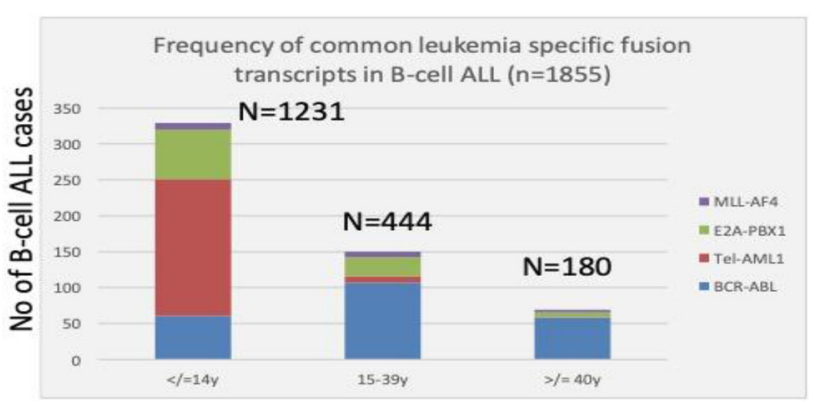

Disclosure of Interest: None Declared.

Keywords: Fusion transcripts, preB ALL, RT-PCR.

\section{Abstract 38. Droplet Digital PCR Assay More Sensitive than Conventional RT-PCR for Monitoring of PML- RARA in APL}

Poonam Santra*1, Sourav Sarma Chowdhury ${ }^{1}$, NiharenduGhara $^{2}$, Shekhar Krishnan ${ }^{2}$, Vivek Radhakrishnan ${ }^{3}$, Reena Nair ${ }^{3}$, MammenChandy ${ }^{3}$, Mayur Parihar ${ }^{1}$, Deepak Mishra ${ }^{1}$, Neeraj Arora ${ }^{1}$

\section{${ }^{1}$ Molecular genetics, ${ }^{2}$ Paediatric Haematology, ${ }^{3}$ Clinical Haematology, Tata Medical Centre, Kolkata, India}

Aims \& Objectives: Acute promyelocytic leukaemia (APL) is associated with chromosomal translocation $\mathrm{t}(15 ; 17)(\mathrm{q} 24 ; \mathrm{q} 21)$ involving the RARA(17q21) and PML(15q24)genes. Measurable Residual Diesease (MRD) monitoring of patients with APL with conventional RT-PCR or RQ-PCR is a well-accepted standard of care. Newer techniques like droplet digital PCR (ddPCR) has advantages like high sensitivity and absolute quantification, without the use of standards. In this study we wanted to standardize and evaluate a ddPCR assay and compare it with conventional RT-PCR assay for diagnosis and follow up of APL patients.

Patients/Materials \& Methods: Archived RNA samples from newly diagnosed APL patients BCR1 $(n=8)$, BCR2 $(n=2)$, BCR3 $(n=6)$ and post treatment follow up samples (nested RT-PCR negative, $n=14$ ) were evaluated using lab developed ddPCR assays for each transcript and the results were compared with conventional RT-PCR assay. RNA samples were converted into cDNA using Invitrogen Superscript VILO cDNA kit (Thermo Fisher Scientific). Then the quality of cDNA was checked by GUS PCR. Standard BioRad assay protocols and in-house primers were used to amplify the PML-RARA fusion gene transcripts and ABL1 gene using droplet Digital PCR platform (QX200). Archived $B C R-A B L 1$ positivesamples $(\mathrm{n}=10)$ were used to generate LOB and LOD for the ddPCR PML-RARA assay.

Results: TheddPCR $P M L-R A R A$ assay was successful in detecting various fusion transcripts in the newly diagnosed cases $(n=16)$ showing $100 \%$ concordance with conventional RT-PCR. However, of the fourteen nested RT-PCR negative post treatment follow up samples evaluated, two samples (bcr1 transcript) were positive by ddPCR assay (FA- $0.07 \%, 3.83 \%)$. Interestingly both these (ddPCRpos, RT-
PCR neg) cases had clinical relapse within 3 months, and were positive by RT-PCR assay at the time of relapse. This assay has been implemented into the clinics and is available for our follow up APML patients. The sensitivity of our ddPCRPML-RARA assay is calculated to be $0.001 \%$.

Discussion \& Conclusion: PML-RARA Droplet digital PCR results were concordant with conventional RT-PCR and FISH results. Additional samples and comparison with quantitative PCR are being carried out.

Disclosure of Interest: None Declared.

Keywords: APL, ddPCR, RT-PCR.

\section{Abstract 39. PRE B- ALL: The Need for Prompt Treatment}

AkanshaGautam $^{* 1}$, AnamSingh ${ }^{2}$, Sachin Kolte ${ }^{1}$, LeelavathiDawson ${ }^{1}$, Rashmi Arora ${ }^{1}$

${ }^{1}$ Pathology, Vardhman Mahavir Medical College and Safdarjang Hospital, ${ }^{2}$ Pathology, Vardhman Mahavir Medical College and Safdarjung Hospital, New Delhi, India

Aims \& Objectives: To study the role of chemotherapy in remission of Pre B-ALL.

Patients/Materials \& Methods: A case of a 5 year old male who presented with bilateral multiple submandibular axillary,cervical and inguinal lymphadenopathy, fever and hepatosplenomegaly.Peripheral smear, bone marrow aspirate,flowcytometry,bone marrow biopsy was examined and evaluated.IHC was also performed.

Results: The peripheral smear examination of the patient showed raised total leucocyte count $(30,000 / \mathrm{cu} . \mathrm{mm})$ comprising mainly of blasts $(85 \%)$.The bone marrow aspirate also showed suppression of trilineage hematopoesis due to increased blast population. The blasts had high N/C ratio, scant basophilic cytoplasm, large nucleus with prominent 1-2 nucleoli. Flow cytometry evaluation done with peripheral blood showed positivity for CD19, CD20, CD10 confirming the diagnosis of Pre B-ALL. The bone marrow biopsy was also packed with immature blasts with suppression of normal hematopoesis.

Discussion \& Conclusion: Pre B-ALL is an aggressive type of leukemia with usually affects childrens. Cytogenetics is usually not required for the diagnosis. However, most of the patients respond well to chemotherapy and the rate of remissions are higher in childrens than in adults. Hence an early and accurate diagnosis is required for better prognosis. Our case of a 5 year old male child received prompt chemotherapy following diagnosis and is responding well to treatment. Disclosure of Interest: None Declared.

Keywords: Bone marrow, Childrens, PRE B-ALL,

\section{Abstract 40. Mutant NPM1 Allelic Ratio in Patients with Acute Myeloid Leukemia}

AmirthavaniGowrinathan ${ }^{* 1,1}$, Sathya Mani ${ }^{1}$, NivedithaRavindra ${ }^{1}$, Uday P Kulkarni ${ }^{1}$, Anup J Devasia ${ }^{1}$, Fouzia NA ${ }^{1}$, Aby Abraham ${ }^{1}$, Alok Srivastava ${ }^{1}$, Biju George ${ }^{1}$, Vikram Mathews ${ }^{1}$, PoonkuzhaliBalasubramanian ${ }^{1}$

\section{${ }^{1}$ Haematology, CMCH, Vellore, India}

Aims \& Objectives: Mutations of the Nucleophosmin (NPM1) gene are the most frequent genetic aberrations seen in $25 \%$ to $35 \%$ of patients with Acute Myeloid Leukemia (AML) and is associated with good prognosis. Most frequently, 4 bp insertions are seen which 
disrupt the nucleolar localization signal (NoLS) at the C-terminus of the NPM1 protein causing cytoplasmic accumulation of truncated NPM1 protein. Recently few reports have shown that similar to mutant FLT3 allelic ratio (AR), mutant NPM1 AR also has prognostic relevance. Here, we analyzed the mutant NPM1 AR and compared it with basic demographics, type of NPMImutations and presence of FLT3 mutation and cytogenetics.

Patients/Materials \& Methods: Patients diagnosed as AML from 2012 to 2019 were included. DNA was extracted from Ficoll separated mononuclear cells obtained from bone marrow or peripheral blood samples at presentation. Mutations in NPM1 exon 11 and FLT3 internal tandem duplication/tyrosine kinase domain were screened using standard protocols and mutant AR was calculated. The type of NPM1 mutation was identified by Sanger sequencing in the NPM1 mutated cases.

Results: Of the 152 cases of AML with mutated NPM1, 93 were males and 59 females with a median age of 40 years (range: 7-79 years).Type-A (insertion TCTG) NPM1 mutations was the most common, seen in 126 patients while 3 patients had Type-B (insertion CATG) mutation and 1 had Type-I (insertion CAGA) mutation. Remaining patients had other rare types of mutations including complex deletion-insertions. NPM1 mutant allelic ratios could be calculated for 148 patients. The median mutant NPM1 AR was $74 \%$ (range: $6-125 \%$ ). The median allelic ratio was similar for Type-A (insertion TCTG)and non-type A cases $(72 \%$ and vs. $75 \% ; p=$ ns).We further compared the basic demographics, type of NPM1 mutation, FLT3 mutation and FLT3 mutant AR in patients with mutant NPM1 AR $<50$ vs. $>50 \%$ ( $\mathrm{n}=34$ and 114 respectively). The median age, sex, platelets and BM blast $\%$ as well as NPM1 mutation type were not significantly different while PB blast \% (median: $19 \%$ vs. $51 \% ; p=0.003$ ) \& total WBC count (median: 10,150 vs. 28,$900 ; p<0.0001$ ) was significantly higher in patients with mutant NPM1 AR $>50 \%$ compared to those $<50 \%$. The incidence of FLT3 ITD and TKD mutations in these 2 groups were comparable however, there was a trend to significantly higher number of patients with mutant NPM1 AR $>50 \%$ also had FLT3-ITD $\mathrm{AR}>50 \%$ ( 3 of 34 vs. 27 of $114 ; p=0.08$ ).

Discussion \& Conclusion: Our results suggest that similar to FLT3 mutant AR, NPM1 AR also will have potential prognostic relevance. The role of the mutant NPMI AR $>50 \%$ on treatment outcome needs to be evaluated.

Disclosure of Interest: None Declared.

Keywords: Allelic ratio, AML, NPM1.

\section{Abstract 41. Clustering of Chromosomal Gain Pattern Reveals Heterogeneity in High Hyperdiploid Paediatric BCP-ALL}

Ashish Gorantla ${ }^{* 1}$, Manish Kumar Singh ${ }^{1}$, Arun $\mathrm{Sr}^{1}$, Neeraj Arora', Deepak Mishra ${ }^{1}$, NiharenduGhara ${ }^{2}$, AnindyajitBanerjee ${ }^{3}$, VaskarSaha ${ }^{2}$, Shekhar Krishnan ${ }^{2}$, Mayur Parihar ${ }^{1}$, Arpita Bhattacharyya ${ }^{2}$

${ }^{1}$ Department of Cytogenetics and Lab Haematology, ${ }^{2}$ Department of Paediatric Haematology and Oncology, ${ }^{3}$ Tata Translational Cancer Research Centre, Tata Medical Center, Kolkata, India

Aims \& Objectives: To study the pattern of gains of chromosomes based on karyotyping and cluster analysis in our cohort of HH ALL. Patients/Materials \& Methods: The data of newly diagnosed BCPALL patients (2011-2018) was retrospectively analyzed. Karyotyping was done on bone marrow aspirate samples as per standard cytogenetic protocols and reported in accordance with the International System for Human Cytogenetic Nomenclature (ISCN) 2009, 2013 and

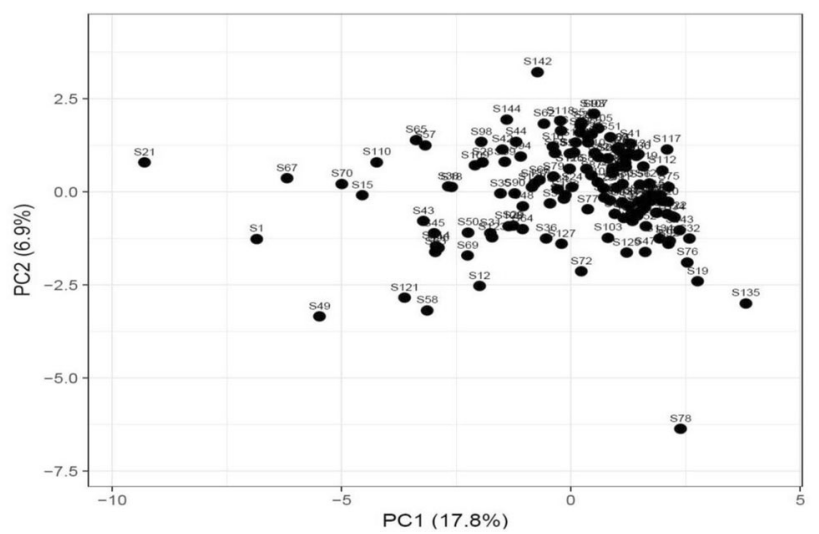

Fig. 1 Clustering of chromosomal gain pattern using Clustvis web tool

2016. The pattern of gains of chromosomes in each of the HH ALL was analysed and principal component analysis was performed using Clustvis web tool.

Results: Of the total 577 paediatric BCP ALL patients HH was identified in 194 patients (34\%) by karyotype and FISH analysis. Karyotyping data was available in $144(24.9 \%)$ patients. The mean age of diagnosis was 4.7 years, with M: F -1.4:1. The most frequent modal number in our study was 55.Chromosome 21 was gained in all the patients. Nine chromosomes $(21>6>X>14>$ $18>4>17>10>8$ ) were gained in more than $50 \%$ of the cases. The combined gain of chromosomes $21, X \& 6$ was seen in $>90 \%$ of the cases. Triple trisomies $(4,10,17)$ were seen in $76(52.7 \%)$ of patients. Gains of $4 \& 10$ was seen in 18 (12.5\%), $10 \& 17$ in 10 (6.9\%), 4 \& 17 in 20 (13.8\%) patients. Additional structural karyotypic abnormalities were seen in $19.4 \%$ of the cases, with duplication 1q being the commonest seen in 17 (11.8\%) cases. Principal component analysis using Clustvis web tool (Fig 1) revealed considerable heterogeneity in the $\mathrm{HH}$ cohort based on modal chromosome number and gain patterns.

Discussion \& Conclusion: The initial event in the development of HH ALL is gains of chromosomes, the pathological consequence being gene dosage effects. Although HH ALL shows non random gains, they form a heterogeneous group. The differences in modal number and gain pattern of chromosomes coupled with more sensitive molecular studies to unravel the heterogeneity in this subgroup may help in understanding the variable response to therapy in this subgroup.

Supporting Document: 87248104-99ab-4e18-840e-da4035fc9ef1. Disclosure of Interest: None Declared.

Keywords: CHROMOSOMAL GAIN PATTERN, CLUSTERING, HIGH HYPERDIPLOID.

\section{Abstract 42. Abnormalities of Short ARM of Chromosome 9 (9P) in Precursor B Cell Acute Lymphoblastic Leukaemia}

Sumanta Kumar Patel ${ }^{* 1}$, Tanvi Gupta ${ }^{1}$, Arun S R ${ }^{1}$, Neeraj Arora ${ }^{1}$, Deepak kumarMishra ${ }^{1}$, NiharenduGhara ${ }^{2}$, Jeevan Kumar', Vivek S RadhaKrishnan ${ }^{3}$, Reena Nair ${ }^{3}$, Shekhar Krishnan ${ }^{2,4}$, Mayur Parihar ${ }^{1}$

${ }^{1}$ Department of Cytogenetics and Lab Haematology, ${ }^{2}$ Department of Paediatric Haematology and Oncology, ${ }^{3}$ Department of 
Clinical Haematology, ${ }^{4}$ Tata Translational Cancer Research Centre, Tata Medical Center, KOLKATA, India

Aims \& Objectives: Structural abnormalities of short arm of chromosome 9 (9p) are seen in 10-15\% of BCP-ALLs. The genes present on 9 p, i.e. PAX5 (transcription factor), $C D K N 2 A / 2 B$ (cell cycle regulation) and JAK2 (Kinase pathway) are frequently involved in BCPALLs. We describe the structural abnormalities identified by karyotyping involving $9 \mathrm{p}$.

Patients/Materials \& Methods: The data of newly diagnosed BCPALL patients (2011-2019) was retrospectively analyzed. Karyotyping was done on bone marrow aspirate samples as per standard cytogenetic protocols and reported in accordance with the International System for Human Cytogenetic Nomenclature (ISCN) 2009,2013 and 2016.

Results: Of the 859 BCP ALL patients, karyotypes were available in 662 patients of which 198 were adults. 9p abnormalities were identified in 120/662 (18\%) with a higher frequency in adult patients (51/ $198,26 \%)$ compared to the pediatric patients $(69 / 464,15 \%)$.

$9 \mathrm{p}$ deletions were seen in $61(51 \%)$ of patients, although the breakpoints were variable. The most common break point in deletions was 9p11.

Unbalanced translocations were seen in $47(39 \%)$ patients and balanced translocations were seen in $12(10 \%)$ patients. Dicentric translocations were noted in $28(23 \%)$ patients. Chromosome $12(12 p)$ was the most common partner involved in dicentric translocation in $12(10 \%)$ patients resulting in deletions of $9 p$ and $12 p$. The other chromosomes involved in dicentric translocations were $7,20,15,4,2$ and 1 . Other balanced translocations included $\mathrm{t}(9 ; 12), \mathrm{t}(9 ; 15), \mathrm{t}(1 ; 9)$, $\mathrm{t}(3 ; 9), \mathrm{t}(9,10)$ and $\mathrm{t}(9 ; 11)$.

Discussion \& Conclusion: Although the prognostic significance of $9 p$ abnormalities is uncertain, deletions, translocations and mutations of the genes housed on 9p have been used to define specific types of B other ALLs with biological and prognostic connotations. The pathogenic effect of dicentric translocations is either due to fusions or deletions of genes on the respective short arms. Dicentric translocations involving chromosome $7 \mathrm{p}$ and $9 \mathrm{p}$ result in deletions of $I K Z F 1$ gene (7p) and $C D K N 2 A / 2 B, P A X 5$ and $J A K 2$ on 9p. Patients with $\operatorname{dic}(7 ; 9)$ can be screened for $E R G$ deletions, in the absence of which these patients can be assigned as IKZFI plus ALL. Karyotyping findings can complement interpreting results of molecular studies used in identifying IKZFI plus patients and those with fusions involving the PAX5 and JAK2 genes.

Disclosure of Interest: None Declared.

Keywords: 9P Abnormalities, BCP ALL, IKZF1.

\section{Abstract 43. A Novel Next Generation Sequencing Assay for Detection of FLT3-ITD Shows Good Correlation}

Rakhi Salve $^{* 1}$, ChinmayeeKakirde ${ }^{1}$, AnamShaikh ${ }^{1}$, SwetaRajpal $^{1}$, Prasanna Bhanshe ${ }^{1}$, Shruti Chaudhary ${ }^{1}$, SwapnaliJoshi $^{1}$, Prashant Tembhare ${ }^{1}$, P G Subramanian ${ }^{1}$, Nikhil Patkar ${ }^{1}$

\section{${ }^{1}$ Tata Memorial Centre, ACTREC, Navi Mumbai, India}

Aims \& Objectives: Next generation sequencing (NGS) technologies have made their way into molecular pathology laboratories and enable us to sequence large number of genes cost effectively. Internal tandem duplications of the FMS-like tyrosine kinase 3 (FLT3-ITD) are critical determinants of outcome as well as therapy in AML. Unfortunately, short read sequencers as well as informatics algorithms are not very accurate in detection of FLT3-ITD. Here we describe a novel assay and use a bioinformatics algorithm for detection of FLT3-ITD in AML.

Patients/Materials \& Methods: Total 393 cases of AML were accrued over 6 years and 7 months period with a median follow up of 25.9 months. FLT3-ITD length (C-Len) and allelic ratio (C-AR) were detected by fragment length analysis. FLT3-ITD positive samples were then subjected to a novel one-step PCR strategy that incorporated instrument specific adapters as well as patient specific indices in the same step. These samples were sequenced on an Illumina MiSeq sequencer using paired-end 500 cycle V2 chemistry. Fastq files were analysed using getITD algorithm. Receiver operating characteristic (ROC) were used to calculate appropriate cut offs for allelic ratio (NGS-AR) as well as for variant allelic frequency (NGS-VAF). A linear regression analysis was used to compare C-AR as well as C-Len when compared to NGS-AR/VAF \& Len. Overall survival (OS) and Relapse Free Survival (RFS) were calculated as per standard recommendations.

Results: Eighty-seven patients harboured FLT3-ITD by conventional techniques but only 71 were subjected to NGS analysis and in remaining 16 patients NGS failed to detect ITDs. Cut-offs for NGSAR $(\geq 0.38)$ and NGS-VAF $(\geq 26.45)$ were determined by ROC analysis. High NGS-AR predicted for inferior OS[median OS: 11.9 months (95\% CI: 8-16), $p=0.006]$ and RFS[median RFS: 11 months (95\% CI: 9-17.4), $p=0.03$ ]. NGS-VAF also correlated with inferior OS[median OS: 14.2 months (95\% CI: 9-17), $p=0.005$ ] and RFS[median OS: 12.4 months (95\% CI: 8-16), $p=0.006]$.A good correlation of NGS-ITD length with C-Len was seen $\left[R^{2}=0.82\right.$ (95\% CI: 0.75-0.95), $\left.p<0.0001\right]$ and also for NGSAR with C-AR $\left[R^{2}=0.83\right.$ (95\% CI: $\left.\left.0.73-0.91\right), p<0.0001\right]$.

Discussion \& Conclusion: We designed a novel bioinformatics algorithm for accurate detection of FLT3-ITD using NGS. Both AR and VAF of FLT3-ITD predicted the survival outcomes in our patients. The limitation of this study is that in 16 cases NGS failed to detect the FLT3-ITD. Most of these patients had longer ITD and few had very low VAF.

Disclosure of Interest: None Declared.

Keywords: AML, FLT3, NGS.

\section{Abstract 44. Study of T and NK Cell Subset in Acute Myeloid Leukemia}

Prasad Dange $^{* 1}$, Seema Tyagi ${ }^{1}$, TulikaSeth ${ }^{1}$, Renu Saksena ${ }^{1}$

${ }^{1}$ Hematology, All India Institute of Medical Sciences, New Delhi, India

Aims \& Objectives: (1) To assess the relative proportion of bone marrow $\mathrm{T}$ cells and NK cells in patients with AML in pre-treatment and post-induction bone marrow.

(2) To compare them with controls and among prognostic subgroups.

(3) To compare pre-treatment with end of induction bone marrow. Patients/Materials \& Methods: We studied 41 patients with acute myeloid leukemia, for CD4 positive helper T cells, CD8 positive cytotoxic T cells and CD16/56 positive NK cells proportion. Quantification was done on bone marrow aspirate sample by flowcytometry. Whenever available, post induction bone marrow was also analysed for the lymphocyte subset.

Results: No significant difference was noted in the percentage of blasts among the three risk categories, favourable, intermediate and adverse. However, there was significant difference in the total lymphocyte among the risk stratification groups, being highest in the favourable group and lowest in the adverse group. CD8 positive cytotoxic T cells were significantly less in APML cases $(p=0.001)$. Total lymphocytes, were however, more numerous in APML 
( $p=0.005$ ). End of induction bone marrow samples for 12 patients could be processed for lymphocyte subset. On comparing the baseline against the post induction bone marrow, it was observed that there was significant increment in the proportion of the CD4 positive $\mathrm{T}$ lymphocytes $(p=0.046)$.

Discussion \& Conclusion: There appears to be difference in the lymphocyte mediated tumour immunity among the different risk stratification groups. Also, the nature of tumour immunity may be different in APML than in non-APML subgroup. There also appears to be change in lymphocyte subset in post induction bone marrow. Disclosure of Interest: None Declared.

Keywords: Acute myeloid leukemia, T and NK lymphocyte, Tumour immunity.

\section{Abstract 45. Correlation Study Between Two Haematology Cell Counters for Complete Blood Count in Cancer Patients}

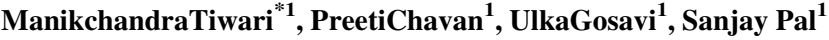 \\ ${ }^{1}$ Hemato-oncology, ACTREC, Tata Memorial Centre, Navi \\ Mumbai, India
}

Aims \& Objectives: To compare performance of hematology cell counters BC6000-Mindray and Advia2120i-SIEMENS for Complete Blood Count (CBC) test in an oncology centre.

Patients/Materials \& Methods: In this correlation study we compared CBC results obtained from two different cell counters BC6000Mindray and Advia2120i-SIEMENS and correlated them statistically. We analysed blood samples of 813 cancer patients which were received for routine analysis in the laboratory on SIEMENS cell counter-Advia 2120i. Left over samples were processed on BC6000-Mindray cell counter as part of validation exercise. Results obtained for WBC, HGB, PLT, Neutrophils, Lymphocytes, Monocytes, Eosinophils, Basophils and RDW-CV parameter were compared between the two analysers.

Result of linear regression analysis

\begin{tabular}{|c|c|c|c|}
\hline Parameters & Units & $\begin{array}{l}\text { Correlation coeffi- } \\
\text { cient }\left(\mathrm{r}^{2}\right)\end{array}$ & $\begin{array}{l}\text { Slope inter- } \\
\text { cept (y) }\end{array}$ \\
\hline WBC & 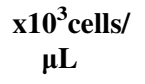 & 0.997 & $0.9337 x$ \\
\hline Neutrophil & $\%$ & 0.9118 & $0.9725 x$ \\
\hline Lymphocyte & $\%$ & 0.8448 & $0.867 x$ \\
\hline Monocyte & $\%$ & 0.6086 & $0.762 x$ \\
\hline Eosinophil & $\%$ & 0.8816 & $0.9111 x$ \\
\hline Basophil & $\%$ & 0.7115 & $2.3848 x$ \\
\hline Hemoglobin & g/dL & 0.9936 & $0.9918 x$ \\
\hline RDW-CV & $\%$ & 0.3989 & $0.9952 x$ \\
\hline Platelet & $\begin{array}{c}\mathrm{x10}^{3} \text { cells } / \\
\mu \mathrm{L}\end{array}$ & 0.985 & $1.0138 x$ \\
\hline
\end{tabular}

Results: CBC results of 813 cancer patients obtained from both the cell counters were evaluated using linear regression analysis. Coefficient of correlation $\left(\mathrm{r}^{2}\right)$ more than 0.9 and a slope of 1 were considered as significant.
Discussion \& Conclusion: For cancer patients, White Blood Cell count, Absolute Neutrophil Count (calculated from Neutrophil), Hemoglobin and Platelet are some of important parameter in the course of treatment. These parameters help the clinicians in evaluating the cell counts, bicytopenia, pancytopenia, anemia status, and guides the clinicians in administering chemotherapy drugs to the patients.

In our study, parameters like WBC, Neutrophil, Hemoglobin and Platelet showed good correlation and minimal bias among the results obtained from the two cell counters.

Among the differential parameters Lymphocyte, Monocyte, Eosinophil and Basophil did not show good correlation. RDW-CV showed no correlation.

It can be concluded that although BC6000-Mindray shows good correlation with Advia 2120i for few important CBC parameters; Advia2120i-SIEMENS showed overall better performance for important parameters and also for differential counts and RDW-CV. Disclosure of Interest: None Declared.

Keywords: Cell counters, Complete Blood Count, Correlation study.

\section{Abstract 46. Comparison of WBC Differential Flags of DXH 800 with ADVIA 2120 I and Peripheral Blood Smear}

\author{
N BHASKAR ${ }^{* 1}$, Deepti $K^{1}$, Shilpa $K^{1}$, Mable $\mathbf{G}^{\mathbf{1}}$, Manasi $\mathbf{D}^{\mathbf{1}}$, \\ Shashikant $\mathbf{M}^{1}$, Subramanian P.G ${ }^{1}$, SumitGujral ${ }^{1}$, \\ Prashant Tembhare ${ }^{1}$
}

\author{
${ }^{1}$ Haematopathology, Tata Memorial Hospital Parel, Mumbai, \\ Mumbai, India
}

Aims \& Objectives: Automated analyzers are an integral part of any busy routine clinical pathology laboratory which provides reliable, rapid, cost-effective results and help in screening for abnormalities. However, automated analyzer generated flags for suspicious abnormal cells still need to be verified by morphological review of peripheral blood smears (PBS), which requires expertise and greatly affects laboratory turn-around-time (TAT). Therefore, effective criteria for review of PBS required. We studied the flags given by two analyzers and its association with PBS findings.

Patients/Materials \& Methods: CBC (complete blood count) and WBC (White Blood Cells) differential counts were done by DxH 800 and ADVIA 2120i in blood samples collected in K2-EDTA. PBS were stained with Wright's stain and interpreted by multiple competent personnel to reduce interobserver variation. All the samples were evaluated within $4 \mathrm{~h}$ of collection. Flagging related to individual parameters were studied along with inter-instrumental concordance as well as diagnostic accuracy by correlating with PBS findings.

Results: 500 blood samples were studied (Male: Female ratio 2.15:1 and age range- 1 to 85 years) for blasts, Left Shift (LS), Immature Granulocytes (IG), Atypical Lymphocytes, and Nucleated Red Blood Cells (NRBC) flags on DxH 800 and ADVIA 2120i These flags showed weak positive correlation (Table 1). We studied the sensitivity and specificity of these flags (Table 2). We found ADVIA gives false Basophilia with high WBC count.

Discussion \& Conclusion: WBC Flags demonstrated in CBC findings showed weak positive correlation between DxH 800 and ADVIA $2120 \mathrm{i}$. However, flags generated by both analyzers had high sensitivity and specificity to detect abnormal cells in PBS, and can be used as effective screening tools.

Disclosure of Interest: None Declared.

Keywords: Peripheral blood smears (PBS), Turn-around-time (TAT)., White Blood Cells (WBC). 
Table 1 Correlation of flags between DxH 800 and ADVIA2120i

\begin{tabular}{|c|c|c|c|c|c|}
\hline Flags & NRBC & Blasts & $\begin{array}{l}\text { Aty } \\
\text { Lymph }\end{array}$ & IG & LS \\
\hline $\begin{array}{l}\text { Correlation } \\
\text { coefficient } \\
\text { "r" }\end{array}$ & 0.4061 & 0.2674 & 0.2585 & 0.5584 & 0.4888 \\
\hline$P$ value & $P<0.0001$ & $P=0.0027$ & $P=0.0037$ & $P<0.0001$ & $P<0.0001$ \\
\hline $\begin{array}{l}95 \% \mathrm{CI} \\
\text { for } \mathrm{r}\end{array}$ & $\begin{array}{r}0.25 \text { to } \\
0.54\end{array}$ & $\begin{array}{r}0.10 \text { to } \\
0.42\end{array}$ & $\begin{array}{c}0.086 \text { to } \\
0.42\end{array}$ & $\begin{array}{r}0.42 \text { to } \\
0.67\end{array}$ & $\begin{array}{c}0.341 \text { to } \\
0.61\end{array}$ \\
\hline
\end{tabular}

Table 2 DxH 800 with PBS

\begin{tabular}{lllll}
\hline Flags & Sensitivity $\%$ & Specificity $\%$ & PPV $\%$ & NPV \% \\
\hline Blast & 97 & 75 & 87 & 93 \\
V. Lymph & 99 & 91 & 90 & 87 \\
IG \& LS & 77 & 89 & 80 & 87 \\
NRBC & 77 & 89 & 35 & 98 \\
\hline
\end{tabular}

\section{Abstract 47. Audit of Fluid Flowcytometry from 2009-2019}

\section{Soma Pradhan ${ }^{* 1}$, JasmitaDass ${ }^{1}$, Jyoti Kotwal ${ }^{1}$, Amrita Saraf ${ }^{1}$, Sabina Langer ${ }^{1}$ \\ ${ }^{1}$ Hematolgy, Sir Ganga Ram Hospital, New Delhi, New Delhi, India}

Aims \& Objectives: To analyse the suitability of FCM in body fluids and correlate the findings with histopathology/Cytopathology if available.

Patients/Materials \& Methods: A retrospective analysis of all fluids specimens sent to the Department of Haematology from 2009-2019. All fluid specimens were analysed using BD FACS Canto II. All patient' medical records were reviewed to identify the histopathology/ Cytopathology samples.

Results: A total of 49 fluids including 24 pleural fluids (PF), 22 ascitic fluids (AF), 2 cerebrospinal fluids (CSF) and one pericardial fluid $(\mathrm{PcF})$ were analysed. Of the PF samples, 10 cases were T-Acute lymphoblastic leukemia (T-ALL) including a case where residual T-ALL was detected,7 were B-cell lymphomas, and one was AML. One case had shown CD4+ CD8+ T-cells. No malignancy was seen in 5 .

Among AFs, 9/22 were B-cell malignancies, 2 plasma cell neoplasms with only one T-ALL and 1 case of Peripheral T cell lymphoma. Nine cases showed no clonal populations. Both CSF samples and $1 \mathrm{PcF}$ also showed no evidence of involvement.

Fluid Cytopathology (FCy) was received in 43 cases and showed discordance in 4 (9.3\%). These were FCM+/FCy- but in all 4, a solid tissue biopsy or bone marrow (BM) was positive for malignancy. FCy was not sent in 7 cases. Of these, 4 samples were FCM+ and a $\mathrm{BM} / \mathrm{solid}$ tissue biopsy was positive for malignancy. One sample was FCM- with BM also being negative. One PF sample had been submitted for only residual leukemia and was positive and the last PF sample was FCM+ for T-ALL but BM was uninvolved indicating extramedullary leukemia.

Discussion \& Conclusion: Fluid FCM is a rapid diagnostic tool and aids cytopathology. In our series, FCy would have missed $9.3 \%$ of all cases. In addition, FCM rapidly types malignancies and leads to early institution of therapy. Even when FCy was not sent, FCM+ could be correlated with other histopathoplogy samples taken from the patients. Fluid FCM should be practiced as a routine diagnostic modality.

Supporting Document: 67f13736-4900-42b0-9257-42952a592da2.

Disclosure of Interest: None Declared.

Keywords: Flowcytometry, Fluids, Leukemia.

\section{Abstract 48. Study of Cytokine TNF-ALPHA in Acute Leukemias}

\author{
Divitasaxena $^{* 1,2}$, LeelavathiDAWSON ${ }^{2}$, Rashmi Arora ${ }^{2}$ \\ ${ }^{1}$ Vardhman Mahavir Medical College and Safdarjung Hospital, \\ Delhi, India, ${ }^{2}$ Pathology, Vardhman Mahavir Medical College \\ and Safdarjung Hospital, Delhi, India
}

Aims \& Objectives:AIM: To study the cytokine TNF- $\alpha$ levels in acute leukemias.

OBJECTIVES:

- To assess the TNF- $\alpha$ levels in acute leukemias.

- To compare the TNF- $\alpha$ levels with that of healthy controls.

Patients/Materials \& Methods: The study samples include all the newly diagnosed cases of acute leukemias and healthy controls from blood bank. The patient samples and the control samples are collected in plain vials and EDTA vials. Then TNF- $\alpha$ levels were assessed by the ELISA method.

Results: The value of TNF- $\alpha$ came out to be high in few patient samples.

Discussion \& Conclusion: Acute leukemia is a heterogenous hematopoietic malignancy having proliferation and accumulation of leukemic blasts in bone marrow and peripheral blood thus severely impairing the hematopoietic system. Although acute leukemia has a low mutational burden but there are very frequent somatic alterations causing a wide variation at the molecular and cytogenetic levels. This heterogeneity is often not reflected in the standard treatment approaches and they fail in particular cases even with high dose multiagent chemotherapy regimens. Another disadvantage is the high treatment related toxicity and the enormous risk of infection during prolonged pancytopenia. In spite of that there are common cytokines and their signalling pathways which show some common alterations irrespective of the type of somatic mutations among the various subtypes of acute leukemias. These alterations lead to the initiation and progression of acute leukemias. So, these cytokines and their pathways can be studied and interventions can be done at this level. TNF- $\alpha$ has importance in the onset and progression of leukemia and also assists leukemia cells in immune evasion, survival and resistance to chemotherapy. It is pleiotropic and is produced mainly by activated macrophages, NK cells, T lymphocytes and neutrophils. TNF- $\alpha$ supports leukemia cell survival and progression through its antiapoptotic and pro-proliferative functions which are mediated by NF$\kappa \mathrm{B}$ and JNK/AP-1 pathways.

In view of the heterogeneous prognostic factors in acute leukemias and variable outcome to the standard chemotherapeutic protocols, study of cytokine TNF- $\alpha$ is definitely of some help in understanding the variable response to the standard chemotherapeutic regimens. Supporting Document: ccdc04bd-ddc6-4f7c-bee7-ad3fd32be660. 


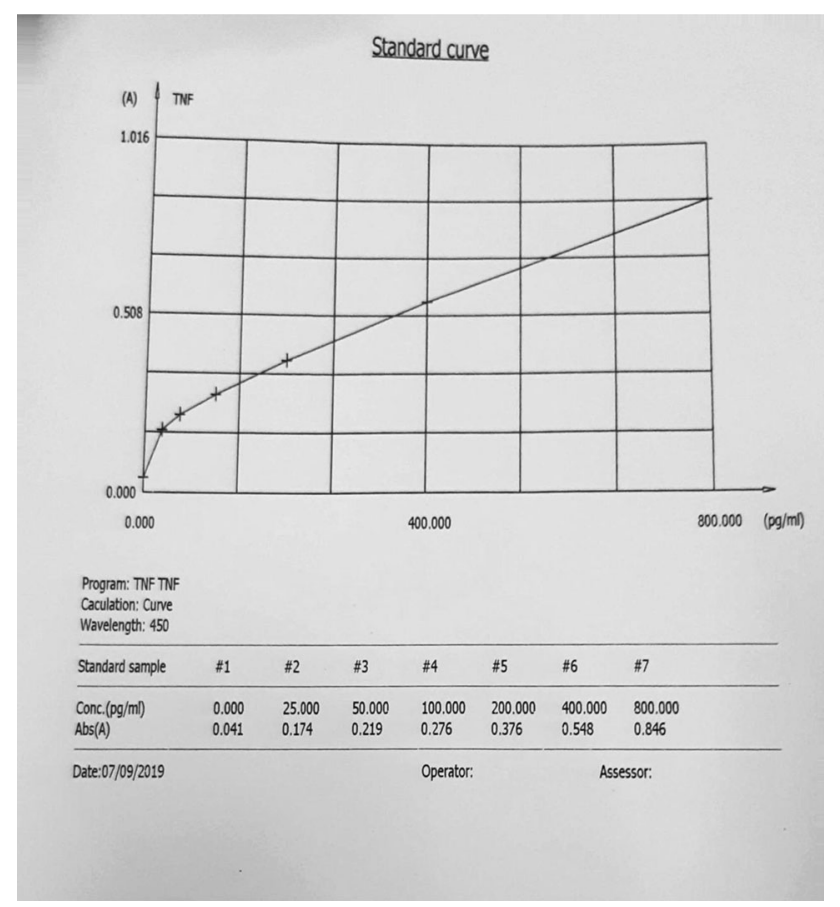

Disclosure of Interest: None Declared.

Keywords: Acute leukemia, levels, tnf-alpha.

\section{Abstract 49. Cotargeting by Venetoclax \& CDK7 Inhibitor Suppresses Transcription of Oncogenes \& Induces Apoptosis}

\section{TarangGaur $^{* 1}$, Rohit Kumar ${ }^{1}$, PG Subramanian ${ }^{1}$, Navin Khattry ${ }^{1}$, Syed Hasan ${ }^{1}$}

\section{${ }^{1}$ ACTREC, Tata Memorial Centre, navimumbai, India}

Aims \& Objectives: Attempts to develop targeted therapy have been very successful in improving outcomes in CML and APL but AML remains a challenge to patients, scientists and clinicians. The development of novel combinatorial therapies that would increase durable responses and survival outcomes is imperative. Based on the available evidences of deregulated pathways of proliferation and apoptosis in AML, we hypothesize that pharmacological modulation of AML blasts by inhibitors of BCL-2 (Venetoclax) and CDK7 (THZ1) will be potently synergistic for the treatment of AML.

Aim of the study: We aim to identify the antumor effects of CDK7 inhibitor and its role in enhancing Venetoclax mediated apoptosis.

Patients/Materials \& Methods: Leukemic cells from OCI-AML2, OCI-AML3, paired venetoclax sensitive and resistant MOLM13 (FLT3-ITD) cells were used. Primary blasts from 20 AML patients which included relapse/refractory were enriched using magnetic-activated cell sorting. Cytarabine, Venetoclax\& THZ1 were purchased from Selleckchem. $\mathrm{IC}_{50}$ calculation was carried out using cell titerglo assay and synergy calculation by Calcusyn. Cell proliferation, apoptosis \& differentiation ability was assessed using colony forming assay, flow cytometry \& dynamic BH3 profiling. qRT-PCR \& Western blot was used to determine mRNA and protein expression of RUNX1, $c-M y c, M C L-1$ respectively.
Results: THZ1 showed mean IC50 at $0.13 \mu \mathrm{M}$ in patient derived AML blasts, it is highly potent compound compared to cytarabine (mean IC50 $=0.37 \mu \mathrm{M}$ ). It suppresses cell proliferation by targeting several oncogenes including $c-M y c \& R U N X-1$ and decreases cyclin B1 expression, leading to cell-cycle arrest at G2/M. The anti-proliferative data derived from leukemic cell lines treated with THZ1 and Venetoclax has shown significant synergism (combination index $=$ 0.159). The annexinV/PI staining has shown significant apoptotic induction after treatment of leukemic cells with $1 \mu \mathrm{M}$ of THZ1. The rate of apoptosis was significantly enhanced after combination with Venetoclax as shown by dynamic $\mathrm{BH} 3$ profiling, expression of cleaved caspases- 3 and cleaved-PARP \& pro survival proteins such as MCL-1, BCL-XL and XIAP.

Discussion \& Conclusion: The preliminary data derived from our in vitro and ex vivo studiessuggest that targeted therapy by Venetoclax and CDK7 inhibitors synergistically modulate apoptosis, proliferation and has greater anti-leukemic activity compared to standard of care in AML.

Disclosure of Interest: None Declared.

Keywords: AML, CDK7 inhibition, targeted therapy.

\section{Abstract 50. Correlation of DNMT3A and IDH1/2 Mutations with Recurrent Genetic Abnormalities in De Novo AML Cases}

\author{
RkBanashreeDevi ${ }^{* 1}$, Neelam Varma $^{2}$, ShanoNaseem ${ }^{2}$, \\ Subhash Varma ${ }^{3}$
}

\section{${ }^{1}$ Pathology Department, JNIMS, Imphal, ${ }^{2}$ Department of Hematology, ${ }^{3}$ Department of Medicine, PGIMER, Chandigarh, India}

Aims \& Objectives: To study the correlation of DNMT3A (R882H), IDH1 (R132) and IDH2 (R140Q)mutationswith clinico-hematological profile and recurrent genetic abnormalities in denovo AML cases.

Patients/Materials \& Methods: The study was conducted in the Department of Hematology, PGIMER, Chandigarh from January 2016 to June 2017. 103 de-novo AML patients were included in the study. Complete blood counts, bone marrow examination and cytochemical stainingwere done. Extraction of genomic DNA for molecular genetic analysis was performed using 'QIAamp ${ }^{\circledR}$ DNA blood Midi kit, Qiagen, Germany. Multiplex RT-PCR assay was then carried out using specific primers to detectinv (16)(p13.1q22); $C B F B$ $M Y H 11, \mathrm{t}(8 ; 21)(\mathrm{q} 22 ; \mathrm{q} 22) ; R U N X 1-R U N X 1-$

T1 and $\mathrm{t}(15 ; 17)(\mathrm{q} 22 ; \mathrm{q} 12) ; P M L-R A R A$ fusion transcripts. Restriction fragment length polymorphism method, amplification-refractory mutation system and high resolution melting curve analysis method were used to identify DNMT3A R882H G $>$ A mutation, IDH2 R140Q and IDH1 R132 mutations respectively. Appropriate statistical tests were performed and analysis was conducted using IBM SPSS STATISTICS (version 22.0).

Results: DNMT3A R882H, IDH1 R132 and IDH2 R140Q mutation was detected in $9(8.7 \%), 9(8.7 \%)$ and $13(12.6 \%)$ cases respectively. $\mathrm{t}(8 ; 21)(\mathrm{q} 22 ; \mathrm{q} 22) ; R U N X 1-R U N X 1 T 1$ fusion gene was detected in 12 (12.1\%), inv(16)(p13.1q22);CBFB-MYH11 fusion gene in $6(6.1 \%)$ and $\mathrm{t}(15 ; 17)(\mathrm{q} 22 ; \mathrm{q} 12) ; P M L-R A R A$ fusion gene in $10(10.1 \%)$ of the patients. None of the DNMT3A mutated AMLcases had concomitant recurrent genetic abnormalities. Among the IDH1 R132 mutated AML patients, $22 \%$ had $\mathrm{t}(15 ; 17)(\mathrm{q} 22 ; \mathrm{q} 12) ; P M L-R A R A$ translocation. Whereas none of the cases harboured inv(16)(p13.1q22);CBFBMYH11 or $\mathrm{t}(8 ; 21)(\mathrm{q} 22 ; \mathrm{q} 22) ; R U N X 1-R U N X 1 T 1$. Among IDH2 
R140mutated cases, $15 \%$ case had inv(16)(p13.1q22); $C B F B-M Y H 11$ and $23 \%$ had $\mathrm{t}(8 ; 21)(\mathrm{q} 22 ; \mathrm{q} 22) ; R U N X 1-R U N X 1 T 1.11 \%$ of IDH1 R132 mutated AML had a coexisting DNMT3A R882H mutation.

Discussion \& Conclusion: None of the recurrent genetic abnormalities[t(15;17)(q22;q12);PML-RARA, inv(16)(p13.1q22);CBFBMYH11 or t(8;21)(q22;q22);RUNX1-RUNX1T1] were observed in the $D N M T 3 A$ mutated cases. Similar finding was reported by Ley et at. Thol et al. noted significant association of DNMT3A mutation inIDH1 mutated cases.

Conclusion: As these mutations have prognostic implications, identification of these may assist in individualised management of AML patients.

Disclosure of Interest: None Declared.

Keywords: AML, DNMT3A, IDH1/2.

\section{Abstract 51. Microbial Spectrum and Their Sensitivity Pattern in Patients with Haematological Malignancy}

\section{KarthikaV $^{* 1}$, Prakas Kumar Mandal ${ }^{1}$ and ShuvraneelBaul, Subham Bhattacharya, Sumit Mitra, Rajib De, T.K. Dolai \\ ${ }^{1}$ Department of Hematology, NRS Medical College, Kolkata, India}

Aims \& Objectives: Febrileneutropeniaisthecommoncauseofmortalityandmorbidityinhaematologicalmalignancy, especially in developing countries like India. Idea about prevalent micro-organism in every hospital and their sensitivity pattern helps proper management. The aim was to determine frequency, spectrum of infection and their sensitivity in febrile neutropeni patients with haematological malignancy.

Patients/Materials \& Methods: Blood specimens were cultured in 535 patients who had 1220 episodes of febrile neutropenic episodes as per IDSA 2010 guidelines in patients with haematological malignancy from July, 2015-October, 2018.

Results: We analysed all febrile neutropenia episodes in 535 patients suffering from different types of haematological malignancies. Out of 1220 febrile neutropenic episodes, positive culture report was obtained in $336(27.54 \%)$ episodes. Overall, $56.84 \%$ of the organisms isolated were gram negative, $39.58 \%$ gram positive and $3.6 \%$ fungal spp. The most common microorganism was Klebsiella pneumoniae in $82(24.40 \%)$ febrile neutropenic episodes, followed by Acinetobacter baumannii in $50(14.9 \%)$ episodes, Pseudomonas aeruginosa in 49 (14.6\%) episodes, Enterococcus $4(2.7 \%)$ and Burkholderia in 1 $(0.76 \%)$ episode. Among the gram positive, Coagulase Negative staphylococci (CoNS) constitute $15(10 \%)$ episodes, Methicillin Resistant staphylococci Aureus (MRSA) (2.7\%) in 4 episodes and among the fungal infection Candida albicans in $6(4 \%)$ neutropenic episodes. Among Gram negative bacteria, majority was sensitive to carbapenems \& polypeptide antibiotics, Among Gram positive organisms, majority were sensitive to Beta lactams, sulphonamides \&glycopeptide.

Discussion \& Conclusion: Most common isolate was gram negative (56.84\%), followed by gram positive $(39.58 \%)$ and fungal spp $(3.6 \%)$. In gram negative most common microorganism isolated was Klebsiella pneumoniae in $24.40 \%$ febrile neutropenic episodes. Among the gram positive Coagulase Negative staphylococci constitute $10 \%$. Among Gram negative bacteria, majority was sensitive to carbapenems \& polypeptide antibiotics, Among Gram positive organisms, majority were sensitive to Beta lactams, sulphonamides \&glycopeptide.
Disclosure of Interest: None Declared.

Keywords: Hematological malignancy, Microbial organism, Neutopenia.

\section{Abstract 52. A Tertiary Care Center Experience with MLPA Technique in Leukemia Diagnosis and Prognostication}

\author{
Prateek Bhatia $^{* 1}$, Minu Singh ${ }^{1}$ \\ ${ }^{1}$ Pediatric Hematology Oncology Unit, Post Graduate Institute Of \\ Medical Education And Research, CHANDIGARH, India
}

Aims \& Objectives: We evaluated the routine usage of MLPA in different paediatric hematological malignancies for its robustness and cost effectiveness.

Patients/Materials \& Methods: A total of 243 pediatric patients suffering from different cancers [B cell acute lymphoblastic leukemia, $\mathrm{n}=203$; $\mathrm{T}$ cell acute lymphoblastic leukemia, $\mathrm{n}=33$; and Le fraumeni syndrome $(n=7)$ ] were analyzed by MLPA assay using MRCHolland kits P335, P383, P327 and P056-C1. A total of 35 controls were run for analysis and normalization of samples in different batches. DNA was extracted from peripheral blood mononuclear cells and processed for MLPA reaction as per protocol. After MLPA-PCR, fragments were resolved by capillary electrophoresis on ABI 3130 Genetic Analyzer (Applied Biosystems) and the peak intensities were analyzed using Coffalyser.net software (MRC-Holland).

Results: CNAs in $I K Z F 1, P A X 5, E B F 1, B T G 1, R B 1, C D K N 2 A / B$ and genes from $P A R 1$ region viz., CSF2RA, IL3RA, P2RY8, SHOX region and $C R L F 2$ were successfully analyzed in pediatric B-ALL patients and were detected in $70 \%(92 / 131)$ of cases, with predominantly deletions found in CDKN2A/B (30\%), PAX5 (20\%) and IKZFI (19\%). CNA detection in B-ALL was helpful in risk stratification and integrated classification upfront and $16 \%$ cases of intermediate risk were upgraded to higher risk based on CNA data. PAR1 deletions suggestive of CRLF2-P2RY8 fusion were detected in 4/131 (3\%) cases and subsequently confirmed on RT-PCR. iAMP 21 was noted in only two of the 105 cases (2\%) screened. EBF1-PDGFRa fusion was detected in 1 case only and targeted therapy (TKI) was added in this patient. T-ALL patients predominantly revealed $C D K N 2 A / B$ gene CNAs in $90 \%$ cases. SIL-TAL1 and NUP-214-ABL1 fusions could be detected in $24 \%(8 / 33)$ and $3 \%(1 / 33)$ cases respectively. For Le Fraumeni syndrome, 2 of the samples showed previously reported deletions in Tp53 gene. MLPA reaction did not fail in any of the samples analyzed. All control reference samples and the control probes within each target probemix had a very low SD of $<2 \%$.

Discussion \& Conclusion: The cost for performing MLPA on patient's samples was around 1500 INR or approximately 22 USD. With such low cost MLPA was found to be highly reliable for analysing and reporting genetic variation in haematological malignancies using kit P335, P383 and P327. It was helpful in B-ALL risk stratification, identification of rare targeted fusions and iAMP21 screening. In T-ALL too, other than prognostic information on CNAs in epigenetic regulatory genes, fusions of SIL-TAL1 and NUP214-ABL1 could be detected. The data shows that MLPA can be utilized as an effective and rapid screening test to make timely therapeutic and prognostication decisions especially in ALL cases. Supporting Document: 4a109183-dd58-48dc-b0ef-1c82b65dc116. 


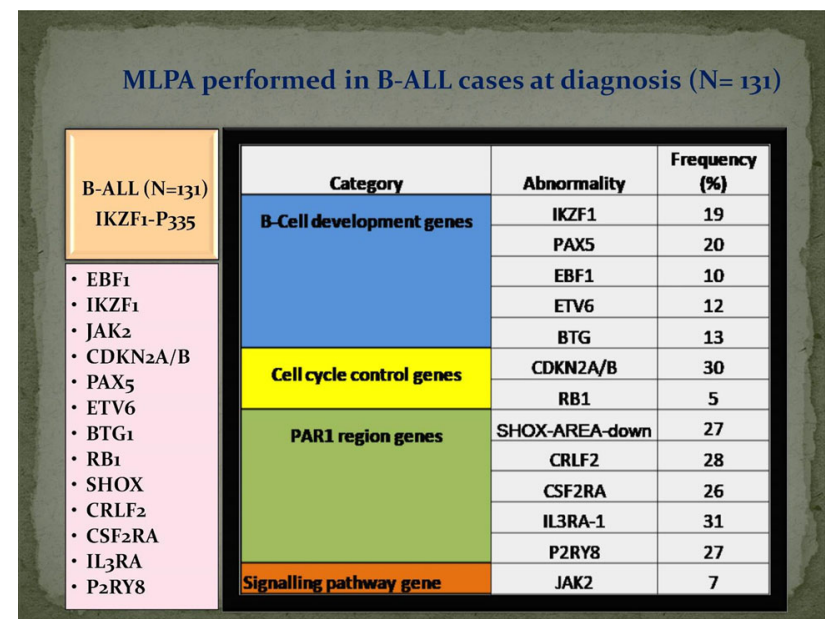

Disclosure of Interest: None Declared.

Keywords: Acute leukemia, CNA, MLPA.

\section{Abstract 53. A Pilot Study Highlighting DNA Methylation Pattern in B-ALL Relapse Cases}

Pankaj Sharma $^{* 1}$, Prateek Bhatia ${ }^{1}$, Minu Singh ${ }^{1}$, AmitaTrehan ${ }^{1}$, Neelam Varma ${ }^{1}$

\section{${ }^{1}$ Pgimer, Chandigarh, India}

Aims \& Objectives: B-cell acute lymphoblastic leukemia (B-ALL) is one of the most common hematological malignancies in children. Despite vast improvement in the treatment of B-ALL, 15-20\% of leukemia cases often relapses and account for more deaths from cancer in children than any other malignancies. DNA methylation changes play a major role in mediating chemo resistance and relapse in pediatric ALL. The aim of the study is to detect, identify, and define the molecular abnormalities at the DNA methylation level to provide vital information on the molecular pathogenesis and clinical aspects of relapse B-ALL.

Patients/Materials \& Methods: A total of 14 DNA samples from 7 B-ALL patients at diagnosis (day 0) and their paired sample at relapse (Day-R) along with 2 controls were selected for methylation studies. DNA samples were run using Infinium HumanMethylation850 K chip (EPIC array that can identify both hypo and hyper methylated sequences within the genome). To evaluate methylation level in each sample, beta values were checked. Beta values greater than 0.70 were regarded as hyper-methylated and less than 0.3 as hypo-methylated. Results: On bioinformatics analysis by Genome Studio software various oncogenes (MAFB, GATA2, KLF4, PAX5, FOXL2 etc.), tumor suppressor genes (CDH11, EXT1, NF2, SMARCB1 etc.) and cell cycle genes (HSPA2, PSMC5, NEK7, CEP250 etc.) were found to be differentially methylated between control and patient samples. Oncogenes such as MYCN, MYB, and ERBB1 were noted to be hypomethylated or overexpressed while tumor suppressor gene such as MDM4, BCL11B were found to be hypermethylated at relapse. Discussion \& Conclusion: Our pilot study suggests that the $850 \mathrm{k}$ EPIC array is particular suitable for DNA methylation studies as it covers 850,000 number of methylation sites on the genome. Our study identified differential methylation in clinically important oncogenes and tumour suppressor genes at relapse and provides data for further studies to define B-ALL relapse disease biology for development of targeted therapeutics.

Supporting Document: 9c51af68-ffc4-4d68-b34a-255c3efa42f5.

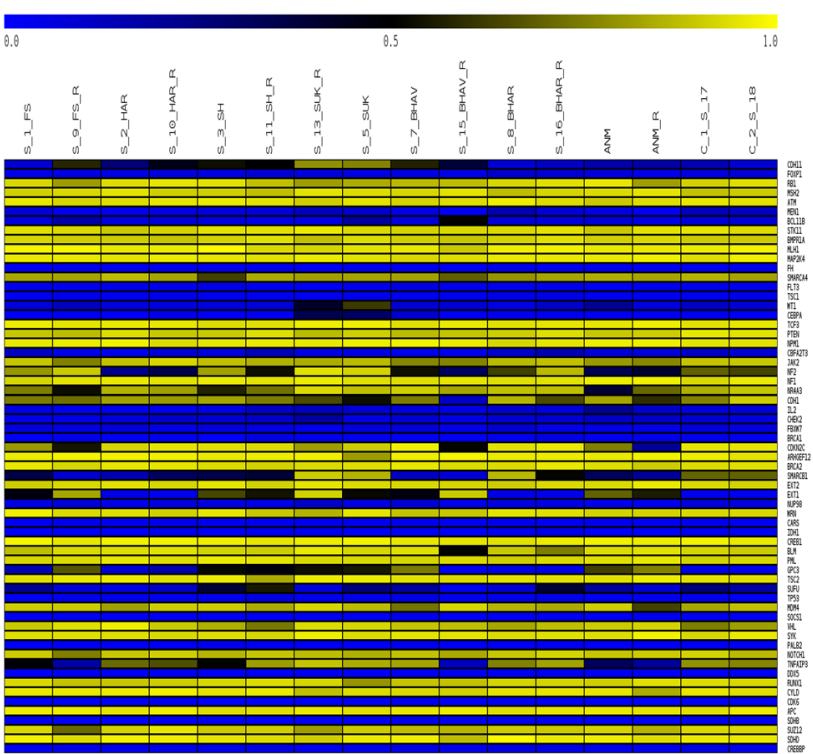

Disclosure of Interest: None Declared.

Keywords: Methylation, Next generation sequencing, Relapse.

\section{Abstract 54. A Comparative Study of Research Parameters on Sysmex XN with the Immuno-Phenotype of Blasts}

\author{
Shruti Mishra ${ }^{* 1}$, Rasheeda Mohamedali ${ }^{1}$, DebasisJash ${ }^{1}$, \\ Gaurav Chhabra ${ }^{1}$
}

${ }^{1}$ Pathology and Lab Medicine, AIIMS Bhubaneswar, Bhubaneswar, India

Aims \& Objectives: The aim of our pilot study was to compare the variations in positional parameters provided by Sysmex XN analyzer with the nature of blasts as determined by multi-color flowcytometry. Patients/Materials \& Methods: This study was conducted in AIIMS, Bhubaneswar. All index acute leukemia cases diagnosed at our center were included. Treated and relapsed cases were excluded from the study. Diagnosis was done either on peripheral blood or bone marrow aspirate. Same sample was processed in Sysmex XN analyzer and Flowcytometer [BD FACS Canto II]. Smears were examined by experienced hematopathologists in all cases. The positional parameters provided by WNR and WDF channels of Sysmex XN were collected. We determined the normal ranges with mean in our laboratory after analyzing 20 normal samples.

Results: 54 cases were included in this pilot study. We had 20 cases each of B-cell precursor Acute Lymphoblastic Leukemia and Acute Myeloid Leukemia. 4 cases of Acute Promyelocytic leukemia, 3 cases of AML-M5a and 7 cases of T-cell ALL out of which 3 were Early T-cell precursor ALL.

Discussion \& Conclusion: We found that NE-WY was the most reliable parameter in predicting acute leukemias. It was uniformly deranged in all types of leukemias. AML and AML with monocytic differentiation, both showed similar pattern of derangement in neutrophilic parameters, while the later showed more increased values in MO-WY. T-cell ALL cases showed lower than normal range values for MO-X while in B-cell ALL cases the value was raised. Acute Promyelocytic leukemia in comparison to AMLs and AMLs with monocytic differentiation showed deranged values for LY-WY and LY-YZ. 
We, conclude in our pilot study that the positional parameters can be used as predictors of the nature of blasts and extended study can help us calculate cut-off values for the same.

Disclosure of Interest: None Declared.

Keywords: blasts, flowcytometry, Sysmex XN.

\section{Abstract 55. Unusual Presentation of an Uncommon Malignancy: Blastic Plasmacytoid Dendritic Cell Neoplasm}

\author{
GurleenOberoi $^{* 1}$, M Prabhu ${ }^{1,2}$, S Venkatesan ${ }^{1,3}$, Seema Tyagi ${ }^{1}$ \\ ${ }^{1}$ Hematology, AIIMS, New Delhi, ${ }^{2}$ Hematology, JIPMER, \\ Pondicherry, ${ }^{3}$ Hematology, AFMC, Pune, India
}

Aims \& Objectives: Blastic plasmacytoid dendritic cell neoplasm (BPDCN) is a rare and highly aggressive hematopoietic malignancy derived from precursors of plasmacytoid dendritic cells. BPDCN without cutaneous involvement is a rare event and only few cases have been reported in literature. We present an unusual case of BPDCN in a young male presented with peripheral cytopenias and without cutaneous involvement.

Patients/Materials \& Methods: We present hematological and morphological features of a 21-year-old male patient with bleeding manifestation, easy fatiguability and weaknwess since 3 months along with intermittent fever since 10 days; diagnosed as Blastic Plasmacytoid Dendritic Cell Neoplasm in the absence of cutaneous manifestations.

Results: This case of BPDCN presented with pancytopenia and atypical cells in bone marrow aspirate examination, which on immunophenotyping by flow cytometry were Lineage negative, CD45 dim, CD4+, CD56+, CD123+ and HLA-DR+ hence a diagnosis of BPDCN was made.

Discussion \& Conclusion: BPDCN is a rare aggressive malignancy typically affects elderly people with cutaneous manifestations. A very high degree of suspicion is required for diagnosis in a young patient presenting with cytopenias and without skin manifestations. Early diagnosis and prompt treatment of this entity may be beneficial, which otherwise has a dismal prognosis.

Disclosure of Interest: None Declared.

Keywords: cutaneous manifestations, cytopenias, Plasmacytoid dendritic cell neoplasm.

\section{Anemia Including Hemolyticanemia}

\section{Abstract 1. Cold Agglutinin Disease: A Grave Masquerader}

\section{K V VinuBalraam*1, Adnan Masood ${ }^{2}$, Venkatesan Somasundaram ${ }^{3}$, Bhushan Asthana ${ }^{3}$ \\ ${ }^{1}$ Pathology, ${ }^{2}$ Medicine, Military Hospital, Roorkee, ${ }^{3}$ Pathology, Armed Forces Medical College, Pune, India}

Aims \& Objectives: Cold Agglutinin Disease is a rare type of Autoimmune Haemolytic Anaemia which usually results due to production of a specific IgM type auto-antibody against the $\mathrm{I} / \mathrm{i}$ and $\mathrm{H}$ antigens on Red Blood Cell membrane. Autoimmune and lymphoproliferative disorders, mycoplasma pneumoniae and other infections can be associated with the production of cold agglutinins. It can have a varied presentation ranging from being incidentally detected to being totally transfusion dependant for a longer or shorter duration.
Patients/Materials \& Methods: Here we present a 54 years old patient who was a known case of chronic obstructive pulmonary disease and was admitted to our hospital in the last winter as a case of alcohol withdrawal syndrome. During the course of the stay, the patient developed respiratory insufficiency and went into Type-II respiratory failure.

Results: Haematological investigations revealed anaemia with disproportionately low RBC count and raised RDW, MCHC and MCV. PBS evaluation showed leucoerythroblastic picture with toxaemia, nRBCs and polychromasia with a reticulocytosis of $12 \%$. Supporting biochemistry showed raised LDH and indirect bilirubin levels. Coomb's test was negative. However, auto-agglutination at $4{ }^{\circ} \mathrm{C}$ was positive confirming the presence of auto-antibodies of cold type.

Discussion \& Conclusion: Cold agglutinin disease is a type of immune mediated haemolysis due to presence of cold type $\operatorname{IgM}$ auto-antibodies. It's quite rare worldwide with an estimated prevalence of 1 per million. Several factors play a role in determining the ability of cold agglutinins in inducing haemolysis such as antibody concentration and temperature. These are usually self-limiting if there is an underlying pathology. However, there may be dreadful complications as well particularly due to acute haemolytic episode which one must be aware of.

Disclosure of Interest: None Declared.

Keywords: Anemia, Cold Antibody, Hemolytic.

\section{Abstract 2. Haemoglobin E Syndromes in Haryana- A 9 Year Experience}

\section{Renuka Verma $^{* 1}$, Sunita Singh ${ }^{1}$, Rajnish Kalra ${ }^{1}$, Rajeev Sen ${ }^{1}$ \\ ${ }^{1}$ Pathology, Pt.B.D.Sharma, PGIMS, Rohtak, Rohtak, India}

Aims \& Objectives: Haemoglobin E is the second most prevalent haemoglobinopathy worldwide. In India it is prevalent in the northeastern states with an average allele frequency of $10.2 \%$. HbE syndromes occur in homozygous, heterozygous, and compound heterozygous states with widely variable clinical phenotypes. This study was designed to find out the incidence of $\mathrm{HbE}$ syndromes in Haryana, to study the clinical profile of these patients and whether RBC indices obtained from automated cell counter can provide a clue to the diagnosis of $\mathrm{HbE}$ syndromes. High Performance liquid chromatography (HPLC) was used as a confirmatory diagnostic test for identification of $\mathrm{HbE}$ variants.

Patients/Materials \& Methods: This epidemiological study was conducted in the Department of Clinical Pathology, Pt.B.D. Sharma, PGIMS, Rohtak (Haryana) from June 2010 to May 2019 for analysis of clinical and haematological profile of $\mathrm{Hb} \mathrm{E}$ syndromes using automated blood cell counter and HPLC.

Results: A total of 5,421 cases that were run on HPLC in the last ten years were screened, out of which 72 cases $(1.32 \%)$ were detected with hemoglobin E syndromes, among which 09 patients were diagnosed as homozygous, 35 as heterozygous, 27 as $\mathrm{HbE} / \beta$-thalassaemia and 01 as $\mathrm{HbE} / \mathrm{HbS}$ double heterozygotes. Range of age of the study subjects was 10 months to 42 years with male: female ratio $1: 1.67$. Majority of the patients $(61.81 \%)$ presented with variable signs and symptoms of anaemia along with microcytic picture with or without hypochromia.

Discussion \& Conclusion: HPLC is an excellent, cost-effective, rapid diagnostic tool, for detection of asymptomatic carriers by accurate quantification of $\mathrm{HbA} 2$ and $\mathrm{HbF}$ levels, because when combined with other variants they may give rise to severe disease. Hence, clinicians and pathologists should consider this haemoglobinopathy in the differential diagnosis of anaemia with or without splenomegaly for early diagnosis and management.

Disclosure of Interest: None Declared.

Keywords: Haemoglobin E, Hemoglobin variants, HPLC. 


\section{Abstract 3. Importance of Peripheral Smear Examination in Hemoglobinopathies}

\author{
Hema GOYAL $^{* 1}$, Molly Joseph ${ }^{1}$ \\ ${ }^{1}$ Pathology, St. Stephen's Hospital, New Delhi, India
}

Aims \& Objectives: Peripheral smear (PS) examination is a primary tool along with other adjunctive tests for diagnosis of various clinically significant haemoglobinopathies.

Patients/Materials \& Methods: Three cases in adolescent age group presented to our emergency department with features of hemolytic anaemia. PS examination was done in all the cases. Two cases (age 18 \& 19yrs females) had sickled RBC in peripheral smear and diagnosis was made with positive sickling test and S-window in HPLC. One case (15 years male) showed marked anisocytosis with many spherocytes and microspherocytes on smear. DCT and ICT were negative. Osmotic fragility test was performed and it showed mean corpuscular fragility of RBC at $5.8 \mathrm{~g} / \mathrm{L}$.

Results: Two cases were diagnosed as Sickle cell disease (One with $\mathrm{HbS}$ homozygous and other with $\mathrm{HbS}$ heterozygous). One case was diagnosed as Hereditary spherocytosis. In all the three cases, Peripheral smear examination was the mainstay for diagnosis and further management.

Discussion \& Conclusion: Even after evolution of high quality haematologyanalysers, PS examination remains to be an efficacious tool for diagnosis of various haemoglobinopathies.

Disclosure of Interest: None Declared.

Keywords: Hemoglobinopathies, HPLC, Peripheral smear (PS).

\section{Abstract 4. Clinico-Pathological Study of Geriatric Anemia in \& Around Varanasi: A Hospital Based Study}

\author{
Vijai Tilak $^{* 1}$, DeepshikhaChaudhary ${ }^{2}$, InderjeetGambhir ${ }^{3}$ \\ ${ }^{1}$ Department of Pathology, Institute of Medical Sciences, \\ ${ }^{2}$ Department of Pathology, Institute of Medical Sciences,BHU, \\ ${ }^{3}$ Department of Medicine, Institute of Medical Sciences, BHU, \\ Varanasi, India
}

Aims \& Objectives: 1 . To study the characteristics of hematological profile in elderly persons presenting with anemia.

2. To undertake clinical \& laboratory workup of elderly patients so as to arrive at the underlying etiology causing anemia.

Patients/Materials \& Methods: It was a hospital based prospective study conducted at the Department of Pathology \& Medicine, IMS, BHU over a period of 36 months on 178 patients above the age of 60 years attending geriatric medicine OPD \& general indoor wards. The patients were evaluated for anemia. After taking consent, a thorough clinical evaluation followed by complete blood count, peripheral blood smear \& bone marrow examination was undertaken. Other pertinent tests were also carried out.

Results: Maximum number of patients belonged to the age group 60-65 years. There was male preponderance. Anemia of chronic disease (ACD) was the commonest cause of geriatric anemia.ACD due to multiple myeloma was the commonest haematological malignancy causing geriatric anemia followed by iron deficiency anemia, myelodysplastic syndrome, aplasticanemia \& megaloblastic anemia. Most of the geriatric patients had severe degree of anemia. However $9.5 \%$ of the cases had mild degree of anemia. Out of 17 cases of mild anemia, 13 cases $(76.47 \%)$ were due to haematological malignancy.
Discussion \& Conclusion: In the present study, patients in the age group 60-65 years were maximally affected which is in concurrence with the study by Mathew Rony et al. \& Tilak et al.

In the present study there was male preponderance. This is in accordance with the study by Guralnik et al., Gary et al. \& Tilak et al. Thus geriatric anemia is a unique anemia since in contrast to anemia in younger people, anemia in elderly persons is more common in men than women. Cessation of blood loss in menstrual in elderly women may be one contributing factor for this observation.

ACD was the commonest cause of anemia. This is in concordance with studies by Matthew et al. \& Mauro Tettamanti et al.

In $76.47 \%$ of mild anemia there was an underlying haematological malignancy. Mildanemia is therefore not a mild but a serious condition in an elderly as it may be a harbinger of a serious disorder which may cause death. Hence every case of geriatric anemia should be thoroughly investigated to reach at a definite diagnosis.

Disclosure of Interest: None Declared.

Keywords: Geriatric anemia, Iron deficiency anemia, Multiple myeloma.

\section{Abstract 5. Quality Indicators in a Hematology Laboratory- A Retrospective Analysis}

\author{
PreetiTripathi $^{* 1}$, AkritiKashyap ${ }^{1}$, Preethi Makam ${ }^{1}$, Arijit Sen ${ }^{1}$ \\ ${ }^{1}$ Indian Airforce, Bengaluru, India
}

Aims \& Objectives: Each laboratory endeavors to deliver reports of highest quality within a reasonable time by putting some checks and balances in their work system. To assess the effectiveness of the final working system, some quality indicators (QIs) which can be assessed objectively and followed up regularly, are required. The authors decided to analyse some commonly studied QIs in the diagnostic hematology laboratory of a tertiary care centre.

Patients/Materials \& Methods: It was a retrospective analysis over a period of two years (July 2017-June 2019). The centre caters to a mix of routine, urgent and critical samples, thereby requiring a fine balance of quantity as well as quality in the delivery system. In order to check the patient safety, effectiveness, timeliness, and efficiency of the system, we decided to study following 7 QIs- sample rejection rates, sample redo rates, routine turnaround time (TAT), critical reports and their TAT, corrected reports, staining quality assessment and concordance in EQAS programme. All samples received for testing in laboratory were included to calculate the denominator for the above rates. The rates were calculated on monthly (or as specified) basis and were compared with previous recorded results. Trend was analysed wherever applicable to assess the process. The authors obtained necessary clearance from institute ethics committee for analysing the data from HIS/hospital records.

Results: Quality indicators assessed monthly included routine turnaround time (mean-4.9 h), critical report TAT (1.5 Hrs), sample Redo rates $(0.05 \%)$, sample rejection rates $(1.5 \%)$, corrected report rates $(0.01 \%)$ staining quality (unsatisfactory days rate-4.5\%). Performance in EQAS was assessed monthly (for coagulation) or quarterly (for CBC) as required and concordance was noted (96\%). Over the study period, a significant downward trend was noticed in TAT, sample rejection and corrected reports rates ( $p$ value 0.01 , $0.007,0.03$ respectively). Number of monthly critical reports and sample redo rates however showed an upward trend ( $p$ value- 0.045 ) which could be attributed to increased awareness amongst lab staff/pathologists.

Discussion \& Conclusion: Quality indicators help not only in objective assessment of any laboratory by external agencies but also in self assessment and self improvement. Their continuous monitoring 
is mandatory to have a tight quality check system and better clientele satisfaction.

Disclosure of Interest: None Declared.

Keywords: quality indicator, sample Redo, turnaround time.

\section{Abstract 6. Screening of Antenatal Women for Beta Thalassemia Carrier State}

\author{
FebeSuman $^{* 1}$, Ravi Teja ${ }^{1}$ \\ ${ }^{1}$ Sri Ramachandra Medical College and Research Institute, \\ Chennai, India
}

Aims \& Objectives: Thalassemias are autosomal recessive disorders consisting of homozygous and heterozygous variants. Heterozygous varieties are symptom free but present with haematological characteristics often useful for their identification. This study was carried out to detect $\beta$ thalassemia heterozygous state in antenatal women and to evaluate the utility of screening indices to filter the samples of persons to be subjected to High performance liquid chromatography (HPLC) and to create a validated flag in the software to alert when $\mathrm{CBC}$ parameters are suspicious of $\beta$ thalassemia.

Patients/Materials \& Methods: This cross sectional study was carried out from July 2015 to August 2017 on women attending the antenatal clinic. A total of 183 antenatal women were screened for haemoglobinopathies by HPLC.

Results: $\beta$ thalassemia trait was detected in $23.5 \%$ of the women. All the women had microcytic hypochromic anaemia as per the haematology analyser reports. The RBC count was either normal or high except in a few cases. Among the 16 indices analysed Sehgal index and Mentzer index both with sensitivities of $97.67 \%$, were found to be suitable. Flags to detect these indices incorporated in the software of the analyser alert to carry out HPLC. $55.81 \%$ spouses of women with $\beta$ thalassemia trait underwent HPLC testing.

$\beta$ thalassemia trait was detected in $23.5 \%$ of the women. All the women had microcytic hypochromic anaemia as per the haematology analyser reports. The RBC count was either normal or high except in a few cases. Among the 16 indices analysed Sehgal index and Mentzer index both with sensitivities of $97.67 \%$, were found to be suitable. Flags to detect these indices incorporated in the software of the analyser alert to carry out HPLC. $55.81 \%$ spouses of women with $\beta$ thalassemia trait underwent HPLC testing.

Discussion \& Conclusion: Antenatal testing by HPLC should be done on all mothers having microcytic hypochromic anemia without iron deficiency. The antenatal screening program to be effective requires spouse testing, if the woman is found as carrier.

Disclosure of Interest: None Declared.

Keywords: Hemoglobinopathy, high performance liquid chromatography, Mentzer index.

\section{Abstract 7. Renal Derangement in Sickle Cell Anemia During Vaso-Occlusive Crisis: Report of 4 Cases}

\section{Jyotsna Pandey $^{* 1}$, Dipika Mohanty ${ }^{1}$, SreeyaDas ${ }^{1}$, SnehalataJagati ${ }^{1}$}

${ }^{1}$ Hematology, Apollo Hospitals., Bhubaneswar, Bhubaneswar, India

\section{Aims \& Objectives:}

AIMS: Paucity of reports regarding renal derangement in sickle cell anemia cases prompted us to undertake this present study. This will help us in taking necessary timely interventions in affected cases of SCD.

Objective: To find out whether the kidney functions are altered in homozygous sickle cell disease cases during vaso-occlusive crisis.

Patients/Materials \& Methods: The present case report includes 4 sickle cell anemia cases, who have moderate to severe renal involvement during vaso-occlusive crisis, presented at Apollo hospitals from June 2018 to July 2019. They were treated accordingly. After the proper management all the cases recovered and they are on follow up.

Results: All 4 were sickle cell anemia (ss) cases who presented with renal derangement, during their vaso-occlusive crisis (Table 1).

Discussion \& Conclusion: This present study brings out the fact that renal involvement during sickle cell crisis is not uncommon. However one has to look for the renal derangement so that proper timely management of the same can be undertaken for recovery of the cases. This study also stresses the utility of routine and microscopic urine examination of SCD patients for early detection of renal involvement, during follow up of the cases in O.P.D.

Supporting Document: e266380c-d23c-4793-b7c1-338d83a3cfd4.

Table-1: Different parameters of kidney function during vaso-occlusive crisis

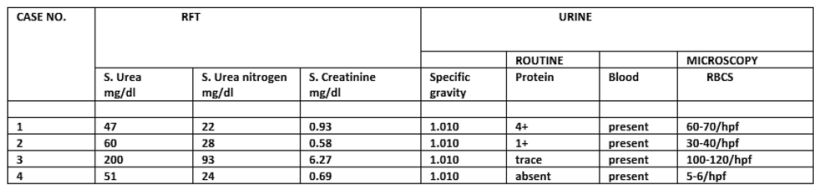

Disclosure of Interest: None Declared.

Keywords: renal derangement, sickle cell anemia, vaso-occlusive crisis.

\section{Abstract 8. Newer Reticulocyte Parameters in Anemia of First Trimester pregnancy: An Observational Study}

UtpalKumar $^{* 1}$, Arvind Gupta ${ }^{1}$, Neha Singh ${ }^{2}$, NilotpalChowdhury $^{2}$, Jaya Chaturvedi ${ }^{3}$, Harish Chandra ${ }^{1}$

${ }^{1}$ Pathology, AIIMS, Rishikesh, ${ }^{2}$ Pathology, AIIMS,Rishikesh, ${ }^{3}$ Obs \& Gynae, AIIMS, Rishikesh, Rishikesh, India

Aims \& Objectives: According to World Health Organization 40.1\% of total pregnant females are anemic worldwide with burden lying mostly in under developed countries and iron deficiency anemia comprising of $50 \%$ of these anemia. Therefore it is essential to diagnose iron deficiency anemia as early as possible in pregnancy so as to prevent complications to both mother and the baby. The present study was done to study role of reticulocyte parameters including reticulocyte hemoglobin content, immature reticulocyte fraction and reticulocyte count which are easily available on routine hematologyanalyzers in evaluating anemia in pregnancy. It was also intended to compare these parameters with serum ferritin, and routine hematological indices in assessment of anemia.

Patients/Materials \& Methods: The prospective study included first trimester pregnant females who were statistically analyzed for anemia using routine hematological indices, serum ferritin and reticulocyte parameters at All India Institute of Medical Sciences, Rishikesh.

Results: The study included total 155 females with hemoglobin less than $11 \mathrm{~g} / \mathrm{dl}$ in 31 and serum ferritin less than $15 \mu \mathrm{g} / \mathrm{l}$ in 64 females. reticulocyte hemoglobin, serum ferritin, immature reticulocyte fraction (IRF) had statistically significant value in differentiating microcytic anemia from other parameters. Reticulocyte count was not statistically significant in differentiating any of these categories. 
Reticulocyte hemoglobin with cut-off of $30.6 \mathrm{pg}$ in Iron deficiency defined by serum ferritin values showed an area under curve (AUCROC) of 0.80 , specificity of $77 \%$, sensitivity of $81 \%$, positive predictive value of $71 \%$ and likelihood ratio of 3.52 .

Discussion \& Conclusion: Reticulocyte hemoglobin is relatively better marker for microcytic anemia compared to ferritin, immature reticulocyte fraction and reticulocyte count in early pregnancy. Reticulocyte hemoglobin and immature reticulocyte fraction which are easily available on automated analyzers are feasible and cost effective surrogate marker for evaluation of anemia in early pregnancy. Further larger studies with serum ferritin and other de facto indicators may be done to establish the role of Reticulocyte parameters in iron deficiency anemia of early pregnancy.

Supporting Document: 25dd13ec-db4f-4813-bdfa-c3056631df75.

\section{Microcytic Anemia}

\begin{tabular}{|l|l|l|l|l|l|}
\hline Parameter & AUC-ROC & $95 \% \mathrm{Cl}$ & Sensitivity & Specificity & Cut-Off \\
\hline Ferritin & 0.786 & $0.642-0.930$ & 0.750 & 0.784 & 10.7 \\
\hline Ret-He & 0.9177 & $0.854-0.982$ & 0.871 & 0.812 & 27.2 \\
\hline IRF & 0.7932 & $0.685-0.901$ & 0.938 & 0.532 & 10.5 \\
\hline Ret Count & 0.5526 & $0.382-0.723$ & 0.438 & 0.763 & 0.054 \\
\hline
\end{tabular}

ROC curve for Serum Ferritin

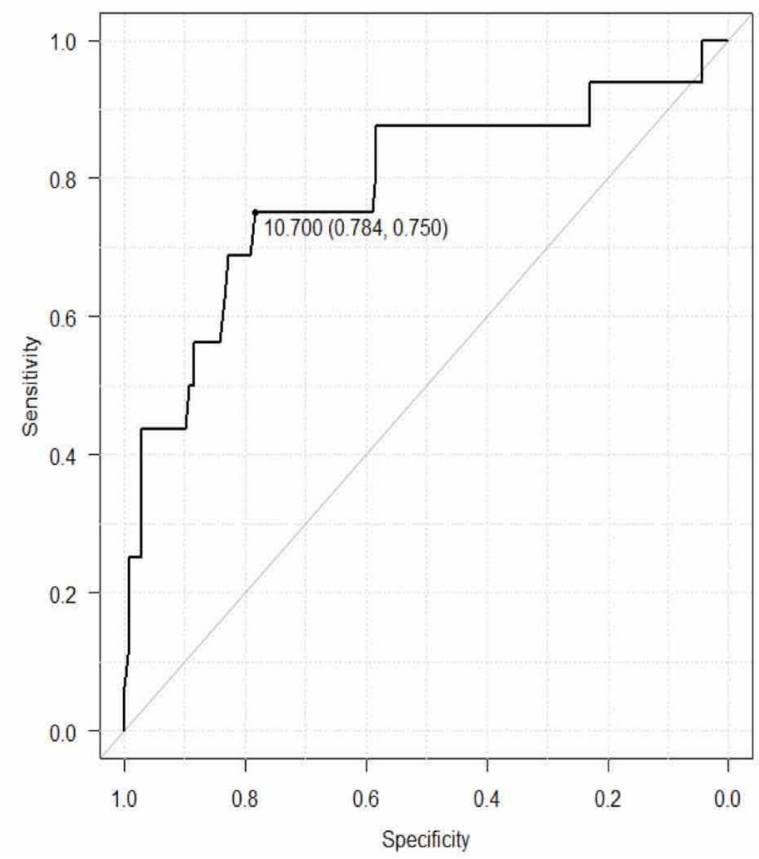

Disclosure of Interest: None Declared.

Keywords: Immature Reticulocyte Fraction, Reticulocyte Hemoglobin, Serum Ferritin.

\section{Abstract 9. Role of Micrornas in Severity of Thalassemia Patients and in Hydroxyurea Mediated HBF Induction}

\author{
Neha Kargutkar*1, Anita Nadkarni ${ }^{1}$ \\ ${ }^{1}$ Haematogenetics, National Institute of Immunohaematology, \\ Mumbai, India
}

Aims \& Objectives: miRNAs act as potent regulators of gene expression. It has been hypothesised that the treatment with hydroxyurea, ameliorates the clinical severity of $\beta$-thalassemia through $\mathrm{HbF}$ induction, however its mechanism still remains unclear. Thus, the aim of this study is to investigate the transcriptional regulatory role of miRNAs in severity of the disease in patients mediated with hydroxyurea treatment and to elucidate its mechanism of action.

Patients/Materials \& Methods: 5 thalassemia intermedia patients prior to hydroxyurea treatment and 5 healthy individuals were selected for the study after informed consent. Hematological analysis was carried out using automated cell counter and by Variant II HPLC, BioRad. miRNAs were extracted from whole blood using MirVana Kit, Ambion and the expression pattern was studied using TaqMan assay on QuantStudio analyser. The patients were further followed up for 6 months to analyse the changes in the expression pattern of micro RNAs.

Results: The hematological analysis of the 5 thalassemia intermedia patients at baseline and after 6 months of treatment showed higher hemoglobin and $\mathrm{HbF}$ levels. Global microRNA expression profile of patients at baseline and post treatment showed a differential pattern of expression. Expression profile of baseline patients showed up regulation of the following miRNAs ( $>10$ folds): $m i R-15 \mathrm{a}, m i R-324$, $m i R-365, m i R-190, m i R-139, \mathrm{miR} 518 \mathrm{f}$ and down regulation of $m i R-$ 532, miR-374, miR-298, miR-127. The expression profile, post HU treatment showed up regulation of microRNA expression of which miR 518f and miR 219 showed relatively higher expression $(>30$ folds) as compared to baseline. Among the down regulated microRNAs miR 425, miR 26a, miR 532 and miR 374 showed 500 folds lower expression as compared to patients at baseline. MicroRNA expression in healthy individuals showed constant expression pattern. Discussion \& Conclusion: The study of MicroRNA expression patterns in patients before and after HU treatment may help in understanding their role and mechanism in $\mathrm{HbF}$ induction in hemoglobinopathy patients. Further the targeted use of exogenous miRNA may act as a novel diagnostic or prognostic strategy for $\mathrm{HbF}$ induction in these patients.

Disclosure of Interest: None Declared.

Keywords: Fetal haemoglobin, hydroxyurea, microRNAs.

\section{Abstract 10. Correlation Between Iron Stores and Platelet Count in Voluntary Blood Donors}

\author{
Leo Stephen $\mathbf{R}^{* 1}$, RanitaDe ${ }^{1}$, Deepak M.B ${ }^{2}$, Joy Mammen ${ }^{2}$, \\ Eunice S Edison ${ }^{1}$ \\ ${ }^{1}$ Department of Haematology, ${ }^{2}$ Immunohaematology and \\ Transfusion Medicine, Christian Medical College, Vellore, \\ Vellore, India
}

Aims \& Objectives: Voluntary blood donation is associated with the risk of iron deficiency. Increase in donation frequency results in depletion of iron stores. Increased platelet count has been observed as depletion of iron stores occur. In this study, we evaluated the association between iron stores and platelet count in a group of voluntary blood donors.

Patients/Materials \& Methods: This study was carried out from May 2018 to March 2019. Voluntary male blood donors fulfilling the inclusion criteria (Age $<50$ years, $\mathrm{Hb}>12.5 \mathrm{~g} / \mathrm{dL}$ ) were included in the study after taking informed consent. Socio demographic parameters like (BMI, smoking etc.) were also documented. Haematological parameters were screened by standard methods. Biochemical parameters (Serum Ferritin, sTfR) were evaluated using immunoassays. 
Results: One hundred and seventy voluntary blood donors were included in this study. The median age of this group were 27.5 years. The mean $\mathrm{Hb}$ was $15.29 \pm 1.65(\mathrm{~g} / \mathrm{dL})$, MCV $86.4 \pm 6.3$ (FL), and platelet count were $2.54 \pm 0.8$ lakh. The median serum ferritin levels were 45.5 (4.6-574) $\mathrm{ng} / \mathrm{ml}$ and the median sTfR was $3.63(1.7-12.73)$ $\mathrm{mg} / \mathrm{L}$. Forty two donors of 170 had sTfR $>4.6(\mathrm{mg} / \mathrm{L})$. Based on ferritin levels, the donors were classified as iron deficient (ferritin $\leq 30 \mathrm{ng} / \mathrm{ml}$ ) and iron replete (ferritin $>30 \mathrm{ng} / \mathrm{ml}$ ). Sixty of them $(60 / 170,35 \%)$ were iron deficient. The sTfR and platelet counts were significantly higher in the iron deficient group than the iron replete group whereas $\mathrm{Hb}, \mathrm{MCV}$ were significantly lower in the iron deficient group. Platelet counts had a negative correlation with serum ferritin levels but was not statistically significant.

Discussion \& Conclusion: This study shows that iron stores and thrombopoiesis are intricately linked. So far studies have shown increased platelet count in iron deficiency anemia. But we show that as iron stores start to decrease, platelet counts increase which indicates that it is a closely knit process. The mechanisms by how this happens are being explored.

Disclosure of Interest: None Declared.

Keywords: Iron deficiency anemia, Iron stores, Thrombocytosis.

\section{Abstract 11. Role of Genetic Modifiers of Fetal Hemoglobin in Clinical Outcome of Hemoglobinopathy Patients}

\author{
Priya Hariharan $^{* 1}$, Anita Nadkarni ${ }^{1}$ \\ ${ }^{1}$ Hematogenetics, National Institute of Immunohematology, \\ Mumbai, India
}

Aims \& Objectives: To investigate the association of single nucleotide polymorphisms (SNPs) located in $3 \mathrm{HbF}$ quantitative trait loci: BCL 11A,HBS1L-MYB intergenic and $\gamma$-globin promoter region with the HbF levels and on ameliorating the clinical severity of the disease.

Patients/Materials \& Methods: Overall 100 SCA, $100 \quad \beta$-thalassemia homozygous patients and 50 healthy controls were studied. SNPs in BCL11 A, MYB-HBSL1 intergenic region, $\gamma$-globin promoter were studied by TaqMan real time genotyping, allele specific PCR and by DNA sequencing.

Results: Hemaotological analysis of $\beta$-thalassemia homozygotes showed that the mean hemoglobin level was significantly higher in milder $\beta$-thalassemia homozygous patients $(7.8 \mathrm{~g} / \mathrm{dL} \pm 1.4)$ as compared to severe patients $(5.4 \mathrm{~g} / \mathrm{dL} \pm 1.1)$. The sickle homozygous patients, the mean $\mathrm{HbS}$ level was $71.3 \% \pm 11.2$ and the mean $\mathrm{HbF}$ level was $18.5 \% \pm 8.2$. A strong negative correlation between the $\mathrm{HbF}$ and $\mathrm{HbS}$ levels (Pearsons correlation R: -0.35, p: 0.0002) was observed in SCA patients. Analysis of the SNPs in the 3 QTLs showed that the $\mathrm{T}$ allele of $\mathrm{rs} 1427407 \mathrm{G} \rightarrow \mathrm{T}(\mathrm{p}: 0.02), 3$ bp deletion allele of rs66650371 (p: 0.02), T allele of XmnI polymorphism (p: $0.04)$ were significantly associated with the $\mathrm{HbF}$ levels in $\beta$-thalassemia patients. Similarly in SCA patients the $\mathrm{C}$ allele of rs $11886868 \mathrm{C} \rightarrow \mathrm{T}$ (p: 0.02), $\mathrm{T}$ allele of $\operatorname{rs} 1427407 \mathrm{G} \rightarrow \mathrm{T}$ (p: 0.01 ), $3 \mathrm{bp}$ deletion allele of rs66650371 (p: 0.0006) were found to be significantly associated with the $\mathrm{HbF}$ levels. m-RNA expression studies of the mutant alleles of BCL11A and MYB intergenic region showed threefold reduced expression as compared to the patients harbouring the normal alleles. It was observed that patients inheriting more number of $\mathrm{HbF}$ modulating alleles showed reduced disease severity, thus suggesting that the elevated $\mathrm{HbF}$ levels may play a potential role in ameliorating the clinical phenotype of hemoglobinopathy.
Discussion \& Conclusion: Inter individual variation of $\mathrm{HbF}$ expression may potentially act as heritable modifier of hemoglobinopathies. Identification of genetic polymorphisms that modulate the $\mathrm{HbF}$ levels may help in understanding the molecular mechanisms that control $\mathrm{HbF}$ expression and on clinical heterogeneity of the disease. Disclosure of Interest: None Declared.

Keywords: BCL11A, Genetic modifier, HBS1L-MYB.

\section{Abstract 12. Prevalence of Anemia and Bone Mineral Density Changes in Chronic Obstructive Pulmonary Disease}

AnshuPalta $^{* 1}$, AmarvirSidhu ${ }^{1}$, Anita Tahlan', Rohit Jindal ${ }^{2}$, Deepak Aggarwal ${ }^{3}$

${ }^{1}$ Pathology, ${ }^{2}$ Orthopaedics, ${ }^{3}$ Pulmonary Medicine, Govt. Medical College And Hospital 32,chandigarh, CHANDIGARH, India

Aims \& Objectives: 1 . To study the prevalence of anemia and bone mineral density changes in COPD patients.

2. To categorize the type of anemia in COPD patients.

3 . To determine the co-relation between anemia and bone mineral density changes in COPD patients.

4. To study the association of anemia and bone mineral density changes with severity of COPD.

Patients/Materials \& Methods: A total of 120 stable COPD patients and 50 healthy age and sex matched controls were selected for the purpose of study. Patients with active cardiac disease, primary haematological disorders, thyroid disorders, parathyroid disorders, other co-morbid conditions and on oral corticosteroids and haematinics were excluded. All patients and controls were subjected to DEXA scan and their blood samples were collected for $\mathrm{CBC}$ including reticulocyte count, iron profile, EPO levels, serum B12, folic acid, Vitamin D and C-reactive protein.

Results: Anemia was detected in $22(18.3 \%)$ COPD patients including 15 (14.7\%) males and 7 (38.8\%) females while polycythemia was found in $10(8.3 \%)$ patients. Further in order to classify anemia, correlation between red cell indices, deranged iron profile, vitamin $\mathrm{B}_{12}$ and folic acid levels was done.

The most common type of anemia detected in COPD was ACD, which was seen in $12(54.5 \%)$ patients. IDA was noted in $7(31.8 \%)$ patients. One $(4.5 \%)$ patient had coexistent IDA and ACD. Two (9.0\%) patients had megaloblastic anemia.

The DXA scan revealed osteopenia in 31 (25.8\%) patients, osteoporosis in $70(58.3 \%)$ patients and was normal in $19(15.8 \%)$ patients.

The correlation of anemia with severity of COPD was found to be significant $(p$ value $=0.001)$. But correlation of decreased bone mineral density with disease severity was insignificant ( $p$ value $=0.500)$. Both the co-morbidities were significantly correlated with each other ( $p$ value $=0.028$ )

Discussion \& Conclusion: The present study thus depicts that anemia and decreased bone mineral density are important co-morbidities associated with COPD, with prevalence of $18.3 \%$ and $84.1 \%$ respectively. Our study is a prospective study which included limited number of patients as compared to other studies which are predominantly retrospective or involved higher number of patients. More studies may be required in future to establish the correlation of decreased bone mineral density with disease severity.

Disclosure of Interest: None Declared.

Keywords: Osteoporosis, Anemia, COPD. 


\section{Abstract 13. Isolated Neutropenia- an Unusual Presentation of Helicobacter pylori Induced Vitamin B12 Deficiency}

\author{
Shiv Kumar ${ }^{* 1}$, PreetiTripathi $^{1}$, AkritiKashyap ${ }^{1}$, Arijit Sen ${ }^{1}$ \\ ${ }^{1}$ Department of Pathology, Command Hospital Air Force, \\ Bangalore, India
}

Aims \& Objectives: Helicobacter pylori is known to be commonly associated with iron deficiency and vitamin B12 deficiency secondary to malabsorption in almost $50 \%$ of the patients. Vitamin B12 deficiency usually presents either with anemia or bi/pancytopenia in most cases. Here, we present a case of $\mathrm{H}$ Pylori induced Vitamin B12 deficiency who presented with isolated neutropenia and responded well to Vitcofol injections and triple therapy for eradication of $\mathrm{H}$ Pylori.

Patients/Materials \& Methods: A 44 years old previously healthy male, was found to have mild low leukocyte count with relative lymphocytosis on multiple occasions in last 7-8 years during annual checkups. Patient is a pilot by profession, vegetarian, teetotaler and asymptomatic otherwise. There was no history of any B symptoms, nodular or abdominal lumps. There was no history suggestive of any intake of herbal medications/dieting or autoimmune disorders. He had been prescribed various oral hematinics intermittently. Physical examination was within normal limits. Hematological workup showed Hb-14.2 gm/dL, TLC-3200/cmm, DLC-N24, E02, B00, M08, L 66, Platelets-2,36,000/cmm, Retic count-1.8\%, MCV-76.1 fl. Liver, renal, thyroid function tests and USG abdomen were within normal limts. Serum Iron: $56 \mathrm{ug} / \mathrm{dL}$, Ferritin: $53.6 \mathrm{ng} / \mathrm{dL}$, TIBC: 276 ug/dL, Folate: $8.29 \mathrm{ng} / \mathrm{ml} \&$ Vitamin B12: $88 \mathrm{pg} / \mathrm{ml}$ (180-914). Autoimmune workup was within normal limits.

Results: Bone marrow examination showed cellular reactive aspirate and biopsy. Flowcytometry on peripheral blood showed normal subset analysis with no clonality. PET scan suggested inflammatory pathology in stomach hence an endoscopy was planned which revealed a nodular mucosa with erosions \& erythema. Antral biopsies were taken which were positive for rapid urease test and also suggested of $\mathrm{H}$ pylori associated gastritis on HPE. He was started on injection vitcofol and $\mathrm{H}$ pylori triple eradication therapy in view of low vitamin B12 and features of antral gastritis. TLC and DLC showed a rapid improvement to injection vitcofol and were corrected within few days. Follow up investigations revealed a sustained normal CBC (Hb-13.3 g/dL, TLC -5700/cmm, DLC -N51, E04, B00, M05, L40, Platelets-2,62,000/cmm, MCV-77.5 fl).

Discussion \& Conclusion: Neutropenia associated with $\mathrm{H}$ pylori infection is generally understood to be autoimmune in nature. However, lack of response to steroids and negative autoimmune markers indicate a different mechanism in this case. Rapid improvemnent of $\mathrm{ANC}$ to vitcofol injections points towards a vitamin B12 deficicncy in a background of Helicobacter pylori associated gastritis. Isolated neutropenia in absence of any anemia/macrocytosis is a rare presentation of Vit B 12 deficiency.

Disclosure of Interest: None Declared.

Keywords: Helicobacter pylori associated gastritis, Isolated neutropenia, Vitamin B12 deficiency.

\section{Abstract 14. Prenatal Diagnosis of Haemoglobinopathies in Odisha: A Preliminary Study}

MaheswarPanda $^{1,1}$, Dipika Mohanty ${ }^{* 1}$, Manas Ranjan Kar ${ }^{2}$

${ }^{1}$ Hematology, ${ }^{2}$ Radiology, Apollo Hospitals, Bhubaneswar, Bhubaneswar, India
Aims \& Objectives: Haemoglobinopathies are the most common monogenic recessive disorders in Odisha. Prenatal diagnosis and selective termination of an affected foetus is a feasible option to decrease the disease burden. Lack of facilities for prenatal diagnosis prompted us to establish the prenatal diagnosis in first trimester of pregnancy by chorionic villi sampling (CVS) analysis. The present communication describes for the first time our experience in last 3 years consisting of 34 cases.

Patients/Materials \& Methods: CVS was obtained by a trained radiologist in the first trimester of pregnancy (10-12 weeks) and the dissection of the same was carried out under the dissecting microscope (Nikon SMZ800N). DNA was extracted using Spin column method (Qiagen). ARMS-PCR was used for detecting different mutations for $\beta$-thalassemia and Sickle Cell Anemia. GAP-PCR was done for detection of $d \beta$-thalassemia. VNTR markers were used to look for maternal contamination.

Results: Eight common mutations in $\beta$-globin gene in Indian population were detected in $33(97 \%)$ cases by ARMS-PCR where as one case (3\%) having rare $d \beta$ thalassemia was detected by GAP-PCR. $33(97.05 \%)$ cases were given confirmed diagnosis using ARMS-PCR where as 1 (2.95\%) case was confirmed by using ARMS \& GAP PCR. Out of 34 cases, 18 (52.9\%) $\beta$ thalassemia, 9 (26.4\%) SCD, 2 (5.8\%) Sickle-thal, 4 $(11.7 \%) \mathrm{HbE}$-thal and $1(2.9 \%) \mathrm{d} \beta$ thalassemia. Fetal diagnosis shows 10 (29.4\%) normal, 6 (17.6\%) HbS trait, $10(29.4 \%) \beta$ thal trait, 1 (2.9\%) $\mathrm{HbE}$ trait, $1(2.9 \%) \mathrm{d} \beta$ thalassemia trait and $6(17.6 \%)$ affected foetus respectively. $19(55.5 \%)$ couples came for prenatal diagnosis who do not have affected children, this is only because of increased awareness in the population. $88.8 \%$ of SCD cases are from western Odisha and specially Aghoria community. All the couples opted for termination of pregnancy if they have affected foetus. IVS 1-5 (GàC) was the commonest mutation $(90 \%)$ and also two rare mutations CD41/42 (-CTTT) \& CD15 (GàA) were encountered.

Discussion \& Conclusion: Utilising ARMS-PCR, GAP-PCR and VNTR, we were able to offer the foetal diagnosis in 2- 3 days time in $97 \%$ of cases.

Disclosure of Interest: None Declared.

Keywords: Prenatal diagnosis, Sickle Cell Anemia., $\beta$-thalassmia.

\section{Abstract 15. Utility of Cellular Hemoglobin Distribution Width (CHDW) Parameter to Differentiate IDA and BTT}

\section{Dinesh Rane ${ }^{* 1}$, Sarika Narkhede ${ }^{1}$, Shashikant Mahadik ${ }^{1}$, Sumeet Gujral ${ }^{1}$, PappagudiSubramanian ${ }^{1}$ \\ ${ }^{1}$ Hematopathology Laboratory, TATA Memorial Hospital, Mumbai, India}

Aims \& Objectives: The overlapping features on morphological evaluation of peripheral blood smear from Beta thalassemia trait (BTT) and iron deficiency anemia (IDA) makes the distinction between these two distinct disorders with completely different etiological, genetic and therapeutic implication difficult. A comprehensive panel of tests including HPLC, $\mathrm{Hb}$ electrophoresis for TT and serum iron, TIBC, serum Ferritin tests for IDA is usually required to make the diagnosis, however these relatively time-consuming, laborious and expensive techniques may not be available in all clinical settings. With this in mind, we sought out to evaluate the reliability of a simple $\mathrm{CBC}$ parameter, cellular hemoglobin distribution width (CHDW), in differentiating IDA from BTT.

Patients/Materials \& Methods: This prospective study was performed using total of 142 fresh blood samples from 100 patients with IDA (conformed to have low serum iron level by TPTZ method) and 42 patients with BTT (diagnosed by HPLC).Blood samples were 
analysed using automated analyser Siemens Advia-2120i. Cellular haemoglobin $(\mathrm{CH})$ was measured with the help of laser optical assembly having illuminator, flow cell and detector. CHDW was determined as the standard deviation of the RBC CH histogram. The in-house reference range of CHDW was established from normal healthy adults of age group 18-63 years with normal hematological parameters at Tata Memorial Hospital, Hematopathology laboratory (3.01-4.07 pg (picograms)).

Results: The patients included in our study showed slight female preponderance ( $\mathrm{M}: \mathrm{F}=1: 1.49)$. Patients with IDA consistently showed CHDW value normal or higher than reference range (Mean $\mathrm{CHDW}=4.35 \mathrm{pg}$, Males $4.43 \pm 1.39 \mathrm{pg}$, females $4.29 \pm 1.26$ ), whereas patients with BTT had CHDW values lower than reference range (Mean $\mathrm{CHDW}=2.57 \mathrm{pg}$, males $2.58 \pm 0.36 \mathrm{pg}$, females $2.56 \pm 0.37 \mathrm{pg}$ ) (Table 1).

Discussion \& Conclusion: Diseases like IDA andTT have strong socio-economic impact and low-cost, rapid screening methods are required to distinguish the two disorders. In this study, we conclude that CHDW parameter in Advia2120i can reliably help in differentiating Iron Deficiency Anemia (IDA) from Beta Thalassemia Trait (ßTT).

Disclosure of Interest: None Declared.

Keywords: BTT- BETA thalassemia trait, CHDW: Cellular Hemoglobin distribution width, IDA- Iron deficiency anemia.

\section{Abstract 16. Evaluation of a Rapid and Cost Effective POCD for Screening Sickle Cell Disease in Indian Population}

Ravi Ranjan ${ }^{* 1}$, HareramPandey ${ }^{1}$, Amit Sharma ${ }^{1}$, Suman Lata ${ }^{1}$,
Kamal Kishor ${ }^{1}$, Seema Tyagi ${ }^{1}$, TulikaSeth ${ }^{1}$, ManoranjanMahapatra ${ }^{1}$, Renu Saxena ${ }^{1}$

\section{${ }^{1}$ Hematology, AIIMS, New Delhi, India}

Aims \& Objectives: Sickle cell disease is one of the most common single- gene disorders in the Indian population. $>312,000$ births are affected annually by sickle cell anaemia (SCA). Early interventions such as newborn screening, penicillin prophylaxis and hydroxyurea can substantially reduce the mortality and morbidity associated with SCD. Nevertheless, their implementation in Asian countries has been mostly limited to pilot projects. Recent development of low-cost point-of-care testing (POCT) devices for sickle haemoglobin ( $\mathrm{HbS}$ ) could greatly facilitate the diagnosis of those affected.

Patients/Materials \& Methods: We conducted the real-world assessment of a low-cost POCT device, HemoTypeSC, in Indian Sickle Cell Disease Patients families. The study involved parallel testing of 50 known cases of Sickle Cell Disease family individuals by using Point of Care device and HPLC between January to June 2019. The discordant samples were analysed by molecular diagnosis. Results: We found that, in optimal field conditions, the sensitivity, specificity, Positive Predictive value (PPV), Negative predictive value (NPV) of the test for SCD were 97.5\%, 100\%, 100\%, 90.9\%, respectively. It suggests that HPLC results might not be as useful and cost effective in a resource-poor setting as usually considered.

Discussion \& Conclusion: The use of such a POCT device can be scaled up and routinely used across multiple healthcare centres in India, which would offer great potential for the identification and management of vast numbers of individuals affected by SCD who are currently undiagnosed.

Disclosure of Interest: None Declared.

Keywords: Capillary Zone Electrophoresis, Point of Care Device, Sickle Cell Disease.

\section{Abstract 17. Effect of Iron Deficiency Anemia on Immune Status and Monocyte Subset Populations in Children}

\author{
Nimisha Dhankar ${ }^{* 1}$, Richa Gupta ${ }^{2}$, Shyam Lata Jain ${ }^{1}$, \\ ShramanaMandal $^{1}$, Monica Juneja ${ }^{3}$, SmitaKaushik ${ }^{4}$ \\ ${ }^{1}$ Pathology, MAMC, ${ }^{2}$ Pathology, UCMS, ${ }^{3}$ Paediatrics, \\ ${ }^{4}$ Biochemistry, MAMC, New Delhi, India
}

Aims \& Objectives: To assess the cell mediated immunity (T lymphocytes and the subsets), humoral immunity (serum immunoglobulin levels) and monocyte population and its subsets in children with iron deficiency anemia (IDA) and to compare the data with age matched healthy children.

Patients/Materials \& Methods: Children between the age of 1 year and 12 years were matched for age and divided into IDA group (40) and Control group (20) after applying stringent inclusion and exclusion criteria. The cell populations were assessed using flow cytometry and immunoglobulin levels by immunoturbidimetry and the data compared between the two groups.

Results: The study group showed relative leukopenia, lymphocytosis and increase in the B lymphocyte population when compared to the control group. The total $\mathrm{T}$ cell population, its subsets ( $\mathrm{T}$ helper cells and cytotoxic $\mathrm{T}$ cells) and CD4/CD8 ratios were found to be unaltered in the two groups. Total monocyte population showed no change, however non-classical subset showed a significant decrease in the IDA group. Immunoglobulin levels were unaltered.

Discussion \& Conclusion: IDA leads to a quantitative decrease in the total leucocytes as well as non-classical monocytes. The defect in T-cell mediated cellular and humoral immunity seen in IDA might be functional rather than quantitative. More studies need to be done to validate the relative lymphocytosis and B cell lymphocytosis seen in the IDA group. Disclosure of Interest: None Declared.

Keywords: Anemia, Immunity, Monocyte subsets.

\section{Abstract 18. A Case of Paroxysmal Nocturnal Hemoglobinuria Mimicking Congenital Dyserythropoietic Anemia}

\section{Udaya Sundarajan ${ }^{* 1}$, Pradeep Arumugam ${ }^{1}$, Rakhee Kar', Prabhu Manivannan ${ }^{1}$, DebdattaBasu ${ }^{1}$}

${ }^{1}$ Pathology, Jipmer, Puducherry, India

Aims \& Objectives: Paroxysmal Nocturnal Hemoglobinuria (PNH) is an acquired clonal hematopoietic stem cell disorder characterized by increased sensitivity to complement resulting in intravascular hemolysis. This case highlights the need for a high clinical suspicion for PNH in patients presenting with features of refractory anemia with dyserythropoiesis.

Patients/Materials \& Methods: A 22-year-old lady presented with easy fatigability and breathlessness on and off for two years. There was one episode of jaundice at fourteen years which resolved spontaneously. Previously she was transfused once and had been on hematinics. Inspite of treatment, she had low haemoglobin $(\mathrm{Hb})$ documented from her school days. On examination, she had pallor, no icterus, no hepatosplenomegaly and no haemolytic facies. Complete hemogram revealed $\mathrm{Hb}-63 \mathrm{~g} / \mathrm{L}, \mathrm{RBC}-2.14 \times 10 \% / \mu \mathrm{L}, \mathrm{MCV}-98.6 \mathrm{fL}$, MCH-29.4 pg, MCHC-29.9 g/dl, WBC count-1190/ $\mu \mathrm{L}$ with ANC$420 / \mu \mathrm{L}$, platelet count $-69,000 / \mu \mathrm{L}$. Biochemical parameters showed total bilirubin/direct fraction-0.77/0.17 mg \%, LDH-1156 IU, Serum Ferritin-17 ng/mL. Coombs' test was negative and Hb HPLC showed borderline $\mathrm{HbA}_{2}$. 
Results: Bone marrow showed cellular marrow with erythroid hyperplasia with prominent dyserythropoiesis (dumb-bell shaped nuclei, nuclear budding, karryorhexis and internuclear bridging) with reduced marrow iron stores. Young age of presentation with prominent dyserythropoiesis made us suspect a possibility of congenital dyserythropoieticanemia. However other cytopenias and low iron stores were misfits. Soon after the patient presented with high coloured urine which made us suspect hemoglobinuria. Urinalysis showed hemosiderin deposits and gel card test for PNH was positive. Flow cytometry showed PNH clone which were detected in granulocytes, monocytes and RBCs.

Discussion \& Conclusion: The present case throws light upon the diagnostic dilemma associated with refractory anemia and emphasises the need for an intensive work up including a simple Perls' stain on the urine sediment. Dyserythropoietic features in marrow are nonspecific and may be seen in a wide variety of haematological disorders including PNH.

Disclosure of Interest: None Declared.

Keywords: Dyserythropoiesis, Paroxysmal nocturnal hemoglobinuria, refractory anemia.

\section{Abstract 19. Reticulocyte Hemoglobin Concentration a Marker of Bone Marrow Iron in Routine Investigation}

Anoushika Mehan ${ }^{* 1}$, ArathiPrabhakar ${ }^{1}$, Neha Singh ${ }^{1}$, Arvind Gupta $^{1}$, NilotpalChowdhury ${ }^{1}$, Harish Chandra ${ }^{1}$

${ }^{1}$ Pathology, AIIMS Rishikesh, Rishikesh, India

\section{Aims \& Objectives: \\ Aim: \\ Correlation between $\mathrm{CHr}$ and bone marrow iron stores. Objectives:}

1. Comparison of $\mathrm{CHr}$ value with diffrent grades of bone marrow iron stores.

2. Cutoff of $\mathrm{CHr}$ in iron deficient patients.

3. Comparison of $\mathrm{CHr}$ in iron deficient cases and non- iron deficient cases.

Patients/Materials \& Methods: All cases of bone marrow aspirates from 2017 till May 2019 in Department of Pathology and Lab Medicine were reviewed. Marrow aspirates of cases with complete blood count sample not run in reticulocyte mode were excluded. We studied 369 cases of which 112 cases were of diluted/aparticulate smears and five cases with dry tap were excluded. In remaining 252 cases iron grading was done based on Gale et al. scale (grade 0 to grade 5).

Results: Cases with bone marrow iron grade 0 and 1 were classified into group A, Grade 2 were classified as group B, Grade 3, 4, 5 as group C. Group A corresponds to depleted iron stores, Group B to functional iron deficiency and Group $\mathrm{C}$ as non-iron deficient. Average mean $\mathrm{CHr}$ of group A was $25.05 \pm 5.6 \mathrm{SD}$, Group B $28.9 \pm 4.5 \mathrm{SD}$ and Group C $29.6 \pm 5.6 \mathrm{SD}$ with a $p$ value of 0.007 .

Out of 252 cases 62 cases were of iron deficient anemia and 190 non-iron deficient. In patients with iron deficient anemia mean $\mathrm{CHr}$ was $26.9 \pm 5.9 \mathrm{SD}$ and was $29.3 \pm 5.2 \mathrm{SD}$ in non-iron deficient patients with a $\mathrm{p}$ value of 0.005 . Cutoff for $\mathrm{CHr}$ in iron deficiency anemia taken as $29 \mathrm{pg}$.

Discussion \& Conclusion: Reticulocyte hemoglobin content $(\mathrm{CHr})$ is measurement of hemoglobin inside reticulocyte. It correlates directly with marrow iron stores and provides an accurate, simple, painless, inexpensive and early measure of marrow iron stores. There is significant difference in $\mathrm{CHr}$ between iron deficient and non-iron deficient cases.

This study highlights the use of $\mathrm{CHr}$ as a marker of iron stores in iron deficient and non-iron deficient cases with multiple myeloma, chronic lympho proliferative disorders, aplastic anemia and post chemotherapy, when iron cannot be assessed in aspirate smears as they are either diluted/aparticulate/clotted or have dry tap. $\mathrm{CHr}$ should be used as a routine investigation for evaluation of patients with hematological illnesses.

Disclosure of Interest: None Declared.

Keywords: Anemia, Bone marrow Iron stores, Reticulocyte Hemoglobin.

\section{Abstract 20. A Retrospective Observational Study of Paroxysmal Nocturnal Hemoglobinuria}

Aditi Shah $^{* 1}$, NatarajK S ${ }^{1}$, Hamza Dalal ${ }^{1}$, AmarnadhPolisetty ${ }^{1}$, ShivakumarKomaravelli ${ }^{1}$, SharatDamodar ${ }^{1}$

\section{${ }^{1}$ Hematology, Narayana Health, Bangalore, India}

Aims \& Objectives: Primary objective: To evaluate flowcytometric finding, clinical parameters and outcome in patients with paroxysmal nocturnal hemoglobinuria $(\mathrm{PNH})$.

Secondary objective: to compare between aplastic and hemolytic variant of PNH.

Patients/Materials \& Methods: 20 patients were analysed from June 2014 to July 2019. Diagnosis of PNH clone and their quantification was done using flow cytometry technique and PNH clone more than $10 \%$ was included in the study. Patients were divided into aplastic and hemolytic variant based on bone marrow cellularity and hemolytic markers like elevated LDH, reticulocyte count and indirect bilirubin levels. Indoor and outdoor case papers and discharge summaries were scrutinised for data collection.

Results: The overall mean age was $38.85 \pm 14.99$ years, $45.1 \pm 14.19$ years in group 1 and $32.6 \pm 13.66$ years in group 2 ( $p$ value 0.06 ). Male to female ratio was $2.33: 1$. The number of patients in group 1 were $10(50 \%)$ and in group 2 were $10(50 \%)$. The median (IQR) \% of overall PNH clone was $42 \%(18.12-93.75 \%), 29 \%(12.23-44.3 \%)$ in group 1 and $90 \%$ (38-97.12\%) in group 2. The PNH clone was significantly higher in hemolytic variant $(p=0.019)$. The overall median (IQR) time of onset of symptoms to diagnosis was 3.5 months (2-6 months), 2.5 months (2-3 months) in group 1 and 6 months (4.75-11.25 months) in group 2 which was significantly longer ( $\mathrm{p}$ value-0.006). Out of 20 patients, $3(15 . \%)$ had thrombotic events (portal vein thrombosis, Budd Chiari syndrome and cerebral venous sinus thrombosis), $1(5 \%)$ in group 1 and 2 (10\%) in group 2. The median overall follow up (IQR) was 26 months (6 to 57 months), it was significantly longer in group 2 compared to group 1 . Amongst aplastic variant $2(20 \%)$ underwent bone marrow transplant, 3 (30\%) ATG therapy, 4 (40\%) ciclosporin plus danazol and 1 (10\%) were on regular transfusion treatment. Amongst hemolytic variant 4(40\%) were on steroid plus danazol, 2 (20\%) were on only danazol, 2 (20\%) on regular blood transfusion and $2(20 \%)$ underwent bone marrow transplantation. 7 (35\%) patients have expired, $4((20 \%)$ in group 1 and $3(15 \%)$ in group 2. Discussion \& Conclusion: $\mathrm{PNH}$ is an uncommon disease, it almost equally presents as aplastic and hemolytic variant. Patients with hemolytic variant have significantly higher PNH clone and longer duration from time of onset of symptoms to diagnosis. Thrombotic events are more common in hemolytic variant. Overall survival is better in hemolytic variant.

Disclosure of Interest: None Declared.

Keywords: aplastic variant, hemolytic variant, Paroxysmal nocturnal hemoglobinuria. 


\section{Abstract 21. Utility of Perls Stain on Archived Bone Marrow Slide in Diagnosing a Case of Sideroblastic Anemia}

\author{
SushmithaSathyanarayanan ${ }^{* 1}$, Pradeep Arumugam ${ }^{1}$, \\ Prabhu Manivannan ${ }^{1}$, JaikumarGR ${ }^{2}$, Rakhee Kar', \\ DebdattaBasu $^{1}$
}

\section{${ }^{1}$ Pathology, ${ }^{2}$ Paediatrics, JIPMER, Puducherry, India}

Aims \& Objectives: Introduction- Sideroblastic anemia is a group of refractory anaemia with ineffective erythropoiesis. This disease, in which iron accumulates in the mitochondria of erythroblasts as a result of defective incorporation into heme, may be inherited or acquired. There are no distinct clinical features to point to the diagnosis and a high index of suspicion is necessary.

Patients/Materials \& Methods: Case report- A nine year-old-male child, symptomatic since six months of age with features of anaemia requiring monthly blood transfusion, presented to our hospital. The bone marrow done outside, when the child was a toddler was reported as pure red cell aplasia (PRCA) and Hemoglobin HPLC done showed normal pattern. On examination, he had pallor and mild hepatosplenomegaly. Investigations revealed haemoglobin of $64 \mathrm{~g} / \mathrm{L}$ with reticulocyte count of $0.5 \%$. Serum Ferritin was $>1500 \mathrm{ng} / \mathrm{ml}$. Bone marrow was done with the idea of confirming PRCA.

Results: Bone marrow aspirate was aparticulate but the imprint smear was cellular and showed active trilineage hematopoiesis. In the meantime, old bone marrow slides ( 2 sets) were submitted for review. It showed showed contrary to the diagnosis, erythroid hyperplasia with normoblastic to micronormoblastic maturation. These slides were destained and restained with Perls' stain which revealed iron stores of grade $5 / 6$ and numerous ring sideroblasts. A final diagnosis of congenital sideroblastic anaemia was made correlating the clinical presentation, presence of numerous ring sideroblasts and high serum ferritin.

Discussion \& Conclusion: The case was challenging for the following reasons. The erythroid cells with basophilic wispy cytoplasm due to defective hemoglobinisation could have been mistaken for lymphoid cells giving rise to an erroneous diagnosis of PRCA. Initially, Perls' stain was not performed by us on the aspirate as it was aparticulate. The almost eight year old Romanowsky stained slides had to be destained and repeat Perls' staining done, which revealed the ring sideroblasts and clinched the diagnosis. This reiterates the importance of a simple iron stain in cases with refractory anemia. It is important to diagnose sideroblastic anaemia as some of the cases may respond to pyridoxine while the management of PRCA involves immunosuppression to which the patient may be unnecessarily subjected.

Disclosure of Interest: None Declared.

Keywords: Perls stain, Refractory anemia, Sideroblastic anemia.

\section{Abstract 22. Peripheral Smear and Bone Marrow Examination in Patients with Spleomegaly: A Tertiary Centre Study}

\section{Jyoti Mishra $^{\text {*1 }}$, Preetisingh ${ }^{1}$, Sadhnamarwah ${ }^{1}$, Vijay kumar ${ }^{1}$ \\ ${ }^{1}$ Dept. Of Pathology,PgimerDr.RmlHospital,new Delhi, NEW DELHI, India}

Aims \& Objectives: To evaluate the causes of splenomegaly based on peripheral smear and bone marrow morphology findings.

To evaluate diagnostic utility of peripheral smear and bone marrow examination in splenomegaly.

Patients/Materials \& Methods: The study is a retrospective analysis conducted in the Department of Pathology, PGIMER \&Dr. RML
Hospital, New Delhi. All cases presenting with splenomegaly for which bone marrow aspiration and/or biopsy done between 2016-2018 are included in the study. Cases where bone marrow aspiration/biopsy are inadequate have been excluded. Peripheral smear findings and bone marrow aspiration/biopsy findings, wherever available are recorded. Data are analysed using descriptive statistics wherever indicated.

Results: A total of 200 patients with splenomegaly were studied over a period of 2 years. The most common finding in peripheral smear is leucopenia $(40 \%)$ followed by microcytic hypochromic (26\%), normocytic normochromic (20\%), megaloblastic (8\%),dimorphic RBC morphology, leukemia and leucocytosis $(2 \%)$.

On bone marrow examination most common finding is erythroid hyperplasia $(26 \%)$ and least common is leukemia $(8 \%)$. Others i.e. infective $(6 \%)$, splenic marginal zone lymphoma (4\%), megaloblastic (4\%). $22 \%$ are diluted and $21 \%$ are normal.

Discussion \& Conclusion: Any patient who presents with unexplained splenomegaly should have an evaluation with respect to its cause, particularly if cancer is in the differential diagnosis. The study emphasizes on importance of peripheral blood smear and bone marrow examination in evaluating patients of splenomegaly. These two investigations are corner stone in etiological diagnosis of splenomegaly.

Supporting Document: fd56c28d-1539-4f7d-8472-83b87e96e5e0.

Disclosure of Interest: None Declared.

Keywords: Bone marrow aspirate, Peripheral smear, splenomegaly.

\section{Abstract 23. Persistent Splenomegaly in Sickle Cell Anemia: An Unusual Finding}

Neha Aggarwal ${ }^{* 1}$, Priya Sahu ${ }^{1}$, Vijay Kumar ${ }^{1}$, SadhnaMarwah $^{1}$

${ }^{1}$ Pathology, PGIMER, Dr RML Hospital, New delhi, India

Aims \& Objectives: To study persistent splenomegaly in sickle cell anemia.

Patients/Materials \& Methods: A 23 year old female presented with complaints of fever, breathing difficulties and pain in upper limbs and back since 2 days. She had past history of similar complaints on and off. On examination, she had pallor, icterus and splenomegaly. Detailed haematological work up including HPLC was done. It's correlation with clinical details will be presented.

Results: She was found to be HbS on hematological workup.

Discussion \& Conclusion: Sickle cell anemia is caused by a globin chain defect leading to hemolyticanemia and recurrent sickling crisis due to increased sludging and thrombosis in small vessels. Patients develop splenic infarcts, which over time cause autoplenectomy. Most patients have autosplenectomy by the age of 8 years. Persistent splenomegaly is found in about $10 \%$ patients after the age of 10 years which may be due to various environmental and genetic factors.

Disclosure of Interest: None Declared.

Keywords: persistent splenomegaly, sickle cell anemia, sickling crisis.

\section{Abstract 24. Assessment of Serum Holotranscobalamin as Early Biomarker in People Prone to Megaloblastic Anemia}

Abhishek Verma*1, Sunita aggarwal ${ }^{1}$, Sandeep garg ${ }^{1}$, Smitakaushik $^{2}$

${ }^{1}$ General Medicine, ${ }^{2}$ Biochemistry, Maulana Azad Medical College, Delhi, India 
Aims \& Objectives: To compare serum holotranscobalamin levels with serum vitamin B12 levels as early biomarker in people prone to megaloblastic anemia.

Patients/Materials \& Methods: 83 healthy adult patients $(\mathrm{Hb} \geq 12$ $\mathrm{gm} / \mathrm{dl}$ ) prone to megaloblastic anemia were studied for routine hematological investigations, serum vitamin B12, serum holotranscobalamin and serum folic acid levels.

Results: Among 83 patients, patients were classified in 6 groups on the basis of risk factors for vitamin B12 deficiency. 29 patients (34.9\%) were on antiepileptic drugs, 26 patients $(31.3 \%)$ were chronic alcoholic, 10 patients (12\%) had malabsorption, 10 patients $(12 \%)$ had ileal tuberculosis, 6 patients $(7.22 \%)$ had chronic pancreatitis and 2 patients $(2.4 \%)$ had history of ileal resection. 7 patients $(8.43 \%)$ had low serum vitamin B12 levels and low serum holotranscobalamin levels were observed in 30 patients (36.14\%), including 7 patients with low serum vitamin B12 level also. Holotranscobalamin estimation unmasked vitamin B12 deficiency in 23 patients (27.7\%). 7 patients $(8.43 \%)$ had $\mathrm{MCV} \geq 100 \mathrm{fL}$ and 8 patients (9.63\%) had vitamin B12 deficiency related changes on peripheral smear. Low levels of both serum vitamin B12 and holotranscobalamin were significantly associated with changes on peripheral smear, with $\mathrm{p}$ value of 0.039 and 0.041 respectively, while no such association seen with MCV.All patients had normal folic acid levels.

Discussion \& Conclusion: Holotranscobalamin level estimation helps in unmasking vitamin B12 deficiency in patients with normal serum vitamin B12 levels, who are prone to develop megaloblastic anemia. Thus, holotranscobalamin estimation can be considered early and reliable marker of vitamin B12 deficiency in patients prone to megaloblastic anemia.

Disclosure of Interest: None Declared.

Keywords: Holotranscobalamin, Megaloblastic anemia, Vitamin B12.

\section{Abstract 25. Haemoglobin Fractions in Indian Paediatric Population: Do We Need To Look Westward?}

\section{Shabnam Singh $^{* 1}$, Pallavi Sinha ${ }^{1}$, Sarika Singh ${ }^{1}$ \\ ${ }^{1}$ Pathology, Maulana Azad Medical College, Delhi, India}

Aims \& Objectives: Introduction: There is a dynamic change in the fraction of hemoglobin $(\mathrm{Hb})$ in paediatric population (age $0-12$ years). To apply haemoglobin analysis as diagnosis of thalassemia and other hemoglobinopathies in children, we need reference values in our population.

Aim: To establish levels of $\mathrm{Hb}$ fractions in Indian Paediatric population and to compare it with patients with anaemia.

Patients/Materials \& Methods: Eleven cord blood samples from normal deliveries were collected for analysis. One hundred and sixty three children from the pediatric outpatient clinic were recruited \& categorized by their ages into cohorts. Complete blood count was done by hematology analyser XT 2000i and proportion of HbA, $\mathrm{HbA} 2$, and $\mathrm{HbF}$ was obtained by high performance liquid chromatography (HPLC variant II). Patients with haemoglobin less than $10 \mathrm{~g} / \mathrm{dl}$ and any haemoglobinopathies were excluded.

Results: There were 125 specimens left for evaluation. Of these 40 children were females and 85 were males. The percentage of $\mathrm{Hb} \mathrm{A}$, $\mathrm{A} 2$, and $\mathrm{F}$ gradually changed with increasing age. The percentage of $\mathrm{HbA}$ was $20.41 \pm 5.14$ (mean $\pm \mathrm{SD}$ ) in cord blood and increased substantially to $84.7 \pm 1.83 \%$ at $6-12$ months of age. The value of $\mathrm{HbA} 2$ was 0 in the cord blood and reached $2.52 \pm 0.3 \%$ at 6-12 months and increased to $2.65 \pm 0.89$ at $2-12$ years. The percentage of $\mathrm{HbF}$ was $79.9 \pm 0.48 \%$ in cord blood and decreased rapidly in the first 6 months.
Discussion \& Conclusion: There is paucity of Indian data pertaining to normal reference range, this can aid in interpretation of haemoglobin analysis in Indian paediatric population and pave a path for larger data base in near future as prevalence of hemoglobinopathies in Indian paediatric population was $6.7 \%$ in this study.

Disclosure of Interest: None Declared.

Keywords: Hemoglobin fraction, Hemoglobin variants, Pediatric population.

\section{Abstract 26. Analysis of RBC Membrane Defects \& Genetic Mutations in Distal Renal Tubular Acidosis and Anemia}

\section{Avantika Gaur $^{* 1}$, R Godbole ${ }^{1}$, Jyoti Sharma ${ }^{2}$, Jyoti Singhal ${ }^{2}$, R Manchanda ${ }^{1}$}

${ }^{1}$ Pathology, ${ }^{2}$ Nephrology, K.E.M Hospital and Research Center, Pune, India

Aims \& Objectives: To study the red cell membrane flow cytometric analysis in cases of distal renal tubular acidosis with anemia.

Patients/Materials \& Methods: We screened 20 cases of distal renal tubular acidosis with anemia and performed a flow cytometric analysis for red cell membrane defect using eosin 5 malemide dye. We also attempted to analyses the type of anemia and associate it with the genetic mutation using Sanger sequencing.

Results: 2 cases demonstrated red cell membrane defects and associated SLC4A1 gene mutations. Both cases had evidence of hemolyticanemia with negative Coomb's test and non contributory osmotic fragility correlating with a diagnostic variant of distal renal tubular acidosis with hereditary stomatocytosis. In the index case, the mutation was positive in the parents as well, confirming genetic transmission. Results of the remaining 18 cases are pending.

Discussion \& Conclusion: Estimated prevalence of this condition in India is unknown. RBC membrane abnormalities can range from asymptomatic anemia to hemolyticanemia. Patients with SLC4A1 mutation associated distal renal tubular acidosis tend to have asymptomatic or hemolyticanemia, and those with AE1 mutation may have severe hemolysis. These mutations have been reported in Thailand and our study attempts to analyze the same in an Indian population. Phenotypic heterogeneity and mutations being region specific, can lead to under diagnosis.

Disclosure of Interest: None Declared.

Keywords: Flow cytometry, RBC Membrane defect, RENAL TUBULAR ACIDOSIS AND ANEMIA.

\section{Abstract 27. Utility of RET-HE in Differentiating Iron Deficiency Anemia from Anemia of Chronic Disease}

\author{
Sunita SHARMA ${ }^{1}$, Renu SINGH ${ }^{* 1}$, PreetiRai $^{1}$ \\ ${ }^{1}$ Pathology, Lady Harding Medical College, Ghaziabad, India
}

Aims \& Objectives: To study levels of Reticulocyte Hemoglobin (RET He) parameter and soluble transferrin. Receptor (sTfR) in children with iron deficiency anemia and anemia of chronic disease. Patients/Materials \& Methods: It was a hospital-based Cross Sectional study in which 54 patients of IDA (group A) and ACD (group B) were included along with 30 age and sex matched controls. All the cases and controls were subjected to the following tests: $\mathrm{CBC}$ including RET He levels was done by Hematology XN- 1000 series. Serum ferritin was performed by chemiluminescence. CRP and sTfR 
levels were performed by ELISA methods. Statistical analysis was performed by the SPSS version 18. Continuos variables were presented as mean $\pm \mathrm{SD}$. For all statistical tests, $p$ value $<0.05$ was taken as significant.

Results: The age group ranged from 6 months to 17 yrs with maximum cases was $<3$ yrs of age with M:F was $0.41: 1$. The mean value of RET He was significantly reduced in both IDA $(15.03 \pm 3.26 \mathrm{pg})$ and ACD $(22.73 \pm 4.62 \mathrm{pg})$ as compared to controls $(30.18 \pm 2.36 \mathrm{pg})(p<0.001$ each). However, the decreased was more in IDA as compared to ACD which was also statistically significant $(p<0.001)$. The sTfR was high in IDA $(10.82 \pm 3.17) \mu \mathrm{g} / \mathrm{ml}$ as compare to ACD $(2.39 \pm 1.08) \mu \mathrm{g} / \mathrm{ml}$ and controls $(2.03 \pm 0.28) \mu \mathrm{g} / \mathrm{ml}$. The RET He in sub group of ACD with concomitant IDA $(\mathrm{sTfR}>3 \mu \mathrm{g} / \mathrm{ml})$ was significantly reduced as compared to pure ACD subgroup $(p<0.001)$. RET He showed positive correlation with serum ferritin and negative correlation with sTfR in both IDA and ACD groups $(\mathrm{r}=0.064,-0.438$, $p 0.646,<0.001, \mathrm{r}=0.380,-0.496, p=0.05,0$ respectively).

Discussion \& Conclusion: These observations suggested that RET $\mathrm{He}$, a new parameter helps to assess iron availability for erythropoiesis. It can be a valuable additional tool apart from other biochemical and hematological parameters for the diagnosis of iron deficiency anemia (IDA) and also to distinguish concomitant IDA with ACD.

Supporting Document: 052675ff-fb53-4480-83fe-8e33ad3355c0.

Disclosure of Interest: None Declared.

Keywords: anemia of chronic disease, Iron deficiency anemia, Reticulocyte hemoglobin equivalent.

\section{Abstract 28. Study of Prevalence of Thalassemia Trait in Asymptomatic Pregnant Females in a Tertiary Care Centre}

\section{Madhukar Kumar $^{* 1}$, PREETI TRIPATHI ${ }^{1}$, Vanmalinitewari ${ }^{1}$ \\ ${ }^{1}$ pathology, command hospital airforce, BANGALORE, India}

Aims \& Objectives: Plethora of haemoglobin variants are prevalent in India owing to ethnic diversity of its population with minimal to major clinical significance. Being recessively inherited from parents, most carriers are asymptomatic. Over 9000 thalassemic children are born every year. Detection of these asymptomatic carriers by reliable laboratory methods is the cornerstone of prevention of this serious health problems. The aim of this study is to analyze the prevalence of asymptomatic hemoglobinopathies in pregnant females.

Patients/Materials \& Methods: It was aprospective study done in 230 pregnant women attending ante natal clinic at our centre. Study period was 16 months from March 2018 to July 2019. All pregnant ladies were were subjected to complete blood counts, mentzer index calculation, peripheral blood smears and serum iron studies. 105 cases of microcytic hypochromic anaemia were identified. All these patients were taken up for Hb HPLC by automated HPLC analyser (BIORAD, D-10).

Results: 105 ANC cases of microcytic hypochromic anaemia were studied. $4.7 \%$ of ANC cases were found to have Beta thal trait \& $1.9 \%$ came under indeterminate zone. Mentzer index was found to be less than 13 in all cases of Beta thal traits. However, patients with indeterminate $\mathrm{Hb} \mathrm{A} 2$ and few cases $(8 \%)$ of iron deficiency were also found to be low indicating towards its limitations in pregnancy. All patients who were identified $\mathrm{B}$ thal trait were counselled \& their spouse were checked for thal trait. Patients with indeterminate $\mathrm{Hb}$ A2 values were followed up \& HPLC was repeated after correction of Iron/vitamin B12/Folic Acid deficiency.

Discussion \& Conclusion: $4.7 \%$ of ANC cases at our centre were found to have Beta thal trait \& $1.9 \%$ came under indeterminate zone. These cases may go undiagnosed in absence of antenatal screening for hemoglobinopatthy. Hence, routine Hb HPLC in all ante natal cases should be mandatory in order to prevent thalassemia.

Supporting Document: 108438c0-caf9-4b33-a796-796926181e5b. Disclosure of Interest: None Declared.

Keywords: Beta thalassemia, High performance liquid chromatography (HPLC), Microcytic hypochromic anaemia.

\section{Abstract 29. Hemoglobinopathy and Electrophoresis: Every Hero Needs Their Side Kicks}

Pradeep Kumar V $^{* 1}$, Sujay Prasad ${ }^{1}$, Kavya Shankaranarayana ${ }^{1}$, Samrat Bordoloi ${ }^{1}$, AnanthvikasJayaram ${ }^{1}$

\author{
${ }^{1}$ Hematopathology, Anand Diagnostic Laboratory, Bengaluru, \\ India
}

Aims \& Objectives: Hemoglobinelectrophoresis (HBEP) is the choice of investigation for diagnosis of hemoglobinopathy. The pattern of electrophoresis may not yield a diagnosis in every case. Additional Parameters including, red cell indices, reticulocyte count and Cell Counter Scatterplot, may often clinch the diagnosis. We describe a series of such cases from our experience.

Patients/Materials \& Methods: Consecutive cases received for HBEP over 1 year (August 2018 to July 2019) were analysed including additional parameters (red cell indices, reticulocyte count and Cell Counter Scatterplot).

Results: A total of 7400 HBEP samples were received during this time period. Among the discordant cases:

(a) (a)150 cases showed thalassemic indices with normal pattern, hinting presence of hemoglobinopathy.

(b) 96 cases of Double Heterozygotes with varying combinations of $\mathrm{HbS} / \mathrm{HbD} / \mathrm{HbE}$ and Alpha and Beta Thalassemias.

(c) (c)Membrane Disorders: 4 cases of Hereditary Spherocytosis, 2 cases of Hereditary Elliptocytosis and 1 case of hereditary pyropoikilocytosis.

(d) 8 cases of Acute Leukemia including 2 APML.

(e) 4 cases of CML.

Most of the cases with thalassemic indices and double heterozygotes were confirmed on molecular studies using a combination of ARMSPCR, PCR-RFLP and Sanger Sequencing, thus confirming the underlying Hemoglobinopathy. 2 cases of APML were further confirmed on Flow Cytometry and Cytogenetics.

Discussion \& Conclusion: Hemoglobin Electrophoresis is often asked for in cases of anemia. But as observed, the combination of Cell Counter Parameters and Scatterplots often identify the true cause of anemia. One must also be alert to identify Leukemias where Anemia may only be the presenting symptom. A systematic approach will ensure error free diagnosis and optimum management.

Disclosure of Interest: None Declared.

Keywords: Cell Counter Scatterplot, Hemoglobin Electrophoresis, Red Cell Indices. 


\section{Abstract 30. The Phenotype-Genotype Spectrum of Indian Patients with Stomatocytosis}

\author{
Manu Jamwal $^{* 1}$, Anu Aggarwal ${ }^{1}$, Prashant Sharma ${ }^{1}$, \\ Arindam Maitra $^{2}$, Deepak Bansal ${ }^{3}$, Pankaj Malhotra ${ }^{4}$, \\ Reena Das ${ }^{1}$ \\ ${ }^{1}$ Hematology, PGIMER, Chandigarh, ${ }^{2}$ NIBMG, Kalyani, \\ Kolkata, ${ }^{3}$ Pediatrics (Hematology-Oncology Unit), \\ ${ }^{4}$ Internal Medicine (Clinical Hematology Division), \\ PGIMER, Chandigarh, India
}

Aims \& Objectives: Presence of stomatocytes in peripheral bloo film with elevated reticulocyte count and other features of hemolysis are often suggestive of red cell membrane permeability defects. Diagnosis is difficult due to the infrequent occurrence of these disorders, variable genotypes and phenotypes. Stomatocytes can also be seen as an artifact. Multiple large and complex genes are involved in pathogenesis making Sanger sequencing time-consuming and expensive. Hence, targeted resequencing is a better approach to find the underlying genetic cause.

Patients/Materials \& Methods: Nineteen patients were encountered in 14 families with the invariable presence of stomatocytes with or without thrombocytopenia and were consistent with the features of stomatocytic disorder. Common and uncommon causes of anemia were excluded. DNA libraries were prepared using Illumina's custom panels (TruSight One Sequencing Panel and TruSeq Custom Amplicon v1.5 panel) and sequencing was done on a MiSeq/HiSeq Sequencing System (Illumina). Data analysis was done using MiSeq Reporter, TruSeq amplicon pipeline (BaseSpace) and VariantStudio software. Variants predicted to be causal were subjected to validation by Sanger sequencing in the patient and family members.

Results: Fourteen families with 19 patients showing a variable degree of anemia, hemolysis, reticulocytosis, splenomegaly and blood transfusion requirement along with presence of stomatocytes were enrolled.

Of these seven families with 11 patients were diagnosed to have Mediterranean stomatocytosis/macrothrombocytopenia. All had the presence of stomatocytes along with macrothrombocytopenia, short stature and continuous abdominal discomfort. Pathogenic variants were found in the $A B C G 5$ gene in five families and $A B C G 8$ gene in one. In one patient no causal variant was found. These patients were mostly misdiagnosed and the condition seems to be probably underdiagnosed in the country.

Two families were found to have overhydrated hereditary stomatocytosis with probably causal variant found in $R H A G$ gene. Potentially causal variants implicated with xerocytosis were found in four families with five patients in PIEZO1 gene.

One patient who was incidentally detected with stomatocytosis and large platelets had probably deleterious variant in the SLC2A1 gene.

Discussion \& Conclusion: Phenotype characterization was decisive in establishing the causality of the variants identified. All patients with Mediterranean stomatocytosis/macrothrombocytopenia were advised to take ezetimibe (sterol-absorption inhibitor) and were given accurate diagnosis after targeted resequencing. Splenectomy was deferred in patients of Overhydrated Stomatocytosis. Hence, this study implies that inherited conditions of stomatocytosis are underdiagnosed in our country and genetic diagnosis can have therapeutic benefits.

Disclosure of Interest: None Declared.

Keywords: Hemolyticanemia, Next generation sequencing, Stomatocytosis.

\section{Abstract 31. Genotype \& Phenotype Correlation in Thalassemia Syndrome: A Case Series from Tertiary Care Centre}

\author{
Nupur Parakh"1, MoumitaSaha ${ }^{1}$, Kruthikabk ${ }^{1}$, Sunita Sharma ${ }^{2}$, \\ Jagdish Chandra ${ }^{3}$ \\ ${ }^{1}$ Pediatrics, Kalawati Saran Children's Hospital, \\ ${ }^{2}$ Pathology, Lady Hardinge Medical College, ${ }^{3}$ Peditarics, \\ Kalawati Saran Children's Hospital, DELHI, India
}

Aims \& Objectives: The patients with "Thalassemia syndrome" are characterized by marked clinical heterogeneity ranging from Non Transfusion Dependent Thalassemia (NTDT) to Transfusion Dependent Thalassemia (TDT) state due to variable modifiers affecting the phenotypic expression in these patients. Here we report case series of four patients of Thalassemia syndrome who presented with diagnostic dilemma due to variable phenotypic expression.

Patients/Materials \& Methods: We retrospectively analyzed 350 patients of thalassemia syndrome (TDT and NTDT), who were under regular follow up from December 2010 to December 2019 and found that four patients had marked phenotypic variable presentation inspite of similar beta gene abnormalites.

Results: Two cases of 5 yrs of age presented with progressive pallor, hepato-splenomegaly \& no history of blood transfusion in past. CBC and HPLC suggested beta Thalassemia Major with both parents being $\beta$ Thalassemia trait ( $\beta T T)$. One case was homozygous for IVS 1-5 (G/C) and both parents were heterozygous for IVS 1-5 (G/C). Alpha mutation analysis revealed $-\alpha 3.7$ gene deletion. Final diagnosis of NTDT (IVS 1-5 (G/C) homozygous with Alpha Gene deletion) was made. The other was found to be compound heterozygous for IVS 1-1 and codon 8-9 mutation. Alpha mutation analysis revealed $-\alpha 3.7$ gene deletion. Final diagnosis of NTDT (compound heterozygous state for IVS 1-1 and codon 8-9 mutation with Alpha Gene deletion) was given.

The third case presented with clinical diagnosis of TDT. The parents CBC and HPLC suggested $3 T$ T in father and mother being normal. The patient and father were heterozygous for IVS 1-5 mutation and mother was normal. Alpha mutation analysis of patient showed homozygous alpha triplication and both parents being heterozygous for alpha triplication. Final diagnosis of TDT (Heterozygous for IVS 1-1 with Alpha Gene homozygous triplication) was made.

A Mother of patient with TDT presented with history suggestive of NTDT, however her HPLC was suggestive of $\beta T T$. She was heterozygous for IVS 1-1 and had triplicated alpha thalassemia allele $(\alpha \alpha \alpha / \alpha \alpha)$. Final diagnosis of NTDT (IVS 1-5 (G/C) Heterozygous with Alpha Gene Triplication) was made.

Discussion \& Conclusion: Coinheritance of $\alpha$-multiplications/deletions with $\beta$-thalassemia alters clinical phenotype. Down regulation of $\alpha$ expression give insight for new therapeutic modality in $\beta$-Thalassemia syndromes.

Disclosure of Interest: None Declared.

Keywords: alpha Thalessemia, Genotype-phenotype-correlation, Thalassemia.

\section{Abstract 32. Pure Red Cell Aplasia (PRCA): Tripod of Cases}

Swati Rao $^{* 1}$, SwastiJain ${ }^{1}$, Vijay Kumar ${ }^{1}$, SadhnaMarwah ${ }^{1}$

\author{
${ }^{1}$ Department of Pathology, PGIMER Dr RML Hospital New \\ Delhi, New delhi, India
}

Aims \& Objectives: To study the clinico-haematological spectrum of Pure red cell aplasia. 
Patients/Materials \& Methods: Case 1: 1 Y/F_chief complaint of fever and cough $\times 7$ days.

Case 2: $17 \mathrm{Y} / \mathrm{M}$ - chief complaint of fever, dry cough, vomiting and loss of appetite $\times 1$ week.

Case 3: $5 \mathrm{Y} / \mathrm{M}$ - chief complaint of fever, generalised bodyache, arthralgia $\times 1$ month.

In the cases discussed above, On examination pallor was present in two cases and hepatosplenomegaly in first case only.

Results: CBC, peripheral smear and bone marrow studies were done and will be discussed further.

Discussion \& Conclusion: PRCA is a rare disorder resulting from isolated depression of erythroid series cells with normal granulopoiesis and megakaryopoiesis, presenting clinically as anaemia. It is characterized by normocytic normochromic anaemia, reticulocyte count $<1 \%$, marrow erythroblasts $<0.5 \%$. It may be congenital or acquired.

The reticulocyte count usually is very low $(<10,000 / \mu 1)$ and hemoglobin levels decrease at a rate of approximately $0.1 \mathrm{~g} / \mathrm{dl}$ per d $(1 \mathrm{~g} / \mathrm{L}$ per d), corresponding to the RBC lifespan; thus, patients rapidly become transfusion dependent.Serum ferritin increases to very high levels $(>1000 \mu \mathrm{g} / \mathrm{L})$ and so does the transferrin saturation $(>70 \%)$.

Understanding the pathophysiology, it has provided us with novel therapeutic options including cyclosporine, cyclophosphamide, rituximab, anti-thymocyte globulin. Therefore, any anaemia of prolonged duration, not responding to conventional therapy should be evaluated for PRCA.

Besides this other causes should be taken into consideration example ineffective erythropoiesis, dysplastic syndromes, infiltrative diseases of marrow or selective erythroid suppression.

Conclusion: Isolated anemia associated with marked reticulocytopenia should raise suspicion of PRCA. The diagnosis requires absence or near absence of erythroblasts from an otherwise normal marrow.

Disclosure of Interest: None Declared.

Keywords: Anaemia, Erythroid series cells, PRCA.

\section{Abstract 33. Association of Genetic Variants with Vitamin B12 Deficiency in Southern Indian Pregnant Population}

SreenithiSanthakumar ${ }^{* 1}$, Neelakandan Kamalanathan ${ }^{1}$, Dino Mathew $\mathbf{C}^{\mathbf{1}}$, Jabasteen $\mathbf{J}^{\mathbf{1}}$, Haritha Raghavan ${ }^{1}$, Syed Tanveer $A^{1}$, Jaya Lakshimi P.R ${ }^{1}$, Anne George ${ }^{2}$, Vinod Abraham ${ }^{2}$, Eunice Sindhuvi ${ }^{1}$

${ }^{1}$ Haematology, ${ }^{2}$ Community Health and Development, Christian Medical College, Vellore, India

Aims \& Objectives: Vitamin B12 is an important cofactor in onecarbon cycle metabolism whose dysregulation affects the fetal growth and is a common public health problem affecting pregnant women. Genome-wide association studies (GWAS) have identified variants in several genes including fucosyl transferase 6 (FUT6), fucosyl transferase 2 (FUT2), transcobalamin (TCN2), methylene tetrahydrofolate reductase (MTHFR) and cubulin (CUBN), that may influence plasma B12 concentrations. However, little is known about the contribution of these variants to plasma B12 concentrations in the Indian population. The aim of the study is to understand the role of these genetic variants in influencing serum B12 concentrations.

Patients/Materials \& Methods: In this descriptive study, we included 68 pregnant women (age $<35$ years and gestational age $\geq 36$ weeks) who presented to the Department of Community health for child birth. Haematological and biochemical parameters were analysed by standard methods.
Peripheral blood of mother was collected at delivery and genomic DNA was isolated and analysed by standard protocol. All variants specific exons were amplified by PCR and sequenced (Applied Biosystems Inc, Foster city, CA, USA). Serum vitamin B12 and ferritin was measured using immunoassay. Serum hepcidin and GDF15 levels were measured using ELISA. Statistical analysis was performed using SPSS software.

Results: Among the 68 subjects included in the study, 34 had vitamin B12 deficiency (vitamin B12 $<200 \mathrm{pg} / \mathrm{ml}$ ) with anemia. The haematological and biochemical parameters of the groups are tabulated (Table 1). Mean corpuscular volume was significantly higher in vitamin $\mathrm{B} 12$ deficient pregnant women (mean $\pm \mathrm{SD}-83.3 \mathrm{fl}, p=0.000$ ). Serum hepcidin and GDF15 concentrations were higher in B12 deficient group $(p=0.000)$. Previously reported genetic variants rs 78060698, rs 3760775 in FUT6, rs281379, rs601338 in FUT2, rs1131603 in TCN2, MTHFR C677T and rs12780845 in CUBN were present in our population. But these variants were not significantly associated with B12 concentrations. In univariate analysis, serum GDF15, hepcidin and MCV were better predictors of vitamin B12 levels.

Discussion \& Conclusion: Based on significant levels of iron status indicators in B12 deficient state, we propose that hepcidin will be useful as a diagnostic marker for vitamin B12 deficiency during pregnancy. The role of genetic variants has to be functionally analysed in larger cohort as it will help in understanding the complex interplay between genetic determinants and B12 concentrations.

Supporting Document: 1166f83c-a7e0-4c2d-a7bd-107bc58ba404.

Table 1: Haematological and Biochemical parameters of the study cohort

\begin{tabular}{|c|c|c|c|c|c|c|}
\hline Cohort & $\begin{array}{c}\mathrm{Hb} \\
(\mathrm{g} / \mathrm{dL})\end{array}$ & $\begin{array}{c}\mathrm{MCV} \\
(\mathrm{II})\end{array}$ & $\begin{array}{c}\text { VitaminB12 } \\
(\mathrm{pg} / \mathrm{ml})\end{array}$ & $\begin{array}{c}\text { Ferritin } \\
(\mathrm{ng} / \mathrm{ml})\end{array}$ & $\begin{array}{c}\text { Hepcidin } \\
(\mathrm{ng} / \mathrm{ml})\end{array}$ & $\begin{array}{c}\text { GDF15 } \\
(\mathrm{pg} / \mathrm{ml})\end{array}$ \\
\hline $\begin{array}{c}\mathrm{B} 12 \\
\text { deficient } \\
\mathrm{N}=34\end{array}$ & $9.9 \pm 1.1$ & $86.7 \pm 16$ & $\begin{array}{c}118.4 \\
(50-197)\end{array}$ & $\begin{array}{c}8.35 \\
(0.9-61)\end{array}$ & $\begin{array}{c}32 \\
(0.25-173)\end{array}$ & $\begin{array}{c}25611 \\
(10558-86105)\end{array}$ \\
\hline $\begin{array}{c}\text { Control } \\
\mathrm{N}=34\end{array}$ & $10 \pm 1$ & $72.5 \pm 7.2$ & $\begin{array}{c}321 \\
(200-763)\end{array}$ & $\begin{array}{c}6.9 \\
(0.2-30)\end{array}$ & $\begin{array}{c}6.7 \\
(0.26-80)\end{array}$ & $\begin{array}{c}12280 \\
(5641-41501)\end{array}$ \\
\hline
\end{tabular}

Disclosure of Interest: None Declared.

Keywords: Anemia, Pregnancy, Vitamin B12 deficiency.

\section{Abstract 34. Predictors of Quality of Life of Beta Thalassemia Major Children: An Epidemiological Study} BijitBiswas $^{* 1}$, Narendra Nath Naskar $^{2}$, KeyaBasu $^{3}$,
AparajitaDasgupta $^{4}$, Bobby Paul $^{4}$, RivuBasu $^{5}$

${ }^{1}$ Community and Family Medicine, All India Institute of Medical Sciences, Patna, ${ }^{2}$ Public Health Administration, All India Institute of Hygiene and Public Health, ${ }^{3}$ Pathology, Calcutta National Medical College and Hospital, ${ }^{4}$ Preventive and Social Medicine, All India Institute of Hygiene and Public Health, ${ }^{5}$ Community Medicine, R.G. Kar Medical College and Hospital, Kolkata, India

Aims \& Objectives: The study was designed to explore the quality of life $(\mathrm{QoL})$ of beta-thalassemia major $(\beta-\mathrm{TM})$ children, its predictors and relationship with care-related quality of life (CarerQoL) of their caregivers.

Patients/Materials \& Methods: It was a cross-sectional, observational study, conducted among $328 \beta$-TM children and their caregivers attending a tertiary care health facility of Kolkata; West Bengal in between May 2016-April 2017 with a structured schedule which consisted of socio-demography; socio-economy; clinico-therapeutic; caregivers' knowledge regarding the disease, perceived level 
of social discrimination and happiness; Peds4QL and CarerQoL 7D for assessment of QoL of the study participants and CarerQoL of their caregivers respectively where higher score indicated higher QoL. The data were analysed using SPSS 16.0.

Results: The median Peds4QL score was found to be 54.3 with an interquartile range of 43.5-67.4 while in case of CarerQoL score, it was 5 and 4-7 respectively. In the final multivariable logistic regression model, thalassemic children who were residing in an urban area [AOR, adjusted odds ratio: 4.1 (1.9-8.8)], had mothers with higher educational level (middle and above) [AOR: $2.2(1.1-4.2)]$, working parents [AOR: $3.0(1.3-7.0)$ ], no family history of thalassemia [AOR: $3.6(1.6-8.3)$ ], received less number of blood transfusion in the previous year $(<12)$ [AOR: $2.2(1.1-4.3)$ ], higher pre-transfusional haemoglobin $(\mathrm{Hb})$ level [AOR: 1.8 (1.1--.7)], no transfusion transmitted infections (TTIs) [AOR: 3.5 (1.8-6.6))], higher body mass index z score [AOR: 1.6 (1.1-2.3)], caregivers level of happiness ( $\geq$ 5) [AOR: 2.8 (1.4-5.7)] and CarerQoL score ( $>5$ ) [AOR: $2.5(1.2-5.1)$ ] were significant predictors of favourable QoL (Peds4QL score > 54.3) of the study participants adjusted with their age, per capita monthly income (PCMI), spleen status, health seeking behaviour, caregivers knowledge level related to the disease and discrimination status. Overall the model explained $57.3 \%$ variability of QoL of the study participants with predictive accuracy rate (PAR) of $82.5 \%$ while CarerQoL independently explained $17.9 \%$ of its variability with PAR of $68.3 \%$

Discussion \& Conclusion: QoL of the study participants was heavily dependent upon CarerQoL of their Caregivers. To improve the QoL of the study participants, their caregivers CarerQoL need to be enhanced too; therefore, a more holistic approach is required to address this problem.

Supporting Document: b6776cd0-2ecc-4222-a52b-a0a77df6b015.

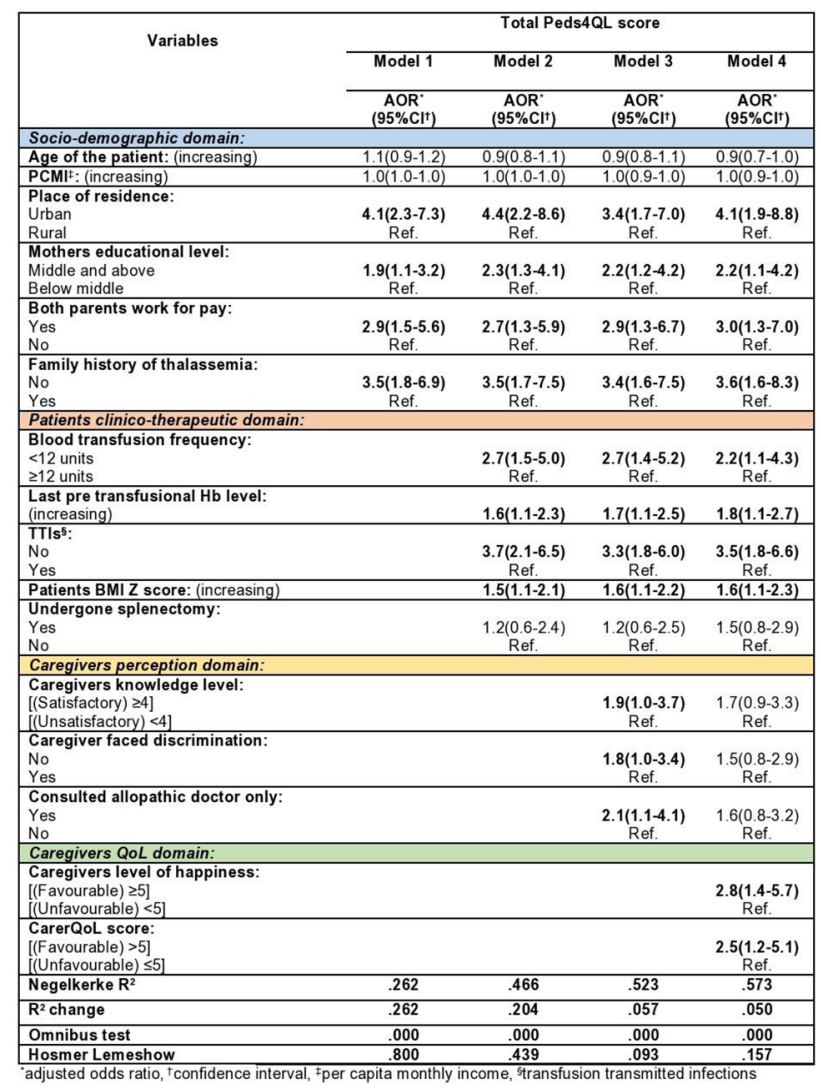

Disclosure of Interest: None Declared.

Keywords: thalassemia, caregivers, quality of life.
Abstract 35. Efficient Molecular Diagnosis of Diamond Blackfan Anaemia

NivedithaRavindra ${ }^{* 1}$, Madhavi Maddali ${ }^{1}$, Prasanth $S^{1}$, Eunice $S$ Edison ${ }^{1}$, Alok Srivastava ${ }^{1}$, Fouzia NA ${ }^{1}$, Biju George ${ }^{1}$, ShajiRV ${ }^{1}$

${ }^{1}$ Department of Haematology, Christian Medical College, Vellore, Vellore, India

Aims \& Objectives: Pure red cell aplasia (PRCA) is a rare cause of anemia in childhood and is attributed to several causes. Diamond Blackfan anaemia (DBA) is an autosomal dominant PRCA caused predominantly by mutations in the ribosome biogenesis genes. A few cases of DBA caused by defects in the genes involved in erythroid differentiation have also been reported. Molecular diagnosis of PRCA cases with suspected DBA helps in establishing the aetiology and can aid in management, prediction of future malignancies and prenatal testing. The aim of this study is to analyze the spectrum of the genes and mutations associated with DBA in the Indian population and to establish genotype-phenotype correlation in this disease.

Patients/Materials \& Methods: Patients suspected with DBA between 2016-2019 were included in the study. Clinical details of the patients and laboratory results such as complete blood count (CBC) and bone marrow findings were analyzed. DNA was extracted from the patients and their parents. DNA was extracted from chorionic villus in one family for prenatal diagnosis. We standardized long amplicon-PCRs (LA-PCRs) for 9 most common genes associated with the pathogenesis of DBA- RPS19, RPL11, RPL5, RPS24, RPS26, RPL35A, RPS17, RPL15 and GATA1. Library preparation and next generation sequencing (NGS) were performed to obtain $100 \mathrm{X}$ coverage of the PCR products. In-house bioinformatics pipeline was used to identify the mutations, and the mutations identified by NGS were confirmed by Sanger sequencing. For the cases that did not have point mutations in the selected genes, MLPA was performed to detect deletions.

Results: During the study period, there were 27 patients whose bone marrow examination was suggestive of PRCA. Out of these, 4 patients $(14.8 \%)$ had systemic/syndromic features characteristic of DBA. Only in one family, there was more than one patient with DBA. The median haemoglobin at presentation in the patients who were not transfused at the time of evaluation was $3.6 \mathrm{~g} / \mathrm{dL}$. Mutations were identified in 14 cases, with point mutations identified by LA-PCRNGS in 11 cases and by MLPA in 3 cases. As reported earlier, RPS19 was found to be the most common gene defective in Indian patients. Mutations in this gene were found in 10 out of 14 cases $(71.4 \%)$, followed by 2 cases with mutations in RPS17. Mutations were detected in RPS26 and RPL5 in 1 case each. One patient had mutations in two genes, GATA1 and RPS19. In none of the cases could the mutations be detected in the parents. Out of the 27 patients, 16 $(59.2 \%)$ responded to treatment with either prednisolone or cyclosporine. Of the remaining patients who did not respond to either treatment, 2 underwent haploidentical transplant and 1 succumbed to complications. There was no significant difference in the number of patients in whom mutations were detected between the patients who responded and did not respond to the treatment. The entire testing could be completed within 3 weeks and this enabled us to perform prenatal testing in one family, where the foetus was normal.

Discussion \& Conclusion: Molecular diagnosis helps in establishing diagnosis of DBA and can be useful in prenatal testing. De novo mutations were observed in all cases. RPS19 is the most common gene involved in our population. The LA-PCR technique is superior to exome sequencing, as it reduces the time and cost involved in the diagnosis of DBA.

Disclosure of Interest: None Declared.

Keywords: Diamond Blackan Anaemia, Long amplicon NGS, MLPA. 


\section{Abstract 36. Comparison of HPLC Systems: Biorad Variant-II And Tosoh HLC-723G11 for Hemoglobinopathy Evaluation}

\author{
ParulChopra $^{* 1}$, Sunanda Bhardwaj ${ }^{1}$, Pushkar Negi ${ }^{1}$, Anil Arora ${ }^{1}$ \\ ${ }^{1}$ Hematology and Immunology, Dr. Lal Path Labs, NRL, Delhi, \\ India
}

Aims \& Objectives: The laboratory diagnosis of hemoglobin abnormalities by HPLC is based on identifying structural variants or abnormal elevations in normal hemoglobins. In the present study we have analyzed two HPLC systems Tosoh HLC-723G11 and Bio-Rad Variant II for the detection of common ( $\mathrm{HbA}, \mathrm{A} 2, \mathrm{~S}, \mathrm{E})$ as well as few uncommon $\mathrm{Hb}$ variants (D-Punjab, D-Iran, Q-India, $\mathrm{HbH}$, Lepore). Patients/Materials \& Methods: This study was carried out at Dr. Lal Path Labs, Delhi in April \& May 2018. EDTA samples from 100 hematologically normal healthy subjects and 107 subjects who were carriers or affected with hemoglobinopathies were analysed using HPLC systems Tosoh HLC-723G11 and Bio-Rad Variant II. Chromatograms generated were studied. Retention time (RT) and area percentage of peaks for $\mathrm{HbA} 2, \mathrm{HbF}$ and other structural variants were compared. Wherever discrepancies were noted, sample was run on Sebia Capillary zone electrophoresis for confirmation of results.

Results: Levels of $\mathrm{HbA} 2$ and $\mathrm{HbF}$ correlated well by both analyzers in normal samples $\left(\mathrm{R}^{2}=0.83\right.$ and 0.9 respectively). On comparison of $\mathrm{HbF}$ values between both analyzers with variant $\mathrm{Hbs}(\mathrm{n}=107)$, a good correlation was seen $\left(\mathrm{R}^{2}=0.99\right)$. HbE co-elutes with $\mathrm{Hb} \mathrm{A} 2$ in Biorad. Correlation done using the apparent $\mathrm{HbA} 2$ concentration from Biorad with $(\mathrm{HbE}+\mathrm{HbA} 2)$ concentration from Tosoh showed a good correlation $\left(\mathrm{R}^{2}=0.97\right)$. Correlation of $\mathrm{HbS}$ (Eluting at $\mathrm{S}$ window at $\mathrm{RT}$ $3.11 \mathrm{~min}$ in Tosoh and $4.33 \mathrm{~min}$ in Biorad) as well as HbD Punjab (Eluting at D-window at RT $2.82 \mathrm{~min}$ in Tosoh and $4.06 \mathrm{~min}$ in Biorad) by both instruments was good. Hb D-Iran (Eluting at E-window at RT 2.69 min in Tosoh and with $\mathrm{HbA} 2$ at 3.53 min in Biorad); $\mathrm{HbQ}$ (Eluting at C-window at RT $3.78 \mathrm{~min}$ in Tosoh and unknown window at $4.7 \mathrm{~min}$ in Biorad), $\mathrm{HbH}$ (Eluting at $\mathrm{P} 00$ window at RT $0.13 \mathrm{~min}$ in Tosoh and giving frontal peak in Biorad), $\mathrm{Hb}$ Lepore (Eluting at P08 window at RT $2.67 \mathrm{~min}$ in Tosoh and with $\mathrm{HbA} 2$ at $3.46 \mathrm{~min}$ in Biorad) gave comparable results. Correlation with findings of $\mathrm{Hb}$ CZE result was done in few cases where identification of $\mathrm{Hb}$ variants by HPLC was difficult.

Discussion \& Conclusion: The comparison between two automated HPLC instruments demonstrated similar usefulness of both in routine screening of patients for presence of hemoglobinopathies. Both instruments give complex elution pattern that may be sometimes difficult to interpret. Also the co-elution of some variants like $\mathrm{HbA} 2$, $\mathrm{HbE}, \mathrm{Hb}$ Lepore, HbD-Iran (in Biorad); HbD-Iran and $\mathrm{HbE}$ (Tosoh) poses difficulty in interpretation. A complementary second method like the use of CZE is required in these cases. Confirmation by molecular tests remains necessary.

Disclosure of Interest: None Declared.

Keywords: Chromatogram, Hemoglobinopathies, HPLC.

\section{Abstract 37. Decitabine vs Hydroxyurea on Hemoglobin F Induction in Patients of E Beta Thalassemia}

\section{Karuna Jha $^{* 1}$, Rudra Ray ${ }^{1}$, MaitreyeeBhattacharyya ${ }^{1}$ \\ ${ }^{1}$ Institute Of Hematology And Transfusion Medicine, Kolkata, India}

Aims \& Objectives: To study efficacy (hemoglobin and hemoglobin $F$ percentage increment) and safety in non-transfusion dependent (NTDT) patients of E-beta thalassemia.
Patients/Materials \& Methods: E-beta Thalassemia patients (age $>12$ years) belonging to NTDT category were randomly assigned into two treatment arms after taking informed consent. One arm received daily oral doses of hydroxyurea (HU) while the other arm received subcutaneous decitabine injections twice weekly for a total period of 12 weeks. Complete hemogram was done every 2 weeks, HPLC was done at the beginning and every month. Follow up period was 12 weeks. Results were studied in terms of increments in hemoglobin and hemoglobin $\mathrm{F}$ from the baseline. Adverse events in terms of infections, cytopenia and deranged biochemical parameters were noted. Effect of underlying molecular factors like beta mutation, associated alpha deletion or XMN1 polymorphism on the response of the drugs on fetalhemoglobin induction also studied.

Results: Peak mean increment in total hemoglobin was $0.98 \mathrm{~g} / \mathrm{dl}$ ( $p<0.001$; range $0.1 \mathrm{~g} / \mathrm{dl}$ to $1.4 \mathrm{~g} / \mathrm{dl}$ ) in $\mathrm{HU}$ arm and $1.28 \mathrm{~g} / \mathrm{dl}$ (range $0.5 \mathrm{~g} / \mathrm{dl}$ to $1.6 \mathrm{~g} / \mathrm{dl})$ in decitabine arm. $53.3 \%$ (8/15) patients on decitabine showed $>1.0 \mathrm{~g} / \mathrm{dl}$ rise in $\mathrm{Hb}$ as compared to $26.6 \%(4 / 15)$ patients in $\mathrm{HU}$ arm. Mean hemoglobin $\mathrm{F}$ percentage increment was $5.13 \%$ in $\mathrm{HU}$ arm and $5.38 \%$ in decitabine arm. Side effects noted were respiratory tract infection $(\mathrm{HU}=66.6 \%$; Decitabine $13.3 \%)$, neurologic ( $\mathrm{HU}$ arm, $6 \%)$, fever $(\mathrm{HU}=13.3 \%)$. No discontinuation of therapy was noted in our study. Associated alpha deletion and XMN1 polymorphism was found to be important determinant of response.

Discussion \& Conclusion: Induction of hemoglobin $\mathrm{F}$ is one of the mechanisms that can alleviate severity of thalassemia. Long term use of hydroxyurea is of concern due to the risk of mutagenicity or carcinogenesis. Low concentrations of decitabine can deplete DNA methyltransferase levels without causing DNA damage. Also it can be more bone marrow sparing, as compared to HU, to sustain a better compensatory marrow erythropoiesis. In our study decitabine was found to be safe and efficacious and better tolerated than hydroxyurea in E-beta thalassemia patients.

Disclosure of Interest: None Declared.

Keywords: decitabine, e beta thalassemia, hydroxyurea.

\section{Abstract 38. Anaemia as a Presenting Feature of Metastatic Prostate Carcinoma: A Case Report}

\begin{abstract}
NongmaithemKaruna $^{* 1}$ on behalf of PGT, RkBanashree ${ }^{1}$ on behalf of associate professor, IromAnil singh ${ }^{2}$ on behalf of ASSISTANT PROFESSOR, LaitonjamSushila devi ${ }^{1}$ on behalf of Professor and Head
\end{abstract}

\section{${ }^{1}$ Pathology, ${ }^{2}$ Medicine, JNIMS, Imphal East, India}

Aims \& Objectives: The most common presenting features of metastatic prostate carcinoma are pain in the back, hips, thighs and other bone pains. Anaemia as a presenting feature in metastatic prostate carcinoma is quite rare.

Patients/Materials \& Methods: A 60 year old man presented to Medicine OPD with anaemia for 3 months, fever and generalised weakness for 4 days who is a T2DM patient under treatment. Patient was evaluated thoroughly and battery of investigations were done. Patient was transfused with 2 units of PRBC however no cause of anaemia was ruled out. Ultimately bone marrow examination was advised as a part of evaluation of an unknown origin of anaemia. During the procedure of the bone marrow examination, bone was extremely hard. Inspite of repeated aspirations no material could be aspirated. Subsequently bone marrow biopsy was tried many a times from both sides and 1 strip of chalky white bone measuring $1.5 \mathrm{~cm}$ was taken out.

Results: On analysis bone marrow aspiration and biopsy imprint were acellular. The trephine biopsy showed hypocellular marrow with 
extensive myelofibrosis and malignant cells arranged in trabecular and acinar pattern could be made out in one of the intertrabecular spaces. On reevaluation the patient gave prolonged history of enlargement of prostate which was asymptomatic at the time of bone marrow examination. Subsequently PSA was sent and it came to be markedly increased $(669.5 \mathrm{ng} / \mathrm{ml})$ which is strongly suggestive of prostate carcinoma.

Discussion \& Conclusion: So this case is presented to highlight the anaemia as an unusual presenting feature of prostate carcinoma metastasizing to bone marrow.

Disclosure of Interest: None Declared.

Keywords: Anaemia, Bone marrow, prostate carcinoma.

\section{Abstract 39. The Clinical and Laboratory Spectrum of Autoimmune Hemolytic Anemia}

\section{SamruddhiMudgal $^{* 1}$, Sushma Belurkar ${ }^{1}$ \\ ${ }^{1}$ Pathology, Kasturba Medical College, Manipal, India}

Aims \& Objectives: To study the prevalence of AIHA in a tertiary care center.

To correlate clinical features and the labparameters in these patients.

To classify AIHA into primary and secondary based on etiology. Patients/Materials \& Methods: This was a 2 years retrospective study. All cases that were DAT positive, with or without Indirect antiglobulin test (IAT), were included in the study. Coombs test was done using Bio-Rad Coombs test gel cards. The peripheral smears were reviewed. The patient clinical details and investigations were retrieved from medical records and Laboratory Information System (LIS). The clinical features and laboratory parameters of the patients diagnosed as AIHA were compared. Statistics was done using SPSS 20.

Results: Total of 453 cases were DAT positive, 74 (16.3\%) of which were classified as primary AIHA and $379(83.6 \%)$ as secondary. The median age was 47 years. M:F ratio was almost equal. The most common etiology for secondary AIHA was SLE (65 cases) followed by HIV (47 cases). The most common presentation was fever and symptoms related to anemia like weakness and easy fatigability. Decreased hemoglobin, increased LDH levels, features of hemolysis on peripheral smear were predominantly seen in secondary AIHA, whereas reticulocytosis and increased bilirubin were more prevelant in primary AIHA.

Discussion \& Conclusion: Study by V. Alwar et al. showed that secondary AIHA was more common than primary and the most common etiology for secondary AIHA was SLE; which is consistent with our study. Though severe anemia and in vivo hemolysis was seen more commonly in primary AIHA. Baek et al. showed that the common presentation of AIHA was anemia and primary was rarer than secondary AIHA.

A diagnosis of AIHA should raise a suspicion to the likelihood of an associated systemic disease. Thus, when faced with a case of AIHA, clinical and laboratory investigations are mandated with the objective of recognizing underlying pathologies such as autoimmune diseases, infectious diseases and neoplasms at the earliest.

Disclosure of Interest: None Declared.

Keywords: Autoimmune HemolyticAnemia (AIHA), Direct Antiglobulin Test (DAT), Hemolyticanemia.
Abstract 40. Molecular Diagnosis of Congenital Dyserythropoietic Anemia by Long Amplicon NGS

Prasanth $\mathrm{S}^{* 1}$, Madhavi Maddali ${ }^{1}$, NivedithaRavindra ${ }^{1}$, Alok Srivastava $^{1}$, Biju George ${ }^{1}$, Eunice $S$ Edison $^{1}$, ShajiRV ${ }^{1}$

${ }^{1}$ Department of Haematology, Christian Medical College, Vellore, India

Aims \& Objectives: Congenital dyserythropoieticanemia (CDA) is an inherited blood disorder that affects the development of red blood cells. The modes of inheritance of CDA may be either autosomal dominant or recessive. Three types of CDA designated as types I, II and III, have been identified based on the morphological characteristics of erythroid cells in bone marrow. Molecular analysis confirms the diagnosis and is a valuable tool for prenatal testing. This study aims to identify the spectrum of mutations causing CDA in Indian population by using the long amplicon NGS (LA-NGS) technique.

Patients/Materials \& Methods: Cases of CDA between 2017-2019 were studied retrospectively. Electronic records were reviewed to study the clinical manifestations and haematological/biochemical parameters including complete blood count, reticulocyte count, HEMPAS test, LDH and bone marrow examination findings. Peripheral blood samples were collected from these patients. DNA was extracted by established protocols. The following genes involved in the pathogenesis of CDA were amplified: CDAN1, c15orf41, SEC23B, KIF23, KLF1 and GATA1. The amplified fragments were pooled and purified using MN kit protocol. Following library preparation, the amplicons were sequenced by NGS to obtain a coverage of 100x. In-house pipelines were used to identify causative mutations. Pathogenicity was established using online tools. Mutations obtained were confirmed by Sanger sequencing.

Results: Five cases of suspected CDA were referred for molecular diagnosis in the study period. Three patients were female $(60 \%)$. The mean age at presentation was 5.55 years. The mean haemoglobin before transfusion was $5.96 \mathrm{~g} / \mathrm{dL}$ with a mean reticulocyte count of $3.46 \%$. Mean LDH level was $684 \mathrm{U} / \mathrm{L}$. The bone marrow examination showed morphology of CDA type I in one case (20\%) and CDA type II in the remaining 4 cases (80\%). HEMPAS test was performed in 2 cases with CDA type II and was positive in one of them. As expected, CDAN1 mutation was identified in the CDA type I case and SEC23B was seen in the CDA type II cases. Three of the SEC23B mutated cases were homozygous for same mutation Y462C which has been reported previously as a common causative mutation in Indian population.

Discussion \& Conclusion: SEC23B is more common than CDAN1 in Indian patients with CDA. LA-NGS was found to be a robust and cost-effective technology for identification of disease causing mutations in CDA.

Disclosure of Interest: None Declared.

Keywords: Congenital dyserythropoietic anaemia, Long amplicon NGS, molecular testing.

\section{Abstract 41. Spectrum of Mutations in Congenital Sideroblastic Anaemia}

NivedithaRavindra $^{* 1}$, EswariS ${ }^{1}$, SumithraS ${ }^{1}$, Uday Kulkarni ${ }^{1}$, ShajiRV $^{1}$, Biju George ${ }^{1}$, Eunice $S$ Edison ${ }^{1}$

${ }^{1}$ Department of Haematology, Christian Medical College, Vellore, Vellore, India

Aims \& Objectives: Congenital sideroblastic anaemias (CSAs) are a group of rare disorders with sideroblasts in the bone marrow. 
Mutations are inherited in an autosomal recessive/X-linked fashion. Genetic diagnosis helps in establishing aetiology and response to pyridoxine supplementation. The aim of this study is to assess the spectrum of mutations in ALAS2 and SLC25A38 genes in patients with CSA in our population.

Patients/Materials \& Methods: Cases with suspected CSA were retrospectively analyzed from 2012 to 2019. Clinical features and laboratory parameters were documented. Peripheral blood was collected from index cases and family members. DNA was extracted followed by sequencing of ALAS2 and SLC25A38 genes. Bioinformatic tools and online databases were used to ascertain pathogenicity. Results: Seventeen patients with suspected CSA were evaluated for molecular diagnosis. There were 13 male and 4 female patients with male:female ratio of $3.25: 1$. Among the 13 male cases, six had mutations in ALAS2; of which 2 cases required regular transfusions. 8 patients had mutations in SLC25A38, all of which were transfusion dependent. Six of the SLC25A38 mutated cases were males and two were females. Overall, 14/17 cases had mutations in these 2 genes $(82.3 \%)$. The SLC25A38 mutated cases presented at an earlier age (Median: 4 months) than the ALAS2 cases (Median: 7 years). The median haemoglobin levels and ringed sideroblast percentage were $5.2 \mathrm{~g} / \mathrm{dL}, 42 \%$ and $5.4 \mathrm{~g} / \mathrm{dL}, 32.5 \%$ in SLC25A38 and ALAS2 mutated cases respectively. A frameshift mutation in SLC25A38 (c.409_410insG) was identified in 4 unrelated cases and appears to be a common mutation in our population. Three cases with ALAS2 mutation became transfusion independent after pyridoxine supplementation (no details for 1 case). One patient in the SLC25A38group underwent haploidentical transplant, but succumbed to transplant related sepsis.

Discussion \& Conclusion: Screening for mutations in ALAS2 and SLC25A38 covers majority of cases of CSA in our population. Molecular diagnosis helps in deciding treatment. Inherited mutations in ALAS2 may present at a later age and should be included in the differential diagnosis when ringed sideroblasts are present in the bone marrow.

Disclosure of Interest: None Declared.

Keywords: molecular testing, pyridoxine, Sideroblastic anemia.

\section{Abstract 42. Efficacy of Thalidomide and Hydroxyurea as HB F Inducer in Non-transfusion Dependent Thalassemia}

\section{Manisha Jain $^{\text {*1 }}$ RajibDe $^{1}$, Ankit Jitani ${ }^{1}$, PrantarChakrabarti ${ }^{2}$, PrakasKumar Mondal $^{1}$, ShuvraneelBaul ${ }^{1}$, TuphanKanti Dolai ${ }^{1}$ \\ ${ }^{1}$ Haematology, Nil Ratan Sircar Medical College and Hospital, ${ }^{2}$ Haematology, Fortis Hospital, West Bengal, India}

Aims \& Objectives: $\mathrm{HbF}$ inducers are known to improve haemoglobin level, and quality of life in NTDT patients. The study was conducted to assess the role of thalidomide and hydroxyurea in these patients.

Patients/Materials \& Methods: A randomized controlled trial of 30 patients with HbE-beta thalassemia was done with 15 patients in each treatment arm. Pregnant females and those planning for pregnancy were excluded. Group A received Hydroxyurea $(10 \mathrm{mg} / \mathrm{kg} /$ day $)$ and Group B Thalidomide (fixed dose-50 mg/day). Response was assessed at 1, 3 and 6 months. Primary end point taken was increment in haemoglobin and $\mathrm{HbF}$ level. Secondary end point was tolerability and safety. Responder was defined as increment of haemoglobin $\geq 1 \mathrm{~g} / \mathrm{dl}$ and non-responder as rise by $<1.0 \mathrm{~g} / \mathrm{dL}$ or drop in haemoglobin level from the baseline.

Results: Demographic details of patients in each group is given in Table. The increment of haemoglobin at 1,3 and 6 months were statistically significant $(p$ value $=0.0007$ ) in the thalidomide arm as compared to hydroxyurea. There was significant reduction ( $p$ value $=0.0001)$ in the blood transfusion requirement in patients in thalidomide. Mean increment in $\mathrm{HbF}$ at 1 and 3 months were not found to be statistically significant in either arm. Adverse effects noted were mild GI upset in hydroxyurea arm, while somnolence and headache in the thalidomide arm, although no medical interventions were required. No severe adverse effects leading to stoppage of drug was reported.

Discussion \& Conclusion: Study shows a statistically significant and sustained increase in haemoglobin with significantly reduction in blood transfusion requirement at 1,3 and 6 months with Thalidomide then with hydroxyurea. Although study including large cohort with long term follow-up is required to depict long term efficacy and safety.

Supporting Document: abe220ec-9b5d-4aba-9efd-459769ab6999.

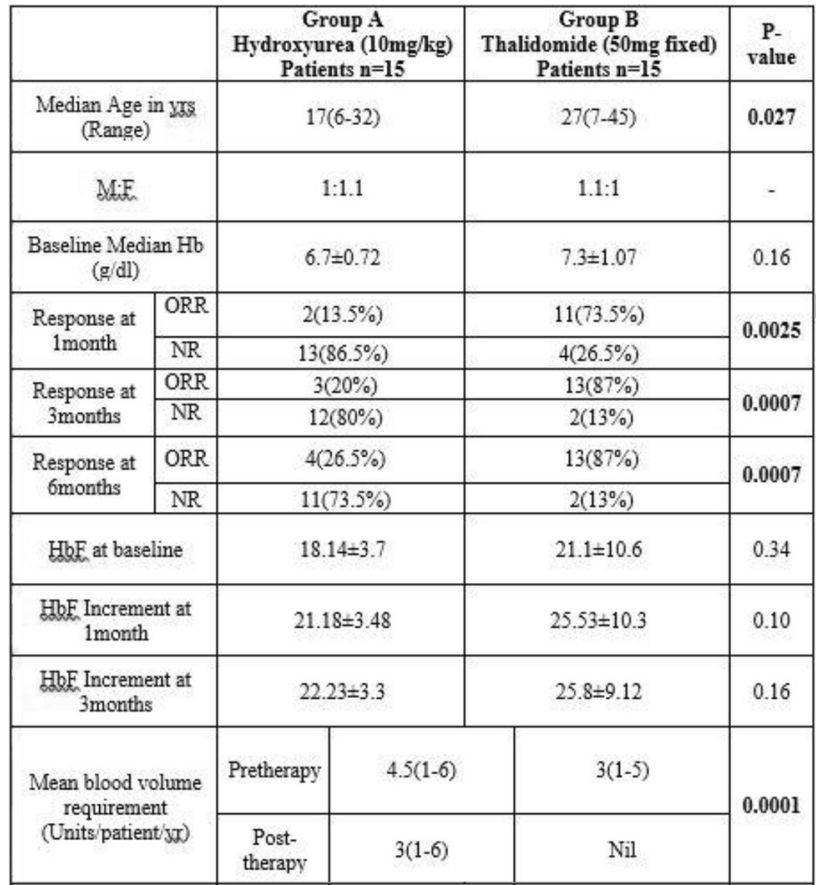

Disclosure of Interest: None Declared.

Keywords: Haemoglobin E-Beta, Hb F: Haemoglobin F, NTDT: Non-Transfusion dependent thalassemia.

\section{Abstract 43. Efficacy of Rituximab in Pediatric Autoimmune Hematological Disorders: A Single Centre Experience}

\section{Bandivarshini*1, Sirisha Ranis $^{1}$ \\ ${ }^{1}$ Hemato Oncology, Rainbow Children Hospital, Hyderabad, India}

Aims \& Objectives: Rituximab is a chimeric monoclonal antibody targeting B cells expressing CD20, which is used for various autoimmune conditions and malignancies refractory to first line drugs including autoimmune haemolytic anemia (AIHA), chronic ITP, Posttransplant lymphoproliferative disease (PTLD), Burkitt's leukemia/lymphoma, SLE etc. This study aims to assess role of rituximab in children aged 1 to 16 years with autoimmune hematologicaldisordes that are refractory to standard modes of treatment. 
Patients/Materials \& Methods: A total of 45 children seen over 6 yrs, between 1 and 16 years with different autoimmune conditions who failed to respond to initial treatment were treated with Rituximab at standard dose of $375 \mathrm{mg} / \mathrm{m}^{2}$ weekly. Response was defined by remission of symptoms, complete or partial reduction in requirement of immunosuppressive drugs.

Results: Total 45 children (male-23, female-22) were screened during study which include Hemolyticanemia $(21 / 45$, 46.6\%) (AIHA were 16, EVAN syndrome were 2, Other anemia 2), ITP $(18 / 45,40 \%)$, Juvenile SLE $(7 / 45,15 \%)$.

In hemolyticanemia group 18/21 (85.7\%) children had complete response (CR) at 2 months after treatment and 19/21 (90\%) after 6 months and all except one became transfusion free. Median haemoglobin raise from $5.6 \mathrm{~g} / \mathrm{dl}$ to $9.3 \mathrm{~g} / \mathrm{dl}$ was noted at 2 months followup.

In ITP group, 5/18 (27.7\%) had CR and 5/18 (27.7\%) had Partial Response (PR) at 2 month follow up. At 6 months follow up 6/18 (33.3\%) had CR.

Among SLE, CR for proteinuria was observed in 4/7 (57\%). At 6 months follow up CR was seen in 5/7 (71\%) along with improvement in haemoglobin level and coagulopathy with subsequent minimal usage of immunosuppressants.

Except two, immediate complications like allergic reactions were not seen. Delayed immunosuppression was seen in one child, requiring IVIG replacement therapy for recurrent gastroenteritis.

Discussion \& Conclusion: In our observation Rituximab was found to be effective and safe treatment option for refractory autoimmune hematological disorders with minimum side effects.

Disclosure of Interest: None Declared.

Keywords: Autoimmune Hemolyticanemia, ITP, rituximab.

\section{Abstract 44. Interesting HB HPLC Findings in a Child with Sickle Cell Disease}

\section{Soma Pradhan $^{* 1}$, Sabina Langer ${ }^{1}$, Jyoti Kotwal ${ }^{1}$ \\ ${ }^{1}$ Hematolgy, Sir Ganga Ram Hospital, New Delhi, New Delhi, India}

Aims \& Objectives: To investigate an unusual pattern of Hb- HPLC of a African origin baby and the parents.

Patients/Materials \& Methods: The peripheral blood examination, Hb-High Performance Liquid Chromatography and gene sequencing of the patients along her parents was done.

Results: The Hemoglobin High Performance Liquid Chromatography (Hb HPLC) of 8 months old boy of African origin was received. The parents were diagnosed as sickle cell trait from outside. The $\mathrm{Hb}$ HPLC using the BioRad 'Variant' System- $\beta$ Thalassemia Short Program revealed 2 major peaks, one constituting $42 \%$ within the S-retention window and the other comprising $22.9 \%$ of abnormal hemoglobin $(\mathrm{Hb})$ with retention time of $4.31 \mathrm{~min}$.

The parental Hb HPLC performed; showed heterozygous state for $\mathrm{Hb}$ A/S (sickle trait) in the father and revealed 3 abnormal peaks in the mother. One abnormal $\mathrm{Hb}(12.5 \%)$ corresponded to the retention time within S- window which indicated heterozygous state of $\mathrm{Hb} \mathrm{S}$. The second and third peaks were at retention time 4.10 and $4.74 \mathrm{~min}$ respectively, constituting $14.5 \%$ and $14 \%$ of total haemoglobins respectively. Since parental HPLC studies was suggestive of sickle trait in both the parents, the possibility of homozygous sickle cell disease with coinheritance of alpha chain variant like HbGPhiladelphia and its hybrid was considered.

The molecular studies of alpha and beta gene sequencing of the baby and parents was done and revealed homozygous state of sickle disease with coexisting heterozygous Hb G-Philadelphia in the baby.
Discussion \& Conclusion: An interesting and rare case of co-inheritance of $\mathrm{Hb} \mathrm{S}$ and HbG-Philadelphia.

Supporting Document: 3d4d09a6-50e9-4b1f-91a0-db77b502f77c. Disclosure of Interest: None Declared.

Keywords: HbG-Philadelphia, HPLC, sickle cell anemia.

\section{Abstract 45. Status of Patients with Autoimmune Hemolytic Anaemia in a Tertiary Care Center of Eastern India}

AvritiBaveja $^{* 1}$, PrakasMandal $^{1}$, ShyamaliDutta ${ }^{1}$, SumitMitra $^{1}$, ShuvraneelBaul $^{1}$, TuphanDolai ${ }^{1}$

\section{${ }^{1}$ Hematology, NRSMCH, Kolkata, India}

Aims \& Objectives: 1 . To assess the Clinico-pathological profile of patient with AIHA.

2. To assess the overall outcome in patients of AIHA.

Patients/Materials \& Methods: The retrospective case record analysis was performed on newly diagnosed patients with auto immune haemolytic anaemia attending the hematology department of NRSMCH, Kolkata with study period July, 2016 to July, 2019. The demographic profile clinical features were documented. A diagnostic baseline workup and workup to determine the secondary causes was performed. Patients were started treatment with first line of therapy with steroids and outcomes were analysed.

Results: There were 80 patients (50 primary; 30 secondary) patients identified with median age of 26 years and of $72(90 \%)$ female patients. The most common etiology was primary $(62.5 \%)$ followed by SLE 20 ( $25 \%$ ) followed by Lympho proliferative disorders $8(10 \%)$. The main clinical features were pallor, jaundice, fever, hepatomegaly, splenomegaly and bleeding manifestations. Onset was acute in 48 (60\%).Median reticulocyte count was $5.8 \%$. DAT positivity was observed in $72(90 \%)$ cases. Evan syndrome was present in $10(12.5 \%)$ cases. Oral prednisolone achieved remission in $68(85 \%)$ cases with median response duration of 16 days. Relapse on steroid tapering was observed in 40 $(50 \%)$ cases. In primary cases, nocorrelation of response was observed with age, sex, presenting haemoglobin, jaundice, TLC, reticulocyte count, hepatosplenomegaly and DAT status.

Discussion \& Conclusion: Autoimmune haemolytic anaemia in this study had heterogenous presentation with female preponderance and younger age group of presentation. Idiopathic causes being the commonest presentation followed by SLE.DAT positivity was common. Steroid induced remission in $85 \%$ cases, but frequent relapses were observed on steroid tapering.

Disclosure of Interest: None Declared.

Keywords: Autoimmune Hemolytic Anemia (AIHA), Direct Antiglobulin Test (DAT), Evan Syndrome.

\section{Abstract 46. Haematological Manifestations in Connective Tissue Disorders: Clinico Pathological Audit}

SiddarthaKasula $^{* 1}$, Sujatha Poduval ${ }^{1}$, Deepika chirumamilla ${ }^{1}$

${ }^{1}$ Pathology, Krishna institute of medical sciences, Hyderabad, India

Aims \& Objectives: Even though cytopenias are common and one of the criteria in the connective tissue disorders like systemic lupus erythematosis; bone marrow abnormalities like hypo/hyper cellularity, marrow lymphoplasmacytosis, myeloid hyperplasia, dyspoietic 
features in erythroid series and abnormal localization of immature precursors are also evident in these Connective tissue disorders. So, the aim is to study the bone marrow findings in the connective tissue disorders. Patients/Materials \& Methods: We examined 110 bone marrow samples (aspirations and biopsies) in a period of two and half years. We set inclusion criteria of positive serology like ANA, RA factor, anti CCP, anti dsDNA etc., and irrespective of the management stage. Symptomatic but seronegative cases are excluded from the study. We studied peripheral blood film and bone marrow for these patients.

Results: Cellular changes are the most common findings with hypocellularity in $56 \%$ of the cases, hypercellularity in $30 \%$ and normocellular in the remaining. Next common finding noted was marrow lymphoplasmacytosis (including lymphoid aggregates) in $33 \%$ of cases followed by dyspoietic changes in erythroid lineage (26\%) and Abnormal localisation of immature precursors (20\%).

Discussion \& Conclusion: Based on these varied changes, we can assume that bone marrow is also the target organ in connective tissue disorders. Like in MDS, dyspoietic features (reactive changes) are also seen in BM of CTD. So, we should not conclude MDS. Similarly, by seeing lymphoid aggregates we should be cautious not to interpret as lymphomatous involvement. Proper history, serological findings should be available for the correct interpretation of these BM findings. So it is our endeavour to study various bone marrow reactions to the CTD and during the course of disease.

Disclosure of Interest: None Declared.

Keywords: Bone marrow, connective tissue disorders, SLE.

\section{Abstract 47. DAT Negative Autoimmune Haemolytic Anemia: Serological Characterization}

\author{
SuryatapaSaha $^{* 1,2}$, Sudipta Sekhar Das ${ }^{1}$ \\ ${ }^{1}$ Transfusion medicine, Apollo Gleneagles Hospital Kolkata, \\ ${ }^{2}$ Transfusion Medicine, Apollo Gleneagles Kolkata, Kolkata, \\ India
}

Aims \& Objectives: A negative DAT does not rule out AIHA. The incidence of this clinical entity known as DAT negative AIHA has been reported to be $2-4 \%$. Sensitive techniques like enzyme linked antiglobulin test (ELAT), flow cytometry (FC), complement-fixation antibody consumption test etc. have been described for diagnosis of DAT negative AIHA. Majority of blood bank laboratories lack these advanced methods. Here we share our experience of diagnosing DAT negative AIHA using simple but sensitive methods which are otherwise less practised.

Patients/Materials \& Methods: The prospective study included 377 anemic patients clinically suspected of AIHA. Blood samples received in blood bank were subjected to polyspecific DAT using both conventional tube test (CTT) and column agglutination technique (CAT). Polyspecific DAT negative results were further evaluated using sensitive but simple methods. Hematological and biochemical parameters of all patients were obtained from hospital information system. In vivo hemolysis was categorized as per the criteria established by previous workers. SPSS statistical software (version 13, USA) was used for all statistical evaluation.

Results: Of the final 353 clinical AIHA patients evidence of autoimmunization by CTT was observed in 335 . Where DAT negative AIHA was observed in $18(5.1 \%)$ patients, 14 showed evidences of autoimmunization by extended sensitive methods. Four patients responded well to AIHA therapy despite DAT negativity by available methods. Severe hemolysis was observed in 4 (22.2\%) DAT negative AIHA patients.

Discussion \& Conclusion: We conclude that DAT negative patients with clinical suspicion of AIHA and positive laboratory evidences should be evaluated for the presence of autoantibody by alternate sensitive methods which are otherwise less practiced. Blood banks may establish these useful simple techniques and stick to the defined protocols to diagnose DAT negative AIHA.

Disclosure of Interest: None Declared.

Keywords: Flow cytometry, Haemolysis, Negative DAT.

\section{Abstract 48. Peripheral Erythrophagocytosis in a Case of Autoimmune Haemolytic Anaemia}

\author{
Kundan Kumar $^{* 1}$, Aparna Ningombam ${ }^{1}$, ShreyamAcharya ${ }^{1}$, \\ AbhirupSarkar ${ }^{1}$, Vijay Antony ${ }^{1}$, Ruchi Tiwari ${ }^{1}$

\section{${ }^{1}$ Department of Laboratory Medicine, All India Institute of} \\ Medical Sciences, New Delhi, India
}

Aims \& Objectives: In peripheral blood smear, erythrophagocytosis by monocytes is an uncommon phenomenon. We would like to report this rare morphological finding in a prospectively diagnosed case of autoimmune haemolytic anaemia.

Patients/Materials \& Methods: A 32-year old male patient, with history of repeated blood transfusions for anaemia, presented with severe pallor, fatigue and icterus. On examination splenomegaly was detected. Peripheral smear was prepared. Direct Coomb's test (DCT) was done, based on clinical suspicion, to confirm diagnosis of autoimmune haemolytic anemia.

Results: Florid erythrophagocytosis by monocytes was seen in peripheral smear. Marked agglutination in red cells, with polychromasia was also evident. DCT came out positive in the patient. The patient recovered within 4 weeks following treatment with corticosteroid, folic acid and iron.

Discussion \& Conclusion: Erythrophagocytosis has rarely been described in cases of paroxysmal cold haemoglobinuria, haemolytic disease of newborn, severe cold agglutinin disease, autoimmune haemolytic anaemia associated with Epstein Barr virus and parvovirus B19 and also in diffuse large B cell lymphoma. In our case, further investigations were not done, hence we could not ascertain the aetiology of autoimmune haemolytic anaemia.

The case highlights the importance of detailed examination of peripheral smear in cases of anaemia where finding like erythrophagocytosis can provide a hint towards possibility of autoimmune haemolytic anaemia. Clinical and prognostic significance of such a finding in these diseases need to be ascertained via long term follow-up of appropriate age and sex matched patient cohorts with valid controls and subsequent relevant statistical analyses.

Disclosure of Interest: None Declared.

Keywords: Agglutination, AIHA, erythrophagocytosis.

\section{Abstract 49. Plasma Hepcidin Level in Diagnosis Of Patients With Microcytic Hypochromic Anemia}

\author{
DebdasBose $^{* 1}$, R Saxena ${ }^{1}$, H P Pati ${ }^{1}$ \\ ${ }^{1}$ Dept. of Hematology, AIIMS, New Delhi, India
}

Aims \& Objectives: Study was done to observe the potential usefulness of plasma hepcidin in the diagnosis of iron deficiency anemia (IDA), beta-thalassemia trait without iron deficiency (BTT) and anemia of chronic disease (ACD), and also to differentiate them from each other. 
Patients/Materials \& Methods: Twenty four cases each of iron deficiency anemia and BTT; and 20 cases each of ACD and healthy controls were taken with age group of 18-60 years with equal male and female distribution. All ACD cases were with adequate to increase bone marrow iron store (grade 3-4+). Controls were taken after excluding anemia, pregnancy, obesity, iron deficiency, thalassemia, and any other diseases by doing batteries of tests, such as alanine \&aspertate transaminase, bilirubin, urea, creatinine, C-reactive protein, erythrocyte sedimentation rate, anti nuclear antibody and rheumatoid factor. Plasma hepcidin was measured by commercial ELISA kit.

Results: Mean plasma hepcidin level among all four groups (Control, IDA, BTT and ACD) are $16.19 \mathrm{ng} / \mathrm{ml}, 1.92 \mathrm{ng} / \mathrm{ml}, 5.02 \mathrm{ng} / \mathrm{ml}$ and $61.20 \mathrm{ng} / \mathrm{ml}$ respectively with a high level of significance of difference in the hepcidin level between these all four groups seen. (ANOVA, $P$ value $<0.001$ ). If plasma hepcidin level is less than $1.95 \mathrm{ng} / \mathrm{ml}$, then IDA is likely rather than ACD, with sensitivity of $100 \%$ and specificity of $95.8 \%$, with $\mathrm{AUC}^{\mathrm{ROC}}$ for hepcidin is 0.996 $(95 \%$ CI $0.985,1.0)$. If hepcidin level is more than $15 \mathrm{ng} / \mathrm{ml}$, is taken as best cut off, then ACD is more likely rather than BTT. \{AUC ROC for hepcidin is $1.00(100 \%)$ with $95 \%$ confidence interval $(1.000$, 1.000 ), with sensitivity of $100 \%$ and specificity of $100 \%$ \}. BTT can be differentiated from IDA using plasma hepcidin level cut off of $3.15 \mathrm{ng} / \mathrm{ml}$ with $\mathrm{AUC}^{\mathrm{ROC}}$ is 0.867 with $95 \%$ confidence interval $(0.759,0.975)$, and sensitivity of $70.8 \%$, and specificity of $91.7 \%$.

Discussion \& Conclusion: Measurement of plasma hepcidin level by ELISA is able to discriminate IDA, BTT and ACD, and may help in diagnosis of these three entities.

Disclosure of Interest: None Declared.

Keywords: Diagnosis, Microcytic hypochromic anaemia, Plasma hepcidin level.

\section{Abstract 50. Clinico-Epidemiological Profile and Correlation with Etiology in Anaemia of Elderly}

\author{
Meghaverma $^{* 1}$, Atul Goel ${ }^{2}$, Sangeeta pahuja ${ }^{3}$ \\ ${ }^{1}$ Clinical Haematology, Sir Ganga Ram Hospital, ${ }^{2}$ Internal \\ Medicine, Lady Hardinge Medical College, Delhi, ${ }^{3}$ Pathology, \\ Lady Hardinge Medical College, New Delhi, India
}

Aims \& Objectives: To study the clinical and etiological profile of anemia in hospitalized geriatric patients in a tertiary care center.

Patients/Materials \& Methods: We studied 50 consecutive admitted geriatric patients diagnosed with anemia on admission. Patients were examined and investigated for clinicohematological profile and aetiology of anaemia and correlations were made in reference to severity, sex and age.

Results: majority of patients had mild anemia, with anaemia of inflammation $(48 \%)$ as the most common etiology. normocytic normochromic $(52 \%)$ was the most common morphology found. No significant correlation was found between severity of anaemia with age or sex.

Discussion \& Conclusion: Most of the hospitalized elderly patients diagnosed with anemia have anemia of inflammation as etiological diagnosis and normocytic anemia as morphological diagnosis. These findings are similar and mostly comparable to the previously conducted studies Etiological profile of anemia in elderly of different from the younger age group. Confirming the type of anemia is critical to direct the investigation for profiling the etiology.

Disclosure of Interest: None Declared.

Keywords: anemia, elderly, Etiology.
Abstract 51. Bone Marrow Dyspoiesis Associated with Severe Refractory Anemia in Liver Cirrhosis

AnnapooraniVaradarajan $^{* 1}$, Chagganbihari ${ }^{1}$

${ }^{1}$ Hematology, ILBS, New Delhi, India

Aims \& Objectives: Peripheral cytopenias and dyspoiesis are common in cirrhosis and pose challenges in management. However, the prevalence of dyspoiesis and its contribution in cirrhosis related cytopenias has not been studied. We aimed to study the bone marrow (BM) dyspoiesis and its impact on peripheral blood cell counts and refractory anemia in patients with cirrhosis.

Patients/Materials \& Methods: We reviewed all the BM aspirates and biopsies of cirrhotic cases, done from 2011-2018 for clinical indications. Dyspoiesis was considered if $>5 \%$ of the precursor cells of any of the 3 lineages showed dyspoietic changes-incoherent nuclear-cytoplasmic maturation, frayed cytoplasmic edges, nuclear budding, bi or multilobulation of the nucleus of erythroid cells, nuclear fragmentation, mitosis, uneven distribution of chromatin, monolobulated megakaryocytes, abnormal hypo or hypergranulation. Cases with neoplasia, primary hematological malignancies, chronic kidney disease, drug intake, acute and chronic hepatitis, granulomatous disease were excluded.

Results: A total of $608 \mathrm{BM}$ of cirrhotic cases were assessed. Of these, 82 cases $(13.2 \%)$ showed dyspoiesis in the BM precursors. There was no difference in age $(p=0.15)$, gender $(p=0.42)$ and spleen size $(p=0.21)$ in cases with or without dyspoiesis. Majority of the cases had dyspoiesis in erythroid series $(60,70.5 \%)$, and megakaryocytes $(11,17.6 \%)$, Dyspoiesis was more prominent in alcoholics (45; $p=0.033)$ and autoimmune diseases $(13 ; p=0.02)$ than other etiologies. Erythroid hyperplasia $(47 \pm 14.5 \%$ vs $39.4 \pm 12.1 \%$; $p<0.001$ was higher in cases with dyserythropoiesis. Patients with dyspoiesis had lower hemoglobin $(6.8 \pm 1.5$ vs $9.5 \pm 2.2 \mathrm{~g} / \mathrm{dl}$, $p<0.001)$, but the total leukocyte count $(p=0.35)$ and platelet counts $(p=0.21)$ were comparable. $52(8.07 \%)$ had refractory anemia $(\mathrm{Hb}<7 \mathrm{~g} / \mathrm{dl}$ for 3 months after nutritional supplementation and required $\geq 3$ transfusions).

Discussion \& Conclusion: BM dyspoiesis, especially dyserythropoiesis, is associated with severe refractory anemia in cirrhotics and requires new therapeutic approaches.

Disclosure of Interest: None Declared.

Keywords: Alcoholic liver disease, Cirrhosis, Dyspoiesis.

\section{Abstract 52. Erythrocytosis Interactions with Beta Thalassemia Trait \& HPFH-3}

Nabhajit MALLIK ${ }^{* 1}$, Prashant SHARMA ${ }^{1}$, Sanjeev CHHABRA ${ }^{1}$, JasbirHIRA ${ }^{\mathbf{1}}$, Neelam VARMA ${ }^{1}$, Pankaj MALHOTRA ${ }^{2}$, Amita TREHAN ${ }^{3}$, Reena DAS ${ }^{1}$

\author{
${ }^{1}$ Hematology, ${ }^{2}$ Internal Medicine, ${ }^{3}$ Pediatrics, PGIMER, \\ Chandigarh, India
}

Aims \& Objectives: We present 3 cases that were being investigated for erythrocytosis, but during the work up were found to have the additional findings of beta thalassemia trait ( 2 patients) and hereditary persistence of fetal haemoglobin (1 patient). We aim to highlight the effect that these haemoglobin disorders have on the underlying erythrocytosis.

Patients/Materials \& Methods: Two of the patients were brothers aged 7 (patient 1) and 22 (patient 2). Both were detected to have erythrocytosis during evaluation for fever. The older sibling had a history of stroke and seizures during infancy, and delayed 
developmental milestones. Patient 3 was 6 years old when he presented with headache, and on evaluation was found to have erythrocytosis.

Results: Patient 1 and patient 2 had Hb levels of $210 \mathrm{~g} / \mathrm{l}$ and $225 \mathrm{~g} / \mathrm{l}$ respectively, and showed microcytic hypochromic RBCs. The RBC counts were $10.26 \times 10^{12} / 1$ and $10.55 \times 10^{12} / 1$ respectively. $\mathrm{Hb}$ HPLC showed normal $\mathrm{Hb} \mathrm{A}$ and $\mathrm{Hb} F$ levels, and increased $\mathrm{Hb}$ A2 levels (5.3\% and 5\% respectively) in both cases. Genetic testing revealed an IVS 1-5 (G-C) heterozygous state in both the patients. Patient 3 had $\mathrm{Hb}$ level of $224 \mathrm{~g} / \mathrm{l}$, normal RBC indices and RBC count of $7.85 \times 10^{12} / 1$. Hb HPLC showed $49.8 \% \mathrm{Hb} \mathrm{A}, 2.3 \% \mathrm{Hb}$ A2 and $35.8 \% \mathrm{Hb} \mathrm{F}$. Genetic testing revealed presence of HPFH-3. In all three patients, the serum erythropoietin levels were within normal limits. Molecular testing for JAK2 V617F, JAK2 exon12 and VHL exon3 were negative.

Discussion \& Conclusion: All three patients had a relatively early age of presentation. The brothers with concomitant BTT showed extremely high RBC counts, with the heterozygous thalassemia mutation possibly contributing to it. A study with a larger number of patients having erythocytosis along with hemoglobinopathies might provide interesting insights on the interplay between the effects of mutations in these disorders.

Disclosure of Interest: None Declared.

Keywords: Beta thalassemia, Erythrocytosis, HPFH.

\section{Abstract 53. Frequency of Alpha Gene Number and HMOX1 Polymorphism in Indian Sickle Cell Disease Patients}

\section{HareramPandey $^{* 1}$, Ravi Ranjan', KanwaljeetSingh ${ }^{1}$, Amit sharma $^{1}$, Kamal kishor ${ }^{1}$, Manoranjanmahapatra', Renu Saxena ${ }^{1}$ \\ ${ }^{1}$ Hematology, All India Institutes Of Medical Science, New Delhi, Delhi, India}

Aims \& Objectives: The aim of the study was to correlate the effect of different genotypic factors with the sickle cell patient's phenotype. To determine the molecular and hematological correlation under influence of inherited co-factors like $\alpha$ deletion and Hmox 1 polymorphism.

Patients/Materials \& Methods: A total 60 patients, presence of $\mathrm{HbS}$ were studied. Their clinical details, hemogram and HPLC findings were noted.GAP PCR was done for detection alpha deletion. Where as allele specific PCR was done for HMOX1 polymorphism.

Results: A total of 60 sickle cell anemia patient's blood sample collected and characterized. After identification of mutation, subjects were categorized in three groups according to the presence of alpha thalassemia genotype. Eighteen patient had alpha deletions with a mean age $10.4 \pm 6.7$ (10 male and 8 female) and 3 patient had anti $\alpha$ $3.7 \mathrm{~kb}$ (alpha triplication) with mean age group $18 \pm 6.08$ (2 male and 1 female) while 39 patients were without any alpha deletions with mean age of $11.41 \pm 8.07$ ( 24 male and 16 female). Patient with presence of alpha deletions had higher hemoglobin, mean corpuscular volume $(\mathrm{MCV})$, mean corpuscular hemoglobin $(\mathrm{MCH})$ levels and mean corpuscular hemoglobin concentration (MCHC). Mean difference of hematological variables amongst patient with alpha deletions and without alpha deletions were statistically significant ( $p$ value $<0.05$ ).Highest frequency of alpha 3.7 heterozygous $(50 \%)$, followed by alpha 3.7 homozygous $(38.89 \%)$ and 4.2 heterozygous $(11.11 \%)$ were found in sickle cell anemia patients.

We report the higher frequency of splenomegaly (19.04\%), acute chest pain $(16.66 \%)$ and painful crisis $(21.42 \%)$ in SCD patients without presence of alpha deletions while SCD patients with co- existence of alpha thalassemia had less frequency of splenomegaly, chest pain and painful crisis.

Out of 60, 27 were heterozygous (AT), 19 were homozygous (TT) and 14 were normal (AA) genotype for HMOX1 polymorphism. Haematological parameters i.e. Reticulocytes, haemoglobin and red cell indices were improved in HMOX1 (TT) carrier. The frequency of clinical condition was severe in Hmox 1 heterozygous phenotype in sickle homozygous patients.

Discussion \& Conclusion: Discussion: The prevalence of splenomegaly is higher but leg ulcer, acute chest syndrome, priapism is lower than the west. This may be due to higher prevalence of alpha and beta gene co-inheritance with $\mathrm{HbS}$ in India than in the West.

Conclusion: This study indicates that in sickle cell disorders, $\alpha$ thalassemia co-existence ameliorates the phenotype and may play pivotal role in understanding the clinicopathological profile of these cases.

Disclosure of Interest: None Declared.

Keywords: Alpha Gene deletion, HMOX1, polymorphism.

\section{Bleeding Disorders}

\section{Abstract 1. Role of Peripheral Blood Smear in Screening of Rare Bleeding Disorder with Unusual Presentation}

\author{
Nidhi Rathi* ${ }^{* 1}$, Ankur Ahuja ${ }^{1}$, TathagatChatterjee ${ }^{1}$, \\ Devika Gupta ${ }^{1}$, KanwaljeetSingh ${ }^{1}$ \\ ${ }^{1}$ Dept of Lab \& Molecular Medicine, Army Hospital RR, Delhi, \\ India
}

Aims \& Objectives: To Assess the importance of peripheral blood smear in a case who presented with capillary hemangioma.

Patients/Materials \& Methods: We present a case who presented with capillary hemangioma and whose sample was sent for complete blood count and peripheral blood smear.

Results: Our case an eight months old fair skinned female child, first born of non consanguinous marriage presented with bluish pigmentation of face and limbs, capilliaryhemangioma on forehead,multiple mongolian spots over back. Her anterior Fontanelle was not closed and had small eyes with epicanthal folds and low set ears are noted. Mother also noted hyperpigmentated spots. No abnormality was detected on systemic examination.

On routine basis her $\mathrm{CBC}$ was sent which showed normal haemogram and peripheral blood smear revealed normocytic normochromic RBCs with no increased polychromasia, Chediak Higashi Granules were seen in neutrophils, eosinophils, lymphocytes and monocytes. No left Shift was noted. Hair Shaft specimen shows regular sized and shaped pigment clumps consistent with Chediak Higashi Syndrome. Fundus examination shows peripheral retinal pigment epithelium atrophy. Her endocrine profile was normal.

The history when elicited revealed that patient was getting multiple upper respiratory infections and was advised repeated nebulisation. The patient had developed a bluish pigmentation in response to nebulisation suggestive of a sensitive skin. Because of her fair looking skin hypopigmentation of skin which was not noticed in this case.

Sequence analysis was done which revealed LYST gene mutation which confirmed the presence of Chediak Higashi syndrome. Her bone marrow was also done which revealed normal cellular reactive bone marrow with no hemopgaocytic activity.

Discussion \& Conclusion: Chediak-Higashi syndrome (CHS) is a rare, inherited, complex, immune disorder that usually occurs in childhood characterized by reduced pigment in the skin and eyes 
(oculocutaneous albinism), immune deficiency with an increased susceptibility to infections, and a tendency to bruise and bleed easily. In a rare disorder like Chediak Higashi disease, when the history is not forthcoming it was the appearance of the presence of 'giant granules' in microscopic analysis of white blood cells that led to the diagnosis. Hence it becomes important in every case to correlate the history and clinical findings with peripheral blood picture so that no cases are missed.

Supporting Document: 6e23ddf0-d0c2-4a5e-ba1a-5fe2bdbc539f.

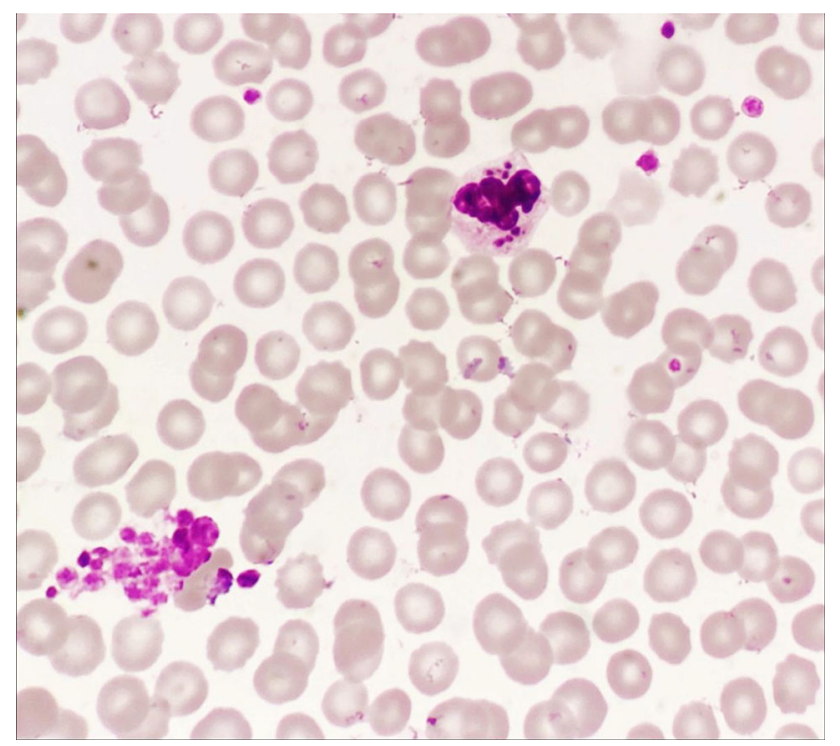

Disclosure of Interest: None Declared.

Keywords: Capillary hemangioma, Chediak Higashi syndrome, Giant granules.

\section{Abstract 2. Spectrum of Transfusion Transmitted Infections in Hemophilia Patients}

\section{Roopa Sharma $^{* 1}$, Naresh Gupta ${ }^{1}$, Sandeep Garg ${ }^{1}$}

\section{${ }^{1}$ Internal Medicine, Maulana Azad Medical College, Delhi, India}

Aims \& Objectives: To determine prevalence of transfusion transmitted infections (TTIs) in adult haemophilia patients and to study clinical spectrum of disease resulting from these infections.

Patients/Materials \& Methods: This study was cross sectional analytic study done on $\mathbf{1 0 0}$ confirmed cases of Hemophilia A and B patients aged 18 years and above at haemophilia day care centre of Loknayak Hospital, Delhi.Patients had to respond to small questionnaire and clinical examination was done. Blood samples were collected for liver function tests and detection of seven transfusion transmitted infections which included Hepatitis C (AntiHCV), Hepatitis B (HbsAg), HIV (ELISA), Syphilis (VDRL), CMV (IgG seroloy), Dengue (IgG serology) and Malaria (peripheral smear).

Results: Eleven percent of patients were found to be TTI positive, however prevalence of TTI in Hemophilia patients was $12 \%$, as one of the patient was coinfected with hepatitis B and C. Prevalence of Hepatitis C was found to be $9 \%$, Hepatitis B $2 \%$, HIV $1 \%$, while none of the patients were found positive for Syphilis (VDRL), Dengue (IgG) and Malaria (peripheral smear). Prevalence for CMV IgG positivity was found to be $76 \%$. Hepatitis $\mathrm{C}$ was the most prevalent TTI and two third patients (6/9) of Hepatitis C were asymptomatic. Patients with viral hepatitis had significantly deranged liver functions tests $(p<0.05 \%)$. Out of two Hepatitis B patients, only one was symptomatic. Only patient with HIV positivity had no clinical evidence of opputunistic infections. Age and number of transfusions were independent risk factors for acquiring TTI $(p<0.05)$.

Discussion \& Conclusion: Hemophilia patients have higher risk of acquiring transfusion transmitted infections with hepatitis $\mathrm{C}$ being most prevalent TTI. Since most of the patients with TTIs remains asymptomatic till the disease progresses, it is thus important to screen these patients. In recent years, due to mandatory blood screening, use of more senstive methods to screen TTIs in blood banks and availability of virally inactivated plasma derived or recombinant factors,prevalence of TTIs is on decreasing trend.

Disclosure of Interest: None Declared.

Keywords: Hemophilia, Hepatitis B and C, Transfusion transmitted infections.

\section{Abstract 3. Factor VIII Inhibitors- IGG Subclass Profile in Different Cohorts of Hemophilia Patients}

\author{
RuchaPatil $^{* 1}$, ShrimatiShetty ${ }^{2}$, Chandrakala $S^{3}$
}

${ }^{1}$ Haemostasis and Thrombosis, Icmr- National Institute Of Immunohaematology, ${ }^{2}$ Haemostasis and Thrombosis, National Institute of Immunohaematology, ${ }^{3}$ Haematology, King Edward Memorial Hospital, Mumbai, India

Aims \& Objectives: To better understand the association of inhibitor kinetics with IgG subclass in haemophilia A (HA) patients.

Patients/Materials \& Methods: Twenty controls, 45 inhibitor negative HA, $17 \mathrm{HA}$ with $<5 \mathrm{BU} / \mathrm{mL}$ and $19 \mathrm{HA}$ with $>5 \mathrm{BU} / \mathrm{mL}$ inhibitors, $13 \mathrm{HA}$ with immediate acting inhibitors (IAIs), 5 acquired HA and 5 lupus anticoagulant (LA) positive, 7 HA on ITI were included in the present study. The inhibitor titer was deduced by Nijmegen Bethesday (NBA) assay.

Results: The results of IgG subclass ELISA are shown in Table 1/ Fig. 1. Among the 30 repetitive samples of 13 patients with IAI, the interesting observation was the evolution of $\mathrm{IgG}$ subclass subclass along with inhibitor kinetics i.e. all these samples were $\operatorname{IgG} 1$ or/and IgG4 positive when they were positive for progressive FVIII inhibitors who gradually developed $\mathrm{IgG} 2$ and $\mathrm{IgG} 4$ when they became positive for IAI. Similar profile was also seen in all the 5 acquired HA patients. In 7 patients on ITI, the 2 successful patients showed IgG4 and IgG1 initially and then a there was a gradual decrease in IgG4; IgG1 prevailing which eventually became negative. All the remaining ITI patients developed IAIs and were positive for IgG2 and IgG4. In 3 of these patients ITI was discontinued as even after 1- 2 years, these patients had IAIs with no improvement in titre. Two of these patients had all subtypes. The $5 \mathrm{LA}$ samples were negative for all the IgG subtypes.

Discussion \& Conclusion: IgG4 subclass is generally found in all patients with high titre inhibitors. In ITI patients the success seems to be synonymous with the disappearance of IgG4 followed by IgG1 subclass. IgG3 seems to be the non-pathogenic and non-neutralizing in HA. FVIII inhibitors are generally time and temperature dependent whereas LAs are IAIs. However there exists the presence of specific FVIII inhibitors in some congenital HA with absence of time dependent inactivation kinetics. The observation that 8 out of $10 \mathrm{HA}$ patients on ITI who initially had classical FVIII inhibitors developed IAIs is interesting and the clinical relevance needs to be looked into. The IgG subclass profile in these patients similar to that of acquired HA raises a question whether immunosuppression along with classical treatment can improve the clinical outcome.

Supporting Document: f4cfac0b-a638-4116-9bc5-3dd2b0aab4c7. 
Table 1: IgG subclass in different cohorts of haemophilia A patients

\begin{tabular}{|l|l|c|c|l|}
\hline $\begin{array}{l}\text { Sr. } \\
\text { No. }\end{array}$ & Controls/ Patients & Number & $\begin{array}{l}\text { FVIII IgG } \\
\text { positive }\end{array}$ & IgG subclass \\
\hline 1 & Healthy controls & 20 & 2 & 2-IgG3 \\
\hline 2 & $\begin{array}{l}\text { Lupus } \\
\text { anticoagulant } \\
\text { positive }\end{array}$ & 5 & 0 & 0 \\
\hline 3 & $\begin{array}{l}\text { Inhibitor negative } \\
\text { patients }\end{array}$ & 45 & 9 & $\begin{array}{l}\text { 6-IgG3 } \\
\text { 3-IgG4 }\end{array}$ \\
\hline 4 & $\begin{array}{l}\text { Low titre inhibitor } \\
\text { patients }\end{array}$ & 17 & 14 & $\begin{array}{l}\text { 5-IgG1 } \\
\text { 1-IgG1 and IgG3 } \\
\text { 6-IgG4 } \\
\text { 1-IgG4 and IgG3 }\end{array}$ \\
\hline 5 & $\begin{array}{l}\text { High titre classical } \\
\text { inhibitor patients }\end{array}$ & 19 & 19 & $\begin{array}{l}\text { 17-IgG4 } \\
\text { 2-IgG4 and IgG3 }\end{array}$ \\
\hline 6 & $\begin{array}{l}\text { Immediate acting } \\
\text { inhibitor patients }\end{array}$ & $\begin{array}{l}30 \text { repetitive } \\
\text { samples in } \\
13 \text { patients }\end{array}$ & 30 & $\begin{array}{l}\text { 23-IgG2 and IgG4 } \\
\text { 4- IgG2, IgG4 and IgG3 } \\
\text { 3- all four subclass }\end{array}$ \\
\hline 7 & $\begin{array}{l}\text { Acquired } \\
\text { haemophilia }\end{array}$ & 5 patients & 5 & $\begin{array}{l}\text { 4- IgG2 and IgG4 } \\
\text { 1- IgG2, IgG4 and IgG3 }\end{array}$ \\
\hline
\end{tabular}

Figure 1: IgG subclass in different cohorts of haemophilia A patients

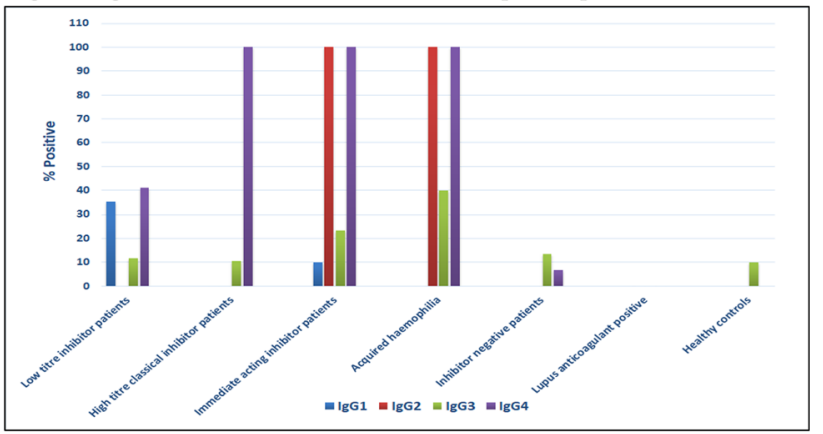

Disclosure of Interest: None Declared.

Keywords: Factor VIII inhibitors, Hemophilia, IgG Subclass.

\section{Abstract 4. Role of Platelet Indices as Predictive Markers of Immune Thrombocytopenic Purpura (ITP)}

\author{
DeepshikhaRana $^{* 1}$, Sunita Singh $^{2}$, Rajeev Sen $^{3}$ \\ ${ }^{1}$ Pathology, PGIMS, Rohtak, GURGAON, ${ }^{2}$ Pathology, PGIMS, \\ Rohtak, ${ }^{3}$ Pathology, PGIMS, Rohtak, Rohtak, India
}

Aims \& Objectives: Thrombocytopenia is considered to be one of the most important causes of abnormal bleedings. The aim of our study was to retrospectively investigate the cause of thrombocytopenia as diagnosed on bone marrow aspiration and to correlate with the values of PIs in peripheral blood. We also aimed to establish PIs as predictive markers of ITP in thrombocytopenic patients and also to critically evaluate if these indices could be helpful enough to avoid an invasive procedure, as a bone marrow aspiration, in discriminating thrombocytopenia due to hypoproductive or hyperdestructive causes. Patients/Materials \& Methods: 365 cases of thrombocytopenia (automated analyser and peripheral blood examination) with platelet counts $<150 \times 10^{9} / 1$ in whom bone marrow aspiration was indicated were taken over 1 year period and divided into two groups: hyperdestruction (i.e. ITP, 68 patients) and hypoproduction (i.e. Non-ITP, 297 patients). Platelet indices of the two groups were compared.

Results: Comparison between the two groups showed that platelet count $\left(10^{\wedge} 9 / \mathrm{L}\right)$ and PCT $(\%)$ were higher in patients in the hypoproductive group and this difference was found to be statistically significant. Comparison of the MPV (fl) and PDW between the two groups showed that both were higher in patients in the hyperdestructive group (not statistically significant). P-LCC (10^9/L) was found to be higher in hypoproductive group and was statistically significant.

Discussion \& Conclusion: Patients with ITP had significantly lower platelet counts, PCT (\%) and P-LCC and higher MPV than patients of non-ITP causes. PCT along with other clinical findings can be a useful tool to the clinician for predicting ITP and hence avoiding BM aspiration.

Disclosure of Interest: None Declared.

Keywords: Bone marrow aspiration, Platelet indices, Thrombocytopenia.

\section{Abstract 5. Guardian 10: Post-approval Safety Study of Turoctocog Alfa in Patients with Haemophilia $a$ in India}

Shashikant Apte ${ }^{* 1}$, Aby Abraham ${ }^{2}$, PrantarChakrabarti ${ }^{3}$, Trine Hansen ${ }^{4}$, Naresh Gupta ${ }^{5}$, M Joseph John ${ }^{6}$, Cecil Ross ${ }^{7}$, S Chandrakala ${ }^{8}$, TulikaSeth ${ }^{9}$, Neeraj Sidharthan ${ }^{10}$, Anne Louise Tølbøll Sørensen ${ }^{4}$ and Dr Aby Abraham, Dr PrantarCharabarti, Ms Trine Hansen, Dr Naresh Gupta, Dr M Joseph John, Dr Cecil Ross, Dr S Chandrakala, Dr Tulika Seth, Dr Neeraj Sidharthan and Ms Anne-Louise TølbøllSørensen

${ }^{1}$ Department of Haematology \& Bone Marrow Transplant Unit, Sahyadri Speciality Hospital, Pune, ${ }^{2}$ Department of Haematology, Christian Medical College, Vellore, ${ }^{3}$ Department of Haematology, NRS Medical College, Kolkata, India, ${ }^{4}$ Novo Nordisk A/S, Søborg, Denmark, ${ }^{5}$ Department of Medicine and The Haemophilia Centre, Maulana Azad Medical College \& Associated Lok Nayak and GB Pant Hospitals, New Delhi, ${ }^{6}$ Department of Clinical Haematolog, Christian Medical College, Ludhiana, ${ }^{7}$ Medicine \&Hematology Department, St John's Medical College Hospital, Bangalore, ${ }^{8}$ Department of Hematology, King Edward Memorial Hospital, Parel, ${ }^{9}$ Clinical Department of Hematology, All India Institute of Medical Sciences, New Delhi, ${ }^{10}$ Department of Haematology and Medical Oncology, Amrita Institute of Medical Sciences, Kochi, India

Aims \& Objectives: Turoctocog alfa (NovoEight ${ }^{\circledR}$, Novo Nordisk) is a B-domain truncated recombinant factor VIII (FVIII) product approved in India for the prevention and treatment of bleeding in patients with haemophilia A. Guardian 10 (NCT03449342) was a post-authorisation, phase 4 , non-randomised, open-label, multicentre trial evaluating the safety and efficacy of turoctocog alfa for prophylaxis and treatment of bleeding episodes in previously treated patients (PTPs) from India with moderate or severe haemophilia A (FVIII activity $\leq 5 \%$ ).

Patients/Materials \& Methods: Sixty PTPs $\geq 12$ years of age were enrolled and received intravenous prophylaxis with turoctocog alfa. Based on inclusion criteria, patients had no history of FVIII inhibitors and $\geq 150$ documented exposure days (EDs) to FVIII-containing products. The duration of the trial including follow-up period was 12 weeks, with an 8 -week treatment period ( $\geq 20$ EDs to turoctocog alfa). The primary endpoint was the incidence of FVIII inhibitors $(\geq 0.6 \mathrm{BU})$ during the treatment period. Secondary endpoints included frequency of adverse reactions (ARs; treatment-related adverse events) and the successful haemostatic effect of turoctocog alfa for treating bleeding episodes (success defined as 'excellent' or 'good' haemostatic response). 
Results: Sixtyadult ( $\geq 18$ years) and adolescent $(12-<18$ years)patients -57 of whom had severe haemophilia A-were dosed with turoctocog alfa. The majority of patients were $\geq 18$ years $(n=50)$ and median age was 25.0 years. Mean prophylactic dose was 38.9 IU/ $\mathrm{kg} / \mathrm{dose}$ (37.7 IU/kg/dose, adults; $45.1 \mathrm{IU} / \mathrm{kg} / \mathrm{dose}$, adolescents). All patients completed the trial, resulting in a cumulative treatment period of 9.77 years. No patient developed FVIII inhibitors. Nine treatmentemergent adverse events were reported in $6(10 \%)$ patients; none were treatment-related. No safety concerns or fatalities were reported. Of the 49 treated breakthrough bleeding episodes observed during the treatment period, $40(81.6 \%)$ had an 'excellent' or 'good' haemostatic response to turoctocog alfa.

Discussion \& Conclusion: Turoctocog alfa demonstrated a favourable safety profile in Indian PTPs. No FVIII inhibitors or ARs were reported in guardian 10 with a prophylaxis regimen of $\geq 20$ EDs per patient. The majority of breakthrough bleeds had an 'excellent' or 'good' haemostatic response to turoctocog alfa.

Disclosure of Interest: S. Apte Grant from: Novo Nordisk, A. Abraham Grant from: Novo Nordisk, P. Chakrabarti Grant from: Novo Nordisk, T. Hansen Grant from: Novo Nordisk, N. Gupta Grant from: Novo Nordisk, M. J. John Grant from: Roche, C. Ross: None Declared, S. Chandrakala: None Declared, T. Seth: None Declared, N. Sidharthan: None Declared, A. L. TølbøllSørensen: None Declared. Keywords: Hemophilia, Prophylaxis, Turoctocog alfa.

\section{Abstract 6. Coagulation Profile in Snake Bite Patients}

\author{
Anita Tahlan ${ }^{* 1}$, Ram Singh ${ }^{2}$ \\ ${ }^{1}$ Pathology, ${ }^{2}$ Medicine, Gmch, Chandigarh, Chandigarh, India
}

Aims \& Objectives: To study the coagulation profile of patients presenting with snake bite contributing to patients mortality and morbidity.

Patients/Materials \& Methods: This prospective study was conducted in the Department of Pathology \& Medicine after the approval of IEC. Twenty five patients presenting to medicine department with the history of snake bite were enrolled. Exclusion criteria were partially treated patients or patients with preexsisting bledding disorder. Patient on long term antibiotic treatment were also excluded.

Results: A total of 26 patients presented of which 04 patients died before the investigations could be undertaken. Of these 03 patients presented with neuroparalytic signs and symptoms and one patient presented with vasculotoxic signs and symptoms with the whole blood clotting time more than $20 \mathrm{~min}$. These cases were excluded from the study. The data analysis was done of the remaining 22 patients.

Among the 22 study subjects 18 subjects i.e. $81.8 \%$ were males and only 4 i.e. $18.2 \%$ were females. The age range was 17 to 80 years.

Majority i.e. 18 subjects had PT ranging between 13.6- $18.5 \mathrm{~s}$. Range of PT in the study subjects was 13- $29 \mathrm{~s}$. Mean \pm SD was $16.7 \pm 4.5$. PT ranged between 13 to $29 \mathrm{~s}$ among the study subjects.

17 out of 22 subjects (77.3\%) had INR value between 1-1.30 that is normal. And only 5 study subjects $(22.7 \%)$ had Prothrombin ratio $>1.30$. Mean \pm SD INR was $1.28 \pm 0.59$. Minimum and maximum INR was 1 and 3.54 respectively.

Maximum of the subjects (20 out of 22) i.e. $90.9 \%$ subjects had normal APTT between 30-40 s. Only one subject had APTT between 50 to $60 \mathrm{~s}$ and 60 to $70 \mathrm{~s}$ each. Mean \pm SD is $34.9 \pm 9.0 \mathrm{~s}$. Minimum APTT was $30 \mathrm{~s}$ and maximum was $70 \mathrm{~s}$.

Discussion \& Conclusion: The coagulation screen showed a normal range prothrombin time in $86.3 \%$ patients. The prothrombin time was prolonged significantly in $13.7 \%$ patients. The prothrombin index was also significantly prolonged in $13.6 \%$ patients. The INR was significantly prolonged in $22.7 \%$ patients. The activated partial thromboplastin time was prolonged in $9.1 \%$ patients. The patients with prolonged coagulogram were $13.7 \%$ and $9.1 \%$ with significantly prolonged prothrombin time and activated partial thromboplastin time. In our local area the patients with prolonged coagulogram are relatively less in number. However they should be actively identified and treated accordingly. Moreover the screening coagulogram should be included in the initial investigation panel for these patients.

Disclosure of Interest: None Declared.

Keywords: INR, PT, snakebite.

\section{Abstract 7. Immune Thrombocytopenia: An Atypical Presentation}

TombingNiangneihching ${ }^{* 1}$, IromAnil ${ }^{2}$, Tungnung Ginzaniang ${ }^{3}$, P Vedanti $^{4}$, Md Mustaque ${ }^{5}$, Daniel Ningthoujam ${ }^{6}$

${ }^{1}$ Pgt, 2nd yr, Medicine, ${ }^{2}$ Assistant Professor, Medicine, ${ }^{3}$ Professor, Medicine, ${ }^{4}$ Associate Professor, Medicine, ${ }^{5}$ Senior Resident, Medicine, ${ }^{6}$ Pgt, 2nd yr, Microbiology, Jnims, Imphal, India

Aims \& Objectives: Immune Thrombocytopenia-An atypical presentation.

Patients/Materials \& Methods: Immune thrombocytopenia (ITP) is an acquired immune mediated destruction of platelets leading to bleeding manifestations. Corticosteroids, IVIG and Anti -D are first line of therapy ${ }^{1}$. Steroid is the preferred first line of therapy because it is cheap and freely available. However it has many side effects if not given properly ${ }^{2}$. We are reporting a case of ITP with atypical presentation.

Results: A 62 years old female patient had presented with skin bleeding and bleeding gum. She was diagnosed as ITP and was put on Prednisolone $40 \mathrm{mg}$. Her platelet count had improved. However, steroids were not tapered for 6 months. She came to JNIMS with fever, loose stool and productive cough. On examination she had fever, puffiness of face, edema, oral candidiasis, crackles bilaterally. Stool examination shows larva of strongyloidesstercoralis (Fig. 1). Chest xray shows consolidation in right middle and lower zone. Pleural fluid was suggestive of Tuberculosis with secondary infection (Table 1). CECT-Thorax shows feature suggestive of Pulmonary Koch with empyema thoracis. The steroid was tapered off quickly over 10 days. The patient was started on ivermectin, albendazole, antitubercular drugs and supportive medications. Patient had improved with this treatment.

Table 1 Pleural fluid report

Appearance Hazy
Coagulum Present
Protein $3.2 \mathrm{gms} \%$
Sugar $75 \mathrm{mg} \%$
Total cell count > 10,000cell/cu.mm
DLC L15\%, Polymorph $85 \%$
ADA $51.2 \mathrm{U} / \mathrm{L}$

ADA $51.2 \mathrm{U} / \mathrm{L}$

Discussion \& Conclusion: Infection with Strongyloides stercoralis in ITP patient has been reported by Suvajdzic N etal ${ }^{3}$ and Sebastian A et $\mathrm{al}^{4}$. However in our case, the patient developed Strongyloidesstercoralis infection along with Pulmonary \& Extrapulmonary Koch, which is not reported and very rare. We need to be careful 
while giving steroids and make sure to taper off quickly so as to minimize the side effect.

Supporting Document: 193aad68-2048-4174-8eea-906d842accf5.

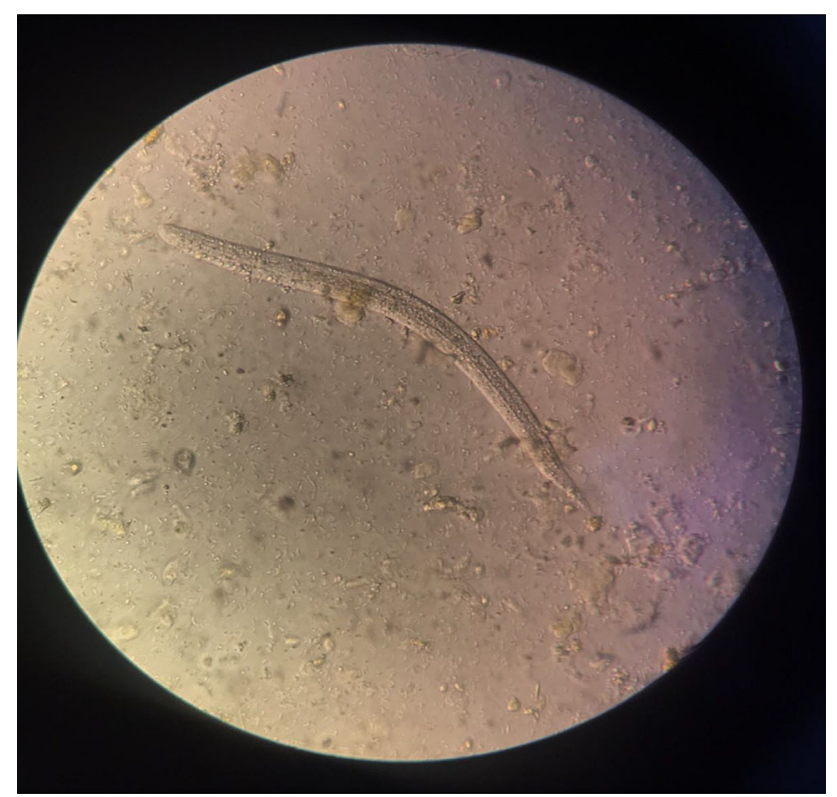

Disclosure of Interest: None Declared.

Keywords: IMMUNE THROMBOCYTOPENIA, IVIG, STEROID.

\section{Abstract 8. Assessment of Quality of Life in Severe Hemophilia Using Generic and Hemophilia Specific Instruments}

\author{
Juhi Singh $^{* 1}$, Naresh Gupta ${ }^{1}$, Sandeep Garg ${ }^{1}$ \\ ${ }^{1}$ Maulana Azad Medical College, Delhi, India
}

Aims \& Objectives: To assess Quality of Life (QoL) in severe hemophilia using generic (SF-36 \& EQ-5D) and hemophilia specific instruments (Haem-A-QoL) and to correlate the assessment outcomes from different QoL instruments.

Patients/Materials \& Methods: A cross-sectional analytical study was done in Hemophilia centre over a period of one year included sixty severe hemophilia A patients aged between 18 to 45 years, who were administered Indian version of generic and specific instruments for self-completion and data on demographic and hemophilia profile was gathered. Responses were transformed into scores using specific scoring system and analysed using SPSS 16.0 version.

Patients with co-morbidities like Rheumatoid arthritis, coronary artery disease, cerebro-vascular accidents and those who were not capable of undertaking QoL assessment were excluded.

Results: In our study, mean age of subjects was $25.37 \pm 6.64$ years, majority were in $21-30$ years $(51.67 \%)$ age group, $26.67 \%(\mathrm{n}=16 /$ $60)$ were married, $36.67 \%(\mathrm{n}=22 / 60)$ were students, $36.67 \%$ $(n=22 / 60)$ were employed while $26.67 \%(n=16 / 60)$ unemployed. According to modified BG Prasad's socio-economic scale, $40 \%$ ( $n=24 / 60$ ) belonged to class II (upper middle class).

Mean age of diagnosis and annual bleed rate (ABR) was $70.68 \pm 81.31$ months and $13.07 \pm 8.02$ per year, respectively.
About 66.67\% $(n=40 / 60)$ had major bleeding and $48.33 \%(n=29 /$ $60)$ had hemophilia related morbidity. Prevalence of transfusiontransmitted infection was 3/60 (5.00\%).

Out of 5 dimensions of EQ5D5L (i.e. mobility, self-care, usual activities, pain/discomfort and anxiety/depression), 90.0\% $(n=54 /$ 60) patients had reported to have pain followed by problem in mobility $61.7 \%(\mathrm{n}=37 / 60)$.

The SF-36 instrument measures two main health concepts (physical and mental health) with 36 items and 8 multi-item scales showed mental health was better than physical health.

Haem-A-QoL consists of 46 items, showed better scores in physical health, sports/leisure, work and family planning and worst in dealing, partnership and sexuality in comparison to contemporary studies.

All the dimensions of generic instruments showed correlation with Haem-A-QoL but was not true vice versa (details of analysis will be discussed).

Discussion \& Conclusion: Hemophilia causes physical and mental health impairment, impacts the patient's employment, relationships, daily activities, and perceived quality of life. Our study highlight these issues and encourage more studies in this dimension using Haem-A-QoL which is much better and comprehensive instrument for assessing QoL in adult hemophilia patients as compared to generic instruments.

Disclosure of Interest: None Declared.

Keywords: EUROQOL 5 Dimension 5 Level EQ-5D-5L, Health Related Quality of Life HRQoL, Short Form 36 SF-36.

\section{Abstract 9. Severity of Injury and Coagulopathy in Patients with Isolated Head Trauma}

\author{
Karishma Tahlan $^{* 1}$, Ruchika Sodhi ${ }^{1}$, Meera Sikka ${ }^{1}$, \\ Mrinalini Kotru ${ }^{1}$, GurubachanSingh ${ }^{2}$ \\ ${ }^{1}$ Pathology, ${ }^{2}$ Neurosurgery, University College of Medical
Sciences, DELHI, India
}

Aims \& Objectives: Traumatic brain injury (TBI) remains a major cause of mortality. Identification of factors which predict prognosis will aid in better management and reduced mortality. Coagulation abnormalities are evident soon after trauma and identify a subset of patients who are likely to develop secondary complications. The abnormalities are reported to be more frequent with increasing severity of TBI. This study aimed to assess the association of coagulopathy with severity of injury in Indian patients with isolated head trauma.

Patients/Materials \& Methods: The study included 100 patients admitted within $12 \mathrm{~h}$ of isolated head trauma. The severity of injury was assessed by Glasgow Coma score (GCS) and graded as mild (13-15), moderate (9-12) and severe (3-8).Complete blood counts including platelet count, PT and APTT were done in all patients. Coagulopathy was defined as a prolonged PT ( $>14 \mathrm{~s}$ ) and/or APTT (>34 s).

Results: The study included $78 \%$ males and $22 \%$ females in the age range of 7-82 years. The severity of injury was mild, moderate and severe in $45 \%, 28 \%$ and $27 \%$ patients respectively. The prevalence of coagulopathy was $39 \%$; associated thrombocytopenia being present in $30(76.9 \%)$ patients. The prevalence of coagulopathy and thrombocytopenia increased with increasing severity of injury and these were also seen in mild TBI. A highly significant $(p<0.001)$ association was seen between GCS and coagulopathy as also thrombocytopenia. 
Discussion \& Conclusion: The study observed an increased prevalence of coagulopathy and thrombocytopenia with increasing severity of TBI. Mild TBI constitutes majority of cases of head injury and these abnormalities were observed in these patients also. As most studies have evaluated coagulopathy in patients with moderate and severe TBI, the results of this study indicate that tests of coagulation must be done in all patients of TBI irrespective of severity for better stratification and management which will result in reduced morbidity and mortality.

Disclosure of Interest: None Declared.

Keywords: COAGULOPATHY, SEVERITY OF INJURY, TRAUMA.

\section{Abstract 10. The Economic Burden of Immune Thrombocytopenia: A Single Center, Cross-Sectional Study}

\section{Shreyas Hanmantgad $^{* 1}$, Rohith Chitrapur ${ }^{1}$ \\ ${ }^{1}$ Bangalore Baptist Hospital, Bangalore, India}

Aims \& Objectives: Immune thrombocytopenia Purpura (ITP) is an immune mediated thrombocytopenia which is characterized by an isolated low platelet count of less than $1,00,000 /$ cumm and is frequently associated with various bleeding manifestations. There are only a handful of studies which throw light on the economic burden of ITP in India. This study was conducted to understand the economic burden of ITP in a single centre.

Patients/Materials \& Methods: A retrospective, cross sectional, non interventional, descriptive study was carried out in Bangalore Baptist Hospital which is a 340 bedded tertiary care hospital in Bengaluru, India. All patients who were diagnosed to have Immune thrombocytopenia either as inpatient or outpatient and age more than 16 years, in a oneyear period from June 2018 to May 2019, were included in the study. ITP was defined as a platelet count of less than 1,00,000/cumm with other causes being ruled out. Patient data was collected from the hospital record system and chart review and the finance department.

Results: 35 patients were included in the study. The required details were available for 34 patients. 20 patients were newly diagnosed ITP (58.8\%, duration of treatment $<3$ months), 8 patients had persistent thrombocytopenia of varying severeity $(23.5 \%$, duration of treatment $\geq 3$ to $<12$ months) ( $24 \%$, persistent ITP) and 4 patients had thrombocytopenia $\geq 12$ months ( $11.7 \%$,refractory/chronic ITP). 25 patients $(71.4 \%)$ presented with bleeding manifestations, including 2 intracranial haemorrhages. 33 patients $(94.2 \%)$ were admitted at some point of time at diagnosis or during treatment. with an average of 8.9 admission days per patient. Of the 30 patients $(85.7 \%)$ who received treatment, all received corticosteroids, 6 patients (20\%) received intravenous immunoglobulin, and 6 patients (20\%) required further therapies like azathioprine, dapsone or eltrombopag. 2 patients $(6.7 \%)$ underwent splenectomy. Average inpatient direct medical costs (mean (standard deviation)) was INR $35,468.12$ per patient with INR $36,587.13$ in 20 newly diagnosed ITP patients. 18 patients $(51.4 \%)$ were classified as low socio-economic status by the hospital social service system.

Discussion \& Conclusion: Immune thrombocytopenia is a benign disorder but still contributes to a significant economic burden to patients especially those belong to the lower socioeconomic group. Inpatient costs and intravenous immunoglobulin were the main cost drivers. Transfusions of platelets in ITP, though not recommended except in severe bleeding manifestations, still contribute to a portion of the cost burden.

Disclosure of Interest: None Declared.

Keywords: bleeding, COST, ITP.

\section{Abstract 11. 'LUPUS ANTICOAGULANT HYPOPROTHROMBINEMIA SYNDROME'- A CaSe Series}

\author{
Kannan $\mathrm{S}^{* 1}$, TulasiGeevar ${ }^{1}$, SukeshChandran Nair ${ }^{1}$, \\ Surendar Singh ${ }^{1}$
}

\section{${ }^{1}$ Transfusion Medicine and Immunohaematology, CMC Vellore,} Vellore, India

Aims \& Objectives: To study the clinical presentation and laboratory parameters of cases diagnosed as 'Lupus Anticoagulant Hypoprothrombinemia Syndrome (LAHS)' and to report an exclusive presentation of LAHS with 'Transient Lupus anticoagulant positivity'. Patients/Materials \& Methods: An electronic search of the archives revealed six patients who were diagnosed as 'Lupus Anticoagulant Hypoprothrombinemia Syndrome' between 2011 to 2019 in our institution. The clinical presentation, lab parameters of the coagulation tests at the time of presentation and follow up were reviewed and analysed. Results: Case 1 and 2 presented with mucosal haemorrhage and skin haemorrhages along with Lupus Nephritis. Case 3 presented with ecchymotic patches over trunk, scalp, upper and lower limbs along with fever and loose stools, whose follow-up after 3 months showed no intrinsic haemostatic defect and disappearance of lupus anticoagulant. Hence LAHS with a transient lupus anticoagulant positivity, possibly following a viral infection was thought of. Case 4 and 6 were female patients with menorrhagia as presenting symptom. Case 5 presented with massive intraperitoneal bleeding following aspiration of liver abscess. Table 1 gives an summary of the lab parameters of the cases.

Discussion \& Conclusion: Antiphospholipid antibody syndrome (APS) is an autoimmune disorder frequently seen in patients developing thrombosis and has a female preponderance. Rare scenarios which predispose an increased risk of bleeding along with thrombosis in APS are catastrophic APS with thrombocytopenia, thrombocytopathies, acquired inhibitors to Prothrombin(II) and Factors VII, VII IX and X. Lupus anticoagulant hypoprothrombinemia syndrome (LA-HS) is a rare acquired hypoprothrombinemia due to non-neutralising antibodies causing a rapid clearance of prothrombin from circulation. This case series is being presented for (i) it's rarity and (ii) a unique presentation of LAHS with transient lupus phenomenon possibly triggered by an infection which has only a handful of references in the existing literature. It has a better prognosis with the disappearance of LA and normalization of PT with time when compared to LAHS associated with autoimmune diseases. The mainstay of treatment for the latter is immunosuppressants. Major problems faced during treatment are relapses and increasing risk of thrombosis with improving prothrombin levels and delicate equilibrium between bleeding and thrombosis in LAHS.

Supporting Document: 0ed58207-b61b-445d-9dcf-439a5119ffd7.

\begin{tabular}{|c|c|c|c|c|c|c|}
\hline TABLE 1 & case 1 & case 2 & case 3 & case 4 & case 5 & case 6 \\
\hline $\begin{array}{l}\text { YEAR OF } \\
\text { DIAGNOSIS }\end{array}$ & 2011 & 2013 & 2017 & 2018 & 2018 & 2019 \\
\hline AGE & 18 & 46 & 6 & 14 & 47 & 11 \\
\hline SEX & Male & Male & Male & Female & Male & Female \\
\hline \multicolumn{7}{|l|}{$\begin{array}{l}\text { LABORATORY } \\
\text { PARAMETERS: }\end{array}$} \\
\hline ANA & $2+$ & $1+$ & - & $1+$ & $2+$ & $2+$ \\
\hline dsDNA & - & 615 & - & 260 & 15 & 20 \\
\hline LA & $\begin{array}{l}\text { Markedly } \\
\text { Positive }\end{array}$ & $\begin{array}{l}\text { Markedly } \\
\text { Positive }\end{array}$ & $\begin{array}{c}\text { Markedly } \\
\text { positive }\end{array}$ & $\begin{array}{l}\text { Markedly } \\
\text { Positive }\end{array}$ & $\begin{array}{l}\text { Markedly } \\
\text { Positive }\end{array}$ & $\begin{array}{l}\text { Markedly } \\
\text { Positive }\end{array}$ \\
\hline PT & $\begin{array}{c}63.8 \mathrm{~s}(\mathrm{~N}: \\
9.5-12.7 \mathrm{~s})\end{array}$ & $\begin{array}{c}15.2 \mathrm{~s}(\mathrm{~N}: \\
10.0-12.5 \\
\mathrm{~s})\end{array}$ & $\begin{array}{c}20.8 \mathrm{~s} \text { (N: } 10.0- \\
12.5 \mathrm{~s})\end{array}$ & $\begin{array}{c}15.6 \mathrm{~s}(\mathrm{~N}: \\
9.8-12.0 \mathrm{~s})\end{array}$ & $\begin{array}{c}15.2 \mathrm{~s}(\mathrm{~N}: \\
9.8-12.0 \mathrm{~s})\end{array}$ & $\begin{array}{c}24.5 \mathrm{~s}(\mathrm{~N}: 9.8 \\
-12.0 \mathrm{~s})\end{array}$ \\
\hline PT MIX & $13.6 \mathrm{~s}$ & 13.3 & 14.3 & 12.2 & $13.0 \mathrm{~s}$ & $9.9 \mathrm{~s}$ \\
\hline INR & 5.96 & 1.37 & 1.90 & 1.44 & 1.52 & 2.49 \\
\hline APTT & $\begin{array}{l}>3 \min (\mathrm{N}: \\
26.6-40.2 \mathrm{~s})\end{array}$ & $\begin{array}{c}55.3 \mathrm{~s}(\mathrm{~N}: \\
25.1-36.7 \mathrm{~s})\end{array}$ & $\begin{array}{r}155.3 \mathrm{~s}(\mathrm{~N}: \\
24.7-37.5 \mathrm{~s})\end{array}$ & $\begin{array}{c}59.3 \mathrm{~s}(\mathrm{~N}: \\
26.5- \\
35.3 \mathrm{~s})\end{array}$ & $\begin{array}{c}75.0 \mathrm{~s}(\mathrm{~N}: \\
26.5-35.3 \mathrm{~s})\end{array}$ & $\begin{array}{c}48.4 \mathrm{~s}(\mathrm{~N}: \\
26.5-35.3 \mathrm{~s})\end{array}$ \\
\hline aPTT MIX & $92.1 \mathrm{~s}$ & $66.3 \mathrm{~s}$ & $112.2 \mathrm{~s}$ & $48.1 \mathrm{~s}$ & $97.3 \mathrm{~s}$ & 43.1 \\
\hline $\begin{array}{l}\text { FACTOR } \\
\text { ACTIVITY }\end{array}$ & $<1 \%$ & $24.90 \%$ & $16.50 \%$ & $11.20 \%$ & $12.30 \%$ & $3.50 \%$ \\
\hline REPEAT LA & $\begin{array}{l}\text { Markedly } \\
\text { Positive }\end{array}$ & $\begin{array}{l}\text { Markedly } \\
\text { Positive }\end{array}$ & Negative & $\begin{array}{l}\text { Markedly } \\
\text { Positive }\end{array}$ & - & - \\
\hline
\end{tabular}


Disclosure of Interest: None Declared.

Keywords: LAHS with transient lupus anticoagulant positivity, LAHS, Lupus anticoagulant hypoprothrombinemia syndrome.

\section{Abstract 12. Assessment of Haematological and Coagulation Profile of Patients Diagnosed as Tuberculosis}

\author{
Sujata Raychaudhuri" ${ }^{* 1}$, ReetikaMenia ${ }^{2}$ \\ ${ }^{1}$ Pathology, ESIC Medical College and Hospital, ${ }^{2}$ Pathology, \\ ESIC Medical College and Hospital, Faridabad, India
}

Aims \& Objectives: Study the haematological and coagulation parameters in cytologically diagnosed cases of tuberculous lymphadenopathy.

Patients/Materials \& Methods: A cross sectional study of 60 newly diagnosed cases of tuberculosis on cytology with no history of prior anti tuberculous treatment was done. Prior ethical committee consent was taken. Venous blood samples were collected in EDTA and Citrate vacutainers. XN 1000 fully automated haematology analyser and Vesmatic were used for haematology parameters and ESR. Stago analyser was used for coagulation studies. Haematological and coagulation parameters were studied and were analysed using t test with significance level of $5 \%$.

Results: 60 cases presenting with tuberculous lymphadenopathy on cytology were analysed which showed a M: F of 1.2:1. The age range of patients was 12 to 74 yrs. Anaemia was noted equally in both sexes. ESR and WBC count was raised in $52 \%$ and $42 \%$ of cases respectively. Prothrombin Time was raised in $95 \%$ of cases while Activated Partial Thromboplastin Time was raised in $82 \%$ of cases. BT and CT were within normal range and platelet count was within normal limits in $80 \%$ of cases. Lymphocytosis was noted in $78 \%$ of cases while $52 \%$ cases revealed neutrophilia. Smoking and literacy rate had positive correlation. Paired $\mathrm{t}$ test shows positive correlation between age and WBC count $(p<0.001)$ and age and $\mathrm{Hb}$ value $(p<0.001)$.

Discussion \& Conclusion: Tuberculosis is a hypercoagulable state with alteration in the haematological profile. These simple blood tests with no additional cost can serve as guide to physicians in establishing the early diagnosis along with clinical findings and ancillary investigations. Such Pilot studies can be extended to larger populations and corporate setups to start early treatment and reduce the morbidity and mortality.

Disclosure of Interest: None Declared.

Keywords: APTT, PT, Tuberculosis.

\section{Abstract 13. Prevalence of Macrothrombocytopenia in West Bengal}

Rudra Ray $^{* 1}$, SamarpanNath ${ }^{1}$, SunisthaBhattacharjee ${ }^{1}$, MaitreyeeBhattacharyya ${ }^{1}$

\section{${ }^{1}$ IHTM, Ihtm, Medical College Kolkata, Kolkata, India}

Aims \& Objectives: Macrothrombocytopenia or Giant Platelet Disorder is an entity characterized by reduced number of abnormally large platelets in the circulation, with or without association with bleeding manifestations. As per previous studies, prevalence of the said entity is high in North Eastern India and particularly West Bengal. It is frequently misdiagnosed as Immune thrombocytopenic purpura and leads to wrong line of treatment and therapeutic misadventure. The objective of this study was to find out the prevalence of macrothrombocytopenia in the population of West Bengal and its clinical determinants.

Patients/Materials \& Methods: In this study, 200 blood samples were taken from healthy residents of West Bengal who were not on antiplatelet drugs or previously diagnosed as ITP. These blood samples were evaluated using 5 part automated hematologyanalyzer and MPV, PDW and TPC values were noted in each case.

Results: Out of 200 subjects, 53 subjects were provisionally diagnosed as MT cases as they had MPV $>=12 \mathrm{fl}$ and TPC $<1,50,000 /$ $\mathrm{ml}$. These were confirmed using peripheral blood smear and their association with possible socio-demographic and clinical determinants was studied. MPV, Platelet count and PDW of these 53 subjects were $13.75 \pm 0.86 \mathrm{fL}, 222.87 \times 10^{3} \pm 27.78 \times 10^{3} / \mathrm{ml}$ and $19.33 \pm 3.15 \mathrm{fL}$ respectively.

Discussion \& Conclusion: Relation with other factors like age, sex, religion (consanguinity was taken as risk factor), geographical distribution and bleeding tendencies have also been established in this study. Possible geographical factors may also be present as the prevalence is highest in a particular district more than the others. Also, out of the 200 subjects, there was also a group of 87 subjects who had giant platelets in their circulation with normal platelet count, suggesting that high MPV may not always be associated with a low platelet count.

Disclosure of Interest: None Declared.

Keywords: ITP, Macrothombocytopenia, MPV.

\section{Abstract 14. A Single-Tube and Singleplex PCR Strategy for the Detection of Common Alpha Globin Gene Deletions}

\section{Rudra Ray $^{* 1}$, Ankita Biswas ${ }^{1}$, SunisthaBhattacharjee ${ }^{1}$, MaitreyeeBhattacharyya ${ }^{1}$ \\ ${ }^{1}$ IHTM, Ihtm, Medical College Kolkata, Kolkata, India}

Aims \& Objectives: The alpha thalassemia syndromes are among the most common single-gene disorders with more than $20 \%$ of the world population to be a carrier of some form of $\alpha$-thalassemia, as estimated by WHO.In India also it is common, and the gene frequency of occurrence is appreciably higher than that of $\beta$-Trait. Alpha $3.7 \mathrm{~KB}$ Deletion and Alpha 4.2 Deletion are commonest reported genotype which exists in India. The existing molecular diagnostic techniques to detect alpha globin gene deletions involve multiplex GAP-PCR system which is a complex method and troublesome to standardize, reproduce as routine investigation. The aim of this study was to establish a PCR method which involves a single-tube, singleplex reaction system to detect both $3.7 \mathrm{~kb}$ and $4.2 \mathrm{~kb}$ alpha gene deletions. Patients/Materials \& Methods: Selection of primers and strategy to develop the singleplex PCR system were done using Snap-Gene Viewers in which the reference sequence of Alpha gene cluster at NCBI was imported. In brief, the strategy involved selection of two primers from the regions upstream to $4.2 \mathrm{~KB}$ deletion and downstream to $3.7 \mathrm{~KB}$ deletions respectively. The targeted amplicon spans a region that covers both the sequence segments affected by $3.7 \mathrm{~KB}$ and $4.2 \mathrm{~KB}$ deletion. Optimal adjustment in the PCR condition and protocol was done.

Results: By this PCR it was possible to identify $3.7 \mathrm{~KB}$ and $4.2 \mathrm{~KB}$ deletion in the alpha globin gene cluster. The normal alpha globin gene and the respective deletion could be separated by their large difference of amplicon size which was detected by running the PCR products in $2 \%$ agarose gel. The single and double deletion could be distinguished by the presence or absence of the amplicon for normal alpha globin gene cluster.

Discussion \& Conclusion: The method is simple and involves only two primers whereas the previous established GAP-PCR method involved a combination of six primers to identify alpha $3.7 \mathrm{~KB}$ and 
4.2 KB deletion. Though the PCR cycle is more time consuming but by the proposed method it is easier and lesser time consuming to clearly identify the separation of band pattern in agarose gel. This method will simplify the detection and diagnosis of alpha thalassaemia.

Disclosure of Interest: None Declared.

Keywords: Alpha Globin, GAP PCR, Gene Deletion.

\section{Abstract 15. Molecular Analysis of Glanzmann's Thrombasthenia: Targeted Resequencing Based Approach}

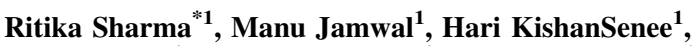 \\ Varun Uppal' ${ }^{1}$, Narender Kumar ${ }^{1}$, JasminaAhluwalia ${ }^{1}$, \\ Reena Das ${ }^{1}$
}

\section{${ }^{1}$ Hematology, PGIMER, Chandigarh, India}

Aims \& Objectives: To determine the molecular basis of GT and to implement the results for prenatal diagnosis, carrier screening, and genetic counseling.

Patients/Materials \& Methods: Seven unrelated patients presenting with bleeding manifestations were recruited in our center. A detailed clinical, bleeding and family history was taken. Basic coagulation screening included peripheral blood smear, prothrombin time, activated partial thromboplastin time and fibrinogen assay. Light transmission aggregometry (LTA) was done for platelet aggregation studies using various physiological agonists. Flow cytometry analysis was done using CD61 and CD41 for studying the expression of glycoprotein IIb/IIIa (GPIIb/IIIa). For further molecular analysis, genomic DNA was isolated using QIAamp DNA midi kit, quantity and quality was analyzed using Qubit fluorimerty and Agilent 2100 Bioanalyzer. The library was prepared using HaloPlex ${ }^{\mathrm{HS}}$ enrichment custom panel (Agilent) and sequencing was done using MiSeq (Illumina). FASTQ files generated were aligned using Agilent's SureCall software and variants were annotated using Variant Studio Software. Various in silico tools were used to predict the pathogenicity of variants.

Results: LTA studies revealed severely reduced or absent platelet aggregation with physiological agonists such as arachidonic acid, adenosine diphosphate, collagen, and epinephrine, but a normal response to ristocetin. Flow cytometry revealed reduced to absent GPIIb/IIIa expression using antibody CD61 and CD41. Next generation sequencing (NGS) data analysis revealed a potentially deleterious variant in all seven cases. Missense and Indels were found in three cases $(42.8 \%)$ each and a nonsense variant was found in one case. In silico analysis using M-CAP, CADD, PolyPhen-2, SIFT, MutPred, PROVEAN predicted all missense variants deleterious. The sequencing results were used to predict a normal fetus in one case. Discussion \& Conclusion: NGS resulted in high diagnostic yield $(100 \%)$ in patients with GT. Molecular characterization of GT patients was helpful in prenatal diagnosis, carrier screening and genetic counseling of families.

Disclosure of Interest: None Declared.

Keywords: Bleeding Disorder, Glanzmann's Thrombasthenia, NGS.

\section{Abstract 16. The Role of Thrombelastography in Diagnosis And Categorizing Fibrinolysis: Report from Pilot Study}

\author{
Ganesh Mohan $^{* 1}$, ShameeShastry ${ }^{1}$, PrethikaP. A. ${ }^{1}$, \\ DhivyaKandasamy ${ }^{1}$
}

${ }^{1}$ Immunohematology and Blood Transfusion, Kasturba Medical College, Manipal, India
Aims \& Objectives: There are various conditions associated with both primary as well as secondary hyperfibrinolysis which are often fatal, however, quantitative diagnosis of the fibrinolysis mediators are not so well developed as compared to the coagulation system. The aim of this study was to assess the utility of thrombelastograph $\left(\mathrm{TEG}^{\circledR} 5000\right)$ in diagnosing and categorizing the fibrinolysis activity. Patients/Materials \& Methods: This was a prospective observational pilot study carried out for six months in a tertiary care centre. All TEG tests done during this period was included in the study. Patients were classified into with and without fibrinolysis based on TEG results and hyperfibrinolysis again subdivided into primary and secondary based on the TEG algorithm. Clinical management were decided upon TEG reports and clinical outcome of the patients were compared to understand the utility of TEG. Parameters analysed were blood component transfusions, antifibrinolytics, anticoagulants, length of hospital stay, invasive procedures and complications.

Results: TEG was done for a total of 161 patients during the study period with a male to female ratio of 2.17 and a mean age of 43.64 (SD18.67). TEG was abnormal in 65.2\% (105/161) patients which included hypocoagulable $(31.67 \%, 51 / 161)$ and hypercoagulable state $(12.42 \%, 20 / 161)$ with or without fibrinolysis. Fibrinolysis was observed in $39.3 \%$ (n-65), of these $49.23 \%$ (n-32) was primary and $50.76 \%$ (n-33) was secondary. Antifibrinolytic was given in $62.5 \%$ of primary fibrinolysis. Commonest diagnosis in fibrinolysis were Trauma $(24.84 \%)$ followed by DCLD with UGI bleed $(14.54 \%)$, Sepsis $(7.87 \%)$ and PPH $(4.96 \%)$. A total of 1632 blood products were transfused, 495 PRBCs (97 patients, Median 4), 503 FFPs (80 patients, Median 4), 286 platelets (41 patients, Median 5) and 348 Cryoprecipitate (25 patients, Median 10).

Discussion \& Conclusion: The result of the pilot study indicate that fibrinolysis can be categorically diagnosed and managed using TEG. Disclosure of Interest: None Declared.

Keywords: Coagulation, Fibrinolysis, Thromboelastogram.

\section{Abstract 17. Characterisation of Analytical Errors in Classical Thromboelastography (TEG) Interpretation}

TapasyapreetiMukhopadhyay $^{* 1}$, ArulselviSubramanian ${ }^{1}$, Renu Saxena ${ }^{2}$

${ }^{1}$ Laboratory Medicine, ${ }^{2}$ ematology, AIIMS, New Delhi, India

Aims \& Objectives: The primary aim was to identify and determine the incidence of analytical errors in interpretation of classical Thromboelastography (TEG) and to assess the nature of analytical errors in terms of their preventability in a clinical diagnostic laboratory.

Patients/Materials \& Methods: This retrospective study was conducted over a period of one month (August, 2019) in the Department of Laboratory Medicine of an apex trauma centre in North India. TEG tracing obtained on TEM-A automated Thromboelastometer (FramarBiomedica, Rome, Italy) from citrated whole blood samples of trauma patients admitted in during February to June, 2019 were reviewed and correlated clinically by at least two laboratory physicians, with differences resolved by consensus. The tracings suspected to have a probable analytical error were compared with the subsequent tracing obtained after taking measures to resolve it, based on the laboratory SOP, thus confirming the chance finding to be an analytical error. Cases with pre-analytical errors (low sample volume, clotted or diluted sample), TEG runs lasting for less than $10 \mathrm{~min}$, cases with missing data and those cases which had obvious technical issues/breakdown of the instrument during the run, were excluded. The analytical errors observed were further classified into (i) Preventable (ii) Non-preventable (iii) Partially preventable. 
Results: Out of a total of 440 TEG tracings that were reviewed over a period of 5 months, 70 cases were excluded based on the exclusion criteria. Analytical errors were observed in 58 tracings (15.6\%) with 2 tracings having more than one error. We observed the following six types of characteristic TEG tracings (in the order of frequency): (i) Tracings of severe hypocoaguable states showing $\mathrm{k}$-time $=0$ (33.3\%) (ii) Tracings with spikes at irregular intervals (30\%) (iii) Tracings with a beak at the origin (11.6\%) (iv) Tracings with falsely high value of maximum amplitude due to exaggerated opening up of the graph after clot formation (10\%) (v) Falsely hypercoaguable appearing graph starting at the origin $(8.3 \%)$ and (vi) Falsely hypocoaguable appearing graph or straight line that improved after the use of freshly prepared calcium chloride (6.6\%). The respective analytical variables identified for the observed errors were namely: (i) instrument configuration in severe hypocoaguable state producing $\mathrm{k}$-time $=0$, (ii) vibrations during the sample run, (iii) incorrect placement of reaction cup (iv) gradual evaporation of the sample during the sample run (v) failure of standard auto-calibration occurring before a run and (vi) not using freshly prepared calcium chloride. Most analytical errors $(83.3 \%)$ were preventable or partially preventable.

Discussion \& Conclusion: Thromboelastography (TEG), being an operator-dependent point of care test, has the potential of misinterpretation of results due to various analytical variables. Most analytical errors observed in our study were preventable in nature. Awareness amongst clinicians, training of laboratory physicians and operators, and regular monitoring of the inaccuracies in the laboratory may prevent serious delays and errors in clinical decisions.

Disclosure of Interest: None Declared.

Keywords: Analytical errors, TEG misinterpretation, Thromboelastography.

\section{Abstract 18. Serum Sclerostin Levels in Patients with Haemophilia}

\section{NavyaJ $^{* 1}$, SatendraSharma ${ }^{1}$, Meera Sikka ${ }^{1}$, Sunil Gomber ${ }^{2}$, LalendraUpreti ${ }^{3}$ \\ ${ }^{1}$ Pathology, ${ }^{2}$ Paediatrics, ${ }^{3}$ Radio-Diagnosis, UCMS \& GTB Hospital, Delhi, India}

Aims \& Objectives: To estimate serum sclerostin levels in patients with haemophilia and to compare it with normal controls.

Patients/Materials \& Methods: This case control study was carried out in Departments of Pathology, Paediatrics and Radio-Diagnosis, UCMS \& GTB Hospital. It included 40 patients from Haemophilia Day Care Centre in the Department of paediatrics, UCMS \& GTB Hospital and 40 age matched healthy male children.

Routine investigations included CBC,PT, APTT, LFT, KFT, serum Calcium and Phosphorus.

Serum sclerostin levels were measured by Human Sclerostin (SOST) ELISA kit (IMMUNOTAG, USA) in 40 cases and 40 controls.

Results: Thirty three $(82.5 \%)$ patients were anemic and $11(27.5 \%)$ patients presented with joint swelling of which 5 showed effusion on X-ray.

Mean serum sclerostin levels in cases was $796.1 \mathrm{pg} / \mathrm{ml}$ with range of $200-4000 \mathrm{pg} / \mathrm{ml}$. Serum sclerostin levels in control subjects ranged from $220-3580 \mathrm{pg} / \mathrm{ml}$ with the mean value of $559 \mathrm{pg} / \mathrm{ml}$. Since the range of serum sclerostin levels was very wide, logarithmic mean \pm SD values were calculated. Logarithmic mean \pm SD sclerostin levels in cases was $2.7 \pm 0.4 \mathrm{pg} / \mathrm{ml}$ and in controls it was $2.6 \pm 0.2 \mathrm{pg} / \mathrm{ml}$. Serum sclerostin levels were higher in cases as compared to controls although this was not statistically significant $(p=0.102)$.
Discussion \& Conclusion: Studies over the past two decades have shown that haemophilic subjects have a low bone mass compared with normal population. Sclerostin is a glycoprotein hormone secreted by osteocytes that downregulates osteoblastic bone formation by inhibiting WNT/ $\beta$-catenin signalling pathway and hence, high serum sclerostin levels result in low bone mineral density.

The role of serum sclerostin in low bone mass remains unclear. The published data in this area are highly inconsistent. Therefore, additional longitudinal studies are needed to evaluate the relation of sclerostin levels in haemophila with low bone density.

Disclosure of Interest: None Declared.

Keywords: ELISA, haemophilia, sclerostin.

\section{Abstract 19. Fluoxetine Induced Bleeding in a Pediatric Case: A Case Report}

GoutomiChatterjee $^{* 1}$, Neha Rastogi ${ }^{1}$, DhwaneeThakkar ${ }^{1}$, Rohit Kapoor ${ }^{1}$, Anil Sharma ${ }^{1}$, Satya Prakash Yadav ${ }^{1}$

${ }^{1}$ Pediatric Hematology, Oncology and Bone marrow transplantation, Medanta, The Medicity, Gurgaon, India

Aims \& Objectives: Mucocutaneous bleeding episodes are usually because of platelet defects. Qualitative platelet function disorders should be suspected when there is muco-cutaneous bleeding in absence of thrombocytopenia and Von Willebrand disease. Drug induced platelet dysfunction is one of the common causes of acquired platelet disorders. Selective Serotonin Receptor Inhibitor (SSRI) like Fluoxetine reduce dense granule serotonin levels in the platelets, resulting in defective platelet plugs and defective thrombus formation which results in clinical bleeding.

Patients/Materials \& Methods: We hereby report an adolescent boy who had fresh bleeding from mouth.

Results: A 14 year old boy presented with spontaneous fresh bleeding from mouth 10-12 times/day without any associated history of cough or vomiting. No history of bleeding from any other site. No significant past history. Family history was unavailable as the child was an adopted son. On examination, no obvious oral lesion, no hepato-splenomegaly, no lymphadenopathy and other systemic examination was insignificant. Platelet counts were normal and peripheral smear did not reveal any abnormality. Coagulation profile was normal. Factor VIII, IX, XIII and von willebrand factor levels were normal. Liver function tests and Renal function tests were normal. Bronchoscopy and Upper GI endoscopy were done to rule out respiratory and gastrointestinal causes. CECT Paranasal sinuses and MR angiography neck were done to rule out local vascular malformation and juvenile angiofibroma. When no cause was ascertained, then a careful detailed history was again taken which revealed that the child was on Fluoxetine for impulsive and aggressive behavior. Platelet aggregation study was performed which revealed reduced response to ADP and Epinephrine while aggregation to Collagen, Ristocetin and Arachidonic acid were normal. Fluoxetine was stopped. Single donor platelet transfusions were done. Eventually the frequency and quantity of bleeds decreased and finally stopped completely after one week of stopping Fluoxetine. No repeat episode of bleeding for last 3 months. Repeat Platelet function test is planned after 6 months to rule out any underlying disorder.

Discussion \& Conclusion: While treating a child with bleeding, if no definitive cause is found thenacquired platelet qualitative disorders should be suspected and drug history should be elucidated meticulously.

Disclosure of Interest: None Declared.

Keywords: Bleeding, Fluoxetine, Paediatric. 


\section{Abstract 20. Commonest Platelet Function Disorder in a Tertiary Health Care Centre in Eastern India}

\author{
Kaushik Paul $^{* 1}$, MaitreyeeBhattacharya ${ }^{1}$, Sandeep Saha $^{1}$ \\ ${ }^{1}$ Clinical Hematology, Institute of Hematology and Transfusion \\ Medicine, Kolkata, India
}

Aims \& Objectives: Our aims and objectives were to study the pattern of platelet function defect in individuals presenting with superficial bleeding and having normal coagulation profile of any age group.

Patients/Materials \& Methods: We have included total 55 patients since December, 2017 to June, 2019. The study was done at institute of hematology and transfusion medicine, MCH, Kolkata. Their presenting complaints were superficial bleeding in the form of epistaxis, gumbleeding, ecchymoses. Their platelet counts were normal and coagulation profile were found to be normal. Therefore platelet function test were done.

Results: 52 pts had normal platelet function. Only 3 had normal aggregation with ristocetin, but, reduced aggregation with ADP, Epinephrine, arachidonic acid S/O Glanzmann thrombasthenia.

Discussion \& Conclusion: In our study commonest platelet function defect was glanzmannthrombasthenia. How ever larger patient cohort is required for determining exact prevalence and incidence of platelet function disorders.

Disclosure of Interest: None Declared.

Keywords: Commonest platelet function disorder, Eastern india, Tertiary health care centre.

\section{Abstract 21. Frameshift Genetic Variants Commonly Occur in B Domain of F8 Gene in Severe Haemophilia A}

SankariDevi G*1, Raj Kumar S.V ${ }^{1}$, ShajiR.V ${ }^{1}$, Aby Abraham Abraham ${ }^{1}$, Alok Srivastava ${ }^{1}$, Eunice $S$ Edison ${ }^{1}$

${ }^{1}$ Clinical Haematology, Christian Medical College, Vellore, India

Aims \& Objectives: Haemophilia A (HA) is caused by a wide spectrum of mutations in the factor 8 gene (F8), leading to deficiency of FVIII activity. Intron 22 and intron 1 inversions account for $50 \%$ of the molecular defects in Haemophila A, while point mutations constitute the other major group. We analysed the distribution and different classes of point mutations in cases with HA.

Patients/Materials \& Methods: Haemophilia A patients who came for molecular diagnosis from the year 2008 to 2019 were included in the study. Common inversions causing intron 1 and intron 22 inversions were screened by PCR and agarose gel electrophoresis. Inversion negative samples were screened for point mutations by multiplex PCR and conformation sensitive gel electrophoresis and/or direct DNA sequencing strategy. Gross deletions and duplications were analysed by MLPA.

Results: Four hundred and twenty HA cases opted for molecular diagnosis between 2008 and 2019. Linkage analysis was carried out in 15 samples and these were excluded from this study. Disease causing genetic defects was identified in $(396 / 405,97.7 \%)$ cases. Intron 22 inversions were identified in $41.6 \%$ and intron 1 inversions in $2.7 \%$ patients respectively. Point mutations were seen in 198/396 (50\%) cases; gross deletion in 21/396 (5.3\%) and gross duplication in one case. Mutations/deletions could not be identified in the remaining 10 patients. Frameshift mutations accounted for one third of the point mutations. They were caused mainly by deletions in $45(72.5 \%)$ and insertions in $17(27.4 \%)$ cases. Among the deletions, a single base nucleotide deletion $(\mathrm{n}=25)$ or insertion $(\mathrm{n}=13)$ was the commonest and was found in 38 patients. Out of the 38 single nucleotide deletions and insertions, 20 were identified in the B domain of factor 8 gene. Nucleotide 'A' deletion and insertions accounts for 14 (36.8\%) different disease causing variants.

Discussion \& Conclusion: Frameshift mutations appear to occur more frequently within B domain of exon 14 than in other exons of F8 gene. Disclosure of Interest: None Declared.

Keywords: B Domain, Frameshift genetic variants, Haemophilia A.

\section{Abstract 22. Does Clot Waveform Analysis Help in Detecting a Bleeding Risk in Chronic Liver Disease}

TapanBiswal $^{* 1}$ and Rajesh Kumar Bhola1, Manoj Kumar Sahu2, Sarita pradhan1, Ripunjaya Mohanty1, Shilpa Anpurba,1 Debahuti Mohapatra1

${ }^{1}$ Department of Pathology, Imsans Sum Hospitals, Bhubaneswar, India

Aims \& Objectives: Prothrombin time (PT) is abnormal but unreliable in chronic liver disease to assess the risk of bleeding its severity. We investigated whether prothrombin time ratio was an independent risk factor for bleeding alongside its clot waveform analysis.

Patients/Materials \& Methods: We studied 40 consecutive chronic liver disease and 40 healthy subjects. A coagulometer Sysmex CS2500 was used for detecting both prothrombin time (PT) and clot waveform analysis (CWA) which included maximum velocity (1st derivative) or min1, time to maximum velocity, acceleration (2nd derivative) or min2, time to maximum acceleration and deceleration or max 2 and time to maximum deceleration.

Results: It is found that there is significant difference $(p<0.05)$ of prothrombin time, time to maximum velocity, time to maximum acceleration, time to maximum deceleration among the normal and chronic liver disease patients. The maximum velocity, maximum acceleration and maximum deceleration doesn't show significant difference between normal and chronic liver disease patients. Among the 40 consecutive chronic liver disease patients 18 had bleeding manifestation. When we compared the PT and CWA among the bleeders and non-bleeders, significant difference $(p<0.05)$ was found for maximum velocity, maximum acceleration, maximum deceleration, prothrombin time, time to maximum velocity, time to maximum acceleration, time to maximum deceleration.

Discussion \& Conclusion: It can be emphasized that clot waveform analysis especially maximum velocity, maximum acceleration and maximum deceleration can predict bleeding risk in chronic liver disease. Disclosure of Interest: None Declared.

Keywords: Bleeding, Chronic liver disease, Clot waveform analysis.

\section{Abstract 23. Platelet Aggregation Studies in Myeloproliferative Neoplasms with Bleeding Diathesis}

Shivangi Harankhedkar ${ }^{* 1}$, Richa Chauhan ${ }^{1}$, ManoranjanMahapatra ${ }^{1}$, Renu Saxena ${ }^{1}$

${ }^{1}$ Hematology, All India Institute Of Medical Sciences, New Delhi, India

Aims \& Objectives: Acquired platelet function defect in Myeloproliferative neoplasms (MPN) can be attributed to disease biology or therapy with tyrosine kinase inhibitors (TKI). We intend to observe 
the platelet function defects in patients with MPN chiefly chronic myeloid leukemia (CML) presenting with bleeding manifestations, irrespective of their treatment status.

Patients/Materials \& Methods: 32 patients with MPN who presented with bleeding diathesis were studied. Screening coagulogram, quantitative plasma fibrinogen level and platelet function test with light transmission aggregometry was performed.

Results: Out of 32 patients, 17 were on tyrosine kinase inhibitor treatment, 13 on imatinib and 2 each on dasatinib and nilotinib. Platelet aggregometry on PRP was carried out in 22 patients where 13 were CML on TKI, and 9 were other MPN not on TKI. Defect in platelet aggregation was observed in $38 \%$ and $44 \%$ of these patients respectively. Five patients with CML-CP (on imatinib) showed single agonist platelet dysfunction. Four patients had reduced platelet aggregation response with AA and one with ADR. An aspirin-like platelet defect was observed in 4 of 9 newly diagnosed MPNs with bleeding. Reversal of defect was demonstrated in 2 of the patients.

Discussion \& Conclusion: Acquired platelet defects are described both in untreated newly diagnosed patients who might present primarily with bleeding and in CML on TKI treatment. Bleeding manifestations in MPN vary from mild mucocutaneous bleeding to life threatening hemorrhage. In presence of normal platelet count, cause of bleeding could be attributed to platelet function defect, as highlighted by platelet aggregometry studies.

Disclosure of Interest: None Declared.

Keywords: Bleeding disorders, Imatinib, Myeloproliferative neoplasm.

\section{Abstract 24. Incidence of $\boldsymbol{H}$. Pylori Infection in Patients with Immune Thrombocytopenia in North India}

\author{
Rajeev Sandal $^{* 1}$, Aditya Jandial ${ }^{1}$, Deepesh Lad ${ }^{1}$, Alka Khadwal ${ }^{1}$, \\ ShalmoliBhattacharyya ${ }^{2}$, JasminaAhluwalia ${ }^{3}$, Savita Kumari ${ }^{1}$, \\ Neelam Varma ${ }^{3}$, Pankaj Malhotra ${ }^{1}$, GouravParkash ${ }^{1}$ \\ ${ }^{1}$ Internal Medicine, ${ }^{2}$ Biophysics, ${ }^{3}$ Haematology, PGIMER, \\ CHANDIGARH, India
}

Aims \& Objectives: Immune thrombocytopenia (ITP) is a heterogenous acquired autoimmune disease, which is characterized by the destruction of platelets by auto antiplatelet antibodies.We prospectively studied the incidence of H. Pylori infection in ITP patients in North India and analyzed the clinical profile of these patients.

Patients/Materials \& Methods: This was a prospective interventional study. Three hundred sixteen consecutive patients with isolated thrombocytopenia (defined as platelets less than $100 \times 10^{9} / \mathrm{L}$ as per ASH guidelines for ITP 2011) were enrolled in the study after taking informed consent. Demographic data including age, place, gender, occupation, socioeconomic status of patients were recorded. A detailed history of patients about bleeding symptoms was documented. It included site and severity of bleeding (as per WHO bleeding scale), duration of bleeding, previous history of prolonged bleeding after surgery, hemostasis during pregnancy in women, systemic symptoms like loss of weight, loss of appetite, and venous thrombosis. History of medications (including indigenous and alternative), which may lead to thrombocytopenia, was documented. Transfusion history and family history of thrombocytopenia were documented. Detailed examination of the patient was done to rule out evidence of secondary ITP or other disorders associated with thrombocytopenia. All eligible ITP patients underwent urea breath testing (UBT) during the study period. Patients who were found to be UBT positive underwent upper gastrointestinal endoscopy with histopathological examination for $\mathrm{H}$. pylori infection.
Results: Among 316 patients, 184 were females (58.2\%) and 132 were males $(41.8 \%)$. The median age of the study population was 36 years (range $13-76$ years). Out of 316 patients, 202 patients $(63.9 \%)$ had a history of bleeding. The urea breath test was performed in 299 patients. 34 patients (11.4\%) were found to have positive UBT. There was no difference in the clinical presentation of UBT positive patients from UBT negative patients. Of these 34 patients, 19 gave consent for an upper gastrointestinal endoscopy. Out of these nineteen patients, eleven patients $(57.8 \%)$ were found to have chronic active H. Pylori gastritis on endoscopic antral biopsy. Median hemoglobin of UBT positive patients was $11.9 \mathrm{gm} / \mathrm{dl}(6.5-15.2 \mathrm{gm} / \mathrm{dl})$, and median platelet was $39,500 / \mu \mathrm{L})(5000-97,000 / \mu \mathrm{L})$. On comparison of UBT positive $(n=34)$ and UBT negative patients $(n=265)$, UBT positive patients were found to have higher mean platelet count as compared to UBT negative patients $(p=0.001)$. UBT positive patients have low reticulocyte count $1.2(0.98-2.7)$ as compared to UBT negative patients $1.8(0.97-5.98)(p=0.000)$. There was no gender wise difference between UBT positive ITP patients.

Discussion \& Conclusion: It was largest prospective study from India on ITP and H. pyloriinfection.Clinical profile of H. Pylori infected ITP patients was similar to non-infected patients. In view of high prevalence of $\mathrm{H}$. pylori infection in India, its association with the ITP and potential therapeutic benefit to patients-routine testing for $\mathrm{H}$ pylori may be considered in all ITP patients in our region.

Disclosure of Interest: None Declared.

Keywords: H. pylori infection, Immune thrombocytopenia, North India.

\section{Abstract 25. Evaluation of Bone Mineral Density in Hemophilia Patients: A Single Institute Observational Study}

Govind R Patel ${ }^{* 1}$, Renu Saxena ${ }^{2}$, PravasMishra ${ }^{3}$, Manisha Jana ${ }^{4}$, ManoranjanMahapatra ${ }^{2}$

${ }^{1}$ Division of Clinical Hematology, Department of Medicine, Dr. S. N. Medical College, Jodhpur, ${ }^{2}$ Department of Hematology, All India Institute of Medical Sciences, ${ }^{3}$ Department of Hematology/ Hemato-oncology, Max Super Speciality Hospital, Patparganj, ${ }^{4}$ Department of Radiodiagnosis, All India Institute of Medical Sciences, New Delhi, India

Aims \& Objectives: To assess the bone mineral density (BMD) in Indian hemophilia patients, to correlate BMD with findings regarding the joint disease (hemophilic arthropathy) and to evaluate the effect of factor replacement therapy (on demand versus prophylaxis) on BMD. Patients/Materials \& Methods: Thirty nine patients of moderate or severe hemophilia A or B aged less than 18 years and 20 healthy control subjects (age and sex-matched) from the same socioeconomic and ethnic background (Asian) as the patients were selected. Anthropometric measurements were carried out in all cases. Joint evaluation for hemophilic patients and controls by total joint ROM score (Sum of the scores at six major affected joints i.e. bilateral ankles, knees, hips, wrists, elbows and shoulders) using criteria of ROM measurement in Colorado PE-1: full point instrument was calculated before using dual energy X-ray absorptiometry (DEXA). DEXA scanning of lumbar spine focusing on L2-L4 vertebrae, total hip and total body was performed in all hemophilic patients and controls. The baseline BMD of these participants was compared with repeat testing after 6 months of follow up period during which many hemophilia patients received factor replacement therapy.

Results: BMD and BMD Z-scores were significantly reduced in hemophiliacs compared to controls at all measured sites $(p=0.017$, $p=0.004$ and $p=0.001$ for $\mathrm{BMD}$, and $p=0.0002, p=0.0006$ and 
$p=0.0001$ for BMD Z-score at the lumbar spine, the total hip and the total body respectively). Based on the WHO T-score criteria, the prevalence of low BMD in hemophilia patients ranged from 30.8 to $41 \%$ at various measured sites whereas it ranged from 10 to $15 \%$ for controls. About half of the patients $(56.4 \%)$ in our study had vitamin D deficiency. Although our study showed low vitamin D level in hemophilia patients as compared to normal population, we did not find any statistically significant correlation of vitamin D level with low BMD $(p=0.489)$. Also, in hemophilic patients, there was a significant inverse correlation between BMD Z-score and number of target joints $(\mathrm{r}=-0.774$ and $p=0.024)$, number of joint bleeds per 6 months $(\mathrm{r}=-0.857$ and $p=0.006)$, number of arthropathic joints $(\mathrm{r}=-0.822$ and $p=0.012)$, total joint ROM scores $(\mathrm{r}=-0.881$ and $p=0.003$ ), interval between first joint bleed and start of prophylaxis $(\mathrm{r}=-0.834$ and $p=0.010)$. No statistically significant relationship was found between various treatment groups as well as other disease related characteristics and BMD at baseline and at 6 months of follow up.

Discussion \& Conclusion: Children with hemophilia may have reduced BMD compared with age- and gender-matched controls. Children with recurrent target joint bleeding resulting into chronic arthropathy exhibited the lowest BMD and BMD Z-score. Effective prophylactic factor replacement started at early age markedly decreases bleeding episodes in joints and consequent immobilization, and thereby may retard the progression of bone mineral loss in these patients.

Disclosure of Interest: None Declared.

Keywords: Bone mineral density (BMD), dual energy X-ray absorptiometry (DEXA), hemophilic arthropathy.

\section{Abstract 26. Efficacy and Safety of Dapsone in the Patients of Persistent and Chronic Immune Thrombocytopenia}

\section{KarthikaV $^{* 1}$, PrakasKumar Mandal ${ }^{1}$ and ShuvraneelBaul, Subham Bhattacharya, Sumit Mitra, Rajib De, T.K. Dolai}

\section{${ }^{1}$ Department of hematology, Nrs Medical College, Kolkata, India}

Aims \& Objectives: Approximately two-thirds of adult patients do not have a sustained response to first-line therapy. For these patients, a number of second line options exist. Dapsone is an inexpensive drug with a well-established safety profile. Aim of the study is to evaluate the efficacy and safetyof dapsone in persistent and chronic Immune Thrombocytopenia (ITP) patients.

Patients/Materials \& Methods: Prospective study, 42 non-pregnant patients aged $>2$ years and $<60$ years, without any comorbidities with normal LFT and G6PD assay, diagnosed as persistent and chronic ITP by published guidelines, with features of bleeding or platelet count $<$ $30,000 / \mathrm{cmm}$, previously treated with steroids and IV Immunoglobulin/ anti-D, started with Dapsone $2 \mathrm{mg} / \mathrm{kg} /$ day. These patients are followed up with clinical and laboratory parameters, twice weekly for 3 months and monthly once for 1 year, from January, 2018 to June,2019.

Results: Out of 42 patients, there are $28(66 \%)$ patients were female.In our cohort, patients aged between $>2-18$ years constitute 29 (69\%), between $19-<60$ constitute $13(31 \%)$, and patients with persistent ITP constitute 17 (40\%) patients, chronic ITP constitutes 25 $(60 \%)$. Overall response rate (ORR) noted at 2 months in $14(33 \%)$ patients, out of which partial response (PR) in $10(23 \%)$ and complete response $(\mathrm{CR})$ in $4(10 \%)$ patients, with medium time to rise the platelet count were 25 days, median raise in platelet count were $30,000 / \mathrm{cmm}$. Out of $14(33 \%)$ patients showing ORR at 40 days, 10 $(71 \%)$ patients are below 18 years, and $4(28 \%)$ patients are between 19-60 years. All the patients were on Prednisolone $1 \mathrm{mg} / \mathrm{kg} /$ day till response, then steroid tapered slowly, continued only on Dapsone. On tapering steroid, relapse noted in $13(92 \%)$ patients. During follow up Grade 2 bleed (WHO bleeding scale) were noted in 13 (32\%) patients, switched to other form of therapy. $12 \%$ patients developed raised SGOT,SGPT; normalized after holding Dapsone for 2 weeks. Then again drug was resumed. 1 patient developed interactable itching, withdrawn from study.

Discussion \& Conclusion: Dapsone in persistent and chronic ITP, ORR was noted in $33 \%$ of patients while the patients are on steroid. ORR is better in paediatric patients. Very minimal adverse effects noted. On tapering of steroid, $92 \%$ of the patients relapsed.

Supporting Document: b2b1ba67-56a0-43e7-a50d-3d2151a7e10a.

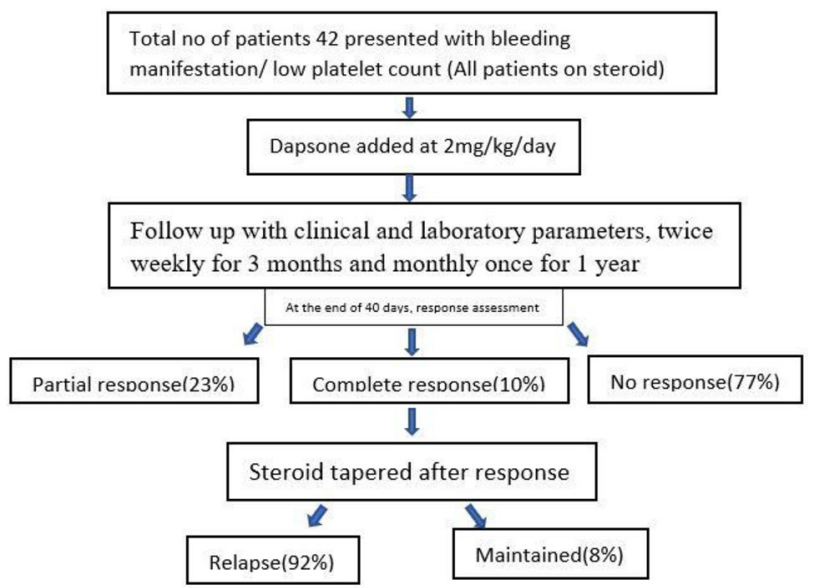

Disclosure of Interest: None Declared.

Keywords: Dapsone, immune thrombocytopenia, safety and efficacy.

\section{Abstract 27. Dohle Bodies May Be:"MAY HEGGLIN" Anomaly!: A Rare Case Report}

Madhuri Gadhekar*1, Ashwini Narayankar', AvinashChalke², RashmitaNevgi $^{3}$, VrindaKulkarni ${ }^{4}$

${ }^{1}$ Fellow in Clinical Haematology, ${ }^{2} \mathrm{Jr}$ Scientific Research Officer, Haematology, ${ }^{3}$ Lab Technician, Haematology, ${ }^{4} \mathrm{Head}$ of Department, Clinical Haematology, Nair hospital, Mumbai, India

Aims \& Objectives: To emphasize on early detection of May Hegglin Anomaly in cases of Macrothrombocytopenia. Genetic analysis should be done to confirm the diagnosis in order to improve perinatal care and to monitor not only the patients but also their children.

Introduction: MYH9 disorders are autosomal dominant platelet disorder characterized by a triad of giant platelets, thrombocytopenia, and characteristic Dohle bodies and are occasionally accompanied by nephritis,cataracts, and difficulty hearing. MYH9 disorders are rare genetic disorders caused by abnormalities in the nonmuscle myosin heavy chain-9 (MYH9) gene located on chromosome 22q12-13 that encodes nonmuscle myosin heavy chain-IIA (NMMHC-IIA).

Patients/Materials \& Methods: CASE REPORT.

Patient: A 32 yr old female, G3P2L1MTP1, 4 month ANC was reffered to Haematology, in view of thrombocytopenia, presented with pervaginal bleeding with mild abdominal pain and generalized weakness. There was history of pervaginal spotting noted in early months of pregnancy. No history of bleeding from any other site. No history suggestive of visual impairment and nephritis. She had never received platelets or any blood constitute, No lymphadenopathy. No organomegaly. Systemic examination-normal, CBC: $\mathrm{Hb}-10 \mathrm{gm} \%$, 
TLC $-11,800 / \mathrm{uL}$, Platelet-37,000/ $\mu \mathrm{L}$. Peripheral smear examination shows large platelet, with Dohle bodies which are small, Lancet shaped, pale-blue to grey structures in cytoplasm of neutrophils. Coagulation profile normal. one marrow study done to rule out Idiopathic thrombocytopenic purpura showing dohle bodies in granulocytes.

Results: In this case study Target gene sequencing revealed heterogeneous non sense variation in exon 41 of MYH9 gene (chromosome 22) confirming morphological diagnosis of May hegglin Anomaly.

Discussion \& Conclusion: MYH9 disorders are rare genetic disorders caused by abnormalities in the nonmuscle myosin heavy chain- 9 (MYH9) gene located on chromosome 22q12-13 that encodes nonmuscle myosin heavy chain-IIA (NMMHC-IIA). The management of pregnant women with May hegglin anomaly consists of an accurate diagnosis, genetic counseling, management of delivery with rapid blood access, and management of complications. As inheritance is autosomal dominant $50 \%$ chance of having baby with the same phenotype. In this case study Target gene sequencing revealed heterogeneous non sense variation in exon 41 of MYH9 gene (chromosome 22) confirming morphological diagnosis of May hegglin Anomaly. Hussein et al. recently conducted a systematic review and reported that $79 \%$ of pregnant patients with MYH9 disorders delivered their babies without hemostatic prophylaxis. Furthermore, a massive postpartum hemorrhage was observed in $5 \%$ of pregnancies. Disclosure of Interest: None Declared.

Keywords: Döhle body, Giant Platelets, May-Hegglin anomaly.

\section{Abstract 28. A Study of Alterations of Immune Cell Subsets in ITP Patients Following Treatment}

\section{Kartik Purohit $^{* 1}$, Jasmitadass ${ }^{1}$, Jyoti kotwal ${ }^{1}$, Ajay Sharma ${ }^{1}$, Nitin Gupta ${ }^{1}$ \\ ${ }^{1}$ Hematology, Sir Gangaram Hospital, New Delhi, New Delhi, India}

Aims \& Objectives: Immune thrombocytopenia (ITP) is an autoimmune disorder occurs due to antibody mediated platelet destruction. Recent studies have also shown important role of cell mediated immunity. We did prospective observational study to measure difference of frequency of immune cell subsets in ITP patients as compared to those with normal controls and post treatment.The objectives of this study was to measure alterations in numbers of Natural Killer (NK) cells and T regulatory cells (Tregs) in ITP patients compared to normal controls and to study the effect of therapy on Natural Killer (NK) cells and T regulatory cells (Tregs) frequency in ITP patients at one month follow up.

Patients/Materials \& Methods: After fulfilling eligibility criteria peripheral blood of 25 patients was collected in EDTA vacutainer. The cells were stained with the following antibody combination: CD45/CD127/CD4/CD25/CD16/CD56/CD8/CD3. The acquisition of events was done by using the BD FACS Canto II flow cytometer. Total 20 Patients were followed up after 1 month and again flowcytometric analysis with same antibody combination was done. Results: We found that T regulatory cells (Tregs) $(p=0.01)$ and NK $\mathrm{T}$ cells $(p=0.01)$ were significantly lower in patients as compared to controls. The CD4+/CD8+ was significantly low in patients as compared to controls $(p=0.001)$. We found that only NK T cells $(p=0.014)$ and Tregs $(p=0.03)$ are statistically significantly different between pre and post treatment group. Both cell numbers were significantly lower in post treatment arm as compared to pre treatment arm.

Discussion \& Conclusion: The immune cell subsets in our study differ significantly from normal control population at diagnosis but they don't differ significantly after treatment from pre treatment. Most of previous studies of immune cell subsets have been done in pediatric population; in whom ITP remains acute and resolves with therapy or spontaneously. In these studies, Tregs and NK cells increased post treatment. In one study of adult chronic ITP there was no change in post treatment Tregs counts. Probably in our study also most likely due to inclusion of adult population; who are likely to develop chronic ITP; there was no change in immune cell subset frequency post therapy. Lack of increase of Tregs may suggest ITP may become chronic.

Supporting Document: 7c0bcd04-a371-419e-b90d-48e6f89a0c59.

\begin{tabular}{|c|c|c|c|c|}
\hline \multicolumn{5}{|c|}{ Results of independent sample $t$ test comparing immune cell subsets of patients and control } \\
\hline variable & Controls & Patients & t score & $\mathrm{p}$ value \\
\hline & $($ mean $\pm \mathrm{SD})$ & $(m e a n \pm S D)$ & & \\
\hline $\mathrm{CD} 4+(/ \mu 1)$ & $702.79 \pm 191.78$ & $605.09 \pm 372.50$ & 1.064 & 0.293 \\
\hline $\mathrm{CD} 8+(/ \mu 1)$ & $58.37 \pm 17.11$ & $491.73 \pm 341.35$ & -6.338 & 0.001 \\
\hline $\mathrm{NK}$ cell $(/ \mu 1)$ & $218.24 \pm 121$ & $150.18 \pm 107.09$ & 2.000 & 0.052 \\
\hline NK T cell $(/ \mu \mathrm{l})$ & $48.78 \pm 38.62$ & $24.45 \pm 27.27$ & 2.474 & 0.017 \\
\hline Tregs $(/ \mu 1)$ & $533.46 \pm 186.26$ & $66.85 \pm 45.95$ & 10.940 & 0.001 \\
\hline 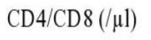 & $12.39 \pm 2.85$ & $1.73 \pm 2.01$ & 14.106 & 0.001 \\
\hline
\end{tabular}

Disclosure of Interest: None Declared.

Keywords: ITP, NK cells, Tregs.

\section{Bone Marrow Failure and MDS (Clinical)}

\section{Abstract 29. Mimicker of Myeloproliferative Neoplasm in Adult-Gaucher Disease} Smriti Gupta $^{* 1}$, Ankur Ahuja
KanwaljeetSingh $^{1}$, YanamandraUdatChatterjee

${ }^{1}$ Dept of Lab \& Molecular Medicine, ${ }^{2}$ Dept of Hematology, Army Hospital RR, DELHI, India

Aims \& Objectives: To assess close mimicker of Myeloproliferative neoplasm.

Patients/Materials \& Methods: Analysis of mimicker of myeloproliferative, a Gaucher disease.

Results: 30 yrs old female presented with generalised lethargy \& weakness along with peripheral neuropathy for last 4-5 years. On physical examination patient was found to have pallor with moderate splenomegaly because of which she was being evaluated as a case of myeloproliferative neoplasm/Lesihmaniasis. Patient didn't have any history of loss of appetite and loss of weight. Her haemoglobin was $10.4 \mathrm{gm} / \mathrm{dl}$ with normocytic normochromic picture with mild anisopoikilocytosis with occasional tear drop cells and a few target cells. Platelets were found to be adequate. Bone Marrow aspirate was 
done as a part of work up which showed particulate cellular bone marrow aspirate with trilineage proliferation with M:E was 3:1. Megakaryocytes were adequate with normal morphology. Both myeloid and Erythroid lineage showed normal maturation. There was numerous collection of crumpled paper appearing histiocytes which were PAS positive. No hemoparasites were seen in the bone marrow aspirate. Her BCR-ABL, JAK-2 mutation profile was negative. Bone marrow biopsy showed an overall cellularity of $70 \%$ with aggregates of gaucher cells showing positivity for PAS and negativity for CD 41 . Since the disease was chronic and has some neuropathy it bests fitted into Gaucher disease Type 3.

Discussion \& Conclusion: Gaucher disease is an Autosomal recessive lysosomal storage disease in which there is deficiency of the enzyme Glucocerebrosidase and which leads to zccumulation of Glucosylceramide and its deacetylated form glucosylsphingosine. It is most prevalent in Ashkenazi Jews. Of all the three types of Gaucher Disease, Type 1 (GD1) is most common and it comprises of $94 \%$ of cases. The prevalence of GD1 is 1 in 75000 births. Here we wanted to emphasise the importance of awareness of unusual age involvement of adult onset Gauchers which closely mimicked myeloproliferative neoplasm clinically and landed up in hematologyward. Interestingly this case appeared to be a rarer type Type 3 . Therefore it is important to be aware of this entity while considering cases presenting with massive splenomegaly.

Supporting Document: 5ef3ef3a-a63a-4eb3-a642-dddacd54f92c.

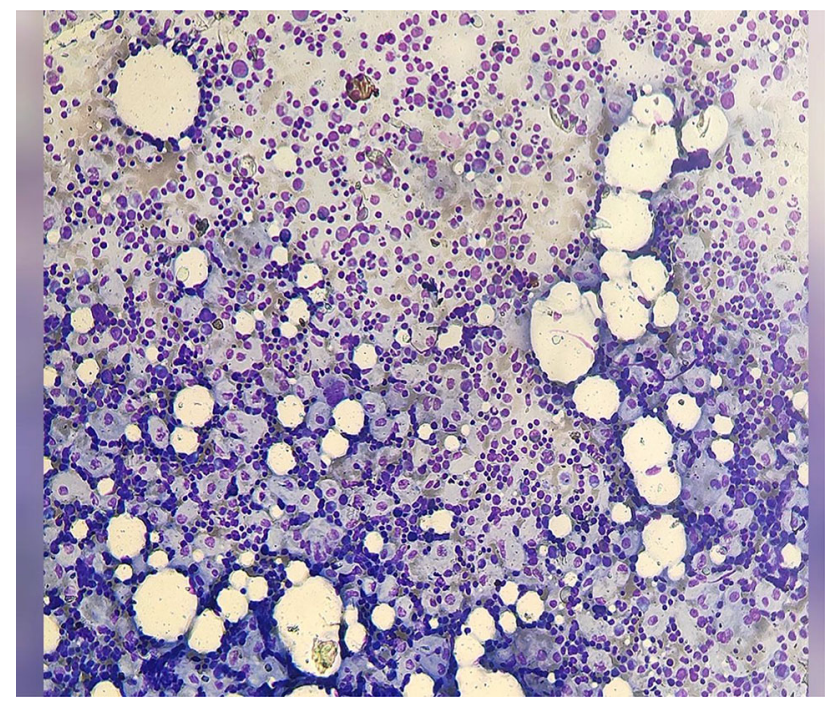

Disclosure of Interest: None Declared.

Keywords: Gaucher disease, Generalized weakness with peripheral neuropathy, Massive splenomegaly.

\section{Abstract 30. Profile of Hemophagocytic Lympohistiocytosis (HLH): Prospective Observational Study}

SirisharaniSiddaiahgari ${ }^{1}$, Archana Rauthan ${ }^{1}$, Ranjith $\mathrm{CS}^{2}$, JoydeepChakrabartty ${ }^{3}$, Anil Aribandi ${ }^{* 2}$

${ }^{1}$ Department of Haemato-Oncology, Rainbow Children's Hospital, ${ }^{2}$ Department of Haemato-Oncology, Care Hospitals, Hyderabad, ${ }^{3}$ Department of Haemato-Oncology, Amri Hospitals, Kolkata, India
Aims \& Objectives: To study epidemiology, clinical features, laboratory findings $\&$ diagnosis of underlying disease associated with HLH and also to assess their response to the treatment.

Patients/Materials \& Methods: We enrolled 53 consecutive patients diagnosed with HLH from January 2012 to March 2015 who satisfied the HLH 2009 diagnostic criteria and were between the age of 1 month and 15 years.

Results: Most children belonged to age group 31 days-12 months (47.16\%). Mean age was 35 months, male (54.71\%) children were slightly more affected than female (45.29\%) children.

The most common presenting symptom in our series was fever (96.2\%) and most common sign was pallor (100\%). Bone marrow hemophagocytes were demonstrated in $92.15 \%$. Raised S. Ferritin levels $>500 \mathrm{ng} / \mathrm{ml}$ was seen in $98.11 \%$ with mean ferritin levels of $4146 \mathrm{ng} / \mathrm{ml}$. Transaminitis was seen in $84.9 \%$. Bicytopenia was noticed in $62.26 \%$ and pancytopenia in $35.84 \%$.

With respect to etiology, Infection was the most common, noticed in $75 \%$ of cases. Dengue was found in 14 patients (26.1\%). Primary HLH was seen in $5(9.5 \%)$ of children.

Out of 53 children enrolled in the study only 47 children received some form of therapy, with prephase steroid alone therapy $(n=31)$ $(65.9 \%)$ had a improvement in clinical parameter, however on follow up $3(9.6 \%)$ children died and 1 left against medical advice.

Among 16 children who had no improvement with steroids were advised complete HLH protocol, but only $12(25.5 \%)$ children parents gave the consent, during course of therapy 7 children died $(14.8 \%$ in 47) $(58.3 \%$ in 12 who completed protocol), 3children discharged against medical advice (6.3\%) at the time of completion of protocol only $2(4.2 \%$ in 47$)(16.6 \%)$ in 12 who completed protocol.

Discussion \& Conclusion: Infection associated HLH has better outcome with early diagnosis and prompt treatment. Primary HLH should be suspected and genetic analysis should be sent for patients $<3$ yrs, with history of consanguinity and sibling death due to similar condition, those presenting with CNS signs and symptoms \& patients who deteriorated rapidly on treatment with Dexamethasone alone.

Disclosure of Interest: None Declared.

Keywords: HLH, Infection, pancytopenia.

\section{Abstract 31. Phenytoin Induced Pure Red Cell Aplasia}

\section{Chandan Kumar" ${ }^{* 1}$, Kailash Kumar', Nilesh Kumar', VibhuKhare ${ }^{1}$, Anurag Rana ${ }^{1}$}

\section{${ }^{1}$ Institute of Medical Sciences- BHU, Varanasi, India}

Aims \& Objectives: Hematological toxicities of phenytoin are well known but it rarely causes pure red cell aplasia. We present a case of Pure red cell aplasia which developed after patient was started on phenytoin therapy for GTCS.

Patients/Materials \& Methods: A 24 years old male presented with complaints of generalized weakness and easy fatiguability for 1 month duration without any history of any bleeding manifestation, fever, or any feeling of heaviness in the abdomen. There was a history of phenytoin intake for GTCS for last 4 months with good compliance. On general examination, pallor was present. There was no other finding in systemic or general examination. On investigations there was normocytic anemia with normal TLC and platelet counts. Iron studies were within normal limits. Reticulocyte count was reduced $(0.6 \%)$.Bone marrow examination was done which showed severe erythroid hypoplasia with normal platelets and WBC lineage. Autoimmune markers, Monospot IgM for EBV, Parvovirus B19 were negative. CECT thorax did not had any evidence of thymoma. With these evidences we finally made a diagnosis of Phenytoin induced pure red cell aplasia and phenytoin was with withdrawn. 
Results: The patient improved clinically and hemoglobin improved on follow up.

Discussion \& Conclusion: Pure red cell aplasia is an uncommon adverse effect of many drugs.Of them phenytoin, azathioprine, and isoniazid has proper evidence for causal association. So drug history is an important aspect of proper evaluation of anemia. In patient presenting with anemia with norml TLC and platelet Pure red cell aplasia is a rare but important differential.

Disclosure of Interest: None Declared.

Keywords: Parvovirus B19, phenytoin, PURE RED CELL APLASIA.

\section{Bone Marrow Failure and MDS (Laboratory)}

\section{Abstract 1. Bone Marrow Mesenchymal Stem Cells of Acquired Aplastic Anemia Patients Retain Basic Properties}

Vandana Sharma ${ }^{* 1}$, SonaliRawat ${ }^{2}$, SuchiGupta $^{2}$, RinkeySharma ${ }^{2}$, Sujata Mohanty ${ }^{2}$, TulikaSeth ${ }^{1}$

\section{${ }^{1}$ Hematology, ${ }^{2}$ Stem Cell Facility, AIIMS, New Delhi, NEW DELHI, India}

Aims \& Objectives: Acquired aplastic anemia (aAA) is a bone marrow (BM) failure disorder. Mesenchymal stem cells (MSCs) are an important component of BM microenvironment that maintain hematopoiesis. In the present study, we investigated whether BMMSCs contribute to the pathogenesis of aAA. We assessed the cellular morphology, surface marker profiling, adipogenic, chondrogenic and osteogenic differentiation potential of BM-MSCs from untreated aAA patients and in age and sex matched controls.

Patients/Materials \& Methods: BM samples were obtained from 5 untreated newly diagnosed aAA patients (mean age 20 years, 3 males, 2 females) and 5 age and sex matched controls. $1 \mathrm{ml}$ of BM sample was seeded in complete growth media and passaged with $0.05 \%$ trypsin-EDTA. Cultured cells were subjected to immunophenotyping (CD105,CD73, CD29, CD90, HLA-ABC, HLA-DR, CD34/45) and trilineage differentiation as per the respective protocols. MSCs were stained with Alizarin Red dye for identifying osteogenic differentiation; Alcian blue staining for chondrogenic differentiation and Oil Red 'O' staining for adipogenic differentiation.

Results: MSCs of aAA and control group shared similar spindle shaped morphology. Both revealed a consistent immunophenotypic profile; being negative for CD34/CD45, HLA-DR and positive for CD105, CD90, CD29, CD73 and HLA-ABC. aAA-MSCs had decreased osteogenic differentiation compared to controls as depicted by less intense alizarin red staining. Under adipogenic condition, aAA-MSCs showed less lipid-containing cells as compared to controls. However, these were not statistically significant. aAA-MSCs and control MSCs showed similar potential to differentiate into chondrocytes. However, the overall differential proliferation of aAA and control MSCs did not show any significant difference.

Discussion \& Conclusion: In acquired aplastic anemia, defective hematopoiesis leads to an empty bone marrow. Mesenchymal stroma supports hematopoiesis and has immune modulating properties. Any flaws in bone marrow mesenchymal stromal cells can affect hematopoiesis. In our study, we analyzed the basic characteristics of aAA-BMMSCs as compared with normal BM samples. We observed that BMMSCs from aAA patients and controls share similar cellular morphology, immunophenotypical and differential properties. Our preliminary results suggest that BM-MSCs are not involved in pathogenesis of aAA. Further investigations on stromal components in aAA would be necessary for better understanding the mechanism of this disease.
Disclosure of Interest: None Declared.

Keywords: Acquired aplastic anemia, Bone marrow faailure, Mesenchymal stem cells.

\section{Abstract 2. Molecular Characterization of Osteopetrosis in the Indian Population} EswariSampath $^{* 1}$, Fouzia N.A
Eunice. S Edison $^{1}$, Anu Korula

${ }^{1}$ Department of Haematology, Christian Medical College, Vellore, India

Aims \& Objectives: Osteopetrosis (marble bone disease) is a group of rare genetic bone disorders characterized by an increased bone mass due to defective osteoclast development or function. The severity of the disease varies from incidental radiological findings to bone marrow failure. It is a very rare disorder with an estimated incidence of one case per 100,000-500,000 population. Haematopoietic stem cell transplant [HSCT] is the only curative treatment option currently available.

Patients/Materials \& Methods: This study represents the experience of testing suspected osteopetrosis cases from 2014 to 2019. Patients were initially screened by haematological parameters [CBC, peripheral blood smear], biochemical parameters [ALP, LDH, PTH, Calcium and Vitamin D levels] and radiological findings. Peripheral blood samples were collected and wherever possible, from the parents. DNA was extracted by established protocols. Molecular analysis of TCIRG1, CLCN7, RANK and RANKL genes were done by direct DNA sequencing of coding and splice site regions.

Results: During this study period, we received samples from 22 suspected cases of osteopetrosis for molecular analysis. We identified mutations in $50 \%$ of these cases. Mutations in TCIRG1 were present in 8 cases; 4 of which were missense variants, 2 splice site variants, 1 nonsense, 1 insertion and 1 deletion frameshift variants. Two missense and one splice site variant were identified in the RANK gene. We have also offered pre-natal diagnosis for osteopetrosis in one family. It is possible that the remaining cases may have mutations in other genes that cause osteopetrosis and may require analysis by next generation sequencing. Discussion \& Conclusion: Osteopetrosis caused by TNFSF11 [RANKL] mutations cannot be treated by HSCT. So, a molecular diagnosis should be made before transplantation to ensure that the pathology is not due to RANKL mutation. None of our cases had a RANKL mutation. Molecular diagnosis paves the way for specific treatment, counselling and prenatal diagnosis in osteopetrosis.

Disclosure of Interest: None Declared.

Keywords: osteopetrosis, Sequencing, TCIRG1.

\section{Abstract 3. Neuroblastoma Mimicking as Lymphoblastic Leukemia- Flow Cytometry as the Initial Clue}

Ankur Ahuja ${ }^{1}$, Juhi Arora ${ }^{* 1}$, Devika Gupta ${ }^{1}$, Suman Pramanik², TathagatChatterjee $^{1}$

\section{${ }^{1}$ Dept of Lab and Molecular Medicine, ${ }^{2}$ Dept of Hematology,} Army Hospital RR, Delhi, India

Aims \& Objectives: To analyze the role of ancillary techniques like flow cytometry, cell block in a case of neuroblastoma who mimicked as a case of lymphoblastic leukemia. 
Patients/Materials \& Methods: A case from pediatric ward at a tertiary centre was evaluated by flow cytometry, BM biopsy, FNAC, cell block and IHC.

Results: 3.5 year old male child admitted in civil hospital in Bihar with chief complaint of fever, swelling right cervical lymph nodes and splenomegaly since 2 months. Child also developed bilateral proptosis and dilated veins over the forehead. His $\mathrm{Hb}$ was $3.3 \mathrm{~g} / \mathrm{dl}$ and TLC was $3800 / \mathrm{mm}^{3}$. His peripheral blood smear \& bone marrow outside there was reported as a case of ALL, while FNAC of lymph node showed reactive lymphadenopathy. CECT also showed anterior mediastinal mass.

Patient was referred to our tertiary centre and his bone marrow sample was sent for the flow cytometry which revealed absent CD 45 and low SS cells which on gating showed positivity for CD 38 and negativity for CD 34, TdT, CD 17, CD 19, cCD3, MPO and other lineage markers. Flow was followed by BMA morphology which revealed small cells showing overlapping and rosettes with neuroprils. On the basis of above a diagnosis of possibility of neuroblastoma was offered. FNAC of lymph node was done and cell block was done. Both cell block and bone marrow biopsy revealed infiltration by similar type of small round blue cells positive for synaptophysin, chromogrannin and CD56 which confirmed it to be metastatic neuroblastoma.

Discussion \& Conclusion: Here we wanted to emphasize the mimicker of lymphoblastic leukemia both clinically and morphologically. Also emphasize was the role of flow cytometry and cell block which can also be used as an essential aid for the final diagnosis of neuroblastoma which mimics lymphoblastic leukemia very closely.

Supporting Document: bdbf9bef-57bc-47a9-9595-d2f919671ced.

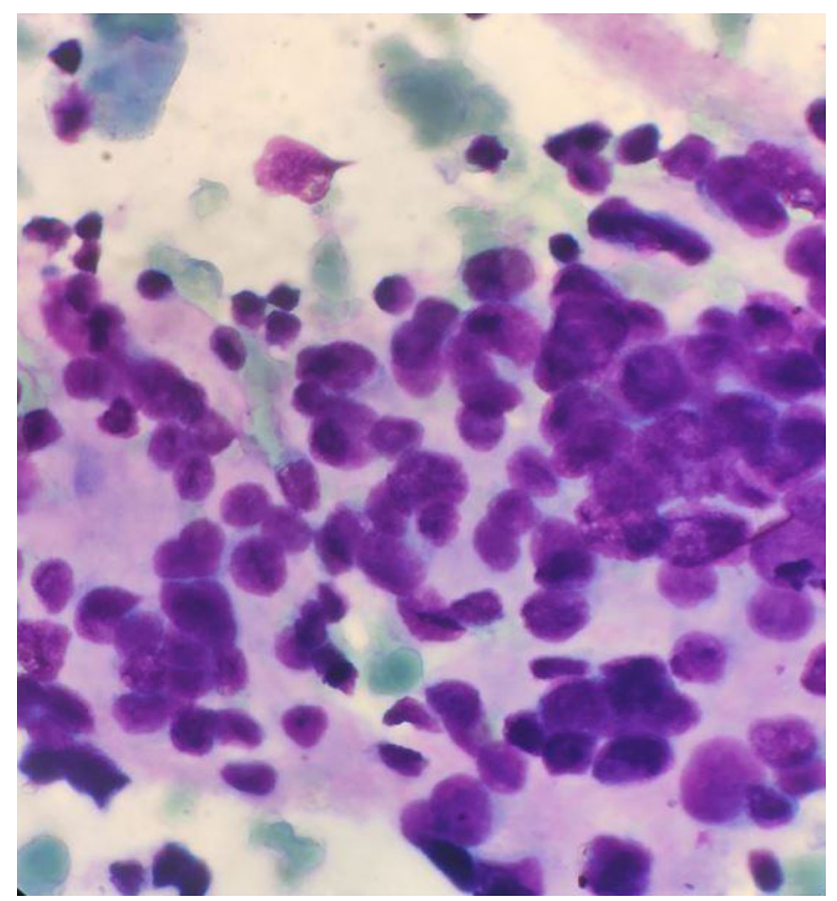

Disclosure of Interest: None Declared.

Keywords: CD 56 positive tumor, Flow cytometry in non hematological malignancy, Neuroblastoma.

\section{Abstract 4. Utility of E-Cadherin, CD71, Glycophorin $a$ in Delineating Erythroid Lineage in Bone Marrow Biopsy}

\section{AnandhiAnbalagan $^{* 1}$, Rakhee Kar ${ }^{1}$, DebdattaBasu ${ }^{1}$ \\ ${ }^{1}$ PATHOLOGY, JIPMER, PUDUCHERRY, India}

Aims \& Objectives: In bone marrow biopsies, immature erythroid cells have an undifferentiated morphology quite similar to immature precursors of other lineages necessitating immunohistochemical distinction. Further, colonies of normal erythroid cells may be confused with lymphoid and other round cell. In certain conditions like pure red cell aplasia (PRCA) identifying the sparse erythroid cells among other elements in bone marrow is essential. It is also of great importance for assessing remission status, differentiating megaloblasts from myeloblasts and in assessing distribution of various cell lineages in myelodysplastic syndromes (MDS).

Objective: To assess the usefulness of E-cadherin, along with glycophorin A and CD71 in delineating erythroid precursors in various haematological conditions.

Patients/Materials \& Methods: We analysed 111 bone marrow biopsies including Acute leukemia on chemotherapy for assessing remission status (47), Acute Myeloid Leukaemia (16), MDS (8), Megaloblastic anemia (5), Aplastic Anemia (6), Primary Myelofibrosis (6), PRCA (3), staging marrows (6), Reactive marrows (9), sideroblastic anemia (1) and Paroxysmal nocturnal hemoglobinuria (PNH) (1). For all cases along with E-cadherin, the following markers were used-CD 34, CD 117, MPO, CD 61 and TdT, as and when indicated. In 21 cases we have analysed for all three erythroid lineage markers-E-cadherin, CD71 and Glycophorin A. The distribution and pattern of positivity in the spectrum of erythroid cells were studied. Results: E-cadherin and CD71 highlighted early erythroid precursors and were absent/weak in more mature erythroid cells. In remission marrows, they helped delineate erythroid precursors from other blasts both myeloid and lymphoid and aided in diagnosis in conjunction with other markers. These markers helped in assessing the proportion of erythroid lineage especially in cases of MDS,PRCA and aplastic anemia. Glycophorin A on the other hand was positive in only late erythroblasts and erythrocytes. It helped in delineating micronormoblastic erythroid islands and in distinguishing them from lymphocytes.

Discussion \& Conclusion: Our results demonstrate the usefulness of E-cadherin and CD71 as markers of early erythroid lineage and Glycophorin A as marker specifically of late erythroid differentiation, in bone marrow biopsies.

Disclosure of Interest: None Declared.

Keywords: Bone marrow biopsy, erythroid lineage, Immunohistochemistry.

\section{Abstract 5. Myelodysplastic Syndrome- Myeloproliferative Neoplasm-Unclassified: A Diagnosis of Exclusion}

\section{Hitesha Bhandari $^{* 1}$; Sashau Bansal, Naresh Somani, Chaitali}

${ }^{1}$ Pathology, Bhagwan Mahaveer Cancer \& Research Hospital, Jaipur, Rajasthan, Jaipur, India

Aims \& Objectives: Myelodysplastic Syndrome-Myeloproliferative Neoplasm-Unclassified meets the criteria for MDS/MPN category at the time of initial presentation with clinical,morphological and laboratory features overlapping with both myelodysplastic and myeloproliferative categories ideally along with the presence of $<$ $20 \%$ blasts in the peripheral blood and bone marrow, distinguished 
with platelet count $>450 \times 10^{9} / 1$,WBC count $>13 \times 10^{9} / 1$ with bone marrow megakaryocytic proliferation. We report a case of MDSMPN-UNCLASSIFIED that was diagnosed at the time of presentation.

Patients/Materials \& Methods: An 84 year non-alcoholic male presented with the history of generalized weakness, recurrent fever with no history of any comorbidity or previous treatment. On clinical examination, no abnormality was detected. Patient's diagnostic workup included COMPLETE BLOOD COUNT that revealed HB-

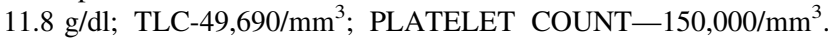
PERIPHERAL BLOOD FILM findings showed neutrophilic leucocytosis with shift to left,BONE MARROW EXAMINATION revealed hypercellular marrow for age with overall cellularity of $>$ 95\% with myeloid and megakaryocytic prominence suggesting features of dysmegakaryopoiesis (micromegakaryocytes with atypia) along with $4.6 \%$ blasts.

Results: The bone marrow biopsy showed morphological features of MDS-MPN-U and molecular workup for this patient was done and was found to be negative for the BCR-ABL-1 gene, JAK-2, EXON 12-15 mutation, C-MPL, CAL-R.

Discussion \& Conclusion: In our case the prompt integration of clinical, morphological and molecular workup led to the diagnosis of entity that's a diagnosis of exclusion on the very first presentation. Hence, in a scenario where a proportion of case meets criteria of CMML, BCR-ABL NEGATIVE ATYPICAL CML, MPN IN ACCELERATED OR BLAST PHASE, MDS OR AML and others classified as MDS-MPN-U,it highlights the importance of morphological and next generation sequencing for TET2, NRAS1, CBL, SET, BP1.

Supporting Document: 2e2c6be1-bc96-49d0-95be-41d48f5bd133.

Disclosure of Interest: None Declared.

Keywords: MDS, MPN, UNCLASSIFIED.

\section{Abstract 6. Role of IHC on Bone Marrow Biopsies in Enhancing the Diagnosis in Suspected Cases of MDS}

\author{
Richa Juneja $^{* 1}$, HaraprasadPATI ${ }^{2}$, Seema Tyagi ${ }^{2}$, \\ Manoranjan MAHAPATRA ${ }^{2}$, Aastha Gupta ${ }^{3}$, Renu SAXENA ${ }^{2}$ \\ ${ }^{1}$ Hematology, AIIMS, New Delhi, ${ }^{2}$ Hematology, AIIMS, \\ ${ }^{3}$ Hematology, Dangs Lab, Delhi, India
}

Aims \& Objectives: To study whether IHC with anti-CD61 antibody would increase detection of micro megakaryocytes and hence diagnosis of MDS compared to H \& E stained biopsy.

To study pattern of P53 expression in cases with and without dysplasia in study cohort.

Patients/Materials \& Methods: We studied 43 bone marrow biopsies from 40 suspected MDS patient having undiagnosed cytopenia. Patients with other possible causes of cytopenia and obvious MDS cases like multilineage dysplasia and excess blasts were excluded. Megakaryocytes were analyzed on H and E and CD61 IHC. Dysplastic megakaryocyte was calculated as percentage of micro megakaryocytes, hypolobate megakaryocytes and multinucleate megakaryocytes out of total megakaryocytes. We studied pattern of P53 expression in cases with and without dysplasia. Antibody obtained from Dako (Clone: DO-7; Isotype: Ig G2b, Kappa) was used for same.

Results: Out of total 43 biopsies of suspected MDS cases-13 (30.2\%) showed $>10 \%$ dyspoiesis on reviewing $\mathrm{H}$ and $\mathrm{E}$ stained slide. None of these cases showed absence of dysplastic megakaryocytes on CD61 IHC. Out of 30 cases with no significant dysplastic megakaryocytes $21 \quad(48.8 \%)$ cases showed $>10 \%$ dysplastic megakaryocytes on CD61 facilitating the diagnosis of MDS with inter observer agreement ( $p$ value 0.0001). 9 cases showed no significant dysmegakaryopoiesis with either H \& E or CD61 IHC.

In our study out of 30 cases with $<10 \%$ dysplastic megakaryocytes on $\mathrm{H}$ and $\mathrm{E} 5$ had $>30 \%$ dysmegakaryopoiesis on CD61. 10 cases showing $>10 \%$ but $<30 \%$ megakaryocytes on $\mathrm{H}$ and $\mathrm{E}-3$ had $>$ $30 \%$ and 6 cases had $>40 \%$ dysplastic megakaryocytes on CD61 IHC. This finding suggests IHC may help in detection of dysmegakaryopoiesis with higher cut off as well.

Out of total 34 cases showing significant dysmegakaryopoiesis 7 cases $(20.6 \%)$ showed positivity for p53 on IHC which is little less than that reported in low grade MDS.

Discussion \& Conclusion: Our study strongly suggests that CD61 IHC significantly increases detection of dysplastic megakaryocytes in suspected MDS patients and facilitate MDS diagnosis with good inter-observer agreement.

P53 positivity rate by IHC is low in such cases with subtle dysplasia but when present may suggest revisiting for possibility of MDS.

Disclosure of Interest: None Declared.

Keywords: IHC, MDS, p53.

\section{Abstract 7. Effect of Telomere Length and T-Cells in Anti-thymocyte Globulin Treated Aplastic Anemia Patients}

Deborah Arul $^{* 1}$, ArunaBarade ${ }^{1}$, Eunice Sindhuvi ${ }^{1}$, Biju George $^{1}$

${ }^{1}$ Haematology, Christian Medical College, Vellore, India

Aims \& Objectives: Aplastic anemia (AA) is a bone marrow failure syndrome characterized by peripheral pancytopenia and hypocellular bone marrow. Clinical and laboratory evidence implicate AA with T-cell mediated immune destruction of bone marrow. Studies have reported abnormal telomere shortening in AA patients. This study aims to investigate the role of telomere length and the pattern of $\mathrm{T}$-cells in patients with $\mathrm{AA}$ in response to antithymocyte globulin (ATG) therapy.

Patients/Materials \& Methods: This study includes 14 patients diagnosed with AA and were treated with ATG at our centre from 2018 to till date. Blood samples were collected at different time points post ATG treatment. T cells markers CD3, CD4, CD8 were analysed using flow cytometry and CD4:CD8 ratio was calculated. DNA was extracted from peripheral blood and relative telomere length (rTL) was measured using quantitative real-time PCR (qPCR). Statistical analysis was carried out using SPSS Software.

Results: The median age of AA patients in the study was 27 (5-49) years and there were 9 males and 5 females. Patients were grouped based on severity as non severe AA $(\mathrm{N}=2)$ and severe AA $(\mathrm{N}=12)$. The median relative TL of AA patients measured at different time points are tabulated (Table 1). Out of 14 patients, 2 patients did not respond to the treatment, 9 partially responded and 3 had complete response at 6 months follow up. In this study, ATG response rate did not vary based on gender $(p=0219)$ neither on severity of the disease $(p=0.523)$. There was no significant association with rTL and CD4:CD8 ratio when compared to the treatment response, at different time points.

Discussion \& Conclusion: There is paucity of literature concerning responses to ATG treatment in AA from India. Varied results ranging from 33.3 to $87.9 \%$ response rates have been reported from previous studies in India. In our study, AA patients receiving ATG treatment, relative TL and pattern of T-cells was not associated with response. Further analysis in large cohort will enable to understand the significance of telomere length with ATG response.

Supporting Document: 0042599e-92fa-4bcd-b606-641b4612c0ca. 


\begin{tabular}{|c|c|c|c|c|}
\hline ATG Treatment & Pre & $1 \mathrm{mo}$ & $2 \mathrm{mo}$ & $3 \mathrm{mo}$ \\
\hline $\mathrm{N}$ & 14 & 8 & 6 & 7 \\
\hline $\begin{array}{c}\text { Relative TL } \\
\text { Median (Range) }\end{array}$ & $0.36(0.19-0.67)$ & $0.40(0.17-0.76)$ & $0.47(0.27-0.55)$ & $0.44(0.30-0.53)$ \\
\hline
\end{tabular}

Disclosure of Interest: None Declared.

Keywords: Aplastic anemia, Immunosuppressive therapy, Telomere Length.

\section{Abstract 8. Clinicopathological Profile of Bonemarrow Findings in Metastatic Solid Tumors: A Series of 4 Cases}

Vaishali Rohilla $^{* 1}$, AnamSingh ${ }^{1}$, Sachin Kolte ${ }^{1}$, LeelavathiDawson ${ }^{1}$, Rashmi Arora ${ }^{1}$

${ }^{1}$ Pathology, Vardhman Mahavir Medical College, New Delhi, India

Aims \& Objectives: To study the clinicopathological profile of bonemarrow findings in 4 cases of metastatic solid tumors.

Patients/Materials \& Methods: 4 cases of metastatic tumor to bonemarrow were evaluated. Their clinical details, bonemarrow aspirates and bonemarrow biopsies were examined and evaluated. IHC was performed wherever necessary.

Results: Case 1: A 67 year old male presented with difficulty in breathing and cough for 1 year. Bonemarrow biopsy showed a packed marrow infiltrated by tumor cells having scant cytoplasm, irregular nuclear membrane and stippled chromatin. Few cells showed moulding and artefacts. IHC was performed to confirm the diagnosis of metastatic neuroendocrine carcinoma.

Case 2: A 32 year old male presented with bilateral pleural effusion with mediastinal and retroperitoneal lymphadenopathy. Bonemarrow biopsy was mild hypocellular and showed clusters of abnormal atypical cells with abundant vacuolated cytoplasm and eccentric nucleus. IHC was performed to confirm the diagnosis of metastatic adenocarcinoma.

Case 3: A 4 month old male child presented with abdominal swelling. Bonemarrow aspirate showed tiny clusters of atypical cells and bonemarrow biopsy showed an infiltration by atypical cells with increased N/C ratio, scant cytoplasm and focal nuclear moulding. IHC panel was put to confirm the diagnosis of metastatic deposits of neuroblastoma to the bonemarrow.

Case 4: A 1 year old male child presented with abdominal distension, fever and vomiting. Bonemarrow aspirate showed few clusters of atypical cells. Bonemarrow biopsy showed necrotic areas with small round tumor cells with increased N/C ratio, scant cytoplasm and dense chromatin. IHC was performed to confirm the diagnosis of metastatic deposits of small round cell tumor to the bonemarrow.

Discussion \& Conclusion: Detection of metastatic tumor in bonemarrow is of great importance for the clinical staging of tumor spread as it will influence the response to treatment and overall survival.

In adults after lymphoma, most common metastasizing tumor to bonemarrow are carcinoma from prostate, breast and lung.

In children majority of metastasizing tumors are neuroblastoma, rhabdomyosarcoma, ewing sarcoma and retinoblastoma.

Trephine biopsy confirmed by IHC remain gold standard in establishing the definitive diagnosis in bonemarrow metastasis.

Disclosure of Interest: None Declared.

Keywords: Bonemarrow, Metastasis, Solid tumor.
Abstract 9. Bonemarrow Angiogenesis and Microvessel Density in Patients with Aplastic Anemia

Richa Sharma $^{* 1}$, Sunita Singh ${ }^{1,1}$, Rajeev Sen ${ }^{1}$, Renuka Verma ${ }^{1}$

${ }^{1}$ Pathology, Pt. B.D. Sharma PGIMS, Rohtak, India

Aims \& Objectives: The present study was conducted with the aim to diagnose and categorize aplastic anemia (AA) according to the severity as non severe (NsAA), severe (sAA) and very severe (VsAA) and to study microvessel density using immunohistochemical stain using CD 34 and morphometric analysis of microvessel density by computer assisted quantitative analyser and to compare it in the three subcategories of aplastic anemia with control group.

Patients/Materials \& Methods: The present prospective study was conducted in the Department of Pathology at Pt. B.D. Sharma PGIMS, Rohtak. Thirty cases of AA patients and 10 cases of control were taken. AA patients were diagnosed on the basis of complete blood count, peripheral blood smear, bone marrow aspiration examination and bone marrow trephine biopsy (using Jamshidi's needle). AA patients were categorized as sAA, NsAA and VsAA using Camitta et al. criteria. IHC using CD 34 was performed to look for angiogenesis and to calculate microvessel density (MVD).

Results: Maximum number of patients $(56 \%)$ of AA were between 1 st and 3rd decade of life. Females were more commonly affected with a male to female ratio (11/19) of 0.58:1. In Aplastic anemia patients (Group I), using Camitta et al. criteria 30 cases were categorized into NsAA (10 cases), sAA (12 cases) and VsAA (8 cases). MVD was calculated using CD 34 which is a good marker of angiogenesis. MVD showed a decreasing trend with increase in severity of the disease.

Discussion \& Conclusion: The study shows that Aplastic anemia is associated with reduced Bone Marrow angiogenesis and mean MVD score shows a decreasing trend from non severe to very severe Aplastic anemia.

Supporting Document: c836cd5c-74eb-429c-9883-d385c21f8999.

Disclosure of Interest: None Declared.

Keywords: Angiogenesis, Aplastic anemia, Microvessel density.

\section{Abstract 10. Intelli-NGS: Using "ARTIFICIAL INTELLIGENCE-INTELLIGENTLY" to Filter Variants from Hematology Panels}

\author{
Aditya Singh $^{* 1}$, Prateek Bhatia ${ }^{1}$ \\ ${ }^{1}$ Paediatrics, Postgraduate Institute of Medical Education and \\ Research, Chandigarh, India, Chandigarh, India
}

Aims \& Objectives: Targeted Next generation sequencing (NGS) is widely being used in current haematology practice. However, handling the voluminous data bioinformatically is a nightmare for most laboratory scientists and we end up relying on available default software pipelines accompanying the platform for reporting good calls. However, this not only takes time and diligent effort but also is vulnerable to false positive and negative errors in calling. In current study we designed and explored artificial intelligence-based algorithm to help us with filtering out good calls seamlessly.

Patients/Materials \& Methods: Inherited bone marrow failure syndrome panel consisting of 33 genes and inherited anaemias panel consisting of 10 genes were used for training of an artificial intelligence framework written in Python 3 using decision tree method of sci-kit learn package. Supervised training was used by assigning good and bad call values after manual scrutiny of the data. A total of 6681 variants were fed to the model training algorithm written in Python 3 
which randomized the various parameters of the decision tree model till the desired accuracy of over $99 \%$ was achieved.

Results: The final decision tree model was able to achieve a total accuracy of $99.94 \%$ with ROC-AUC of 1.0 which makes it theoretically $100 \%$ accurate. IntelliNGS takes around 5-7 s per VCF file for filtration on personal computer.

Discussion \& Conclusion: IntelliNGS is capable of sorting good from bad calls based on 25 parameters that make it aggressively stringent and reduces the chance of errors. It is humanly impossible to find a relationship between all these parameters per call per patient. This artificial intelligence makes it easier for less adept users to reduce type 1 and type 2 errors and to advanced users it adds an additional layer of confidence. IntelliNGS is "ever evolving", it can be used to retrain the model once additional data becomes available over time, hence allowing it to adapt to updated algorithms. We are currently in the validation phase of our AI using genome in a bottle consortium (GIAB) high confidence data. This tool, once released, will be able to significantly reduce the turnaround time and increase the confidence of reports, ultimately benefitting patients and doctors alike. Also, as it is capable of running efficiently on personal computers, it'll not be adding any capital to the institution/individual and will not require any knowledge of programming or editing codes.

Supporting Document: 14d4dbee-41d9-462a-b126-9d1f0504f473.

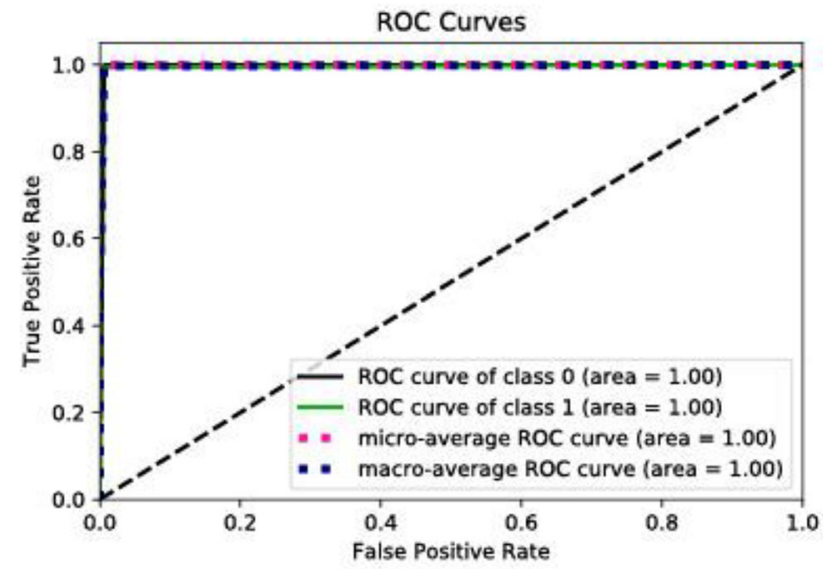

Disclosure of Interest: None Declared.

Keywords: artificial intelligence, deep learning, Next generation sequencing.

\section{Chronic Leukemia (Clinical)}

\section{Abstract 1. Treatment Free Remission in Indian Patients of Chronic Myeloid Leukemia on Generic Imatinib Therapy}

\section{Naveen $\operatorname{Yadav}^{* 1}$, SanjeevanSharma ${ }^{1}$, Venkatesan $S^{2}$ \\ ${ }^{1}$ Medicine, ${ }^{2}$ Pathology, AFMC, Pune, India}

Aims \& Objectives: Treatment Free Remission in Indian Patients of Chronic Myeloid Leukemia (CML) on Generic Imatinib therapyPreliminary report.

Patients/Materials \& Methods: $27 \mathrm{CML}$ patients with Undetectable bcrabl transcript levels were prospectively enrolled in this study between Aug 2017 to June 2019. All patients were CML in CP having major bcrabl transcript (p210). All patients received Indian Generic Imatinib upfront and continued till enrolment in the trial, except in one patient, where the TKI was switched to Dasatinib due to Imatinib intolerance. Minimum duration of Imatinib therapy was 07 years. After stopping Imatinib, patients were monitored once a month for six months, then two monthly for next six months and then once in three months. At each visit peripheral blood quantitative bcrabl/abl ratio on international scale with sensitivity of $4.5 \log$ or above by RQPCR was measured and recorded. Loss of molecular response (bcrabl/abl ratio $>0.1$ on IS) was taken as cut off for restarting Imatinib therapy. Molecular monitoring was continued as scheduled even after restarting Imatinib.

Results: 27 patients (19 males \& 8 females) with mean age of 47 years (18-71), took initial therapy with Indian Generic Imatinib for a median of 126 months (84-190). 17 patients have completed more than 12 months since enrolment, and 07 patients have completed 6 to 11 months. 03 patients have completed 4-5 months. Three patients $(11 \%)$ developed significant TKI withdrawal syndrome. 8 patients $(30 \%)$ lost their major molecular response at a median of 3 months (2-10) and were restarted on Imatinib. All these 8 patients have regained their major molecular response within a median of 3 months (2 -6). The other 19 patients $(70 \%)$ continue to remain in deep molecular remission and are asymptomatic with normal hematological and biochemical parameters.

Discussion \& Conclusion: Patients of CML (CP) on Indian generic Imatinib therapy can be taken off Imatinib therapy under stringent and controlled setting for monitoring their molecular response. The treatment free remission (TFR) rates and patterns are similar to those reported in western world.

Disclosure of Interest: None Declared.

Keywords: CML, generic Imatinib, treatment free remission.

\section{Abstract 2. Steroid Induced Chronic Myeloid Leukemia: Rare Coincidence or Etiology, A Diagnostic Dilemma?}

\author{
Rakesh $^{* 1}$, PrernaArora ${ }^{1}$, Naresh Gupta ${ }^{2}$ \\ ${ }^{1}$ Pathology, ${ }^{2}$ Medicine, MAMC, New Delhi, India
}

Aims \& Objectives: Anabolic Steroids induced Chronic Myeloid Leukemia: rare coincidence or etiology, a diagnostic dilemma?

Patients/Materials \& Methods: Chronic Myeloid Leukemia rarely occurs with steroids intake.

A 25 year old body builder presented with bleeding via glans and priapism with history of intravenous steroids intake for 10 months. On clinical examination there was no organomegaly. Hemogram revealed leukocytosis with TLC count of 3 lakh/cumm. Peripheral smear revealed shift to left and myeloid preponderance, nucleated RBC (2/100 WBC), blast constituted $6 \%$ of mononuclear cells. NAP score was markedly low (10). BMA smears were hypercellular for age, with increase in myeloid precursors, eosinophils and eosinophilic precursors with erythroid suppression, Blasts cell constitute $8 \%$ of mononuclear cells. Retiulin stain shows grade $0-1$ fibrosis.

Results: Case diagnosed as Chronic Myeloid Leukemia.

Discussion \&Conclusion: The androgenic anabolic steroids use by athelets in the therapeutic dose range is well documented. There are increasingly frequent reports of potentially fatal diseases that occur with the therapeutic use of these agents. Chronic myeloid leukemia occurring due to use of these agents is a rare manifestation and a clinician should be aware of such atypical presentation of these agents.

Disclosure of Interest: None Declared.

Keywords: atypical, leukemia, STEROID. 


\section{Abstract 3. COX-2 and ABC Transporters as Putative Predictive Biomarkers of Imatinib Resistance in CML Patients}

Anil Aribandi ${ }^{* 1}$, GangappaDharmapuri $^{2}$, JoydeepChakrabartty ${ }^{3}$, ArunasreeKalle $^{4}$

${ }^{1}$ Department of Haemato-Oncology, Care Hospitals, ${ }^{2}$ Department of Animal Biology, University of Hyderabad, Hyderabad,

${ }^{3}$ Department of Haemato-Oncology, Amri Hospital, Kolkata,

${ }^{4}$ Departmant of Animal Biology, University of Hyderabad, Hyderabad, India

Aims \& Objectives: Efflux of chemotherapeutic drugs by ATPBinding Cassette $(\mathrm{ABC})$ transporter proteins is one of the several mechanisms known to be involved in the development of resistance. To evaluate the possibility of COX-2, MDR1, MRP1 and ABCG2 as predictive biomarkers for imatinib-resistant CML.

Patients/Materials \& Methods: A total of 50 peripheral blood samples were collected from patients suffering with CML both from newly diagnosed patients and who are on imatinib therapy of various doses $(300 \mathrm{mg}, 400 \mathrm{mg}, 600 \mathrm{mg}$, and $800 \mathrm{mg}$ ) between 18 to 55 years of age only after obtaining informed consent from the patient. Lymphocytes were isolated from the blood using Ficollhistopaq density gradient and total RNA was isolated using TRI Reagent ${ }^{\circledR}$ (sigma) according to manufacturer's protocol and stored at $-80{ }^{\circ} \mathrm{C}$ until further use.

Results: The real-time PCR results indicated significant increase in the expression of COX-2, MDR1, MRP1, ABCG2, b-catenin, GSK-3b and LEF1 genes with increase in the imatinib dosage confirming COX-2-dependent efflux pump expression and thus imatinib resistance.

Discussion \& Conclusion: The present study indicates that the expression of the COX-2 and $\mathrm{ABC}$ transporters during follow-up visits can be used as predictive biomarkers to monitor development of imatinib resistance. Furthermore, the present study signifies the efficacy of combinatorial treatment of celecoxib and imatinib as a treatment strategy to overcome imatinib resistance.

Disclosure of Interest: None Declared.

Keywords: CML, COX-2, Imatinib resistance.

\section{Abstract 4. Chemotherapy Induced Pemphigus in Chronic Lymphocytic Leukemia}

\section{Ekta Devi ${ }^{* 1}$, Sudhir Kumar Atri ${ }^{1}$ \\ ${ }^{1}$ Department of Medicine and Hematology, Pgims Rohtak, Rohtak, India}

Aims \& Objectives: A rare case of chemotherapy related cutaneous lesions in chronic lymphocytic leukemia (BENDAMUSTINE induced Pemphigus vulgaris) is being presented.

To study chemotherapy related cutaneous lesions in chronic lymphocytic leukemia. (BENDAMUSTINE induced Pemphigus vulgaris)To study chemotherapy related cutaneous lesions in chronic lymphocytic leukemia. (BENDAMUSTINE induced Pemphigus vulgaris).

Patients/Materials \& Methods: 51 year female, known case of chronic lymphocytic leukemia since past 18 months, on chemotherapy (BR regimen), developed multiple painful fluid filled blisters over skin after two days of completion of $5^{\text {th }}$ cycle of chemotherapy. No history of fever, joint pain, insect bite or trauma. On examination Multiple painful cutaneous bullous lesions present over extremities, trunk and face. Nikolsky sign and bulla spread sign were positive. Lesions ruptured to form painful skin erosions. Rest of the examination was normal.

Results: Complete hemogram was suggestive of eosinophilia. Tzank smear showed acantholytic cells. Skin biopsy showed suprabasilar bullae with focal rupture leading to marked dermal edema. Single row of keratinocytes attached to basal layer. Diffuse infilterates comprised of neutrophils, eosinophils. Immunofluroscence showed Ig G-Fishnet pattern positivity on intercellular squamous cell. Ig M, Ig A, C3 were Negative. features were suggestive of Pemphigus vulgaris likely drug induced (eosinophilic infilterates).

Discussion \& Conclusion: Though paraneoplastic pemphigus is common in CLL, based on biopsy findings diagnosis of Drug induced Pemphigus vulgaris secondary to chemotherapeutic agent (? Bendamustine? Rituximab) was made.

Patient was started on treatment for pemphigus vulgaris and after healing of lesions she was given chemotherapy in form of rituximab only. Lesion did not recur with rituximab. Thus the final diagnosis of Bendamustine induced Pemphigus vulgaris, a rare cutaneous adverse drug effect of bendamustine was made.

Supporting Document: 06e14f35-3cd3-46f8-b431-d6e5d943c7c5.

Figure 2. Skin biopsy showing diffuse infiltrates of neutrophils and eosinophils (High power field)

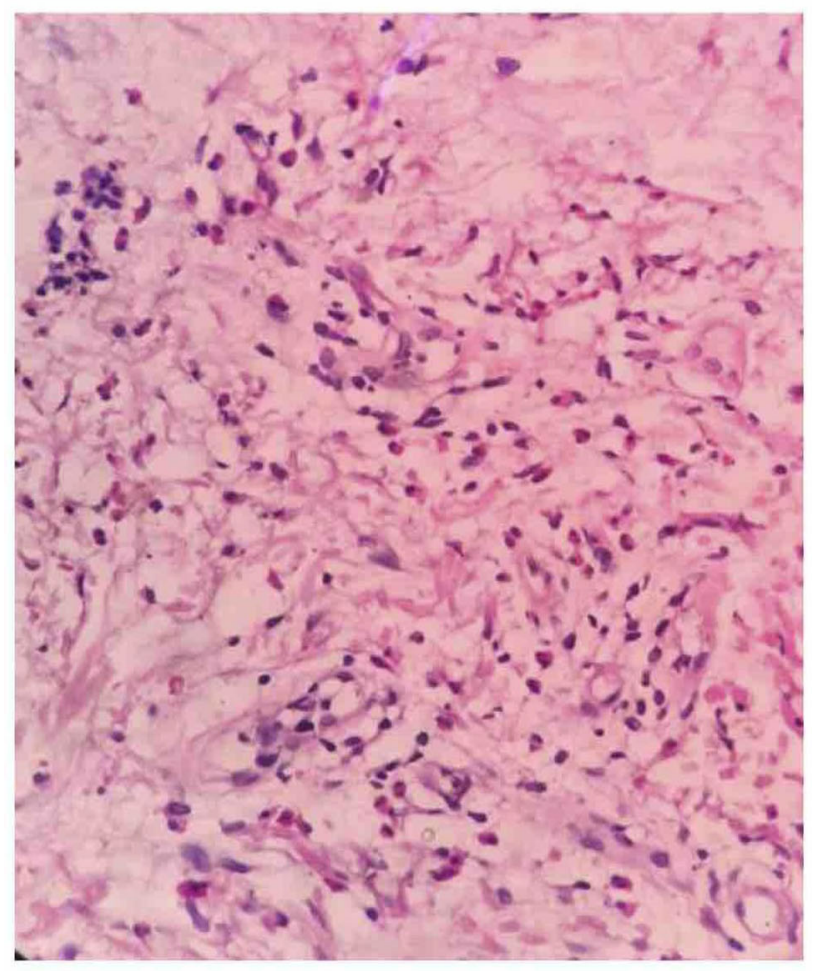

Disclosure of Interest: None Declared.

Keywords: adverse event, CHRONIC LYMPHOCYTIC LEUKEMIA, Pemphigus. 


\section{Abstract 5. Eosinophilic Folliculitis in Chronic Lymphocytic Leukaemia: An Unrecognised Entity}

\section{Ranjit kumar $^{1}$, Aribandianilkumar ${ }^{* 1}$ and Dr.Krishnamanikalpathi,Dr.ArunkumarLingutla}

\section{${ }^{1}$ Department of Haemato Oncology, Care Hospitals, Hyderabad, India}

Aims \& Objectives: In Chronic lymphocytic leukaemia (CLL) As though we think dermatological involvement un common it has varied dermatological features, reported in about $25 \%$ of CLL cases either by neoplastic cells or by a non-metastatic phenomenon. Cutaneous manifestations of CLL as generalized pruritis vasculitis, paraneoplastic pemphigus, erythema nodosum, erythroderma, sweet's syndrome and insect bite like reactions. A less commonly recognized subtype of EF described in hematological malignancies, in particular leukaemia's and non-Hodgkin lymphomas. Here we are reporting two such patient presented to our institute.

Keywords: CLL, Eosinophilicfolliculitis, Chemotherapy.

Patients/Materials \& Methods: Case series:

Patient 1: 60 years old gentleman diagnosed CLL Stage A Binet in Dec2013 progressed to stage C Binet in August 2018, was initiated on Bendamustin and Rituximab chemoimmunotherapy, after completion of $6^{\text {th }}$ cycles in Feb 2019, he developed urticarial papules on forehead and face initially, recurring later on arms and legs. Biopsy of skin lesion was suggestive of eosinophilic follulitis exaggerated insect bite response.

Patient 2: A 73 years old gentleman diagnosed as CLL, was initiated on bendamustin and rituximab-based chemo-immunotherapy after $2^{\text {nd }}$ cycle of chemotherapy 3 weeks later he developed eosinophilia accompanied by pruritus without dermatological manifestations, within 2 weeks after this event he developed erythematous papules over the trunk, limbs and face with intense itching. A skin biopsy was done suggestive of eosinophilic folliculitis.

Results: Patient-1, was treated with topical steroids initially later inview of poor response and recurring lesion was started on topical steroids, Indomethasone, Mometasone F cream and Atarax, he also received a dose of IvIG at $400 \mathrm{mg} / \mathrm{kg}$.

Patient-2, was treated with topical steroids, Ivermectin and Albendazole.

Discussion \& Conclusion: The EF most commonly seen in CLL among the all hematological malignancies, difficult to differentiate from many other clinical dermatosis and difficult to treat hence multidisciplinary approach and skin biopsy will give us clue.

Disclosure of Interest: None Declared.

Keywords: chronic lymphocytic leukemia, dermatopathy, Eosinophilic folliculitis.

\section{Abstract 6. Lenalidomide Maintenance an Effective Strategy in CLL: Outcomes of a Randomized Controlled Trial}

Nishant Jindal $^{* 1}$, Man Updesh Singh Sachdeva ${ }^{2}$, ArihantJain ${ }^{1}$, Gaurav Prakash $^{1}$, Alka Khadwal ${ }^{1}$, SreejeshSreedharanunni ${ }^{2}$, Neelam Varma ${ }^{2}$, Pankaj Malhotra', Deepesh Lad ${ }^{1}$

${ }^{1}$ Department of Internal Medicine, ${ }^{2}$ Department of Hematology, Postgraduate Institute of Medical Education and Research, Chandigarh, India

Aims \& Objectives: Chemo-immunotherapy (CIT) continues to be an acceptable treatment option in CLL without deletion 17. Patients with early progression $(<2$ years) have poor response to second line chemoimmunotherapy and a reduced PFS with every subsequent relapse. In developed countries, novel agents like ibrutinib and venetoclax are the standard of care for relapsed CLL and are finding approval as part of frontline therapy as well. In middle to low SDI countries, these drugs cost 4 to 40 times the per capita GDP and are unlikely to be affordable to a majority in near future. Therefore, strategies to prolong PFS and the time to next treatment are still relevant in these settings. Lenalidomide has shown promise as maintenance treatment in CLL in two large randomized trials (CLLM1 and CALGB). In this randomized trial, we evaluated the efficacy and safety of individualized, low dose, fixed duration, lenalidomide maintenance versus observation in non- deletion 17 CLL.

Patients/Materials \& Methods: The study period was from January 2018 to June 2019. CLL patients who had achieved at least partial remission (iwCLL response criteria) after frontline CIT were included in the study. Patients with deletion 17 or mutated TP53 were excluded. Baseline and follow-up bone marrow MRD assessment was done using 8-color flow cytometry with a detection threshold of 10-4. MRD was classified into three groups; nil: $<10-4$, intermediate: 10-4 to 10-2 and high: $>10-2$. Patients were randomly assigned to lenalidomide maintenance or observation. Lenalidomide was started at $5 \mathrm{mg}$ every alternate day and escalated to a maximum dose of $10 \mathrm{mg}$ daily in a 20/28 day schedule as per patient tolerance. Maintenance therapy was given for a fixed duration of 6 cycles. PFS was defined as the time from study recruitment to progression or death due to any cause. Haematological toxicity was graded using the iwCLL guidelines for grading of toxicity and other adverse events were graded according to the Common Terminology Criteria for Adverse Events version 5.0 (CTCAE).

Results: A total of 32 patients were included in the study and randomized to lenalidomide maintenance $(n=19)$ or observation $(n=13)$. The two arms were matched for cytogenetics, comorbidity burden, disease status and MRD at recruitment. At a median follow up of 10.5 months, median PFS was not reached for either arm with no significant difference between the two arms $(p=0.338)$. High MRD at the end of treatment was associated with an increased risk of early progression $(p=0.165)$. Lenalidomide showed PFS benefit in the high MRD subgroup (median PFS 7 months vs. not reached), with a trend towards statistical significance $(p=0.067)$ (see figure). Skin toxicity was the most common adverse event and was more common in the lenalidomide arm $(63.2 \%)$. Grade 3 neutropenia was reported in only $5.3 \%$ patients in the lenalidomide arm. Incidence of grade 3 or more severe infections was not different between the two arms.

Discussion \& Conclusion: Maintenance therapy with 6 cycles of dose-escalated lenalidomide is safe and may afford PFS benefit in patients with non- deletion 17 CLL and high MRD at the end of frontline chemo-immunotherapy. The CLLM1 and the CALGB trials had a fixed ramp up dosing schedule of lenalidomide and reported more adverse events ( $38 \%$ and $71 \%$ grade $3 / 4$ neutropenia) compared to our individualized, low dose lenalidomide protocol. The encouraging results of this study warrant further investigation into the role of lenalidomide maintenance in CLL in larger trials, especially in low and middle SDI setting where access to novel agents is limited and is going to remain so in the foreseeable future.

Supporting Document: dbda8a5f-9acf-42b8-8383-5a77d0c0442c. 


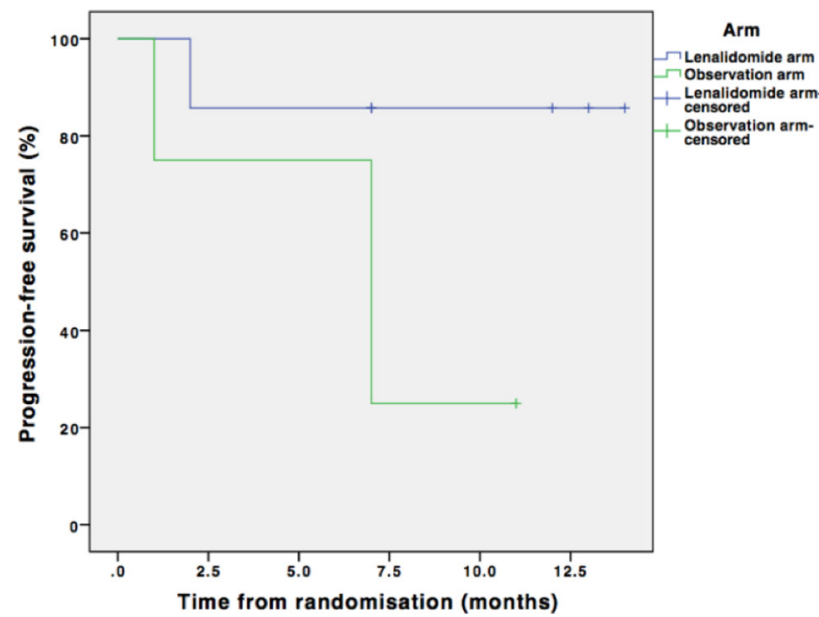

Disclosure of Interest: None Declared.

Keywords: CLL, Lenalidomide, Maintenance.

\section{Abstract 7. Hand Foot Syndrome: An Uncommon Adverse Effect of a Common Drug}

\section{Ashwini Narayankar ${ }^{* 1}$, Madhuri Gadekar ${ }^{1}$, RashmitaNevagi ${ }^{1}$, AvinashChalke ${ }^{1}$, VrindaKulkarni ${ }^{1}$ \\ ${ }^{1}$ Clinical hematology, NAIR HOSPITAL, MUMBAI, India}

Aims \& Objectives: To review rare dermatologic toxicity due to Imatinib and general principles of management.

Patients/Materials \& Methods: 50 year old female case of chronic myeloid leukaemia-Accelerated phase on tablet Imatinib $600 \mathrm{mg}$ per day developed red raised lesions on bilateral hands and feet associated with pain and burning sensations. Also associated with conjunctival redness. Diagnosed clinically as Hand foot syndrome. Skin biopsy was done which was consistent with interstitial granuloma annulare. Discontinuation of Imatinib alongwith antihistaminic, local steroids and wax paraffin application was advised. Follow up with RT-PCR for BCR-ABL1 advised to know cytogenetic response as drug discontinuation was done.

Results: Imatinib mesylate was discontinued for 2 weeks, and the patient showed improvement. Patient was restarted on the same dose but due to poor intolerance in the form of vomiting and redness of eyes dose reduction was done.

Discussion \& Conclusion: The cutaneous adverse reactions other than maculo-papular eruptions are rare with imatinib. Severe and lifethreatening cutaneous reactions can occur in 5\% cases. Hand-foot syndrome (HFS), synonymous with hand-foot skin reaction and palmar-plantar erythrodysesthesia is the least known cutaneous adverse effect of Imatinib. In a literature review lower incidence of skin rash $(11 \%$ and $15 \%)$ was found in patients with blast crisis (myeloid and lymphoid, respectively), compared with $22 \%$ and $27 \%$ of the patients with accelerated and chronic phases of CML. Skin rash usually disappears after discontinuation of Imatinib. Recognition of cutaneous toxicities related with Imatinib is very important as it can be a dose limiting adverse effect or may necessitate the discontinuation of implicated agent altogether in severe cases.

Disclosure of Interest: None Declared.

Keywords: adverse event, Hand foot syndrome, Imatinib.

\section{Abstract 8. Treatment Free Remission in CML with Generic Imatinib}

Deepak Goni $^{* 1}$, ArihantJain', Deepesh lad ${ }^{1}$, Gaurav prakash ${ }^{1}$, Alka khadwal $^{1}$, Shanonaseem ${ }^{2}$, Neelam varma ${ }^{2}$, Pankaj malhotra $^{1}$

${ }^{1}$ Internal Medicine, ${ }^{2}$ Haematology, PGIMER, Chandigarh, India

Aims \& Objectives: To study the outcome of treatment free remission (TFR) in patients with CML-CP who are on generic forms of Imatinib in sustained deep molecular response.

Patients/Materials \& Methods: In a prospective single centre study, CML-CP patients who are on generic Imatinib for $>3$ years duration and in $\mathrm{MR}^{4}$ for $>2$ years were recruited from adult haematology clinic PGIMER Chandigarh. After informed consent Imatinib was stopped and patients were followed up with monthly CBC and RQPCR for BCR-ABL from peripheral blood. Imatinib was restarted if there was a loss of MMR at any single point of time.

Results: Since January 2017 a total of 24 patients (11 females, 13 males) were enrolled. Twenty patients were on the same form of generic Imatinib Veenat ${ }^{\mathrm{TM}}$ and 4 were on other variable generic brands. After a median follow-up of 15 months (range, 1-29 months) out of 24, 14 patients had to be restarted on Imatinib due to loss of MMR within a median of 3 months (range, 3-21 months) of treatment discontinuation. All of the 14 patients who were restarted on Imatinb had re-attained MMR within a median duration of 8 weeks (range, 4-16 weeks). Ten patients $(41 \%)$ are still in treatment free remission with a median followup of 15 months (range, 1-29 months).

Discussion \& Conclusion: The current study suggests TFR is possible with generic forms of Imatinib.

Disclosure of Interest: None Declared.

Keywords: generic Imatinib, India, TFR.

\section{Abstract 9. Treatment of CML-CP Patients with Nilotinib Followed by Imatinib, Compared to Upfront Imatinib}

Aditi Jain $^{* 1}$, ManoranjanMahapatra ${ }^{1}$, TulikaSeth ${ }^{2}$, Anil Irom¹, Sudha Sazawal ${ }^{3}$

${ }^{1}$ Clinical Hematology, ${ }^{2}$ Clinical Hematology, ${ }^{3}$ Hematology, AIIMS, New Delhi, India

Aims \& Objectives: To compare the molecular responses and longterm outcomes in treatment naïve CML-CP patients treated with Nilotinib for the initial 3 or 6 months followed by Imatinib compared to treatment with Imatinib alone.

Patients/Materials \& Methods: CML CP patients were divided into 3 groups at the time of daignosis. Group 1 received Imatinib $400 \mathrm{mg}$ OD upfront, Group 2 and 3 received Nilotinib $300 \mathrm{mg}$ BD for $1^{\text {st }} 3$ and 6 months respectively then switched over to Imatinib 400 OD. Quantitative real time PCR for BCR-ABL (RQ PCR for BCR-ABL) was done at $3,6,12,18,24,30$, and 36 months to assess response. 3 early molecular responses were defined for analysis. EMR 1 was defined as Bcr-abl RQPCR $<10 \%$ at 3 months, EMR 2 as $<1 \%$ at 6 months and EMR 3 as $<0.1 \%$ at 6 months.

Results: Patients on Nilotinib had deeper responses than patients on Nilotinib at 3 and 6 months. However, subsequent molecular responses were similar in all 3 groups. The rates of Early Molecular responses were significantly better in the Nilotinib group compared to the Imatinib group. Significantly lesser patients in Group 1 had EMR 1 compared to Groups 2 and 3 [80.49\% vs $100 \%$ vs $95.56 \%$, p value 0.001]. Results were similar for EMR 2 and 3 [EMR 2: $34.15 \%$ vs 
$42.11 \%$ vs $60 \%, p$ value 0.017 , EMR 3: $25.71 \%$ vs $25.53 \%$ vs $44.44 \%, p$ value Group 1 vs Group 3: 0.030]. TKI failure/progression rates were significantly better amongst patients who achieved EMR vs those who did not achieve EMR for EMR 2 and 3 but not EMR 1. For EMR 2, 42.68\% who did not achieve EMR eventually had TKI failure compared to $18.03 \%$ of those who achieved EMR ( $p$ value 0.002 ). Similarly, $32.95 \%$ who failed to achieve EMR 3 progressed vs $11.9 \%$ of those who achieved EMR 3 ( $p$ value 0.011). 3 patients in group 1 and 1 patient in group 2 developed T315I mutation while none did in Group 3.

Discussion \& Conclusion: Patients on Nilotinib upfront achieved deeper responses and EMR more than patients on upfront Imatinib. However, this early advantage was not sustained after switching to Imatinib. Nevertheless, target responses were still achieved and similar failure rates were present in all 3 groups.

Disclosure of Interest: None Declared.

Keywords: CML, Imatinib, Nilotinib.

\section{Chronic Leukemia (Laboratory)}

\section{Abstract 1. Prognostic Impact of CD4:CD8 Ratio in Patients with Chronic Lymphocytic Leukemia}

\author{
Monika Gupta ${ }^{* 1}$, Sakshi Dhaiya ${ }^{1}$, Rajeev Sen ${ }^{1}$ \\ ${ }^{1}$ Pathology, Pt BDS, PGIMS, Rotak, Rohtak, India
}

Aims \& Objectives: Chronic lymphocytic leukemia (CLL) is a lymphoproliferative disease characterized by clonal expansion of Bcells. The clinical course of CLL patients is highly variable. These patients have dysregulated immune functions due to profound quantitative and qualitative defects of $\mathrm{T}$ cells resulting in impaired antitumor immunity and increased risk for infections. Both subsets of CD4 and CD8 T cells are increased, while the CD4/CD8 ratio is reversed in some patients, mostly in advanced Rai stages. However, the precise mechanisms that attribute to this immunosuppressive phenotype in CLL in not understood.

We analysed the T-cell subset (CD4:CD8 ratio) and correlated with the Rai staging.

Patients/Materials \& Methods: Immunophenotyping was performed in 50 new cases of CLL on 8 Colour Flow cytometer BD FACS Canto II using CD19/SSC gating. The CD panel used was CD45, CD19, CD20, CD23, CD5, CD200, CD38, CD4, CD8, CD3, FMC7, CD10, CD79b, Kappa, Lambda. The Rai classification for the estimation of clinical stages was used. Clinical characteristics of patients including age, sex, and complete hemogram were performed.

Results: There was slight male predominance with male to female ratio of 1.6:1. Average age of the patients was 60.8 years (range $43-85$ years). The largest numbers of patients were in Rai stage III and IV $(n=26)$. The mean helper to suppressor ratio $(C D 4 / C D 8)$ was normal in 11, equal in 23 and reversed in 16 patients. 24 patients with equal or reversed CD4:CD8 ratio were in Rai stage III/IV due to a decrease of the absolute number of CD4+ cells.

Discussion \& Conclusion: This study provides an attempt to correlate simple immune function parameters and clinical status in this entity. CD4/CD8 ratio was decreased in advanced form of CLL. The therapeutic modulation of $\mathrm{T}$ cell response in these patients may play an important role in the disease behaviour and may be a key event compensating the immunodeficiency characteristic for the advanced stages of the disease.

Disclosure of Interest: None Declared.

Keywords: CD4/CD8, Chronic Lymphocytic leukaemia, flowcytometry.

\section{Abstract 2. Role of Flow Cytometry in Chronic Lymphoproliferative Disorders}

\author{
Anita Tahlan ${ }^{1}$, NayanKoul ${ }^{* 1}$, AnshuPalta ${ }^{1}$, Sanjay D Cruz ${ }^{2}$ \\ ${ }^{1}$ Pathology, Gmch, Chandigarh, ${ }^{2}$ Medicine, Gmch, Chandigarh, \\ India
}

Aims \& Objectives: To analyse the profile of B and T cell markers in patients with treatment naive CLPD and to subcategorize CLPD using flow cytometry and to correlate flow cytometric antigenic expression in FNA and bone marrow infiltration.

Patients/Materials \& Methods: Thirty cases of CLPD diagnosed on FNA and/or peripheral blood/bone marrow were subcategorized using flow cytometry.FNA was done on patients with palpable lymphadenopathy diagnosed as CLPD/NHL and BM staging was done. Flow cytometric evaluation was done on FNA and BM/peripheral blood (PB) material.

Results: Out of 30 CLPD cases, a frequency of $80 \%$ B-CLPD and $20 \%$ of T- CLPD was found. Half of the cases were grouped in CD5+ CD10-CLPD followed by CD5+ CD10+ CLPD in 25\% and CD5- CD10+ CLPD in 25\% cases. Final diagnosis of 30 cases were 15cases were CLL, 6 cases were DLBCL, 4 cases of SLL, 1 case of SMLZ, 1 case of FL and 3 cases of T-CLPD. Bone marrow infiltration was noted in $20 \%$ of CLPD cases. In one of the case (3.33\%) marrow infiltration was noted by FC which was undetected on morphological examination of bone marrow aspirate and trephine biopsy.

Discussion \& Conclusion: FC helps to subcategorise the CLPD and this differentiation is important as the treatment and prognosis differs in the two subcategories. Staging of the disease is conventionally done by bone marrow examination which further predicts the treatment and prognosis. We also conclude that if flow is added as an ancillary investigation to morphology we would add to staging, which would be missed by conventional morphological examination.

Disclosure of Interest: None Declared.

Keywords: CLL, clpd, NHL.

\section{Abstract 3. Concurrent Mature B Cell Neoplasm with Prostatic Adenocarcinoma: An Unusual Case Report}

\author{
Seema Sharma ${ }^{* 1}$, Nandita Chaudhary ${ }^{1}$ \\ ${ }^{1}$ Pathology, SGPGIMS, Raibareli Road, Lucknow, Lucknow, \\ India
}

Aims \& Objectives: The concurrent presence of adenocarcinoma of the prostate with mature B cell neoplasm is rather unusual. We hereby report a case of synchronous mature B cell neoplasm and high grade prostatic adenocarcinoma in an elderly patient.

Patients/Materials \& Methods: A 65 year male presented with complaints of lower urinary tract symptoms including dysuria and increased frequency of micturition for five years, for which he was on alpha blocker therapy. Patient a chronic cigarette smoker was a diagnosed case of COPD since past 3 years. There was no superficial lymphadenopathy or organomegaly.

Results: On investigations serum PSA (63.6 ng/ml) and TLC (30x 10 ${ }^{9} / \mathrm{L}$ ) were found to be raised. Peripheral blood smear showed marked lymphocytosis (60-74\%) and the lymphocytes were small sized with scanty cytoplasm, round to indented nuclei, condensed chromatin and inconspicuous nucleoli.

MRI- prostate showed enlarged prostate and diagnosis of prostatic adenocarcinoma, Gleason score $5+5$, grade group $\mathrm{V}$ was made on transrectal ultrasound guided prostate biopsy. 
Flow cytometry on bone marrow aspirate sample showed B lineage and expressed CD 19, CD20, CD23, CD200 and CD22 and were negative for CD5, CD10, CD103, CD123, CD11c, CD25 and FMC-7. In view of the morphological findings combined with flow cytometry results, a diagnosis of CD5-ve CLL, was favored.

Patient was treated for prostatic cancer and his urinary tract symptoms improved and serum PSA level was normalized at three months. For B cell neoplasm being in early stage close monitoring was advised. The patient is in follow-up for past 2 years with periodical PSA evaluation and complete blood count.

Discussion \& Conclusion: The simultaneous evidence of an adenocarcinoma prostate and mature B cell neoplasm is rather unusual. A very few case reports on synchronous solid tumors and hematologic malignancies are available on literature search.

Disclosure of Interest: None Declared.

Keywords: Concurrent, Mature B Cell neoplasm, Prostatic adenocarcinoma.

\section{Abstract 4. Role of Flow Cytometry in CML Blast Crisis: Essentiality Can not be Negated}

\section{VindhyaprabhaHegde $^{* 1}$, Sarika Singh ${ }^{1}$, Pallavi Sinha ${ }^{1}$ \\ ${ }^{1}$ Pathology, Maulana Azad Medical College, New Delhi, India}

Aims \& Objectives: To evaluate the immunophenotypic nature of blasts in CML blast crisis.

Patients/Materials \& Methods: A retrospective study was conducted in the department of pathology, Maulana Azad Medical College. Cases of CML in Blast crisis was done over a time period of 5 years (2014 to 2019) were subjected for multiparametric flow cytometry. Data of CML BC was compiled and analyzed for the immunophenotypic categorization of blasts. The antibody panel comprised of CD19, CD20, CD10, Ccd79a, CD3, CD4, CD7, CD8, HLADR, CD34, CD117, CD56, CD33, CD14, CD38, CD41, CD123, CD16, CD64, and CD11c.

Results: 20 cases of CML BC were analyzed retrospectively. Mean age of presentation was 39.84 years. Male to female ratio 1:1.5. Blast count ranged from $20-92 \%$, TLC from $6630-5,14,620$, platelet count from 6000-19.31 lakhs. 5 cases showed circulating micromegakaryocytes ranging from 2-9/100 WBC. Out of 20 cases 5 (25\%) showed blasts of myeloid lineage, $8(40 \%)$ myelomonocytic, 4 (20\%) B lymphoid and $3(15 \%)$ showed mixed lineage $\mathrm{Bc}$ with myelomonocytic and megakaryocytic differentiation.

Discussion \& Conclusion: Discussion: Chronic myeloid leukemia (CML) is triphasic. Patients with CML might progress to blast crisis (BC) after undergoing through chronic phase and accelerated phase. Blast lineage in blast crisis of CML is heterogeneous and may show antigens of more than 1 lineage. Hence, it is necessary to evaluate the immunophenotypic nature of blasts for further correct management of patient, as prognosis and treatment is dependent on it.

Conclusion: Immunophenotyping is an important and mandatory adjunct in cases of CML BC including accelerated phase as the lymphoid and mixed phenotypic blast lineages are harbinger of poor prognosis and treatment response and they need to be triaged.

Disclosure of Interest: None Declared.

Keywords: CML blast crisis, Flow cytometry in CML blast crisis, immunophenotyping in CML blast crisis.

\section{Abstract 5. Flow Cytometry Evaluation of B Chronic Lymphoproliferative Disorders}

Jyoti Sawhney ${ }^{* 1}$, Birwa Rahiya ${ }^{2}$, BirenParikh ${ }^{1}$, BeenaBrahmbhatt $^{1}$, HemanginiVora $^{2}$, PritiTrivedi $^{1}$ ${ }^{1}$ Pathology, ${ }^{2}$ Flow cytometry Division, Gujarat Cancer And
Research Institute, Ahmedabad, India

Aims \& Objectives: In this study we evaluated the role of CD200 and CD43 new markers that have been introduced in the Euroflow panel in the separation between chronic lymphocytic leukemia (CLL) and all other mature B cell malignancies.These markers were also correlated with the markers used in the Matutes score like FMC7 and Surface membrane Immunoglobulin.

Patients/Materials \& Methods: Patient samples between October 2017-July 2019 were studied prospectively, peripheral blood or bone marrow aspirates of patients with suspected B-cell lymphoproliferative disorders were subjected to evaluation by flow cytometry. Immunophenotyping of peripheral blood or bone marrow spirate was subjected to erythrocyte lysis by ammonium chloride. After washing, samples were stained by antibodies targeting the antigens CD45, CD19, CD5, CD10, CD20, CD23, CD43, CD79b, CD200, FMC7, CD25, CD103, CD11c sIgM, kappa and lambda. Corresponding isotype controls were used. Immunophenotyping was performed using a BD FACS Canto flow cytometer (Lymphocytes were selected by gating on CD45high/sideways scatter (SSC)low events. All subsequent analyses were then performed on CD19+ B cells. The modified Matutes score was calculated with positivity defined as $\geq 20 \%$ positive cells. Mean Fluorescence Intensity (MFI) ratio (MFI sample/ MFI isotype) was calculated as a measure of expression intensity.

Results: There were a total of 170 cases of B- CLPD that were analysed by flow cytometry. Mean age was 65 years. There were 122 males and 48 female patients. There were 126 cases of typical CLL, 6 cases of atypical CLL, 5 cases of Mantle cell lymphoma (MCL), 6 cases of Follicular lymphoma (FCL), 9 cases of Splenic lymphoma with villous lymphocytes (SLVL), 6 cases of Hairy cell leukemia (HCL), 10 cases of unclassifiable B-NHL and 2 cases of lymphoplasmacytic lymphoma. All our cases of typical CLL showed bright expression of CD200. This marker was also brightly expressed in all our atypical cases of CLL. CD43 was also brightly positive in all our cases of typical and atypical CLL. The expression of CD200 negativity along with expression of CD43 was helpful in diagnosis of MCL. CD200 negativity was not exclusively seen in Mantle cell lymphoma. It was also seen in two of our cases of hairy cell leukemia variant However we faced diagnostic difficulties in differentiating atypical CLL from B-NHL unclassifiable. The lack or dim expression FMC7 expression along with absent or dim expression of SIgM were useful in differentiating atypical CLL from B-NHL unclassifiable.

Discussion \& Conclusion: The Euroflow panel is helpful in the distinction of chronic lymphocytic leukemia from Mantle cell leukemia. However CD200 negativity should be analysed in the background of clinical details, peripheral blood morphology and lymph node biopsy. To distinguish cases of atypical CLL from B-NHL unclassifiable, markers that are evaluated in Matutes score are useful and can be used as a secondary panel.

Disclosure of Interest: None Declared.

Keywords: CD200, CD43, CLPD. 


\section{Abstract 6. Genomic Landscape of Chronic Myelomonocytic Leukemia}

Tanmay Wairkar $^{* 1}$, ShrinidhiNathany ${ }^{1}$, ShriyaVaidya ${ }^{1}$, Prasanna Bhanshe $^{1}$, PapagudiSubramanian ${ }^{1}$, Nikhil Patkar ${ }^{1}$

${ }^{1}$ Hematopathology, Tata Memorial Hospital, Navi Mumbai, India

Aims \& Objectives: Chronic myelomocyticleukemia (CMML) is a clonal haematopoietic malignancy with features of both myeloproliferative neoplasm and myelodysplastic syndrome. It is mainly characterised by peripheral blood monocytosis, $B C R-A B L 1$ negative, and blasts $<20 \%$ in blood/bone marrow. We aimed to study the genomic landscape of CMML patients by comprehensively evaluating the mutational profile using high through-put next generation sequencing (NGS) which will help us in identifying therapeutically targetable mutations.

Patients/Materials \& Methods: This was a retrospective study. Patients whose requisition for NGS was raised in Tata memorial centre from June 2018 to May 2019 were enrolled in the study. Clinical and cytogenetics testing data were retrieved from the electronic database. DNA extracted from blood/bone marrow samples using Qiagen Gentra-Pure gene method was used as template. For mutational screening we developed a 51 gene $(151.5 \mathrm{kB})$ low-cost hybrid capture based targeted sequencing myeloid panel involving single molecule molecular inversion probes implicated in myeloid malignancies. All samples were sequenced at high coverage using this assay on an Illumina Miseq and data was analysed using custom pipeline.

Results: Total of 18 patients were included with median age at diagnosis of 53.5 years (range 20-70 years). Among the study cohort, mutation was identified in 16 cases. The most frequent mutation was found in the RUNX1 gene (n-6, 33\%) (Figure 1). This was followed by mutations in the $C B L(\mathrm{n}-4,22 \%), K R A S(\mathrm{n}-4$, $22 \%)$, and $J A K 2(\mathrm{n}-3,17 \%)$ gene. Interestingly, $68.75 \%(\mathrm{n}-11)$ of cases had more than one mutation. Two $(11.11 \%)$ cases showed coexistence of RUNX1,CBL and CSF3R gene mutation. Less frequently mutated gene that we found were NPM1, CUX1, ZRSR2, PTPN11, KIT and DNMT3A.

Discussion \& Conclusion: In our study, $88.89 \%$ of CMML cases harbour at least one mutation. Mutation in the $R U N X 1$ gene was most frequent in our cohort unlike the Western studies where mutation in the ASXL1 gene is most common. This may indicate that the genetic profile of CMML cases in our ethnic group may differ compared to the Western literature. NGS will help to identity the presence of mutations that can be therapeutically targetable as well help in predicting prognosis.

Supporting Document: d0ea35e7-1207-4e7c-b106-9d0052573aec.

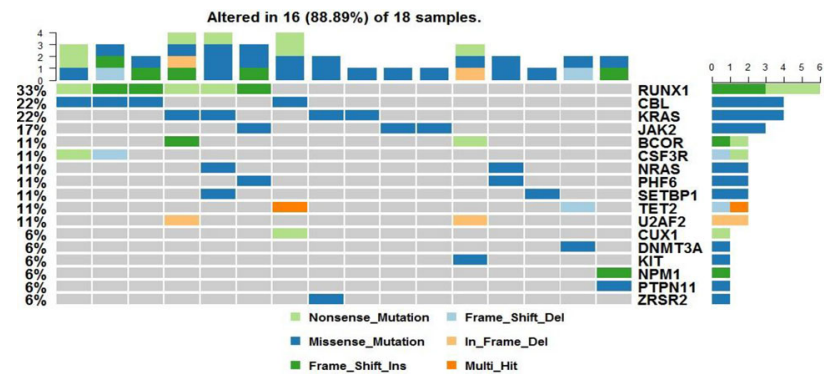

Disclosure of Interest: None Declared.

Keywords: Chronic myelomocyticleukemia, Next generation sequencing, RUNX1 gene.
Abstract 7. Binucleated \& Trinucleated Lymphocytes in Chronic B-Lymphoproliferative Disorder- A Rare Case Report Anirban Kundu ${ }^{* 1}$, Gayatri Behera $^{1}$, Gaurav Chhabra
Ashutosh Panigrahi

${ }^{1}$ Pathology, ${ }^{2}$ Haematology, AIIMS, Bhubaneswar, Bhubaneswar, India

Aims \& Objectives: To describe the presence of binucleate, trinucleate and quadrinucleate lymphocytes in a case of chronic lymphocytic leukemia (CLL) and to interpret the clinical significance of this morphological variation in CLL on the disease progression. and inguinal lymphnodes.

Patients/Materials \& Methods: A 45-year-old male presented with fever for 15 days, cough, blood in stool \& multiple tender erythematous nodules on palms for 10 days. CECT abdomen showed hepatosplenomegaly with enlarged retroperitoneal and inguinal lymphnodes. He was a diagnosed case of CLL, Rai stage II with complete remission after completion of $\mathrm{BR}$ regimen 2 years back. The immuneprofile was $\mathrm{CD} 20+, \mathrm{CD} 23+, \mathrm{BCl} 2+, \mathrm{CD} 3-, \mathrm{CD} 10-$, Cyclin D1- with $28 \%$ cells showing heterologous $13 \mathrm{q}$ deletion, $25 \%$ cells showing trisomy 12 and absence of TP53 deletion on FISH study.

Results: On investigation, CBC revealed leukocytosis (WBC count$120.95 \times 103 / \mathrm{cmm})$, normal platelet and RBC count indicating relapse of the disease after more than one year of achieving remission status. PS revealed lymphocytosis with $84 \%$ abnormal lymphocytes and $12 \%$ mature lymphocytes. Smudge cells- 50-60/100 lymphoid cells. The morphology of abnormal lymphocytes showed bi-nucleation (64\%), tri-nucleation (26\%) and quadri-nucleation (10\%).

Discussion \& Conclusion: CLL is a common lymphoproliferative disorder in adults with absolute lymphocyte count $>5 \times 10^{9} / \mathrm{cmm}$. The leukemic cells are monoclonal, small and mature appearing lymphocytes with regular nuclear contour, scant peripheral basophilic cytoplasm. In our patient, the leukemic cells are small, mature predominantly comprising of binucleate and trinucleate lymphocytes. Binucleated lymphocytes are previously described in "Persistent polyclonal B-cell lymphocytosis (PPBL)" with an isochromosome for the long arm of chromosome 3, $+\mathrm{i}(3 \mathrm{q})$ which is absent in our case. Only four studies have been performed regarding the presence of bi-nucleated and tri-nucleated lymphocytes in CLL. However, this is the index case showing quadri-nucleated lymphocytes in CLL. Mechanism of this morphological subtype of CLL is not described anywhere nor its prognostic value on the disease.

Bi-nucleated, tri-nucleated and quadri-nucleated lymphocytes in B-cell CLL is extremely rare. The etiopathogenesis of this morphological feature is enigmatic. This should be distinguished from other differentials i.e. PPBL. Clinical significance of this morphological alteration needs to be reported to see impact on cytogenetics and on the disease course.

Disclosure of Interest: None Declared.

Keywords: Binucleated lymphocytes, CLL, Trinucleated lymphocytes.

\section{Abstract 8. Immunophenotypic Characterisation of Blast Crisis in Chronic Myeloid Leukemia}

\author{
Shivani Singhal ${ }^{* 1}$, Monika Gupta ${ }^{2}$, Sunita Singh ${ }^{3}$, \\ Lovekeshmonga ${ }^{3}$, Rajeev sen $^{3}$
}

${ }^{1}$ pathology, Pt B.D. Sharma, PGIMS, ${ }^{2}$ Pathology, Pt B.D. Sharma, PGIMS, ${ }^{3}$ Pathology, Pt. B.D. Sharma, PGIMS, Rohtak, India

Aims \& Objectives: Chronic myeloid leukemia (CML) is a clonal stem cell disorder that is hallmarked by the presence of a $t(9 ; 22)$, also 
known as the Philadelphia chromosome. The natural history of CML is typically triphasic: an initial indolent chronic phase, followed by an accelerated phase and usually a terminal, highly aggressive blast phase. Immunophenotypic studies have a limited role in the diagnosis of CML but are increasingly being used in CML blast transformation. It is most helpful to document lineage and aberrant immunophenotype in a blast crisis. Determination of the cell lineage of CML blasts is clinically important for a better response to chemotherapy and longer survival. With improvement in management protocols, many patients of CML-BC are expected to show remission after initial chemotherapy and a better survival. Hence, it becomes imperative to correctly classify the nature of BC for further course of management.

Patients/Materials \& Methods: Bone marrow or peripheral blood smears from 15 CML-BC patients during the years 2014 to 2019 were studied by morphological and cytochemical stain analysis. Lineage of blasts were determined in each case depending upon the expression of markers by comprehensive immunophenotypic analysis performed on 8 Color Flowcytometer BD FACS Canto II using 19 antibody panel. Results: Out of 15 cases of CML-BC, male to female ratio was 2:1 and a median age was 38.3 yrs. Blast percentage ranged from $16 \%$ to $90 \%$. Flowcytometry revealed 8 cases were of lymphoid BC and 7 cases were of myeloid BC. Out of 7 cases of myeloid BC, 2 cases showed monocytic differentiation. Aberrant myeloid antigen expression was common in cases with lymphoid BC while CD7 was the most common aberrant antigen seen in myeloid BC.

Discussion \& Conclusion: Immunophenotyping is important in distinguishing between a myeloid and lymphoid blast crisis, thus providing clinically useful information for the further treatment protocols and prognosis.

Disclosure of Interest: None Declared.

Keywords: blast crisis, Chronic Myeloid Leukemia, Immunophenotyping.

\section{Abstract 9. Genomic Landscape of Juvenile Myelomonocytic Leukemia (JMML): A Real World Context}

\author{
ShrinidhiNathany $^{* 1}$, Shruti Ghai ${ }^{1}$, Gaurav Chatterjee ${ }^{1}$, \\ Gaurav Narula ${ }^{2}$, Prashant Tembhare ${ }^{1}$, PapagudiSubramanian', \\ Sumeet Gujral $^{1}$, Shripad Banavali ${ }^{3}$, Nikhil Patkar ${ }^{1}$

\section{${ }^{1}$ Hematopathology, ACTREC, Tata memorial centre, Navi Mumbai, ${ }^{2}$ Pediatric Oncology, ${ }^{3}$ Medical Oncology, Tata memorial hospital, Mumbai, India}

Aims \& Objectives: Juvenile myelomonocytic leukaemia (JMML) is a clonal haematopoietic disorder occurring in pediatric age group and is characterised by aberrant signal transduction of the RAS signalling pathways (NF1, NRAS, KRAS, PTPN11 and CBL). Recent highthroughput studies have started to push the horizon of JMML associated mutations wider and have proposed molecular-based risk algorithms. Our study was carried out to determine the genomic landscape of JMML in 50 patients who were diagnosed at our centre. Patients/Materials \& Methods: We developed a 51 gene low-cost targeted sequencing myeloid panel based on single molecule molecular inversion probes. This focussed panel interrogated sets of genes implicated in pathogenesis of myeloid malignancies. A total of 50 children with clinical and pathological features of JMML were sequenced at high coverage using this assay on an Illumina MiSeq. Results: The median age of our cohort was 2 years, with a male preponderance. Among the 50 patients, 43 (86\%) harboured mutations in one of the RAS/MAPK pathway genes. The most frequently mutated gene was PTPN11 (14, 28\%), and NRAS $(14,28 \%)$, followed by NF1 mutation in $22 \%$ (11) cases (Figure 1). Interestingly, 20\% (10) of children $>1$ mutation, with 5 cases harbouring two RAS pathway mutations. Other non-RAS pathway mutations involved ABL1, ASXL1, ATRX, SETBP1, SH2B3 and ZRSR2 genes. The median variant allele frequency of RAS pathway mutations was $40.75 \%$. Monosomy 7 was detected in $32 \%$ (16) patients. Follow-up data revealed that 37 (74\%) patients have succumbed to the disease. The median time period from diagnosis to death was 7.2 months. Patients with PTPN11 mutations showed a trend to shorter overall survival, compared to their wildtype counterparts ( $p=0.7$, median OS 10.6 months vs 38.1 months). Patients harbouring more than one mutation also showed a trend to shorter OS. Discussion \& Conclusion: Our study represents the largest cohort of JMML from a single centre in the Indian subcontinent. This study depicted that almost $90 \%$ cases of JMML harbor at least one mutation with $86 \%$ harbouring at least one RAS pathway mutation. Presence of PTPN11 mutations and co-existence of more than one mutation may be the major determinants of outcome in JMML.

Disclosure of Interest: None Declared.

Keywords: JMML, PTPN11, RAS.

\section{Abstract 10. Basophilic Blast Phase of CML}

\author{
Soma Pradhan $^{\text {*1 }}$, JasmitaDass ${ }^{1}$, Jyoti Kotwal ${ }^{1}$, Amrita Saraf ${ }^{1}$ \\ ${ }^{1}$ Hematology, Sir Ganga Ram Hospital, New Delhi, New Delhi, \\ India
}

Aims \& Objectives: To describe a rare morphological finding in a case of Chronic Myeloid Leukemia.

Patients/Materials \& Methods: The peripheral blood examination, bone marrow examination and immunophenotyping of a patient of Chronic Myeloid Leukemia.

Results: CBC showed leucocytosis with $69 \%$ blasts with prominent basophilic granules on peripheral blood, basophilia and schistocytes. BM showed presence of $67 \%$ blasts which were large with basophilic granules in cytoplasm, round nuclei with nuclear convolutions, fine to coarse clumped chromatin. On immunophenotyping, blasts were dim CD34, bright CD117, HLA-DR, dim CD11b, dim CD33, CD 123, CD38, dim CD 22, CD25, bright CD7 and bright CD9 positive and lack expression of MPO, cCD3, cCD79a. Bone marrow biopsy showed diffuse marrow fibrosis, distorted marrow architecture with cellular areas comprised of predominantly blasts.

Discussion \& Conclusion: This case is being presented because of unusual morphological presentation of Chronic Myelodleukemia in blast crisis.

Supporting Document: 63882bba-c399-4bd9-92e9-5f44faf38b21.

Disclosure of Interest: None Declared.

Keywords: basophils, CML blast crisis, MPO.

\section{Abstract 11. Prognostic Value of P2X7 1513 (A-C) and BAX G(-248)A Gene Polymorphisms in CLL Patients}

Ravi Kumar $^{\text {*1 }}$, Amit Sharma ${ }^{1}$, Kamal Kishor ${ }^{1}$, Bharat Bhushan ${ }^{2}$, Sudha Sazawal $^{1}$, ManoranjanMahapatra ${ }^{1}$, Renu Saxena ${ }^{1}$

\section{${ }^{1}$ Haematology, ${ }^{2}$ Medical Oncology, AIIMS, New Delhi, India}

Aims \& Objectives: CLL is characterized by defective apoptosis and development of drug resistance. Polymorphism in $\mathrm{P} 2 \mathrm{X} 7$ receptor gene [1513 (A-C)] results into non-functional receptor proteins which leads to defective apoptosis, whereas polymorphism in the promoter region of the BaxG(-248)A leads to low expression of Bax protein, treatment resistance and short survival in CLL. However, several studies 
suggested contradiction in the potential role of these polymorphisms in the pathogenesis of CLL. Furthermore, scanty information is available on the prognostic value of these polymorphisms in CLL and not yet studied in Indian CLL patients. Therefore present study was designed to assessed the prevalence as well as prognostic impact of P2X7 1513 (A-C) and BaxG(-248)A gene polymorphisms in CLL.

Patients/Materials \& Methods: 100 CLL patients and 50 healthy controls were studied. P2X7 1513 (A-C) and BaxG(-248)A gene polymorphisms were evaluated by PCR-RFLP. Outcomes were analyzed by Kaplan-Meier curves for PFS and OS.

Results: The frequency of P2X7 1513 (A-C) polymorphism was found to be higher in CLL compared to controls (46 vs. 38\%, $p=0.35$ ), however it was not statistically significant. Similarly, no significant difference was observed in the frequency of BaxG(-248)A gene polymorphism between CLL patients and controls (22 vs. $24 \%$, $p=0.78$ ). Furthermore, mean PFS for the CLL patients with P2X7 1513 AC/CC genotypic was found to be higher compared to wild type P2X7 1513AA (32.3 vs. 26.0 months, $p=0.33$ ), although not significant, but showing a trend towards higher PFS in CLL patients with mutant genotype. Whereas, in case of BaxG(-248)A gene polymorphism, no significant difference was found in mean OS between Bax (-248) GA/AA and wild type GG genotype in CLL patients (62.2 vs. 59.0 months, $p=0.61$ ).

Discussion \& Conclusion: We conclude that P2X7 1513 (A-C) gene polymorphism may have role in pathogenesis due to its high prevalence in CLL patients and higher PFS in CLL patients with mutant genotype showed possibility of its prognostic role in CLL. Further, more prospective study with large sample size is required to assess the impact of P2X7 1513 (A-C) polymorphisms in Indian CLL patients. However, BaxG(-248)A gene polymorphism is neither associated with an increased susceptibility for CLL nor associated with prognosis.

Disclosure of Interest: None Declared.

Keywords: CLL, P2X7, Prognostic markers.

\section{Abstract 12. Changes in CLL Immunophenotype on Follow-up- A Tale of Two Cases}

\section{AsishRath $^{* 1}$, ManoranjanMohapatra ${ }^{1}$, Renu Saxena ${ }^{1}$}

\section{${ }^{1}$ Haematology, AIIMS, New Delhi, India}

Aims \& Objectives: Introduction: Chronic lymphocytic leukaemia/ small lymphocytic lymphoma (CL1/SLL) is a neoplasm of monomorphic small B lymphocytes in the peripheral blood (PS), bone marrow (BM), spleen and lymph nodes. CLLs are usually diagnosed on a peripheral blood/BM immunophenotyping on flow cytometry, expressing CD20, CD22, co-expression of CD5/CD19, co-expression of CD5/CD23 and light chain restriction. CD79b and FMC7 are negative or weakly expressed. CD10 is negative. However atypical phenotype with CD5 or CD23 negativity may be seen. We present here two cases of CLL in which immunophenotype have changed post chemotherapy in one and on follow-up in the other.

\section{Patients/Materials \& Methods:}

\section{Results: Case History.}

CASE-1 73 year old male presented with incidentally found high TLC (total leucocyte count) from outside. On examination mild pallor and sub-centimetric cervical lymphadenopathy was noted. On hemogram TLC was $87,000 / \mathrm{cmm}$ with $\mathrm{Hb}-9.8 \mathrm{gm} / \mathrm{dl}$ and $1.6 \mathrm{lac} / \mathrm{cmm}$ platelets. Peripheral blood immunophenotyping revealed a classical CLL immunophenotype with a matutes score 5/5. In view of significant weight loss and worsening anemia patient received BR chemotherapy. 10 years post therapy patient presented with anemia with normal TLC (62\% polymorphs) which responded to iron therapy. Bone marrow revealed $25 \%$ small lymphocytes which on immunophenotype showed loss of CD5, CD23 and gain of IgM, CD79b. Patient did not have any complaints except for fatigue and had no organomegaly or lymphadenopathy.

CASE-2 68 year old male presented with fatigue and weight loss. On investigation he found to have high TLC $(80,000 / \mathrm{cmm})$ with normal platelet counts and hemoglobin level. On peripheral blood immunophenotyping a classical CLL immunophenotype. Patient was advised for a follow-up. After one year patient present with anemia and mild splenomegaly. A repeat immunophenotyping from peripheral blood was ordered. CD5 was negative and all other markers were same as at diagnosis.

Discussion \& Conclusion: Previous studies has shown a statistically significant decrease in CD5 and CD23 expression in both treated and untreated patients on follow-up. IgM positivity in IgGV mutated CLL may indicate higher chance disease progression.

Conclusion: Changes in immunophenotype in CLL post treatment or an untreated case should be appropriately evaluated along with clinical details to avoid making new diagnosis in an already known case. Disclosure of Interest: None Declared.

Keywords: CD5, CLL, Immunophenotype.

\section{Infections and support care}

\section{Abstract 1. Utility of VCS Parameters as a Cost Effective and Sensitive Marker of Sepsis: A Hospital Based Study}

\section{Hema Goyal $^{* 1}$, Molly Joseph ${ }^{1}$}

${ }^{1}$ Pathology, St. Stephen's Hospital, Tis Hazari, New Delhi, India

Aims \& Objectives: Due to increased burden of complications and mortality due to sepsis, there is a need to evaluate and validate more accurate and early predictor of sepsis in critically ill patients. We analyzed VCS (Volume, Conductivity \& Scatter) parameters of leukocytes in sepsis patients and compared them with control group with no illness.

Patients/Materials \& Methods: It was a prospective case-control study. The patients were divided in two groups, the "sepsis group" with positive blood culture and "control group" with no illness. Total of 80 patients were studied with forty cases in each group. CBC reports were generated by Beckman Coulter LH series (LH 750 \& 780). VCS (Volume, Conductivity, Scatter) parameters for neutrophils, lymphocytes \& monocytes were compared between both groups. The results were analyzed using SPSS software (16.0 version).

Results: The age group was $20-85$ years with male predominance. The mean neutrophil volume (MNV) \& mean monocyte volume (MMV) were higher in sepsis group when compared with control group. The mean neutrophil scatter was lower in sepsis group and comparatively higher in control group. MNV values were higher in sepsis group even with low TLC. There is no change in Mean Lymphocyte VCS parameter and Mean monocyte conductivity, scatter in both the groups. ROC curves were analyzed. For predicting sepsis, with a cut off of $150, \mathrm{MNV}$ had a sensitivity of $72.5 \%$ and specificity of $70 \%$ and for MMV, setting cut off of 170 , sensitivity was $83.3 \%$ and specificity was $77.3 \%$.

Discussion \& Conclusion: MNV and MMV are found to be a sensitive and cost effective marker in prediction of sepsis. Even when TLC was low in few patients, these parameters predict sepsis accurately. This helps clinicians to assess sepsis in patients at an early stage and has an important practical implication.

Disclosure of Interest: None Declared. 
Keywords: Mean monocyte volume (MMV), Mean neutrophil volume (MNV), Volume, Conductivity, Scatter (VCS).

\section{Abstract 2. Unusual Outcome In a Post- transplant Case of Wiskot Aldrich Syndrome}

\author{
NeetiSindhwani" $^{* 1}$, Ankur Ahuja ${ }^{2}$, TathagatChatterjee ${ }^{1}$, \\ Devika Gupta ${ }^{1}$, RajanKapoor ${ }^{3}$
}

\begin{abstract}
${ }^{1}$ Dept of Lab and Molecular Medicine, ${ }^{2}$ Dept of Lab and Molecular Medicine, Army Hospital RR, Delhi, ${ }^{3}$ Dept of Hematology, Command Hospital, Kolkata, India
\end{abstract}

Aims \& Objectives: Evaluation of the cause of mortality due to unknown infection in post -transplant case of Wiskot Aldrich syndrome.

Patients/Materials \& Methods: Introduction-Free-living amoebas are environmental protozoan parasites with worldwide distribution. Four genera cause disease in humans: Naegleria, Acanthamoeba, Balamuthia, and Sappinia. All these species cause central nervous system (CNS) infections.

Material \& Methods- Study of limited autopsy Wiskot Aldrich syndrome case in post-transplant.

Results: 02 yrs old male child, was presented with pancytopenia after infection. On evaluation peripheral blood smear was found to have microplatelets with MPV of $4 \mathrm{fl}$ and hemophagocytosis and was confirmed to have Wiskott-Aldrich Syndrome by molecular methods. $\mathrm{Pt}$ was evaluated for transplant. However, the conditioning regimen was postponed as the child developed fever. The blood culture was suggestive of Burkholderia spp. Further, the deceased was managed on injectable antibiotics as per culture and sensitivity report till the patient was afebrile, active and alert. Further PBSCT was done. On day +3 of transfusion, the patient developed fever along with loose stools, for which he was managed on injectable antibiotics. Subsequently, he had an episode of seizure on day +14 preceded by fever. He was initiated on antiepileptics and other supportive measures. On evaluation patient was found to have altered sensorium with a GCS score of E3M4V2.

NCCT Head was done which revealed multiple hypodensities over parietal, frontal and temporal lobes, likely abscess/fungal. He was managed with antiepileptics, antifungals upgraded and intubated and mechanically ventilated. The condition of the patient kept deteriorating in the subsequent days. Finally, patient succumbed to death. On limited brain autopsy patient was found to have necrotic areas grossly with microscopic confirmed Acanthomoeba spp.

Discussion \& Conclusion: Amoebic encephalitis should be considered when assessing an immunocompromised host with neurological abnormalities. Establishing a timely diagnosis of Acanthomoebaand promptly initiating targeted therapy may provide the best chance to alter the clinical course of this severe CNS infection. Preventive steps like avoidance of exposure of patient with soil, use of filter tap in water taps, use of RO water for hydrotherapy and regular service of air conditioning cooling towers is required.

Supporting Document: 588ab673-1ffd-4e29-a467-678f08ebe098.

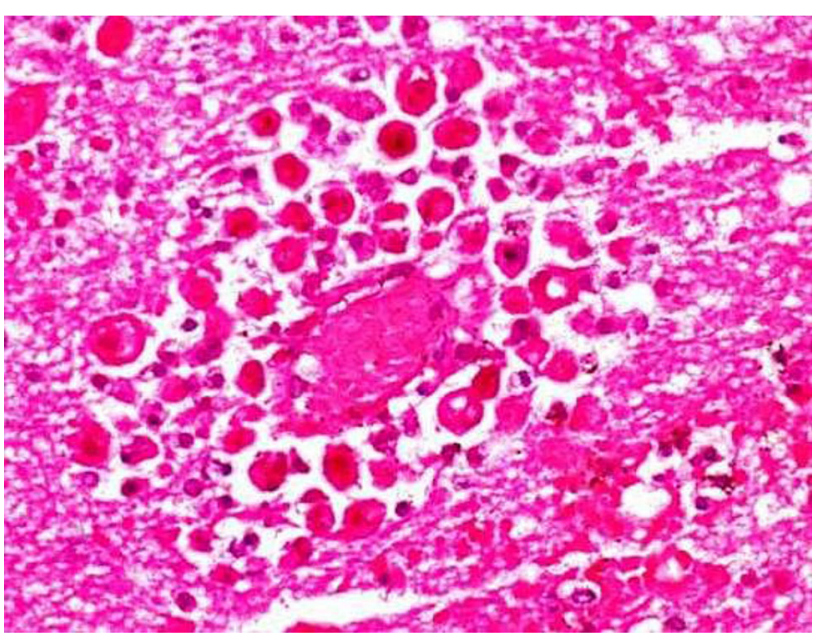

Disclosure of Interest: None Declared.

Keywords: Acanthomoeba, CNS infections post transplant, Wiskot Aldrich syndrome.

\section{Abstract 3. Variable Spectrum of HLH Presentation with Two Different Outcomes}

\author{
Ankur Ahuja ${ }^{1}$, TathagatChatterjee ${ }^{2}$, RajanKapoor ${ }^{3}$, Anish \\ Sharma $^{* 2}$, KanwaljeetSingh ${ }^{2}$
${ }^{1}$ Dept of Lab and Molecular Medicine, ${ }^{2}$ Dept of Lab and Molecular Medicine, Army Hospital RR, DELHI, ${ }^{3}$ Dept of \\ Hematology, Command Hospital, Kolkata, India
}

Aims \& Objectives: To assess the spectrum of two HLH cases reported in 2018-2019.

Patients/Materials \& Methods: Retrospectives study in 2018-2019 of primary HLH cases.

Results: Case Report 1: A 6 years old girl born of non-consanguineous marriage presented with complaints of continuous high grade fever not responding to antibiotics for past 1 month with no significant past or family history. The patient had splenomegaly along with pancytopenia. Her bone marrow revealed significant hemophagocytosis. Her entire infection spectrum was negative except for Brucella IgM which was positive. Her S. ferritin was $1363 \mathrm{ng} / \mathrm{ml}$, S.LDH was $923 \mathrm{IU} / \mathrm{ml}, \mathrm{S}$. Triglycerides were $464 \mathrm{mg} / \mathrm{dl}$.

The patient was started on antibiotics and treatment as per HLH-2004 protocol. On Day $8^{\text {th }}$ of treatment patient deteriorated all of a sudden and succumbed to her disease. Subsequently after a month her molecular profile of patient revealed homozygous missense variant in the exon 2 of STX11 gene (p.Leu135Pro). Thus the final diagnosis of primary HLH (FHL4) was given.

CASE REPORT 2: A 14 yr old boy presented with fever since 12 days associated with vomiting since 10 days and history of a single episode of GCTS seizure. The child was found to have hepatosplenomegaly with pancytopenia, hypofibrinogenemia. Blood culture showed Pseudomonas aerugnosa. His bone marrow aspirate revealed significant Hemophagocytosis along with increased serum ferritin, triglycerides. The child was started on meropenem after which the child became afebrile and his counts along with symptoms improved. His molecular profile showed variant in intron 14 of STXBP2 gene in homozygous state (FHL-4) and is awaiting Hematopoetic Stem Cell Transplant.

Discussion \& Conclusion: Hemophagocytic lymphohistiocytosis (HLH) is characterized very low survival rate of $<10 \%^{(1)}$ in patients 
who do not receive immunochemotherapy. HLH includes a spectrum of inherited or acquired defects. As of now HLH-2004 criteria serve as a definition for $\mathrm{HLH}^{(1)}$. Here we are presenting two cases reports of HLH with FHL-4 and FHL-5.

Familial Hemophagocytosis needs more study as they have varied outcomes as we observed in our cases. It should be taken in urgency due to its known fatality. Every hemophagocytic case should be sent for molecular profile to study the spectrum of various familial hemopgaocytic disorders.

\section{References.}

Supporting Document: 40766a0a-69a0-45cb-b52a-a3d97ba40fbe.

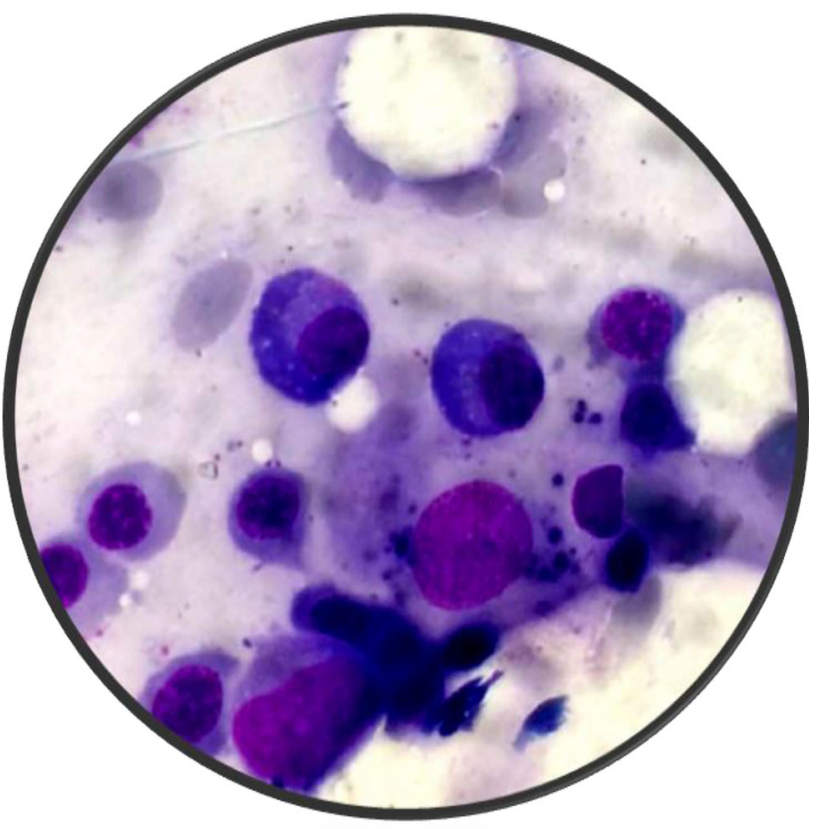

Disclosure of Interest: None Declared.

Keywords: FHL-4, FHL-5, Syntaxin mutation.

\section{Abstract 4. Diagnostic Utility of Pap Stain in Examination of Bone Marrow Aspirate: A Comparative Study}

\section{Sri Krishna Sai ${ }^{* 1}$, Prosenjit Ganguli ${ }^{1}$ \\ ${ }^{1}$ Indian Army, Command Hospital, Kolkata, India}

Aims \& Objectives: Pap stain being a universal cytology stain used for routine analysis of various body fluids. Bone marrow aspiration (BMA) study is conducted for studying cytological analysis of bone marrow. This study aim to evaluate the utility of using Pap stain in bone marrow aspirates and assess its outcomes in comparison with that of Leishman Giemsa (LG) stain.

Patients/Materials \& Methods: A total of 75 cases were included in this prospective study with adequate cellularity as inclusion criteria. Both air dried and alcohol fixed BMA slides were subjected to
Leishman Geimsa (LG) and Papanicolaou (Pap) stains in which findings such as M:E ratio, cellular changes in erythroid, myeloid, lymphoid and plasma cells series were compared between the two stains. Interclass correlation coefficient for continuous variables along with reliability and validity measurements were carried outusing SPSS v22.

Results: The diagnostic outcomes in both stains were grouped into six categories for statistical convenience. Between the two stains techniques concordance was high for reactive marrow, ALL and erythroid hyperplasia with other diagnoses showing a variable outcome. It was observed that blast cells, erythroid and neutrophil series had good correlation and agreement which was significant statistically also a good linear correlation was observed among plasma cells and eosinophil series. Pap stain showed $85.9 \%$ specificity and $89.1 \%$ negative predictive value in determining $\mathrm{M}: \mathrm{E}$ ratio, thus being more accurate in differentiating erythroid and myeloid series.

Discussion \& Conclusion: Plasma cells and eosinophils were easily identified in Pap stain with more distinct staining features. Pap stain for hematological disorders were more precise in diagnosing nonmalignant lesions and malignant lesions especially in cases like plasma cell dyscrasias, idiopathic thrombocytopenic purpura and megaloblastic anemia, where only BMA is sufficient for diagnosis. Conclusion: Most lineages showed more than good correlation and linear agreement when compared to LG stained slides also Pap stain was more accurate in differentiation of myeloid and erythroid series.Hence,Pap stain can be used as an additional stain in examination of BMA cytosmears routinely.

Disclosure of Interest: None Declared.

Keywords: Bone marrow aspirate, Diagnostic utility, Pap stain.

\section{Abstract 5. Histoplasma Detected on Peripheral Smear in Patient of Disseminated Tuberculosis}

\section{MalvikaShastri $^{* 1}$, Neha Garg ${ }^{1}$, Mrinalini Kotru' ${ }^{1}$, Meera Sikka ${ }^{1}$ \\ ${ }^{1}$ Pathology, UCMS and GTBH, Delhi, India}

Aims \& Objectives: Histoplasmosis is rare fungal disease in India, caused by Histoplasma capsulatum. Tuberculosis (TB), caused by Mycobacterium tuberculosis is fairly common in India. They cooccur mostly in immunocompromised state like HIV or immunosuppressive therapy. We report a case of disseminated TB with histoplasmosis in peripheral smear (PS) in absence of both these conditions.

Patients/Materials \& Methods: 45yo-female was referred for evaluation of COPD, massive splenomegaly \&pancytopenia. She had generalised weakness, fever on \& off for 4 months \& now presented with easy fatigability, shortness of breath and 1-episode of malaena. There was past history of pulmonary TB with treatment default. She had pallor with massive spleen palpable $8 \mathrm{~cm}$ below costal margin. $\mathrm{X}$ ray chest \& ultrasound abdomen showed findings suggestive of infective etiology. In view of above picture, disseminated TB and malignancy (CML/lymphoma) were offered as probable clinical differentials.

Results: We received venous blood sample along with bone marrow aspirate (BMA) and biopsy.CBC revealed Hb6.7 g/dl, TLC1.2 $\times 10^{9} /$ $\mathrm{L}$ and platelet count $54 \times 10^{9} / \mathrm{L}$.PS showed pancytopenia with presence of budding yeast-like organisms, $2-4 \mu \mathrm{m}$ in diameter with eccentric chromatin and clear halo. These were seen both intracellularly in neutrophils and monocytes and extracellularly and came as PAS+ve. BMA smears were normocellular-mildly hypocellular for age. Trilineage hematopoietic cells were within normal limits. Histiocytes were increased containing similar organisms as in PS.They were positive for both PAS \& SM in BMA slides. Plasma cells were 
not increased. No abnormal cells/granulomas were detected. ZN stain was negative. Biopsy also revealed similar picture. Final impression offered was cellular reactive marrow with increased histiocytes and presence of intracellular and extracellular organisms, morphologically consistent with histoplasma. Possibility of disseminated histoplasmosis (DH) with bone marrow involvement was considered and work up was advised for immunocompromised state. Further investigations including dengue serology, widaltest, rapid diagnostic tests for malaria and rk39 antigen along with viral markers (HbsAg, HBV, HCV1,2) and HIV came out as negative. The patient later succumbed to chronic illness.

Discussion \& Conclusion: PS is not routinely examined for definitive diagnosis of DH. Few case reports mention incidental discovery of organism on PS specially with HIV/immunosuppressive therapy but none in setting of TB alone. This finding also signifies severely low levels of immunity resulting in involvement of peripheral blood by causative organism. Thus, high degree of suspicion and careful examination of PS can prove to be simple, inexpensive and preliminary step towards definitive diagnosis of $\mathrm{DH}$.

Supporting Document: cc61cb7a-640e-4152-bad8-792388599493.

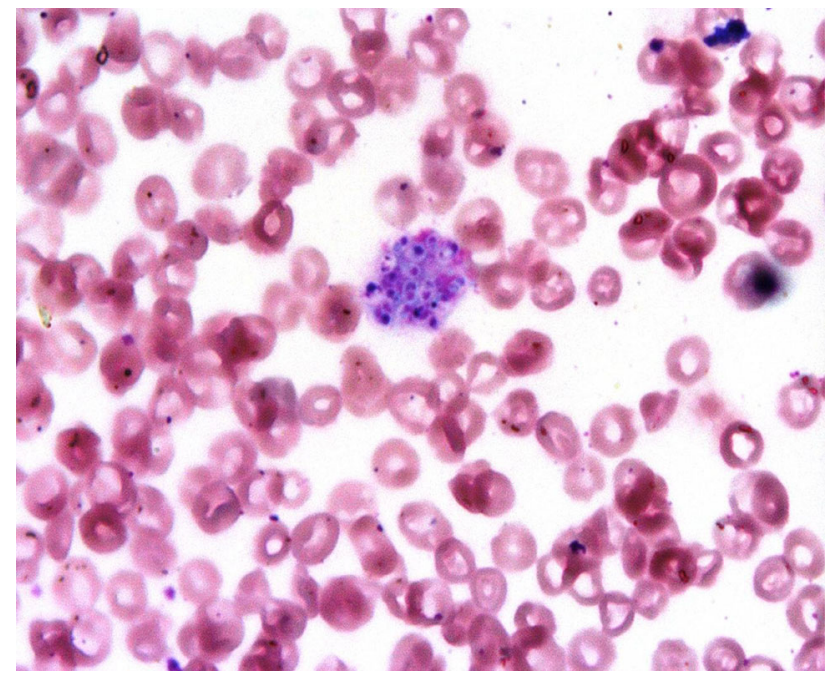

Disclosure of Interest: None Declared.

Keywords: Disseminated TB, Histoplasma, Peripheral smear (PS).

\section{Abstract 6. Acquired Pure Red Cell Aplasia Caused by Parvovirus B19 Infection in a Renal Transplant Patient}

\section{NeelofarMansuri $^{* 1}$, KeyuriPatel ${ }^{1}$, Mustafa Ranapurwala ${ }^{1}$, Rohit Kumar Singh ${ }^{1}$}

\section{${ }^{1}$ Pathology, Pramukhswami Medical College, Karamsad, India}

Aims \& Objectives: Acquired Pure Red Cell Aplasia caused by Parvovirus B19 Infection in a Renal Transplant Patient-A Case Report.

Patients/Materials \& Methods: We report a 54 years old male patient who developed severe and persistent anaemia after a kidney transplantation while being on immunosuppression therapy. The patient was currently on erythropoietin therapy and presented with worsening anemia (hemoglobin, $7.8 \mathrm{~g} / \mathrm{dL}$ ). His bone marrow aspirate showed erythroid hypoplasia with the classic morphological changes induced by parvovirus B19 infection which is the presence of giant proerythroblasts with viral inclusions. Parvovirus B19 immunoglobulin $\mathrm{M}$ antibody was positive (2.4; positive index $>1.10$ ) by enzyme immunoassay, and qualitative polymerase chain reaction detected DNA for parvovirus.

Results: Parvovirus B19 is a single-stranded DNA virus and selectively replicates in erythroid precursors in BM or peripheral blood causing transient or permanent suppression of erythropoiesis in immunocompromised patients. Parvovirus B19, transmitted in humans through exposure to infected respiratory droplets or blood products, can propagate only in erythroid progenitor cells. The infection may remain asymptomatic or cause aplastic crisis, pure red cell aplasia and prolonged erythroid suppression in immunodeficient patients as seen in the present case.

This aetiology must be kept in mind especially when a chronic anaemia, refractory to treatment, is accompanied by a reticulocytopenia. Early recognition and prompt treatment spares the patient unnecessary exposure to blood transfusions, erythropoietin and renal disease caused by the virus.

Discussion \& Conclusion: Parvovirus B19, transmitted in humans through exposure to infected respiratory droplets or blood products, can propagate only in erythroid progenitor cells. The infection may remain asymptomatic or cause aplastic crisis, pure red cell aplasia and prolonged erythroid suppression in immunodeficient patients as seen in the present case.

Disclosure of Interest: None Declared.

Keywords: Acquired red cell aplasia, parvo virus infection, renal transplant patient.

\section{Abstract 7. Utility of Lymphocyte-Monocyte Ratio and Neutrophil-Lymphocyte Ratio as Screening Tools For H1N1}

\author{
Vineeth G Nair ${ }^{* 1}$, AnupaDixit ${ }^{2}$, Sanjay Arora ${ }^{2}$ \\ ${ }^{1}$ Suburban Diagnostics, Porvorim, ${ }^{2}$ Suburban Diagnostics, \\ Andheri, Mumbai, India
}

Aims \& Objectives: To evaluate the utility of Neutrophil-Lymphocyte Ratio (NLR) and Lymphocyte-Monocyte Ratio (LMR) as screening tools for suspected H1N1 cases.

Patients/Materials \& Methods: This was a retrospective study conducted between January 2016 and June 2019, on patients who were tested for H1N1 Influenza (by RT-PCR) and who also had a complete blood count performed concurrently. Detailed statistical analysis was then performed to determine the utility of NLR and LMR as screening tools for H1N1 Influenza.

Results: Out of 7467 patients tested for H1N1, CBC was concurrently performed in 2198 cases during the period of Jan 2016-June 2019 (42 months). Of these, 26.3\% ( $n=578)$ tested positive for H1N1 Influenza. The incidence was statistically higher in the 40-49 and 50-59 age groups with almost equal distribution among males and females. On analysis, a statistically significant decrease in the median platelet count $(p<0.001)$, WBC count $(p<0.001)$ and LMR $(p<0.001)$ was seen in H1N1 positive when compared to the H1N1 negative patients. No such statistical significance was seen with regards to NLR. Neutrophil predominance $(>70 \%)$ was seen in $37.5 \%$ of the $\mathrm{H} 1 \mathrm{~N} 1$ positive patients suggesting a secondary bacterial infection. An ROC analysis of LMR as a screening tool showed a less than desirable AUC of 0.61. An LMR cut-off, (derived from Youden's Index), of 3.5 showed Sensitivity $=68.69 \%$, Specificity $=44.5 \%$, Positive predictive value $=30.63 \%$ and Negative predictive value $=79.93 \%$. A similar ROC analysis of NLR showed an AUC of 0.48 , which means it has little to no discrimination as a screening test. 
Discussion \& Conclusion: While some studies in the past have shown that NLR/LMR may have some utility as screening toolswith lower ratios suggesting a higher probability of $\mathrm{H} 1 \mathrm{~N} 1$ - our study reveals that it is not so. This is probably due to the increased susceptibility to, and thereby higher prevalence of secondary bacterial infections in H1N1 affected individuals. While it is possible that these ratios may have some discriminatory capacity in patients with purely uncomplicated $\mathrm{H} 1 \mathrm{~N} 1$, the results of our study indicate that, in general, they have limited to no utlilty as screening tools in suspected cases. Disclosure of Interest: None Declared.

Keywords: H1N1, Lymphocyte-Monocyte Ratio, NeutrophilLymphocyte Ratio.

\section{Abstract 8. Finding a New Marker for Sepsis by Coagulation Screen}

\author{
Jayalakshmi B $^{* 1}$, FebeRenjitha Suman ${ }^{1}$, Sri Gayathri ${ }^{1}$, \\ RithikaRajendran ${ }^{1}$, Binoj. ${ }^{1}$
}

${ }^{1}$ PATHOLOGY, Sri Ramachandra Institute of Higher Education \& Research, Chennai, India

Aims \& Objectives: To compare the APTT clot waveform in Systemic Inflammatory Response Syndrome (SIRS) and sepsis patients. The global epidemiological burden of sepsis is estimated to be affecting more than 30 million people worldwide every year, leading to 6 million deaths. Detecting sepsis at an early stage can reduce mortality and morbidity. As conventional methods require more than a day to prove sepsis, there is a need to develop markers that can detect early sepsis.

Patients/Materials \& Methods: 1. The demography details of patients whose blood has been sent for blood cultures are retrieved from microbiology department.

2. Whether aPTT is requested on the same day will be checked.

3 . If aPTT is requested the clot wave is observed.

4. The clot wave form is extracted as dot file.

5. Statistical analysis done for the parameters (i) $\min 1$, (ii) $\min 2$, (iii) clot time, (iv), $\max 2$ (v) first derivative $\operatorname{Min} 2 / \min 1$, (vi) second derivative $\max 2 / \min 1$. The above parameters are compared between sepsis (culture positive) and SIRS (culture negative) patients using welcht two sample t- test. A $p$ value of $<0.05$ is considered to be significant.

Results: There is no statistically significant difference in clot time, Min2, Min2/Min1 and Max2/Min1between sepsis and SIRS group. But statistically insignificant difference was found in clot time. Min1 and Max2 were significantly increased in sepsis group when compared to SIRS group.

Discussion \& Conclusion: Min1 and Max2 are additional early predictive markers of sepsis. However multicentric laboratory studies need to be done. Equipments with clot wave analyzer and expertise are needed for the trials.

Disclosure of Interest: None Declared.

Keywords: aPTT, CLOT WAVE, SEPSIS.

\section{Abstract 9. Primary Immunodeficiency: Case Report \\ Neha Parashar ${ }^{* 1}$, Pallavi Sinha ${ }^{1}$, Sarika Singh ${ }^{1}$, Seema Kapoor ${ }^{2}$ \\ ${ }^{1}$ Pathology, ${ }^{2}$ Paediatrics, Maulana Azad Medical College, New Delhi, India}

Aims \& Objectives: Primary immunodeficiency (PID) are rare with an estimated prevalence of 1 in 1200 live births in the United States.
In India, there is a paucity of published literature about PID as very few centres have the clinical experience, laboratory facilities and technical wherewithal for diagnosis and management of PID. PID can affect any of the components of immune system including $\mathrm{T}$ or $\mathrm{B}$ lymphocytes, antibody production, phagocyte number or function and complement components. In the past few years flow cytometry has been used for diagnosis of PID as it allows enumeration of B-cells, T-cells, NK cells, and the evaluation of lymphocyte markers, T-cell variability, and adhesion receptors that may be associated with specific immune defects.

Patients/Materials \& Methods: A 9-year-old girl presented to the OPD with complaints of short stature, recurrent abscess, chronic osteomyelitis, chronic eczematoid dermatitis, chronic oral thrush, diarrhoea and recurrent blood in stools. History of delayed milestones and failure to thrive since birth was present. Patient had a history of disseminated tuberculosis (skeletal and pulmonary) at 3 years of age. No significant family history of any such complaints. On examination, features of dysmorphism: triangular facies, posteriorly rotated ears, flat nasal bridge, maxillary hypoplasia, macrocephaly, malocclusion of teeth and prominent chin were present. Nystagmus was present. Skin showed generalised eczematous lesions. Weight: $15 \mathrm{~kg}(-3.20 \mathrm{SD})$ and height: $99 \mathrm{~cm}(-4.63 \mathrm{SD})$. Peripheral blood sample was taken. Haemoglobin: $13.2 \mathrm{~g} / \mathrm{dL}$ and TLC: $9410 / \mathrm{cc}$. No abnormal cells or haemoparasite was seen. Flow cytometry was done for $\mathrm{B}$ cell, T cell and NK cell enumeration.

Results: Flowcytometry showed absolute lymphocyte count of 2380/ $\mathrm{mm}^{3}$. T lymphocyte count $=1863 / \mathrm{mm}^{3}$ (normal value: $1239-2611$ / $\mathrm{mm}^{3}$ ), B lymphocyte count $=285 / \mathrm{mm}^{3}$ (normal value: $276-640 / \mathrm{mm}^{3}$ ). Ratio of $\mathrm{T}$ helper cells: cytotoxic $\mathrm{T}$ cells $=0.47: 1$ (normal value: 1.18-2.65). Overall features were suggestive of enhanced cytotoxic $\mathrm{T}$ cell activity and suppressed B cell/humoral immunity. Patient was advised further Ig assay and genetic work up for type of PID.

Discussion \& Conclusion: Detailed clinical history and examination with flow cytometry, along with clinical suspicion for immunodeficiency state brought out the index case from myriad diagnostic dilemma and improved her quality of life reiterating the need of systemic approach in all such cases.

Disclosure of Interest: None Declared.

Keywords: Flow cytometry, hematology, immunodeficiency.

\section{Abstract 10. Clinical Profile of Patients with Bacteremia in Haemato-Oncological Settings}

Nitin Bansal ${ }^{* 1}$, Sumeet Mirgh ${ }^{2}$, Narendra Agarwal $^{2}$, RayazAhmed $^{2}$, Dinesh Bhurani ${ }^{2}$

${ }^{1}$ Infectious Diseases, ${ }^{2}$ Hematology, Rajiv Gandhi Cancer Institute $\&$ Research Center, Delhi, India

Aims \& Objectives: Bacterial infections cause significant morbidity and mortality in haemato-oncological setting. Worsening patterns of resistance is making management of these bacterial infections difficult. Few studies have co-related bacteriological and resistance patterns with clinical severity in bacteremic hosts with hematological malignancies.

Patients/Materials \& Methods: Retrospective study was conducted from Jan 2019 to June 2019 in hematology unit of tertiary level oncology centre in North India. Any patient with positive blood culture who was admitted in hematology unit was included in the study and medical records were analyzed. Multiple positive blood cultures in the same febrile episode showing same organism were taken as one. Patients were divided in 2 groups (Group A: Mortality due to bacteremia, Group B: survived that bacteremia episode and discharged). Intergroup analysis was done using Student T-test and Chi square test. 
Results: A total of 80 records (Males $=48$; Females $=32$ ) were analyzed. Median age was 52 years (Range $=4-89$ years). AML (35\%) was the most common baseline disease followed by Lymphoma (18.75\%), ALL (12.5\%), Multiple Myeloma (12.5\%), and chronic leukemia $(3.75 \%)$. Gram negative (GNB) organisms $(72.5 \%)$ were predominant cause of bacteremia and Klebsiella pneumoniae (38.5\%) was the most common organism, followed by E. coli, Pseudomonas aeroginosa and Acinetobacter spp. Among gram positives (27.5\%), coagulase negative staphylococci were most common. Over all, carbapenem resistance (CR) rate among gram negatives was $70.6 \%$. Resistance to cefoperazonesulbactam was seen in $66.25 \%$ cases. Overall mortality rate was $11.25 \%$. On intergroup analysis (Table 1); infection with CR-GNB, high procalcitonin and high SOFA (sequential organ failure assessment score) was associated with mortality.

Discussion \& Conclusion: This study emphasizes that carbapenem resistance is alarmingly high at our centre. Procalcitonin and SOFA score may be utilized in escalating empirical antibiotics to cover carbapenem resistance in haemato-oncological settings.

Supporting Document: d65167f7-e9df-4a4d-abce-9de2bbaef555.

Table 1: Various parameters in two groups
\begin{tabular}{|l|l|l|l|}
\hline Variables & Mortality group (n=9) & Survival group (n=60) & P value \\
\hline Age & $52(29-58)$ & $52(4-89)$ & 0.634 \\
\hline Males & $4(44.4 \%)$ & $39(65 \%)$ & 0.235 \\
\hline Neutropenia & $5(55.5 \%)$ & $45(75 \%)$ & 0.103 \\
\hline Organisms isolated in blood culture & $9(100 \%)$ & $43(71.66 \%)$ & 0.065 \\
\hline Gram-negative ( $)$ & $30(69.76 \%)$ & 0.004 \\
\hline CR Gram-negative ( $n)$ & $9(100 \%)$ & $17(28.3 \%)$ & 0.065 \\
\hline Gram - -positive (n) & 0 & $5.63(0.15-76.02)$ & 0.015 \\
\hline $\begin{array}{l}\text { Median Procalcitonin } \\
\text { (Range) }\end{array}$ & $23.14(0.45-68)$ & $4(1-10)$ & 0.00019 \\
\hline $\begin{array}{l}\text { Median SOFA Score } \\
\text { (Range) }\end{array}$ & $8(6-11)$ & & \\
\hline Pvalue of < $<0.05$ was taken as significant &
\end{tabular}

Disclosure of Interest: None Declared.

Keywords: Bacteremia, Carbapenem Resistance, Febrile Neutropenia.

\section{Abstract 11. Dengue and High Fluorescence Lymphocyte Count Percentage in Blood: A Fascinating Association}

ShreyamAcharya $^{* 1}$, Kundan Kumar ${ }^{1}$, AbhirupSarkar ${ }^{1}$, Aparna Ningombam ${ }^{1}$, MeghaBrijwal ${ }^{2}$, Aashish Choudhary ${ }^{2}$, S. Arulselvi ${ }^{1}$, Lalit Dar ${ }^{2}$

${ }^{1}$ Laboratory Medicine, ${ }^{2}$ Department of Microbiology, AIIMS, New Delhi, India

Aims \& Objectives: Study aim is to retrospectively evaluate high fluorescence lymphocyte count percentage (HFLC \%) by Sysmex XN series haematology analyser in peripheral blood of patients with serologically positive dengue infection and to compare this with HFLC \% in patients without dengue infection.

Primary objective is finding out an association between increased HFLC \% with non-structural protein 1 Antigen (NS1 Ag) or immunoglobulin $\mathrm{M}(\mathrm{Ig} \mathrm{M})$ positivity in dengue patients.

Patients/Materials \& Methods: 500 peripheral blood samples, already subjected to serological evaluation for dengue were recruited for screening. Complete haemogram report by Sysmex XN series auto-analyser on the day of serological testing, were collected to evaluate HFLC \% for total 102 serologically positive dengue cases and 70 non dengue controls. Dengue cases were further stratified into NS1Ag and Ig M positive sub groups with 35 and 67 cases respectively, and compared with controls separately.

Results: Receiver operating characteristic curve analysis established significant association and showed a cut off HFLC $\%$ of $>2 \%$ having
$97.18 \%$ specificity and $71.43 \%$ sensitivity while $>2.7 \%$ with $100 \%$ specificity and $71.43 \%$ sensitivity for NS1Ag positivity (at $95 \%$ confidence interval). Same analysis comparing Ig M positive cases with negative controls showed $>1.1 \%$ cut off having $95.77 \%$ specificity and $85.29 \%$ sensitivity, while $>2.7 \%$ cut off having $100 \%$ specificity and $85.29 \%$ sensitivity.

Study population was further sub grouped into high and normal HFLC $\%$ for NS1Ag and Ig M positive cases based on HFLC \% cut off value $>2.7 \% .71 .4 \%$ of NS1Ag positive cases and $86.5 \%$ of IgM positive cases showed high HFLC \%.

Discussion \& Conclusion: Literature review suggested high fluorescence scatter detected in lymphocyte population in Sysmex autoanalyser scatter plot and percentage data generated can be correlated with increased reactive lymphocyte population in peripheral blood. Recent studies in dengue patients showed evidence of increased HFLC $\%$ as well as reactive lymphocytes in peripheral smear.

This study focuses to a specific cut off for HFLC \% for suspicion of dengue, which is always needed keeping days of fever in consideration. Serological conversion to Ig M is statistically more associated with increased HFLC \%. This may help in early suspicion and diagnosis of dengue cases.

Supporting Document: 5718e723-5fce-4d79-aa33-e7ceae1f6218.

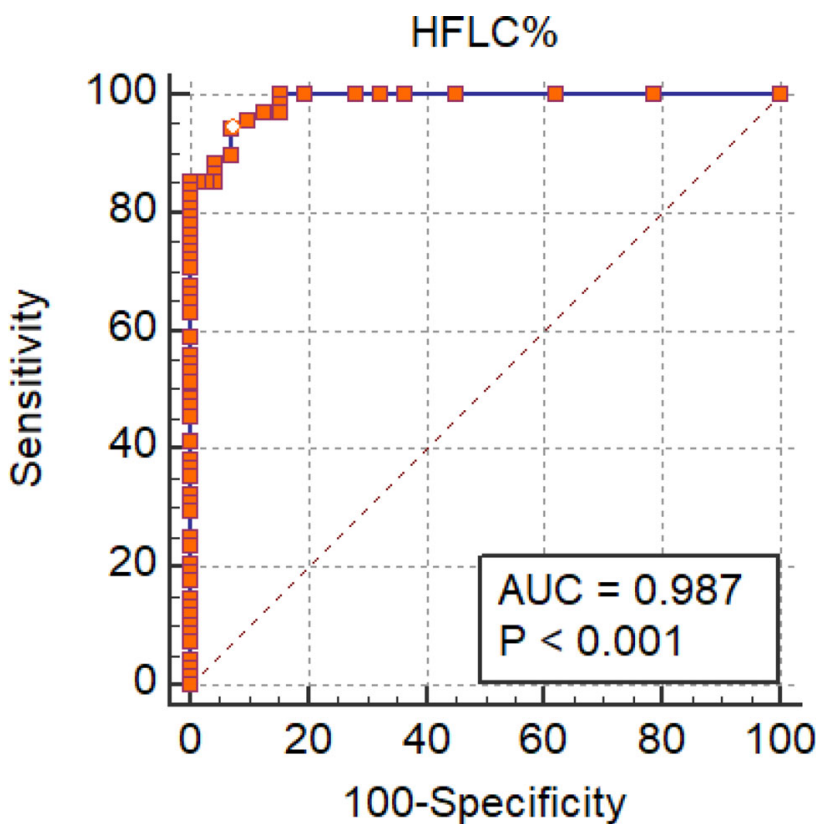

Disclosure of Interest: None Declared.

Keywords: Dengue, High Fluorescence Lymphocyte Count, Sysmex $\mathrm{XN}$.

\section{Abstract 12. Plasmodium Vivax Infection Presenting as Immune Thrombocytopenia: An Unusual Occurrence}

SonaliDixit $^{* 1}$, Neha Garg' ${ }^{1}$, Richa Gupta ${ }^{1}$, Mrinalini Kotru' ${ }^{1}$, Meera Sikka ${ }^{1}$

${ }^{1}$ University College of Medical Sciences \& G.T.B. Hospital, Delhi, India

Aims \& Objectives: Plasmodium vivax infection commonly presents with fever with chills and rigors. However a $P$. vivax infection without fever is extremely rare, also a secondary immune 
thrombocytopenia due to $P$. vivax infection has never been reported. Here we report a case of $P$. vivax malaria with unusual presentation. Patients/Materials \& Methods: A 31 year old male reported with petechial haemorrhages, gum bleeding, and melena for 3 months, which were not associated with fever. Splenomegaly and cervical lymphadenopathy were also present. Patient was found to have thrombocytopenia $\left(7 \times 10^{9} / \mathrm{L}\right)$ and few atypical/monocytoid cells on peripheral smear. However flowcytometry did not reveal atypical cells. Clinically a haematological malignancy or Immune thrombocytopenia was suspected. Four units of platelet concentrate were transfused but platelet count did not improve. Tests for antinuclear antibodies/Rheumatic factor, dengue, Hepatitis B/C and HIV were found to be negative.

Results: Bone marrow aspiration and biopsy were suggestive of megakaryocytic thrombocytopenia.

A diagnosis of immune thrombocytopenia was made and intravenous immunoglobulin was started. On follow up thrombocytopenia persisted but trophozoites of P. vivax found on peripheral smear. Then patient started on antimalarial drugs (Artesunate) along with IV immunoglobulins and responded well on subsequent follow up (Platelet count: $\left.160 \times 10^{9} / \mathrm{L}\right)$. Considering an atypical presentation and afebrile malaria, immune disturbance was suspected.

A review of literature revealed that in a malaria patient, fever might be suppressed due to immunosuppression. Thus a basic T, B and NK cell panel flowcytometry was done along with CD4 and CD8 ratio. There was an increase of $\mathrm{T}$ lymphocytes (90\%) as compared to normal with a reversal of CD4 to CD8 ratio (0.95). Thus it may be postulated that immune dysregulation may be the cause of afebrile nature of disease, also this is the first case where malaria resulting in immune thrombocytopenia.

Discussion \& Conclusion: Unusual presentation with absence of fever and reversal of CD4:CD8 ratio is suggestive of an immunocompromised stage. Therefore fever as a surrogate indicator for the diagnosis of malaria should be used with caution in patients with immunosuppression. Thrombocytopenia in $P$. vivax infection usually occur due to increased platelet phagocytosis. However its association with immune thrombocytopenia has never been reported.

Disclosure of Interest: None Declared.

Keywords: Immune Thrombocytopenia, Megakaryocytic thrombocytopenia, Plasmodium Vivax.

\section{Abstract 13. Unusual Association of Pure Red Cell Aplasia with Myelofibrosis}

\author{
Shubhra Singhal $^{* 1}$, Prernaarora ${ }^{1}$, Nita Khurana ${ }^{1}$, \\ Ashima Dabbas', Sangeeta Yadav ${ }^{2}$ \\ ${ }^{1}$ Pathology, Maulana Azad Medical College, ${ }^{2}$ Paediatrics, \\ Maulana Azad Medical College and Lok Nayak Hospital, New \\ Delhi, India
}

Aims \& Objectives: To study unusual manifestation of pure red cell aplasia.

Patients/Materials \& Methods: Pure red cell aplasia (PRCA) is an uncommon disorder in which maturation arrest occurs in the formation of erythrocytes. It may be secondary to a host of neoplastic, autoimmune or infectious diseases. The association of PRCA and myelofibrosis is very rare with only two such cases reported in the literature.

We present an unusual case of PRCA in a 3 year old female presenting with high grade fever and associated with myelofibrosis, with evidence of parvovirus infection.

Results: Hemogram revealed $\mathrm{Hb}=8.9 \mathrm{gm} / \mathrm{dL}, \mathrm{TLC}=3410 / \mathrm{mm}^{3}$, Platelets $=1.2 \mathrm{lacs} / \mathrm{mm}^{3}$.
On examination, pallor was present, Liver was palpable $3.5 \mathrm{~cm}$ below the costal margin, spleen tip was palpable. Chest $\mathrm{X}$ ray was within normal limit.

Bone marrow aspirate smears revealed hemodilution with prominence of stromal fibroblasts, giant proerythoblasts, few plasma cells, rare erythroid precursors with dyserythropoeisis and occasional megakaryocytes with dysmegakaryopoeisis. Myeloid precursors were unremarkable. Provisional diagnosis of?MDS?PRCA? Tuberculosis was suggested. Bone marrow biopsy showed suppression of erythroid precursors, focal lymphoid aggregates, increased plasma cells and areas of fibrosis. Final diagnosis of PRCA with myelofibrosis with? Autoimmune etiology. On Immunohistochemistry, presence of proerythroblasts was confirmed by $\mathrm{CD} 71$. Patient serology was strongly positive for parvovirus infection.

Discussion \& Conclusion: Conclusion: Manifestations of PRCA may be complex and secondary to multiple etiologies. The clinician needs to be aware of the broad differential and PRCA with parvovirus infection may be the cause of unusual bone marrow findings of myelofibrosis with pancytopenia.

Disclosure of Interest: None Declared.

Keywords: Myelofibrosis, Parvovirus, Pure Red Cell Aplasia.

\section{Abstract 14. Profile of Venous Cathters Inhaemato- Oncology Patient}

\author{
Ranjit kumar ${ }^{* 1}$, Ranjit Kumar $\mathrm{CS}^{2}$ on behalf of \\ Dr,Krishnamanikalapti, Dr. ArunLingitla, Dr Harshita Aribandi \\ and Dr.Krishnamanikalpathi, Dr. ArunkumarLingutla, \\ Dr.HarshitaAribandi,Mrs Suguna kumar,Mannakumari
} ${ }^{1}$ Department of Haemato Oncology, Care Hospitals, Hyderabad,
${ }^{2}$ Department of Heamato Oncology, Care Hospitals, Hyderabad,
India

Aims \& Objectives: To study complications of centrally inserted catheters in haematological malignancies and solid malignancies over 18 months period.

Patients/Materials \& Methods: A retrospective study of 74 centrally inserted catheters and possible complications. These included 39 PICC lines, 26 chemoports, 7 Haemodialysis sheath lines and one each of Hickman line and other central lines. The study group included male to female ratio of 4:5 between 13 years to 69 years of age.

Results: Main indications for line insertions were chemotherapy for carcinoma of breast (23\%), AML (20\%), ALL (13.5\%), Multiple myeloma $(6.75 \%)$, Non Hodgkins lymphoma (6.75\%), Aplastic Anaemia (6.75\%), Carcinoma Colon (6.75\%), CML (2.7\%), TTP (2.7\%) and one case each of Hodgkins lymphoma, pure red cell aplasia, primary plasma cell leukemia, oesophageal carcinoma, carcinoma of lung, squamous cell carcinoma of left maxilla, Ewings Sarcoma and carcinoma of cervix.

- Among 7 Haemodialysis central lines, one was removed suspecting infection (culture and sensitivity showed staphylococcus capitis).

- One Hickman line reported infection which was removed (culture and sensitivity showed MDR).

In total $8(11.4 \%)$ lines were reported infection of which 6 lines were removed. In addition, one line $(1.35 \%)$ was removed due to Lumen fracture.

Discussion \& Conclusion: Incidence of line infections was 8 (11.4\%) and incidence of line removal for reasons (other than the end of treatment) was $7(9.45 \%)$. This is lower compared to other studies, with asepsis, more infectious complications can be avoided, and lines can be salvaged. 
Disclosure of Interest: None Declared.

Keywords: cancer, central venous catheter, complications.

\section{Abstract 15. Immune Thrombocytopenia (ITP) in an Adult Following Dengue}

\author{
Anup Halappanavar ${ }^{* 1}$, SanjeevanSharma ${ }^{2}$ \\ ${ }^{1}$ Internal Medicine, Armed Forces Medical College, ${ }^{2}$ Clinical \\ Haematology, Command Hospital (Southern Command), Pune, \\ India
}

Aims \& Objectives: Dengue fever can have some atypical manifestations of the disease, like hepatitis, encephalitis and immune thrombocytopenic purpura (ITP). It is known that ITP, following viral infection generally, occurs in children and has an acute course. On the other hand, ITP in adults has a chronic course and is not often associated with viral infections. In this article, a case of chronic ITP in an adult man that occurred after dengue fever is described.

Immune thrombocytopenia (ITP) can be both primary and secondary. The secondary form of this disease may occur in association with systemic lupus erythematosus, antiphospholipid antibody syndrome, immunodeficient states, lymphoproliferative disorders, viral infections and drugs such as quinidine, sulfa and heparin. ITP can also be classified as either acute or chronic. The acute forms of ITP are more common among children, generally following a viral infection and tend to have a self-limited nature in up to $80 \%$ of the cases. On the other hand, ITP in adults almost invariably has a chronic course and requires treatment to obtain remission of signs and symptoms. ITP in adults is rarely associated with viral infections. In this article we report a case of chronic ITP in an adult that appeared following viral infection (classic dengue) in a way that was similar to what happens in majority of acute ITP cases among children.

Patients/Materials \& Methods: In Jul 2019, a 27-year-old male presented with fever of 05 days duration, associated with headache, myalgia, anorexia, retro-orbital pain, nausea and prostration. He gave history of taking over the counter paracetamol but not made any regular use of any other drug before the onset of symptoms. Physical examination revealed blanchable erythema over anterior chest wall. Systemic examination was essentially normal. His investigations revealed $\mathrm{Hb} 12.3 \mathrm{~g} \%$, leukocytes 3410, haematocrit (Ht) $28.7 \%$, platelets 24100 , Total bilirubin $0.7 \mathrm{mg} \%$, AST 35, ALT 21, urea $17.4 \mathrm{mg} / \mathrm{dl}$, creatinine $0.6 \mathrm{mg} \%$, calcium $7.9 \mathrm{mg} / \mathrm{dl}, \mathrm{K}+4.4 \mathrm{mEq} / \mathrm{l}$, $\mathrm{Na}+143 \mathrm{mEq} / \mathrm{l}$, PT 13.6, PTTa 29, INR 1.0, His dengue serology revealed NS1Ag positive, IgM/IgG Dengue Negative.

Results: Immune thrombocytopenia generally affects adults in an idiopathic and chronic manner, and it is found twice as frequently among women as among men. In contrast, ITP is frequently acute among children, with a condition of petechia or purpura appearing a few days or weeks after an infection that, in most cases, is viral. Thrombocytopenia associated with viral infection may be either due to a reduced production of platelets from megakaryocytes or a reduced half-life of platelets, the latter being principal mechanism. Platelets that are sensitized by autoantibodies are destroyed by cells of the reticuloendothelial system, particularly in spleen. These autoantibodies against glycoproteins of the platelet membrane can be identified in $80 \%$ of the patients. The presence of signs of skinmucosal bleeding only later on in the course the disease supports this diagnosis, given that immune thrombocytopenia characteristically first appears between the seventh and tenth day of illness, which is different from the thrombocytopenia inherent to an infectious condition, which occurs earlier.

Discussion \& Conclusion: ITP in adults is generally a chronic and idiopathic disease. However, as could be seen in our patient, it may also occur in a chronic form, following viral infection, between the adult and child forms of ITP. The factors that determine whether postviral thrombocytopenia will follow an acute or chronic course remain unknown.

Supporting Document: d933732c-978d-4088-8cb9-da1bcd11d003. Disclosure of Interest: None Declared.

Keywords: Adults, Dengue, Immune thrombocytopenic purpura (ITP).

\section{Abstract 16. Flow Cytometry Based Diagnosis of LAD, ALPS and CGD}

Gayathri Kuppuswamy $^{* 1}$, KotteswariKathirvel ${ }^{1}$, Merlin Priyanka $^{1}$, Anup Devasia ${ }^{1}$, Uday Kulkarni', Aby Abraham $^{1}$, Biju George ${ }^{1}$, Fouzia NA ${ }^{1}$, Anu Korula ${ }^{1}$, Arun Kumar Arunachalam ${ }^{1}$, Vikram Mathews ${ }^{1}$

\section{${ }^{1}$ Department of Haematology, Christian Medical College \& Hospital. Vellore, Vellore, India}

Aims \& Objectives: Primary immunodeficiency (PID) is a broad disease spectrum comprising more than 350 distinct disorders with distinct and overlapping clinical and laboratory features. Flow cytometry plays an important role both for screening and diagnosis of various PID. Here we describe our experience with flow cytometry based diagnosis of Leucocyte Adhesion Defect (LAD), Autoimmune Lymphoproliferative Syndrome (ALPS) and Chronic Granulomatous Disease (CGD).

Patients/Materials \& Methods: This retrospective study includes 421 samples analysed for LAD $(n=48)$, ALPS $(n=270)$ and CGD $(\mathrm{n}=103)$. Flow cytometry acquisition was done with BD FACSCanto and $\mathrm{BC}$ Navios 10 colour flow cytometers while the analysis was done with BD FACSDiva and Kaluza software. The following antibodyfluorochrome conjugate panels were used: CD18-FITC, CD11a-FITC, CD11b-APC, CD11c-PE, CD19-PerCP-Cy5.5 and CD56-APC for LAD, CD3-APC, TCR $\alpha \beta-F I T C$, CD4-PE, CD8-PE, CD45-PerCP for ALPS while Phorbol Myristate Acetate (PMA) and Dihydrorhodamine (DHR) were used for stimulation and cell dependent oxidation respectively in CGD.

Results: Among the 48 patients evaluated for LAD, 18 patients showed complete loss of CD11a, CD11b, CD11c and CD18 expression in the neutrophils which is consistent with a diagnosis of LAD. The median (range) percentage of neutrophils expressing CD11a, $\mathrm{CD} 11 \mathrm{~b}, \mathrm{CD} 11 \mathrm{c}$ and $\mathrm{CD} 18$ were $1.2 \%(0-5.6 \%), 0.85 \%(0.1-6.1 \%)$, $0.2 \%(0-3.1 \%)$ and $0.1 \%(0.1-15.7 \%)$ respectively while the expression were normal in LAD negative patients [CD11a-98.2\% (90.3-99.9\%), CD11b-99.3\% (87.6-99.9\%), CD11c-99.2\% (89.5-99.9\%) and CD18-99.7\% (96.4-100\%)]. Among the 270 patients evaluated for ALPS, 53 patients (19.6\%) had an increase in CD3+ TCR $\alpha \beta+$ CD4-CD8- (Double negative T cells) consistent with a diagnosis of ALPS. The median percentage of double-negative $\mathrm{T}$ cells among total lymphocytes and CD3+ T cells were $2.38 \%$ $(1.6-26.2 \%)$ and $3.31 \%(2.38-51.1 \%)$ respectively. CGD is defined by the lack of increase in the DHR mean fluorescence intensity (MFI) following stimulation of neutrophils with PMA and was seen in 18 (median patient/control MFI: $0.485 \quad(0.227-0.799)$ vs. 1.002 (0.945-1.086); $p<0.001)$ among the 103 patients. The median MFI post PMA stimulation in CGD patients and normal controls were432.5 (202-686) and 902.5 (760-955) respectively.

Discussion \& Conclusion: While genetic analysis is required for the definitive diagnoses of PIDs, flow cytometry provides a rapid costeffective tool to effectively diagnose ALPS, CGD and LAD.

Disclosure of Interest: None Declared.

Keywords: ALPS, CGD, LAD. 


\section{Abstract 17. A Quality Improvement Initiative to Improve Critical Value Notification in the Hematology Laboratory}

\section{TapasyapreetiMukhopadhyay $^{* 1}$, ArulselviSubramanian ${ }^{1}$ \\ ${ }^{1}$ Laboratory Medicine, JPNATC, AIIMS, New Delhi, India}

Aims \& Objectives: To determine the rate of critical value notification in the haematology section of the laboratory, and to improve the rate to more than $50 \%$ over a period of one month by the application of the principles of Quality Improvement (QI).

Patients/Materials \& Methods: The pre-test/post-test intervention study was conducted in the haematology section of the Department of Laboratory Medicine, JPNATC, AIIMS, New Delhi over 3 months, one month each of pre-intervention, intervention and post intervention phase. A quality improvement team (QI) was made that constituted the faculty in charge of the clinical laboratory and three senior residents. Critical value notification was defined as notifying the critical value (laboratory defined) to a healthcare provider in the respective ward telephonically by the laboratory technician after checking for analytical reliability followed by documenting the details including name of the patients, UHID, location of the patient, parameter and value, and the name of the receiver.

Rate of critical value notification $(\mathrm{CVN})=$ Number of documented critical value/Total reportable critical value $\times 100$.

The samples without barcode (care-of sample, internal quality control samples) or with incomplete information were rejected. Systematic auditing of the test laboratory records for critical values and fishbone analysis for identifying the barriers were carried out in the pre-intervention phase. QI team directly observed the CVN process and regularly motivated and spread awareness amongst the laboratory staff about CVN as a part of good laboratory practice in the intervention phase. Systematic auditing of CVN was continued during the intervention and post-intervention phase and analysed for improvement. Descriptive statistics was calculated for all the data of variables collected during critical value notification.

Results: A total of 712 critical values in the hematology section were analyzed during the study period of three months of which 13 were rejected based on exclusion criteria. Of the total 699 critical values included, 208 were studied in the pre intervention phase and 215 during the intervention and 276 in the post-intervention phase. The QI team achieved the goal within the time frame successfully. The rate of critical value notification improved from $2.8 \%(\mathrm{n}=6)$ in the pre- intervention phase to $38.13 \%(\mathrm{n}=82)$ during the intervention phase and to $68.1 \%$ $(n=188)$ in the post-intervention phase. Lack of awareness of the critical values, lack of interest from the receiver's end and telephone line to be busy were the prominent barriers for CVN. Highest notification was for abnormal platelet count i.e. $42 \%(n=79)$, followed by for abnormal hemoglobin i.e. $37.7 \%(\mathrm{n}=71)$ and least for abnormal leucocyte count i.e. $20.2 \%(\mathrm{n}=38)$. Out of the informed critical values in the post-intervention phase, $51.6 \%(\mathrm{n}=97)$ were patients with test request coming for the second time or more during the hospital stay and $54.7 \%(\mathrm{n}=103)$ critical values were notified during the night shift. Maximum patients belonged to general ICU $(n=45)$ followed by the emergency department $(n=37)$ amongst others.

Discussion \& Conclusion: A laboratory critical value represents a life threatening condition. It needs urgent notification to the clinician for which some corrective actions could be taken promptly. The purpose of the study was to improve the ability to communicate the critical test results in a timely manner to the clinicians and was accomplished successfully within time. Quality Improvement principles proved effective to improve critical value notification in the clinical Hematology laboratory.

Disclosure of Interest: None Declared.
Keywords: Critical value notification, Laboratory Management, QI in Hematology.

\section{Abstract 18. Molecular Microbiology Improves Outcome in Febrile Neutropenia Compared to Blood Culture}

\author{
AninditaPaul $^{* 1}$, Ravi Kumar ${ }^{2}$, Sachin Jadhav ${ }^{1}$ \\ ${ }^{1}$ Clinical Hematology and BMT, HCG Cancer Hospital, \\ ${ }^{2}$ Microbiology, XCYTON Diagnostics, Bangalore, India
}

Aims \& Objectives: High risk febrile neutropenia is a life-threatening condition which needs emergent and accurate antibiotics therapy to reduce mortality. We have performed a retrospective analysis to compare the clinical outcomes between using a multiplex PCR- based molecular diagnostic test, Syndrome Evaluation System (SES) vs. blood culture, to decide antibiotic therapy for high risk febrile neutropenia in hematological conditions.

Patients/Materials \& Methods: Retrospective review of inpatient charts of patients from BGS Global and Fortis Hospital, Bengaluru, who had been admitted for high-dose chemotherapy or haematopoietic stem cell transplant (HSCT) between Sept 2013 to March 2019. All of these had suffered from high-risk febrile neutropenia.

Results: 271 patients underwent testing with SES over a period of 66 months. $66.7 \%$ (183) patients were male with a median age of 39 years (1-86 years).

Majority of the samples were blood samples 224 (82.6\%) while others were CSF 8 (2.95\%), Baylor wash 4 (1.47\%), pleural fluid 4 (1.47\%), nasopharyngeal swab $2(0.73 \%)$.

SES results were positive in $152(56 \%)$ patients while the blood culture was positive in $33(19.6 \%)$ of patients among the 168 in whom the data was available, $p=<0.00001$.

Out of 71 patients in whom the site of infection was recorded, the sites were respiratory $54(76 \%)$, diarrhoea $3(4.2 \%)$, intravenous cannula $1(1.4 \%)$, urinary tract $1(1.4 \%)$, other sources $11(15.4 \%)$.

101 patients had a change in antibiotics and the reason for change was the SES result in $43(42.5 \%)$ patients, culture report in $22(21.7 \%)$ patients, and clinical judgement in $36(35.6 \%)$ patients, $p=<0.00001$.

Data on mortality was available on 161 patients out of which 135 $(83.8 \%)$ were live and $26(16.1 \%)$ had died. $20(76 \%)$ of these were SES positive and $6(24 \%)$ were SES negative, $p=<0.00001$.

Discussion \& Conclusion: Compared to blood culture, molecular microbiologic testing with SES gives a significantly higher positive rate of identification of the infecting organism. It also significantly helps in the appropriate change in treatment and also predicts mortality.

Disclosure of Interest: None Declared.

Keywords: culture, molecular, neutropenia.

\section{Abstract 19. MDRO Associated Sepsis In Neutropenic Patients with Hematological Malignancy: A Cause of Concern}

Malini Garg ${ }^{* 1}$, Ankit Jitani ${ }^{1}$, Prakash Singh Shekhawat ${ }^{1}$, RajibDe $^{1}$, Prakas Kumar Mandal ${ }^{1}$, ShuvraneelBaul, SubhamBhattacharya ${ }^{1}$, TuphanKantiDolai ${ }^{1}$

${ }^{1}$ Hematology, NRS Medical College, Kolkata, India

Aims \& Objectives: To study the patterns of microbial sepsis in patients of hematological malignancies receiving chemotherapy and their outcome. 
Patients/Materials \& Methods: Cultures were sent for patients with febrile neutropenic episodes from July 2018 to June 2019. The cultures were analysed using Bactalert $3 D$ Biomeriux analyser and their antimicrobial sensitivity patterns were studied. Multidrug resistant organism (MDRO) was defined as organisms in which only two or fewer unrelated antibiotics were active.

Results: A total of 444 cultures were sent during febrile episodes. Majority of these cultures were sent from blood (69.8\%) followed by oral swab (13.2\%) and PICC line tip (10.2\%). Out of which $23 \%$ (105/444) showed growth of different pathogenic microorganisms. The majority of the cultures were isolated from ALL (43\%) followed by AML $(23 \%)$. Out of all positive isolates, majority were gram negative organisms $(69.91 \%)$. Klebsiella spp was the predominant isolate with $29 \%$ cases. MRSA was positive in $23 \%$, Acinetobactor in $14 \%$, E coli in $14 \%$ and Pseudomonas in $12 \%$. Candida grew in only $1.8 \%$ of the cultures. Majority $(66 \%)$ of these cultures belonged to MDRO. Overall infection associated mortality was $25.5 \%$ (27/105) of which $88.8 \%$ (24/27) was due to MDRO sepsis.

Discussion \& Conclusion: Gram negative organism is a major cause of sepsis in neutropenic patients. MDRO constitute $66 \%$ of the cases and are major reason for infection associated mortality in hematooncology ward.

Disclosure of Interest: None Declared.

Keywords: Hematological malignancy, MDRO, neutropenia.

\section{Abstract 20. Utility of Haematology Analyser in Identification of Microclots and Apoptotic Bodies}

\author{
Ruchi Tiwari $^{* 1}$, Aparna $\mathbf{N}^{2}$, Vijay Antony ${ }^{1}$ \\ ${ }^{1}$ Laboratory Medicine, ${ }^{2}$ Laboratory Medicine, AIIMS New Delhi, \\ New Delhi, India
}

Aims \& Objectives: Identification of micro clots and apoptotic cells by haematology analyser SYSMEX XN 9000.

Patients/Materials \& Methods: We analysed the routine blood samples for a duration of 3 months in sysmex haematology analyser $\mathrm{XN}$ 9000. The peripheral smears of the same were examined prospectively. Apoptotic cells and microclots were identified morphologically via light microscopy. Respective scatterplots of these cases were retrieved and analysed post-peripheral smear examination. Results: On analysing the scatterplot of the cases with evident microclots and apoptotic bodies in the peripheral smear we found in the WDF (SSC-SFL) plot the area of neutrophils was starting much below and showed more left ward shift as compared to the normal expected position of neutrophils. Other cell populations were represented on the scatterplots within normal limits.

Discussion \& Conclusion: Automated haematology analysers can rapidly provide accurate blood cell counts and white blood cell differentials. The development of highly sophisticated and accurate WBC differential counters represents the greatest advance in haematology analyser instrumentation till date. Methods involving concurrent use of principles of electrical impedance, laser light scattering, conductivity and cytochemistry, combined with use of cell-specific lysing reagents, have provided the requisite tools for rapid automated assessment and delineation of a composite WBC population into its cytomorphological subtypes.

Apoptosis, or programmed cell death, plays a key role in many physiological processes including embryonic morphogenesis, immune cell maturation and maintenance of adult tissue architecture. Micro Clots show fibrin strands, platelets and white cells clumped together. Found in In vitro artefact caused by poor venesection technique and leads to false low counts-can influence white cell, red cell and platelet counts.
Therefore, the concordance between the scatterplot and peripheral smear finding will assist laboratory physicians in prompt requisition for a fresh sample.

Supporting Document: 36e8ea2b-ae4f-4791-a0a5-99dc5d3ee8f8.
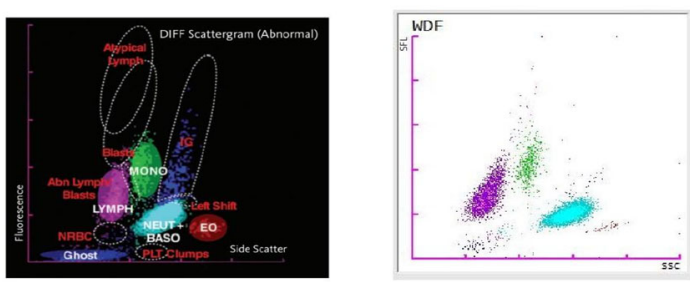

SCATTERPLOTS

"APOPTOTIC BODIES AND MICROCLOTS"
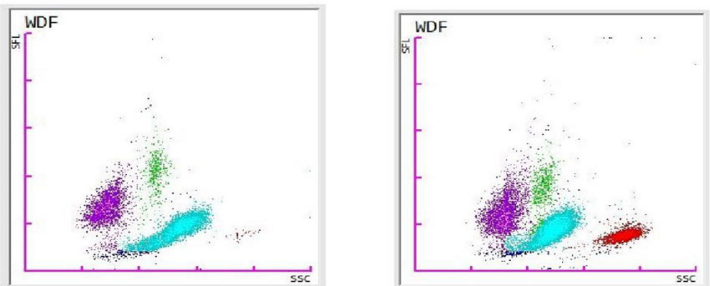

Disclosure of Interest: None Declared.

Keywords: Microclots, Sysmex, XN.

\section{Abstract 21. Klebsiella producing Carbapenemase: A Bug Giving Nightmares to Haematologists in Hemato-Oncology Ward}

Malini Garg ${ }^{* 1}$, Ankit Jitani ${ }^{1}$, Prakash Singh Shekhawat ${ }^{1}$, ShuvraneelBaul ${ }^{1}$, Prakas Kumar Mandal ${ }^{1}$, RajibDe ${ }^{1}$, SubhamBhattacharya ${ }^{1}$, TuphanKantiDolai ${ }^{1}$

\section{${ }^{1}$ Hematology, NRS Medical College, Kolkata, India}

Aims \& Objectives: To study the pattern of Klebsiella positive cultures in febrile neutropenic episodes in haematological malignancies.

Patients/Materials \& Methods: All patients receiving chemotherapy in different haematological malignancies from July 2018 to August 2019 were analysed and cultures were sent from various sites during febrile neutropenic episodes. The cultures were analysed using $\mathrm{Bac}$ talert $3 D$ Biomeriux analyser and their antimicrobial sensitivity patterns were studied. Klebsiella isolates were classified into drug sensitive Klebsiella pneumoniae and Klebsiella producing carbapenemase (KPC).

Results: During July 2018 to June 2019, a total of 476 blood cultures were sent during febrile episodes. Out of which $25 \%(119 / 476)$ showed growth of different pathogenic microorganisms. Out of all positive cultures, Majority (30\%) showed growth of Klebsiella. $83 \%$ (30/36) were KPC and $17 \%(6 / 36)$ were drug sensitive Klebsiella pneumoniae. All the KPC showed sensitivity to Colistin and tigecycline and were resistant to most of the $1^{\text {st }}$ line antibiotics used in our wards. The majority of the cultures of Klebsiella were isolated from Acute Myeloid Leukemia patients (18/36) out of which 55.5\% (10/18) were isolated during induction chemotherapy and 46\% (8/10) during consolidation. 38\% (14/36) cultures of Klebsiella were isolated from 
Acute Lymphoblastic leukemia patients, out of which 33\% (5/14) were isolated during induction and 66\% (9/14) during consolidation phase of chemotherapy. Overall mortality associated with Klebsiella infection was $44.4 \%(16 / 36)$.

Discussion \& Conclusion: Majority of the cultures isolated belonged to Klebsiella pneumoniae. Majority of the Klebsiella pneumoniae infections were due to KPC isolates. These organisms were resistant to most of the $1^{\text {st }}$ line antibiotics and are a major emerging cause of mortality in neutropenic patients with haematological malignancies. Disclosure of Interest: None Declared.

Keywords: Hematological malignancy, Klebsiella pneumoniae, KPC.

\section{Abstract 22. Langerhan's Cell Histiocytosis in Skin in an Adult-A Case Report}

\author{
DaminiSingh $^{* 1}$, Rashmi Kushwaha ${ }^{1,1}$, Geeta Yadav', \\ ParulVerma $^{2}$, S.p.verma ${ }^{3}$, Madhu kumar ${ }^{1}$, Ashutosh kumar ${ }^{1}$ \\ ${ }^{1}$ Pathology, ${ }^{2}$ Skin and v.d., ${ }^{3}$ Clinical Hematology, KGMU, \\ Lucknow, India
}

Aims \& Objectives: Langerhan'S Cell Histiocytosis is a rare pathology characterised by an abnormal clonal proliferation of langerhan cells that infiltrates different organs of the human body. Among different organs cutaneous involvement is seen in $40 \%$ of cases but the disease limited to skin is uncommon among reported case of adult Langerhan Cell Histiocytosis.

Patients/Materials \& Methods: A 24 year old female presented with persistent, soft to firm swelling over nape of neck with scarring along with pustules and papules over bilateral axilla and trunk over a period of 1.5 years. FNAC was performed from the lesion and later on biopsy was also received.

Results: FNAC smears showed infiltration by large sized atypical cells with eccentric round nuclei, open nuclear chromatin and occasional prominent nucleoli with scant cytoplasm. Multinucleated giant cells and histiocytes were also seen. CD 68 was done on FNAC slides. CD 68 was positive in large cells.

Biopsy was performed. Microscopy of showed keratinized squamous epithelium. Papillary dermis was densely infiltrated by abnormal medium sized cells with coffee bean nuclei, vesicular chromatin, 1 to 2 prominent nucleoli and scant to moderate amount of cytoplasm along with mature lymphocytes and few foamy histiocytes. IMMUNOHISTOCHEMISTRY showed CD1a positivity of langerhan's cells. So a final diagnosis of Langerhan's Cell Histiocytosis was made.

Discussion \& Conclusion: Cutaneous lesions maybe the sole presenting feature of Langerhan's Cell Histiocytosis. Diagnosis is based on demonstration of CD1a positive histiocytes. Langerhan 's Cell Histiocytosis may present with unusual cutaneous manifestations limited to skin in adult.

Disclosure of Interest: None Declared.

Keywords: Biopsy, FNAC, Langerhans Cell Histiocytosis.

\section{Abstract 23. Abdominal Infections in Immunocompromised Hosts; Aclinical, Radiological \& Microbiological Co Relation}

Sachin Bansal $^{* 1}$, ManoranjanMahapatra ${ }^{1}$, Priyanka Naranje ${ }^{2}$, Sarita Mahapatra ${ }^{3}$, Immaculata Xess ${ }^{3}$

${ }^{1}$ Hematology, ${ }^{2}$ Radiodiagnosis, ${ }^{3}$ Microbiology, AIIMS New Delhi, Delhi, India
Aims \& Objectives: Abdominal infections are a significant cause of mortality and morbidity amongst immunocompromised hosts. Diagnosis of an infectious episode in neutropenic patients is often difficult as inflammatory response is inadequately mounted and the clinical symptoms are subtle- thus there is need for clinical,microbiological and radiological assessment and correlation to diagnose an infectious cause, its treatment and further follow up. Our aim is to study and correlate clinical, microbiological and radiological characteristics of abdominal infections in immunocompromised patients.

Patients/Materials \& Methods: It's a prospective observational study in 198 neutropenic episodes of hemato-lymphoid malignancies or aplastic anemia, admitted with febrile neutropenia (ANC $<500$ / $\mathrm{mm}^{3}$ aged $1-60$ years.

Results: 88 (44.44\%) out of 198 episodes had abdominal symptoms with total 31 (35.22\%)episodes had documented etiology on CECT or USG abdomen. 23 (74.2\%) episodes had NEC, 3 (9.6\%)episodes had pancreatitis. Appendicitis, mesenteric panniculitis, Ileoileal intussusceptions, PID, SAIO were present in 1 episodes each. Microbiological isolation shows gram negative bacteremia in 4 episodes with K.pneumoniae in 3 and E.coli in 1 NEC episode. The median duration of symptoms was significantly longer in NEC group (14 days) than among patients without mural thickening ( 11 days, $p<.244$ ), and the NEC related mortality rate was higher $(34.78 \%$ v $30.68 \%)$. Patients with bowel wall thickness of more than $10 \mathrm{~mm}$ had a significantly higher duration of neutropenia $(p<0.311)$ and higher median duration of symptoms (14 vs 13 days).

Discussion \& Conclusion: This is a prospective study of abdominal infections in patients with acute leukaemia and myelo suppressive diseases. A combination of US/CECT, microbiological and clinical investigations was applied. Clinically and/or microbiologically diagnosed abdominal infectious diseases occurred in $31(15.65 \%)$ out of 198 episodes of neutropenia (23 had neutropenic enterocolitis, 3 patient had pancreatitis and appendicitis, mesenteric panniculitis, ileoileal intussusceptions, PID, SAIO present in 1 episode each.

Disclosure of Interest: None Declared.

Keywords: BWT, NEC, USG.

\section{Abstract 24. A Curious Case of Leukopenia: Not Always a Disease}

Rajeev Ranjan ${ }^{* 1}$, Aparna Ningombam ${ }^{1}$, JayastuSenapati ${ }^{2}$, AbhirupSarkar ${ }^{1}$, UpinderSingh ${ }^{1}$, S Arulselvi ${ }^{1}$

${ }^{1}$ Laboratory Medicine, ${ }^{2}$ Haematology, AIIMS, New Delhi, India

Aims \& Objectives: Pseudoleukopenia is an uncommon phenomenon and can lead to change in clinical management and severity assessment. Rarer than pseudothrombocytopenia, this phenomenon is usually due to pre-analytical errors based on the method of sample collection and storage or due to disease characteristics as can be seen in Chronic liver disease (CLD) and autoimmune conditions due to antibody mediated aggregation of neutrophils. We present the case of a 42 year old lady of CLD with pseudoleukopenia.

Patients/Materials \& Methods: A 42 y/f patient of autoimmune hepatitis related CLD with celiac disease presented with complaints of fever, worsening liver function and pancytopenia. She had been on intermittent immunomodulation with azathioprine and steroids for 15 years. For evaluation of prior episodes of pancytopenia, and with differentials of autoimmune cytopenia versus hypersplenism, bone marrow had been done which showed normal cellularity with preserved cell lines.

The following were her present parameters, run on Coulter LH750:

Hb- 5.9 g/dl; WBC- 900/cumm (P-32/L-38/M-19/E-11); Platelet$1.1 \mathrm{lac} / \mathrm{cumm}$ 
$\mathrm{P} / \mathrm{S}$ (Geimsa stained)- Clusters of WBC throughout smear, suspicious of neutrophil autoagglutination.

For further evaluation the sample was re run on Sysmex XE-2100 and Abbott Ruby CELL-DYN in both EDTA and citrated tubes. Parallel finger prick samples were run to rule our preanalytical errors. Results: Citrated tube sample run on Sysmex and Abbott and finger prick samples correlated as follows:

WBC- 3200-4000/cumm (P-76, L-16, M-6, E-2).

$100 \times$ magnification: Tailing, mild neutrophil agglutination.

Discussion \& Conclusion: We represent an important laboratory vignette of pseudolekuopenia due to pre analytical and analytical error. Given the rarity of this situation, especially when fresh blood samples are analysed, it can be overlooked leading to management changes for the patient. While EDTA can cause leukoagglutination, antibody mediated neutrophil agglutination has been described in autoimmune conditions, patients of CLD and those on chronic immunosuppression. In these group of patients finger prick samples for PS or Citrated samples for automated counters can give more accurate data.

Supporting Document: e22fab87-ac24-4a8d-8829-486440d8e941.

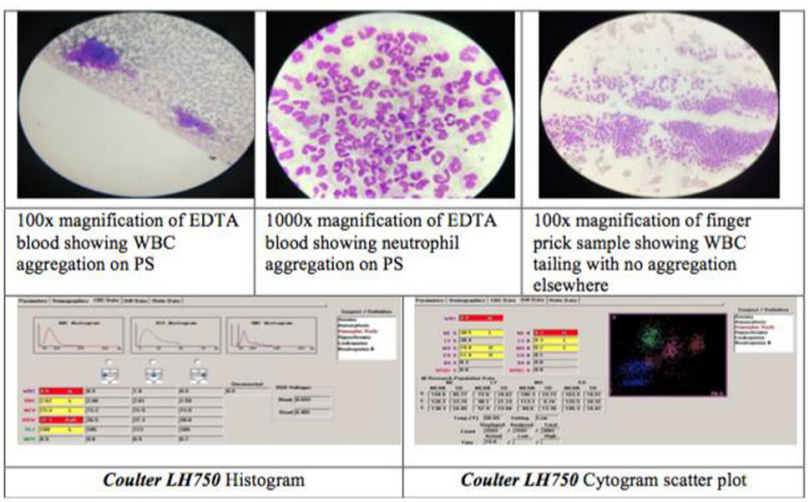

Disclosure of Interest: None Declared.

Keywords: Chronic liver disease, Leukoagglutination, Pseudoleukopenia.

\section{Abstract 25. Bilirubin Crystals in the Neutrophils of Patient with Sepsis}

UpinderSingh $^{* 1}$, ShreyamAcharya ${ }^{1}$, Aparna Ningombam ${ }^{1}$, AbhirupSarkar', Rajeev Ranjan', Renu Saxena ${ }^{1}$

${ }^{1}$ Laboratory Medicine, All India Institute of Medical Sciences, New Delhi, India

Aims \& Objectives: Presence of bilirubin crystals in neutrophils from peripheral blood smear has been reported in neonatal population with jaundice and sepsis. Here we are reporting a case of an adult patient with incidental finding of bilirubin crystals in neutrophils. Presence of bilirubin crystals in neutrophils from peripheral blood smear has been reported inneonatal population with jaundice and sepsis. Here we are reporting a case of an adult patient with incidental finding ofbilirubin crystals in neutrophils.
Patients/Materials \& Methods: A 24-year old male patient was admitted in AIIMS, with High fever, abdominal pain and vomiting with MODS. Laboratory finding showed HB-5.1 mg/dl,WBC count 22.800/microlitres, platelet count. 5000/microlitre. Serum Bilirubin$31 \mathrm{mg} / \mathrm{dl}$, liver functions were derranged. Peripheral blood smear was made from EDTA anticoagulated sample incubated at room temperature for $40 \mathrm{~min}$ to look for atypical cells. No atypical cells were found rather with the neutrophilic predominance, an incidental finding of golden yellow refractile crystals, rhomboid in shape within the cytoplasm of neutrophils (magnification 100×, Wright Giemsa stain). A 24-year old male patient was admitted in AIIMS, with High fever, abdominal pain and vomiting with MODS. Laboratory finding showed HB-5.1 mg/dl, WBC count 22.800/microlitres, platelet count $5000 /$ microlitre. Serum Bilirubin- $31 \mathrm{mg} / \mathrm{dl}$, liver functions were deranged. Peripheral blood smear was made from EDTA anticoagulated sample incubated at room temperature for $40 \mathrm{~min}$ to look for atypical cells. No atypical cells were found rather with the neutrophilic predominance, an incidental finding of golden yellow refractile crystals, rhomboid in shape within the cytoplasm of neutrophils. (magnification $100 \mathrm{X}$, Wright Giemsa stain).

Results: Patient was managed in Intensive care unit with iv fluids, iv broad spectrum antibiotics and other supportive care. After 2-3 days, patient improved clinically and his liver parameters also improved, $\mathrm{S}$. Bilirubin dropped (Unconjugated fraction also decreased). Repeat EDTA anticoagulated blood sample incubated for $40 \mathrm{~min}$ at room temperature did not reveal bilirubin inclusions in the neutrophils.

Patient was managed in Intensive care unit with iv fluids, iv broad spectrum antibiotics and other supportive care. After 2-3 days, patient improved clinically and his liver parameters also improved, S. Bilirubin dropped (Unconjugated fraction also decreased). Repeat EDTA anticoagulated blood sample incubated for $40 \mathrm{~min}$ at room temperature did not reveal bilirubin inclusions in the neutrophils.

Discussion \& Conclusion: Bilirubin crystals are seen mostly in neonates with Unconjugated hyperbilirubinemia and septicemia. These crystals are golden yellow refractile and rhomboid in shape. These are found in the cytoplasm of neutrophil. The decreased solubility of unconjugated bilirubin in EDTA sample causes crystallization in vitro (not in heparinized sample) it usually occurs in higher bilirubin levels. Literature is inconclusive about a cut off value, above which they are seen. We are the first to report Bilirubin crystals in an adult patient with unconjugated hyperbilirubinemia and sepsis.

Bilirubin crystals are seen mostly in neonates with Unconjugated hyperbilirubinemia and septicemia. These crystals are golden yellow refractile and rhomboid in shape. These are found in the cytoplasm of neutrophil. The decreased solubility of unconjugated bilirubin in EDTA sample causes crystallization in vitro. (not in heparinized sample) it usually occurs in higher bilirubin levels. Literature is inconclusive about a cut off value, above which they are seen. We are the first to report Bilirubin crystals in an adult patient with unconjugated hyperbilirubinemia and sepsis.

Supporting Document: 957c13f8-36e8-42f0-a2f7-e9f9d7d6b4c3. 


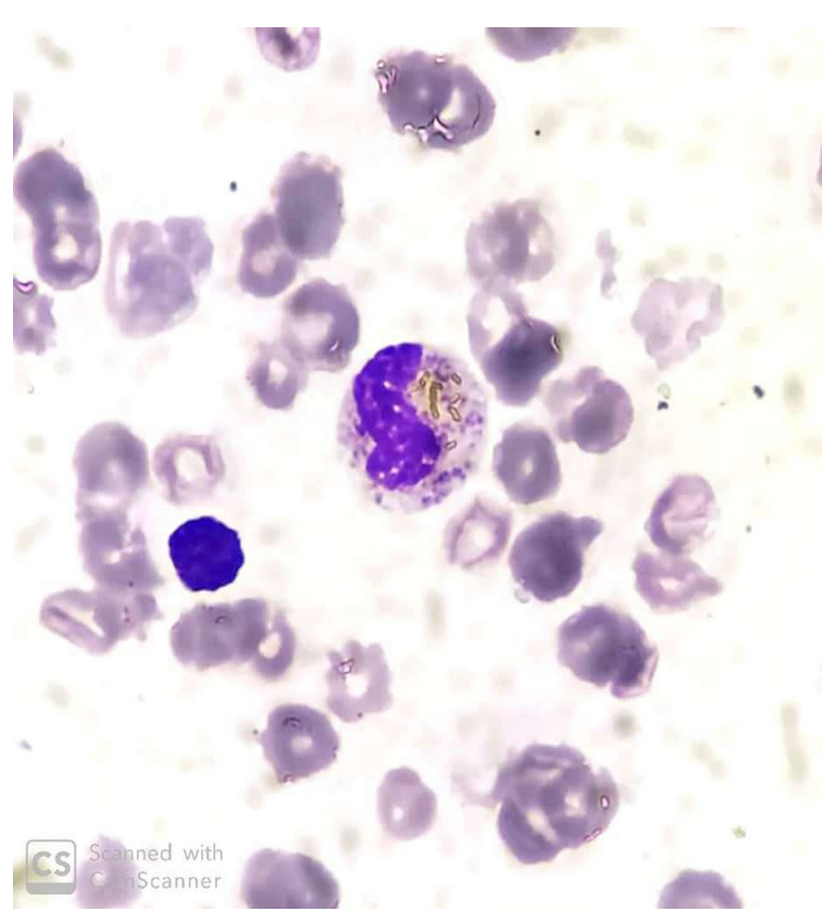

Disclosure of Interest: None Declared.

Keywords: Bilirubin crystals, Peripheral blood smears (PBS), Unconjugated Hyperbilrubinemia.

\section{Lymphoma and Myeloma (Clinical)}

\section{Abstract 1. Primary Intramedullary Spinal Cord Lymphoma: A Rare Entity}

\author{
Pradeep Kumar $^{* 1}$, Sachin Bansal ${ }^{2}$, Rajesh Kashyap ${ }^{3}$, \\ SoniyaNiyanand ${ }^{4,4}$
}

\begin{abstract}
${ }^{1}$ Hematology/Medical Oncology, NayatiMedicity, Mathura, ${ }^{2}$ Hematology, All India Institute of Medical Sciences, New Delhi, ${ }^{3}$ Sanjay Gandhi Post Graduate Institute of Medical Sciences, Lucknow, India, ${ }^{4}$ Hematology, Sanjay Gandhi Post Graduate Institute of Medical Sciences, Lucknow, India
\end{abstract}

Aims \& Objectives: Primary intramedullary spinal cord lymphoma (PISCL) is a rare entity and here, we report a case of PISCL.

Patients/Materials \& Methods: A 25 years old female presented with gradual onset of progressively increasing weakness of both lower limbs associated with below chest sensory loss and funicular pain. On examination: motor- b/l lower limb 0/5 power, hypertonic, DTR hyperactive $3+$ with clonus at both ankle; sensory-pain \& touch $10 \%$ loss at D6, $20 \%$ at D10, 30\% at L1 and 50\% at L3 with impaired posterior column sensation. MRI cervico-dorsal spine revealed intramedullary mass lesion extending from C6-8, irregular in shape, causing cord dilatation with hypo-intense on T1w and hyper-intense on $\mathrm{T} 2 \mathrm{w}$ with heterogeneous contrast enhancement and enhancing part extended from D1-5. Rest of the imaging studies (MRI of brain \& rest of spine and CT thorax \& abdomen) and Bone marrow examination with in normal limit except raised LDH. She underwent D3-5 laminectomy \& decompression surgery and biopsy revealed CD 20 positive NHL (T cell rich DLBCL).
Results: Patient was advised chemotherapy with methotrexate (Mtx), cytarabine (Ara-c) with radiation (DeAngelis protocol) but she was lost to follow up after 1st cycle.

Discussion \& Conclusion: Spinal cord lymphoma is defined as primary if there is no e/o systemic lymphoma. MRI showed enlargement of the spinal cord with hyper intense on T2w section. PISCL is usually involved lower cervical and upper thoracic region. Systemic chemotherapy with radiotherapy showed good results in comparison to alone but overall survival is poor.

Disclosure of Interest: P. Kumar Conflict with: None, Grant from: None, S. Bansal Conflict with: None, Grant from: None, R. Kashyap Conflict with: None, Grant from: None, S. Niyanand Conflict with: None, Grant from: None.

Keywords: Methotrexate, Cytarabine \& radiation, Primary intramedullary spinal cord lymphoma, T cell rich DLBCL.

\section{Abstract 2. Primary Bone Marrow Lymphoma- Case Series and Diagnostic Dilemma}

Ankur Ahuja ${ }^{1}$, Meghna Yadav ${ }^{* 1}$, TathagatChatterjee ${ }^{1}$, Gaurav Gahlot ${ }^{1}$, Suman Pramanik ${ }^{2}$

${ }^{1}$ Department of Lab and Molecular Medicne, ${ }^{2}$ Dept of Hematology, Army Hospital RR, Delhi, India

Aims \& Objectives: To assess the clinicopathology profile of primary bone marrow lymphoma.

Patients/Materials \& Methods: Case series of primary bone marrow lymphoma from tertiary centre.

Results: Case 1- 26 years old male was admitted with complaints of headache for 03 months and low grade fever for 01 month. Patient also complained of low backache for 05 days. There was no organomegaly and no lymphadenopathy. Few atypical lymphocytes were noted on PBS. There was positivity for IgG EBV and Leptospira IgM/ . BM biopsy was hypercellular with diffuse infiltration was seen by large lymphoid cells and one intertrabecular space was completely replaced by these cells which were positive for CD79a, CD30 and Alk, EBV and EMA. Increased mitotic activity was seen. PET scan was done later which revealed verbal and lytic lesion with increased FDG avidity in axial as well as appendicular skeleton. Based on these findings patient was diagnosed as a case of high grade lymphomaunclassified. Patient was started on chemotherapy CHOP regime and responded well.

Case 2- A 58 yrs old lady came to accident and emergency room with history of severe lower backache since past 7 days. There was no history of trauma, fever, loss of weight. However there was history of loss of appetite since past 3-4 months. On examination her vitals were stable however her mobility was severely compromised. Patient was found to have slurred speech. CT brain showed multiple calvarial deposits. MRI lumbosacral area showed multiple lytic lesions in spine, sacrum and bilateral humerus with associated paraspinal and epidural component with fracture vertebrae at DV-11, DV-12 and LV-1. Interstial distribution of these atypical lymphocytes in BM biopsy which were positive for CD 45, CD 20, Vimentin, mum-1 and bcl-2 while negative for CD3, CD10, bc16, cycin D1, PAN-CK, CD 138 and CD 5. On the basis of above diffuse large B cell lymphoma (Non Germinal centre type). Patient was given six cycles of CHOP. After six cycles of CHOP patient went into remission.

Discussion \& Conclusion: Primary bone marrow lymphoma (PBML) is a rare entity whose clinicopathological significance are not well defined. Criteria to diagnose PBML include isolated infiltration of bone marrow, without involvement of nodal or extranodal tissues, including the bone. We present such two cases which presented as diagnostic dilemna. 
Disclosure of Interest: None Declared.

Keywords: DLBCL, Lytic lesion, Primary Bone marrow lymphoma.

\section{Abstract 3. Isolated Soft Tissue Amyloidoma of Nasal Cavity: A Rare Presentation}

SuvirSingh $^{* 1}$

${ }^{1}$ Clinical Haematology and Stem Cell Transplantation, Dayanand Medical College, Ludhiana, Ludhiana, India

Aims \& Objectives: Amyloidosis is a heterogeneous group of disorders characterized by extracellular deposition of amyloid fibrils in various organs, leading to end organ dysfunction with a variable rate of progression. Localized collection of amyloid material in the form of a mass is extremely rare.

Patients/Materials \& Methods: Mr. M presented with unilateral epistaxis for one week. On initial evaluation, he was found to have a friable soft tissue mass in the right maxillary antrum, attached to its anterior wall. He underwent excision of the nasal mass, which was followed by resolution of symptoms. Pathologic examination revealed a friable mass with a mucoid surface, mixed with blood clots. Microscopic examination showed the presence of normal airway epithelium, with subepithelial tissue showing large areas of amorphous pink material. Fibrin thrombi were noted in vessels and no fungal elements or mucin identified on the same. The pink material was positive for methyl violet and Congo red stain and showed apple green birefringence. Staining for SAA was negative, and kappa/ lambda staining was equivocal. Evaluation for systemic amyloidosis and multiple myeloma, including bone marrow examination, immunofixation electrophoresis and a PET scan was negative. No other mass was seen on PET scan. He had mild proteinuria ( $\sim 300 \mathrm{mg} / 24 \mathrm{~h}$ ), which resolved on its own. The patient was kept under follow up and continues to be asymptomatic with no evidence of systemic involvement.

Results: Amyloidosis is characterized by systemic deposition of extracellular protein, commonly leading to skin, renal, gastrointestinal or neurologic involvement. Presentation as an isolated mass is extremely rare and has been described to occur as a lung mass or in the extremities in relation to soft tissue. Such presentation as a single isolated mass in the nasal cavity has been described in less than 11 patients so far in literature.

Discussion \& Conclusion: Amyloidosis can have a variable clinical presentation and can present as an isolated mass. A high index of suspicion must be kept, and systemic involvement should be ruled out based on nature of amyloid tissue noted.

Supporting Document: 9f9db6fc-9ca5-4763-93e1-d807ee8713d8.

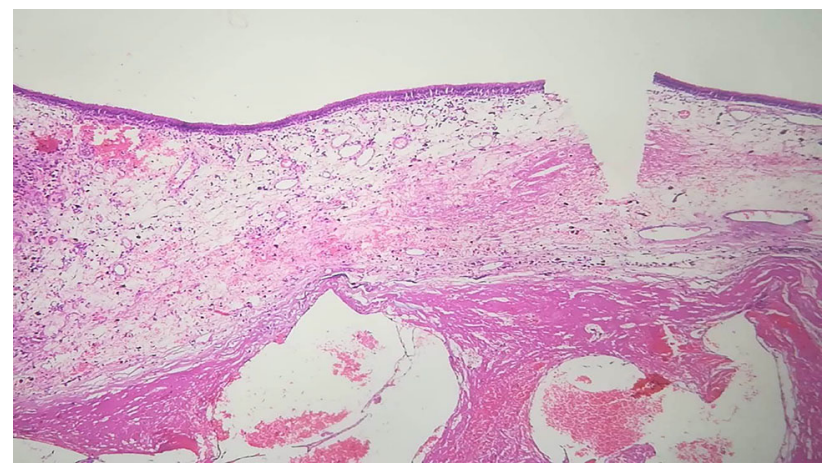

Disclosure of Interest: None Declared.

Keywords: amyloid, bleeding, myeloma.
Abstract 4. Significance of Pattern of CD200 Expression in Plasma Cell Myelomas in Indian Population

\author{
Ankur Ahuja ${ }^{1}$, Roma Hanjura ${ }^{* 1}$, YanamandraUday ${ }^{2}$, \\ KanwaljeetSingh $^{1}$, TathagatChatterjee ${ }^{1}$ \\ ${ }^{1}$ Dept of Lab and Mol Medicine, ${ }^{2}$ Dept Of Hematology,
Army Hospital RR, Delhi, India
}

Aims \& Objectives: 1. To study the prevalence and pattern of expression of CD 200 by flow cytometry.

2. To correlate the expression of CD 200 with the other lab prognostic markers.

Patients/Materials \& Methods: Total of 35 cases of plasma cell myeloma enrolled in a tertiary centre were incorporated in the study in 2018-2019. Routine clinical bone marrow samples were screened morphologically and immunophenotypically to confirm the diagnosis of plasma cell myeloma. Further, serum protein electrophoresis and serum immunofixation of these patients was done as a routine work up for myeloma. Plasma cell myeloma was diagnosed as per the International Myeloma working foundation 2014 diagnostic criteria. Samples were then analyzed for CD 200 expression by Flow Cytometer along with other plasma cell myeloma panel immunomarkers. CD 200 expression was compared with other immunomarkers and prognostic markers like $\beta 2$ microglobulin, serum creatinine, serum free light chain ratio, lactate dehydrogenase, albumin, haemoglobin, bony lesions, bone marrow plasma cells.

SPSS software, version 24.0 was used for statistical analysis and $P$ value $<0.05$ was considered statistically significant.

Results: (1) $65-70 \%$ cases of plasma cell myeloma showed positivity for CD200 with variable expression pattern.

(2) In our study significant correlation of CD200 negativity with anaemia $(\mathrm{Hb}<10 \mathrm{gm} / \mathrm{dl}),>40 \%$ plasma cells in bone marrow aspirate, diffuse sheet like pattern involvement in bone marrow biopsy and also with abnormal serum free light chain ratio was noted.

(3) Similarly, patients were showing significant correlation of moderate to bright CD200 expression with normal serum Beta 2 microglobulin level, normal serum LDH, normal serum BUN and creatinine, normal serum albumin level.

Discussion \& Conclusion: CD200 negativity can be taken as indirect evidence of poor prognosis in myeloma cases and can be thus be used as future targeted therapy in these patients to improve clinical outcome. However, further more studies in larger subset of patients is required.

Disclosure of Interest: None Declared.

Keywords: CD 200, myeloma, Prognostic factor in myeloma.

\section{Abstract 5. Comparison of Whole Body MRI and Conventional Radiography in Multiple Myeloma Related Bone Disease}

\author{
Nishilgowda $^{* 1}$, Sudhir Kumar Atri ${ }^{1}$, Seema Rohilla ${ }^{2}$ \\ ${ }^{1}$ Department of medicine and Hematology, ${ }^{2}$ Department of \\ Radiodiagnosis, Pt.B.D.SharmaPgims, Rohtak, Rohtak, India
}

Aims \& Objectives: Bone disease is one of the multiple myeloma defining events and it is usually established using the radiographic skeletal survey. Due to the low sensitivity of radiography, whole body magnetic resonance imaging (WBMRI) has emerged. MRI has the ability to detect diffuse and focal bone marrow infiltration even in the absence of osteolytic lesions on skeletal survey. The main objective of this study is to compare the skeletal survey with WBMRI in detection of skeletal lesions in multiple myeloma. 
Patients/Materials \& Methods: Thirty newly diagnosed cases of multiple myeloma underwent standard laboratory investigations, radiographic skeletal survey and whole body MRI (T1, STIR and diffusion weighted sequences) from vertex to ankles.

Results: Skeletal survey detected skeletal lesions in $70 \%$ and WBMRI in $93 \%$ of patients. $77 \%$ of patients had concordant findings between the two imaging techniques. WBMRI was found to be superior than skeletal survey in detection of skeletal lesions in cervical spine ( $33 \%$ vs. $3 \% ; P=0.004)$; thoracic spine (50\% vs. $20 \%$; $P=0.004)$; lumbar spine (63\% vs. $17 \% ; P<0.001)$; pelvis (73\% vs. $33 \% ; P<0.001)$ and clavicles $(27 \%$ vs. $3 \% ; P<0.05)$, while WBMRI was found to be inferior than skeletal survey in skull $(10 \%$ vs. $33 \% ; P=0.015)$. There was no difference $(P>0.05)$ between the imaging techniques in ribs $(13 \%$ vs. $7 \%)$; sternum $(13 \%$ vs. $0 \%)$; scapulae (10\% vs. $0 \%$ ); humeri (37\% vs. $23 \%)$ and femora (57\% vs. $40 \%$ ). In patients with lesions on WBMRI, $43 \%$ had focal pattern, $23 \%$ had diffuse pattern, $23 \%$ had both focal and diffuse pattern and $3 \%$ had variegated pattern.

Discussion \& Conclusion: WBMRI is more powerful in the detection of skeletal lesions in multiple myeloma at diagnosis than conventional skeletal survey. WBMRI is better in visualizing the lesions of spine and pelvis, while skeletal survey is better for lesions in skull. Due to the better detection of skeletal lesions at diagnosis, prognostic significance of skeletal lesions and its utility in monitoring the response to the treatment, WBMRI must be considered routinely in the management of multiple myeloma in our population.

Disclosure of Interest: None Declared.

Keywords: magnetic resonance imaging, Multiple myeloma, X-ray.

\section{Abstract 6. Experience with Brentuximab in Treatment of Hodgkin Lymphoma: A Single Centre Experience}

\author{
Nitin YashasMurthy ${ }^{* 1}$, Amit Rauthan ${ }^{1}$, Poonam Patil ${ }^{1}$, \\ Ashish Dixit ${ }^{1}$, Mallikarjun Kalashetty ${ }^{1}$

\section{${ }^{1}$ Medical Oncology \& Haematology, Manipal Hospital, Bangalore, India}

Aims \& Objectives: To describe the impact of Brentuximab in treatment of Hodgkin Lymphoma in an Indian Setting.

Patients/Materials \& Methods: Patients of Hodgkin lymphoma who received Brentuximab between 2013-2019 were reviewed. Response and Survival trends were analysed.

Results: A total of 9 patients received Brentuximab from 2013-2019. 5 received it after having relapsed post autologous stem cell transplant (auto-SCT). 3 patients received it at 1 st relapse. 1 patient is receiving it as consolidation in first line setting.

In our institute, out of the 10 patients of hodgkin lymphoma who relapsed again post auto-SCT, 5 received Brentuximab and 5 received salvage chemotherapy/best supportive care. Out of the 5 who received Brentuximab, 4 patients achieved complete response (CR) after 3 cycles and went onto receive 3 more cycles and have a ongoing $\mathrm{CR}$ at 6 years, 4 years, 1 year and 6 months of follow up respectively without any subsequent therapy, 1 patient achieved partial Response (PR) and proceeded to Allogenic stem cell transplant. In comparison, the 5 who received conventional chemotherapy/supportive care have all expired with a median survival of 18 months.

Among the 3 patients who received Brentuximab at first relapse, 1 patient recieved it with chemotherpay for 3 cycles, achieved CR and proceeded to auto-SCT. The other 2 patients received it as single agent and achieved CR after 3 cycles and received 3 more cycles and continue to remain in $\mathrm{CR}$ at follow up of 4 years and 18 months without further treatment/auto-SCT. Common toxicities noted were
Grade 2 peripheral neuropathy in 4 patients (44\%) and Leucopenia in 3 patients $(30 \%)$.

Discussion \& Conclusion: Outcomes of those with hodgkin lymphoma who relapsed post auto-SCT were poor when treated with conventional therapy as compared to brentuximab which showed high response rates and improved PFS and OS without subsequent therapy Superior outomes were also seen in patients who received Brentuximab upfront in relapsed setting as a single agent.Those achieving CR early can be considered for a shorter duration of therapy. Considering low toxicities it can be considered upfront in the relapsed/refractory setting ahead of auto-SCT.

Disclosure of Interest: None Declared.

Keywords: Brentuximab, Hodgkins Lymphoma, Relapsed hodgkins.

\section{Abstract 7. Types of B-NHL \& their Treatment Outcomes: A Tertiary Care Government Hospital Report}

Ankita Sen ${ }^{* 1}$, ShuvraneelBaul ${ }^{1}$, PrakasMandal ${ }^{1}$, RajibDe $^{1}$, PrantarChakrabarti $^{2}$, ShyamaliDutta ${ }^{1}$, TuphanDolai ${ }^{1}$

${ }^{1}$ Haematology, NRS Medical College, ${ }^{2}$ Haematology, VIMS, Kolkata, India

Aims \& Objectives: The presentation ofNon-Hodgkin lymphoma (NHL) varies with age and ranges from indolent to aggressive. B-cell NHL (B-NHL) (80-85\%) are more common than T-NHL. ${ }^{1,2}$ Lowgrade B-NHL is more common in high-income regions, while, lowand middle-income regions have more of high-grade B-NHL, T/NKcell NHL. ${ }^{3}$ Rituximab alone or as combination chemotherapy is the commonly used therapy in patients of B-NHL who require therapy. ${ }^{4}$ Our paper aims to analyse the spectrum of B-NHL and their treatment outcomes, among patients of varied physical characteristics and social demographics, who attended a tertiary care government hospital.

Patients/Materials \& Methods: Retrospective analysis of 204 patients with B-NHL, out of total 249 patients in an ongoing lymphoma registry at NRS Medical College, Kolkata over a span of 4 years (May 2014-May 2018).

Results: The median age of our patients was 47.5 years (range 2-80 years) with a male predominance (male: female 2.8:1). Diffuse Large B-Cell Lymphoma (DLBCL) was the commonest subtype (43.1\%), followed by Mantle cell lymphoma (9.3\%) and High-grade B-NHL (including Burkitt lymphoma) comprised 5.4\%. The indolent lymphomas included, Follicular Lymphoma (14.7\%), Chronic Lymphocytic Leukemia/Small Lymphocytic Lymphoma and Prolymphocytic Leukemia (2.4\%), Waldenstrom's lymphoma and Lymphoplasmacytic Leukemia (3.4\%) and Marginal Zone lymphoma (7.8\%). A group of patients were diagnosed as CD20+ B-NHL (13.7\%) and they behaved mostly as indolent lymphomas. The demographics of our patients are outlined in the included Table.

Not all patients of DLBCL could be evaluated for COO by immunohistochemistry or molecular studies. Out of those who were evaluated, Double Expressor/Double Hit Lymphomas (DE/DH) were 14/88 $(15.9 \%)$. Different treatment regimens were used for the different types of B-NHL and their variable response rates are shown in the Table.

Discussion \& Conclusion: The patients attending a government medical college mostly belong to the lower socio-economic strata, and our findings of corroborate with other studies. ${ }^{1,2}$ Male predominance noted in our study is also noted in other studies. ${ }^{1}$ DLBCL is the commonest subtype of NHL and CR rates range from $60-70 \%$, like our findings of $60.7 \% .^{4}$ The treatment outcomes and patients' loss to follow up, are primarily due to dissimilar social and economic conditions. This needs to be further studied before a conclusion can be drawn with regards patient characteristics and treatment outcomes. 
Supporting Document: 5346643a-24fb-443a-ad82-43e3fa40982c.

Table: Demographic distribution of B-NHL subtypes and their Treatment Outcomes among patients from a tertiary care hospital in eastern India

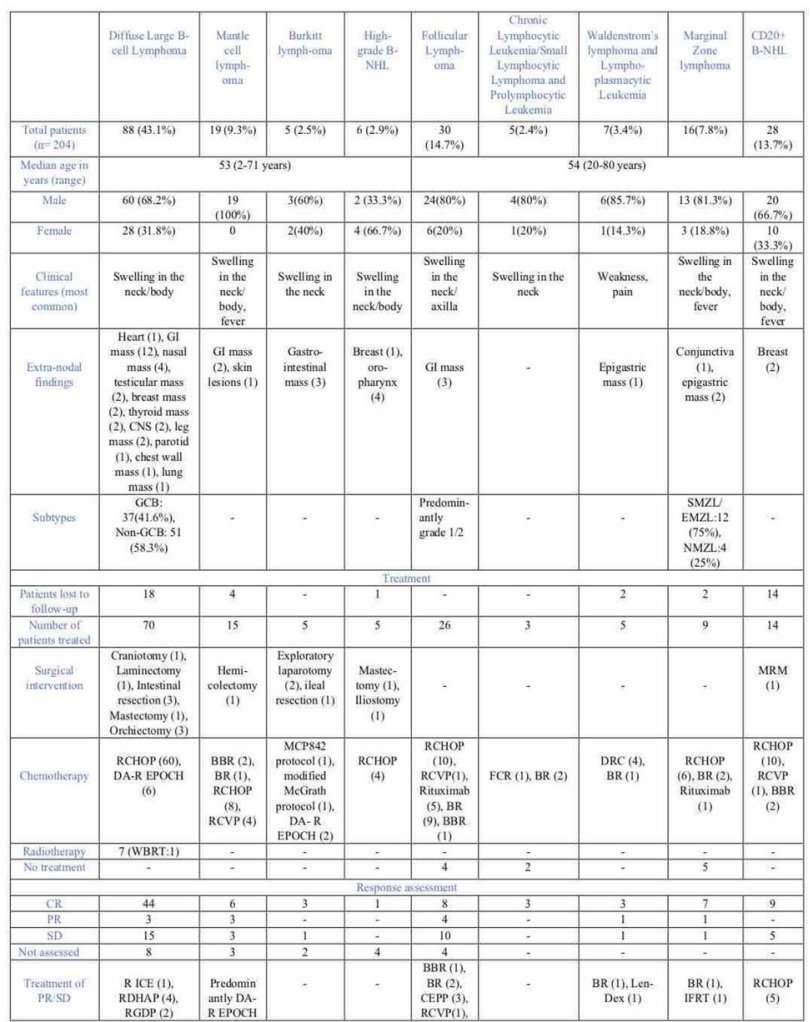

Disclosure of Interest: None Declared.

Keywords: B-NHL, Patient characteristics, treatment outcomes.

\section{Abstract 8. Cytogenetic Abnormalities in Multiple Myeloma Patients: A Single Center Experience}

\author{
LekshmiVK ${ }^{* 1}$, SanjeevanSharma ${ }^{2}$, Venkatesan $S^{3}$ \\ ${ }^{1}$ Internal Medicine, ${ }^{2}$ Haematology, ${ }^{3}$ Pathology, AFMC, Pune, \\ India
}

Aims \& Objectives: Multiple myeloma represents a malignant proliferation of plasma cells derived from a single clone. The risk stratification criteria of multiple myeloma (2014 IMWG revised) includes cytogenetic analysis of bone marrow plasma cells at diagnosis. Since 2016, our centre is sampling all initial marrows of multiple myeloma patients for a cytogenetic analysis by FISH and karyotyping.

Patients/Materials \& Methods: All patients with newly diagnosed multiple myeloma, had their initial diagnostic marrow sampled for cytogenetics by karyotyping and by FISH, using a predefined panel of markers-Del 13q, Del 17p, t(4,14), t(11,14). Cytogenetic data was co-related with clinical course, nature of monoclonal paraprotein, and response to induction therapy. Outcome data was evaluated at $\mathrm{d}+120$, $\mathrm{d}+365$ or death at any time point.

Results: Out of 36 patients ( 24 males and 12 females), with mean age of 66 (55-84) years, who had their bone marrow cytogenetic analysis done, 12 (33\%) had some detectable cytogenetic abnormality. Del $13 \mathrm{q} 14$ (intermediate risk) is the most prevalent among our patients $(\mathrm{n}=6)$. Others being $\mathrm{t}(11 ; 14)$ (standard risk) $(\mathrm{n}=3), \mathrm{t}(4 ; 14)$ (intermediate $=$ risk $)(\mathrm{n}=3)$ and del17p13 (high risk) $(\mathrm{n}=2)$. The corelation of these cytogenetic abnormalities with other parameters and clinical outcomes will be discussed.

Discussion \& Conclusion: Among various prognostic markers in multiple myeloma, cytogenetic abnormality detected by conventional cytogenetics and FISH studies are major factors deciding clinical outcome. Cytogenetic aberrations have a variable prevalence reported all over the world (33-66\%). Our study is also showing an almost same prevalence.

Despite having lesser number patients, renal dysfunction prevalence is higher in those with positive cytogenetics as compared to patients with normal cytogenetics.

Disclosure of Interest: None Declared.

Keywords: Cytogenetic abnormalities, FISH, Risk stratification.

\section{Abstract 9. Management of HLH Secondary to Advanced Hodgkin's Lymphoma in the Era of Targeted Therapy}

Shiva Kumar komaravelli ${ }^{* 1}$, NatarajKS ${ }^{1}$, Amarnadhpolisetty ${ }^{1}$, Hamza dalal $^{2}$, Aditi shah ${ }^{1}$, Sharatdamodar ${ }^{1}$

${ }^{1}$ Hematology, Narayanahrudayalaya Hospital, ${ }^{2}$ Hematology, Narayana Health, Bangalore, India

Aims \& Objectives: Hemophagocytic lymphohistiocytosis (HLH) is an uncommon disease that often presents with nonspecific findings. There are few case reports who presented with HLH secondary to Hodgkin's lymphoma, but treatment outcome was poor. Aim of this paper to reveal the role of targeted therapy in advanced Hodgkin's lymphoma with HLH.

Patients/Materials \& Methods: Collected data from medical records.

Results: Case no. 1. 22 yr old female presented with fever, neck swelling and weight loss for 1 month, evaluated elsewhere and treated as tubercular lymphadenitis. After 2 months, there was no response and she developed hyperbiliruinemia with cholestatic pattern of liver injury. On re-evaluation LN biopsy and immunohistochemistry was suggestive of Hodgkin's lymphoma, in view of worsening liver injury liver biopsy was done which showed erythrophagocytic activity with cholestasis and bone marrow examination showed HLH. She was referred to our center for further management. On admission she was in sepsis with AKI, her total bilirubin was $29 \mathrm{mg} / \mathrm{dl}$ with direct bilirubin of $22.7 \mathrm{mg} / \mathrm{dl}$, hence she was started on HLH protocol with steroids and etoposide (CSA was avoided in view of $\mathrm{AKI}$ ). But her liver parameters worsened and she developed hepatic encephalopathy, hence planned for treatment of primary disease with chemotherapy. But in view of active infection, liver failure and cytopenia ABVD chemotherapy was avoided. She was treated with Inj.Brentuximab which is an antibody and drug conjugate, she tolerated well to brentuximab. Later her sensorium improved, liver parameters improved and total WBC count recovered. After complete recovery she was discharged. Later she received 2cycles of brentuximab plus AVD chemotherapy then switched to ABVD. At present she is on regular follow up from OPD basis.

Case no.2, $39 \mathrm{yr}$ old male presented with intermittent fever, weakness, tiredness for 2 to 3 months, with past history of biopsy proven EBV related lymphadenitis. On initial evaluation he found to have pancytopenia with hepatosplenomegaly. he was not responded to antibiotics. Later he developed acute livery injury with worsening cytopenias. On further evaluation PET CT showed hepatosplenomegaly with increased FDG uptake and lymphadenopathy. Bone marrow examination was suggestive of HLH and liver biopsy was suggestive of hodgkins lymphoma. Meanwhile he developed 
hepatic encephalopathy with sepsis, hence he was started on steroids for HLH. But his liver parameters were not improved, later he was started on Inj. Brentuximab for hodgkins lymphoma. At present his sensorium improved and liver parameters are improving, he is planned for brentuximab plus AVD chemotherapy after complete recovery.

Discussion \& Conclusion: HLH is a syndrome known to have high mortality. Prognosis can markedly improve with prompt initiation of appropriate treatment, therefore it is imperative to diagnose and begin therapy early.

Disclosure of Interest: None Declared.

Keywords: Brentuximab, hemophagocytic lymphohistiocytosis, HODGKINS LYMPHOMA.

\section{Abstract 10. Histomorphological Assessment of Marrow Fibrosis in Multiple Myeloma}

\section{Snigdha Goel ${ }^{* 1}$; Ankita Jaiswal Govil, Tejinder Singh \\ ${ }^{1}$ Department of Hematology, Surgical Pathology, Oncquest Labs, Delhi, India}

Aims \& Objectives: To assess and correlate marrow fibrosis with tumor load and differentiation of plasma cells in multiple myeloma. Patients/Materials \& Methods: 97 cases of multiple myeloma were examined from January to August 2019 out of which 24 cases showed presence of fibrosis on $\mathrm{H} \& \mathrm{E}$ and was further graded on Reticulin stain.

Results: 24/97 (25\%) cases revealed presence of fibrosis on the bone marrow biopsy.

Out of these 24 cases, 10 cases $(41.6 \%)$ showed mature type of plasma cells, 5/24 (21\%) showed intermediate differentiation, $7 / 24$ (30\%) were of immature differentiation while 1 case each (4\%) was of plasmablastic and anaplastic differentiation.

On studying the pattern of infiltration, it was found that $14 / 24$ cases $(60 \%)$ involved the marrow in interstitial/nodular pattern while $10 / 24(41 \%)$ cases demonstrated complete or partial replacement of the marrow.

Reticulin stain on these biopsies revealed that 14/24 (60\%) casesshowed lower grade of fibrosis (Reticulin grade 1-2) while 10/24 $(41 \%)$ cases showed a higher grade of fibrosis (Reticulin grade 3-4).

On correlating grade of fibrosis with the pattern of infiltration (tumor load), $41 \%$ (10/24) cases showed a high tumor load (partial replacement or diffuse marrow involvement) and 8 out of these 10 cases showed a higher grade of reticulin fibrosis.

$14 / 24$ cases with low tumor load also showed lower grade of reticulin fibrosis in 12/14 cases.

Correlating fibrosis with differentiation, it was seen that $15 / 24$ (63\%) showed mature/intermediate type out of which 11 were associated with a lower grade of fibrosis.

9/24 (38\%) cases demonstrated immature/plasmablastic/anaplastic differentiation and 6 of these revealed higher grade of fibrosis.

On correlating pattern of involvement with differentiation, it was found that $15 / 24(63 \%)$ cases showed mature to intermediate differentiation, out of which $80 \%$ (12/15) showed low tumor load (interstitial/nodular).

9/24 cases $(38 \%)$ showed immature/plasmablastic/anaplastic differentiation and 07 out of these had a high tumor burden.

Discussion \& Conclusion: In our study, we found that higher tumor load in the biopsy is associated with dedifferentiated plasma cells like plasmablastic/immature/anaplastic type. Higher grade of fibrosis with increased plasma cell number on aspirate and biopsy were also recorded.

Supporting Document: 101ec602-b8ab-420a-962b-b57cd8874345.
Disclosure of Interest: None Declared.

Keywords: Fibrosis, Myeloma, Plasma cell.

\section{Abstract 11. Nivolumab as Bridge Therapy for Autologoussct in Relapsed/Refractory Hodgkin Lymphoma: Our Experience}

\author{
JaikumarPatel $^{* 1}$, Sandip Shah ${ }^{1}$, Akanksha Garg ${ }^{1}$, Kinnari Patel ${ }^{1}$, \\ Kamlesh Shah ${ }^{1}$, Asha Anand ${ }^{1}$
}

${ }^{1}$ Department of Medical Oncology, Gujarat Cancer Research Institute, Ahmedabad, Ahmedabad, India

Aims \& Objectives: To study the response rates and adverse events with use of Nivolumab in relapsed/refractory (R/R) Hodgkin lymphoma (HL) prior to autologous stem cell transplant (ASCT).

Patients/Materials \& Methods: We analyzed reponse rate and toxicity profile in 8 eligible patients with $\mathrm{R} / \mathrm{R}$ HL treated with Nivolumab before ASCT. Written informed consent was taken. Nivolumab was administered as a $3 \mathrm{mg} / \mathrm{kg}$ IV infusion in $100 \mathrm{ml} \mathrm{NS}$ over 30-45 min every 2 weeks. All study patients had disease evaluation before starting $\&$ after 4 to 6 cycles of Nivolumab and response rate assessed. The adverse events were noted if any and documented. The cost of Nivolumab was covered by state government schemes along with patient assistance program or self payment.

Results: In study 6 patients were male and 2 were female with all having ECOG Performance status 1. Six cases had Stage III or IV at diagnosis. All patients received front-line ABVD. One patient was primary refractory; others had relapsed disease and received multiple lines of therapy. The median age at Nivolumab initiation was 16 years. The median number of treatment lines prior to Nivolumab were 4 (range, 3-6). All patients received a median of 6 cycles (range 4-8) of Nivolumab. Four patients had Complete Response (CR), 2 had Partial response (PR), 2 had Stable Disease (SD). No patient had disease progression. The drug was well tolerated, with infrequent adverse events. None required discontinuation, interruption or dose reduction.

Discussion \& Conclusion: We studied outcomes and toxicity profile of $8 \mathrm{R} / \mathrm{R}$ HL patients treated with Nivolumab used as a bridge therapy for transplant. Our cohort resembles those published by Bekos et al. and Ansell et al. in terms of the patients' performance status, and median number of previous lines of therapy. Our response rates were comparable to their results. Out of 8 patients 6 patients had Overall Response Rate (ORR), 2 had SD.Only few adverse events were seen with nivolumab therapy and all of grade 1 (ctcae v5.0). Nivolumab was evaluated in a Phase I study (CHECKMATE 039) of 23 patients with $\mathrm{R} / \mathrm{R}$ HL who were heavily pre-treated with systemic therapy, including post ASCT patients. It had an ORR of $87 \%$ and a CR of 17\%.In the subsequent Phase II (CHECKMATE 205) study, the median duration of response and PFS to nivolumab was 17 months and 15 months, respectively, which compares favorably to the median PFS of 6 months in patients treated with BrentuximabVedotin.

Conclusion: Nivolumab is effective and tolerated well in patients with R/R HL. To our knowledge, this is the first study from India where Nivolumab has been used as a bridging therapy for ASCT in heavily pre-treated patients.

Disclosure of Interest: None Declared.

Keywords: bridge therapy, nivolumab, Relapsed hodgkins. 


\section{Abstract 12. Multiple Myeloma Mimicking an Abdominal Malignancy: An Unusual Presentation}

\section{Beauty Sarkar*1 $^{* 1}$, Sonalidixit ${ }^{1}$, Mrinalini kotru ${ }^{1}$, Richa Gupta ${ }^{1}$, Meera sikka ${ }^{1}$ \\ ${ }^{1}$ Pathology, University College of Medical Sciences, New Delhi, India}

Aims \& Objectives: Multiple myeloma (MM) is a progressive neoplastic proliferation of plasma cells, accounting for $10 \%$ of hematological malignancies. Typical symptoms include bone pain, malaise, anemia, renal insufficiency and hypercalcemia. We herewith report the case of a 65 year old lady who presented with hepatosplenomegaly and melena which was worked up for GI malignancy.

Patients/Materials \& Methods: A 65 year old female presented in the medicine outpatient department with fever, loss of appetite, weight loss, pedal edema for 2 months and melena 2 days prior to admission. 1 unit of RCC was transfused and patient was on vitamin B12 and folic acid.

On physical examination and ultrasonography, hepatosplenomegaly and abdominal lymphadenopathy was documented.

Results: On lab results, white blood cell count, $21.8 \times 10^{9} / 1$; platelet count, $150 \times 10^{9} / 1$, ESR $70 \mathrm{~mm} / \mathrm{hr}$. Kidney function test, serum calcium were within normal limits.

Clinically, abdominal malignancy was suspected.

On peripheral blood smear pancytopenia with microcytic hypochromic to normocytic normochromic anemia was seen.

On bone marrow aspirate, there were increase in plasma cells (26\%) with few binucleate and multinucleate forms. 6\% Plasma blasts were also seen.

On bone marrow biopsy, there were increase in plasma cells as well as plasma blasts present interstitially and paratrabecular region. So, a final diagnosis of plasma cell dyscrasia possibly multiple myeloma was made.

Discussion \& Conclusion: In rare cases, extra medullary disease may be the first symptom, making diagnosis and management more challenging. Involvement of gastrointestinal (GI) system, particularly GI haemorrhage in the course of MM is rare and is mainly due to high serum viscosity due to accumulation of immunoglobulins. Such presentation may represent poor biology of the disease or the advanced stage.

Disclosure of Interest: None Declared.

Keywords: abdominal malignancy, gastrointestinal haemorrhage, Multiple myeloma.

\section{Abstract 13. Spectrum of Renal Involvement in Patients of Multiple Myeloma}

RintuSharma $^{\text {*1 }}$, ArihantJain ${ }^{1}$, Deepesh Lad ${ }^{1}$, Gaurav Prakash ${ }^{1}$, Alka Khadwal ${ }^{1}$, RitambhraNada ${ }^{2}$, Raja Ramachandran ${ }^{3}$, Pankaj Malhotra ${ }^{1}$ and Department of Hematology

\section{${ }^{1}$ Hematology, ${ }^{2}$ Pathology, ${ }^{3}$ Nephrology, PGIMER, Chandigarh, India}

Aims \& Objectives: Renal involvement can occur in $20-30 \%$ of newly diagnosed patients with multiple myeloma. Myeloma cast nephropathy is the most common form of renal injury. We prospectively studied newly diagnosed multiple myeloma patients who presented with any form of renal injury and studied the renal outcome after institution of anti-myeloma therapy.
Aim of this study is to study spectrum of renal involvement in patients of multiple myeloma.

Patients/Materials \& Methods: We prospectively studied all newly diagnosed multiple myeloma patients who presented with renal injury from July 2018 to March 2019. Patients with baseline serum creatinine of $>2 \mathrm{mg} / \mathrm{dl}$ or eGFR $<40 \mathrm{ml} / \mathrm{min}$ by MDRD equation or $24 \mathrm{~h}$ urine protein $>300 \mathrm{mg} / \mathrm{d}$, were defined to have renal impairment (RI). Renal biopsy was done wherever possible. All patients with serum creatinine $>2.0 \mathrm{mg} / \mathrm{dl}$ received 4 drug induction regimen for one month as per institution protocol. The regimen consisted of high dose dexamethasone for 4 days followed by 4 days interval, thalidomide 50-200 mg/day and bortezomib $1.3 \mathrm{mg} / \mathrm{m} 2 \mathrm{D} 1,4,8,11$ regimen along with weekly cyclophosphamide $300 \mathrm{mg} / \mathrm{m}^{2}$. In addition, patients with cast nephropathy were offered five sessions of plasmapheresis on alternate days. After the first cycle, patients were switched to three drug regimen consisting of weekly dexamethasone, an Imid and weekly bortezomib.

Results: Among 101 patients of newly diagnosed multiple myeloma seen during thisperiod, $45(44.55 \%)$ patients had renal impairment. Median age of the patient's withRI was 61 years (range 40 to 75 years). $19(42.2 \%)$ were females while $26(57.7 \%)$ were males. 16 patients required dialysis at presentation and 5 patients received 5 sessions of plasma exchange. Twelve patients underwent renal biopsy. Most common pattern seen in renal biopsy was mixed- cast nephropathy along with proximaltubulopathy, amyloidosis or podocytopathy. In patients where biopsy couldn't bedone $(n=33)$, probable cast nephropathy was attributed as the pathology in 20 (60\%) patients followed by hypercalcemia, seen in $18(54.4 \%)$ patients. Out of these, 7 patients were assumed to have dual pathology-hypercalcemia and probable cast nephropathy. 4 patients had isolated proteinuria of $>1 \mathrm{~g}$ - likely light chain proteinuria. Complete renal response was seen in $31(73 \%)$ patients and partial/minor renal response in 7 patients after institution of appropriate anti-myeloma therapy. Out of 16, 11 patients became dialysis independent. 7 patients died, all the deaths being in first two months of diagnosis.

Discussion \& Conclusion: Wide spectrum of renal impairment is seen in myeloma patients who present with renal injury. The most common manifestations was cast nephropathy followed by hypercalcemia. Prompt identification and aggressive management with early institution of anti-myeloma therapy can help in restoration of renal function in view of potential reversibility. However further studies are required to decrease early mortality.

Disclosure of Interest: None Declared.

Keywords: Cast Nephropathy, Multiple myeloma, Renal Impairment.

\section{Abstract 14. A Comprehensive Serum Microrna Profiling in Indian Multiple Myeloma Patients}

\section{HarshiniSriram $^{* 1}$, Syed Hasan ${ }^{2}$, Sitaram Ghogale ${ }^{2}$, Nilesh Deshpande ${ }^{1}$, Badrinath Yajamanam ${ }^{1}$, Nikhil Patkar ${ }^{1}$, Manju Sengar ${ }^{3}$, NavinKhattry ${ }^{2}$, PapagudiSubramanian ${ }^{1}$, Sumeet Gujral ${ }^{4}$, Prashant Tembhare ${ }^{1}$ \\ ${ }^{1}$ Hematopathology, ${ }^{2}$ ACTREC, ${ }^{3}$ Tata Memorial Centre, Navi Mumbai, ${ }^{4}$ Tata Memorial Centre, Mumbai, India}

Aims \& Objectives: To study a comprehensive serum microRNA (miRNA) profiling in Indian multiple myeloma (MM) patients treated with VCD-protocol to explore their clinical relevance in the prediction of initial response.

Patients/Materials \& Methods: Serum miRNA-profiling was performed in a discovery cohort of 20 newly diagnosed MM patients and 4 age-matched healthy controls. Affymetrix 4.1 24-Array 
platform was used for expression profiling and data was analyzed using GeneSpring.

Results: Our study included 20 newly diagnosed MM patients (10 males and 10 females) with age-range 35-75 years. A comprehensive miRNA-profiling revealed $\mathbf{1 3}$ miRNAs were differentially expressed between MM patients and healthy controls. Among these, 10 miRNAs were significantly down-regulated, and 3 were up-regulated, with $\boldsymbol{p}$ values $<\mathbf{0 . 0 5}$. Down-regulated miRNAs include let-7a-5p, miR-23a-3p, miR-150-5p, miR-320a, miR-342-3p, miR-320b, miR$320 \mathrm{c}, \mathrm{miR}-320 \mathrm{~d}, \mathrm{miR}-4467$ and miR-4485. Up-regulated miRNAs are miR-1281, miR-4440 and miR-4801. Expression pattern of these 13 miRNAs divided MM patients in the study into $\mathbf{2}$ clusters. However, the cohort of 20 was too small for any statistical correlation with the initial response. Hence, miRNA profiling is being performed in an additional 65 newly diagnosed MM patients.

Discussion \& Conclusion: Our results revealed dysregulationof a new set of miRNAs as compared to reports published in western studies. It may be due to the ethnic variations of Indian patients from the western population. To validate our finding, we are studying the expression of these in an additional $65 \mathrm{MM}$ patient serum samples by NGS, and the results of the same will be presented in the meeting.

Disclosure of Interest: None Declared.

Keywords: Biomarker, microRNAs, Multiple myeloma.

\section{Abstract 15. Rare Initial Presentation of Autonomic Dysfunction in Cardiac Amyloidosis with Multiple Myeloma}

\author{
Anil Kumar Behera ${ }^{* 1}$, Sarat C. V Talluri \\ ${ }^{1}$ Internal Medicine and Critical Care, Care Hospital, \\ Banjara hills, ${ }^{2}$ Internal Medicine and Critical Care, \\ Care hospital, Hyderabad, India
}

Aims \& Objectives: Amyloid cardiomyopathy is a rare disease characterised by the extracellular deposition of insoluble fibrils resistant to proteases. Patient presenting autonomic dysfunction intially then diagnosed to have amyloid cardiomyopathy and multiple myeloma is rare. so we are presenting a case intially presenting orthostatic hypotension diagnosed to have multiple myeloma and cardiac amyloidosis.

Patients/Materials \& Methods: A 65 year old healthy man with previous no comorbidities came with chief complain of giddiness and significant weight loss. On examination, no evidence of lymphadenopathy, organomegaly and all other systems are fine except he had orthostatic hypotension. On evaluation, haemogram showed anemia and high ESR. Renal parameters showed increased creatinine and normal electrolytes. Liver parameters showed albumin globulin reversal. 2D ECHO showed characteristic restrictive LV filling with hypertrophied LV with characteristic sparkling appearance of the septum. Cardiac MRI showed restrictive cardiomyopathy and T1 mapping showed evidence of cardiac amyloidosis. Serum electrophoresis showed grossly elevated kappa chains. Bone marrow aspiration and biopsy was done which showed increased plasma cells. Pet scan showed no increased uptake for except marrow. Serum B2 microglobulin and albumin was done and diagnosed to have multiple myeloma (ISS Stage 3).

Results: Patient was diagnosed to have cardiac amyloidosis with multiple myeloma initially presenting as orthostatic hypotension. Chemotherapy patient was started with 3 drug regimen chemotherapy with alpha agonist midodrine. Patient improved symptomically and on regular follow up.

Discussion \& Conclusion: Amyloid cardiomyopathy remains a challenging condition to diagnose and treat. Mostly supportive therapy along with chemotherapy drugs patient initially presenting autonomic dysfunction evaluated to have cardiac amyloidosis underlying plasma cell disorders is rare. So patient presenting autonomic dysfunction, cardiac amyloidosis should be kept as differential though rare.

Disclosure of Interest: None Declared.

Keywords: Amyloid, Autonomic Dysfunction, Multiple myeloma.

\section{Abstract 16. Interphase Fish in Multiple Myeloma: A Retrospective Institutional Analysis of 126 Patients}

\author{
Sumeet Mirgh $^{* 1}$, AnveshikaSoni ${ }^{1}$, Jyotsna Kapoor ${ }^{1}$, \\ VishvdeepKhushoo $^{1}$, FaranNaim ${ }^{1}$, RayazAhmed ${ }^{1}$, \\ Narendra Agrawal ${ }^{1}$, Dinesh Bhurani ${ }^{1}$

\section{${ }^{1}$ Hematology and BMT, Rajiv Gandhi Cancer Institute and Research Centre, New Delhi, India}

Aims \& Objectives: To observe and analyse the clinico-pathological profile and therapy outcomes amongst the various cytogenetic risk groups in Multiple Myeloma.

Patients/Materials \& Methods: Retrospective single-centre study conducted at a single tertiary care centre from North India. Multiple Myeloma interphase FISH analysis was performed on bone marrow samples on enriched plasma cells using specific DNA probes-Del 13q14.3 (LSI D13S25), t(4;14) (KreatechIgH/GFR3 DC-DF), t $(11 ; 14)$ (Zytovision directly labelled IgH/CCND1 DC-DF), $t(14 ; 16)$ (KreatechIgH/MAF DC-DF), Del 17p13.1 (LSI TP53). Recently, a probe for chromosome 1 (1q gain/1p deletion) has been introduced. A cut off for detection of deletion/fusion signal in normal individuals was taken as $3 \%$. Records of patients in the last 2.5 years (November 2016-March 2019) with a minimum follow-up of 4 months were analysed.

Results: MM FISH panel was done in 126 patients. Amongst these, 112 were newly diagnosed and 14 were relapsed. Of these, abnormalities were detected in nearly half $(n=61 ; 48.4 \%)$ of the patients. The distribution of patients is ISS-1/2/3 were $20.6 \%(n=26) / 40.5 \%$ $(n=51) / 34.2 \%(n=43)$,respectively. Majority of patients belonged to R-ISS $2(57.9 \% ; n=73)$ group. Frequency of cytogenetic abnormalities were as follows: $13 \mathrm{q}$ deletion $(\mathrm{n}=43 ; 34.2 \%), \mathrm{t}(11 ; 14)(\mathrm{n}=9 ; 7.2 \%)$, $\mathrm{t}(4 ; 14) \quad(\mathrm{n}=8 ; 6.4 \%), \quad \mathrm{t}(14 ; 16) \quad(\mathrm{n}=17 ; 13.5 \%), \quad 17 \mathrm{p} \quad$ deletion $(\mathrm{n}=10 ; 7.9 \%)$, 1q gain $(\mathrm{n}=1 ; 0.7 \%)$. Nearly, one-fifth $(\mathrm{n}=25 ; 19.8 \%)$ patients had dual/triple abnormalities. Most common symptom across all groups was bone pains present in $94.5 \%$ patients $(n=119)$, followed by anemia $(n=64 ; 50.3 \%)$. Renal dysfunction was commonly seen in $58.8 \%(\mathrm{n}=10)$ patients in $\mathrm{t}(14 ; 16)$ group with MAF translocation.Data regarding treatment response was available in 95 patients, which were graded as per IMWG uniform response criteria. Induction regimens used were VRd $(\mathrm{n}=94 ; 74.6 \%)$, VTd $(\mathrm{n}=4 ; 3.2 \%)$, VCd $(\mathrm{n}=15 ; 12 \%)$, doublet $(\mathrm{n}=10 ; 7.9 \%)$, Others $(\mathrm{n}=2 ; 1.6 \%)$. Amongst 95 patients, ORR was observed in $61 \%(\mathrm{n}=58)$ patients. The ORR (CR/VGPR/PR) to first line induction therapy of patients in different groups were-Normal, 13q deletion, $\mathrm{t}(4 ; 14), \mathrm{t}(11 ; 14), \mathrm{t}(14 ; 16), 17 \mathrm{p}$ deletion, were $69.2 \%(\mathrm{n}=45), \quad 62.7 \%(\mathrm{n}=27), 62.5 \%(\mathrm{n}=6)$, $77.8 \%(n=7), 52.9 \%(n=9), \quad 40 \%(n=4), \quad$ respectively. Similarly, amongst patients with dual/triple abnormalities-ORR was $64 \%(\mathrm{n}=16)$.

Discussion \& Conclusion: In Indian setting, frequency of chromosomal aberrations are less common than the western counterpart. Both iFISH with selective molecular profiling may further improve classification and contribute in risk stratification of disease which should be adapted in standard routine care of MM patients in clinical practice.

Disclosure of Interest: None Declared. 
Keywords: Cytogenetic abnormalities, FISH, Multiple myeloma.

\section{Abstract 17. Role of Flowcytometry in Diagnosis of Angioimmunoblastic T-Cell Lymphoma}

\section{Fatima Khan $^{* 1}$, Rashmi Kushwaha ${ }^{2}$ and King GEORGE MEDICAL COLLEGE}

\section{${ }^{1}$ Pathology, King Georges Medical University, ${ }^{2}$ Pathology, King George's Medical University, Lucknow, India}

Aims \& Objectives: Angioimmunoblastic T-cell Lymphoma is a neoplasm of mature TFH cells characterized by systemic disease. It is a rare form of Peripheral T-cell Lymphoma representing only 1 to $2 \%$ of all cases of Non Hodgkin's lymphoma and nearly 1 in 5 cases of Peripheral T-cell lymphoma diagnosed per annum. Its diagnosis remains a challenge as clinical presentation as well as pathological findings are frequently misleading. We present a case of Angioimmunoblastic T-cell Lymphoma diagnosed on FNAC Flowcytometry. Patients/Materials \& Methods: A 64-year-old male presented with bilateral cervical, axillary and intra abdominal lymphadenopathy with splenomegaly $3 \mathrm{~cm}$ below costal margin. His CBC parameters were Hb: 8 gm \%, TLC: 6500/cu.mm, DLC: P60, L30, E4, M6, Platelet: 2,10,000/cu.mm. Biochemical parameters were Serum Proteins: $11.6 \mathrm{~g} / \mathrm{dl}$, Albumin $4.5 \mathrm{~g} / \mathrm{dl}$, Globulin: $7.1 \mathrm{~g} / \mathrm{dl}$, LDH: $600 \mathrm{U} / \mathrm{L}$. FNAC done from cervical node was suggestive of Non Hodgkin's Lymphoma. Flowcytometric immunophenotyping was done from fine needle aspirate sample using BD FACS Calibur.

Results: Cells were gated on CD45 versus side scatter. Approx $60 \%$ cells gated were found to be positive for CD3, CD5, CD4, CD10 and negative for CD20, CD8, CD19, CD34. So diagnosis of T cell lymphoma with TFH cell origin was made. Mean while we also received biopsy of lymph node. Histomorphology showed effaced architecture with partially preserved follicles. Stroma has numerous high endothelial venules. IHC showed neoplastic cells positive for CD3, CD5, CD10 and BCL6. CD20, CD30, CD56, BCL2 were negative. So diagnosis of Angioimmunoblastic T cell lymphoma was made.

Discussion \& Conclusion: Angioimmunoblastic T-cell Lymphoma is a systemic disease characterised by monoclonal proliferation of $\mathrm{T}$ cells expressing CD3 and CD4. Characterstically, the tumor cells show immunophenotype of normal TFH cells, expressing CD10, CXCL13, BCL6. Flowcytometry on fine needle aspirate material helps in providing prompt diagnosis.

Disclosure of Interest: None Declared.

Keywords: Flowcytometry, Angioimmunoblastic T-cell lymphoma, Immunohistochemistry.

\section{Abstract 18. To Study the Role of FDG PET as a Tool of Disease Burden Estimation in Multiple Myeloma Patients}

\author{
Saurabh Pandey ${ }^{*}$, SanjeevanSharma ${ }^{1}$, Anil Kumar ${ }^{2}$ \\ and Armed Forces Medical College
}

\section{${ }^{1}$ Internal Medicine, ${ }^{2}$ Nuclear Medicine, AFMC, Pune, India}

Aims \& Objectives: To study the role of FDG PET Scan as a tool of disease burden estimation in multiple myeloma patients.

Patients/Materials \& Methods: All subjects meeting the eligibility criteria were included in the study. After obtaining written consent, a brief history and case-file review for noting the time of onset and clinical and laboratory parameters at the time of diagnosis, and clinical examination was performed. The patients were given induction chemotherapy as per institutional protocol. Disease evaluation at end of induction was done using the standard bone marrow morphology and serum paraprotein estimation. All patients also underwent FDG PET scan for disease assessment at end of induction therapy. Concordance in remission status was compared between the standard disease assessment tools and that given by FDG PET. The PET data was scored on the basis of the no of lesion and max and avg SUV. All patients were followed up till discharge or death in hospital. Results: 36 patients ( 32 males and 04 females), with mean age of 51 (32-72), underwent FDG PET after diagnosis of MM at least once at time of diagnosis or after completion of chemotherapy to document remission. 34 (94\%) who had evidence of clinical disease activity and all of them showed metabolically active lesions on FDG PET. All 36 patients when they achieved disease remission on bone marrow examination, also showed PET negative images, thus having complete concordance in predicting remission.

Discussion \& Conclusion: FDG PET can be used as a non-invasive tool with good correlation of disease activity for follow up avoiding the need for invasive procedures like BM studies.

Supporting Document: ef1166bb-dddb-4e10-811a-ab5fe8684450. Disclosure of Interest: None Declared.

Keywords: Disease burden, FDG PET, Multiple myeloma.

\section{Abstract 19. Results of Interim PET-Guided Therapy of Classical Hodgkin Lymphoma: A Single-Center Experience}

Ashok RAJOREYA ${ }^{* 1}$, Sudeep Vaniya ${ }^{1}$, Anamika Bakliyal, DebranjaniCHATTOPADHYAY ${ }^{1}$, RituparnaChetia ${ }^{1}$, JivakBansal $^{1}$,.deepak Sundriyal ${ }^{1}$, Uttam Kumar Nath ${ }^{1}$

\section{${ }^{1}$ AIIMS Rishikesh, Rishikesh, India}

Aims \& Objectives: Classical Hodgkin lymphoma (CHL) is a highly curable neoplasm. ABVD still remains the gold standard chemotherapy regimen in CHL. Assessment of response to $1^{\text {st }}$ line chemotherapy by interim positron-emission tomography (PET)-CT could potentially identify CHL patients who may benefit from therapy escalation. The present study describes the results of interim PET-CT guided therapy in patients with classical Hodgkin lymphoma at AIIMS Rishikesh.

Patients/Materials \& Methods: Our prospective study enrolled total 37 patients of CHL between July 2016 \& July 2019. International prognostic score (IPS) was calculated for patients with stage III-IV disease. All patients were initially treated with ABVD chemotherapy. Interim PET-CT with Deauville score was done after 2 cycles. Patients with Deauville score $\leq 3$ received ABVD (2-4 additional cycles according to stage). Patients with Deauville score 4-5 on interim PET-CT received 4 cycles of Escalated BEACOPP chemotherapy if deemed fit, followed by end-of-treatment PET-CT for response assessment. Patients with Stage I-II disease received radiation therapy as per standard guidelines.

Results: Median patient age was 34 years (range 5-70 years). Nine patients $(24 \%)$ had early stage disease [stage I, II non-bulky], and 28 patients $(76 \%)$ had advanced stage disease [stage II bulky, III, IV]. Nineteen out of 28 patients $(68 \%)$ with advanced stage disease had IPS score $\geq 4$. Interim PET-CT could be done in 31 patients. Twentytwo patients $(71 \%)$ had complete metabolic response (CMR) [Deauville score $\leq 3$ ] on interim PET-CT, \& received total 6 cycles of ABVD. Therapy was intensified to Escalated BEACOPP regimen in 5 out of 9 patients with Deauville score $4-5$ on interim PET-CT, \& the rest were continued on ABVD therapy as they were not deemed fit for more aggressive chemotherapy due to advanced age, poor 
performance status or significant comorbidity. One patient with Deauville 5 on interim PET was refractory to Escalated-BEACOPP \& died of relapse post-autologous transplantation. Treatment results are summarized in Table 1.

Discussion \& Conclusion: Interim PET-CT identifies classical Hodgkin lymphoma patients who have suboptimal response to $1^{\text {st }}$ line ABVD chemotherapy, \& are likely to benefit from escalation of therapy. Therefore, interim PET-CT should be incorporated in the CHL treatment algorithm for risk-adapted management.

Supporting Document: 4c48c06d-5fa2-47e0-a57e-31bcf9666bae.

Table 1

\begin{tabular}{|c|c|c|c|c|c|}
\hline \multirow{2}{*}{ CHL Stage } & \multicolumn{3}{|c|}{ Interim PET-CT result } & \multicolumn{2}{c|}{ Subsequent chemotherapy } \\
\cline { 2 - 6 } & $\begin{array}{c}\text { Deauvi ABVD } \times 2 \text { cycles [n=31] } \\
1-3\end{array}$ & $\begin{array}{c}\text { Deauville } \\
4-5\end{array}$ & $\begin{array}{c}\text { Not } \\
\text { done }\end{array}$ & $\begin{array}{c}\text { ABVD } \times 4 \\
\text { cycles }\end{array}$ & $\begin{array}{c}\text { Escalated BEACOPP } \\
\text { x 4 cycles }\end{array}$ \\
\hline $\begin{array}{c}\text { Limited stage } \\
\text { (I, II non-bulky) } \\
\text { [n=9] }\end{array}$ & 7 & 2 & -- & 8 & 1 \\
\hline $\begin{array}{c}\text { Advanced stage } \\
\text { (II bulky, III, IV) } \\
\text { [n=28) }\end{array}$ & 15 & 7 & 6 & 24 & 4 \\
\hline
\end{tabular}

Disclosure of Interest: None Declared.

Keywords: Classical Hodgkin lymphoma (CHL), interim positronemission tomography (PET)-CT, International prognostic score (IPS).

\section{Abstract 20. Results of DA-Epoch-R Chemotherapy in High-Grade B-Cell Lymphomas: A single-Center Experience}

Sudeep Vaniyath $^{* 1}$, RituparnaChetia ${ }^{1}$, DebranjaniChattopadhyay ${ }^{1}$, Ashok Rajoreya ${ }^{1}$, Anamika Bakliwal $^{1}$, Uttam Kumar Nath ${ }^{1}$

\section{${ }^{1}$ Department of Clinical Haematology, AIIMS RISHIKESH, RISHIKESH, India}

Aims \& Objectives: High-grade B-cell Non-Hodgkin Lymphomas (HGBL) are a heterogeneous group of aggressive lymphomas, and Rituximab + CHOP chemotherapy is associated with inferior outcomes. There is scarcity of data on efficacy \& safety of Dose-adjusted $\mathrm{EPOCH}+$ Rituximab (DA-EPOCH-R) regimen in HGBL patients in India. Our study describes the results of DA-EPOCH-R chemotherapy in HGBL patients at AIIMS Rishikesh.

Patients/Materials \& Methods: We analyzed data of 12 patients of HGBL treated with DA-EPOCH-R (dose-adjusted etoposide, prednisolone, vincristine, cyclophosphamide \& doxorubicin, plus rituximab) regimen between November 2017 \& July 2019. Staging PET-CT, bone marrow biopsy \& CSF analysis were done in all patients. C-MYC rearrangement was done in willing patients. Chemotherapy was initiated at dose level 1 , and subsequent dose levels were escalated/de-escalated based on nadir absolute neutrophil count (ANC) \& nadir platelet count, as per protocol. Pegylated-Filgrastim was routinely administered on day 6 for febrile neutropenia prophylaxis. Total eight doses of intrathecal methotrexate were administered for CNS prophylaxis as per protocol. End of treatment PET-CT was done for assessment of treatment response.

Results: Median age of patients was 46 years (range 22-64 years). Nine patients $(75 \%)$ were male. Majority $(80 \%)$ had advanced stage disease (stage III-IV). Median Ki-67 index was 80\% (range 70-100\%). Eight patients $(67 \%)$ had poor-risk R-IPI (score $\geq 3$ ). Seven patients $(58 \%)$ had extra-nodal involvement, \& seven patients (58\%) had bulky disease $(\geq 7.5 \mathrm{~cm})$. Median number of DA-EPOCH-R cycles administered was
4 (range 4-6 cycles); 50\% patients tolerated up to dose level 3. Eight patients $(67 \%)$ achieved complete metabolic response (CMR) on PET$\mathrm{CT}$, three patients had residual PET positivity, \& one patient had refractory disease requiring salvage chemotherapy. Grade 4 neutropenia $\&$ grade 4 thrombocytopenia occurred in $67 \%$ \& 50\% patients respectively. The commonest grade 3 non-hematological toxicity was nausea/ vomiting $(25 \%)$. Dose level de-escalation was required in 4 patients due to febrile neutropenia; three patients had septic shock. There was no incidence of treatment discontinuation due to toxicity, and no treatmentrelated mortality.

Discussion \& Conclusion: Our short-term experience with DAEPOCH-R chemotherapy regimen in high-grade B-cell lymphoma shows encouraging results, with satisfactory response rates \& manageable toxicity. Long term follow-up and enrollment of larger number of patients is planned.

Disclosure of Interest: None Declared.

Keywords: da-epoch-r, hgbl, toxicity.

\section{Abstract 21. Multiple Myeloma with Chromosomal Aberrations: Retrospective Study from Eastern India}

\section{Karuna Jha $^{* 1}$, Sandeep Saha ${ }^{1}$, MaitreyeeBhattacharyya ${ }^{1}$ \\ ${ }^{1}$ Institute of Hematology and Transfusion Medicine, Kolkata, India}

Aims \& Objectives: To study the clinical and biochemical behavior and response to therapy in myeloma patients with chromosomal aberrations.

Patients/Materials \& Methods: We conducted a retrospective study at our institute to identify patients diagnosed as MM from 2015 to 2018. We studied the epidemiological, clinical and biochemical profile and the outcome of the treatment in terms of response rates. The diagnosis, risk stratification, prognostic scoring and response evaluation were done as per the guidelines by the International Myeloma Working Group.

Results: $51.14 \%$ patients (179/350) were evaluable at the time of the study. The median age at diagnosis was 57 years (range 40-73 years) with a male: female ratio of 2.4:1. $16 \%$ of the patients had karyotypic abnormalities done by FISH. Most common presentation was with anemia and low back pain $(100 \%)$. Lytic lesions, hypercalcemia and renal failure were present in $34 \%, 17 \%$ and $17 \%$ respectively. Bone marrow plasma cells ranged from $25-55 \%$, IgG-K being most common immunoglobulin. The patients having $13 \mathrm{q}$ deletion, $17 \mathrm{p}$ deletion, $\mathrm{t}(4 ; 14), \mathrm{t}(14 ; 16)$ and multiple aberrations were $31 \%, 13 \%, 20 \%, 3 \%$ and $31 \%$ respectively. In patients with $13 \mathrm{q}$ deletion, $88 \%$ belonged to R-ISS-II stage, median duration of therapy being 18 months; response was VGPR in $77.7 \%$ and PR in $11.11 \%$ cases. In patients with $17 \mathrm{p}$ deletion, all patients were in R-ISS-III stage, responses achieved were VGPR $(50 \%)$ and CR $(50 \%)$. In $\mathrm{t}(4 ; 14)$ group: $50 \%$ were in R-ISS-III stage; interestingly the light chain secreted in this group along with IgG was lambda; VGPR and CR achieved were $66.6 \%$ and $33.3 \%$ respectively. In the category with multiple aberrations, $13 \mathrm{q}$ del coexisted with $\mathrm{t}(4 ; 14)$ in $33.3 \%$, with $17 \mathrm{p}$ del in $33.3 \%$, with $\mathrm{t}(11 ; 14)$ in $22.2 \%$ cases; $55 \%$ patients belonged to R-ISS-III category; $88.8 \%$ achieved VGPR response.

Discussion \& Conclusion: Comprehensive cytogenetic data in MM are lacking in the Indian population.In our study, 13q deletion was the most common aberration occurring singly or with other genetic mutations. $17 \mathrm{p}$ deletion was found in the younger age group as compared to the rest. The response rates were variable in the different groups depending on many patient related and therapy factors.

Disclosure of Interest: None Declared.

Keywords: chromosomal aberrations, Multiple myeloma, therapy response. 


\section{Abstract 22. Real World Scenario of Bortezomib- Thalidomide-Dexamethasone(VTD) in Newly Diagnosed Multiple Myeloma}

Prakash Shekhawat ${ }^{* 1}$, Ankit Jitani ${ }^{1}$, Malini Garg ${ }^{1}$, PrakasMandal $^{1}$, SubhamBhattacharya ${ }^{1}$, ShuvraneelBaul ${ }^{1}$, RajibDe $^{1}$, TuphanDolai ${ }^{1}$

\section{${ }^{1}$ haematology, NRS Medical College, Kolkata, India}

Aims \& Objectives: Although myeloma management has evolved significantly over the last few years with better response rate and prolonged progression free survival with the advent of novel agents. VTD still holds a place as standard induction regimen in myeloma. We retrospectively analysed newly diagnosed myeloma patients with VTD. The primary end points were depth of response. The secondary end point was safety.

Patients/Materials \& Methods: All myeloma patients treated upfront with VTD chemotherapy from January 2014 to July 2019 were analysed. Regimen consisted of bortezomib $1.3 \mathrm{mg} / \mathrm{m}^{2}$ and dexamethasone $40 \mathrm{mg}$, weekly on day $1,8,15,22$ or day $1,4,8,11$; and thalidomide $100 \mathrm{mg}$ dailyper 28-day cycle. Response assessment was done by standard IMWG criteria.

Results: A total of 188 patients with median age of 51 years (range $25-74)$, male: female ratio of $2.24: 1$ were analysed. The most common symptom was anaemia $69 \%$ (131/188), followed by bone lesions $48 \%$ (92/188), renal impairment 34\% (64/188), and hypercalcaemia $23 \%$ (44/188). Involved immunoglobulin Isotype were IgG 56\% (106/ 188); IgA 20\% (38/104); and light chain $15.95 \%$ (30/188). ISS staging in decreasing frequency were, ISS-III 52.1\% (98/188); ISS-II $24.4 \%$ (46/188); and ISS-I $21.8 \%$ (41/188). Patients with response rate $\geq$ VGPR was $65.95 \%(124 / 188)$ after 4 cycles and $76.65 \%(146 /$ 188 ) after 6-8 cycles. Autologous stem cell transplant was performed in $24.27 \%$ (25/103) eligible patients. Maintenance therapy was given to $81.25 \%(65 / 80)$ patients. The major non-hematological toxicity were peripheral neuropathy (overall $45.2 \%$, grade $3 / 4: 6.38 \%$ ), constipation $(31.9 \%)$, infection $(11.7 \%)$, rash $(10.63 \%)$, anddiarrhoea (overall 9.24\%, grade 3/4: 1.56\%). The major hematological toxicities (all grades)were neutropenia (18.61\%), thrombocytopenia $(11.17 \%)$, and thrombosis $(6.38 \%)$. Allcause mortality was $5.3 \%$ (10/188).

Discussion \& Conclusion: VTD is a cheap, effective and safe drug regimen for newly diagnosed multiple myeloma patients, with good depth of response and manageable toxicity.

Disclosure of Interest: None Declared.

Keywords: NDMM, VGPR, VTD.

\section{Abstract 23. A Retrospective Comparitive Study of Paediatric and Adult Burkitts Lymphoma in Last 10 Years}

SreeVamsee Chetana Panthula ${ }^{* 1}$, Arun Philip ${ }^{1}$, PavithranK ${ }^{1}$, Wesley jose $^{1}$, Neeraj Sidharthan ${ }^{1}$, InduNair ${ }^{2}$

${ }^{1}$ Medical Oncology, ${ }^{2}$ Pathology, Amrita Institute of Medical Sciences, Kochi, India

Aims \& Objectives: A Retrospective comparative study of Paediatric and Adult Burkitts Lymphoma in a Single Institution over last 10 years.

Patients/Materials \& Methods: All patients with the proven histological diagnosis were included in the study. Patients were divided and Paediatric $(<18 \mathrm{yr})$ and Adult $(>18 \mathrm{yr})$ populations. Age at time of diagnosis, nodal/extra nodal status, LDH at time of diagnosis, albumin level and status at last follow up were noted for evaluation.
Results: A total of 20 patients were analyzed retrospectively. 4 patients were excluded from the analysis due to incomplete data. $43.75 \%$ patients were below 18 years and $56.25 \%$ patients above 18 years. The male to female ratio was 3.1. All paediatric patients were males.

$71 \%$ of paediatric and $42 \%$ of Adult patients had extranodal disease.

$22.5 \%$ of adult patients presented with the B symptoms. B symptoms were not noted paediatric population. $28 \%$ of patients were Immuno suppressed. One patient was HIV positive. Other patient was a renal allograft recipient.

The Median value for $\mathrm{LDH}$ for adult population was 1500 . The Median LDH for Paediatric population was 2178. The Median value for Serum albumin in paediatric and adult population was 3.4 and 2.8 respectively. Mortality was $33.3 \%$ in Paediatric and $30 \%$ in Adult population.

The Mean Survival time for patients $<18$ yrs was $417 \pm$ 51.45 days and $2073 \pm 479$ days for Adults. The overall survival was better in Adults ( $p$ value 0.7)

The Mean survival time for albumin $>3.5$ was $1955 \pm 516$ days than for albumin $<3.5 \mathrm{gm}$ of $1189+446$ days ( $p$ value 0.9 ).

The Mean survival time for patients treated before 2010 was $357+70$ days when compared to patients treated after 2010 with $2303+473$ days ( $p$ value .29 )

The Mean Survival time for patients with extranodal disease was $1209+411$ days when compared to those with nodal disease of $2182+521$ days ( $p$ value 0.2 )

Discussion \& Conclusion: The Survival time was better in adult patients treated. Improved survival noted for patients treated after 2010 due to availability of better supportive care. However the results were not significant due to small sample size.

Supporting Document: 0407f93b-67c5-42c6-912c-693f20ed22d3.

Survival Functions

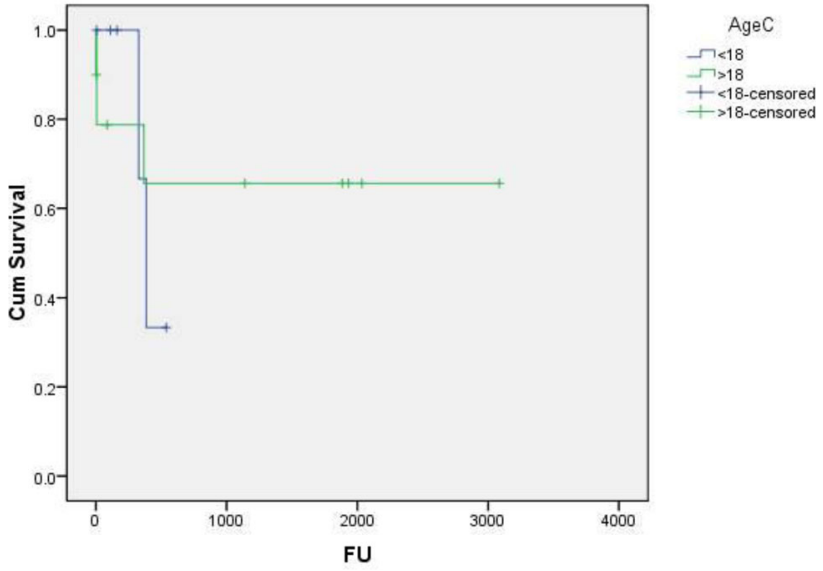

Disclosure of Interest: None Declared.

Keywords: Adult Burkitts, Comparative study, Paediatric Burkitts.

\section{Abstract 24. Clinical Spectrum of Patients with Primary GI Lymphoma in a Tertiary Care Centre from North India}

CharanpreetSingh $^{* 1}$, ArihantJain ${ }^{1}$, Deepesh Lad ${ }^{1}$, Ashok Pannu ${ }^{1}$, Alka Khadwal $^{1}$, Vikas Suri ${ }^{1}$, Ashish Bhalla ${ }^{1}$, Pankaj Malhotra ${ }^{1}$, Savita Kumari $^{1}$, Gaurav Prakash ${ }^{1}$

${ }^{1}$ Internal Medicine, PGIMER, Chandigarh, India 
Aims \& Objectives: The gastro-intestinal tract is the most common extra-nodal site of involvement for Non-Hodgkin lymphomas. Though $5-20 \%$ of NHLs may involve the gastro-intestinal tract, primary GI NHL remains a relatively uncommon malignancy in comparison to the epithelial neoplasms and they account for only $1-4 \%$ of GI tract cancers. We aimed to study the clinical profile demographic features, treatment and response profile and outcomes of patients with gastro-intestinal NHL at our centre.

Patients/Materials \& Methods: We retrospectively analysed the case records of patients diagnosed with a primary gastro-intestinal lymphoma over the course of 7 years from 2011-2017.

Results: A total of 73 patients of primary gastro-intestinal NonHodgkin lymphoma were found in the electronic database in the study period. Most of the patients were male $(55 / 73,75.3 \%)$, with the male to female ratio being over $3: 1$. The mean age of the patients was 50.16 years $( \pm 15.94)$ and ranged from 14 to 66 years. The median duration of symptoms was 4 months (range $0.5-72$ months). At presentation, $50(68.5 \%)$ patients had B symptoms while $6(8.2 \%)$ patients had bulky disease. Twenty-Eight patients (38.4\%) had lymphadenopathy at baseline, while $11(15.1 \%)$ had hepatomegaly and 9 $(12.3 \%)$ had splenomegaly. Most patients (58 patients, $66.6 \%)$ presented with limited stage disease (Stage IE and IIE) while 26 patients (33.4\%) had advanced disease at presentation (Stage III and IV). Thirty-Six $(49.3 \%)$ patients had a high LDH at baselines and most patients belonged to the Low Risk $(22 ; 30.1 \%)$ and Low Intermediate Risk $(14 ; 19.2 \%)$ groups of the IPI. The most common site of involvement was the Stomach (34 patients; $46.6 \%$ ) followed by the Ileum (17 patients, 23.3\%). Sixty Seven of the 73 patients received therapy, with the most common regimen being RCHOP/CHOP (45 patients, 68.1\%). Forty-Six patients $(68.7 \%)$ received Rituximab containing regimens. Final outcome assessment was available for 63 patients from the total cohort. Fifty patients achieved an ORR (79.3\%) with 45 patients achieving a CR (71.4\%). 3 patients had progressive disease $(4.8 \%)$ and 9 patients $(14.3 \%)$ died before completion of chemotherapy.

Discussion \& Conclusion: The primary gastro-intestinal NonHodgkin Lymphoma was more common in male than females in our study. The most common organ involved was the stomach and most patients had a good risk disease according to IPI at baseline. Nearly $70 \%$ of the patients received Rituximab based regimens, and the patients in our cohort had a high CR and ORR of $71 \%$ and $79 \%$ respectively.

Disclosure of Interest: None Declared.

Keywords: Gastrointestinal lymphoma, NHL, rituximab.

\section{Abstract 25. Primary AL Amyloidosis: Clinical Presentation \& Outcome from a Tertiary Care Hospital in North India}

\author{
Arijit Bishnu ${ }^{* 1}$, Sanjeev Yadav', Dinesh Chandra ${ }^{1}$, \\ Manish Singh' ${ }^{1}$, Anshul Gupta ${ }^{1}$, KhaliqurRahman ${ }^{1}$, \\ Ruchi Gupta $^{1}$, Rajesh Kashyap ${ }^{1}$, SoniyaNityanand ${ }^{1}$ and \\ Sanjay Gandhi Post Graduate Institute of Medical Sciences
}

\section{${ }^{1}$ Department of Hematology, Sanjay Gandhi Postgraduate Institute Of Medical Sciences, LUCKNOW, India}

Aims \& Objectives: To study the pattern of clinical presentation \& outcome in North Indian patients with primary AL amyloidosis.

Patients/Materials \& Methods: Clinical and laboratory data of primary AL amyloidosis retrieved from the medical records were retrospectively analyzed from January 2017 to June 2018.

Results: 18 patients were diagnosed during the study period, with $\mathrm{M}$ : $\mathrm{F}$ of $1.3: 1$ and mean age of presentation being 54 years (range:
34-73 years). Most common presentation was edema (66\%) followed by proteinuria of varying degrees in nine $(50 \%)$ patients. Renal insufficiency was seen in $33 \%$ patients. $80 \%$ had a biopsy proven renal involvement. Cardiac involvement was seen in $28 \%$ patients with restrictive cardiomyopathy seen in two cases $(11 \%)$. Congo red positivity with apple green birefringence was seen in $78 \%$ patients. Total of 13 patients $(72 \%)$ showed lambda light chain restriction. M-band was seen in $55 \%$ patient with median value of $0.56 \mathrm{~g} / \mathrm{dl}$ (Range: $0.31-1.78 \mathrm{~g} / \mathrm{dl}$ ). M-protein noted in patients was primarily IgG-lambda (53\%) followed by IgA lambda (27\%). Nine patients tested M-protein negative. Lambda $\mathrm{AL}$ amyloidosis patients presented at a younger age and had more severe renal impairment requiring dialysis. 2 patients presented at stage IV disease. $6(33 \%)$ patients succumbed to gram negative septic shock. Seven patients were treated with VCD regime, 1 patient was treated with VCD but later shifted to DVd regime owing to disease progression. Overall survival in patients who opted for therapy is $57 \%$ with a median survival of 5.14 months. None of our patient opted for ASCT in CR1 due to financial constrains.

Discussion \& Conclusion: Primary AL amyloidosis is a rare disease and since presentation is variable and often nonspecific, diagnosis is often delayed resulting in organ damage which impairs the ability to deliver effective treatment and has a negative impact on prognosis. There is no single diagnostic test for this disease and diagnosis requires a high index of suspicion. Data available from Indian population is very limited. Treatment is difficult with early mortality being the major hurdle. Disclosure of Interest: None Declared.

Keywords: primary amyloidosis, prognosis, treatment outcomes.

\section{Abstract 26. HLH Masquerading Lymphoma: Diagnostic Dilemma and Treatment Outcomes}

\author{
MeghaVerma ${ }^{* 1}$, Nitin Gupta ${ }^{2}$, JasmitaDass ${ }^{3}$, Jyoti Kotwal ${ }^{3}$, \\ Ajay Sharma ${ }^{3}$ \\ ${ }^{1}$ Haematology, Sir Ganga Ram Hospital, Delhi, ${ }^{2}$ Clinical \\ Haematology, ${ }^{3}$ Haematology, Sir Ganga Ram Hospital, New \\ Delhi, India
}

Aims \& Objectives: a case series to illustrate difficulties faced in diagnosis, management and subsequent therapeutic approach patients presenting with HLH secondary to lymphoma.

Patients/Materials \& Methods: A retrospective review of patients treated in clinical hematology department of Sir Ganga Ram Hospital New Delhi for HLH and lymphoma was performed from Jan 2017 to April 2019. Diagnosis of HLH was made using HLH 2004 criteria along with $\mathrm{H}$ score. Only patients who fulfilled HLH 2004 criteria were included.

Results: Nine patients were treated during above period, four patients with Hodgkins lymphoma, one patient had DLBCLand four patients had T-cell lymphoma. All patients presented with features of HLH and underlying lymphoma was detected on further evaluation. All patients had $\mathrm{H}$ score above the cut off value for diagnosis of HLH.Out of 9 patients, 5 received chemotherapy and 2 were given only steroids, 1 received IVIG with steroids. 1 died early before institution of therapy. Out of the 5 patients who received chemotherapy, 3 attained $\mathrm{CR}$ but one patient relapsed within a month of completion of treatment. In the remaining 4 patients who could not be started on chemotherapy, all died within 2 weeks of presentation.

Discussion \& Conclusion: Underlying lymphoreticular malignancy should be actively searched in adult patients presenting with HLH. As early diagnosis and initiation of disease specific therapy with or without specific HLH directed treatment can improve the historical poor prognosis. 
Disclosure of Interest: None Declared.

Keywords: HLH, lymphoma, MANAGEMENT.

\section{Abstract 27. Renal Outcomes in Biopsy Proven Light Chain Cast Nephropathy: A Real World Experience}

Niranjan khaire ${ }^{* 1}$, Naga Ganesh Kasinadhuni $\mathrm{K}^{1}$, Manish rathi ${ }^{2}$, Uday Yanamandra ${ }^{1}$, Alka Khadwal ${ }^{1}$, Gaurav Prakash ${ }^{1}$, Subhash Varma $^{1}$, RitambhraNada ${ }^{3}$, Vinay Sakhuja ${ }^{2}$, Pankaj Malhotra ${ }^{1}$

${ }^{1}$ Internal Medicine, ${ }^{2}$ Nephrology, ${ }^{3}$ Histopathology, PGIMER, Chandigarh, India

Aims \& Objectives: To study the renal outcomes of multiple myeloma patients with biopsy proven light chain cast nephropathy in a real world setting in a tertiary care institution in North India.

Patients/Materials \& Methods: We reviewed patients of multiple myeloma with renal involvement who had kidney biopsy proven light chain cast nephropathy from January 2010 to July 2013. The baseline renal and myeloma parameters, the free light chain load, therapy received including the anti myeloma regimen given or plasmapheresis given were recorded and are presented in Table 1 and correlated with renal outcomes including $>50 \%$ reduction in baseline serum creatinine and/or dialysis independence. All decisions regarding investigations, kidney biopsy, treatment modalities were taken by the primary treating team in conjunction with the consent and affordability of the patient, making this a real world experience. The study was approved by our institutional review board.

Results: Out of a total of 29 cases with biopsy proven cast nephropathy who were identified during the study period, 6 were excluded from analysis (one early death before any treatment, 5 lost to follow up) and a total of 23 cases were analysed and followed up for six months from start of therapy. All patients received either VTD or CyBorD anti myeloma therapy and $13(56 \%)$ patients received plasmapheresis (5 to 7 cycles of plasma exchange).

Characteristics

Total Cohort

Plasmapheresis Group

Non plasmapheresis group

$\mathrm{n}$

23

13

10

Age in years (mean)

(Range)

53.76

(39-67)

54.2

(39-67)

53.2

(42-65)

Male: Female ratio

13:10

4:9

9:1

Baseline serum creatinine in $\mathrm{mg} / \mathrm{dL}$ (mean) continued

7.24

6.8

7.8

Requiring dialysis (\%)

$22(96 \%)$

$13(100 \%)$

$9(90 \%)$

$24 \mathrm{~h}$ urine protein in gm (mean)

$1.27(\mathrm{n}=17)^{*}$

$1.28(\mathrm{n}=11)^{*}$

$2.55(n=6)^{*}$

Bence Jones Proteinuria

4 out of $14(29 \%)$

1 out of $7(14 \%)$

3 out of $7(42 \%)$

Bone marrow plasmacytosis (mean)

$36.8 \%$

$45.75 \%$

$25.3 \%$

International Staging System score

ISS I

ISS II

ISS III

0

$2(12 \%)$

$15(88 \%)$

$(\mathrm{n}=17)^{*}$

0

$1(10 \%)$

$9(90 \%)$

$(\mathrm{n}=10)^{*}$

0

$1(14 \%)$

$6(85 \%)$

$(\mathrm{n}=7)^{*}$

VTD (at least 2 cycles)

$13(72 \%)$

$9(82 \%)$

$4(58 \%)$

CyBorD (at least 2 cycles)

$5(28 \%)$

$2(18 \%)$

$3(43 \%)$

Dialysis Independence

$12(70.5 \%)$

$8(72.7 \%)^{*}$

$4(66.6 \%)$

$(\mathrm{n}=6)^{*}$

At least $50 \%$ creatinine reduction

$11(61.1 \%)^{*}$ 
continued

$8(72.7 \%)^{*}$

$3(43 \%)^{*}$

Mortality prior to 2 cycles therapy

$5(21.7 \%)^{*}$

$2(15.3 \%)^{*}$

$3(30 \%)^{*}$

Overall mortality

$8(34.8 \%)$

$4(30.7 \%)$

$4(40 \%)$

Table 1: Summary of patient characteristics and outcomes. *: data may not be available or eligible for analysis from the entire cohort, hence the denominator will be a value different to the sample size of the cohort.

Thus amongst patients who completed at least two cycles of chemotherapy, $72.7 \%$ of patients receiving plasmapheresis achieved dialysis independence and at least a partial renal response (defined as $>50 \%$ reduction in serum creatinine) as compared to $66.6 \%$ patients with dialysis independence and $43 \%$ with partial renal response in the non plasmapheresis group.

Discussion \& Conclusion: The real world experience suggests plasmapheresis in conjunction with bortezomib based chemotherapy lead to improvement in renal response, dialysis independence and overall mortality as compared to patients who received only bortezomib based therapy.

Disclosure of Interest: None Declared.

Keywords: Cast Nephropathy, MYELOMA, PLASMAPHERESIS.

\section{Abstract 28. Fear of Recurrence and Somatic Symptom Severity in Multiple Myeloma Patients}

Praveen SHENOY*1, VineethaRaghavan ${ }^{1}$, ManuprasadAvaronnan ${ }^{2}$, Chandran Nair ${ }^{2}$ and Sajeev Kumar PB, Zoheb Raj

${ }^{1}$ Department of Clinical Hematology and Medical Oncology, ${ }^{2}$ Malabar Cancer Center, Thalassery, India

Aims \& Objectives: 1. To study prevalence of Fear of cancer recurrence among multiple myeloma survivors.

2. To determine the prevalence of somatic symptoms among the same population.

Patients/Materials \& Methods: This study was done between July 15 to July 30, 2015 among patients with myeloma who had been on treatment for at least one year. Patients were asked to fill fear of cancer recurrence inventory (FCRI) questionnaire and somatic symptom severity assessment using Physical Health Questionnaire-15 (PHQ-15) questionnaire with interviewer assistance.

Results: Sixty-four patients were included in this study. Median age was 60 yrs. Majority of them were females $(\mathrm{N}=38,60 \%)$. At the time of this study $15(23 \%)$ were in complete remission, $25(39 \%)$ had very good partial response, $11(17 \%)$ had partial response, $2(3 \%)$ had stable disease and $7(11 \%)$ had progressive disease. Somatic symptom severity assessed by PHQ- 15 revealed that majority $(\mathrm{N}=54,84 \%)$ had mild or even lesser somatic symptom burden. Median total FCRI score was 21 (Range 0-117). Median score was slightly higher in males compared to females (25.5 Vs 18.5$)$. Reliability analysis was performed on our data and overall Cronbach alpha of 0.86 was obtained indicating good internal consistency.

Discussion \& Conclusion: Fear of cancer recurrence scores and somatic symptom severity are lower compared to those seen in other solid tumors. Such a study exploring fear of cancer recurrence among myeloma patients is first of its kind.

Disclosure of Interest: None Declared.

Keywords: Fear of cancer recurrence, myeloma, Physical Health Quality.

\section{Abstract 29. Treatment Outcomes of Aggressive Lymphomas with Daepoch-R: A Single Center Audit}

Ankur Mittal $^{* 1}$, Ketan Modak ${ }^{1}$, Neeraj Arora ${ }^{2}$, Saurabh Bhave ${ }^{1}$, Lateef Zameer $^{3}$, InduArun ${ }^{3}$, RimpaBasuAchari ${ }^{4}$, Deepak Mishra ${ }^{2}$, MammenChandy ${ }^{1}$, Reena nair ${ }^{1}$

${ }^{1}$ Department of Clinical Haematology \& BMT, ${ }^{2}$ Department of Haematopathology, ${ }^{3}$ Department of Histopathology, ${ }^{4}$ Department of Radiation Oncology, Tata Medical Center, Kolkata, India

Aims \& Objectives: We undertook an audit of our patients treated with da EPOCH-R.

Patients/Materials \& Methods: EMR records of 2887 lymphomas ( $>18$ years) were evaluated retrospectively. Demography, Clinical features, staging, prognostic stratification, associated co-morbidities and first line treatment response and outcomes of 68 patients who were treated with daEPOCH between 2013 to 2017 have been analyzed. Available paraffin blocks were subjected to immunophenotyping to subtype DLBCL into Germinal centre (GCB) and non-GCB.

Response rates, progression free survival (PFS) and OS were evaluated for these patients who were followed up till August 2019. Patients in remission were censored at last follow-up. Patients in whom there was no follow-up information available (physical/telephonic) after progression were considered dead.

Results: The median age of presentation in our cohort is 46 years, with $17(25 \%)$ elderly patients more than 60 years of age. Gender ratio is $2: 1$. The median duration of symptoms was 3 months. The lymphoma subtypes treated included 45 patients with DLBCL, 15 \# BL, 3 \# Grey zone lymphoma and 5 \# PMBCL.

Patients presented with late stage disease [3 and 4] in $70 \%$, intermediate and High R-IPI in 90\%, extranodal disease in $69 \%$ at presentation. The commonest extra nodal sites were bone $34 \%$, followed by Gastro-intestinal (30\%), and Genitourinary (30\%).

The Overall response rate $(\mathrm{CR}+\mathrm{PR})$ is $83 \%$, and $10 \%$ patients progressed on therapy. The median follow up for this cohort is 30 months. PFS for this cohort is $65 \%$ at 5 years. The PFS for the various subtypes is $70.4 \%$ for DLBCL, $66.7 \%$ for BL and Grey zone lymphomas and $40 \%$ for PMBCL. The OS for the DLBCL subtype was $70 \%$ for GCB and $60 \%$ for non GCB subtype. OS for the patients above 60 years is $64 \%$ at 5 years and for those below 60 years is $68 \%$.

Discussion \& Conclusion: This single centre retrospective analysis showed that intensive treatment for aggressive NHL is feasible. Dose escalation was possible in most of the patients below 60 years though maximum level of dose escalation was possible only in 2 patients thus suggesting the challenges of dose escalation in real world setting. Hospitalisation for febrile neutropenia added to cost and delayed the treatment. Patients above the age of 60 years tolerated the treatment with equivalent outcomes.

Results for use of RdaEPOCH in BL, gray zone, c-myc positive DLBCL and DLBCL with high MIB index are encouraging in our hands and future randomized trials should evaluate da EPOCH-R in 
aggressive B cell lymphomas with high R-IPI BEFORE WE ABANDON THE PROTOCOL.

Disclosure of Interest: None Declared.

Keywords: Aggressive DLBCL, daEPOCH-R, NonHodgkins Lymphoma.

\section{Abstract 30. Baseline Characteristics and Therapeutic Outcomes of Patients with Myeloma: Indian Perspective}

GopilaGupta $^{* 1}$, ManoranjanMahapatra ${ }^{1}$, TulikaSeth ${ }^{1}$, Mukul Aggarwal $^{1}$, Rishi Dhawan ${ }^{1}$, HaraprasadPati ${ }^{1}$, Seema Tyagi ${ }^{1}$, Renu Saxena ${ }^{1}$

\section{${ }^{1}$ Hematology, AIIMS, New Delhi, India}

Aims \& Objectives: Multiple myeloma is an incurable plasma cell dyscrasia with an annual incidence of $1 \%$ of all malignancies. Because of lack of adequate healthcare and problems with access to novel drugs in our country, treatment of MM is still a challenge. The aim of this study is to determine the baseline characteristics and therapeutic outcomes in Indian population.

Patients/Materials \& Methods: We retrospectively collected data from medical records of MM patients treated at our institute's Hematology department from January 2015 to December 2018. Baseline characteristics of patients were noted. Event free survival (EFS) was defined from the date of first remission to date of first relapse, death or date of last follow up. Responses were defined by IMWG 2014 criteria.

Results: A total of 115 patients were recruited for our study. Median age was 56 years (range 28-79 years). $16 \%$ patients were younger than 40 years of age. Majority $(68 \%)$ were males. The most common symptoms at presentation was fatigue $(88.7 \%)$, followed by pain in back $(80 \%)$, fever $(18 \%)$, weight loss $(11.3 \%)$ and pathological fracture $(11 \%)$. Lytic lesions on imaging (majority $\mathrm{X}$ - ray), hemoglobin $<10 \mathrm{~g} / \mathrm{dl}$, serum creatinine $>2 \mathrm{mg} / \mathrm{dl} \quad \& \quad$ serum calcium $>11 \mathrm{mg} / \mathrm{dl}$, were found in $84 \%, 83.5 \%, 18.26 \%$ \& $15.65 \%$ patients respectively. The mean bone marrow plasma cell percentage and SPEP before therapy was found to be $39.2 \pm 24.5 \%$ and $3.3 \pm 2.33 \mathrm{~g} / \mathrm{dl}$. On serum IFE majority $(39 \%)$ patients had IgG kappa subtype and $22 \%$ patients had only light chains. ISS stage $1,2,3$ was seen in $39 \%, 29 \%$ and $31 \%$ patients respectively. Majority received bortezomib based triplet therapy (64\%) rest received lenalidomide based triplet or doublet therapies. 38 patients $(33 \%)$ underwent autologous stem cell transplant. $83 \%$ patients attained VGPR and higher response. Median EFS was 45.2 months (95\%CI34.8-58.8). 37\% patients relapsed after first line therapy. On univariate analysis, female sex ( $p$ value 0.033 ) and albumin $<3.5 \mathrm{~g} / \mathrm{dl}$ ( $p$ value 0.042 ) were found to be statistically significant predictors of relapse.

Discussion \& Conclusion: Based on our results, Indian population has onset of MM at relatively younger age and higher proportion of symptomatic patients at presentation. Majority patients received bortezomib based therapy. Transplant could be done in only one third of patients.

Disclosure of Interest: None Declared.

Keywords: autologous stem cell transplant, Bortezomib, Multiple myeloma.

\section{Abstract 31. Oculomotor Palsy- An Unusual Presentation of Multiple Myeloma}

\author{
TejasviniVaid"1, $^{* 1}$ Mukul Aggarwal $^{1}$, ManoranjanMahapatra ${ }^{1}$ \\ ${ }^{1}$ Hematology, AIIMS, Delhi, India
}

Aims \& Objectives: Oculomotor palsy- An unusual presentation of multiple myeloma.

Patients/Materials \& Methods: HISTORY:

A $50 \mathrm{yr}$ old female with no pre-morbidities presented with complaints of fatigue and body aches for 6 months and ptosis of the right eye for 10 days. Examination revealed pallor and the presence of right oculomotor palsy. Remaining systemic examination was normal. Initially a possibility of acute leukaemia with CNS involvement or lymphoma with CNS involvement was considered.

Results: INVESTIGATION AND THERAPY:

Investigations revealed $\mathrm{Hb}: 4.3 \mathrm{~g} \%$, TLC 6700 (P75,L25,E1,M2) and platelet count of $3 \mathrm{lakh} / \mathrm{mcl}$. Creatinine was $2.3 \mathrm{mg} / \mathrm{dl}$, corrected calcium: $15.1 \mathrm{mg} / \mathrm{dl}$, globulin: $12 \mathrm{mg} / \mathrm{dl}$ and $\mathrm{A}: \mathrm{G}$ ratio of 0.1 . Although the clinical picture of oculomotor palsy did not fit with the possibility of myeloma, the presence of hypercalcemia, renal dysfunction and anemia warranted a work up for myeloma. Bone marrow biopsy revealed sheets of abnormal plasma cells. Serum M protein was 5.88 g, IFE: IgG kappa, SFLC ratio: 35.8 thus confirming the diagnosis of Myeloma. MRI Brain done to delineate the cause for the oculomotor palsy revealed a space occupying lesion arising from the right clivus and extending into the right cavernous sinus suggestive of a plasmacytoma. CNS cytospin was normal. Patient was treated with hydration and pamidronate for hypercalcemia correction. She was started on CyBorD regimen and showed drastic improvement on therapy with recovery of the palsy. She also received RT 25 Gy to the base of skull in divided doses. She continues to be on regular follow up 1 year after her diagnosis.

Discussion \& Conclusion: CNS myeloma is a rare entity which occurs in 1 to $1.8 \%$ of all myeloma cases. Most cases present with CNS manifestations at relapse $(76 \%)$ and rarely at the time of diagnosis (24\%). The pattern of CNS involvement may be leptomeningeal, parenchymal and osteodural. The data on its management is sparse and no standard of care is available. Since iMiDs are known to cross the BBB, they must be included in therapy regimen. Radiotherapy is reported to be the best therapy for CNS myeloma but many studies report improved outcomes only in combination with chemotherapy. OS is 1.5 months to 6.7 months with osteo-dural subtype showing best outcomes.

Disclosure of Interest: None Declared.

Keywords: CNS disease, myeloma, oculomotor palsy.

\section{Abstract 32. Serum Free Light Chain Assay as a Predictor of Response to Induction Therapy in Multiple Myeloma}

\section{Lakshmi Mythili Mulam ${ }^{* 1}$, Sabina Langer ${ }^{1}$, Jyoti Kotwal ${ }^{1}$, Ajay Sharma ${ }^{2}$, Nitin Gupta ${ }^{2}$ \\ ${ }^{1}$ Hematology, ${ }^{2}$ Clinical Hematology, Sir Ganga Ram Hospital, Delhi, India}

Aims \& Objectives: To study the predictive value of reduction of involved free light chain level on Day 21 of chemotherapy for achievement of VGPR after 4 cycles of induction chemotherapy. Patients/Materials \& Methods: In 28 patients of newly diagnosed Multiple Myeloma with baseline involved FLC more that $100 \mathrm{mg} / \mathrm{L}$, we measured the involved FLC level on Day 21 of start of treatment 
and patients were followed up till the end of induction to assess the response to treatment. The above sample size provided a $90 \%$ power to study.

Results: After the induction chemotherapy, out of 28 patients, 13 patients achieved CR, 8 patients achieved VGPR, 4 patients achieved PR, 2 patients had stable disease and one patient died after receiving $2^{\text {nd }}$ cycle of chemotherapy. The mean per cent reduction in iFLC 21 days after start of treatment as compared to baseline was $91.5 \%$ and $57.1 \%$ in patients achieving $\geq$ VGPR and.

Discussion \& Conclusion: Monitoring iFLC levels on Day 21 can be used as an important tool for early identification of responders/non responders to anti-myeloma therapy.

Disclosure of Interest: None Declared.

Keywords: Multiple myeloma, Prognostic marker, serum free light chain assay.

\section{Abstract 33. FGFR3 Expression by Flow Cytometry in Multiple Myeloma}

JagrutiPatil $^{* 1}$, Sitaram Ghogale ${ }^{1}$, YajmananBadrinath ${ }^{1}$, Nilesh Deshpande ${ }^{1}$, DevasisPanda ${ }^{1}$, Subramaniam PG $^{1}$, Gaurav chatterjee ${ }^{1}$, Nikhil Patkar ${ }^{1}$, Prashant Tembhare ${ }^{1}$ and Flowcytometry lab,Hematopathologydepartment,TMH MUMBAI

\section{${ }^{1}$ Hematopathology, Tata Memorial Centre, Kharghar, India}

Aims \& Objectives: Multiple Myeloma (MM) is a haematological malignancy of clonal plasma cells genetically characterized by recurrent aneuploidy patterns and translocations. The $\mathrm{t}(4 ; 14)(\mathrm{p} 16 ; \mathrm{q} 32)$ translocation, found in $15 \%$ of multiple myeloma cases, indicates a poor prognosis. Plasma cells (PC) with $\mathrm{t}(4 ; 14)$ ectopically express the fibroblast growth factor receptor 3 (FGFR3) tyrosine kinase receptor, which has potential transforming activity and may represent a therapeutic target. Flow cytometry (FC) analysis of FGFR3 expression can be a reliable method for the detection alongside molecular studies. Herein, we present our study related to standardization of FGFR3 expression by flow cytometry in cases of MM.

Patients/Materials \& Methods: We studied 80 clinically suspected cases of multiple myeloma. Wright stained bone marrow aspirate smears were evaluated for morphology and quantitation of plasma cells. Smears with a differential count of $>10 \%$ plasma cells were included in the study for FGFR3 expression by FC. Samples were processed using bulk-lyse-stain-wash technique and a three tube 13 color antibody panel was used for enumeration of plasma cells and FGFR3 expression. Data was acquired using a 3 laser 13 color flow cytometer i.e. CytoFlex (Beckman Coulter) and was analysed in Kaluza-v 2.1 software. Cytogenetic analysis by FISH was done to identify $\mathrm{t}(4 ; 14)$ in all cases.

Results: Our study included62 cases of MM. The median age was 57 years (22-76). The median $M$ band at presentation was $4 \mathrm{~g} / \mathrm{dl}$ (0.3-10.6). Median value of plasma cells by flow cytometry was $7 \%$ $(0.034-70.45)$. FGFR3 expression was considered as positive if more than $5 \%$ of abnormal plasma cells express FGFR3. 5 out of 62 cases $(8.1 \%)$ was positive for FGFR3. The sensitivity and specificity of FGFR3 detection by flow cytometry as compared to FISH was $80 \%$ and $100 \%$ respectively.

Discussion \& Conclusion: We standardized FGFR3 expression byFC which is highly useful for rapid detection of $t(4 ; 14)$ cases of $\mathrm{MM}$ and early risk stratification. Therefore, it should be included in routine practice as an alternative method.

Disclosure of Interest: None Declared.

Keywords: FGFR3, Multiple myeloma, Plasma cell.

\section{Lymphoma and Myeloma (Laboratory)}

\section{Abstract 1. Synchronous Malignancies: A Diagnostic Conundrum}

\section{PreetiTripathi $^{* 1}$, Preethi Makam ${ }^{1}$, Pragya Sharma ${ }^{1}$, Arijit Sen ${ }^{1}$}

${ }^{1}$ Laboratory Medicine CHAF Bengaluru, Indian Airforce, Bengaluru, India

Aims \& Objectives: In todayser a, patients with multiple tumors detected simultaneously are progressively increasing. Such cases are not only difficult to diagnose but also pose a therapeutic dilemma to clinicians due to complexities of multiple protocols being practised on one body. We present a case of an elderly female who presented with a small swelling on base of tongue and during evaluation was found to have multiple pathologies in tongue, ovary, breast and thyroid.

Patients/Materials \& Methods: A 60 years old, previously healthy female presented with a small mass $(2.5 \mathrm{~cm} \times 2 \mathrm{~cm})$ on base of tongue for past 2-3 weeks which was causing mild dysphagia. There was no history of fever, weight loss, loss of appetite, abdominal fullness, mass/nodule anywhere else in body. Aspiration cytology of the mass base of tongue was suggestive of high grade non hodgkins lymphoma (NHL). Biopsy confirmed the tongue mass as diffuse large B cell lymphoma on immunohistochemistry.

Results: A PET scan to look for extension revealed FDG uptake in base of tongue mass, left level II cervical nodes, superolateral quadrant of left breast, left ovary, multiple pelvic and iliac lymph nodes, omental nodules. USG neck showed multiple hyperechoic lesions in both lobes (TIRADS III). Suspecting an atypical spread for NHL, further biopsies of individual organs were done which revealed serous adenocarcinoma in left ovary with omental mets, fibroadenoma in left breast and features of thyroiditis in thyroid lobe aspiration. In view of the complex clinical picture, patient was given "tailor made" chemotherapy along with Rituximab which significantly reduced her tongue growth. Currently, patient awaits surgery for ovarian mass and breast lesion.

Discussion \& Conclusion: The tendency of some individuals to develop multiple tumors (synchronous or metachronous) may be explained either by an individual predisposition or by the action of carcinogenic factors acting on different organs at similar/different times. The diagnosis of such cases need an "open mind" to expect the unexpected and successful patient's management require an individual specific approach.

Supporting Document: 3b8eb989-dc0e-4385-80c9-8012daad82b1.

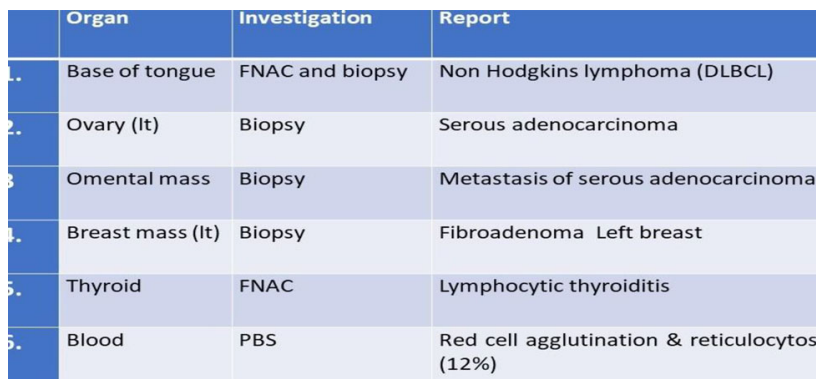

Disclosure of Interest: None Declared.

Keywords: DLBCL, non hodgkins lymphoma, synchronous malignancy. 


\section{Abstract 2. Unusual Rainbow Presentation of Myeloma- Does It Have Poor Prognostic Significance}

\author{
Ankur Ahuja ${ }^{* 1}$, TathagatChatterjee ${ }^{1}$, Dwarika Tiwari ${ }^{1}$, Uday \\ Yanamandra $^{2}$, PrernaGuleria ${ }^{1}$ \\ ${ }^{1}$ Dept of Lab and Molecular Sciences, ${ }^{2}$ Dept of Hematology, Army \\ Hospital RR, DELHI, India
}

Aims \& Objectives: To review Myeloma cases with an unusual site manifestation.

Patients/Materials \& Methods: 3 case reports of Myeloma with unusual sites at tertiary hospital in 2018 .

Results: Case 1.

38 yrs old male an old case of relapsed Multiple Myeloma after both Autotransplant and Allotransplant remained symptomatic throughout the course of treatment with partial response to chemotherapy.Patient succumbed to the disease and autopsy was done which revealed a nodular mass on the serosal surface of duodenum, both kidneys, extradural surface occupying the left frontal, temporal and occipital portions. On microscopy these tumor masses resembled large plasmacytoid cells and were immunopositive for CD38, CD138, CD56, EMA, EBV; and immunonegative for CD10 and CD20 leaving possibilities of Plasmablastic lymphoma/Anaplastic myeloma.

Case 2.

46 years old lady presented with history of mass over the periorbital area which on FNAC revealed to be comprised of plasma cells. Flow cytometry was also done on the same sample which revealed these cells to be positive for CD 38, CD 56, CD 117, CD 20, monoclonal restriction of lambda chain. Autotransplant was done followed by BM remission of disease with MRD positivity of $0.58 \%$ but unfortunately on day 45 patient had relapsed disease with more than $20 \%$ circulating plasma cells. Patient succumbed to illness 2 weeks after the disease.

Case 3.

A 62 years old lady presented with Rt cervical lymphadenopathy since last 3 months. Her lymph node biopsy revealed replacement by plasmablasts which were positive for CD 38, CD 138, CD 56 and negative for EMA, CD 20. Bone marrow, Skeletal survey was advised which revealed involvement by sheets of plasma cells and multiple lytic lesions respectively.

Discussion \& Conclusion: Here we wanted to emphasise the importance of awareness of unusual site involvement of myeloma as it will not be helpful in diagnosis when they are presented as an unusalmanfestations but also adding into prognosis aspect ${ }^{2}$. Interestingly all these cases were young patients and two of the patient despite of transplant died in the subsequent course.

Disclosure of Interest: None Declared.

Keywords: Myeloma involving bilateral kidney, Myeloma involving dura surface of brain, Myeloma involving periorbital surface.

\section{Abstract 3. Flow Cytometric Immunophenotyping of Normal and Abnormal Plasma Cells in Multiple Myeloma Patients}

\section{NAMRATA AWASTHI ${ }^{* 1}$, Abhishek Bajpai ${ }^{1}$, Swati kumari ${ }^{1}$, PradyumnSingh $^{1}$, Gaurav Gupta ${ }^{2}$, NuzhatHusain ${ }^{1}$ \\ ${ }^{1}$ Pathology, ${ }^{2}$ Medical Oncology, RMLIMS, Lucknow, India}

Aims \& Objectives: Identification of plasma cells into abnormal (APC) and normal (NPC) compartment is the initial step in flow cytometric (FC) analysis of Multiple myeloma (MM) for diagnosis, prognosis and follow up. No single phenotypic marker is sufficient to distinguish between NPC vs APC. The present study was aimed to determine the immunophenotype (IP) of APC and NPC in bone marrow samples of MM patients. The sensitivity and specificity of various markers (CD19, CD45, CD117, CD56, CD200 and CD81) and their combinations for identification of APC/NPC was evaluated. Patients/Materials \& Methods: 43 newly diagnosed cases of MM and 13 controls were included in the study. Bone marrow sample from $2^{\text {nd }}$ pass was processed on the same day with antibodies against CD38, CD138, CD19, CD81, CD45, CD117, CD200, CD56, cytoKappa and cytoLambda in a four colour experiment with CD38 and CD138 as gating antibodies. Sample acquisition and analyses were done on BD FACS Calibur flow cytometer.

Results: Mean APC \% in cases was $96.5 \%$. Classical IP of APC which is CD19-/56+/45-/81-/117+/200+ with monoclonal light chains was found in only 13/43 MM cases. In 30/43 cases APC revealed deviation from expected IP either for single or a combination of markers. Of the 13 control cases classical IP of NPC which is CD19+/56-/45+/81+/117-/200- with polyclonal expression of light chains was seen in $8 / 13$ cases. Small subpopulations of normal non neoplastic plasma cells with an aberrant IP were noted in 5/13 controls.Sensitivity for APC detection was highest for CD19 (95.2\%) followed by CD56 (90.4\%) and CD81 (83.7\%). Specificity was highest for CD19 (100\%), CD56 (100\%) and CD81 (100\%) followed by CD117 $(92.3 \%)$. Difference in mean values of CD19-56+ and CD19+56- PC in cases and control was highly significant $(p<0.0001)$. Combination of markers with maximum sensitivity to detect APC (97.6\%) was CD81neg or CD19neg and CD200pos or CD56pos and for NPC (92.3\%) was CD81pos/CD19pos/CD56neg/ CD117neg.

Discussion \& Conclusion: Plasma cell IP can be highly variable and therefore $8-10$ colour approach is more informative than a 4 colour approach. Evaluation of sensitive and specific markers along with simultaneous cytoplasmic light chain expression is the ultimate proof for the presence of APC.

Supporting Document: c997d3d0-f88a-4824-8626-f974b255e7b1.

\begin{tabular}{|c|c|c|c|c|c|c|c|}
\hline Marker & & $\begin{array}{l}\text { PRESENT } \\
\text { STUUY } \\
\text { 2019 }\end{array}$ & $\begin{array}{l}\text { TEMBHARE } \\
\text { et al. } \\
2014\end{array}$ & $\begin{array}{l}\text { GUPTA et } \\
\text { al. } \\
2009\end{array}$ & \begin{tabular}{|l|} 
RAWSTRON \\
et al, \\
2008 (Review)
\end{tabular} & \begin{tabular}{|l} 
JUAN-FLORES MONTERO et \\
al,2016 (Review)
\end{tabular} & Othor studios \\
\hline \multirow{2}{*}{ CD81 } & $\begin{array}{l}\text { Sensitivity } \\
\%\end{array}$ & 83.7 & 95 & NE & -1 & 55 & \\
\hline & $\begin{array}{l}\text { Specificity } \\
\%\end{array}$ & 100 & 100 & NE & - & - & \\
\hline \multirow[t]{2}{*}{ CD200 } & $\begin{array}{l}\text { Sensitivity } \\
\%\end{array}$ & 79.0 & NE & NE & - & $>70$ & $\begin{array}{l}61.8 \% \text { Olteanu } \\
\text { et al. 2011, } \\
71 \% \text { Alapat et } \\
\text { al.2012 }\end{array}$ \\
\hline & $\begin{array}{l}\text { Specificity } \\
\%\end{array}$ & 53.8 & NE & NE & - & $\begin{array}{l}49 \%(31-68 \%) \text { PC in normal } \\
\text { express CD200 }\end{array}$ & \\
\hline \multirow{2}{*}{ CD45 } & $\begin{array}{l}\text { Sensitivity } \\
\%\end{array}$ & 71.4 & 90 & 84.1 & - & 73 & \\
\hline & $\begin{array}{l}\text { Specificity } \\
\%\end{array}$ & 84.6 & 59 & NE & - & $\begin{array}{l}22 \%(0-69 \%) \text { PC in normel are } \\
\text { negative }\end{array}$ & \\
\hline \multirow{2}{*}{ CD117 } & $\begin{array}{l}\text { Sensitivity } \\
\%\end{array}$ & 52.3 & NE & 50.5 & 30 & 30-32 & $\begin{array}{l}32 \text { Ocqueteau } \\
\text { Met al, } 1996\end{array}$ \\
\hline & $\begin{array}{l}\text { Specificity } \\
\%\end{array}$ & 92.3 & NE & NE & - & $\begin{array}{l}\text { No cells with CD117 expression in } \\
\text { normal }\end{array}$ & 100 \\
\hline \multirow{2}{*}{ CD19 } & $\begin{array}{l}\text { Sensitivity } \\
\%\end{array}$ & 95.2 & 100 & 96.3 & 95 & 96 & \\
\hline & $\begin{array}{l}\text { Specificity } \\
\%\end{array}$ & 100 & 50 & NE & - & $\begin{array}{l}30 \% \mathrm{pc} \text { in normal are negative for } \\
\mathrm{CD} 19\end{array}$ & \\
\hline \multirow{2}{*}{ CD56 } & $\begin{array}{l}\text { Sensitivity } \\
\%\end{array}$ & 90.4 & 69 & 82.2 & 75 & 60-75 & \\
\hline & $\begin{array}{l}\text { Specificity } \\
\%\end{array}$ & 100 & 74 & NE & - & 6-9\% pc in normal express CD56 & \\
\hline
\end{tabular}

Disclosure of Interest: N. AWASTHI Grant from: RESEARCH CELL RMLIMS, A. Bajpai: None Declared, S. kumari: None Declared, P. Singh: None Declared, G. Gupta: None Declared, N. Husain: None Declared.

Keywords: FLOW CYTOMETRIC, IMMUNOPHENOTYPING, PLASMA CELL. 


\section{Abstract 4. Impact of Stem Cell Transplantation on Immunofixation Electrophoresis in Multiple Myeloma}

\section{ChandramallikaPaul ${ }^{* 1}$, S. Sugumar ${ }^{1}$, Debasish Adhya ${ }^{1}$, Arijit Roy $^{1}$, SubhosmitoChakraborty ${ }^{1}$ \\ ${ }^{1}$ Clinical Biochemistry, Tata Medical Center, Kolkata, Kolkata, India}

Aims \& Objectives: Bone marrow reconstitution is a hallmark feature of Hematopoietic Stem Cell Transplantation (HSCT). In multiple myeloma (MM), often, the patients are managed by HSCT. We present a MM patient who exhibited different serum protein electrophoresis (SPEP) and Immunofixation electrophoresis (IFE) restriction patterns before and after HSCT.

Patients/Materials \& Methods: The patient is a 61 year male from Kolkata, INDIA diagnosed as multiple myeloma ISS III and $\mathrm{t}(4 ; 14)$, intermediate-risk cytogenetics. The initial SPEP demonstrated one large $\mathrm{M}$ spike $(6.92 \mathrm{~g} \%)$ and one faint band in the gamma region while IFE demonstrated IgA kappa restriction pattern. The patient was initially managed with conventional chemotherapy, followed by auto HSCT. Electrophoresis was performed by high-resolution Agarose gel electrophoresis in a semi-automated instrument (Hydrasys 2 scan from Sebia).

Results: Three months post-HSCT, the SPEP demonstrated two faint $\mathrm{M}$ bands in the gamma region while the IFE demonstrated unusual oligoclonal bands with $\operatorname{IgG}$ lambda $+\operatorname{IgG}$ kappa $+\operatorname{IgA}$ kappa + additional kappa light chain restriction.

Discussion \& Conclusion: In MM, an uncontrolled proliferation of monoclonal proteins with a characteristic heavy and light chain restriction pattern occurs. Periodic recording of the location and the characteristic of isotype on IFE is vital in both diagnosis and management. The appearance of abnormal protein bands distinct from the presenting paraproteins in due course of treatment may be associated with bone marrow reconstitution or the emergence of a new malignant clone; which may complicate the assessment of disease response to treatment.

Such cases of appearances of multiple additional abnormal protein bands in the form of oligoclonal bands in MM post-HSCT in India is worth to mention which is approximately $10 \%$ in our institution in a period of 7 years (January 2012-January 2019).

Conclusion: The emergence of abnormal protein bands in IFE unlike those observed at the time of diagnosis may pose a problem in long term immune-surveillance. Higher-resolution protein separation methods are needed to differentiate the oligoclonal bands from malignant monoclonal proteins effectively. This is a case demonstrating multiple additional bands or oligoclonal bands on IFE, a unique impact of HSCT in multiple myeloma.

Supporting Document: 5b7e5853-bf59-4618-8e3c-d81faec5486c.

Disclosure of Interest: None Declared.

Keywords: Immunofixation electrophoresis, Multiple myeloma, Stem cell transplantation.

\section{Abstract 5. Role of Flowcytometry in Predicting the Probability of Leukemic Spill Over of Myeloma}

\author{
ChumbeniNgullie $^{* 1}$, Sarika singh ${ }^{1}$, Pallavi Sinha ${ }^{1}$, \\ Sunita aggarwal ${ }^{2}$, D P Bhadoria $^{2}$ \\ ${ }^{1}$ Pathology, ${ }^{2}$ Medicine, Maulana Azad Medical College, New \\ Delhi, India
}

Aims \& Objectives: To study the exprssion of CD56, CD27 \& CD81 along with CD19, CD20 \& CD28 in both peripheral blood as well as in bone marrow aspirate samples synchronously, in predicting the probability of leukemic spill over of clonal plasma cells in blood. Patients/Materials \& Methods: This study was conducted in the department of Pathology, Maulana azad medical college, Delhi, where paired samples of blood as well as bone marrow were taken and flowcytometry was performed synchronously at the time of diagnosis. 5/6 cases had plasma cells in both blood and marrow aspirate samples with plasma cell count ranging from $0-23 \%$ in peripheral blood and 25-90\% in marrow aspirate. Multiparametric flowcytometry five color with three laser FC500 Beckman Coulter, following panel was used: CD45,CD19,CD20, $\kappa, \lambda, C D 38, C D 138$, CD27,CD28,CD200,CD117 \& CD81.

Results: Expression of CD56, CD117 \& CD27 was found to be positive in $4 / 6$ cases, $1 / 6$ cases $\& 3 / 6$ cases respectively in peripheral blood while one case had dim positivity for CD19 in both blood and marrow aspirate sample. One case had expression CD200, CD28 \& CD81 in blood and loss of CD28 in marrow aspirate sample.

Discussion \& Conclusion: Plasma cell leukemia (PCL) is leukemic state of myeloma. This could be primary or secondary where the circulating clonal cells in blood are $>2000 / \mu \mathrm{L}$ or $20 \%$ of all nucleated cells. Myeloma cells spill over to blood is hypothesized due to reduced expression cell adhesion molecule CD56, overexpresion of CD27 and loss of CD81. Along with CD19, CD20 \& CD28 positivity, this phenomenon is often observed among patients with primary PCL, while positive expression of CD28, IL-6 \& CD27 are considered important in secondary PCL. This study proposes the utility of doing syncronous analysis of paired sample of blood and marrow aspirate in plasma cell myeloma cases to predict the probability of circulating plasma cells in blood and thus plasma cell leukemia as the latter has poor prognosis and needs correct sub-categorization.

Supporting Document: a2594bb2-9270-4915-a68e-a3c373a770fa. Disclosure of Interest: None Declared.

Keywords: flowcytometry, leukemia, myeloma.

\section{Abstract 6. A Rare Case of Paediatric T-Cell/Histiocyte Rich Large B-Cell Lymphoma -A Diagnostic Challenge}

\author{
NazmaK $^{* 1}$, Lalita Jyotsna $\mathbf{P}^{\mathbf{1}}$, KushaSharma ${ }^{1}$, Tanvi Arora ${ }^{1}$, \\ Jagdish Chandra $^{2}$, Rajiv Chadha ${ }^{3}$

\section{${ }^{1}$ Pathology, ${ }^{2}$ Paediatrics, ${ }^{3}$ Paediatrics Surgery, Lady Hardinge} \\ Medical College, New Delhi, India
}

Aims \& Objectives: With only a few cases reported in literature, T cell/histiocytic rich large B cell lymphoma (THRLBCL) is very rare in paediatric population. It is an uncommon subtype of DLBCL commonly seen in adults in $6^{\text {th }}-7^{\text {th }}$ decade. Accurate diagnosis depends on the careful immunohistochemical analysis of tumour cells and inflammatory mileu. We report a case of an 11 year old boy with $\mathrm{T}$ cell/histiocytic rich large B cell lymphoma.

Patients/Materials \& Methods: An 11 year old male child presented with fever, easy fatiguability and abdominal distention for 6 months. Examination revealed generalized lymphadenopathy and hepatosplenomegaly. Complete blood counts revealed pancytopenia whereas BMA showed a few atypical cells. On FNAC, a diagnosis of Hodgkin's lymphoma was made and surgical excision was advised. Axillary lymph node dissection was done and sent for histopathological examination.

Results: Ten lymph nodes were dissected and sections showed diffuse effacement of lymph node architecture with dispersed large cells (a few of them binucleate and multinucleated) in a background of small lymphocytes and histiocytes. Immunohistochemistry (IHC) 
revealed that the large cells were positive for $\mathrm{CD} 20$ and negative for CD15 \& CD30. Hence a differential diagnosis of diffuse variant of Nodular lymphocyte predominant Hodgkin Lymphoma \& THRLBCL was kept. Further IHC revealed among others, predominantly CD3 positive reactive $\mathrm{T}$ lymphocytes and macrophages along with absence of dendritic follicular cells and this tilted the balance in favour of THRLBCL.

Discussion \& Conclusion: T cell/histiocytic rich large B cell lymphoma is currently underdiagnosed in paediatric population. The diagnosis is challenging as it may be confused morphologically with nodular lymphocyte predominant Hodgkins lymphoma, Hodgkins lymphoma and peripheral $\mathrm{T}$ cell lymphoma. Immunohistochemistry plays a significant role when it presents with such a diagnostic dilemma. Reaching an accurate diagnosis is of paramount importance as treatment modalities differ and children respond better to the therapy and have a better prognosis as compared to adults.

Disclosure of Interest: None Declared.

Keywords: Nodular lymphocyte predominant Hodgkin lymphoma, Paediatric, T-cell/histiocyte rich large B-cell lymphoma.

\section{Abstract 7. Panorama of Lymphomas in India: A Tertiary Care Center Review}

\section{Surabhi Jain ${ }^{* 1}$, SaumyaranjanMallick ${ }^{1}$, Prashant Ramteke ${ }^{1}$, Ajay Gogia $^{2}$, Sameer Bakhshi ${ }^{2}$, Atul Sharma ${ }^{2}$ \\ ${ }^{1}$ Pathology, ${ }^{2}$ Medical oncology, Aiims, New Delhi, New delhi, India}

Aims \& Objectives: To characterise the epidemiological profile of lymphoma cases among Indian population at our center.

Patients/Materials \& Methods: This was a retrospective study. All lymphoma related biopsies from 2015 to 2018 were reviewed from database of Department of Pathology, AIIMS, New Delhi for epidemiological purposes. Detailed histomorphological and immunohistochemical evaluation was done and the cases were classified according to WHO classification 2017.

Results: A total of 3620 lymphoma cases were diagnosed between 2015 to 2018 . Hodgkin lymphomas comprised of $30.55 \%(\mathrm{n}=954)$ and Non-Hodgkin lymphomas were $69.44 \%(\mathrm{n}=2168)$. On subtyping of NHL, $6.68 \%$ were Precursor lymphoid neoplasm which further were B-cell (1.6\%) and T- cell (5.07\%), 82.97\% Mature B-cell neoplasm, $9.64 \%$ Mature NK-/T- cell neoplasm and $0.7 \%$ were atypical lymphoproliferative disease. The mature B-cell neoplasms comprised of DLBCL (49.53\%), Follicular lymphoma (9.73\%), CLL/SLL (5.53\%), Mantle cell lymphoma (4.5\%), Marginal zone lymphoma $(3.8 \%)$ and others. The mature NK/T-cell lymphomas were classified into PTCL, NOS (2.76\%), ALCL-ALK + (2.16\%), Extranodal NK-T cell lymphoma $(1.89 \%)$ and others. The Hodgkin lymphomas were subtyped comprising of $82.38 \%$ Classical type, $2 \%$ Nodular lymphocyte predominant type and $15.5 \%$ were unclassifiable. Classical subtype further comprised of 37\% Nodular sclerosis, 37.5\% Mixed cellularity, $0.8 \%$ Lymphocyte rich, $0.1 \%$ Lymphocyte depleted and rest could not be classified. $72.56 \%(\mathrm{n}=2627)$ were primary nodal lymphomas whereas others were diagnosed as primary at other sites like brain, spleen, testis, skin, mucosa. Male predominance was seen $(\mathrm{M}: \mathrm{F}=3: 1)$ and the presentation was spread across all age groups.
Discussion \& Conclusion: Indian studies indicate that NHL presentation in India differs in younger median age of presentation, higher male preponderance, higher number of patients with B-symptoms, poor performance status, proportion of the various subtypes and increased mortality. In order to help the scientific research, patient management and planning of health services, generation of epidemiological data is essential.

*The study was done under the projects.

-SERB/ECR/452/2015.

-SERB/EEQ/402/2016.

Disclosure of Interest: S. Jain: None Declared, S. Mallick Grant from: SERB/ECR/452/2015 and SERB/EEQ/402/2016, P. Ramteke: None Declared, A. Gogia: None Declared, S. Bakhshi: None Declared, A. Sharma: None Declared.

Keywords: Epidemiology, India, lymphoma.

\section{Abstract 8. Spurious Leukocytosis Leads to a Diagnosis}

\author{
Jayalakshmi B $^{* 1}$, FebeRenjitha Suman ${ }^{1}$, Sri Gayathri ${ }^{1}$, \\ Emmanuel Baskar ${ }^{2}$
}

${ }^{1}$ Pathology, ${ }^{2}$ General Medicine, Sri Ramachandra Institute of Higher Education and Research, Chennai, India

Aims \& Objectives: Spurious leukocytosis occurs in many conditions and it is the responsibility of the pathologist to differentiate it from leukocytosis. Careful evaluation of histogram and scatterogram may help in directing the clinician to further evaluation. An interesting case of spurious leukocytosis which leads to evaluation and final diagnosis is presented here with.

Patients/Materials \& Methods: A 50 year old female, presented with single cervical lymphadenopathy and headache, for 2 weeks. On general examination the patient was pale and cervical lymph node measuring $3 \times 3 \mathrm{~cm}$, firm in consistency was observed. Purpuric spots were noted in the arms. Complete blood count with hematologyanalyzer revealed: Hemoglobin- $5.9 \mathrm{~g} / \mathrm{dl}$, Total WBC count$1,68,000$ cells/cu.mm, platelets- 4,70,000/cu.mm. The WBC histogram showed interference before $35 \mathrm{fl}$. Presence of cryoglobulins was suspected. Fresh sample was incubated at $37{ }^{\circ} \mathrm{C}$, which when processed, showed hemoglobin- $6 \mathrm{~g} / \mathrm{dl}$, total WBC count of 4800 cells/cu.mm and platelets- $1,45,000 /$ cu.mm. The presence of headache, lymphadenopathy with purpura and spurious leukocytosis due to cryoglobulin interference suggested lymphoplasmacytic lymphoma.

Results: Cervical lymph node on excision was $2 \times 1.5 \times 1 \mathrm{~cm}$, firm with grey white cut surface. Microscopically paracortical expansion by monotonous population of atypical small lymphocytes along with plasmacytoid lymphocytes was noted. By immunohistochemistry, the lymphoblasts were positive for CD45, CD20, CD79a, PAX-5, MUM1 \& Kappa. Plasma cells were focally positive for CD-138 \& CD20. CD3 \& lambda were negative. Ki-67 labelling index was $20 \%$. The final diagnosis was given as LYMPHOPLASMACYTIC LYMPHOMA.

Discussion \& Conclusion: Careful analysis of WBC scatter plots \& histogram is necessary to differentiate spurious leukocytosis; evaluation of which reveals a hidden diagnosis. Clinico-pathological correlations and discussions are essential for effective patient care. 
Disclosure of Interest: None Declared.

Keywords: Cryoglobulins, Lymphoma, Spurious leukocytosis.

\section{Abstract 9. Dilemma of CD 45 Bright Expression by Flowcytometry in Leukemic Presentation of Lymphoid Neoplasms}

Pradeep Arumugam ${ }^{* 1}$, Rakhee Kar ${ }^{1}$, Prabhu Manivannan ${ }^{1}$, DebdattaBasu

\section{${ }^{1}$ Pathology, JIPMER, Puducherry, India}

Aims \& Objectives: This case series highlights the diagnostic challenges faced in cases with leukemic presentation and blastoid morphology but a bright CD 45 expression by flowcytometry.

Patients/Materials \& Methods: We selected four cases which had leukemic presentation in peripheral blood. Three of these cases had blastoid morphology and a diagnosis of acute leukemia was tendered on peripheral smear. The other case had morphology of mature lymphoid cell with a diagnosis of chronic lymphoproliferative disorder. Bone marrow studies including aspirates \& biopsies with immunocytochemistry and immunohistochemistry were performed. Flowcytometry analysis was done by CD19, CD3, and CD45 vs. side scatter gating strategy. Lymph node biopsies were available in two patients. Clinical profiles of the cases are as follows:

Case 1: 76yrs/male with fever, weight loss and lymphadenopathy. No splenomegaly/hepatomegaly.

Case 2: 44yrs/female with left cervical lymphadenopathy fever on and off $\times 2$ months, presented with altered sensorium, reduced urine output and puffiness.

Case 3: $34 \mathrm{yrs} / \mathrm{male}$ with inguinal and cervical lymphadenopathy for 2 weeks and bleeding from tooth extraction site for 1 week.He also had hepatosplenomegaly.

Case 4: $65 y$ rs/male with easy fatiguibility, hematochezia and significant blood loss. O/E splenomegaly $+12 \mathrm{~cm}$. No lymphadenopathy.

Hematological profile of these cases is tabulated (Table 1).

Results: As can be seen from the Table 1, all the cases had a bright CD 45 expression in the non blast region on flow cytometry. Two turned out to be Non Hodgkin lymphoma with peripheral leukemic spillover and included a follicular grade $3 \mathrm{~A}$ with diffuse large $\mathrm{B}$ cell lymphoma and a mantle cell lymphoma -blastoid type. The case with the mature morphology was actually T-Lymphoblastic Lymphoma/ Acute leukemia and was positive for CD99 with a high Ki67 index. Interestingly this case was negative for CD34 as well as TdT. The last case was diagnosed as Burkitt leukemia which had distinct CD45 expression in the monocytoid region.

Discussion \& Conclusion: This case series highlights the need for awareness of a bright CD45 expression having a varied diagnosis from an immature to more mature lymphoid neoplasms.

Disclosure of Interest: None Declared.

Keywords: CD45 bright expression, Flow cytometry, Non hodgkins lymphoma.

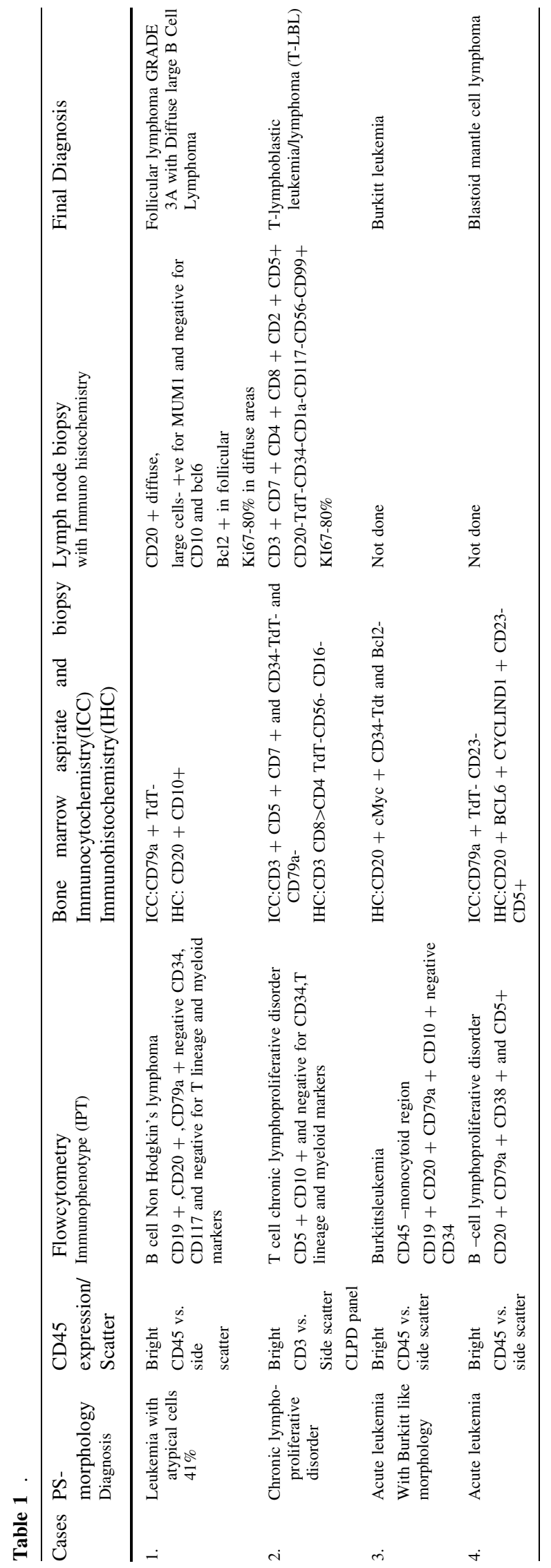




\section{Abstract 10. Cyclooxygenase-2 (COX-2) Expression in Newly Diagnosed Plasma Cell Myeloma}

\section{Sandeep Abhijit Pattnaik ${ }^{* 1}$, SomanathPadhi ${ }^{1}$, AsutoshPanigrahi ${ }^{2}$,} PritinandaMishra ${ }^{1}$, Mukund N. Sable ${ }^{1}$

\section{${ }^{1}$ Pathology, ${ }^{2}$ Hematology, AIIMS, Bhubaneswar, India}

Aims \& Objectives: Aim- To explore the cyclooxygenase (COX) pathway in Plasma Cell Myeloma (PCM).

Objectives:

1. To study the expression of COX-2 on trephine biopsy sections in newly diagnosed cases of PCM by immunohistochemistry (IHC).

2. To correlate the pattern of COX-2 expression with the bone marrow morphology and with selected hematological parameters and response to therapy.

Patients/Materials \& Methods: Four micron EDTA decalcified trephine biopsy sections from 31 newly diagnosed cases of plasma cell myeloma (PCM) (25 males, 6 females) were subjected to immunohistochemistry (IHC) by using COX-2 rabbit monoclonal antibody.Four cases of reactive plasmacytosis in cases of aplastic anemia, four cases of polyclonal plasmacytosis associated reactive marrow changes, and one case of post radiotherapy reactive plasmacytosis in a case of esophageal squamous cell carcinoma were taken as external controls. COX-2 expression was studied in trephine biopsies of PCM cases, each case was assigned a proportionate score (a) and intensity score (b), and an overall 'histoscore' (axb) was calculated. Based on the median histoscore, COX-2 expression was designated as low expression or high expression.

Results: The median age at diagnosis was 60 years (range; 40 to 75 years). On histomorphology, $19 / 31$ (61.3\%) had low-intermediate grade phenotype and remainder 12 cases $(38.7 \%)$ had a blastic morphology; and kappa to lambda light chain ratio was $9 / 22$. COX-2 expression was observed in 30/31 (96.7\%) cases. Low COX-2 expressor group was significantly associated with higher histologic grade and weak to heterogenous cytoplasmic positivity $(P<0.0001$, Fisher exact test). There was no significant association between COX2 expression and MIB-1 proliferative index $(P>0.42)$.

Discussion \& Conclusion: COX-2 expression was noted in a higher proportion of myeloma patients in our study. Similar to CD138, COX2 can be considered as plasma cell marker, and increased expression in myeloma cells may be a surrogate marker of disease activity which needs to be further explored in future prospective studies.

Supporting Document: a31cfaf6-75c6-409e-b2c1-a952d0a07f29.

\begin{tabular}{|c|c|c|c|}
\hline Parameters & Cox-2 low expressor & Cox-2 high expressor & P value \\
\hline Number of cases $(N)$ & $16(51.6 \%)$ & $15(48.4 \%)$ & - \\
\hline Male/female & $14 / 2$ & $11 / 4$ & $>0.05$ \\
\hline $\begin{array}{l}\text { Age in years } \\
: \quad \text { Median age (years, range) } \\
\text { Mean }\end{array}$ & $\begin{array}{l}60(40-75) \\
59.37 \pm 9.27\end{array}$ & $\begin{array}{l}56(40-71) \\
56.0 \pm 8.61\end{array}$ & $>0.05$ \\
\hline $\begin{array}{l}\text { Histological grading } \\
: \quad \text { Low intermediate } \\
\quad \text { High }\end{array}$ & $\begin{array}{l}7 / 16(43.75 \%) \\
9 / 16(56.25 \%)\end{array}$ & $\begin{array}{l}12 / 15(80 \%) \\
3 / 15(20 \%)\end{array}$ & $\begin{array}{l}<0.0001 \\
\text { (Fisher's } \\
\text { exact test) }\end{array}$ \\
\hline $\begin{array}{c}\text { Mib-1 proliferation index } \\
-\quad \text { Median (range) }\end{array}$ & $40 \%(5-85 \%)$ & $55 \%(10-75 \%)$ & 0.46 \\
\hline $\begin{aligned} \text { Pattern of Cox-2 expression } \\
\text { : Membrane accentuation } \\
\text { Heterogenous }\end{aligned}$ & $\begin{array}{l}2 / 16(12.5 \%) \\
14 / 16(87.5 \%)\end{array}$ & $\begin{array}{l}8 / 15(53.3 \%) \\
7 / 15(46.7 \%)\end{array}$ & $\begin{array}{l}<\mathbf{0 . 0 0 0 1} \\
\text { (Fisher's } \\
\text { exact test) }\end{array}$ \\
\hline
\end{tabular}

Disclosure of Interest: None Declared.

Keywords: Histological score, outcome, stromal microenvironment.

\section{Abstract 11. Role of Serum Protein Electrophoresis in Clinically Suspected Cases of Plasma Cell Dyscrasias}

Anu Singh ${ }^{* 1}$, Priya Sahu ${ }^{1}$, Garima Baweja ${ }^{1}$, Vijay Kumar ${ }^{1}$, SadhnaMarwah $^{1}$, MinakshiBhardwaj $^{1}$

\section{${ }^{1}$ Dr Rml Hospital, New Delhi, India}

Aims \& Objectives: This study was aimed to assess serum protein electrophoresis patterns in all clinically suspected cases of plasma cell dyscrasia with usual and unusual clinical presentations and to correlate with ancillary investigations.

Patients/Materials \& Methods: We analysed serum protein electrophoresis (SPE) patterns in clinically suspected cases of plasma cell dyscrasia in 2 year duration. Based on SPE pattern these cases were divided into two groups: Group I, with M-component and Group II, without M-component. These cases were reviewed with respect to clinical presentation and correlated with immunofixation electrophoresis and bone marrow aspiration/biopsy findings wherever available, especially in the $\mathrm{M}$ band positive cases to differentiate multiple myeloma (MM) from the other conditions.

Results: A total of 80 samples were received for SPE, out of these, $56.3 \%$ were clinically suspected to have plasma cell dyscrasia. Among these cases $60 \%$ were in group I and $40 \%$ were in group II. In group II, $39 \%$ cases were essentially non neoplastic after relevant investigations. In group I, M-band was observed in $88.9 \%$ cases in $\gamma$-region and $11.1 \%$ cases in $\beta$-region. Interestingly, 2 cases showed more than one M-Band. The mean fraction of the $\mathrm{M}$ protein was $4.7 \mathrm{~g} / \mathrm{dl}$ with a range of $2.0 \mathrm{~g} / \mathrm{dl}$ to $9.8 \mathrm{~g} / \mathrm{dl}$. In group II $72.2 \%$ showed polyclonal hypergammaglobulinemia, $22.2 \%$ showed normal electrophoretic pattern and 5.6\% showed hypogammaglobulinemia. Bone marrow aspiration and biopsy were available in $88.9 \%$ of all suspected cases. Various haematological neoplasms observed in both the groups, their clinical presentations with interesting facets of the cases have been studied.

Discussion \& Conclusion: Serum protein electrophoresis is one of the most important diagnostic modality in clinical chemistry. It helps to resolve cases with bone marrow plasmacytosis particularly in differentiating between reactive non-neoplastic plasmacytic proliferations and clonal plasma cell disorders. Moreover, it also facilitates the work-up of cases with atypical presentations and thereby paves way to clinch the diagnosis in these ambiguous cases.

Disclosure of Interest: None Declared.

Keywords: monoclonal gammopathy, Plasma cell dyscrasias, serum Protein electrophoresis (SPEP).

\section{Abstract 12. Plasma Cell Leukemia- Experience at AIIMS Rishikesh}

\author{
ArathiK $^{* 1}$, Sudeep Vaniyath ${ }^{2}$, Neha Singh ${ }^{1}$, Arvind Gupta ${ }^{2}$, \\ UttamNath $^{2}$, Harish Chandra ${ }^{1}$ \\ ${ }^{1}$ Pathology, ${ }^{2}$ AIIMS Rishikesh, Rishikesh, India
}

Aims \& Objectives: Plasma cell leukemia (PCL) is a rare, very aggressive form of malignant monoclonal gammopathy with poor treatment response, characterized by the presence of plasmacytes in peripheral blood. The criteria for diagnosis is presence of $>20 \%$ circulating plasma cells and an absolute number of minimum $2 \times 109 / \mathrm{L}$ plasma cells in peripheral blood. It is classified as primary PCL occurring 'de novo', or as secondary PCL in patients with relapsed/refractory multiple myeloma. Induction therapy aimed at disease control has to be started immediately post diagnosis.

Patients/Materials \& Methods: Of 457 bone marrow aspirates assessed in 1.5 years in the department of hematology, 45 cases were 
plasma cell lesions of which 3 were plasma cell leukemias. First case is a 42 year old female patient who presented with complaints of fever, loose stools and mild abdominal pain. CBC showed a TLC of 9440 with presence of $25 \%$ plasma cells in peripheral smear. Bone marrow aspirate showed $62 \%$ plasma cells. Case 2 is a 71 year male, diagnosed with multiple myeloma in 2013 with relapse of the disease in 2018, 10 months after which he showed a TLC of 6100 and 34\% plasmacytes. 3rd patient is a diagnosed case of IgA Multiple Myeloma diagnosed in Oct 2017 and had undergone chemotherapy. She had reappearance of M Spike in Jan 2019 and treatment refractoriness since then. 7 months later she developed ascites with presence of numerous plasma cells in the ascetic fluid cytology and CBC showed A TLC of 9200 with presence of $38 \%$ plasmacytes.

Results: Case one as was diagnosed as primary plasma cell leukemia and rest two as secondary plasma cell leukemia.

Discussion \& Conclusion: Plasma cell leukemia is a rare yet aggressive disease demanding a prompt beginning of induction therapy aimed to rapid disease control and to minimize the risk of early death, which makes proper understanding and early diagnosis of it a matter of utmost importance.

Disclosure of Interest: None Declared.

Keywords: Multiple myeloma, Plasma cell leukemia, Primary and secondary.

\section{Abstract 13. Lymphoid Neoplasm with FGFR1 Rearrangement}

\section{Shruti Chaudhary ${ }^{* 1}$, Nikhil Patkar ${ }^{1}$, ShrinidhiNathany ${ }^{1}$, PrassannaBhanshe $^{1}$, SwapnaliJoshi ${ }^{1}$, P G Subramanian ${ }^{1}$ \\ ${ }^{1}$ Hematopathology Laboratory, TATA Memorial Centre, ACTREC, Navi Mumbai, India, Mumbai, India}

Aims \& Objectives: Myeloid/lymphoid neoplasms with fibroblast growth factor receptor-1 (FGFR1) rearrangement is consider as distinct category in the WHO 2017 classification of Hematolymphoid Neoplasms. It has been reported that FGFRl fusions can present as a myeloproliferative neoplasm, AML or T-lymphoblastic leukemia/lymphoma. The patients having FGFR1 rearrangement have poor prognosis and poorly respond towards tyrosine kinase therapy. Hence, we aimed to look at the clinical characteristics and behaviour of the patients with $F G F R 1$ rearrangement.

Patients/Materials \& Methods: This was a retrospective study where database search of cases with FGFRl rearrangement in all the cases, for which RNA sequencing were done in a Tata Memorial Centre during 2018-2019. RNA extracted from Peripheral blood, bone marrow or FNAC from lymph node. cDNA synthesis followed by RNA sequencing was done using anchored multiplex PCR (24 different gene specific primers).

Results: Three cases with FGFR1 fusion were found during 2018-2019. These cases had different fusion partners such as ZMYM2, BCR and LRRFIP1 with FGFR1. Clinical characteristics of these patients have been summarized in Table 1 .

Discussion \& Conclusion: Myeloid/lymphoid neoplasm with FGFR1 rearrangement is highly under recognized entity in our resource constraint setting. ZMYM2 is the commonest partner of FGFR1 and is seen in approximately $40 \%$ cases with this fusion. Although, TKI are not effective in patients harbouring this rearrangement, Midoustaurin has shown to be effective with this fusion. HoweverBCR-FGFRI \& LRRFIP1- FGFR1 also seen in this study. Thus, RNA sequencing not only helps in making correct diagnosis, but also helps in risk stratification and deciding therapy.

Supporting Document: 5eb81ba3-e761-4580-8854-1d8ee722e07f.
Table 1. Clinical characteristics of patients with FGFR1 fusion

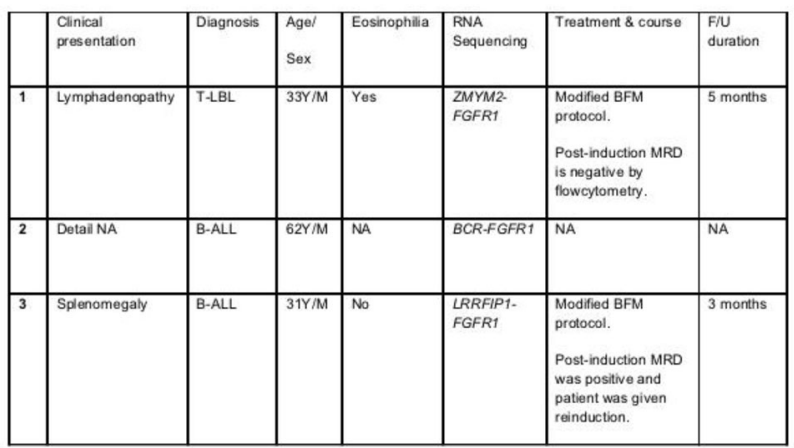

T-LBL:-T-Lymphoblastic Iymphoma; B-ALL:- Precursor B lineage Acule Lymphoblastic Leukemia, NA:-Not Avallable.

Disclosure of Interest: None Declared.

Keywords: FGFR1, Lymphoid neoplasm, RNA sequencing.

\section{Abstract 14. Plasmablastic Myeloma Masquerading Leukemia in an Immunocompetent Patient}

Amrita Anand"1, Smitasingh ${ }^{1}$, Kiran Agarwal ${ }^{1}$, JeevithaGovindswamy ${ }^{1}$, Atul goel ${ }^{2,2}$, Sanjay Kumar Meena ${ }^{2,2}$

${ }^{1}$ Pathology, Lady Hardinge Medical College, ${ }^{2}$ Medicine, Dr. Ram Manohar Lohia Hospital Post Graduate Institute of Medical Education and Research, Delhi, India

Aims \& Objectives: To typify myeloma in absence of clinical and biochemical parameters.

Patients/Materials \& Methods: A 70-year-old man presented to our outpatient department with complaints of easy fatiguability and lower backache for 6 months. Patient was hypertensive showing facial puffiness. There was no lymphadenopathy/organomegaly/lytic lesions on X-ray and MRI. Serum total proteins, albumin and globulin were $6.5 \mathrm{~g} / \mathrm{dl}, 3.2 \mathrm{~g} / \mathrm{dl}$ and $1.54 \mathrm{~g} / \mathrm{dl}$. Patient had a urine output of $250 \mathrm{ml} /$ day. Complete blood count revealed haemoglobin level of $8.9 \mathrm{~g} / \mathrm{dl}$ and total leucocyte count of 12,000 per $\mathrm{cmm}$ with peripheral smear showing 8 percent blasts. Marrow aspirate smears comprised of $74 \%$ blasts. These blasts showed a high nuclear/cytoplasm ratio, central to eccentric nuclei and single macronucleoli. Serum protein and urine electrophoresis revealed no mononoclonal band and urine Bence Jones proteins were negative. Flowcytometric analysis revealed majority of blasts strongly expressing CD38, CD138 with kappa restriction. Blasts were found to be negative for CD45 (leukocyte common antigen), CD20 and CD3.

Results: A final diagnosis Multiple myeloma (plasmablastic type) was made.

Discussion \& Conclusion: Discussion: Plasma cell myeloma (PCM) is a germinal centre-derived monoclonal neoplasm with mainly post switch B-cell phenotype. PCM with absent serum and urine monoclonal M-band have been classically described as non-secretory. These myelomas have been found to have elevated FLC levels and/or abnormal FLC ratios. The serum assay is diagnostically more accurate than the urine FLC assay.

Conclusion- The absence of serum M-band and lytic lesions in a patient does not exclude the possibility of PCM. A thorough work-up with serial evaluation of serum FLC assay is useful for diagnosis. It is also important to identify plasmablastic morphology as it confers poor prognosis.

Disclosure of Interest: None Declared. 
Keywords: FLC- Free Light Chain, MM- Multiple Myeloma, PCMPlasma cell myeloma.

\section{Abstract 15. Small Lymphocyte Like Plasma Cell Myeloma}

Priya Murthy ${ }^{* 1}$, TejindarSingh ${ }^{2}$ and Hematology

${ }^{1}$ Oncquest Laboratory, Bangalore, ${ }^{2}$ Oncquest Laboratory, New Delhi, India

Aims \& Objectives: Multiple myeloma (MM) has variable presentation clinically and morphologically. They show varied morphology and one subset shows small lymphocyte-like features. This category is a rare morphologically challenging variant distinguished from B-cell lymphoma by lack of CD45 and presence of CD138 and the clinical presentation of MM. Many of these cases are known to be associated with $\mathrm{t}(11,14)$ translocation along with CD20 expression and carry a better overall survival.

1. To assess the incidence of small lymphocyte like variant of MM.

2. To distinguish these cases from Lymphoproliferative disorders by immunophenotyping.

Patients/Materials \& Methods: A total of 335 patients diagnosed with MM over a period of 2 years were included in the study out of which 32 cases were reported as Small Lymphocyte-like Multiple Myeloma. Plasma cell morphology was classified as Plasmacytic/Intermediate/Immature/Plasmablastic/Small Lymphocyte-like based on specific morphologic characters. The last subset had prominent small lymphocyte-like or lymphoplasmacytoid features and closely mimicked a B-cell Lymphoma. IHC with CD138, Kappa, Lambda, CD20, CD3, Cyclin D1 and CD56 etc. was carried out to type the Lymphoid/ Lymphoproliferative Neoplasm.

Results: The overall incidence of Small Lymphocyte like Myeloma was found to be $9.5 \%$.

The neoplastic plasma cells were slightly larger than mature lymphocytes with round to oval nucleus and mild to moderate basophilic cytoplasm. In the biopsy the cells showed a diffuse involvement of the marrow with relatively little difference from a diffuse lymphocytic infiltrate. Only three cases showed increased reticulin.

In these cases neoplastic Plasma cells were CD45 $5^{\text {Negative }}$ and strongly positive for CD138, MUM1 \& CD20 with light chain restriction. $68.8 \%$ of cases showed kappa light chain restriction and the rest $28.1 \%$ showed lambda restriction. Only one case was nonsecretory. Cyclin D1 was done in two cases and were negative in both.

All the cases were differentiated from Waldenstorm's Macroglobulinemia and other B Lymphoproliferative Disorders by IHC.

Correlation between morphological subtype and prognosis could not be established because of lack of follow up.

Discussion \& Conclusion: Small Lymphocyte-like morphologic features are uncommon in Multiple Myeloma. Although the morphology causes diagnostic difficulty, however clinical features are of a typical MM. Use of a panel of markers including CD45 and CD138 on all Lymphoproliferative disorders would help in picking up this variant of Myeloma so that appropriate therapy could be instituted.

Disclosure of Interest: None Declared.

Keywords: Kappa Lambda, Multiple myeloma, small lymphocyte like.
Abstract 16. Diagnostic Capabilities of SPEP, IFE and SFLC Assay in Intact Immunoglobulin Plasma Cell Dyscrasia

Vinita Paswan"1, Dinesh Chandra1, KhaliqurRahman, Ruchi Gupta ${ }^{1}$, Sanjeev Kumar', Anshul Gupta ${ }^{1}$, SoniyaNityanand ${ }^{1}$, Manish Singh ${ }^{1}$

${ }^{1}$ Hematology, SGPGIMS, Lucknow, India

Aims \& Objectives: To analyze the diagnostic performance and concordance of serum protein electrophoresis (SPE), Serum immunofixation (IFE) with Serum free light chain (SFLC) assay in Intact Immunoglobulin Plasma Cell Dyscrasia patients (II-PCD).

Patients/Materials \& Methods: This was a retrospective observational study of all the newly diagnosed patients with Intact Immunoglobulin plasma cell dyscrasia over a period of 10 months (Sept 2018-July 2019). The baseline clinical and laboratory parameters were retrieved from electronic medical records. SPE was performed on gel electrophoresis system HYDRASYS ${ }^{\mathrm{TM}}$ (SEBIA) and the monoclonal component was identified by IFE with HYDRAGEL ${ }^{\mathrm{TM}}$ (SEBIA) kit. SFLC assays were performed by nephelometry using a latex-enhanced immunoassay (Freelite $^{\mathrm{TM}}$; The Binding Site Group Ltd, Birmingham, UK).

Results: Total 128 patients with intact immunoglobulins plasma cell dyscrasia were analysed which included. 13/128 (10.2\%) patients of IIMGUS and 115/128 (89.8\%) patients of multiple myeloma (IIMM). No patient of Smouldering myeloma was diagnosed. In IIMGUS group, majority of the patients $(11 / 13,84.6 \%)$ had normal SFLC ratio. In contrast, $12.2 \%$ (14/115) of IIMM patients had normal SFLC ratio. This normal SFLC ratio was more frequently observed with IgG lambda subtype of M-protein ( $52 \%$ vs. $48 \%$ with other subtypes). Median age, $\mathrm{Hb}$ and TLC was higher in patients of IIMGUS than patients of IIMM, with normal SFLC ratio. In seven (of the 14) patients of IIMM with normal SFLC ratio, the baseline S. Creatinine level was high. In addition, it has been observed that the dFLC was higher in patients with IIMM than IIMGUS with normal SFLC ratio (23.8 vs. 11.7 , respectively).

Discussion \& Conclusion: The baseline SFLC assay can reveal a normal SFLC ratio in IIMM patients, both with normal and deranged renal function. It has been pointed out that this could be the outcome of clonal heterogeneity in myeloma patients. It also reinforces the fact that SFLC and Intact immunoglobulins are independent tumor markers. The serial SFLC monitoring in this small group of patients, during treatment, will not be as informative as expected. In this study, the increased presence of normal SFLC ratio in association with IgG lambda type M-protein and a higher dFLC value warrants a detailed follow-up study of such IIMM patients, in order to extract more insights.

Disclosure of Interest: None Declared.

Keywords: Intact Immunoglobulin, Myeloma, SFLC.

\section{Abstract 17. Risk Stratification of Multiple Myeloma Based on Fish Studies in a Tertiary Care Centre}

\section{UdayakumarDS $^{* 1}$, Akshay Gore², Nitin Mathur²,} Manorama Bhargava ${ }^{2}$

${ }^{1}$ Hematology, Cytogenetics and Molecular Hematology, ${ }^{2}$ Medanta-The Medicity, Gurugram, India

Aims \& Objectives:

1. To perform FISH studies on patients of diagnosed multiple myeloma (MM).

2. Risk stratification of each patient based on FISH findings.

Patients/Materials \& Methods: 32 patients (Male: 22, Female: 10, Age range: 32-82 Years) diagnosed with $\mathrm{MM}$ in Medanta- 
The Medicity from November 2018 to August 2019 were included in the study, which includes 30denovo and 2 relapsed myeloma cases. Interphase FISH was performed in all the patients. Mononuclear cells from BM aspirate were enriched by purifying plasma cells using CD 138 coated magnetic beads according to manufacturer's instructions (Miltenyi Biotech, Paris, France).Interphase FISH was performed on plasma cells using locus-specific probes, LSI13 (D13S319), LSI 13q34 (control), LSI 17 (p13.1)(TP53)/CEN17, LSI breakapart dual colour $5^{\prime} 3^{\prime} \mathrm{IgH}$, dual fusion translocation probes MYEOV/IgH, FGFR3/TgH, MAF/ TgH (Metasysytems probe, Germany).

Results: The cohort of $32 \mathrm{MM}$ patients had a median age of 57 years (Age range: 32-82 years) including two young patients with 32 and 40 years of age. $29(90.6 \%)$ patients had chromosome abnormalities. The frequencies of abnormalities were as follows: Monosomy $13(17 / 32$, $53.12 \%)$, Del(17p) $(13 / 32,40.6 \%)$, trisomy 11 (14/32, 43.75\%), IGH rearrangements (10/32,31.25\%), Monosomy 16 (9/32,28.12\%), Monosomy 14 (8/32, 25\%), Del(13q) (4/32, 12.5\%), Monosomy $11(3 / 32$, $9.37 \%), \mathrm{t}(4 ; 14) \quad(3 / 32, \quad 9.37 \%)$, Tetrasomy $13 \quad(2 / 32,6.2 \%)$, Tetrasomy $14 \quad(2 / 32,6.2 \%)$, Trisomy $17 \quad(2 / 32,6.2 \%), \quad t(14 ; 16) \quad(1 / 32$, $3.1 \%)$, Monosomy 17 (1/32, 3.1\%), Trisomy 4 (1/32, 3.1\%), Tetrasomy $4(1 / 32,3.1 \%)$, Tetrasomy $11(1 / 32,3.1 \%)$.

Discussion \& Conclusion: FISH findings in our cohort of MM patients showed differences in the prevalence of cytogenetic abnormalities in comparison to published literature. Monosomy $13(53.1 \%)$ and $\operatorname{Del}(17 \mathrm{p})(40.6 \%)$ were the most frequent abnormalities. Using FISH, 13 patients $(40.6 \%)$ each were classified as Standard risk and High risk myeloma and one with $\mathrm{t}(4 ; 14)$ was classified as Intermediate risk myeloma. Five patients who showed IgH rearrangement other than $\mathrm{t}(4 ; 14), \mathrm{t}(14 ; 16), \mathrm{t}(11 ; 14)$ could not be classified into a specific risk group. The two young myeloma patients had a high risk abnormality of Del(17p).

In a small cohort of $32 \mathrm{MM}$ patients from a tertiary care centre FISH abnormality Del(17p) associated with High risk myeloma with a median OS of 3 years was commoner than the reviewed literature. Supporting Document: 48d4dc31-2c92-4b47-aa6c-f3c56fba0df3.

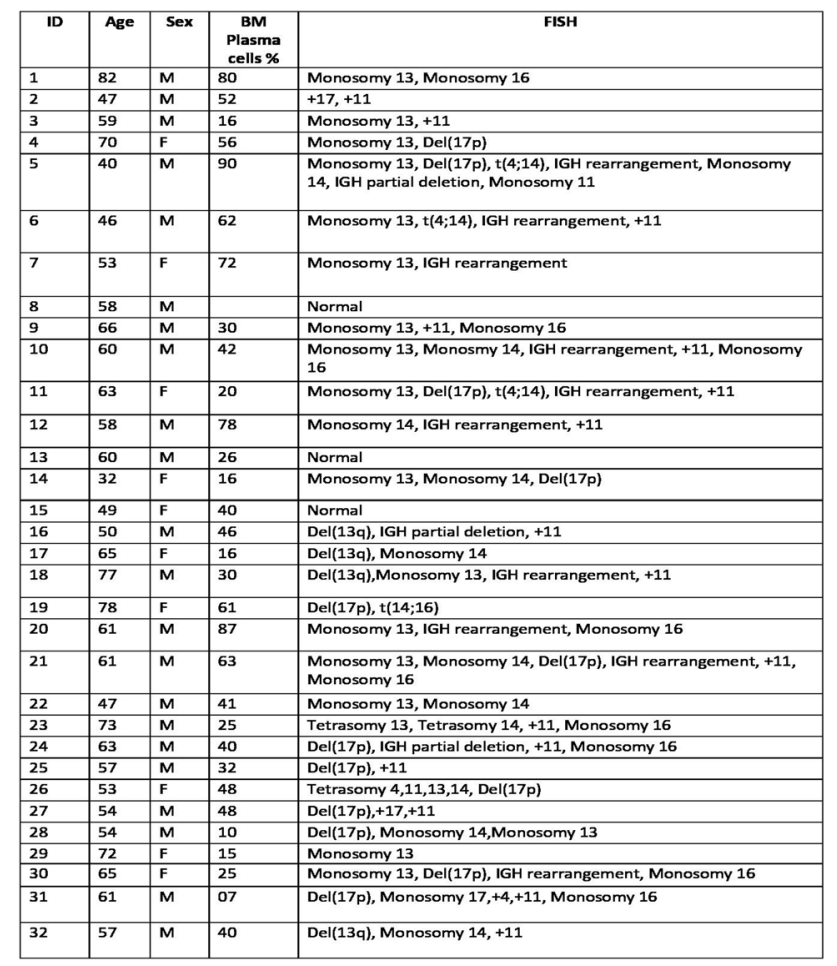

Disclosure of Interest: None Declared.
Keywords: FISH, Multiple myeloma, Myeloma.

\section{Abstract 18. A Novel DNM2-ETV6 Fusion in a Case of T-Lymphoblastic Lymphoma}

\author{
DurvaRaut $^{* 1}$, SwetaRajpal ${ }^{1}$, PrassanaBhanshe ${ }^{1}$, Shruti \\ Chaudhary $^{1}$, SwapnaliJoshi ${ }^{1}$, Gaurav Chatterjee ${ }^{1}$, Prashant \\ Tembhare $^{1}$, P G Subramanian ${ }^{1}$, Sumeet Gujral ${ }^{1}$, Nikhil Patkar ${ }^{1}$
}

${ }^{1}$ Hematopathology, Tata Memorial Centre Advanced Centre for Treatment, Research and Education in Cancer, Navi Mumbai, India

Aims \& Objectives: ETV6 gene encodes for ETV6 protein which is a strong transcriptional repressor. Translocations involving the ETV6 gene are one of the most frequent translocation occurring in leukemias. Similar to $A B L$ and KMT2A genes, ETV6 gene is known to have multiple fusion partners, however, DNM2 gene as a fusion partner for ETV6 gene has not been reported in the literature.We describe a novel fusion between DNM2 and ETV6 in a 14-year-old male with T-lymphoblastic leukemia.

Patients/Materials \& Methods: RNA extraction was doneusing bone marrow sample of the patient.For RNA sequencing, double stranded cDNA was synthesized and then fragmented, A-tailed and ligated using half functional sequencing motifs. Customized panel locally designed in the laboratory using $A B L 1, A B L 2, C S F 1 R, C R L F 2$, DUX4, EPOR, ETV6, EBF1, FGFR1, IKZF1, JAK2, KMT2A, MLLT4, MEF2D, NTRK3, NUP214, NUP98, P2RY8, PAX5, PDGFRA, $P D G F R B, R U N X 1, T C F 3, T Y K 2$ and ZNF384 to detect any putative partner of a fusion gene where one of the partners is known was used for sequencing. Illumina Miseq (300-cycle chemistry) was used to perform paired end sequencing. Custom scripts and bedtools-2.25 were used to determine the coverage and construct the pipeline.Data was analysed using FusionCatcher and Oncofuse.

Results: A 14-year-old male, presented with groin swelling for 3 months duration. The diagnosis of T-lymphoblastic lymphoma was made on inguinal lymphnode biopsy. Bone marrow morphologic \& immunophenotype revealed $2.1 \%$ abnormal lymphoid blasts consistent with bone marrow involvement by T-lymphoblastic leukemia (Near early $\mathrm{T}$ precursor like immunophenotype). RNA sequencing revealed DNM2-ETV6 fusion with breakpoint at exon 12 of DNM2 on chromosome 19 and exon 3 of ETV6 on chromosome 12. Patient was started on modified BFM 90 protocol.

Discussion \& Conclusion: ETV6 translocations have been reported in a diverse array of fusion partners. However literature search has not revealed DNM2 gene as a fusion partner. Mutations in DNM2 gene have been reported in approximately $17 \%$ cases of acute T-lymphoblastic leukemia. This novel fusion might help in refining our knowledge on the disease pathogenesis.

Disclosure of Interest: None Declared.

Keywords: Fusion, LYMPHOMA, RNA sequencing.

\section{Abstract 19. Immunophenotypic Profile of Non Hodgkins Lymphoma by Flow Cytometry and Immunohistochemistry}

Manali SATIZA $^{* 1}$ and Pg resident

${ }^{1}$ Pathology, Pgimsrohtak, Rohtak, India 
Aims \& Objectives: The present study was done to compare the role of flow cytometry and immunohistochemistry and their concordance in the diagnosis and classification of various peripheral NHL.

Patients/Materials \& Methods: The present study was done to compare the role of flow cytometry and immunohistochemistry and their concordance in the diagnosis and classification of various peripheral NHL. Thirty newly diagnosed cases of NHL were evaluated by FCM on peripheral blood or bone marrow aspirate samples and by IHC on bone marrow biopsy sections using a panel of monoclonal antibodies including CD19, CD20, CD5, CD10, CD23, CD79, CD45, kappa and lambda.

Results: All the thirty cases in our study comprised of B-cell NHL. These cases were classified into CLL and Non-CLL group based on morphology and primary panel. An extended panel was used for further sub-typing of non-CLL cases which comprised of FMC7, CD200, CD25, CD103, CD123, CyclinD1, BCL2, BCL6. The overall concordance rate between FCM and IHC regarding the diagnosis of various B-cell lymphomas was $86.6 \%$. The concordance rate between FCM and IHC for all the comparable markers ranged from $76 \%$ to $100 \%$.

Discussion \& Conclusion: We propose a two-tier approach for immunophenotypic analysis of newly diagnosed NHL cases by either method with a minimum primary panel including CD19, CD20, CD5, CD23, CD79, CD10, CD45 for differentiation into CLL/non-CLL group and Kappa and lambda for clonality assessment.An extended panel may be applied wherever required for further sub-typing and stratification.

Disclosure of Interest: None Declared.

Keywords: Bone marrow biopsy, Flow cytometry, non hodgkins lymphoma Immunohistochemistry flow cytometry.

\section{Abstract 20. Hepatosplenic T Cell Lymphomas (HSTCL): A Not 'SO RARE' Entity}

Sadaf Khan $^{* 1}$, JasmitaDass ${ }^{1}$, Amrita Saraf ${ }^{1}$, Sabina Langer ${ }^{1}$, Ajay Sharma ${ }^{1}$, Nitin Gupta ${ }^{1}$, Jyoti Kotwal ${ }^{1}$

\section{${ }^{1}$ Hematology, Sir Ganga Ram Hospital, New Delhi, India}

Aims \& Objectives: To discuss the clinicopathological, immunophenotypical (IPT) findings, and diagnostic difficulties in HSTCL.

Patients/Materials \& Methods: All cases of HSTCL with bone marrow (BM) involvement diagnosed between 2012 and 2019 at Sir Ganga Ram Hospital were selected and studied.

Results: 8 cases, all males, were diagnosed as HSTCL on bone marrow studies. The mean age at diagnosis was $32.6 \pm 12.5$ years (18-58 years). The mean duration of illness was 3.2 months. Six cases presented with fever, three had pancytopenia and two had skin rash.

On examination, all had massive splenomegaly, six had hepatomegaly and two cases had lymphadenopathy. A single case had past history of tuberculosis and another had lung nodule which demonstrated small round cell tumor on biopsy.

All had pancytopenia, mean $\mathrm{Hb}-6.5 \pm 2.1 \mathrm{~g} / \mathrm{dL}$, platelet count$35,800 \pm 2105 / \mu \mathrm{L}$ and TLC- $3486 \pm 2384.7 / \mu \mathrm{L}$

Peripheral smear showed large sized abnormal lymphoid cells, constituting $9.25 \pm 8.5 \%$. BMs were normocellular to hypercellular with $40.45 \pm 25.9 \%$ large sized abnormal lymphoid cells. Two cases had coexistent hemophagocytosis.

IPT in six cases, showed bright CD45 and CD3 positive cases, loss of CD5 in all cases, CD8 in three cases, TCR $\gamma \delta$ in five/six cases and $\mathrm{TCR} \alpha \beta$ in a single case.
Two cases were treated with 1 cycle of CHOP followed by 4 cycles of CHOEP, a third received CHOP followed by splenectomy. The last case was given solumedrol.

\section{Abstract 21. Discussion \& Conclusion: Hepatosplenic T-cell lymphoma was defined in 1990. HSTCL is a rare entity, simulates acute leukemia, and is derived from cytotoxic T-cells typically expressing $\gamma \delta$ T-cell receptor (TCR); represents $1 \%$ of all non-Hodgkin lymphomas and $1 \%>2 \%$ of all $\mathrm{T} / \mathrm{NK}$ cell lymphomas}

The lymphoma presented in varying age groups; ranging from 18-58 years of age. The most common prsenting symptom was fever along with massive splenomegaly, followed by skin rash. Though IPT analysis typically showed a bright TCR $\gamma \delta$ expression; there can be an unusual bright TCR $\alpha \beta$ expression as was seen in one of our cases.

The disease carried a poor prognosis; three out of five cases that were treated succumbed.

Disclosure of Interest: None Declared.

Keywords: Hepatosplenic T Cell Lymphoma, Poor prognosis, RARE.

\section{Abstract 22. Spectrum of Bone Marrow Findings in Chronic Kidney Disease Presenting at a Tertiary Care Center}

NidhinRehman ${ }^{* 1}$, Priyanka Mishra ${ }^{1}$, Deep Kumar Raman ${ }^{1}$, Navneet Puar ${ }^{1}$, Samir Agarwal ${ }^{1}$, Anamika Sinha ${ }^{1}$

${ }^{1}$ Pathology, Command Hospital, Lucknow, India

Aims \& Objectives: We aimed to study the bone marrow aspirate and biopsy findings in Chronic Kidney Disease III, IV and V patients presenting at a tertiary care centre.

Patients/Materials \& Methods: Case files of 50 CKD patients from June 2017-June 2019 whose bone marrow studies were available were retrieved and their clinico-hematological profile studied.

Results: Of $50 \mathrm{CKD}$ patients, the commonest indication for bone marrow studies was refractory anaemia in 40percent (20 out of 50 ),followed by pyrexia of unknown origin $24 \%$ (12 of 50) and pancytopenia in $10 \%$ (05 of 50). Of the $50 \mathrm{CKD}$ patients, patients having glomerulonephritis on renal biopsy are-MPGN (02), Fibrillary glomerulonephritis (01), RPGN (01), immunotactoid glomerulonephritis (01), monoclonal immunoglobulin deposition disease (01). 01 patient was being worked up for POEMS.

In patients with refractory anemia, 11 had anemia of chronic disease, 01 each had AIHA, MDS, PRCA and renal osteodystrophy. Of the 06 patients with Pancytopenia, 02 had hypocellular marrow (? drug induced) and 01 had a reactive bone marrow. In PUO cases, 04 had sepsis related changes and 01 had histoplasma infection (also immunocompromosed).

Out of the 25 patients having plasmacytosis, the plasma cells were ranging from 5 to 60 percent. On further evaluation- 11 had multiple myeloma, 14 were Monoclonal gammopathy of renal significance, 01 had POEMS, and only one had Mixed Connective Tissue Disorder. Discussion \& Conclusion: The commonest indication for bone marrow biopsy in CKD patients is refractory anemia and pancytopenia. Anemia of chronic disease is the commonest hematological abnormality found on bone marrow studies in CKD patients. However, a significant proportion of patients with monoclonal gammopathy present as CKD. Hence, plasmacytosis on bone marrow 
biopsy warrants SPEP, IFE and sFLC assay as well as renal biopsy to identify MGRS.

Disclosure of Interest: None Declared.

Keywords: Bone marrow, Chronic kidney disease, Monoclonal gammopathy.

\section{Abstract 23. A Rare Case of IGG Kappa Lymphoplasmacytic Lymphoma with Associated Amyloidosis}

\author{
Gurpreet Kaur $^{* 1}$ on behalf of AIIMS, Hara Prasad Pati ${ }^{2}$ \\ and AIIMS
}

\author{
${ }^{1}$ Haematopathology, AIIMS, Delhi, ${ }^{2}$ Haematopathology, AIIMS, \\ New Delhi, India
}

Aims \& Objectives: To highlight a rare case of IgG kappa Lymphoplasmacytic lymphoma with associated amyloidosis.

Patients/Materials \& Methods: 65/F presented with low back ache weight loss and easy fatigability of three months duration. There was weight loss of around $20 \mathrm{kgs}$ over 3 months. On examination pallor was present. There was axillary lymphadenopathy around $0.5 \mathrm{~cm}$ along the anterior axillary group, non matted, non tender. Her spleen was just palpable. Rest of the general examination was within normal limits.

Results: There was normocytic normochromic anemia with $\mathrm{Hb}$ of $6 \mathrm{~g}$ $\%$, Peripheral smear showed Rouleaux formation with presence of lymphoplasmacytoid cells (10\%). The bone marrow biopsy revealed a predominance of Lymphoplasmacytic cells (70\%). These cells were positive CD 138 + ve, CD 20 +ve,CD 23 -ve, CD 5-ve, CD3-ve, Cyclin D1 -ve on IHC.Large amyloid deposits were seen which were confirmed by polarizing microscopy. Laboratory examination revealed an M spike of $2.1 \mathrm{~g} / \mathrm{dl}$ with IFE revealing IgG kappa Flow cytometry revealed population of $\mathrm{CD} 200, \mathrm{Cd} 79 \mathrm{~b}$ positive kappa restricted cells. In view of the amyloidosis her Beta 2 microglobulin, NT-pro BNP levels were done which were raised Troponin T however was negative. MYD 88L265P mutation done on bone marrow was positive. FISH myeloma panel was negative. Based on these she was diagnosed as IgG kappa LPL with amyloidosis.

Discussion \& Conclusion: Although the WHO acknowledges their existence, patients with non-IgM LPL are rare and poorly characterized. And only a handful of case reports are seen in literature.The discovery of MYD88 L265P mutations in more than 90\% of LPL/ WM has aided our understanding of this disease entity. WM-associated symptoms are not often seen in the non-IgM case as in our case. Therapeutic perspective, the presence of mutations in MYD88 guides treatment with Ibrutinib, a small-molecule inhibitor of BTK.

Disclosure of Interest: None Declared.

Keywords: amyloidosis, IgG kappa Lymphoplasmacytic lymphoma, MYD88 L265P.

\section{Abstract 24. Post Radiotherapy Testicular Lymphoma (Second Cancer): Coincidence or Side Effect?}

\section{Sucheta - ${ }^{* 1}$, Sant Prakash Kataria ${ }^{1}$, Rajnish Kalra ${ }^{1}$, Rajeev Sen ${ }^{1}$ \\ ${ }^{1}$ Pathology, Pt. B. D. Sharma Pgims Rohtak, ROHTAK, India}

Aims \& Objectives: Primary testicular lymphoma accounts for approximately $5 \%$ of all testicular malignancies. However testicular lymphoma developing as second malignancy after radiotherapy of some other first malignancy is even rarer. We report a case of post radiotherapy testicular lymphoma in 60 years old man.

Patients/Materials \& Methods: A 60 year old man, known case of squamous cell carcinoma of larynx for which he was taking radiotherapy, developed right testicular swelling. Orchidectomy was performed. Histopathological and immunohistochemical examination of orchidectomy specimen revealed Diffuse Large B-cell Lymphoma. Results: Although testicular lymphoma is rare, this is the most common testicular malignancy occurring in men aged more than 60 years. Primary extranodal lymphoma of testes is a lethal lymphoma second only to primary CNS lymphoma. The dominant histological subtype is diffuse large B-cell lymphoma. The usual presentation is a painless unilateral testicular mass or swelling, with bilateral disease present in some cases. In our case patient was already taking radiotherapy for carcinoma larynx and developed testicular lymphoma after approximately 10 months of therapy. The common known sites that develop radiotherapy induced malignancy are breast, thyroid and skin; testes being rare. However it is difficult to conclude that whether it was a coincidence or side effect of radiotherapy. More studies need to be done to evaluate the role of radiotherapy in the development of testicular lymphomas.

Discussion \& Conclusion: Primary testicular lymphoma should always be kept in the differential diagnosis of testicular mass in an elderly patient. In addition patients already taking treatment for a malignancy should be examined for side effects of the therapy and occurrence of a second malignancy whether coincidentally or due to side effects of therapy must be kept in mind for early diagnosis and treatment.

Disclosure of Interest: None Declared.

Keywords: Non hodgkins lymphoma, Radiotherapy, Testicular Lymphoma.

\section{Abstract 25. Hepatosplenic T Cell Lymphoma with Isochromosome 7Q Abnormality}

\section{Ashwini N S ${ }^{* 1}$ and Institute of Liver \& Biliary Sciences \\ ${ }^{1}$ Hematology, Institute of Liver \& Biliary Sciences, New Delhi, India}

Aims \& Objectives: Hepatosplenic T-cell lymphoma (HSTCL) accounts for $1.4 \%$ of all known peripheral T-cell lymphoma.This is an aggressive lymphoma that primarily affects young adults with a strong male predominance. It is characterized by frequent involvement of splenic red pulp, liver sinusoids and bone marrow; nodal and other extra nodal sites are rarely affected.

Patients/Materials \& Methods: A 34-year-old male patient presented with history of episodes of high-grade fever with chills and left upper quadrant pain for the past 1 year. He had history of weight loss and easy fatiguability. On examination he had massive hepatosplenomegaly and no significant lymphadenopathy. Peripheral blood film revealed pancytopenia. On computed tomography (CT) scan, large hepatosplenomegaly with heterogeneous, hypodense areas within the splenic parenchyma and features of hepatosplenic fusion in left lobe of liver were noted. Liver biopsy showed diffuse infiltration of atypical lymphoid cells within the dilated sinusoids. Immunohistochemical analysis revealed these atypical lymphoid cells as CD3 epsilon and CD56-positive, and CD20-negative. Bone marrow biopsy demonstrated sinusoidal infiltration by atypical lymphomatous cells in the background of trilineage haematopoiesis. Subsequent cytogenetic analysis revealed the abnormality of isochromosome 7 .

Results: Diagnosis of Hepatosplenic T Cell Lymphoma was considered in this case. 
Discussion \& Conclusion: HSTCL is a rare entity of peripheral T-cell lymphomas that is characterized by hepatosplenomegaly without significant peripheral lymphadenopathy. It is defined as an extranodal and systemic neoplasm derived from cytotoxic $\mathrm{T}$ cells. Clinically, patients with HSTCL present with B-symptoms, jaundice and hepatosplenomegaly. The predominant laboratory findings include pancytopenia and deranged liver chemistry. Primary involvement of the spleen and the liver with a typical sinusal or sinusoidal infiltration pattern respectively. Associations with chromosomal abnormalities of Isochromosome 7q and trisomy 8 have been reported. HSTCL is an exceedingly rare subtype of mature T-cell lymphomas with dismal outcome despite combination chemotherapies, the accurate diagnosis of which can be challenging. Disclosure of Interest: None Declared.

Keywords: Extranodal, lymphoma, Peripheral T Cell Lymphoma.

\section{Abstract 25. CD200 as a Diagnostic AID IN Differentiating CLL from Mantle Cell Lymphoma by Flow Cytometry}

\author{
Gauri Munjal $^{* 1}$, Monika Gupta ${ }^{2,2}$ \\ ${ }^{1}$ Pathology, Pgims, Hisar, ${ }^{2}$ Pathology, Pgims, Rohtak, India
}

Aims \& Objectives: To assess the role of CD200 in various Mature B-cell Neoplasms.

Patients/Materials \& Methods: The study was conducted in Department of Pathology, PGIMS, Rohtak during the period of July 2018 to June 2019. Immunophenotyping was performed on peripheral blood samples of 50 cases of MBN on 8 ColorFlowcytometer using standard CLPD panel and SSC versus CD19 gating.

Results: Out of 50 cases of MBN, there were 30 cases (60\%) of CLL, 4 cases $(5 \%)$ of MCL, 2 cases (3\%) of marginal zone lymphoma (MZL), 2 cases (2\%) of hairy cell leukemia (HCL) and 12 cases (20\%) of NHL. CD200 was uniformly and strongly expressed in CLL, MZL and HCL but was negative in all the cases of MCL. It was found to be an excellent marker to distinguish CLL from MCL besides CD23.

Discussion \& Conclusion: - Multiparameter flow cytometry is the gold standard for diagnosis of mature B-cell neoplasms (MBN). CD200 has already been proven as a diagnostic marker for chronic lymphocytic leukemia (CLL), it has currently been used as a diagnostic marker to diffrentiate CLL from Mantle cell lymphoma (MCL), as they share common clinical and phenotypic features. The role of $\mathrm{CD} 200$ in other MBN is yet to be established.

-In our study, cases of CLL showed high expression of CD200 as compared to cases of MCL where there was no expression. Since MCL is a more aggressive disease and generally treated differently than B-CLL, it is important to differentiate them. We recommend adding CD200 in routine CLPD panel because of its diagnostic utility in differentiating MCL from CLL and also to aid in targeted immune therapy in the near future.

Disclosure of Interest: None Declared.

Keywords: CD200, Chronic lymphocytic leukemia, Mantle cell lymphoma.

\section{Abstract 26. Chronic Lymphocytic Leukemia and Multiple Myeloma- Two Swords in One Scabbard}

\section{GurleenOberoi $^{* 1}$, Seema Tyagi ${ }^{1}$, ManoranjanMahapatra ${ }^{1}$,} H. P. Pati ${ }^{1}$

\footnotetext{
${ }^{1}$ Hematology, AIIMS, New Delhi, India
}

Aims \& Objectives: Coexistence of chronic lymphocytic leukemia (CLL) and Multiple Myeloma (MM) in the same patient is quite rare. We report a case of synchronous presentation of these two entities. Patients/Materials \& Methods: A 71 years old male was incidentally detected with high peripheral blood white blood cell (WBC) count and absolute lymphocytosis.He was asymptomatic and his physical examination was unremarkable. On subsequent work up, a diagnosis of a rare combination of dual pathology, Multiple Myeloma and Chronic Lymphocytic Leukemia was made.

Results: On workup, his peripheral blood immunophenotyping was suggestive Chronic Lymphocytic Leukemia (Matute's score- 4/5). Bone marrow aspirate showed increased plasma cells (65\%). Bone marrow biopsy showed increased CD138 positive plasma cells infiltrate with kappa restriction along with multiple lymphoid aggregates. Hence a diagnosis of Multiple Myeloma and Chronic lymphocytic Leukemia was made.

Discussion \& Conclusion: The prevalence of CLL was higher in MM patients compared with the general population $(0.05 \%)$. The coexistence of CLL and MM is rare and should be considered. Extensive literature search yielded four reported manuscripts in English literature describing 22 patients. As both CLL and MM are B Cell disorders their coexistence takes on more significance than coexistence of unrelated tumor.

Disclosure of Interest: None Declared.

Keywords: Chronic Lymphocytic Leukemia, Immunophenotype, Multiple myeloma.

\section{Myeloproliferative neoplasms}

\section{Abstract 1. Bone Marrow Histomorphology in Polycythemia Vera}

\author{
Manjula Murari $^{* 1}$, Seema Sharma ${ }^{1}$ \\ ${ }^{1}$ Pathology, SGPGIMS Lucknow, Lucknow, India
}

Aims \& Objectives: Myeloproliferative neoplasms (MPN) are clonal hematopoietic stem cell disorders. In the updated WHO classification bone marrow histopathology constitutes one of the major diagnostic criteria. Aim of the study was to assess the bone marrow biopsy histomorphology in Polycythemiavera.

Patients/Materials \& Methods: Study included 46 cases of Polycythemiavera from January 2013 to December 2017.Marrow biopsies were collected in B-5 fixative, paraffin- embedded, 3-5 micron sections evaluated for cellularity, architectural alterations, proliferation and maturation of trilineage hemopoiesis, dysplastic changes and associated fibrosis. Besides H\&E, VG, Reticulin and Perls stain were done where indicated.In all cases complete hemogram with MGG stained peripheral blood and marrow aspirate smears were also reviewed.

Results: The study showed male preponderance (males 40, females 6), age range $18-75$ years. Clinical history and findings characteristic of polycythemia were seen in majority of cases with headache and plethoric symptoms, splenomegaly was present in $60 \%$ cases. All cases were diagnosed in overt polycythemic phase. Bone marrow cellularity ranged from $60-95 \%$, erythroid cells showed hyperplasia with confluent sheets of erythroid precursors without significant dyserythropoiesis. Concurrent megakaryocytic hyperplasia was seen in $54 \%$ cases with pleomorphic small monolobated to large hyperlobated megakaryocytes, presence of loose clusters. Granulocytic hyperplasia was less prominent with no significant dysplasia or excess of blasts. Sinusoidal dilatation or fibroblastic proliferation was not conspicuous, eight cases showed slight increase in reticulin with no 
collagen fibrosis. Molecular work-up was available in 31cases and of these 22 were positive for JAK2 Axon 14 mutation. Marrow biopsy ruled out evidence of inflammation, myelodysplasia, myelofibrosis or malignant infiltrations.

Discussion \& Conclusion: Diagnosis of Polycythemiavera requires integration of clinical and laboratory findings. Bone marrow histomorphology is valuable as a major diagnostic criterion for Polycythemiavera according to the WHO scheme for differentiation from other MPN particularly Essential Thombocythemia and Primary Myelofibrosis, as well as to rule out some possible causes of reactive erythrocytosis.

Disclosure of Interest: None Declared.

Keywords: Marrow trephine biopsy, Myeloproliferative neoplasm, Polycythemiavera.

\section{Abstract 2. Correlation of JAK2V617F with Thrombosis, Hematological and Biochemical Parameters IN CMPN}

\section{Pradeep $V^{* 1}$, KanwaljeetSingh ${ }^{1}$, TathagatChatterjee ${ }^{1}$,} Ankur Ahuja ${ }^{1}$, S Pramanik ${ }^{2}$

\section{${ }^{1}$ Dept of Lab sciences \& Molecular medicine, ${ }^{2}$ Dept of Clinical Hematology, Army Hospital Research \&referral (AHRR), New Delhi, NEW DELHI, India}

Aims \& Objectives: To correlate JAK2V617F mutation with thrombosis, hematological parameters particularly platelet indices and biochemical parameters in CMPN patients.

Patients/Materials \& Methods: In this study, 160 CMPN patients were enrolled and JAK2V617F mutation analysis was performed. DNA was extracted using EDTA whole blood/bone marrow samples and quality check of this DNA was done using Nanodrop spectrophotopmeter1000. Further real time qualitative PCR was performed to check for JAK2V617F mutation. This mutation was the correlated with complications mainly thrombosis and bleeding, hematological parameters and biochemical parameters.

Results: Among 160 patients, 60 were females and 100 were males (M/F ratio-1:0.6) with age range of $27 \mathrm{yrs}$ to 85 yrs. 80 cases were Polycythemia Vera (PV), 33 were Essential thrombocythemia (ET)\& 47 were Primary Myelofibrosis (PMF). The correlation of JAK2V617F mutation with clinical, hematological, biochemical parameters is summarized in Table 1.

Discussion \& Conclusion: In our study we observed significant correlation of clinical parameters (age \& splenomegaly), hematological parameters (hemoglobin, TLC \& MPV), S.Creatinine\& thrombosis in JAK2V617F positive patients as compared to JAK2V617F negative patients and these findings are in concordance with few studies, though sparsely documented especially for platelet indices \& biochemical parameters. Further, the correlation of platelet indices especially MPV may serve as indirect evidence of JAK2V617F mutational status, however more studies in larger subset of patients are needed to confirm our findings. This study may open new horizons for clinicians to prognosticate and further setting of treatment strategy in CMPN cases.

Supporting Document: 71ad891c-ed4a-4a8e-badf-6ae327dcf099.

\begin{tabular}{|c|c|c|c|}
\hline Clinical parameters & JAK2 positive(91/160) & JAK2 negative( $(69 / 160)$ & pvalue \\
\hline Age(Yrs)(Median) & 58 & 49 & 0.001 \\
\hline Thrombosis & 65 & 29 & 0.037 \\
\hline $\mathrm{Hb}(\mathrm{gm} / \mathrm{dl})$ (Median) & 12.6 & 12 & 0.027 \\
\hline TLC( (cumm))(Median) & 10,500 & 8,000 & 0.031 \\
\hline PIt((cumm) )(Median) & 3.8 & 3.9 & 0.657 \\
\hline MPV(fl)(Median) & 9.2 & 9 & 0.034 \\
\hline PDW(fl)(Median) & 13.7 & 13.6 & 0.484 \\
\hline BUN(mg/dl))(Median) & 18 & 19 & 0.563 \\
\hline s. Creatinine(mg/dl) & 1.1 & 1 & 0.04 \\
\hline S. Bilirubin(mg/dl) & 0.8 & 0.8 & 0.887 \\
\hline LDH(iu/t) & 410 & 398 & 0.135 \\
\hline
\end{tabular}

Disclosure of Interest: None Declared.

Keywords: Chronic myeloproliferative neoplasms (CMPN), JAK2V617F mutation, Thrombosis.

\section{Abstract 3. Rare Atypical CML-How Rare in a Tertiary Care Centre-A Two Year Study}

ChaitaliSingh $^{* 1}$, Shashi Bansal ${ }^{2}$, Upendra Sharma ${ }^{3}$, Surabhi shrivastava ${ }^{4}$

${ }^{1}$ Pathology, ${ }^{2}$ Senior Consultant, Pathology, ${ }^{3}$ Hemato Oncologist, Bhagwanmahaveer Cancer Hospital, ${ }^{4}$ DNB Resident Pathology, BMCHRC, Jaipur, India

Aims \& Objectives: This study aims to find out incidence of the cases of Atypical Chronic Myeloid Leukemia at tertiary care hospital among all the patient who presented to us with Myeloproliferative Neoplasm.

Patients/Materials \& Methods: This is a retrospective two year study of patients who reported at BMCHRC, Jaipur, during the study duration. CBC, peripheral smear \& bone marrow of these patients suggestive of Myeloproliferative Neoplasm.

Results: 2 out of 178 cases of MPN have features of aCML (1.12\%) $\&$ molecular workup shows absence of BCR-ABL gene,JAK-2 exon 12-15 mutation, MPL\&CALR.Out of the 2 cases of aCML, NSG for ETNK1 \& SETBP1 was done in one of them.

Discussion \& Conclusion: aCML is difficult to distinguish from other subtypes of MPN (CML,CNL \& CMML) which is easy to misdiagnose clinically.The presence of granulocytic proliferation associated with marked dysgranulopoeisis \& absence of molecular marker of other MPN including BCR-ABL 1 translocation are defining feature of aCML.

Supporting Document: 38f442 cd-a4f8-4abd-a2e4-96bc9df2b693.

Disclosure of Interest: None Declared.

Keywords: aCML, Myeloproliferative neoplasm, SETBP1 mutation.

\section{Abstract 4. Triple Negative Myeloproliferative Neoplasms and Bone Marrow Fibrosis}

Shashikant Apte ${ }^{1}$, Kannan Subramanian', ReashmaRoshan ${ }^{1}$, Rajesh Phatale $^{2}$, Abhijit Giram ${ }^{* 3}$

${ }^{1}$ Clinical Hematologist, ${ }^{2}$ Hematopathologist, ${ }^{3}$ Hematology Resident, Sahyadri Speciality Hospital, Pune, Pune, India 
Aims \& Objectives: To study the correlation between triple negative mutations- Janus kinase 2 (JAK2), calreticulin (CALR) and myeloproliferative leukemia virus oncogene (MPL) with degree of bone marrow fibrosis and thrombosis.

Patients/Materials \& Methods: 73 BCR-ABL negative MPN patients were included in study. Each case was subjected to screening for JAK2p.V617F, CALR,MPL, and JAK2 exon 12 mutations.Wright stained bone marrow smears and $\mathrm{H} \& \mathrm{E}$ stained trephine biopsies were assessed by hematopathologists. BM fibrosis was graded on a scale of MF-0 to MF-3 (WHO 2008).

Results: We studied 73 cases of BCR-ABL negative MPN with a median age of 57 years (range 18-77 years) and included 57 males and 16 females. Among total 73 cases, 54 are triple negative MPNs. We have 47 cases of PRV, 7 cases of ET and 14 of PMF. 5 cases were categorized as MPN unclassifiable (MPN-U). Among 47 PRV patients, 12 are JAK2 positive, 1 CALR positive and 34 are triple negative. Among $7 \mathrm{ET}$, one is JAK2 positive while 6 are triple negative. Out of 14 PMF patients, 10 are triple negative, 2 has JAK2, 1 has MPL and 1 has CALR mutation positive. 21 patients presented with past or present history of thrombotic events such as Budd Chiari syndrome, cerebrovascular accidents, CVST, portal vein thrombosis, splenic vein thrombosis, Acute MI. Patients with a history of thrombosis included $16 \mathrm{PRV}, 3 \mathrm{ET}$ and 2 PMF cases. Out of 21 patients with thrombotic events, 14 were triple negative. Among 16 PRV with thrombosis, 5 patients are JAK2 positive and 1 CALR positive while 10 are triple negative. Among 3 ET with thrombosis, 2 are triple negative. Both PMF with thrombosis are triple negative.Out of 54 triple negative MPN patients, 35 patients underwent bone marrow examination. Among them 16 had no BM fibrosis, 11 had grade I fibrosis, 2 had grade II fibrosis. 6 patients with triple negative mutations has grade III/III bone marrow fibrosis as compared to one each with JAK2, CALR \& MPL positivity.

Discussion \& Conclusion: Degree of bone marrow fibrosis and thrombotic events were observed more in triple negative MPNs.

Disclosure of Interest: None Declared.

Keywords: Bone marrow fibrosis, Thrombosis, Triple Negative MPN.

\section{Abstract 5. Evaluation of JAK2V617F Mutation in Indian Patients with BCR ABL Negative CMPN}

\section{Suman Lata ${ }^{* 1}$, Sudha Sazawal ${ }^{1}$, Ravi RANJAN ${ }^{1}$, ManoranjanMAHAPATRA ${ }^{1}$, Seema TYAGI ${ }^{1}$, Renu SAXENA ${ }^{1}$ \\ ${ }^{1}$ Hematology, AIIMS, New Delhi, India}

Aims \& Objectives: The JAK2 V617F somatic mutation is present in the majority of patients with myeloproliferative cancer (polycythaemia vera, essential thrombocytosis, and primary myelofibrosis).This study has been introduce to understand the significance of JAK2 mutation for clinico-pathological features, treatment, outcomes and complications of this disorder in Indian patients.

Patients/Materials \& Methods: Mutation screening for JAK2 in patients with polycythemiavera, essential thrombocythemia and primary myelofibrosis was performed in total 800 CMPN suspected patients.

All the patients were analyzed for JAK2V617F mutation using ARMS-PCR and the detail investigations, including measurements of white blood cell (WBC) count, red blood cell (RBC) count, platelet (PLT), hemoglobin (Hb), hematocrit (Hct), and clinical characteristics, were performed whenever required and correlated with mutation results.
Results: We have observed significant association of JAK2V617F mutation in CMPD (PV, PMF and ET) patients.

Discussion \& Conclusion: Our findings has presented the importance of JAK2-V617F mutation in the initial evaluation of patients with suspected BCR-ABL negative CMPN patients. The relationship between the JAK2 mutation and hematological indices can be used in new diagnostic and therapeutic strategies.

Disclosure of Interest: None Declared.

Keywords: JAK2V617F mutation, MYELOFIBROSIS, Polycythemiavera.

\section{Abstract 6. Study on Efficacy of Imatinib with Pioglitazone in Chronic Phase of Chronic Myeloid Leukaemia}

\author{
Ankit Kumar $^{* 1}$, Siddhartha Sankar Ray ${ }^{1}$ \\ ${ }^{1}$ Haematology, Medical college, Kolkata, Kolkata, India
}

Aims \& Objectives: To evaluate the therapeutic efficacy of combination of Imatinib along with pioglitazone in patients with chronic myeloid leukaemia in chronic phase.

Patients/Materials \& Methods: An interventional study done in a tertiary care hospital at Kolkata from April 2018. A total of 30 patients of CML in chronic phase of either gender aged $\geq 18$ years were enrolled. All patients included in this study were naïve, in chronic phase of CML. The enrolled patients were treated with imatinib along with pioglitazone from beginning of therapy and followed up every 3 monthly with the help of BCR-ABL1 transcripts. Primary end points were assessed by early molecular response (EMR), major molecular response (MMR) and molecular response 4.5 (if any).

Results: Thirty patients were included with age range 18-63 years (mean 39.4 years) with male to female ratio of 1.25:1. No pharmacological interaction was observed between the two drugs. The main adverse event was weight gain in 14 patients. The drugs were interrupted in 7 patients for $\geq 2$ times for a total period of 25-30 days in view of thrombocytopenia and/or leucopenia. All patients achieved EMR at 3 month $(<10 \%)$. Almost $66 \%$ patients achieved MMR $(<0.1 \%)$ prior to 12 month of therapy including 2 patients achieving prior to 6 month duration.

Discussion \& Conclusion: All patients who were included in our study received the combination drug from the day one only i.e. all patients who were previously on imatinib were excluded. All patients in our study achieved EMR at 3 month and $66 \%$ of them achieved MMR prior to 12 months of therapy. Rousselot P. et al. observed an early MMR to $\mathrm{MR}^{4.5}$ spontaneous conversions over 12 months in $56 \%$ of patients with combination therapy in comparision to patients on imatinib only achieving the same in 48 months. We conclude, pioglitazone with imatinib combination is an effective and well tolerated therapy for chronic CML patients providing early response rate when Imatinib started early with pioglitazone compared to imatinib only therapy.

Disclosure of Interest: None Declared.

Keywords: Chronic Myeloid Leukemia, Imatinib, Pioglitazone. 


\section{Abstract 7. Clinical Profile and Symptom Burden in Primary Myelofibrosis at a Tertiary Care Centre in India}

Uday Kulkarni" ${ }^{* 1}$, Arvind Venkatraman ${ }^{1}$, Anu Korula ${ }^{1}$, Anup Devasia $^{1}$, Fouzia N A ${ }^{\mathbf{1}}$, Sharon Lionel ${ }^{1}$, Alok Srivastava ${ }^{1}$, Aby Abraham ${ }^{1}$, PoonkuzhaliBalasubramanian ${ }^{1}$, Biju George ${ }^{1}$, Vikram Mathews ${ }^{1}$

\section{${ }^{1}$ Department Of Haematology, Christian Medical College,} Vellore, Vellore, India

Aims \& Objectives: There is paucity of data from developing countries on the clinical profile, symptom burden and treatment access in patients with primary myelofibrosis (PMF).

Patients/Materials \& Methods: We conducted a retrospective analysis of patients diagnosed with PMF from January, 2008 to April, 2019 at our center using hospital records. From January, 2015, patients with PMF were administered the Myeloproliferative Neoplasm Symptom Assessment Form Total Symptom Score (MPN-SAF TSS) on routine hospital visits.

Results: During the study period, a total of 270 patients were diagnosed to have PMF. The median age was 54 years (IQR: 44-61 years). Seventy-five (27.8\%) were females. One-hundred and sixty-two patients $(60 \%)$ had a mutation in $J A K 2,55(20.4 \%)$ in $C A L R$ and $9(3.3 \%)$ in MPL gene. Amongst the remaining, 28 patients $(10.4 \%)$ were triple negative and in 16 patient's mutation results for at least one of these three genes was not available. The median hemoglobin at presentation was $99 \mathrm{~g} / \mathrm{L}$ (IQR: $78-116 \mathrm{~g} / \mathrm{L}$ ), WBC count was $9.4 \times 10^{9} / \mathrm{L}\left(\mathrm{IQR}: 5.7-21.6 \times 10^{9} / \mathrm{L}\right)$ and platelet count was 184 $\times 10^{9} / \mathrm{L}$ (IQR: $\left.78-320 \times 10^{9} / \mathrm{L}\right)$. Ninety-four patients $(34.8 \%)$ had circulating peripheral blood blasts. As per the International Prognostic Scoring System (IPSS) score $(\mathrm{n}=203), 29$ patients $(14.3 \%)$ were low risk, $57(28.1 \%)$ were intermediate-1, $61(30 \%)$ were intermediate2 and $56(27.6 \%)$ were high risk. The MPN-SAF TSS score $(\mathrm{n}=110)$ was recorded at a median time of 5.7 months from diagnosis (IQR: 0-29.8 months). The median total score was 20 (IQR 3-40) with 92 patients $(84.5 \%)$ having at least one symptom. The incidence of the symptoms was: fatigue $65.5 \%$, early satiety $43.6 \%$, abdominal discomfort $47.3 \%$, inactivity $54.5 \%$, concentration problems $30.9 \%$, night sweats $13.6 \%$, itching $13.6 \%$, bone pain $18.2 \%$, fever $8.2 \%$, and unintentional weight loss $31.2 \%$. The common treatment modalities used $(n=201)$ were thalidomide + prednisolone (106 patients), hydroxyurea \pm aspirin (76 patients) or ruxolitinib (6 patients).

Discussion \& Conclusion: In the present study, the clinical profile and the total symptom burden in patients with PMF were similar to those reported in literature. However, most patients did not have access to ruxolitinib due to resource constraints.

Disclosure of Interest: None Declared.

Keywords: MPN-SAF TSS, Myeloproliferative neoplasm, primary myelofibrosis.

\section{Abstract 8. juvenile Myelomonocytic Leukemia: A Series of Three Cases}

\section{IshaSharma*1, Sanjeevansharma $^{2}$, Ajay malik ${ }^{1}$, Venkatesan Somasundaram ${ }^{3}$ \\ ${ }^{1}$ pathology, AFMC, ${ }^{2}$ haematology, CHSC, ${ }^{3}$ haematopathology, AFMC, pune, India}

Aims \& Objectives: We here collate the clinical, hematological, cytogenetic and molecular parameters of an unusual hematolymphoid malignancy namely juvenile myelomonocytic leukemia (JMML).
Patients/Materials \& Methods: 3 JMML cases presented to this hospital between 2016 and 2019. Symptoms varied from fever to respiratory distress and diarrhoeal episodes. They were subjected to routine clinical and peripheral blood examination, Hemoglobin HPLC, bone marrow studies, cytogenetic analysis, molecular genetics and FISH for BCR-ABL fusion.

Results: The patients were between 8 months to $1 \mathrm{yr}$. Out of the three patients, two were girls. Peripheral blood and bone marrow showed features of JMML. Monosomy 7 was found in two out of three cases. FISH for BCR-ABL fusion was negative in all three cases. Somatic NRAS mutation was detected in all three cases. $\mathrm{Hb} \mathrm{F}$ was increased in two out of three cases highest being $6 \%$. Patients were managed with azacytidine, with one patient underwent HSCT. Two of the patients succumbed to their illness after an admission period of 2-3 months, while one is still under follow up.

Discussion \& Conclusion: JMML is a clonal hematopoietic disorder of childhood characterized by proliferation of granulocytic and monocytic lineages with blasts and promonocytes accounting for less than $20 \%$ of the WBC's in the peripheral blood and bone marrow. BCR-ABL fusion is absent, however RAS mutations are characteristic. It accounts for less than 2 to $3 \%$ of all leukemias in children with annual incidence of 0.13 cases $/ 100,000$ children aged 0 to 14 yrs with peak incidence between 1 month to 3 yrs of age. JMML with NRAS/ KRAS mutations have an aggressive course as brought out in this series.

Disclosure of Interest: None Declared.

Keywords: JMML, MONOSOMY, N-RAS.

\section{Abstract 9. Characteristics and Transplant Outcomes of Multiple Myeloma Patients: A Tertiary Care Center Study}

Remi Remakanth ${ }^{* 1}$ on behalf of department of medical oncology $\&$ transfusion medicine, Biswajit dubashi ${ }^{2}$ and medical oncology \& transfusion medicine

${ }^{1}$ Transfusion Medicine, ${ }^{2}$ Medical Oncology, JIPMER, Pondicherry, Pondicherry, India

Aims \& Objectives: To assess the pre \& post-transplant characteristics in patients with Multiple Myeloma (MM) who underwent Autologous Stem Cell Transplantation (ASCT) and to derive the overall survival (OS) and progression free survival (PFS) outcomes in the same patients.

Patients/Materials \& Methods: In our retrospective observational study, all patients with proven diagnosis of MM during the period of January 2013 and January 2019 who underwent ASCT were included [H1]. Data was collected from patient records and registers and hospital information system. The characteristics and outcomes of 38 patients were analysed. PFS and PS of patients were also estimated. Kaplan-Meier estimates of OS and PFS were obtained along with the survival curves for both using SPSS software version 19. 0 .

Results: A total of 38 patients with a mean age of $50.9 \pm 7.9$ years were available for analysis. The number of patients with $\operatorname{IgG}, \operatorname{IgA}$ and LCD types were $19(50 \%), 4(10.5 \%)$ and $8(21 \%)$ respectively and others had unidentified subtypes. The patients with Pre ASCT status were distributed as $9(23.6 \%)$ in very good partial response (VGPR), 8 $(21 \%)$ in partial response (PR), $18(47.3 \%)$ in complete remission (CR) and stringentCR (sCR) and $3(8 \%)$ in progressive disease (PD). Five patients had Comorbidity Index of 1 , six with a CI of 2 , four patients with an index of 3 and 23 had no comorbidities [H1] in the pre transplant phase. Indication for ASCT in $35(92 \%)$ of patients was in view of Consolidation while 3 were in Relapse prior to transplant.Mobilisation with G-CSF alone yielded CD34 counts of 
$3.97 \pm 2.24 \times 10 * 6 / \mathrm{kg}$ while G-CSF with pre-emptive Plerixafor had a mean CD34 yield of $6.29 \pm 2.43 \times 10 * 6 / \mathrm{kg}$ which is significantly higher than G-CSF alone ( $p$ value $<0.004)$. On assessment of post ASCT response among alive patients, $19 \%$ patients were in VGPR, $19 \%$ patients in PR, 39\% in CR \&sCR and rest having progressive disease and not responding to Salvage Therapy. The median Progression -Free Survival time was 56.5 months with 95\% CI [34.2, 78.3] and the median Overall Survival time was 55.6 months with 95\% CI [39.9, 71.0].

Discussion \& Conclusion: Based on our results, PFS and OS in our study were comparable with published literature. The limitation of the study includes inadequate study participants to derive at conclusions and the study population may only represent a selected subset of population. Such registry data analysis however helps us in analysing the long term survival outcomes and various other characteristics that may guide us in clinical decision making in Multiple Myeloma. The median OS is 55.6 with $95 \%$ CI $[39.9,71.0]$ while the median PFS was 56.5 months with $95 \%$ CI [34.2, 78.3] with survival benefits being more apparent among the younger patients.

Disclosure of Interest: None Declared.

Keywords: autologous stem cell transplant, Multiple myeloma, SURVIVAL OUTCOME.

\section{Abstract 10. Study of Clinical and Laboratory Profile of JAK 2 Mutation Negative Primary Polycythemia Patients}

\section{PurushottamChaudhari $^{* 1}$, SanjeevanSharma ${ }^{2}$}

${ }^{1}$ Internal Medicine, ${ }^{2}$ Hematology, AFMC, Pune, India

Aims \& Objectives: Aims: To study the clinical and laboratory profile of JAK 2 mutation negative PrimaryPolycythemia patients in Indian patients.

Patients/Materials \& Methods: Materials and Methods: 130 patients with primary polycythemia presenting to a tertiary care hospital in western India between Jan 2010 to July 2019, and were negative for JAK2 V617F mutation by PCR on initial screening, were included in the study. All secondary causes of polycythemia were excluded. Hemogram, Serum EPO levels, and Bone Marrow studies were done and was documented.

Results: Results: The 130 primary polycythemia patients (117 males $\& 13$ females)had a median age of $44(20-77)$ years at diagnosis. The mean $\mathrm{Hb}$ was $18.5 \mathrm{gm} \%$ (17.0-22.2 gm \%). Bone Marrow studies revealed a hypercellular marrow in $75 \%$ patients.

Discussion \& Conclusion: Discussion: Our study was done at a large tertiary centre which provides free consultation, laboratory investigations and medicines for its patients. Hence all patients who are diagnosed, are treated and followed up and thus collection of data was systematic. All consecutive patients have been included in the study. Our study presented last year at Hematocon 2018 on prevalence of JAK2 mutation had revealed a very low (30\%) level. Similar results were reported recently by another Indian center in Bangalore. We want to highlight the preponderance of this specific entity of JAK2 mutation negative primary polycythemia.

Disclosure of Interest: None Declared.

Keywords: Indian patients, JAK2V617F mutation, Polycythemiavera.
Abstract 11. Primary Myelofibrosis: Histomorphology and Subtypes

Ankita Jaiswal $^{* 1}$, SnigdhaGoel ${ }^{1}$, TejindarSingh ${ }^{1}$

${ }^{1}$ Hematopathology and Surgical Pathology, Oncquest Main Laboratory, New Delhi, New Delhi, India

Aims \& Objectives: Primary myelofibrosis (PMF) is a BCR-ABL1 negative clonal Myeloproliferative Neoplasm (MPN) characterised by proliferation of megakaryocytes and granulocytes in the bone marrow with reactive deposition of fibrous connective tissue and agnogenic myeloid metaplasia and carries the worst prognosis of non-CML, MPNs.

A. To assess the frequency of PMF in cases of marrow fibrosis (MF).

B. To subtype the PMF as per WHO (2016) criteria.

C. To assess the number of cases of PMF in accelerated phase (AP)/blasticphase (BP).

Patients/Materials \& Methods: A retrospective study was carried out from January to August 2019 and 118 casesof MF were diagnosed which included cases of Acute Leukaemia, Lymphoproliferative disorders, Multiple Myeloma, Granulomatous Disease, Metastasis and MPNs. Out of these 118 cases, 42 were of MPNs. These 42 cases of MPN included 26 cases of PMF, 10 cases of Chronic Myeloid Leukemia-Advanced phase, 4 cases of Polycythemia Vera-MF and 2 cases of Essential Thrombocythemia-MF. 26 cases of PMF were subclassified as 3 cases of cellular phase, 14 of fibrotic phase (F), 4 of osteomyelosclerosis (OMS) and 3 of AP and 2 of BP.

Results: All patients except two had splenomegaly.

The 3 cases of cellular phase had granulocytic and megakaryocytic hyperplasia with dense clustering, bulbous nuclei, change in topography to paratrabecular location and features of dysmegakaryopoiesis. Reticulin was grade $0-1$.

The fourteen cases of fibrotic phase showed megakaryocytic proliferation with clustering and atypia with reticulin/collagen fibrosis.

Four cases showed thickening and distortion of bony trabeculae with new bone formation and with marrow space $<50 \%$ were labelled as PMF-OMS.

5 cases of PMF showed clusters of blastoid cells $(\mathrm{AP}+\mathrm{BP})$.

19/26 patients were tested for JAK2/CALR and 18/19 patients were positive.

Discussion \& Conclusion: All cases of splenomegaly and leucoerythroblastic blood picture should undergo bone marrow biopsy for confirmation of PMF. Awareness of Cellular phase -PMF is important and megakaryocytes with dense clustering and bulbous nuclei should be a clue to diagnosis. All cases of PMF should be examined for $\mathrm{CD} 34$ positive cells to rule out $\mathrm{AP}$ as these cases progress to $\mathrm{BP}$ and have a poorer prognosis.

Disclosure of Interest: None Declared.

Keywords: JAK2/CALR, Megakaryocytic Hyperplasia, Primary myelofibrosis.

Abstract 12. JAK2 EXON12 Mutations in JAK2V617F Negative Myeloproliferative Neoplasms- A Case Series

\author{
Hema Malini $^{* 1}$, NivedithaRavindra ${ }^{1}$, Madhavi Maddali ${ }^{1}$, \\ Ekta Jajodia $^{1}$, Arun Kumar A, Kumar A, ${ }^{1}$, \\ Arvind Venkatraman', Kulkarni Uday Prakash', Biju George ${ }^{1}$, \\ Vikram Mathews ${ }^{1}$, PoonkuzhaliBalasubramanian ${ }^{1}$
}

${ }^{1}$ HAEMATOLOGY, CMCH, Vellore, India 
Aims \& Objectives: $B C R-A B L 1$ negative myeloproliferative neoplasms (MPN) are a group of clonal hematopoietic disorders characterized by an increase in differentiated myeloid cells.JAK2V617F mutation is the main driver mutation implicated in $\sim 95 \%$ of patients with polycythemia Vera (PV) and $\sim 50 \%$ of essential thrombocytosis (ET) and primary myelofibrosis (PMF) cases. Mutations located in JAK2 exon12 resulting in deregulated JAK STAT pathway are reported in $2-5 \%$ cases of PV. There are limited reports describing the mutation spectrum of JAK2 exon12 mutations in Indian patients. Here, we describe a series of MPN cases with $J A K 2$ exon 12 mutation identified in our patient cohort.

Patients/Materials \& Methods: JAK2 exon12 mutation screening was done in all JAK2V617F negative cases of MPN sent for molecular diagnosis from 2016-2019. Patient demographics and basic haematological parameters such as complete blood picture including peripheral smear and bone marrow morphology were obtained from electronic case records. Biochemical parameters such as serum erythropoietin (EPO) and LDH levels were also documented. JAK2 exon12 mutation analysis was done on DNA samples extracted from whole blood followed by direct sequencing. Mutations obtained were analyzed for pathogenicity by using bioinformatic tools and published databases.

Results: Mutations in JAK2 exon12 were detected in 8 patients of which 6 were PV and 2 had myelofibrosis. Demographics of the patients is listed in the table.

Unlike a previous report (N Engl J Med 2007; 356: 459-468) that suggest that $50 \%$ of these patients have suppressed erythropoietin levels, none of the PV patients in this series had low or suppressed erythropoietin levels (normal range: 3.7 to $31.5 \mathrm{mIU} / \mathrm{mL}$ ). The spectrum of mutations identified in JAK2V617F negative PV included complex insertion- deletions (indel) in three cases (c.1613_1616ACAA $>$ T, c.1612_1616CACAA $>$ TT \& c.1619_1627TCAGAAATG $>$ GAA), two cases of deletions (c.1622_1627 delGAAATG\& c.1624_1629delAATGAA) and one case of substitution (c.1586 C>A). Among these there were two novel mutations: c.1586 $\mathrm{C}>\mathrm{A}$ andc.1619_1627TCAGAAATG $>$ GAA. In addition, JAK 2 exon 12 mutations were detected in two patients with features of myelofibrosis at presentation (c.1614_1616CAA $>$ ATT \& c.1613_1617ACAAA $>$ TC).

Discussion \& Conclusion: In addition to the targetable hotspot $J A K 2 V 617 F$ mutation in exon 14 , mutations in exon 12 are seen in $2-5 \%$ cases of PV. This is the first case series reporting JAK2 exon 12 mutations in JAK2V617F negative MPN cases including 2 cases of myelofibrosis at presentation. These two cases of myelofibrosis could have been cases of undetected PV that transformed into myelofibrosis. The characteristics and spectrum of mutations identified in this study are similar to previous reports. The functionality of the two novel mutations reported need to be evaluated.

Supporting Document: b2731460-c815-4152-ad61-d3a0f812bf50.

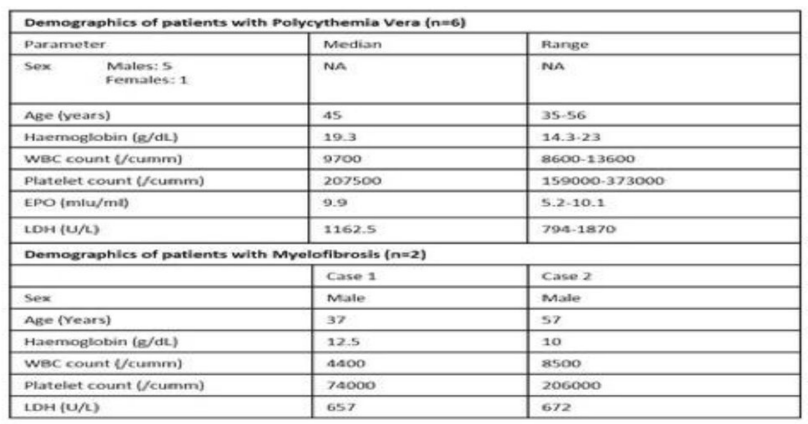

Disclosure of Interest: None Declared.

Keywords: EPO, jak2v617f, MPN.

\section{Abstract 13. Myeloproliferative Neoplasm and Myeloproliferative/Myelodysplastic Neoplasm: An Institutional Study}

\section{DepikaSanasam $^{* 1}$, BanashreeR.K. ${ }^{2}$, Anil Irom ${ }^{3}$, SushilaLaitonjam $^{2}$}

${ }^{1}$ Department of Pathology, ${ }^{2}$ Jawaharlal Nehru Institute of Medical Sciences, Imphal, India, ${ }^{3}$ Department of Medicine, Jawaharlal Nehru Institute of Medical Sciences, Imphal, India

Aims \& Objectives: Myeloproliferative neoplasms (MPN) are a group of hematopoietic stem cell disorders leading to clonal myeloproliferation characterized by granulocytosis, erythrocytosis and/or thrombocytosis. Myeloproliferative/Myelodysplasticneoplasms (MPN/MDS) are neoplasms of the hematopoietic stem cells which show features of both myeloproliferative and myelodysplastic neoplasms. Incidence of MPN and MPN/MDS are limited due to lack of proper data or registry. The study has been done to evaluate the spectrum of MPN and MPN/MDS cases diagnosed in a tertiary referral hospital in a two and half years period.

Patients/Materials \& Methods: It is a retrospective cross-sectional study. All the information was obtained from the bone marrow and clinical OPD registers maintained in the concerned departments. Correlation of complete hemogram \& bone marrow findings with molecular and genetics analysiswere also done in some of the cases. The 2016 WHO criteria was adopted for categorization.

Results: A total of 33 cases were studied including 31 cases of MPN and 2 cases of MPN/MDS. Out of the 31 cases of MPN, 23 cases of chronic myeloid leukemia (CML), 4 cases of polycythemiavera (PV), 2 cases of primary myelofibrosis (PMF), and 2 cases of essential thrombocythaemia (ET) were observed. Amongst the CML cases, $26 \%$ were in blast crisis at presentation. All the cases with lymphoid blast crisis were of B-cell phenotype. Molecular study for BCR-ABL1 was done for all the cases. CALR mutation was noted in 1 case each of JAK2 V617F negative PMF and ET case. Monosomy7 was detected in 1 case of juvenile myelomonocytic leukemia (JMML).

Discussion \& Conclusion: In our study, CML cases had the highest incidence while Srour et al. stated that PV had highest incidence in their study. The difference in the incidence may be due to the difference in geographical and racial distribution.

Disclosure of Interest: None Declared.

Keywords: Chronic Myeloid Leukemia, Myeloproliferative neoplasm, MPN/MDS, BCR-ABL1, Polycythemiavera.

\section{Abstract 14. Abnormal Scattergram \& Cell Population Data by Hematological Analyzer: Clue to Hematopoietic Tumour?}

Urvashi Ghosh $^{* 1}$, Rajesh Kumar Bhola ${ }^{1}$, Sarita Pradhan ${ }^{1}$, Shilpa Anupurba ${ }^{1}$, RipunjayMohanty ${ }^{1}$, DebahutiMohapatra ${ }^{1}$

\section{${ }^{1}$ Department of Pathology, Institute of medical sciences, SUM Hospital, Bhubaneshwar, India}

Aims \& Objectives: All suspected haematological neoplasms and automated blood counts with quantitative abnormality or flags necessitate peripheral smear reviews for morphological evaluation. However the chance of failure to differentiate benign versus malignant aetiology cannot be ruled out even by the expert morphologists. Automated analyser-derived quantitative cell population data of leukocytes and the pattern of histograms or scatter plots has been under tested for distinguishing haematopoietic neoplasm. We tested 
the diagnostic usefulness of leukocyte cell population data together with complete blood count (CBC) parameters in such scenarios.

Patients/Materials \& Methods: We compared Sysmex XN 3000 derived cell population data (CPD) available in the cell counter as research parameters such as Forward side scatter [FSC], Side fluorescence [SFL], Side scatter [SSC],Width of FSC dispersion [WX], side fluorescence [WY], and side scatter [WZ], of neutrophils, lymphocytes and monocytes. Novel reportable parameter like immature granulocyte percentage, absolute immature granulocyte count, NRBC percentage, NRBC count, high fluorescent lymphocyte count and percentage are also compared among the groups.

Results: We analyzed data of patients samples with acute lymphoblastic leukemia (ALL) $(\mathrm{n}=32)$, acute myeloid leukemia (AML) $(n=26)$, acute promyelocytic leukemia (APL) $(n=6)$, myelodysplastic syndrome $(\mathrm{n}=10)$, chronic myelomonocytic leukemia (CMML) $(\mathrm{n}=2)$, chronic myeloid leukemia in chronic phase (CML) $(n=20)$, and chronic lymphoproliferation $(n=26)$; versus 40 controls from healthy volunteers, 20 cases with reactive lymphocytosis due to viral infection and 20 cases with myeloid leukemoid reactions. The cell population data showed difference from the reactive or normal cases in different combination. Hence, combined approach of CBC, CPD along with histogram and scatter plot is highly successful in distinguishing haematopoietic neoplasm.

Discussion \& Conclusion: Though a $\mathrm{CBC}$ along with analyzer generated flags are sensitive, it is not specific and leads to unnecessary slide review. A combined approach of $\mathrm{CBC}$ with the research CPD data and histogram or scatter plot evaluation is of immense help in differentiating haematopoietic neoplasm and help under-resourced laboratory to better triage slide review or referral.

Disclosure of Interest: None Declared.

Keywords: Cell population data, Haematopoietic neoplasms, Scattergrams.

\section{Abstract 15. Refractory Thrombocytopenia: As Clear as Mud}

\section{DipshaKriplani $^{* 1}$, Sneha Janjal ${ }^{1}$, Anu Singh ${ }^{1}$, VistaspAntia ${ }^{1}$}

${ }^{1}$ Department of Haematology, Breach Candy Hospital, MUMBAI, India

Aims \& Objectives: INTRODUCTION.

Refractory thrombocytopenia (RT) is classified by the WHO as a subtype of refractory cytopenia with unilineage dysplasia and is an unusual subtype of MDS that initially presents as chronic pure thrombocytopenia. RT is often misdiagnosed as idiopathic thrombocytopenic purpura (ITP) because the dysplasia in ITP is indistinguishable from that in RT.

Cytogenetic abnormalities associated with RT include del(20q), +8 , and abnormalities of 5 and/or 7 [3]. RT with chro-mosome 5 abnormality should be differentiated from MDS with isolated del $(5 q)$ (5q-syndrome). Since the prognoses and treatments for RT, ITP, and $5 \mathrm{q}$-syndrome are very different, the accurate diagnosis of patients presenting with thrombocytope-nia or chromosomal abnormalities is essential. Here, we report a patient with RT harbouring a 5q deletion. Patients/Materials \& Methods: CASE:

A 62 year old asymptomatic female patient consulted us for a second opinion regarding isolated thrombocytopenia with normal immature platelet fraction. Repeated serial counts showed a declining trend in her platelet counts. She had no fever and no episodes of abnormal bleeding. She was not taking any incriminating drugs. On examination, she had no lymphadenopathy or hepatosplenomegaly. Bone marrow studies were done by her then treating haematologist and was reported to have normocellularity with increase in megakaryocytes with dysplastic changes suggestive of peripheral destruction. Her ANA was negative. She was started on T. Revolade $50 \mathrm{mg}$ OD which was increased to $75 \mathrm{mg}$ OD in view of poor response. In view of inadequate response to ELTROMBOPAG, she consulted us for a second opinion.

Her Complete Blood Count showed the following: $\mathrm{Hb} 11.8 \mathrm{~g} / \mathrm{dl}$, TLC 7800 cumm, platelet count of 39,000 cumm with an Immature Platelet Fraction of $22 \%$. Thrombocytopenia was confirmed on peripheral smear.

Results: On reviewing her bone marrow aspirate and biopsy slides, significant dysplastic changes were noted and a diagnosis of Myelodysplastic Syndrome was considered. Cytogenetic studies by FISH were sent and showed to have a normal karyotype along with $5 q$ deletion present in $8 \%$ cells. A diagnosis of ITP was ruled out. $-5 q$ syndrome usually presents with macrocytic anaemia along with a normal or elevated platelet counts. However, our patient had normal haemoglobin with thrombocytopenia. Hence, a diagnosis of Refractory Thrombocytopenia was made.

Discussion \& Conclusion: RT is a rare subtype of MDS and no clear guidelines for its treatment are laid. Whether to consider starting LENALIDOMIDE for our patient is a thoughtful decision to be taken. Disclosure of Interest: None Declared.

Keywords: $-5 q$ syndrome, Immune thrombocytopenic purpura (ITP), Refractory Thrombocytopenia.

\section{Abstract 16. Chronic Myeloid Leukemia (CML) in Chronic Phase Presenting as Pleural Effusion}

\section{Vibhu Ranjan Khare ${ }^{* 1}$, Kailash Kumar Gupta ${ }^{1}$, Nilesh Kumar ${ }^{1}$, Chandan Kumar', Anurag Rana ${ }^{1}$}

${ }^{1}$ Department of General Medicine, Institute Of Medical Sciences, Banaras Hindu University, Varanasi, India

Aims \& Objectives: In Chronic Myeloid Leukemia (CML), only around $10 \%$ of the patients have extramedullary involvement out of which lymph nodes and skin are most commonly affected. Amongst it, pleural involvement is very rare and poorly understood. Here, we present a patient of CML in chronic phase who presented to us as a case of pleural effusion.

Patients/Materials \& Methods: A 42 year male presented with complaints of breathlessness, cough and chest pain for 1 month and dragging sensation in left upper abdomen for 20 days. On general examination, the patient was tachypneic and was found to have pallor without icterus or lymphadenopathy. On systemic examination he had a dull note on percussion and decreased breath sounds below the left $3^{\text {rd }}$ intercostal space and a $14 \mathrm{~cm}$ palpable spleen. On investigation, Total Leucocyte Count (TLC) was found to be 5.2 lakhs with cells of myeloid lineage predominantly. Peripheral Blood Smear and Bone Marrow Aspiration showed myelocytes, metamyelocyte and a few blasts. Chest X-Ray showed a massive left sided pleural effusion which on cytology and microscopy also showed myelocytes and metamyelocytes. Philadelphia chromosome was positive and BCRABL translocation was quantified. Diagnosis of CML was made and the patient was started on Imatinib and other conservative measures. Results: The patient was continued on Imatinib. He improved clinically, the spleen regressed in size and the pleural effusion also decreased markedly on follow-up.

Discussion \& Conclusion: Pleural involvement in CML is very rare and can be caused by many mechanisms like the leukemic infiltration of pleura, obstruction of pleural capillaries, extra-medullary hematopeoisis among others. Therefore, in cases of non-thoracic causes of pleural effusion, CML should be considered as one of the differential diagnosis especially if splenomegaly is present. 


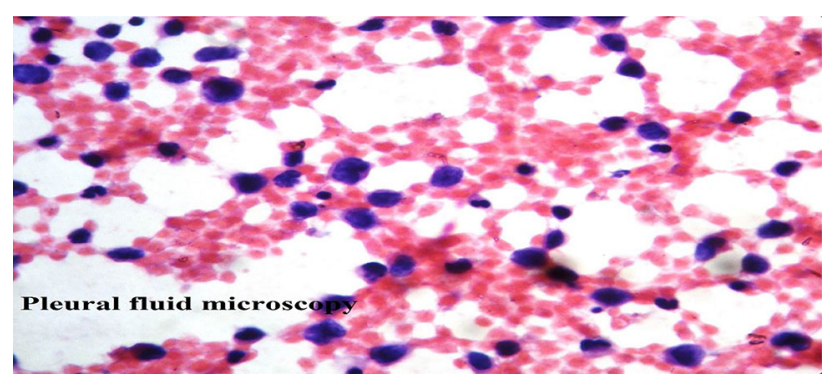

Disclosure of Interest: None Declared.

Keywords: CML, Imatinib, Philadelphia chromosome.

\section{Abstract 17. BCR-ABL Positive Essential Thrombocytosis or an Unusual Presentation of Chronic Myeloid Leukemia}

\section{MeghaVerma ${ }^{* 1}$, Nitin Gupta ${ }^{2}$, Vandana Arya ${ }^{3}$, Sabina Langer ${ }^{4}$, JasmitaDass $^{4}$, Amrita Saraf ${ }^{1}$, Jyoti Kotwal ${ }^{4}$, Ajay Sharma ${ }^{4}$ \\ ${ }^{1}$ Sir Ganga Ram Hospital, New Delhi, India, ${ }^{2}$ Clinical Haematology, ${ }^{3}$ Molecular Medicine, ${ }^{4}$ Haematology, Sir Ganga Ram Hospital, New Delhi, India}

Aims \& Objectives: Implications of testing BCR-ABL in patient with essential thrombocytosis with JAK2,CALR,MPL negative status. Patients/Materials \& Methods: We hereby report a case of 33 years old lady who presented with isolated extreme thrombocytosis with BCR-ABL positivity.

Results: A 33 years old lady was referred to clinical haematology department of Sir Ganga Ram hospital New Delhi for management of extreme thrombocytosis which was detected incidentally during the evaluation of easy fatigue, weakness and loss of appetite of 1-2 months duration. General and systemic examination was unremarkable. Hemogram revealed haemoglobin $10.5 \mathrm{~g} / \mathrm{dl}$, TLC$10.4 \times 10^{3} / \mu 1$ and platelet count of $2100 \times 10^{3} / \mu 1$. Bone marrow aspiration and biopsy revealed hypercellular marrow with M:E ratio of 4.9:1, marked increase of megakaryocytes with pleomorphic forms and reticulin grade 2 fibrosis was reported, suggestive of essential thrombocytosis. USG abdomen revealed normal sized liver and spleen. RT PCR for JAK2, MPL, CALR was negative. Interestingly BCR-ABL by RT-PCR came positive for p210 transcript. Patient was then treated with imatinib which lead to normalization of platelet count after 2 weeks.

Discussion \& Conclusion: BCR-ABL positive ET, a rare entity has been described previously in few case reports with some reports suggestive of aggressive nature of disease and early evolution to blast crisis. Whether this holds true in the present era of TKI therapy is not known. Our patient has shown good response to imatinib therapy but longer follow up is needed.

Conclusion-Every case of essential thrombocytosis without JAK2, CALR and MPL mutation should be tested for BCR- ABL mutation before being designated as triple negative essential thrombocytosis. Disclosure of Interest: None Declared.

Keywords: bcrabl positive, CML, essential thrombocytosis.

\section{Stem Cell Transplantation}

\section{Abstract 1. Impact of KIR \& Their Cognate HLA Ligands on HLA Matched HSCT Outcomes in Hematological Malignancies}

\section{Selma Dsilva ${ }^{* 1}$, Manisha Tambe ${ }^{1}$, NavinKhattry ${ }^{1}$,} Meenakshi Singh ${ }^{1}$

\section{${ }^{1}$ TMC, Navi Mumbai, India}

Aims \& Objectives: Hematopoietic stem cell transplantation (HSCT) is a choice of treatment for hundreds of leukemia patients. The main criteria for selecting a donor is the level of Human Leukocyte Antigen (HLA) matching. Even after finding a HLA matched donor, conditions such as relapse and Graft vs Host Disease (GvHD) result in high mortality. Recently, Killer Immunoglobulin like receptor (KIRs) genes has been implicated in predicting transplant outcomes. We studied the role of KIR receptors and their cognate HLA ligands on post transplant outcomes in HLA matched HSCT.

Patients/Materials \& Methods: A total of 55 HLA matched patient donor pairs were included in this study. HLA and KIR genotyping was carried out by the PCR-SSP method. KIR ligands were assigned to patients using the freely available KIR Ligand calculator tool ( http://www.ebi.ac.uk/ipd/kir). KIR genotypes $\mathrm{B} / \mathrm{x}$ or $\mathrm{A} / \mathrm{A}$ were assigned to each donor depending on the specific KIR genes present. Results: The median follow up period was 36 months. There were a total of 13 patients who relapsed. There was no significant difference between the baseline characteristics like donor gender, donor age, conditioning regimen, or disease status pre transplant with post transplant outcomes. When patients were divided based on the HLA C and $\mathrm{B}$ ligands for KIR that they co-expressed, it was observed that patients carrying the HLA $\mathrm{C} 1 \mathrm{C} 1$ and missing the HLA Bw4 ligand had significantly lower cGvHD $(p=0.017)$ probably leading to significantly better OS $(p=0.036)$. It was also observed that transplantation involving donors with the B/X KIR haplotype (more activating haplotype) resulted in lower risk of acute GvHD $(p=0.007)$. Similarly, donors with higher B content score $(>1)$ resulted in lower aGvHD $(p=0.006)$. Donors with higher number of activating KIR genes (KIR2DS1, KIR2DS2) resulted in lower risk of $\operatorname{aGvHD}(p<0.05)$ and cGvHD $(p<0.05)$.

Discussion \& Conclusion: Our data indicates that absence of the $\mathrm{Bw} 4$ ligand along with the presence of the $\mathrm{C} 1 \mathrm{C} 1$ HLA $\mathrm{C}$ ligand contributes to improved survival in HLA matched related HSCT. Furthermore, donor centromeric and telomeric KIR B haplotype protects against acute and chronic GvHD. This study highlights the importance of KIR genotyping and HLA ligands in predicting transplant outcomes in HLA matched related HSCT.

Disclosure of Interest: None Declared.

Keywords: HLA, HSCT, KIR.

\section{Abstract 2. To Evaluate Stem Cell Yield, Engraftment, Chimerism \& Patient Outcome in Allogeneic PBSC Transplants}

\author{
Neerja Kushwaha' ${ }^{1}$, Anantpreet Kaur Bajaj ${ }^{* 2}$, Joseph Philip ${ }^{1}$ \\ ${ }^{1}$ Immunohaematology \& Blood Transfusion, \\ ${ }^{2}$ Immunohaematology and blood transfusion, Armed Forces \\ Medical College, PUNE, India
}

Aims \& Objectives: The donor characteristics affect the CD34+ stem cell yield. Donor's age,sex,weight, pre procedure WBC and 
platelet counts have been studied as possible predictors of adequate PBSC collection in healthy donors.Correlation of CD34+ stem cell yield of the PBSC product has been studied with haematopoietic recovery, percentage donor chimerism in recipient and GVHD till day 100 post transplant.

Patients/Materials \& Methods: A total of 25 allogeneic PBSC transplants done over a period of 3 years at our center were included in the study. All 25 allogeneic donors underwent mobilization with filgrastim. Leukapheresis was performed followed by flowcytometric CD34+ cell enumeration. Chimerism analysis was done post-transplant and was correlated with the recipient outcome. Statistical analysis was performed using SPSS software and a $p$ value of $<0.05$ was considered significant.

Results: Age had a negative correlation with CD34+ counts while donor weight, pre-procedure platelet \& WBC count, neutrophil \& platelet engraftment,which, though not statistically significant, had a positive correlation with CD34+ count of the product transfused. The chimerism analysed had a mild positive correlation with the CD34+ count of the stem cell product, but this was not statistically significant.

Discussion \& Conclusion: Donor parameters in our study did not affect the CD34+ yield. Healthy individuals can be potential PBSC donors in MUD transplants with adequate yields, irrespective of donor demographics. The engraftment kinetics correlated with the CD34+ yield, but due to the small sample size this could not reach statistical significance.The patient outcome and the incidence of GVHD did not depend on the percentage donor chimerism.

Disclosure of Interest: None Declared.

Keywords: allogeneic PBSC transplant, CD34+ yield and engraftment, chimerism analysis and GVHD.

\section{Abstract 3. A Cell Dose of $>5 \times 10^{6} / \mathrm{kg}$ CD34 Cells is More Cost Effective for Autologous SCT in Multiple Myeloma}

Hamza Dalal $^{* 1}$, NatarajKS ${ }^{1}$, AmarnadhPolisetty ${ }^{1}$, Aditi Shah $^{1}$, ShivakumarKomaravelli ${ }^{1}$, SharatDamodar ${ }^{1}$

${ }^{1}$ Hematology, Mazumdar Shaw Medical Center, Narayana Health, Bangalore, India

Aims \& Objectives: Primary: To evaluate a threshold CD34 cell dose of $>5 \times 10^{6}$ cells $/ \mathrm{kg}$ with respect to anti-microbial use, supportive care, time to engraftment and hospital stay.

Secondary: To assess efficacy of this intervention in reducing overall transplant cost.

Patients/Materials \& Methods: All patients who underwent Autologous stem cell transplant at our center between January 2015 and July 2019 were recruited for the study. BMT records, case papers and discharge summaries were scrutinized for data collection. Patients who underwent transplant on out-patient basis were excluded. Patients who received cryopreserved stem cells were not included due to difficulty in ascertaining accurate CD34 dose in these patients. Patients were divided into 2 groups-Group 1: Conditioning with Melphalan (200 mg/m2) and Group 2: Conditioning with Melphalan $(<200 \mathrm{mg} / \mathrm{m} 2)$. Dose of Melphalan was decided on the basis of performance status and co-morbidity index. Patients in both groups were assigned to 2 sub-groups (A/B) according to stem cell dose (A: $<5 \times 10^{6}$ versus B: $>5 \times 10^{6} \mathrm{CD} 34$ cells $/ \mathrm{kg}$ ). Data was collected pertaining to Antimicrobial use including higher antibiotics (Colistin/Teigecycline), use of Total Parenteral Nutrition, time to engraftment, hospital stay and subsequently compared between the two groups.
Results: A total of 89 patients were recruited for the study. Patients were divided into 2 groups and data was analyzed.

GROUP 1 (Melphalan $=200 \mathrm{mg} / \mathrm{m}^{2}$ ).

53 patients included in this group were subdivided into sub-group A (27 patients) and group B (26 patients). Median age was comparable (58 years) and both groups had 4 patients transplanted at relapse. Total days of antibiotic use, use of higher antibiotics and rates of pre-emptive anti-fungal use were higher in sub-group A. Neutrophil engraftment was comparable between the 2 sub-groups but platelet engraftment was prolonged in Sub-group A. Patients in group A had average of 2 more days hospital stay as compared to group B. Cumulative cost of transplant in group B patients was Rs. 39,400 lower than group A, thereby reducing transplant costs by $10 \%$.

Group 2 (Melphalan $<200 \mathrm{mg} / \mathrm{m}^{2}$ ).

36 patients included in this group were subdivided into sub-group A (16 patients) and group B (20 patients). Median age was 58.5 years for Group A and 56 years for group B. Antibiotic use and pre-emptive anti-fungal prophylaxis was similar in the two groups. Mucositis was more severe in group B with higher TPN use. Median Neutrophil engraftment was 1 day delayed in group A than group B which also translated into 1 additional day of hospital stay for group A patients. Cumulative cost of transplant in group B patients was Rs. 12,500 lower than group A. Major limitation in this group was due to varied doses of Melphalan (140 to $180 \mathrm{mg} / \mathrm{m}^{2}$ ) used in different patients.

Discussion \& Conclusion: A previous study using $>5 \times 10^{6}$ cells/ $\mathrm{kg}$ showed similar benefit in limiting antibiotic use and hospital stay in Autologous transplant recipients. Improved stem cell mobilization strategies have resulted in collection of adequate doses in most patients. Findings of our study assume greater significance with widespread emergence of antibiotic resistant infection in hospital settings. Majority of Indian population cannot afford an Autologous transplant at present. This necessitates use of economic and safe interventions which would enable widespread availability of this treatment modality.

Disclosure of Interest: None Declared.

Keywords: CD34+ yield and engraftment, Multiple Myeloma Autologous SCT, plerixafor mobilisation.

\section{Abstract 4. Correlation of Methods of Hematopoietic Progenitor Cell (HPC) Enumeration in HPC Harvest by Apheresis}

AanchalLuthra $^{* 1}$, Aseem Kumar Tiwari', Swati Pabbi',
GeetAggarwal $^{1}$, Gunjan Bharadwaj

${ }^{1}$ Transfusion Medicine, Medanta -The Medicity, Gurgaon, India

Aims \& Objectives: The primary aim of the study was to correlate a cheaper and easy-to-do HPC (Hematopoietic Progenitor Cell) count obtained from automated cell counter with the current standard of testing by flowcytometric CD34+ enumeration in HPC harvest by apheresis i.e. HPC (A). The importance of HPC enumeration lies in deciding the Time-To-In-Harvest (TTIH) and Adequacy-Of-HarvestDose (AOHD) in HPC (A) transplant setting.

Patients/Materials \& Methods: An observational, prospective study was conducted in year 2018-2019. 42 HPC (A) donors (allogeneic and autologous) were subjected to HPC harvest. 42 peripheral blood (PB) samples, obtained before the start of harvest and 84 apheresis (APH) samples (mid-harvest and product) were included. The samples were analyzed first on Sysmex automated cell analyzer and then on flowcytometer by BD FACSVERSE for CD34+ enumeration. Statistical analysis comprised of correlation coefficient (r) of HPC count, WBC count, MNC count and IG index with CD34+ count and 
Receiver Operator Curve (ROC), to determine the cut-off point for PB HPC count to obtain a target dose of $5^{*} 10^{6} \mathrm{CD} 34+$ cells $/ \mathrm{kg}$.

Results: The main indications of HPC (A) in our study subjects were hematological malignancies (25) and marrow failure states (11). The number of allogeneic and autologous HPC (A) harvest were $29(69 \%)$ and $13(31 \%)$ respectively. The correlation coefficient of HPC with CD34 flowcytometer was 0.617 and 0.699 for peripheral and apheresis sample respectively, which was statistically significant. The correlation with MNC, IG and WBC count was not statistically significant. A cut-off value of PB HPC was established to be 66 cells $/ \mu \mathrm{L}$ for a target dose of $5 * 10^{6} \mathrm{CD} 34+$ cells $/ \mathrm{kg}$, with a sensitivity of $48 \%$ and specificity of $89 \%$.

Discussion \& Conclusion: In India, the challenge with enumeration of HPC by flowcytometer is the cost, limited availability and the longer turn-around time (TAT). This study, possibly the first report from North India, demonstrated a statistically significant correlation of the HPC count with $\mathrm{CD} 34+$ count. The positive predictive value (PPV), that is, if the donors have a PB HPC count of $66 \mathrm{cells} / \mu \mathrm{L}$ or more, the target dose would be obtained, was almost $94 \%$. Thus HPC count, which is $1 / 4$ th the cost of CD34 count, can be used to determine the TTIH and AOHD.

Supporting Document: 01b21bd4-f558-48d1-b216-62edc5ae32eb.

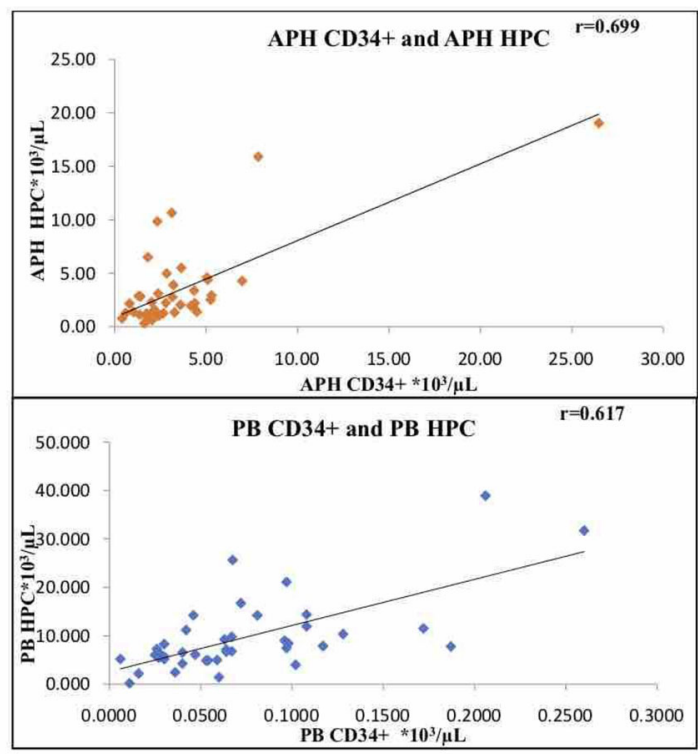

*PB- Peripheral Blood, APH- Apheresis sample, $\mathrm{r}$ - correlation coefficient

Disclosure of Interest: None Declared.

Keywords: apheresis, CD34, HPC.

\section{Abstract 5. Frequency of HLA Matching with the Parents in Indian Population}

\section{Manisha Tambe $^{* 1}$, Selma DSILVA ${ }^{1}$, ShalakaKadam ${ }^{1}$, Meenakshi Singh ${ }^{1}$}

\section{${ }^{1}$ Tme, Navi Mumbai, India}

Aims \& Objectives: Background: Getting a HLA matched donor is a key factor for successful hematopoietic stem cell transplantation. Patients are usually haplomatched with their parents. The chance of finding a full HLA-match among parents is very rare, if so, in cases where there is consanguinity between parents. Availability of HLA $6 / 6$ matched donor with parents in Indian family is more common than the other population. Reports suggest, finding a HLA matched sibling (6/6) is $40 \%$ where HLA matched parent is $25.21 \%$.

Aim: The aim of the study was to evaluate the frequency of finding a full HLA match among parent donors.

Patients/Materials \& Methods: DNA was extracted from peripheral blood sample of the patient (recipient) and donor/s. Low resolution PCR sequence specific primers (PCR-SSP/SSO) method was used for identifying HLA alleles for HLA A, B and DRB1 loci in 1000 families from different communities all over India.

Results: Out of the 1000 families studied, 124 patients were typed with parents as donors. Among these there was no consanguinity in $115(92.75 \%)$ whereas, 9 had a consanguinity history (7.25\%). In the non consanguinous group, 54 had parents as potential 6/6 HLA matched donors $(46.95 \%)$. and haploidentical donors in 29 families (25.21\%). In 11 families parents werehaplomatched with each other (8.87\%). In 32 families, there was sharing of one allele $(27.82 \%)$, sharing of 2 alleles in 27 cases (23.47\%), 3 alleles sharing in (19.13\%) \& 4 allele sharing in (4.34\%) families. Among 115 families, 54 $(46.95 \%)$ patients were matched in both GVH \& HVG direction with one of the parents. In 49 families, parents were either $5 / 6(77.55 \%)$ or $6 / 6(22.44 \%)$ with patient in HVG/GVH direction.

Discussion \& Conclusion: Our results suggest that in the Indian population, other than the siblings there is a higher chance of finding a HLA matched donor among parents. Hence, parents should also be screened as a potential donor for hematopoietic stem cell transplantation.

Disclosure of Interest: None Declared.

Keywords: Allele frequency, HLA, HSCT.

\section{Abstract 6. Safety and Efficacy of Donor Type RBC Transfusion Prior to Major ABO Incompatible BMT}

Shailesh Lavana ${ }^{* 1}$, Dinesh Bhurani ${ }^{2}$, RayazAhmed ${ }^{2}$, Narendra Agarwal ${ }^{2}$, VishvdeepKhushoo ${ }^{2}$, Sumeet Mirgh ${ }^{2}$

${ }^{1}$ Clinical Haemtology, KCHRC, Vadodra, ${ }^{2}$ Clinical Haemtology, RGCRI, Delhi, India

Aims \& Objectives: Retrospective single centre analysis of two children with thalassemia major, to investigate the safety, efficacy and tolerability of donor-type red blood cell transfusion prior to allogeneic bone marrow stem cell transplantation in children with Major ABO mismatch.

Patients/Materials \& Methods: 2 children with thalassemia major received donor type RBC transfusion, in gradually increasing aliquots during conditioning regimen (pre transplant) under antihistaminic and steroid cover. Reaction to donor type RBC and graft transfusion, number of donor type RBC transfusions, haemolysis parameters, and trend of isoagglutinin titers, and engraftment data were observed.

Results: Efficacy of mismatched RBC transfusion: A significant reduction of isoagglutinin titers was observed.

Patient 1: First patient isoagglutinin titre prior to starting of conditioning regimen was clinically significant (Anti B Ig M-1:32, Ig G1:256). After administration of Donor type RBC for four days titre was reduced to clinically insignificant level (Anti B Ig M-1:16, Ig G- 1:16).

Patient 2: First patient isoagglutinin titre prior to starting of conditioning regimen was clinically significant (Anti B Ig M-1:16, Ig G1:128). After administration of Donor type RBC for four days titre was reduced to clinically insignificant level (Anti B Ig M-Nil, Ig G$1: 4)$.

Safety and tolerability of mismatched RBC transfusions: Compared with transfused RBC no severe complications are observed. One child developed stage I hypertension requiring treatment. 


\section{Engraftment data:}

Patient 1: Day of Neutrophil engraftment: D +15 .

Day of platelet engraftment: $\mathrm{D}+16$.

Day +30 chimerism: Complete (Donor-100\%).

Patient 2.

Day of Neutrophil engraftment: D +16 .

Day of platelet engraftment: D +18 .

Day +30 chimerism: Complete (Donor-100\%).

Haemolysis parameter post mismatched RBC transfusion.

Patients did not show significant increase of bilirubin or LDH levels.

Discussion \& Conclusion: Donor type RBC transfusion is a safe, tolerable and effective procedure to reduce the isoagglutinin titers prior to allogeneic $\mathrm{ABO}$ mismatched bone marrow transplantation in children.

Disclosure of Interest: None Declared.

Keywords: ABO missmatch, Donor type Red blood cell transfusion, Major.

\section{Abstract 7. Initial HSCT in Non- HEPA Filter AHU Rooms: A Single Center Experience}

\section{Rohit Upreti $^{* 1}$, SanjeevanSharma ${ }^{1,1}$ \\ ${ }^{1}$ internal medicine, afmc, pune, India}

Aims \& Objectives: To analyse initial HSCT outcomes of a nonHEPA filtered BMT unit.

Patients/Materials \& Methods: A 2 bedded non HEPA filter HSCT unit was started at our 800 bedded tertiary care hospital in Western India in January 2016. 90 HSCTs (55 Autologous and 35 Allogeneic) were carried out from Jan 2016 to Aug 2019. All allogeneic transplants were from family donors except one unrelated donor transplant. Stem cell source was PBSC in 89 and BM in 01. Double and reverse barrier nursing was employed for all patients, and all were put on antifungal prophylaxis at start of conditioning regime. Outcome data was evaluated at $d+30, d+100, d+365$ or death at any timepoint.

Results: 90 patients (52 males and 38 females), with mean age of 49 (1-72), underwent transplants. 88 (98\%) engrafted, with median neutrophil engraftment at $\mathrm{d}+11(8-17)$, and platelet engraftment at $\mathrm{d}+13$ (8-90). 12 patients died before $\mathrm{d}+365$ (3 before $\mathrm{d}+30$ \& 9 between $d+101$ to365). There were no sepsis related mortality till $\mathrm{d}+30$, and no occurrence of peri-transplant pneumonitis. Ac GVHD grade 3-4 occurred in 6 patients (02 steroid refractory), and chronic GVHD (extensive) in 5 patients. Overall survival was $84 \%$ at a median follow up of 16 months, with $6 \%$ TRM at $\mathrm{d}+100$.

Discussion \& Conclusion: HSCTs can be done in non-HEPA filter AHU equipped BMT units with low TRM rates. This option is particularly applicable in resource constrained settings.

Disclosure of Interest: None Declared.

Keywords: Autologous, HSCT, Non HEPA Filter.

\section{Abstract 8. Role of Intravesical RH-GMCSF in Controlling Haemorrhagic Cystitis in Patients Undergoing HSCT}

Roshni Dasgupta ${ }^{* 1}$, Sandip Shah ${ }^{2}$, Akanksha Garg ${ }^{2}$, Harsha Panchal ${ }^{1}$, Kinnari Patel ${ }^{2}$, Kamlesh Shah ${ }^{2}$

\section{${ }^{1}$ Medical Oncology, ${ }^{2}$ Haematology, GCRI, Ahmedabad, India}

Aims \& Objectives: To assess the clinical effects of Intravesical Recombinant Human-Granulocyte Macrophage colony-stimulating factor (rh- GM CSF) in controlling haemorrhagic cystitis in patients undergoing HSCT.

Patients/Materials \& Methods: Nine patients who underwent HSCT and developed haemorrhagic cystitis in our institute were retrospectively studied and data was acquired from case files and written in pre-designed proforma.

A grading system and pre defined criteria were used to define the severity and response of haemorrhagic cystitis respectively.

All patients received prophylaxis for haemorrhagic cystitis and levofloxacin prophylxis.

Therapeutic strategies included hydration, blood component support, bladder irrigation and intravesical rh- GMCSF.

Results: Nine patients who underwent HSCT in our institute were retrospectively studies. There characteristics are shown in Table 1. Median age was 10 years and seven out of nine patients.

Median interval from stem cell transfusion (Day 0) to onset of HC was +9 day (range Day +6 to Day +66 ). Six patients developed grade III HC, 2 of them had grade II and 1 of them had grade I HC. On persistence of Grade II or more hematuria beyond a median time of 3.5 days, intravesical rh-GMCSF was used to control HC.

Six patients achieved CR within an average time of $56 \mathrm{~h}$ from GMCSF administration and three patients failed to respond despite 3 days of intravesical rh-GMCSF. Time to response was within 3 days. Average duration of bladder exposure was $3.6 \mathrm{~h}$.

Localised hypogastric pain bladder was the only major side effect, no systemic side effects were observed.

None of the patients had recurrence during a follow up period of 3 months.

Discussion \& Conclusion: Allogenic BMT, use of high dose cyclophosphamide in conditioning regimens and young age were observed to be predictive risk factors of developing HC in HSCT patients. Anticipation of onset of $\mathrm{HC}$ early in the course may help strategise more rigorous prophylactic measures. Apart from standard of care including hyper hydration, blood component support, antiviral, levofloxacin prophylaxis acknowledging possible BK virus infection and GVHD prophylaxis, early administration of intrvesical rh -GM CSF helped control HC in majority of the patients, thus decreasing the morbidity and duration of hospital stay.

Supporting Document: 03e18fb7-a207-49a0-a392-a21270a903b5.

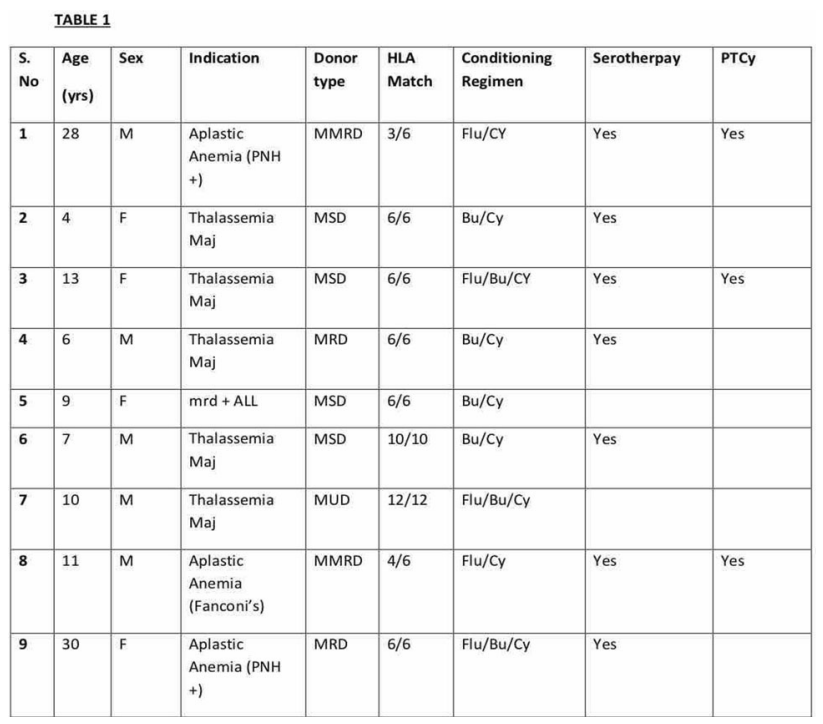

Disclosure of Interest: None Declared.

Keywords: Haemorrhagic cystitis, HSCT, Intravesical rh GM CSF. 


\section{Abstract 9. Cord Blood Stem Cells: Collection, Enumeration and Cryopreservation}

\author{
SummiGanguli $^{* 1}$, Mayurika S Tyagi ${ }^{1}$, Swati Singh ${ }^{1}$, SubirMitra ${ }^{1}$ \\ ${ }^{1}$ Pathology, Santosh Medical College and Hospital, Ghaziabad, \\ U.P., Ghaziabad, India
}

Aims \& Objectives: The present study was conducted in the department of Haematology, Santosh Medical College, Ghaziabad with the aim:

1. To develop techniques for the collection of cord blood maximising the blood volume without compromising the sterility and quality of stem cell yield.

2. To separate and enumerate the Haematopoetic Stem Cells (HSC) in the cord blood and study the effect of cryopreservation on stem cell count and viability.

Patients/Materials \& Methods: Informed consent was taken from pregnant mother undergoing delivery before the collection of umbilical cord blood (UCB). After delivery, the cord was clamped under aseptic conditions, and the umbilical cord was cleaned with betadine and alcohol swabs. Cannulation of umbilical cord just above the clamp was done, and UCB was collected in blood bag containing $22 \mathrm{ml}$ CPDA-1. Blood bag was shaken gently for proper mixing. Processing of cord blood was done using hydroxyl ethyl starch sedimentation. Stem cells were transferred in $25 \mathrm{ml}$ cryoprotectant bag containing $10 \%$ dimethyl sulphoxide. Enumeration of Total nucleated cell (TNC) count and CD34+ cell count was done and using haematology analyser and flow cytometry respectively. The cryo bags were preserved at $-86{ }^{\circ} \mathrm{C}$ deep freezer. Viability count of stem cells was done by using tryptan blue dye exclusion test.

Results: Forty-eight samples of UCB were collected and cryopreserved. Information regarding antenatal history, delivery, newborn weight, placental weight and any complications were noted. The average cord blood volume collected was $92 \mathrm{ml}$. Total nucleated cell count range from $14 \times 10^{7} / \mathrm{ml}$ to $27.5 \times 10^{7} / \mathrm{ml}$. Viability of cord blood stem cells was $98.7 \%$.

Discussion \& Conclusion: Umbilical cord blood banking is one of the significant advances in transplant therapy. Umbilical cord blood, which is usually discarded, can be easily collected without discomfort to the mother or infant. A number of different procedures for collection of UCB have been proposed.

The present study was undertaken to standardise the collection, processing and cryopreservation of cord blood stem cells for setting up cord blood bank for transplantation of cord blood in haematological, genetic and immunological disorders.

Supporting Document: 6d543fc0-5eda-4019-ae3d-3e2eb0389bdc.

Disclosure of Interest: None Declared.

Keywords: Cord blood stem cells, Transplant therapy, Umbilical cord blood bank.

\section{Abstract 10. Haploidentical Stem Cell Transplant in Refractory Langerhans Cell Histiocytosis: A Case Report}

\section{Dr Saroj Bala ${ }^{* 1}$, Vipin Khandelwal ${ }^{1}$}

${ }^{1}$ Dept.of Haematology And Bmt,blk Super Speciality Hospital, Old rajindernagar, India

Aims \& Objectives: Background: Children with multisystem Langerhans cell histiocytosis (MS-LCH) failing to respond to conventional chemotherapy have poor outcomes. Hematopoietic stem cell transplant (HSCT) represents a potential salvage approach.HSCT can achieve greater disease control than chemotherapy, but it carries a high risk of transplant-related mortality; thus, the haploidentical parental HSCT is used infrequently in pediatric refractory LCH. We report a first case of successful haploidentical transplantation in a 4 year old male child with refractory MS-LCH using T cell replete graft from father as a donorandpost transplant Cyclophosphamide. Donor specific antibodies (DSA) by luminex were negative for him. Patients/Materials \& Methods: A 4 year old male child, lymph node biopsy,immunohistochemistryprovenLCH with PET-CT showing multi system involvement treated with cytarabine and cladiribinein Iraq presented to BLK SS Hospital,with repeat PET-CT (done before starting maintenance phase) showing multisystem involvement suggestive of relapsed/refractory multisystem LCH. Patient received 3 cycles of clofarabine with partial response so child was taken for Haploidentical Allogenic HSCT as no fully HLA matched donor was available in family and registries.

Conditioning regimen.

Injection thiotepa $5 \mathrm{mg} / \mathrm{kg}$ on day- 7 ,

InjectionMelphalan $100 \mathrm{mg} / \mathrm{m}^{2}$ on day- 6 .

InjectionFludarabine $40 \mathrm{mg} / \mathrm{m}^{2}$ from day- 5 to day -2 .

Thestemcellsweresourcedfromperipheralbloodandcelldoseof $15 \times 10^{6} \mathrm{CD} 34+/$ kilogrambodyweight infused. Post-transplant in vivo Tcell depletion was done bycyclophosphamide $50 \mathrm{mg} / \mathrm{kg} / \mathrm{day}$ on days +3 and +4 . Graft versus host disease (GVHD) prophylaxiscomprised cyclosporine and mycophenolatemofetil (MMF).

Results: Engrafted on day +15 and nine months post HSCT child is having complete donor chimerism and doing fine, with PET-CT incomplete resolution.

Discussion \& Conclusion: Our case report strongly suggests that T-cell replete haploidentical donor transplantation with cyclophosphamide is a feasible and potentially curative option for Patients with refractory MS-LCH who have no matched donor.

Disclosure of Interest: None Declared.

Keywords: HAPLOIDENTICAL TRANSPLANT, LANGERHANS CELL HISTIOCYTOSIS, PAEDIATRIC REFRACTORY.

\section{Abstract 11. Effect of Stem Cell Dose on Outcome in Autologous Hematopoietic Stem Cell Transplant}

\author{
Preethi Jeyaraman $^{* 1}$, Rahul Naithani ${ }^{2}$, Nitin Dayal ${ }^{3}$, \\ Sangeeta Pathak ${ }^{4}$
}

${ }^{1}$ Max Superspecialty Hospital, ${ }^{2}$ Hematology and bone marrow transplant division, Max SuperspecialtyHospital,Saket, New Delhi, ${ }^{3}$ Department of Pathology, ${ }^{4}$ Department of Transfusion Medicine, Max Superspecialty Hospital, New Delhi, India

Aims \& Objectives: Although the minimum stem cell dose required for autologous stem cell transplant has been established as CD34 dose of $2.5 \times 10^{6}$ cells $/ \mathrm{kg}$, sustained engraftment has been reported to occur at a dose of even lesser than $1 \times 10^{6}$ cells $/ \mathrm{kg}$. In this study we proposed to study the influence of stem cell dose using $2.5 \times 10^{6}$ cells $/ \mathrm{kg}$ as cut off on hematopoietic reconstitution and incidence of infections in patients undergoing autologous stem cell transplant. Patients/Materials \& Methods: We retrospectively analyzed 108 patients who underwent autologous stem cell transplant for myeloma and lymphoma from April 2012 to June 2019. Patients were grouped based on their CD34 dose of $<2.5 \times 10^{6}$ cells $/ \mathrm{kg}$ or $\geq 2.5 \times 10^{6}$ cells $/ \mathrm{kg}$. The effect of stem cell dose on neutrophil and platelet engraftment and length of hospital stay was analysed. Also the effect of stem cell dose on day 100 transplant related mortality and incidence of proven bacterial infections were analysed. 
Results: One hundred and eight patients who underwent autologous stem cell transplant were included in the study out of which lymphoma patients constituted $22.2 \%(\mathrm{n}=24)$ and myeloma patients constituted $77.8 \%(\mathrm{n}=84)$. Median age was 53 years (range: 14 to 68 years) with a male:female ratio of 1.51. Mean CD34 dose of the whole group was $4.96 \pm 4.2 \times 10^{6}$ cells $/ \mathrm{kg}$. CD34 dose correlated with the mobilising regimen used with higher doses obtained with GCSF + Plerixafor mobilisation as compared with only GCSF or chemo-mobilisation $(p=0.001)$. Thirty three out of $108(30.5 \%)$ patients had stem cell dose $<2.5 \times 10^{6}$ cells $/ \mathrm{kg}$. Neutrophil engraftment was significantly faster in patients with CD34 dose of $\geq 2.5 \times$ $10^{6} / \mathrm{kg} \quad\left(\mathrm{CD} \quad 34 \geq 2.5 \times 10^{6} / \mathrm{kg}-10.34 \pm 3.4\right.$ days versus $12.97 \pm 4.7$ days in $<2.5 \times 10^{6} / \mathrm{kg} ; p=0.002$ ). Platelet engraftment was also faster in patients with CD34 count above the threshold value $(10.59 \pm 6.4$ days versus $14.53 \pm 10.8$ days; $p=0.023)$. Patient with higher $\mathrm{CD} 34$ count had shorter duration of hospital stay $(20.46+7.3$ days versus $23.55+6.7$ days, $p=0.043)$. Day 100 transplant related mortality was not significantly different between the 2 groups $\left(\right.$ CD $34 \geq 2.5 \times 10^{6} / \mathrm{kg}$ - 7deaths versus CD $34<2.5 \times$ $10^{6} / \mathrm{kg}$ - 1 death, $p=0.430$ ). There was no significant difference in the incidence of proven bacterial infections between the 2 groups $(p=0.820)$.

Discussion \& Conclusion: In conclusion a CD34 ${ }^{+}$cell dose of $\geq 2.5 \times 10^{6} / \mathrm{kg}$ led to improved hematopoietic recoveries and shorter hospital stay. However, no significant effect was observed in transplant related mortalities and incidence of proven bacterial infections. Disclosure of Interest: None Declared.

Keywords: autologous stem cell transplant, stem cell dose, Stem cell transplantation.

\section{Abstract 12. Autologous Stem Cell Transplantation in Relapsed Hodgkin Lymphoma: Single Centre Study From India}

\section{Jayachandran Perumal Kalaiyarasi ${ }^{* 1}$, Nikita Mehra ${ }^{1}$, Venkatraman Radhakrishnan ${ }^{1}$, Manikandan Dhanushkodi ${ }^{1}$, Tenali Gnana Sagar', KrishnarathinamKannan ${ }^{1}$ \\ ${ }^{1}$ Medical Oncology, Cancer Institute (WIA), Adyar, Chennai, India}

Aims \& Objectives: Background: Hodgkin Lymphoma (HL) earlier called as Hodgkin Disease is a type of B-cell Lymphoma. About one third of the patients with HL will have relapse of the disease. The standard of care for patients with relapsed HL is salvage chemotherapy followed by autologous stem cell transplantation (ASCT). Here we have shared our experience of ASCT in relapsed HL.

Patients/Materials \& Methods: The treatment details and outcomes of patients who underwent ASCT for Relapsed HL during the period of January 1997 to June 2018 were retrospectively collected from the patient records and analyzed. The survival analysis was done by Kaplan-Meier analysis and the comparison was done by Log Rank test.

Results: Median age at diagnosis and at transplant of 41 patients included in the study were 22 years (Range: $4-47$ years) and 24 years (Range: 4-50 years) respectively. Males constituted 63\% $(\mathrm{n}=26)$. ABVD/AVD was the most common upfront regimen $(75 \%, \mathrm{n}=31)$. Advanced stage at presentation was in $63 \%(\mathrm{n}=29)$. The stage at relapse was $2,3 \& 4$ were in $34 \%, 34 \%$ \& $27 \%$ respectively. Bone marrow involvement at relapse was seen in $10 \%(n=4)$. Out of 41 patients, 23 received GVD, 12 received DHAP and 6 received ICE/ miscellaneous as salvage chemotherapy respectively. Complete response before transplant was seen in $51 \%(\mathrm{n}=21)$. ASCT was done post $2^{\text {nd }}$ line in 29 patients $(69 \%)$. LACE, BEAM, CBV was used as conditioning regimen in $51 \%(\mathrm{n}=21), 24 \%(\mathrm{n}=10)$ and $22 \%$ $(n=9)$ respectively. The stem cell dose was available for all those who underwent ASCT after $2008(\mathrm{n}=34)$, the median stem cell dose was $5.36 \times 10^{6}$ cells $/ \mathrm{kg}$ (Range: $1.5 \times 10^{6}-14.8 \times 10^{6}$ cells $/ \mathrm{kg}$ ). Febrile neutropenia $(\mathrm{FN})$ was seen in $97 \%(\mathrm{n}=40)$. Septic shock was seen in $5 \%(\mathrm{n}=2)$. It took a median of 12 days and 16 days for neutrophil and platelet recovery. There was only one treatment related mortality due to FN and refractory septic shock. The median duration of follow up was 28.5 months $(0.5-186$ months). The median relapse free survival (RFS) and overall survival (OS) was not reached in the whole study group. The 3 year RFS and OS were $67.5 \%$ and $71.5 \%$ respectively. The significant factors adversely influencing the RFS were time to treatment failure after $1^{\text {st }}$ line $>12$ months, primary progressive HL, partial response before ASCT, ASCT done at $3^{\text {rd }}$ line or after. The significant factors adversely influencing the OS were primary progressive HL and ASCT done at $3^{\text {rd }}$ line or after.

Discussion \& Conclusion: ASCT in patient with relapsed HL is the standard of care and has shown to induce long term remission in $2 / 3^{\text {rd }}$ of relapsed HL patients. The relapse free survival of primary progressive HL undergoing ASCT is very dismal and hence the role of upfront allogenic stem cell transplantation in that subset has to be further explored.

Supporting Document: 2ce61bda-aa94-4b84-8631-1cc5b78faaba.
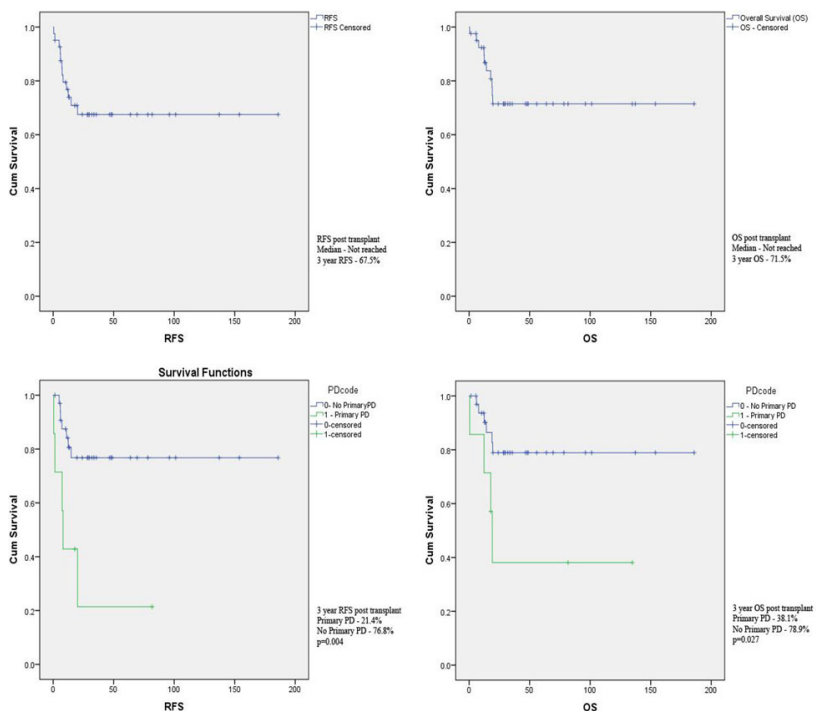

Disclosure of Interest: None Declared.

Keywords: AUTOLOGOUS, Hodgkins Lymphoma, Relapsed hodgkins.

\section{Abstract 13. Impact of ABO Incompatibility on Patient Outcome in Allogeneic HSCT}

\section{AmeePatel $^{* 1}$ \\ ${ }^{1}$ hemato-oncology and BMT unit, BLK SUPER SPECIALITY HOSPITAL, DELHI, India}

Aims \& Objectives: 1. To evaluate risk of acute and delayed hemolytic reactions, white blood cells and platelet engraftment, requirement of $\mathrm{RBC}$ and platelet transfusion and risk of GVHD in ABO incompatible allogeneic HSCT.

2. To evaluate risk of pure red cell aplasia (PRCA) in ABO incompatible allogeneic HSCT. 
Patients/Materials \& Methods: This study included total of 123 patients who underwent hematopoietic stem cell transplantation at Dr. BLK superspeciality Hospital, New Delhi, between July 2017 to December 2018. Out of 123 HSCT, $58(47.15 \%)$ HSCT were done for benign diseases \& $65(52.85 \%)$ for malignant diseases. A total of 54 patients were $\mathrm{ABO}$ incompatible and 69 were $\mathrm{ABO}$ compatible. All patients were followed up till a period of 100 days post HSCT.

Results: Results are summarized in Table 1 in attached document.

Discussion \& Conclusion: In hematopoietic stem cell transplantation, HLA matched allogeneic stem cell donor may have some degree of $\mathrm{ABO}$ incompatibility in approximately $25-50 \%$ of transplants. The impact of $\mathrm{ABO}$ incompatibility has been studied on various transplant outcomes, including neutrophil \& platelet engraftment, PRCA, acute GVHD etc. yet there are conflicting data reported by various studies. Our study showed that only 1 patient of major incompatible group from bone marrow graft source developed acute hemolysis, none of the patients from PBSC graft group developed acute or delayed hemolysis. Moreover, we observed that there were no statistically significant differences in neutrophil or platelet engraftment, incidence of acute GVHD, PRBC and platelet transfusions associated with $\mathrm{ABO}$ compatibility status using any graft source ( $p$ value $>0.05$ ). However, incidence of PRCA was higher in major $\mathrm{ABO}$ incompatible group ( $p$ value $<0.05$ ).

Supporting Document: d61a6190-c7bd-4ba9-9af6-8c948698076e.

\begin{tabular}{|c|c|c|c|c|}
\hline \multirow[b]{2}{*}{ Variable } & \\
\hline & $\begin{array}{l}\text { ABO compatibi } \\
\text { Compatible }\end{array}$ & Bidirectional & Major & Minor \\
\hline $\begin{array}{l}\begin{array}{l}\text { Age } \\
\pm S D\end{array} \text { (Mean } \\
\text { SD }\end{array}$ & $28.98 \pm 16.51$ & $38.44 \pm 24.40$ & $22.68 \pm 16.21$ & $23.20 \pm 15.66$ \\
\hline Male No. (\%) & $50(72.5)$ & $8(88.9)$ & $18(72)$ & $14(70)$ \\
\hline Female No. $(\%)$ & $19(27.5)$ & $1(11.1)$ & $7(28)$ & $6(30)$ \\
\hline $\begin{array}{l}\text { Acute hemolytic } \\
\text { reactions No. } \\
(\%)\end{array}$ & $17-10)$ & (11.1.) & 0 & 0 \\
\hline $\begin{array}{l}\text { Delayed } \\
\text { hemolytic } \\
\text { reactions No. } \\
(\%)\end{array}$ & 0 & 0 & 0 & 0 \\
\hline $\begin{array}{l}\text { WBC } \\
\text { engraftment } \\
\text { median (range) }\end{array}$ & $14(8-25)$ & $\begin{array}{c}14 \\
(11-16)\end{array}$ & $\begin{array}{c}15 \\
(9-21)\end{array}$ & $\begin{array}{c}12 \\
(8-18)\end{array}$ \\
\hline $\begin{array}{l}\text { Platelet } \\
\text { engraftment } \\
\text { median (range) }\end{array}$ & $19(11-60)$ & $\begin{array}{c}20 \\
(13-40)\end{array}$ & $\begin{array}{c}22 \\
(14-66)\end{array}$ & $\begin{array}{c}19 \\
(16-64)\end{array}$ \\
\hline $\begin{array}{l}\text { PRBC } \\
\text { Transfusion } \\
\text { median (range) }\end{array}$ & $8(2-26)$ & $7(2-23)$ & $8(2-20)$ & $7(2-14)$ \\
\hline $\begin{array}{l}\text { Platelet } \\
\text { transfusion } \\
\text { median (range) }\end{array}$ & $24(2-129)$ & $20(3-90)$ & $21(3-122)$ & $\begin{array}{c}31 \\
(2-146)\end{array}$ \\
\hline PRCA No $(\%)$ & 0 & $1(11.1)$ & $4(16)$ & 0 \\
\hline $\begin{array}{l}\text { Acute GVHD } \\
\text { No. (\%) }\end{array}$ & $17(24.63)$ & $1(11.11)$ & $4(16)$ & $4(20)$ \\
\hline
\end{tabular}

Disclosure of Interest: None Declared.

Keywords: ABO incompatibility, Allogeneic hematopoietic stem cell transplantation (HSCT), Engraftment, PRCA.

\section{Abstract 14. Regulatory $T$ Cells in the Graft and the Risk of Acute GVHD After Allogenic HSCT}

\section{Kunal Chhattani ${ }^{* 1}$}

\section{${ }^{1}$ Dr. B. L. Kapur Memorial Hospital, New Delhi, New Delhi, India}

Aims \& Objectives: To study the correlation between the Regulatory $\mathrm{T}$ cells (Tregs) and acute GVHD in allogenic stem cell transplant patients.

Patients/Materials \& Methods: It was a single centre prospective observational study of 30 patients undergoing allogeneic HSCT (MSD and Haploidentical) between June 2017 and December.
Unrelated donor HSCTs were excluded. Tregs frequencies were measured by flow cytometry in graft sample and on Day 30 and Day 60 post transplant blood samples. Tregs Frequency measured was of CD4 + CD25+ CD127lowFoxP3 + cells out of CD3+ CD4+ cells. Patient's post-transplant course was monitored till 100 days. Patients were divided into 2 groups- GVHD group and non-GVHD group. Median Tregs frequencies at Graft, day +30 and Day +60 were compared between two groups and analysed.

Results: Out of 30 patients studied, 18 patients underwent MSD HSCT while 12 patients underwent Haploidentical HSCT. In this study, 7 patients developedaGVHD. There was no difference between the two groups in CD34 stem cell dose infused and Day of engraftment. Median Tregs frequency of Graft sample in GVHD group was $0.48 \%$ while in NonGVHD group it was $0.46 \%$. Day 30 sample showed frequencies of $0.26 \%$ and $1.33 \%$ respectively in the two groups while Day 60 samples showed $0.41 \%$ and $1.93 \%$ median Tregs frequencies respectively. Statistical significance was found in Day 30 $(p=0.028)$ and Day $60(p=0.002)$ samples but not in graft samples. Discussion \& Conclusion: Our study confirms that Regulatory $\mathrm{T}$ cells play a crucial role in promotion of immunological tolerance and they have an inverse relationship with acute GVHD. The results obtained are comparable to previous studies in post-transplant samples. We did not find any correlation between Graft Tregs and Acute GVHD, which is in contrast to many of the previous studies. Further studies are required to confirm graft components influencing incidence of aGVHD. The study also opens up a promise of the utility of regulatory $\mathrm{T}$ cells as biomarker predicting risk of aGVHD, as well as cell therapy product.

Disclosure of Interest: None Declared.

Keywords: Acute Graft Versus Host Disease, Allogenic Hematopoietic Stem Cell Transplant, Regulatory T cells.

\section{Abstract 15. Pre-Harvest CD34 Cut-Off for Optimum Dose in a Single Procedure in Patients with Multiple Myeloma}

\section{Swati Pabbi ${ }^{* 1}$, Nitin Sood ${ }^{2}$, AseemKumar Tiwari ${ }^{1}$, GeetAggarwal $^{1}$, Anand Upadhyay ${ }^{1}$ \\ ${ }^{1}$ Department of Transfusion Medicine, ${ }^{2}$ Department of Haematology and stem cell transplant, Medanta,TheMedicity, Gurugram, India}

Aims \& Objectives: To define cut off points for pre-harvest peripheral blood CD34 counts (PB-CD34) to initiate stem cell apheresis to achieve target dose in a single apheresis session for autologous stem cell transplant in patients with multiple myeloma.

Patients/Materials \& Methods: We retrospectively analysed the stem cell collection records of 51 patients with multiple myeloma (MM) who underwent autologous stem cell transplant between January 2015 to December 2018 at our centre. Receiver operating characteristic curve (ROC) analysis was used to define cut off points for pre-harvest PB CD34 counts to predict the final dose.

Results: Total 55 leukapheresis sessions were performed on 51 patients on day 5 of mobilization with volumes processed ranging from 1.3 Total blood volume (TBV) to $8 \mathrm{TBV}$ (median $3.2 \mathrm{TBV}$ ). Pre-harvest PB CD34 ranged from $6-256$ cells $/ \mu 1$ (median 38 cells $/ \mu 1$, daily final CD34 cells collected ranged from $0.73-31.75$ million $/ \mathrm{kg}$ (median 6.06 million $/ \mathrm{kg}$ ). The correlation coefficient of pre-harvest CD34 with final CD34 cells collected was 0.77 , which was statistically significant. Cut off value of PB CD34 was established to be 13.50 cells $/ \mu \mathrm{l}, 23.5$ cells $/ \mu \mathrm{l}$ and 87.5 cells $/ \mu 1$ for target dose of 2 million cells $/ \mathrm{kg}, 5$ million cells $/ \mathrm{kg}$ and 10 million cells $/ \mathrm{kg}$ respectively. 
Discussion \& Conclusion: Multiple myeloma is the most common indication for autologous stem cell transplant using peripheral blood stem cells. The minimum intended dose of stem cells for autologous transplant has been defined as $2-5$ million cells $/ \mathrm{kg}$. Dose of 5 million cells $/ \mathrm{kg}$ and above has been considered as an optimal harvest and is associated with rapid engraftment. For cryopreservation and second transplant, dose of around $8-10$ million cells $/ \mathrm{kg}$ is desired. As there is a strong correlation between the PB CD34 and the final CD 34 cells collected, we suggest that cut off points defined should be used for initiating apheresis procedures to allow for early intervention and prevent collection failures. Reduction in apheresis sessions will result in decrease in cost, time and discomfort associated with the stem cell collection for the patients.

Supporting Document: 823fda71-5f91-4996-9a7f-87b1d465316d.

ROC Curve Analysis

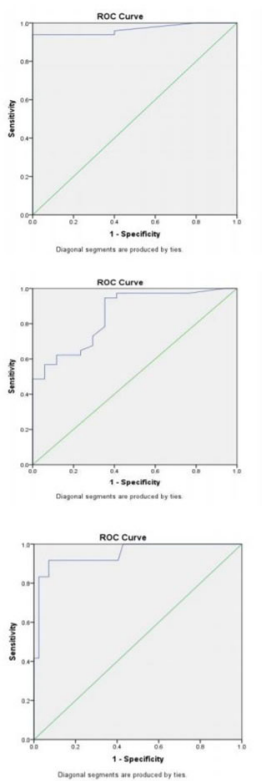

\begin{tabular}{|c|c|c|}
\hline $\begin{array}{c}\text { Cut off for } \\
\text { dose of } 2 \\
\text { million } / \mathrm{kg}\end{array}$ & Sensitivity & specificity \\
\hline 13.50 & 0.939 & 1.000 \\
\hline
\end{tabular}

\begin{tabular}{|c|c|c|}
\hline $\begin{array}{c}\text { Cut off for } \\
\text { dose of 5 } \\
\text { million } / \mathrm{kg}\end{array}$ & Sensitivity & specificity \\
\hline 23.50 & 0.946 & 0.647 \\
\hline
\end{tabular}

Disclosure of Interest: None Declared.

Keywords: Multiple Myeloma Autologous SCT, Peripheral blood stem cell count, Stem cell dose-CD34.

\section{Abstract 16. Clinical Outcomes of Allogeneic Stem Cell Transplant for Fanconi Anaemia- A Single Centre Experience}

SohiniChattopadhyay ${ }^{* 1}$ and Aby Abraham, Anu Korula,
Anup J Devasia, Biju George, Eunice Sindhuvi, Kavitha,
Uday Kulkarni, Fouzia NA, Alok Srivastava, Vikram Mathews

${ }^{1}$ Haematology, Cmc Vellore, Vellore, India

Aims \& Objectives: This abstract aims to describe outcomes of allogenic stem cell transplants (SCT) in patients with Fanconi anaemia (FA).

Patients/Materials \& Methods: This was a retrospective analysis describing the outcomes of SCT in 69 patients with FA who underwent 75 transplants between 1999 and 2018 using a Fludarabine based conditioning regimen. The median age of the patients in our cohort was 40 months (range: $3-420$ ). At time of SCT, 64 patients (85\%) had aplastic anaemia (AA) while $8(10.6 \%)$ had myelodysplastic syndrome (MDS) and $3(0.4 \%)$ had acute myeloid leukaemia (AML). Sixty-two $(85 \%)$ patients underwent matched donor (sibling/non-sibling) transplant while $13(17.3 \%)$ patients underwent haploidentical transplant. The conditioning regimen given was Fludarabine and low dose cyclophosphamide $(10 \mathrm{mg} / \mathrm{kg}$ for 2 days) $(66 \%)$, Fludarabine and busulfan $(13 \%)$ and Fludarabine with melphalan $(0.2 \%)$. While in haploidentical transplants conditioning included Fludarabine, low dose cyclophosphamide with 200 cGy single fraction total body irradiation. Graft versus host disease (GVHD) prophylaxis consisted of cyclosporine and methotrexate in fully HLA matched transplants and use of post-transplant cyclophosphamide with mycophenolate mofetil and tacrolimus in haploidentical transplants. Peripheral blood stem cells was the predominant graft source $(86.6 \%)$.

Results: Of the 75 transplants, primary engraftment failure was noted in 6 transplants $(0.8 \%)$ of which 5 patients expired prior to engraftment. The remaining showed engraftment at a median of 13 days (range: 9-29). Among the patients that engrafted, Day 28 chimerism analysis showed complete chimerism (CC) in $78.1 \%$ while $23.4 \%$ had a mixed chimerism (MC). Acute graft versus host disease (GVHD) occurred in 33 subjects (44\%) out of which $39 \%$ (13 patients) had grade III- IV GVHD. Chronic GVHD occurred in $65 \%$ of patients. The median follow-up in our study is 34 months (range: 1-150). The 5 year estimated overall survival (OS) for the entire group is $75.6 \pm 5.2 \%$. The 2-year OS for fully HLA matched transplants is $80.2 \pm 5.4 \%$ and for haploidentical transplants is $47.0 \pm 15.1 \%$. No significant difference in 2-year OS was noted between matched sibling $(82.9 \pm 5.9 \%)$ and matched non-sibling donor transplants $(65.2 \pm 9.1 \%)[p=0.12]$. Secondary malignancies were noted in 3 patients.

Discussion \& Conclusion: SCT in FA provides good outcomes in patients having fully HLA matched transplants.

Disclosure of Interest: None Declared.

Keywords: allogeneic stem cell transplantation, Fanconi Anaemia, Fludarabine.

\section{Abstract 17. Role of NOD2 Gene Variants in Predicting Sepsis Related Outcomes in Indian HSCT Recipients}

Aditya Jandial $^{* 1}$, ShanoNaseem ${ }^{2}$, JogeshwarBinota ${ }^{2}$,

Kundan Mishra' ${ }^{1}$, Rajeev Sandal ${ }^{1}$, Deepesh Lad ${ }^{1}$, Alka Khadwal, PallabRay $^{3}$, Neelam Varma ${ }^{2}$, Pankaj Malhotra ${ }^{1}$, Gaurav Prakash ${ }^{1}$

${ }^{1}$ Internal Medicine, ${ }^{2}$ Hematology, ${ }^{3}$ Microbiology, PGIMER Chandigarh, Chandigarh, India

Aims \& Objectives: NOD2 protein (nucleotide-binding oligomerization domain containing 2) is known to regulate inflammatory immune response by modulating cytokine production and NF- $\kappa \mathrm{B}$ activation. NOD2 gene single nucleotide polymorphisms (SNPs) also correlate with non-relapse mortality and severe acute GVHD. However, consensus on the role of NOD2 gene SNPs in predicting transplant related outcomes is not yet clear. We studied the role of three NOD2 gene SNPs in predicting sepsis related outcome in Indian HSCT recipients.

Patients/Materials \& Methods: We prospectively studied patients undergoing allogeneic and autologous HSCT at PGIMER Chandigarh from January 2017 to December 2017. Peripheral blood samples collected at baseline were tested for three NOD2 gene SNPs [SNP8 (2104C $>$ T, Arg702Trp), SNP12 (2722G $>$ C, Gly908Arg), and SNP13 (3020insC, Leu1007fsinsC)] with the help of RT-PCR and RFLP. The PCR products were digested with specific restriction endonucleases 
(Euryx, Gdansk, Poland). Regimen related toxicity and GVHD were graded as per standard criteria.

Results: Forty patients were enrolled. Their median age was 38 years (range 3-64); 52.5\% were males. Twenty patients underwent autologous HSCT [multiple myeloma (16) and relapsed/refractory Hodgkin's lymphoma (4)]. Twenty patients underwent allogeneic HSCT [AML (6), CML (4), ALL (4), aplastic anemia (2), thalassemia major (2), CLL (1) and Wiscott-Aldrich syndrome $(\mathrm{n}=1)$ ]. Thirtyeight patients $(95 \%)$ developed febrile neutropenia in the post-transplant period and 4 patients (10\%) died within 100 days of stem cell infusion due to overwhelming sepsis. Nine allogeneic HSCT recipients developed acute GVHD [grade I-II $(n=3)$ and grade III-IV $(n=6)]$. None of the 40 patients showed any of the three NOD2 gene SNPs.

Discussion \& Conclusion: We conclude that the three NOD2 gene SNPs may be uncommon in Indian HSCT recipients. It may be possible that other NOD2 variants are more prevalent in our population. Small sample size and non-enrolment of controls are the major limitations of our study. Larger studies targeting multiple SNPs at the same time may convincingly rule out or confirm the role of NOD2 gene variants in Indian population.

Disclosure of Interest: None Declared.

Keywords: Hematopoietic stem cell transplantation, NOD2, single nucleotide polymorphism.

\section{Abstract 18. Role Of 18F-FLT Imaging in Assessing Hematopoietic Response in Recipients of Allogeneic HSCT}

\author{
Ashok Meshram ${ }^{* 1}$, Anish Bhattacharya ${ }^{2}$, RajenderKumar ${ }^{2}$, \\ Ashwin Singh Pariwar ${ }^{2}$, Jaya Shukla ${ }^{2}$, Gaurav Prakash ${ }^{2}$, \\ Deepesh Lad $^{3}$, ArihantJain ${ }^{3}$, Pankaj Malhotra ${ }^{3}$, Alka Khadwal ${ }^{3}$ \\ ${ }^{1}$ Department of Internal medicine $1,{ }^{2}$ Nuclear Medicine, \\ ${ }^{3}$ Department of Internal medicine, PGI Chandigarh, Chandigarh, \\ India
}

Aims \& Objectives: 18F-Flurothymidine PET/CT assesses the thymidine kinase activity, reflecting cellular proliferation and therefore can be used as a marker for proliferation of hematopoietic stem cells (HSC) in the post-HSCT patients.This technique can be useful in the analysis of proliferative activity in the bone marrow and monitoring response. We undertook this study to find out role of ${ }^{18} \mathrm{~F}$-FLT PET/CT imaging in assessing hematopoietic activity in recipients of allogeneic hematopoietic stem cell transplant.

Patients/Materials \& Methods: We prospectively studied eighteen patients undergoing allogeneic HSCT at PGIMER Chandigarh from January 2018 to June 2019. Primary objective of the study were to compare the hematopoietic activity assessed from bone marrow biopsy specimen with that obtained on ${ }^{18} \mathrm{~F}$-FLT PET/CT by means of standardized uptake values in HSCT recipients. Allogeneic transplant patients were subjected to bone marrow examination at $\mathrm{D}+30$ and ${ }^{18} \mathrm{~F}$-FLT PET/CT at $\mathrm{D}+30$ and $\mathrm{D}+100$. Bone marrow cellularity was compared with mean SUVmax values on ${ }^{18}$ F-FLT PET/CTscan (axial and appendicular skeleton) and also with appendicular skeletal score by visual analysis of 18 F-FLT uptake.

Results: The median age of the enrolled patients was 28 years (range: $7-57) ; 38.9 \%$ were males and $61 \%$ were females. Eighteen patients underwent allogeneic HSCT (aplastic anemia (6), CML (5), AML (3), CLL (1), CMML (1), primary myelofibrosis (1), thalassemia major (1)).The study showed the increased distribution of transplanted marrow into appendicular long bones, previously abandoned bone marrow sites. Follow-up scans showed shifting of tracer activity from the appendicular skeleton to axial skeleton reflecting the normal pathway taken by engrafting hematopoietic cells similar to fetal ontogeny. No statistically significant correlation was found between bone marrow cellularity and mean SUV max at illac crest and sternum ( $p$ value $<0.140$ and 0.125 respectively).

Discussion \& Conclusion: The present study showed that 18F-FLT was a useful non-invasive biomarker in monitoring the proliferative activity of the transplanted HSCs. 18F-FLT PET/CT could show the active state of marrow proliferation in the patients.

Disclosure of Interest: None Declared.

Keywords: Allogenic Hematopoietic Stem Cell Transplant, Bone marrow cellularity, Flurothymidine PET CT.

\section{Abstract 19. Effect of KIR Mismatch on the Clinical Outcomes in Haploidentical Transplantation for Malignancies}

Uday Kulkarni $^{* 1}$, Sushil Selvarajan', Sharon Lionel, Anup Devasia ${ }^{1}$, Anu Korula ${ }^{1}$, Fouzia N A ${ }^{1}$, Kavitha Lakshmi ${ }^{1}$, Aby Abraham ${ }^{1}$, Alok Srivastava ${ }^{1}$, Vikram Mathews ${ }^{1}$, Biju George ${ }^{1}$

\section{${ }^{1}$ Department of Haematology, Christian Medical College, Vellore,} Vellore, India

Aims \& Objectives: To study the effect of Killer immunoglobulin like receptor (KIR) mismatch according to the ligand-ligand model on the clinical outcomes in patients undergoing haploidentical stem cell transplantation (haplo-SCT) for malignancies with the use of post-transplant cyclophosphamide.

Patients/Materials \& Methods: This is a retrospective study conducted using hospital records wherein patients with hematologic malignancies who underwent haplo-SCT using post-transplant cyclophosphamide from January 2010 to December 2018 were included. KIR mismatch was calculated using an online calculator ( www.ebi.ac.uk/ipd/kir/ligand). Descriptive statistics and survival analysis were used.

Results: During the study period, 187 patients underwent haplo-SCT of which 103 were for malignancies. Of these, high resolution HLA typing results were not available for 7 patients. The remaining 96 patients were analyzed. The median age was 21 years (range: 2-57 years). Sixty-seven (69.79\%) were males. Indications for transplant were acute lymphoblastic leukemia $(n=33)$, acute myeloid leukemia $(n=34)$, chronic myeloid leukemia $(n=7)$, non-hodgkin lymphoma $(n=6)$, myelodysplastic syndrome $(n=11)$, hodgkin lymphoma $(\mathrm{n}=4)$ and blastic plasmacytoid dendritic cell neoplasm $(n=1)$. Thirty one $(32.3 \%)$ patients were not in remission at the time of transplant. The disease risk index was low in 6 patients, intermediate in 39 patients, high in 34 patients and very high in 17 patients. The type of conditioning was myelo-ablative for $73(76 \%)$ patients, reduced intensity for $18(18.8 \%)$ patients and non-myeloablative for 5 (5.2\%) patients. All patients had peripheral blood stem cell grafts except for $4(4.2 \%)$ who had bone marrow as the graft source. The mean CD34 cell dose infused was $10.25\left(\right.$ SD 5.18) $\times 10^{6} / \mathrm{kg}$. Sixteen patients died before engraftment. Three patients had primary engraftment failure, of which two died and 1 underwent a second transplant and is alive; one patient had secondary graft failure and subsequently died post second transplant. Forty-one $(42.7 \%)$ patients were KIR matched while $25(26 \%)$ had KIR mismatch in host versus graft (HVG) direction and $30(31.3 \%)$ had KIR mismatch in the graft versus host $(\mathrm{GVH})$ direction. Acute GVHD occurred in $45(46.9 \%)$ patients while $23(24 \%)$ had chronic GVHD. Disease relapse was noted in $26(27.1 \%)$ patients while $61(63.5 \%)$ patients died. For the entire cohort, the 2 -year overall survival was $34.5 \% \pm 5.1 \%$ while the 2 -year event free survival was $29.7 \% \pm 4.9 \%$. The 2 -year overall 
survival for patients with KIR match, KIR mismatch in HVG direction and KIR mismatch in GVH direction was $28.5 \% \pm 7.6 \%$, $18.7 \% \pm 8.1 \%$ and $56.1 \% \pm 9.2 \%$ respectively (log rank p 0.024) (attached figure). On multivariate analysis, factors associated with worse overall survival were non-engraftment, absence of chronic GVHD, non-remission before transplant and KIR mismatch in HVG direction.

Discussion \& Conclusion: In our cohort, KIR mismatch as per the ligand-ligand model was independently associated with the overall survival and hence seems to be a potentially useful tool for donor selection for malignant haploidentical transplantation while using post-transplant cyclophosphamide.

Supporting Document: 03681bbf-a963-4dde-b162-0707ddff4fb5.

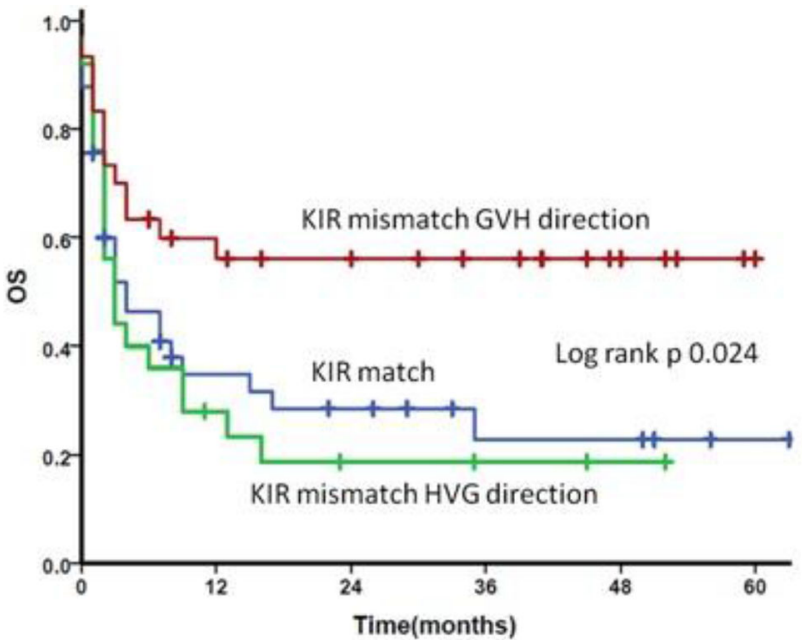

Disclosure of Interest: None Declared.

Keywords: Haploidentical Transplant, Killer immunoglobulin like receptor, natural killer cell alloreactivity.

\section{Abstract 20. BU/CY/ATG vs TREO/THIO/FLU Conditioning Regimen for Allogenic HSCT in Thalassemia Major Patients}

\section{Gunjan Loney $^{* 1}$, SantanuSen ${ }^{2}$, Sameer Tulpule ${ }^{2}$ \\ ${ }^{1}$ Clinical Haematology ad BMT, ${ }^{2}$ Clinical Haematology and BMT, KokilabenDhirubhai Ambani Hospital, Mumbai, India}

Aims \& Objectives: Allogeneic hematopoietic stem cell transplantation (HSCT) remains the only treatment that can correct the hematological manifestations in patients with thalassemia major. The safety and efficacy of conditioning regimen with treosulfan/thiotepa/ fludarabine vs busulfan/cyclophosphamide/ATG were explored in the two groups of ten thalassaemia patients each and outcomes were evaluated.

Patients/Materials \& Methods: Twenty thalassemia major patients underwent allogeneic HSCT. Ten patients after busulfan, cyclophosphamide and ATG based conditioning and ten patients after thiotepa, treosulfan and fludarabine based conditioning. Seventeen patients were transplanted from an HLA-identical sibling, one from matched paternal donor, one from mismatched sibling donor and one from an unrelated donor.

Results: $\mathrm{Bu} / \mathrm{Cy} / \mathrm{ATG}-\mathrm{based}$ conditioning regimen:-

The median age was 5.5 years (range 1-14 years) and the mean CD34+ stem cell dose was $7.93 \times 10^{6} / \mathrm{kg}$. Two patients developed acute graft versus host disease (GVHD), one patient had limited chronic GVHD and two patients experienced graft rejection. There was one treatment-related mortality (TRM) in this group from severe acute steroid resistant GVHD.

Treo/Thio/Flu-based conditioning regimen:-

The median patient age was 5 years (range 2-9 years) and the mean CD34+ stem cell dose was $7.1 \times 10^{6} / \mathrm{kg}$. Two patients developed acute GVHD and one patient had limited chronic GVHD. There was no graft rejection or TRM.

Discussion \& Conclusion: There were no significant differences between the two groups (Treo/Thio/Flu vs Bu/Cy/ATG) in terms of the incidence of acute GVHD and chronic GVHD.

The treosulfan-based preparation found to be safe and effective than busulfan based preparation for thalassemia patients given allogeneic HSCT. Since this is a small set of data, it is difficult to extrapolate the results but certainly the treosulfan based regimen had no graft failures or severe GVHD. The difference is significant, but a larger data set is needed to prove the differences.

Disclosure of Interest: None Declared.

Keywords: Conditioning regimen, Haematopoeitic stem cell transplantation, Treatment related mortality.

\section{Thrombosis}

\section{Abstract 1. Significance and Comparison of LAC with ACLA in High Risk Pregnancy CASES}

\author{
BlessyMathews $^{* 1}$, Ankur Ahuja ${ }^{1}$, TathagatChatterjee ${ }^{1}$, \\ Devika Gupta ${ }^{1}$, KanwaljeetSingh ${ }^{2}$ \\ ${ }^{1}$ Dept of Lab \& Molecular Medicine, ${ }^{2}$ Dept of Lab And Molecular \\ Medicine, Army Hospital RR, DELHI, India
}

Aims \& Objectives: To study the Lupus Anticoagulant assay i.e. aPTT-LA and dRVVT and anticardiolipin antibody- IgM, IgG in High Risk Pregnancy Clinics.

Patients/Materials \& Methods: 200 patients of high risk pregnancy attending the high risk pregnancy clinic at tertiary hospital were enrolled in this prospective. Equal numbers of normal antenatal patients matched for age that did not have any history of previous fetal losses or obstetric complications and had at least one live birth were included as controls. Presence of any infections or drug intake for any disease condition was considered as exclusion criteria in the controls. Duration of study was 15 months.

Each of these samples was run for aCL IgG/IgM, aPTT (screening and confirmatory) and dRVVT (screening and confirmatory) and also positive tests repeated after period of 12 weeks.

The results were analysed using SPSS version 16. Chi square test was used to analyse the significance. $\mathrm{p}$ value of $<0.05$ was taken as significant. 
Results: (1) The prevalence of aCL was $28.8 \%$ and that of LAC was $24.6 \%$.

(2) No difference was seen in the results of aCL IgG and aCL IgM assays.

(3) When used together, more percentage of patients could be detected $(24.6 \%)$, than each of the assays used singly $(\mathrm{dRVVT}=$ $10.8 \%$, aPTT-LA $=18.1 \%$ ).

(4) Repeated LAC and aCL testing is required even to exclude transient positivity.

(5) aCL showed a significant positive correlation with history of early abortions, hypertension in pregnancy, IUGR and history of thrombotic events.

(6) LAC showed a significant positive association with early abortions, still births, hypertension in pregnancy and thrombotic events.

(7) dRVVT and not aPTT-LA showed a significant association with the high risk pregnancy cases like still births, hypertension and thrombosis.

Discussion \& Conclusion: With the help of more than one assay as mentioned in the Revised Sapporo guidelines 2006, the pregnancy cases can be screened for presence of aPL, thereby aiding in prediction of a possible adverse pregnancy. Anticardiolupin was significantly better than isolated LAC tests thus justifying an importance of the former. There is a justified reason to repeat the test after 12 weeks.

Disclosure of Interest: None Declared.

Keywords: Anticardiolupin, APTT-LA, dRVVT.

\section{Abstract 2. Comprehensive Genomic Study of Factors Influencing CAD \& MI in Young patients: Preliminary Data}

\section{Bipin KULKARNI"1, HetanShah ${ }^{2}$, Nikesh Kawankar', Santosh Palled ${ }^{2}$, Aniruddha Pawar ${ }^{2}$, ShrimatiShetty ${ }^{1}$}

${ }^{1}$ Department of Haemostasis and Thrombosis, Icmr- National Institute OfImmunohaematology, ${ }^{2}$ Department of Cardiology, KEM Hospital, Mumbai, India.

Aims \& Objectives: To investigate and functionally correlate the involvement of genomic variations in CAD and MI. The objective is to identify possible specific genomic variants that may alter gene expression profiles in the development and progression of cardiovascular diseases.

Patients/Materials \& Methods: So far 7 patients below 40 yrs, with AMI, without conventional risk factors have been studied for Clinical Exome by NGS. Insilico analysis of variants was done using bioinformatics tools.

Results: 8 clinically significant variants were detected in 6 patients, of which 6 were novel. Insilico analysis of the variants is given in Table attached.

Discussion \& Conclusion: The preliminary data has yielded novel variants in young patients with MI. It will be interesting to perform further functional studies for an understanding of specific markers or mechanisms that may be either independently or synergistically associated to AMI/CAD. Knowledge of these specific markers/ mechanisms may help in designing appropriate prevention strategies. Some of the biomarkers observed could be functionally significant, and could be of therapeutic implications.

Supporting Document: 733219bb-2982-415a-bb1c-bf4af8298ca2.

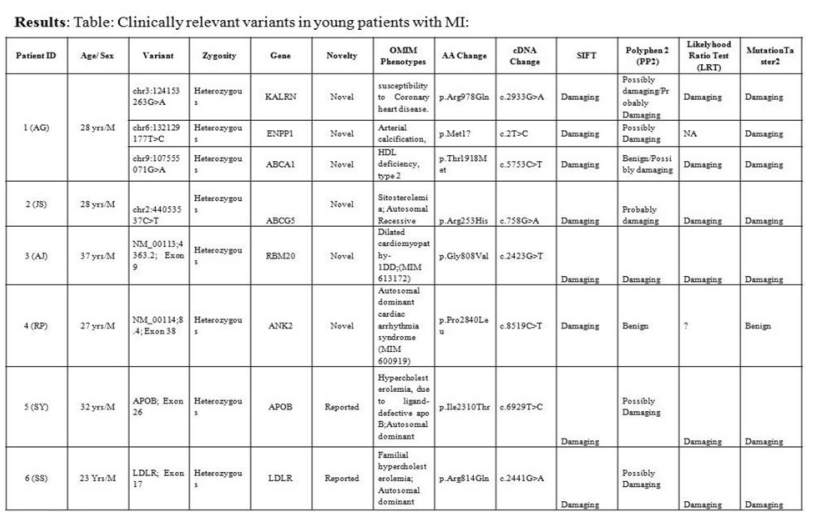

Disclosure of Interest: None Declared.

Keywords: Myocardial Infarction, NGS, Young patients.

\section{Abstract 3. IS HEP Scoring System Superior to 4T'S Scoring System in Diagnosis of HIT?}

\author{
AmarnadhPolisetty ${ }^{* 1}$, SharatDamodar ${ }^{2}$, NatarajK S $^{2}$, \\ Hamza Dalal ${ }^{2}$
}

${ }^{1}$ Dept of Heamatology, ${ }^{2}$ Dept of Heamatology, Narayana health, Bangalore, India

Aims \& Objectives: To correlate Heparin induced Thrombocytopenia Expert Probability Score and the most commonly used 4T's clinical scoring system with the standard test.

Patients/Materials \& Methods: (a) We recruited 100 patients with suspected HIT, for whom antibody testing was ordered at our centre (Narayana Health City, Bangalore, India) between November 2017 and May 2018.

(b) Data were collected at baseline diagnosis in the form of clinical and laboratory data. 4T's score and the HEP score was calculated based on the above details before the availability of antibody test.

(c) HIT antibody testing was done using ID-PaGIA Heparin/pF4 Antibody Test Kit with control. In this 10 millilitre of serum is pippeted into the upper chamber of the appropriate microtube. Incubate the ID card at room temperature for $5 \mathrm{~min}$ at room temperature $\left(18-25^{\circ} \mathrm{C}\right)$. Later centrifuge the ID-card for $10 \mathrm{~min}$ in the ID-centrifuge then read and records the results.

(d) Patients were treated according to the current standard of care, modified as per patient's age and performance status based on the test results and clinician judgment to proceed with Heparin or change to non-heparin based anti-coagulants.

Area under the curve (AUC) for the receiver operating curve (ROC) of HEP and 4T scores was calculated and $\mathrm{p}$ value was obtained based on these curves.

Results: Out of the 100 suspected patients 37 were proven to have HIT by using ID-PaGIA Heparin/PF4 rapid gel agglutination assay. In this series, $91 \%$ patients had undergone cardiothoracic surgery forming the majority. In 87 patients who received UFH, who presented with thrombocytopenia during their perioperative period, 30 were proven to have HIT $(34.4 \%)$.We also observed during that the total leucocyte count at the nadir of platelet was higher in the HIT positive group. However, it was not statistically significant ( $p$ 0.283).Out of 100 patients with suspected HIT 49\% expired. Of the 37 cases proven to have HIT 20 patients expired (54\%). There was no statistically significant association between the occurrence of HIT and mortality $(p$ value $=0.438)$. In this study, the areas under the curve for predicting HIT by $4 \mathrm{~T}$ score was more than HEP score $(0.754$ and 
0.66) with $P$ value-0.093. As the HEP score was not superior to $4 \mathrm{~T}$ score we have evaluated 2 subgroup analysis. Among 36 subjects with the intra-arterial device (included in HEP score), 12 were positive for HIT $(33.3 \%)$. Area under the Curve for the 4T score (0.698) was higher than that for HEP score (0.599) although the difference was not statistically significant ( $p$ 0.3906)In this study, the incidence of renal replacement therapy (not included in HEP score) was 43\%. In this patient population, $46 \%(\mathrm{n}=20)$ are HIT positive. Among subjects on RRT, 4T score (0.814) had higher Area under the curve compared to HEP score (0.607) in the diagnosis of HIT positivity and the difference was statistically significant ( $\mathrm{p}$ value 0.035 ).

Discussion \& Conclusion: The newly diagnosed HEP scoring system, which includes additional causes of thrombocytopenia was not superior to the 4T's score in this study. The inclusion of intra-arterial device in the HEP score did not make a difference in prediction of HIT. Conversely the $4 \mathrm{~T}$ score was superior to HEP score in the evaluation of the subset of patients on renal replacement therapy, a significant cause of thrombocytopenia, which was not included in the scoring system.

Supporting Document: 9d6f17b0-212d-4800-85b8-0bad63178235.

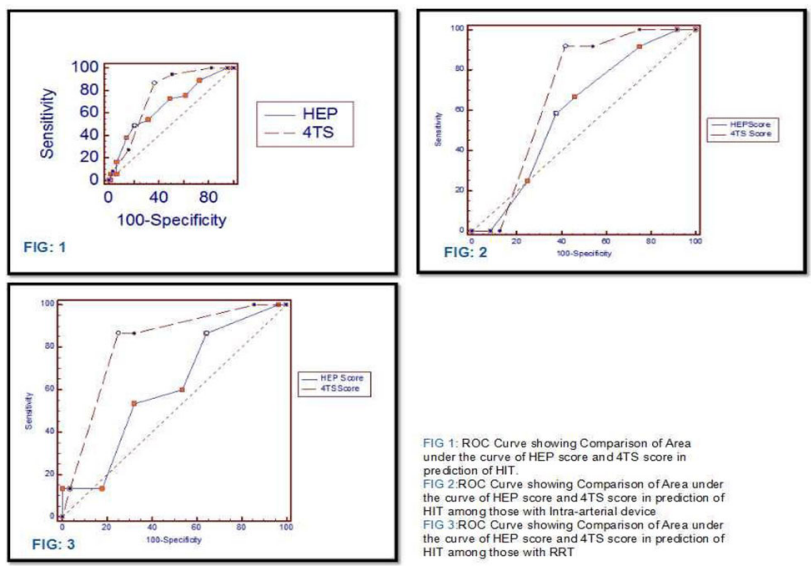

Disclosure of Interest: None Declared.

Keywords: HEP SCORE, HEPARIN, HIT.

\section{Abstract 4. Thrombosis and Angiogenesis in Breast Cancer}

Pankaj Raj ${ }^{* 1}$, Shailaja Shukla ${ }^{1}$, Sunita Sharma ${ }^{1}$, ShinjiniChoudhury ${ }^{1}$, O.p. Pathania ${ }^{2}$

\section{${ }^{1}$ Pathology, ${ }^{2}$ Surgery, Lady Hardinge Medical College, New Delhi, India}

Aims \& Objectives: Cancers often lead to a hypercoagulable state and many times clinically evident coagulation disorder may be the first sign of malignancy. Cancer induces a prothrombotic switch of the host hemostatic system, and in turn, blood clotting activation stimulates tumor growth and dissemination. Tumor angiogenesis is important for its growth and progression. Vascular Endothelial Growth Factor (VEGF) is a potent proangiogenic cytokine expressed by many cancer cells. There appears to be physiological relationship between coagulation activation and angiogenesis in cancers. The aim of this study was to determine the relation of serum VEGF levels to various markers of coagulation abnormalities like plasma fibrinogen and D-dimer levels and to various clinico-pathologic characteristics of breast cancer.
Patients/Materials \& Methods: Preoperative serum VEGF levels and plasma D-dimer and fibrinogen levels were determined in 60 women with breast cancer and compared with 30 healthy female controls. Enzyme linked immunosorbent assay was performed for VEGF estimation. Coagulation tests were performed on a fully automated STA-Compact (Stago, France) coagulation analyser. Levels of VEGF, fibrinogen and D-dimer were correlated with various clinico-pathologic characteristics of breast cancer including age, menopausal status, tumor size, lymph node metastasis,TNM stage, histological grade and hormone receptor status.

Results: Serum VEGF levels in breast cancer were significantly elevated compared with those of controls $(p<0.001)$. VEGF levels were significantly higher in postmenopausal women with breast cancer $(p=0.032)$ and in those having larger tumors $(p=0.044)$.

Plasma fibrinogen and D-dimer levels in breast cancer patients were significantly higher as compared to controls $(p<0.001)$ but showed no significant association with various clinico-pathologic characteristics of the tumor. In addition, there was a positive correlation of serum VEGF levels with plasma D-dimer and fibrinogen levels in women with breast cancer. All cases with elevated VEGF levels also showed raised plasma fibrinogen and D-dimer levels.

Discussion \& Conclusion: Preoperative serum VEGF detects breast cancer with a sensitivity of $72.3 \%$ and specificity of $93.8 \%$. Its levels are increased in breast cancer along with elevated plasma fibrinogen and D-dimer levels.

Additional long term studies are required for a better understanding of the relationship between markers of coagulation and angiogenesis and their prognostic significance in breast cancer.

Disclosure of Interest: None Declared.

Keywords: Angiogenesis, Breast cancer, Thrombosis.

\section{Abstract 5. Pravastatin, A New Anticoagulant Ameliorates In Vivo Thrombus Outcome by Inhibition of FXa}

Swati Sharma $^{* 1}$, Zahid Ashraf ${ }^{1,2}$, ItiGarg ${ }^{1}$, BhuvneshKumar ${ }^{1}$, Mohammad Aman Jairajpuri ${ }^{3}$

${ }^{1}$ Genomics, DIPAS, ${ }^{2}$ Biotechnology, ${ }^{3}$ Biosciences, Jamia Millia Islamia, Delhi, India

Aims \& Objectives: Anticoagulants have been keystone of contemporary therapy used for the prevention and treatment of thromboembolic disorders. Nonetheless, the recent studies identified the novel oral anticoagulants targeting FXa and thrombin, that can overcome the limitations of traditional drug therapy (warfarin). Natural remedies have their own advantages and are considered by most consumers to be both safe and effective. Their use is on the rise as herbal medicines are thought to cause fewer adverse and toxic effects than conventional modern drugs. The on-going studies reveal that plant secondary metabolites and functional foods possess remarkable antithrombotic properties both in clinical and experimental phases. However, their crude extract from plants may act on multiple targets exerting pleiotropic and synergistic effect making the mechanism of action more complex. Pertaining this in mind, a natural compound library was prepared. The library consisted of 158 distinct compounds. Each compound was docked with identified molecular target i.e. FXa, which is a FDA approved molecular target for thrombosis. Patients/Materials \& Methods: Each compound was docked with identified molecular target i.e. FXa, which is a FDA approved molecular target for thrombosis. It is a serine protease and it connects the extrinsic and intrinsic pathway of coagulation. The blind docking study gave 11 natural compounds as probable leads that could inhibit 
FXa. These leads were further analysed by SWISS-ADME and FXa activity assay.

Results: The four compounds, pravastatin, wogonin, hyperoside and diosmetin where shortlisted after above mentioned assays. They were further investigated for their biophysical characterisation and effects on thrombosis by in vivo experiments.

Discussion \& Conclusion: Pravastatin showed antithrombotic potential as evident by decrease in thrombus size in IVC ligated animals injected with pravastatin as compared to IVC controls.

Disclosure of Interest: None Declared.

Keywords: Factor Xa, IVC LIGATION, Pravastatin.

\section{Abstract 6. Assessment of Behavioral Role of Coagulation Factors in Several Malignancies: A Prospective Study}

\section{Lalit Kumar $^{* 1}$, Mona Bargotya ${ }^{1}$, PinkeyKachhap ${ }^{1}$, PayelDas ${ }^{1}$, Ankita Mehta ${ }^{1}$ \\ ${ }^{1}$ Department of Pathology, Rajiv Gandhi Super Speciality Hospital, New Delhi, India}

Aims \& Objectives: Malignancies affect the coagulation pathway as there is an increased tendency for both hemorrhage and thrombosis in patients with cancer. The objective of the study is to assess the effects of pulmonary, gastrointestinal (GI) and urothelial malignancies on coagulation cascade and identify their role as diagnostic as well as prognostic markers.

Patients/Materials \& Methods: All malignant cases received between October 2018 and March 2019 were enrolled in the prospective study. The study was conducted in the Department of Pathology of Rajiv Gandhi Super Speciality Hospital, Tahirpur, Delhi. Prothrombin time (PT), activated partial thromboplastin time (APTT), D-dimers, Fibrinogen and Factor VIII were measured for each sample.

Results: Total 121 malignant cases including 44 cases of urothelial malignancies, 43 cases of GI malignancies and 34 cases of pulmonary malignancies were included in the study. A control group of 35 nonmalignant cases was also included. Specific pattern of increased coagulation factors in various malignancies was observed that was statistically significant. Prothrombin time was raised only in GI malignancies (16.3 s) as compared to the control group (14.2 s). APTT was elevated in all malignancies in the study; $34.9 \mathrm{~s}, 35.9 \mathrm{~s}$ and $33.4 \mathrm{~s}$ for pulmonary, GI and urothelial malignancies, respectively, as compared to $29.8 \mathrm{~s}$ in the control group. D-dimers and fibrinogen were elevated in pulmonary cancers. A higher level of D-dimers \& fibrinogen was associated with higher tumor grade. D-dimers were also increased in GI malignancies. A mild increase in fibrinogen was noted in urothelial malignancies. Factor VIII was unequivocal across all malignancies.

Discussion \& Conclusion: The coagulation cascade was affected in all the studied malignancies. Elevated APTT across all malignancies indicate underlying coagulation abnormalities. Elevated fibrinogen and D Dimer levels in pulmonary malignancies hint at impending thromboembolic events and at a higher tumor grade. A similar observation was noted in colonic adenocarcinomas; increasing levels of PT, APTT, D-dimers and fibrinogen was associated with higher tumor grade and worse prognosis. Monitoring of specific panel of coagulation factors in early cancers could prevent thromboembolic events. Further research has to be undertaken to establish the role of coagulation profile as prognostic markers.

Disclosure of Interest: None Declared.

Keywords: Coagulation factors, Prognosis, Tumor markers.

\section{Abstract 7. Comparative Study of Coagulation Parameters in Breast Cancer}

Dr Seema Jathapi ${ }^{* 1}$, Rajni Choudhary ${ }^{2}$

${ }^{1}$ Patholoy, ${ }^{2}$ Pathology, Gandhi medical college, Bhopal, India

Aims \& Objectives: To evaluate and compare the coagulation profile of breast cancer patients with that of the control group.

Patients/Materials \& Methods: This study included 50 cases of breast cancer and 25 control group were selected to compare the results. After processing of blood samples, various test like complete blood count, platelet count, PT, APTT, Fibrin degradation product, and D-Dimer were done.

Results: A total of 75 cases were evaluated in the study. Significant differences were noted in the coagulation parameters of both the groups. Malignant cases show altered coagulation parameters as compared to control group.

Discussion \& Conclusion: Cancer is associated with a high risk of thrombotic complications. Altered coagulation parameters significantly correlate with malignant nature of tumour and also their spread. Preventing this complications is clinically relevant because they significantly contribute to morbidity and mortality of these patients.

Disclosure of Interest: None Declared.

Keywords: Cancer, Coagulation, Fibrin degradation products.

\section{Abstract 8. Drvvt in APLA- as a Single Test and in Combination with ANTI B2-Glycoprotein I and Anti Cardiolipin}

PriyavadhanaBalasubramanian $^{* 1}$, TulasiGeevar ${ }^{2}$, John Antony Jude Prakash ${ }^{3}$, John Mathew ${ }^{4}$, AnujaAbraham ${ }^{5}$, Melvin Joy ${ }^{6}$, Sukesh Chandran Nair ${ }^{7}$

${ }^{1}$ Transfusion medicine $\&$ immunohematology, ${ }^{2}$ Transfusion medicine and immunohematology, ${ }^{3}$ Microbiology, ${ }^{4}$ Rheumatology, ${ }^{5}$ Obstetrics \&Gynecology, ${ }^{6}$ Biostatistics, ${ }^{7}$ Transfusion medicine and Immunohematology, CMC, Vellore, Vellore, India

Aims \& Objectives: To study the significance of dilute russel viper venom test (DRVVT) towards the diagnosis of antiphospholipid antibody syndrome (APLA) - As a stand alone test and in combination with antibodies to $\beta 2$-glycoprotein I (anti- $\beta 2-\mathrm{GPI}$ ) and cardiolipin (aCL).

Patients/Materials \& Methods: This was a six month retrospective study. The electronic medical records of 451 patients referred for lupus anticoagulant (LAC) test were reviewed. LAC was done by DRVVT. Anti IgG, IgM $\beta 2$-GPI and IgG aCL were done by enzyme linked immunosorbent assay (ELISA). 
Results: 451 patients were referred for LAC test in view of arterial thrombosis $(12.4 \%)$, venous thrombosis $(10.1 \%)$, thrombocytopenia $(7 \%)$, pregnancy morbidity $(8.6 \%)$, systemic lupus erythematosus $(16 \%)$, other autoimmune causes $(6.8 \%)$. DRVVT positivity was seen in 49 cases (23\%). Mild positivity in 29, moderate in 14 and marked in 6 cases. In DRVVT positive patients, IgG anti- $\beta 2$-GPI was positive in 8 cases, IgM anti- $\beta 2$-GPI in 9 and IgG aCL in 15 cases. Repeat LAC testing was sent only for 12 cases. Triple positivity was seen in 10 out of 29 cases. In DRVVT negative patients (89\%), IgG anti- $\beta 2$-GPI was negative in all cases, IgM anti- $\beta 2$-GPI was positive in 21 cases and $\mathrm{IgG}$ aCL in 5. In other indications (38.5\%) referred for LAC, only two cases were mild positive for DRVVT.

Secondary causes were excluded for analysis of sensitivity, specificity of different test modalities (Table 1). DRVVT had highest sensitivity and specificity in cases presenting with thrombosis. IgG aCL was more specific in arterial thrombosis and anti $\operatorname{IgG} \beta 2$ GPI was more specific in venous thrombosis,thrombocytopenia and abortions. Also, the specificity increased when combination of tests were done.

Discussion \& Conclusion: APLA is diagnosed based on clinical and laboratory criteria. DRVVT as a single test has higher sensitivity and specificity in thrombosis but simultaneous testing for other antiphospholipid antibodies increases the specificity.

Supporting Document: 9a093141-94f0-4597-9a5b-995fb974b15e.

Table1 : Specificity, sensitivity of DRVVT, IgG, IgM anti $\beta 2$-glycoprotein I ( $\beta 2$-GPI) and anticardiolipin (aCL) with clinical features of antiphospholipid antibody syndrome.

\begin{tabular}{|c|c|c|c|c|}
\hline $\begin{array}{l}\text { SL. } \\
\text { NO }\end{array}$ & $\begin{array}{l}\text { CLINICAL } \\
\text { MANIFESTATION }\end{array}$ & ANTIPHOSPHOLIPID TEST & $\begin{array}{l}\text { SENSITIVITY } \\
\text { (\%) }\end{array}$ & $\begin{array}{l}\text { SPECIFICITY } \\
\text { (\%) }\end{array}$ \\
\hline \multirow[t]{9}{*}{1} & \multirow{9}{*}{$\begin{array}{l}\text { ARTERIAL THROMBOSIS } \\
(\mathrm{N}-56)\end{array}$} & DRVVT & 25 & 77.8 \\
\hline & & $\operatorname{lgG}$ anti- $\beta 2-G P I$ & 10 & 92.8 \\
\hline & & IgM anti- $\beta 2-G P \mid$ & 20 & 82.3 \\
\hline & & $\mathrm{aCL}$ & 10.8 & 88.3 \\
\hline & & DRVVT + IgG anti- $\beta 2-G P I$ & 12.3 & 99.5 \\
\hline & & DRVVT + IgM anti- $\beta 2-G P I$ & 14.3 & 98.8 \\
\hline & & $\mathrm{DRVVT}+\mathrm{aCL}$ & 11.6 & 97.6 \\
\hline & & IgG anti- $\beta 2-G P I+a C L$ & 14.0 & 99.5 \\
\hline & & IgM anti- $\beta 2-G P I+a C L$ & 13.6 & 98.1 \\
\hline \multirow[t]{9}{*}{2} & \multirow{9}{*}{$\begin{array}{l}\text { VENOUS THROMBOSIS } \\
(\mathrm{N}-46)\end{array}$} & DRVVT & 32.6 & 80.3 \\
\hline & & IgG anti- $\beta 2-G P \mid$ & 14.8 & 94.4 \\
\hline & & IgM anti- $\beta 2-G P \mid$ & 25 & 84.1 \\
\hline & & $\mathrm{aCL}$ & 15.6 & 89.9 \\
\hline & & DRVVT + IgG anti- $\beta 2-G P I$ & 12.3 & 99.5 \\
\hline & & DRVVT + IgM anti- $\beta 2-G P I$ & 14.3 & 98.8 \\
\hline & & $\mathrm{DRVVT}+\mathrm{aCL}$ & 11.6 & 97.6 \\
\hline & & IgG anti- $\beta 2-\mathrm{GPI}+\mathrm{aCL}$ & 14.0 & 99.5 \\
\hline & & IgM anti- $\beta 2-G P \mid+a C L$ & 13.6 & 98.1 \\
\hline \multirow[t]{9}{*}{3} & \multirow{9}{*}{$\begin{array}{l}\text { THROMBOCYTOPENIA } \\
(\mathrm{N}-32)\end{array}$} & DRVVT & 28.1 & 78 \\
\hline & & IgG anti- $\beta 2-G P I$ & 0 & 90.4 \\
\hline & & IgM anti- $\beta 2-G P \mid$ & 0 & 78.7 \\
\hline & & $\mathrm{aCL}$ & 20.8 & 90.7 \\
\hline & & DRVVT + IgG anti- $\beta 2-G P I$ & 0 & 96.5 \\
\hline & & DRVVT + IgM anti- $\beta 2-G P I$ & 0 & 95.6 \\
\hline & & $\mathrm{DRVVT}+\mathrm{aCL}$ & 16.7 & 96.7 \\
\hline & & IgG anti- $\beta 2-G P I+a C L$ & 0 & 96.3 \\
\hline & & IgM anti- $\beta 2-G P \mid+a C L$ & 0 & 95.3 \\
\hline \multirow[t]{9}{*}{4} & \multirow{9}{*}{$\begin{array}{l}\text { ABORTIONS } \\
(\mathrm{N}-39)\end{array}$} & DRVVT & 5.1 & 71.6 \\
\hline & & IgG anti- $\beta 2-G P \mid$ & 1 & 90.4 \\
\hline & & IgM anti- $\beta 2-G P \mid$ & 19.2 & 82 \\
\hline & & $\mathrm{aCl}$ & 2.6 & 84.9 \\
\hline & & DRVVT + IgG anti- $\beta 2-G P I$ & 3.8 & 96.8 \\
\hline & & DRVVT + IgM anti- $\beta 2-G P I$ & 3.8 & 95.8 \\
\hline & & $\mathrm{DRVVT}+\mathrm{aCL}$ & 2.6 & 95.7 \\
\hline & & IgG anti- $\beta 2-G P I+a C L$ & 3.8 & 96.6 \\
\hline & & IgM anti- $\beta 2-G P \mid+a C L$ & 3.8 & 95.5 \\
\hline
\end{tabular}

Disclosure of Interest: None Declared.

Keywords: Antibodies to beta 2 glycoprotein and cardiolipin, Antiphospholipid antibody syndrome, dilute russel viper venom test.
Abstract 9. Genetic Variations Associated with Venous Thrombo-Embolism (VTE)

ItiGarg $^{* 1}$, BabitaKumari ${ }^{1}$, Swati Srivastava ${ }^{1}$, Usha Panjawani ${ }^{1}$, Anju Bansal $^{1}$, BhuvneshKumar ${ }^{1}$

${ }^{1}$ Defence Institute of Physiology and allied Sciences (DIPAS), Defence Research and Development Organization (DRDO), Lucknow Road, Timarpur, Delhi-110054., Delhi, India

Aims \& Objectives: Venous thrombosis (VT) is a major health problem and has a high incidence in several populations across the world. It is a complex multi-factorial disease with unknown pathophysiological mechanism driven by various environmental, acquired and inherited risk factors. Gene polymorphisms play a seminal role in the development of VTE and could be an approach for the identification of predisposing genetic risk factors. These are important determinants for individual's susceptibility towards disease and drug response.

Patients/Materials \& Methods: This study was performed on Indian Army personnel as per ethical guidelines and written consent was obtained from them at the time of study. In present study, large cohort of Deep Vein Thrombosis (DVT) patients along with sex and aged matched healthy control subjects were taken. Basic demographic and physiological information was collected in both the groups. An association based on single nucleotide polymorphism (SNP) mutations were studied in genes related to established risk factors (MTHFR and PAI-1), warfarin metabolism (VKORC1 and CYP2C9 gene) and endothelin gene by genotyping studies using PCR-RFLP method followed by statistical analysis.

Results: The mutation in the MTHFR gene $(677 \mathrm{C} / \mathrm{T})$ was observed only in patients. Contrarily, higher frequency of mutation in the PAI-1 $-844 \mathrm{G} / \mathrm{A}$ was observed in controls in comparison to the patients. SNPs of VKORC1 $-1639 \mathrm{G}>\mathrm{A}$ and $-497 \mathrm{~T}>\mathrm{G}$, showed statistically significant difference between patients and control group at genotypic and allelic level. For CYP2C9 gene, CYP2C9*2 $416 \mathrm{C}>\mathrm{T}$ and CYP2C9*3 $1061 \quad \mathrm{~A}>\mathrm{C}$, showed statistically significant difference both at genotypic and allelic level between the patients and control group. The genotype and allele frequency between control and patients with VTE were significantly altered for EDN T1370G polymorphism.

Discussion \& Conclusion: Current view on genetic predisposition towards venous thrombosis suggests that SNP may confer increased risk or protection towards the disease and play a significant role in predictability of a VTE event in male Indian population.

Disclosure of Interest: None Declared.

Keywords: Deep vein thrombosis, GENOTYPING, SNP.

\section{Abstract 10. Plasmic Score as a Screening Test For Thrombotic Thrombocytopenic Purpura}

Deepika Gupta ${ }^{* 1}$, JasmitaDass ${ }^{1}$

${ }^{1}$ Hematology, Sir Ganga Ram Hospital, New Delhi, India

Aims \& Objectives: To assess the utility of PS to differentiate TTP from other causes of MAHA.

Patients/Materials \& Methods: All MAHA cases from January 2018 to July 2019 were isolated from critical alert records. Data from 61 
adult patients was analysed for calculation of PS. PS of 0-4, 5, 6-7 were labelled as low, intermediate and high risk. ADAMTS13 values and response to TPE was recorded wherever available.

Results: High PS was seen in 12, Intermediate in 11, and low in 38 patients. Of the 12 high PS, there were 9 cases of TTP with ADAMTS $13<3 \%$. Of the remaining 3 patients, 2 were TTP. ADAMTS13 was $25 \%$ in 1 case (sent post transfusion), ADAMTS was not sent in 1 but clinical behaviour was TTP and the last patient was Dengue with bilateral lower limb gangrene. Of these, 11 patients could be diagnosed as TTP correctly on PS. None of the patients in Intermediate and low risk PS category were TTP. Sensitivity, specificity, positive predictive value and negative predictive value of PS to identify TTP was $100 \%, 98 \%, 91.6 \%$ and $100 \%$ respectively.

Discussion \& Conclusion: PS performed very effectively in this cohort of patients. Patients with high PS are likely to have TTP and mortality can be reduced markedly with TPE. Therefore, it can serve as an early decisive tool to identify patients who are likely to have TTP and triage the use of TPE before the ADAMTS13 levels are available.

Supporting Document: 230a6cb9-d945-4006-8314-242b2b2f542f.

Disclosure of Interest: None Declared.

Keywords: ADAMTS13, PLASMIC score, Thrombotic thrombocytopenic Purpura.

\section{Abstract 11. Evaluation of Association Between Hematologic Variables and Deep Vein Thrombosis}

\author{
Govind R Patel ${ }^{* 1}$, ManoranjanMahapatra ${ }^{2}$, Hara Prasad Pati ${ }^{2}$, \\ TulikaSeth $^{2}$, Renu Saxena ${ }^{2}$
}

${ }^{1}$ Division of Clinical Hematology, Department of Medicine, Dr. S. N. Medical College, Jodhpur, ${ }^{2}$ Department of Hematology, All India Institute of Medical Sciences, New Delhi, India

Aims \& Objectives: To evaluate the association between hematologic variables (leukocytes, erythrocytes and red cell indices) and deep vein thrombosis (DVT), and to correlate these hematologic variables with classical thrombotic risk factors.

Patients/Materials \& Methods: We prospectively analyzed data from 76 patients of first episode of DVT and 30 healthy controls. The Hematologic parameters assessed were: total leukocytes, granulocytes, lymphocytes, monocytes, hematocrit, hemoglobin, erythrocytes and red cell indices including mean corpuscular volume, mean corpuscular hemoglobin, mean corpuscular hemoglobin concentration and red cell distribution width (RDW). These parameters were compared at baseline, monthly for first 3 months and then 3 monthly till 12 months of follow up.

Results: We found significant association between high RDW ( $>14 \%)$ and DVT. This association was maintained for all subgroup analyses and remained persistent throughout the study period at different time points. The odds ratios were 4.52 (95\% CI 1.84-11.09), 3.24 (95\% CI 1.33-7.88) and 3.04 (95\% CI 1.23-7.50) at baseline, 3 months and at 12 months respectively for RDW $>14 \%$ ). This association was prevailed after adjustment for age, sex, other hematologic variables (including the hemoglobin level, leukocyte count, platelet count), CRP, malignancy and co-morbidities. Significant correlation was found between the high RDW and comorbid conditions $(p=0.015)$ or patients who developed recurrent DVT during follow up $(p=0.001)$ at all time points of observation. Patients with both high RDW and malignancy $(\mathrm{OR}=3.40,95 \% \mathrm{CI}=0.39$ to $29.04)$, surgery $(\mathrm{OR}=2.00,95 \% \mathrm{CI}=0.21$ to 18.20$)$, pregnancy $(\mathrm{OR}=2.29,95 \% \mathrm{CI}=0.25$ to 20.79$)$, immobilization $(\mathrm{OR}=1.16$, $95 \% \mathrm{CI}=0.21$ to 6.27$)$, thrombophilia $(\mathrm{OR}=1.16,95 \% \mathrm{CI}=0.21$ to 6.27) or comorbid conditions $(\mathrm{OR}=3.70,95 \% \mathrm{CI}=0.97$ to 14.12$)$ had increased association with DVT. No significant association was observed for other hematologic variables investigated.

Discussion \& Conclusion: We have shown that high RDW, a parameter that is inexpensive and easily obtainable, was independently associated with DVT. Whether this association is causal or not, requires further investigation. Future studies should evaluate the underlying mechanism and the use of high RDW in prediction models for first and recurrent thrombosis.

Disclosure of Interest: None Declared.

Keywords: Deep vein thrombosis (DVT), Hematocrit, Red cell distribution width (RDW).

\section{Abstract 12. Influence of TFPI Gene Polymorphisms $(33 \mathrm{~T} / \mathrm{C}$ and $536 \mathrm{C} / \mathrm{T})$ on DVT in Young Indians}

\section{Kamal Kishor ${ }^{* 1}$, Amit Sharma ${ }^{1}$, Ravi Ranjan ${ }^{1}$, Ravi Kumar ${ }^{1}$, HareramPandey $^{1}$, ManoranjanMahapatra ${ }^{1}$, Renu Saxena ${ }^{1}$}

${ }^{1}$ Hematology, All India Institute of Medical Sciences, New Delhi, New Delhi, India

Aims \& Objectives: Deep vein thrombosis (DVT) is one of the major causes of morbidity and mortality in India. Low Tissue factor pathway inhibitor (TFPI) is a predisposing factor of DVT. However, its role in Indian DVT is not yet defined. Furthermore, the role of gene polymorphism affecting its plasma level is controversial. Therfore we look for association of TFPI with the DVT in Indians and to elucidate the role of TFPI polymorphisms with its plasma levels.

Patients/Materials \& Methods: Doppler proven young DVT patients (age $<45$ years) and age and sex matched healthy controls were studied. Exclusion criteria included patients on anticoagulants or those with history of malignancy, pregnancy or sepsis. Plasma TFPI levels were determined by ELISA and its normal range determined (Mean \pm 2SD of TFPI levels in controls). TFPI gene polymorphisms $-33 \mathrm{~T} / \mathrm{C}$ and $536 \mathrm{C} / \mathrm{T}$ were detected by PCR-RFLP method.

Results: 100 DVT subjects, median age 35 years range (20-43 years) were recruited. DVT was seen in lower limb in 86 patients and upper limb in 14 patients. Mean TFPI levels in DVT patients, $(33.55 \pm 11.7 \mathrm{ng} / \mathrm{ml})$ was significantly lower than controls $(48.05 \pm 13.6 \mathrm{ng} / \mathrm{ml}, p=0.001)$. Moreover, 19 patients $(19 \%)$ with DVT had low TFPI $(<20.85 \mathrm{ng} / \mathrm{ml})$, whereas no control had low TFPI. $536 \mathrm{C} / \mathrm{T}$ polymorphism was not detected in our study population. The prevalence of $33 \mathrm{~T}>\mathrm{C}$ polymorphism was significantly higher in controls than patients $(67 \%$ and $50 \%$ respectively $p=0.038$, $\chi^{2}$ : 6.56). Patients with $33 \mathrm{~T} / \mathrm{C}$ polymorphism had decreased OR of 0.521 (95\% CI: 0.289-0.938), 0.33 (95\% CI: $0.103-1.05$ ) in TC and CC genotypes respectively as compared to TT genotype, which showed its protective role against DVT. Mean TFPI levels were significantly lower in TT and TC than CC genotype in patients as well as in controls (patients: TT; $27.08 \mathrm{ng} / \mathrm{ml}, \mathrm{TC} ; 37.24 \mathrm{ng} / \mathrm{ml}, \mathrm{CC}$; $65.0 \mathrm{ng} / \mathrm{ml}, \quad p=<0.001$, and controls: TT; $39.90 \mathrm{ng} / \mathrm{ml}, \mathrm{TC}$; $48.76 \mathrm{ng} / \mathrm{ml}, \mathrm{CC} ; 72.0 \mathrm{ng} / \mathrm{ml}, p<0.001)$.

Discussion \& Conclusion: Low TFPI level predisposes to DVT in young Indians. 536C $>\mathrm{T}$ polymorphism is absent in Indian population. 
33T $>$ C polymorphism is associated with increased TFPI level and has a protective role against DVT in Indian patients.

Disclosure of Interest: None Declared.

Keywords: DVT, PCR RFLP, polymorphism.

\section{Abstract 13. A Single Centre Experience of Diagnosis of Heparin Induced Thrombocytopenia with Thrombosis (HITT)}

\section{Kartik Purohit $^{* 1}$, Chetan Agarwal ${ }^{1}$, Jyoti kotwal ${ }^{1}$ \\ ${ }^{1}$ hematology, Sir Gangaram Hospital, New Delhi, New Delhi, India}

Aims \& Objectives: Conventionally heparin is considered as the most potent anticoagulant but paradoxically it also causes deadliest thrombotic disease-Heparin induced thrombocytopenia with thrombosis (HITT).The objective of this study was to find out the prevalence of HITT in suspected cases of HITT at our institute and to measure sensitivity and specificity of 4T score in our patients.

Patients/Materials \& Methods: It was a retrospective observational study. The rapid ID-gel microtyping system- card test was performed in all suspected cases. The platelet function test was performed on gel card positive samples- heparin induced platelet aggregation test (PAT). For platelet aggregation study; Chronolog model 490-2D with aggro/link ${ }^{\circledR}$ version 5 software was used. The platelet aggregation response of normal platelets (control) to saline; and patient's serum + various dilution of heparin $(0.1 \mathrm{U} / 1 \mathrm{U} / 100 \mathrm{U} / \mathrm{ml})$ was assessed. The platelet aggregation response of $\geq 20 \%$ with low concentration of heparin $(0.1 \mathrm{U}, 1 \mathrm{U} / \mathrm{ml})$ along with no spontaneous aggregation and no aggregation to $100 \mathrm{U} / \mathrm{ml}$ of heparin was considered diagnostic.

Results: We received total 66 samples of suspected HITT patients from 2016 to 2019 and 11 patients $(16.66 \%)$ were diagnosed to have HITT. Out of 11 patients diagnosed to have HITT; 4 patients $(36.4 \%)$ had osteoarthritis, 2 patients (18.2\%) had underwent cardiopulmonary bypass and myocardial infarct each; 1 patient $(9.1 \%)$ with sepsis, stroke and aortic aneurysm each. The median duration to develop thrombocytopenia in the diagnosed HITT was 7 days (range: 5-9). 4T score of 5.5 having $73 \%$ sensitivity and $98 \%$ specificity for diagnosis of HITT.

Discussion \& Conclusion: The pharmacological action of heparin is anticoagulation and so major anticipated adverse effect is bleeding. However, in some patients it leads to life or limb threatening ischemia. It is a clinicopathologic diagnosis; so, both clinical history with laboratory parameters and their correlation is important for diagnosis. Conventionally $4 \mathrm{~T}$ score is used for estimating pre test probability of the disease; relatively low scores $(0-4)$ have very high negative predictive value $(\approx 99 \%)$ to rule out the disease. In our study we found that 8 out of 9 patients $(89 \%)$ with $4 \mathrm{~T}$ score $\geq 6$ were diagnosed to have HITT while only 3 out of 54 patients $(5.3 \%)$ with 4 T score $<6$ were diagnosed to have HITT. World wide it is an over diagnosed disease but, in our country, probably due to lack of awareness it is under diagnosed entity.

Supporting Document: 48b0a0fc-627b-469d-9c99-710a0926e1ba.

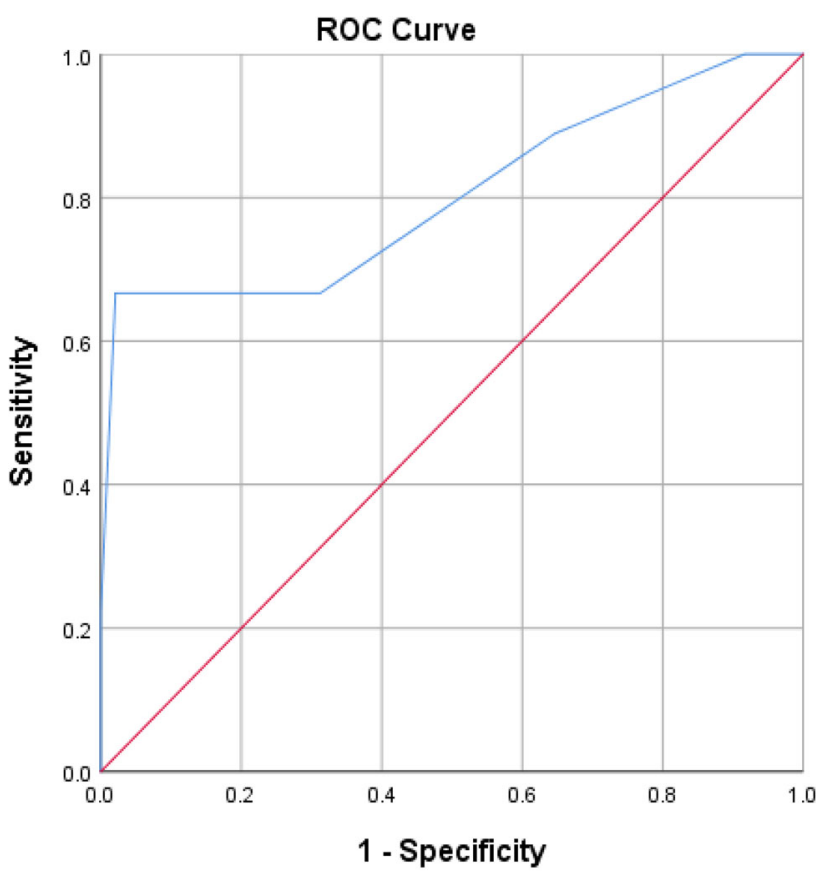

Diagonal segments are produced by ties.

Disclosure of Interest: None Declared.

Keywords: 4T score, HITT, PAT.

\section{Transfusion medicine}

\section{Abstract 1. Serum Ferritin and Serum Transferrin Receptor Levels in Voluntary Blood Donors}

Ashish Philip ${ }^{* 1}$, Meera Sikka ${ }^{1}$, Priyanka Gogoi ${ }^{1}$, PreetiDiwaker ${ }^{1}$, Mrinalini Kotru ${ }^{1}$

\section{${ }^{1}$ Department of Pathology, UCMS and GTB, Delhi, India}

Aims \& Objectives: Each blood donation causes substantial loss of iron. A normal hemoglobin used conventionally for screening of donors does not rule out preclinical iron deficiency. However, collection of blood from such a donor can exacerbate preclinical iron deficiency leading to iron deficiency anemia and their subsequent deferral. This study aimed to assess the iron status of voluntary blood donors and to evaluate the proportion of first time and regular donors with iron deficiency (ID) as detected by serum ferritin (SF) and serum transferrin receptor (sTfR) levels.

Patients/Materials \& Methods: This cross-sectional comparative study was done on 144 voluntary blood donors who were divided into first time and regular donors.Complete blood counts, SF and sTfR were measured in all donors. ID was diagnosed if SF was $<16 \mathrm{ng} / \mathrm{ml}$ or sTfR was $>1.6 \mathrm{ng} / \mathrm{L}$. Proportion of first time and regular donors developing ID was calculated and compared.

Results: Reduced SFwas identified in a significantly $(p<0.001)$ higher proportion of regular donors $(25.7 \%)$ as compared to first time donors $(5.5 \%)$. Increased sTfR levels were also identified in a significantly $(p<0.001)$ higher proportion of regular donors $(34.7 \%)$ as compared to first time donors (18.0\%). Either reduced SF or elevated STfR was detected in $43.0 \%$ of all donors.

Discussion \& Conclusion: SF, the single best indicator of iron stores has been used most frequently to identify ID in donors. Only an 
occasional study has used sTfR to diagnose ID in donors. As SF is an acute phase reactant, sTfR was also employed in this study. Either parameters detected ID in $43 \%$ donors.A significantly higher proportion of regular donors had ID as compared to first time donors. Identification of ID is hence important as iron supplementation of these donors will increase the donor pool.

Regular donation leads to ID which can be detected by SF and STfR. Hence these tests should be used in donors for early identification of ID which can be used for appropriate adjustment of donation interval and help guide iron supplementation.

Disclosure of Interest: None Declared.

Keywords: iron deficiency, serum ferritin and serum transferrin receptors, voluntary blood donors.

\section{Abstract 2. Transfusion Related Change in Plasma Free Hemoglobin Level \& Its Impact on Microvascular Oxygenation}

\section{Akshaya TOMAR ${ }^{* 1}$, Joseph PHILIP ${ }^{1}$, Neerja kushwaha' \\ 1immunohematology \& Blood Transfusion, Armed Forces Medical College, Pune, India}

Aims \& Objectives: Transfusion of stored packed red blood cells (PRBCs) is a common therapeutic intervention however development of hemolysis during storage might contribute to some negative consequences due to release of free hemoglobin ( $\mathrm{fHb}$ ), a potent nitric oxide (NO) scavenger, which may impair vasodilation and microcirculatory perfusion after transfusion. We undertook this study to find out change in fHb levels in recipients and whether it affect microvascular oxygenation post transfusion.

Patients/Materials \& Methods: 80 stable transfusion recipients were selected for this prospective observational study and were divided into 3 groups as per the age of blood transfused $(<7$ days, $7-14$ days and $>14$ days). Blood samples were collected to analyze plasma levels of $\mathrm{fHb}$ of the bag. Patient's circulating plasma $\mathrm{Hb}(\mathrm{FHb})$ and $\mathrm{pO} 2$ by venous blood gas analysis was studied prior to transfusion, and after $1 \mathrm{~h}$ of transfusion. Data was analyzed through paired T test, ANOVA and Pearson's correlation coefficient using SPSS v23.

Results: Overall, there was a significant difference between the three groups in terms of change in plasma free hemoglobin $(\mathrm{gm} / \mathrm{dL})$ from pre-transfusion to the 1 Hour post-transfusion time points $(p<0.05)$. Significant increase in pO2 Levels was noticed from the pre-transfusion to $1 \mathrm{~h}$ post- transfusion time points in bags $<7$ days old $(\mathrm{t}=-4.6, p<0.001)$. Also strong negative correlation was obtained between $\mathrm{FHb}$ and $\mathrm{pO} 2$ using Pearson's correlation coefficient ( $\mathrm{r}$ $-0.738 ; p<0.001)$.

Discussion \& Conclusion: This study concludes that RBC transfusion can significantly increase post-transfusion plasma $\mathrm{fHb}$ levels depending upon the age of PRBC units and has an implication on microvascular oxygenation. However effect on clinical outcome of the patient was not studied and it would be worthwhile to study whether the age of the blood transfused affects the patient morbidity or mortality.

Disclosure of Interest: None Declared.

Keywords: blood transfusion, microvascular oxygenation, plasma free hemoglobin.
Abstract 3. A Prospective Study of Optimization of Antibody Screening During Pre-transfusion Testing

\section{DivyaSetya $^{* 1}$, Prashant Pandey ${ }^{1}$, Mukesh Singh ${ }^{1}$}

${ }^{1}$ Transfusion Medicine, Jaypee Hospital, Noida, Noida, India

Aims \& Objectives: Antibody screening (AS) is considered superior to antihuman globulin (AHG) cross match during pretransfusion compatibility testing. Inspite of knowing the utility and superiority of AS, it has not been adopted uniformly in India. Therefore, scarce data is available in terms of optimisation of red cell antibody detection during pretransfusion testing in form of "Type and screen". The main objective was to study the benefit of performing AS along with blood grouping simultaneously during the first hospital visit to the hospital. Other objectives were to study the prevalence of clinically significant antibody among the Indian population and to follow up the patients who were transfused antibody screen negative but cross match incompatible blood. We also studied some other relevant quality indicators related to efficiency of blood transfusion services.

Patients/Materials \& Methods: This was a prospective study which was carried out at a tertiary healthcare centre in India between July 2014 and Dec 2018 (54 months). The study protocol was submitted to institutional review board and permission was granted. Blood grouping and AS were done during patients' first hospital visit, which we called "type and screen". When the patient got admitted to the hospital and required blood transfusion, a blood request form was generated by the user and sent to blood bank. Depending upon the results of antibody detection, further course of action was chosen. If patient was found to have no antibody, immediate spin test (IST) cross match compatible blood was issued and transfused. In such cases the procedure of AHG crossmatch testing was continued even after issue of blood. Cases where AHG cross match test was found negative no further follow-up of the patient was done whereas when AHG cross match was found positive, patients were followed after the transfusion.

Results: A total of 22,888 patients were "type and screened". Majority were from hemato-oncology, BMT, liver transplant, paediatric cardiac surgery, and medical ICU units. Clinically significant allo-antibody was detected in 145 patients and autoantibody was detected in 53 patients. Alloantibody was detected mainly against Rh and kell blood group systems. In diagnosed AIHA cases, majority were in the form of Warm AIHA (58\%) and $20 \%$ of AIHA cases were having hidden single or multiple alloantibody. Significantly higher proportion of patients in AS positive group required blood transfusion than AS negative group ( $45 \% \mathrm{VS} 34 \%, p<0.05$ ). in both the groups, in planned cases, most of the time blood was issued immediately within the defined turnaround time except in 21 where either transfusion was delayed or surgery was postponed. It happened only in trauma or surgical bleed cases. Expiry of blood was decreased significantly due to no usage of blood $(1.2 \%$ vs. $5 \%, p<0.05)$. During the period of study we obtained 03 cases where the IST cross match was compatible but the AHG cross match was incompatible. During follow up none of the cases demonstrated any sign of hemolysis.

Discussion \& Conclusion: In developing countries like India, optimization of AS during pretransfusion testing increases operational efficiency and significantly decreases the expiry of blood.

Disclosure of Interest: None Declared.

Keywords: antibody screening, pre-transfusion testing, turn around time. 


\section{Abstract 4. Anti- M(A) Antibody in M(A)N Patient: A Rare Case Report}

\section{Atif Khan ${ }^{* 1}$, Afreen Karimkhan ${ }^{1}$, Gopal Patidar ${ }^{1}$, Anjali Hazarika ${ }^{2}$ \\ ${ }^{1}$ Dept of Transfusion Medicine, ${ }^{2}$ CNC Blood Bank, AIIMS, New Delhi, New Delhi, India}

Aims \& Objectives: $M$ antigen is the major antigen of MNS blood group system. Anti-M antibodies are naturally occurring cold reacting saline agglutinins, with both $\operatorname{IgM}$ and $\operatorname{IgG}$ component, rarely may cause hemolytic transfusion reactions, decreased red cell survival or hemolytic disease of newborn. We report an unusual case of $1.5 \mathrm{yrs}$ old patient who contained $\mathrm{M}$ antigen on red cell with Anti-M antibodies in serum with negative auto-control.

Patients/Materials \& Methods: 1 y-5 m male child was planned for routine surgery. His blood was incompatible on routine AHG crossmatch. Further immunohematological tests like antibody screening, identification, antibody titer, red cell phenotyping of child and parents, DCT, autocontrol tests were performed.

Results: Patient was $\mathrm{O} \mathrm{Rh} \mathrm{D}$ positive but there was agglutination $\mathrm{O}$ reagent cells in reverse blood grouping. After antibody identification test and after exclusion of the possibilities of other antibodies by select cell panel it was revealed that patient had Anti-M alloantibody in his serum. Antibody was of both $\operatorname{IgM}$ and $\operatorname{IgG}$ type with titre of 1:2. On further workup, we performed patient's and his parent's red cell phenotyping for $\mathrm{M}$ and $\mathrm{N}$ antigens. On phenotyping it was found that all three were $M$ antigen positive. Patients sera showed no reaction with his father's RBC, but positive reaction with other M-positive RBC's Patient cross matched with $\mathrm{M}-\mathrm{N}+$ and $\mathrm{M}-\mathrm{N}-$ donors and crossmatch compatible blood was given. Patient was successfully transfused 2 units of packed cells with no reaction.

Discussion \& Conclusion: Possibly, patient had some genetic alteration leading to a missing $\mathrm{M}$ antigen (called as $\mathrm{Ma}$ antigen) and antibody directed against missing part (anti-MA antibody). Here, the lack of reaction sera with father's RBC's keeps plausibilty that both father and son have the same defective $\mathrm{M}$ antigen (Ma), elucidating that is an inherited phenomenon.

Disclosure of Interest: None Declared.

Keywords: Anti M antibody, Immunohematology, MNS Blood group system.

\section{Abstract 5. Critical Audit of FFP transfusion Practices in OBS \& GYNE Departments in a Tertiary Care Hospital}

Priyanka Singh $^{* 1}$, Vandana Puri $^{1}$, IpsitaDalal ${ }^{1}$, ArunaChhikara ${ }^{1}$, GeetikaSharma $^{1}$, PreetiRai $^{1}$

${ }^{1}$ Pathology, Lady Hardinge Medical College and Associated Hospitals, Delhi, India

Aims \& Objectives: The administration of Fresh frozen plasma (FFP) is inappropriate without any scientific basis or clinical indication in many hospital settings. Although many FFP transfusion guidelines which has been published till now.

Aim- To determine the appropriate and inappropriate usage of FFP in a tertiary care hospital.
Patients/Materials \& Methods: A retrospective study was carried on between February 2016 to August 2016 in the regional blood transfusion centre of our institution. It was done using the FFP transfusion guidelines based on British Committee for Standards in Hematology (BCSH) and College of American Pathologists (CAP). Blood bank requisition forms received from patients of Obstetrics and Gynecology department during this period were screened. Indications for transfusion requests were studied and further divided into appropriate and inappropriate requests.

Results: A total of 901 units of FFP were issued to 250 patients during this period. Appropriate usage of FFP was found in $67 \%$ and inappropriate usage in $33 \%$ of the 250 patients evaluated. Bleeding related to surgery with deranged coagulation profile constituted the most common appropriate indication for FFP infusion and severe anemia was the most inappropriate indication for FFP use.

Discussion \& Conclusion: The usage of FFP requires a proper understanding and knowledge about the appropriate and inappropriate usages. It also involves training and supervising the medical staff at regular intervals. Computerized audit programs are required to do a prospective monitoring of the FFP issue and usage in any clinical setting. Further studies are required on usage of FFP specifically in Obstetric and Gynecology patients in order to improve the utilization of this precious blood products.

Disclosure of Interest: None Declared.

Keywords: Audit, FFP, obs and gyne.

\section{Abstract 6. Effect of Grey Zone Sample TTI by ELISA on Blood Safety of a Tertiary Care Center}

Anjali Patel $^{* 1}$, KeyuriPatel ${ }^{1}$, Mustafa Ranapurwala ${ }^{1}$, Rohit Kumar Singh ${ }^{1}$, NeelofarMansuri ${ }^{1}$

${ }^{1}$ Pathology, Pramukhswami Medical College, Anand, India

Aims \& Objectives: Estimation of a Grey zone in ELISA testing and repeat testing of Grey zone samples can further help in reducing the risks of TTI in countries where nucleic acid amplification testing for TTIs is not feasible.

Patients/Materials \& Methods: 4286 blood donors screened during the study period (JAN2019-AUG2019). On performing repeat ELISA testing on Grey zone samples in duplicate, the samples showing both OD values below Grey zone were marked nonreactive, and samples showing one or both OD value in the Grey zone were marked indeterminate. The samples on repeat testing showing one or both OD above cut-off value were labeled.

Results: Of the 4286 blood donors screened during the study period, a total of 08 blood donors were found to be in Grey zone. On repeat testing of these Grey zone samples, 02 were found to be reactive for TTIs. And 01 found again in Grey zone.

Discussion \& Conclusion: Though NAT technology is an advanced screening method for screening of all sero-positive donors in the context of reducing the window period still in developing countries like India, installation of this technique in all blood bank centers is not feasible due to cost factor. So, Elisa test still remains a prominent screening tool in most blood bank centers in India. Proper donor screening, sensitive screening assays and effective pathogenic inactivation procedures can minimize the risk of TTI to a great extent. Repeat testing of Grey zone samples will help in improving the safety of blood transfusion.

Disclosure of Interest: None Declared. 
Keywords: Enzyme linked immunosorbent assay, Grey zone, Transfusion transmissible infections.

\section{Abstract 7. A Study on the Impact of Facebook on Voluntary Blood Donations}

Dr IrfanaNikhat ${ }^{* 1}$, Srinivas Manne', Rehana Parveen ${ }^{1}$, Narasimha Anantha Rao ${ }^{1}$

\section{${ }^{1}$ Blood Bank, Star Hospitals Hyderabad, Hyderabad, India}

Aims \& Objectives: In 2017, Facebook introduced a new feature that would prompt users to sign up to be voluntary blood donors. This new way would connect people and organizations to find blood donors when needed. This study was planned to evaluate the impact of the Facebook feature on voluntary blood donors in our hospital.

Patients/Materials \& Methods: The study was conducted at Star Hospital, Hyderabad, Telangana. All the voluntary blood donors were included in this study from October 2018 to March 2019. This data was compared with the retrospective six months' data before we registered our blood bank with the new feature of Facebook. The volunteers who had come for voluntary blood donation but did not donate blood due to various deferral criteria were excluded from the study. The outcome measures were the percentage change in the number of walk-in donors from April to September 2018 and October 2018 to March 2019. Data analysis was performed by using Medcalc ${ }^{\circledR}$ Software and Microsoft Excel 2016.

Results: The total blood donations from Oct ' 18 to Mar '19 were lower than the total blood donations from Apr 18 to Sep 18 with a $3.53 \%$ decrease between the periods. However, the voluntary blood donors increased by more than fivefold between the study periods since the time our blood bank was registered with the new feature of Facebook $(p<0.001)$. It was also observed that there was a linear rise in the Facebook donors from Oct 18 to Mar 19.

Discussion \& Conclusion: The social media also plays a pivotal role in creating support groups for voluntary blood donation. It was observed that there was an overwhelming response through the new feature of Facebook. This is the first study conducted on the impact of Facebook on voluntary blood donations. We hope that by raising awareness and letting people know where and when they can donate, we can increase blood donations and contribute to a more sustainable blood supply.

Disclosure of Interest: None Declared.

Keywords: Facebook feature, Sustainable blood supply, Voluntary blood donors.

\section{Abstract 8. Comparison of ELISA and CLIA for Detection Infectious Disease Markers; HBV, HCV and HIV}

Anand Upadhyay $^{* 1}$, AseemTiwari ${ }^{1}$, GeetAggarwal ${ }^{1}$, Swati Pabbi ${ }^{1}$, Gunjan Bharadwaj ${ }^{1}$, AanchalLuthra ${ }^{1}$

${ }^{1}$ Department of transfusion medicine, Medanta-The Medicity, Gurgaon, India

Aims \& Objectives: Aim of the study was to do head-to-head comparison of Enzyme Linked Immunosorbent Assay (ELISA) and Chemiluminescence Immuno Assay (CLIA) for detection of infectious disease markers (IDM); in terms of various parameters; prevalence, sensitivity, specificity, Cohen's kappa value and strength of agreement for IDM; HBV, HCV and HIV in blood donors.
Patients/Materials \& Methods: Head-to-head comparison of 10,164 healthy blood donors for TTI markers was done using latest generation of CLIA and ELISA in year 2018. All donor samples were screened for mandatory TTI. Screening for the presence of marker of HBV, HCV and HIV was done using CLIA (VITROS 3600; Ortho Clinical Diagnostics, USA) and ELISA (EVOLIS, BioRad, France). All reactive and discordant results were reconfirmed using the gold standard test; neutralization, DeciScan HCV plus and Western Blot for HBsAg, anti-HCV and anti-HIV. Statistical analysis was done for different parameters.

Results: Out of 10,164 healthy donors total 223 samples were found reactive; out of which 93 (55 HBV, $34 \mathrm{HCV}$ and four HIV) samples were concordant reactive and tested positive by both methods while 130 discordant reactive samples were reactive either by ELISA or CLIA. Out of 130 discordant reactive samples ELISA-reactive and CLIA-nonreactive samples for HBV, HCV and HIV were 29, eight, and 15 respectively while CLIA-reactive ELISA-non-reactive samples for HBV, HCV and HIV were three, 36 and 39 respectively. Three hundred concordant non-reactive samples were also tested negative by their respective gold standards. Prevalence of HBV, HCV and HIV was $0.54,0.33$ and 0.04 respectively. Sensitivity of CLIA and ELISA was $100 \%$ for both methods for all three TTI markers, while specificity was 95.24 and $89.55 \%$ for HBV; 97.40 and $89.29 \%$ for $\mathrm{HCV}$ and 91.90 and $99.01 \%$ for HIV, respectively. Kappa statistics (k) for HBV, HCV and HIV was 0.748 and $0.968,0.882$ and $0.605,0.334$ and 0.168 for ELISA and CLIA respectively. Strength of agreement for ELISA and CLIA compared to the gold standard test was good and very good for HBV, very good and good for HCV, and fair and poor for HIV. Diagnostic-accuracy and inter-assay precision was better in CLIA while cross-reactivity, interference and dilution sensitivity were comparable.

Discussion \& Conclusion: In this study we compared a relatively newer technique CLIA; which is gradually replacing approximately five decade older and established ELISA technique at advance BTS due to high throughput, lesser TAT, higher reproducibility and no restriction of batch. ELISA and CLIA were comparable in statistical terms. Different statistical parameters indicate that both tests are equally acceptable for donor screening purposes. CLIA and ELISA did not miss any IDM and are comparable. Blood transfusion service can choose either ELISA or CLIA for screening of blood units according to their need and resources.

Supporting Document: 92bd17fb-cc2c-4146-aed7-11b07b00ca84. 
Table1: Comparison of sensitivity, specificity and accuracy of ELISA and CLIA for HIV, HCV and HBV

\begin{tabular}{|l|r|r|r|r|r|r|}
\hline & \multicolumn{2}{|c|}{ HIV } & \multicolumn{2}{c|}{ HCV } & \multicolumn{2}{c|}{ HBV } \\
\hline & \multicolumn{1}{|c|}{ ELISA } & \multicolumn{1}{c|}{ CLIA } & \multicolumn{1}{c|}{ ELISA } & \multicolumn{1}{c|}{ CLIA } & \multicolumn{1}{c|}{ ELISA } & \multicolumn{1}{c|}{ CLIA } \\
\hline SENSITIVITY & $100 \%$ & $100 \%$ & $100 \%$ & $100 \%$ & $100 \%$ & $100 \%$ \\
\hline SPECIFICITY & $95.24 \%$ & $89.55 \%$ & $97.40 \%$ & $89.29 \%$ & $91.90 \%$ & $99.01 \%$ \\
\hline ACCURACY & $95.30 \%$ & $89.68 \%$ & $97.66 \%$ & $90.27 \%$ & $92.45 \%$ & $99.16 \%$ \\
\hline
\end{tabular}

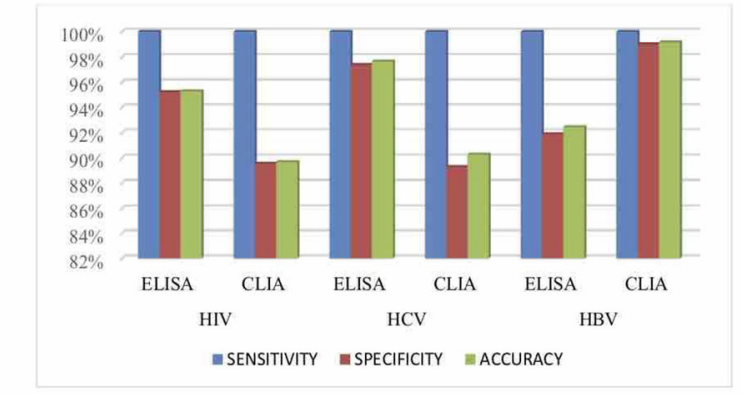

Fig 1: Comparison of sensitivity, specificity and accuracy of ELISA and CLIA for HIV, HCV and HBV

Disclosure of Interest: None Declared. Keywords: CLIA, ELISA, IDM.

\section{Abstract 9. Efficacy of Leukapheresis in Hemato- Oncology Patients-Experience from Tertiary Care Centre}

Rekha Hans*1, Satya Prakash', Ratti Ram Sharma', Pankaj Malhotra ${ }^{2}$, Neelam Marwaha ${ }^{1}$

${ }^{1}$ Transfusion Medicine, ${ }^{2}$ Internal Medicine, Post Graduate Institute Of Medical Education And Research, Chandigarh, Chandigarh, India

Aims \& Objectives: To access the efficacy of therapeutic leukapheresis as life-saving emergency procedure in symptomatic patients. Patients/Materials \& Methods: A retrospective analysis of all therapeutic leukapheresis procedures in symtomatic patients was done over a period of 10 years (2004-2014). Procedures were done on different apheretic devices (CS-3000 Plus, Baxter, USA and Cobespectra,Terumo BCT, Lakewood Co. USA).Patients' pre and post procedural hematological profiles were analyzed by applying paired $\mathrm{T}$ test and pearson's correlation to assess the efficacy of leukapheresis in them.

Results: We performed 80 Leukapheresis procedures in 68 patients with mean age of 31.62 years (range-7-75 yrs). The patients presented in emergency medicine department of our tertiary care centre with acute symptoms of leukastasis such as severe pulmonary and central nervous system symptoms. Male to female ratio was $2: 1$. The range of procedure per patient was 1 to 4 . The mean post procedure decline in total leukocyte count was $33 \%$ (declining from a mean pre count of $254 \pm 166 \times 10^{9} / \mathrm{L}$ to $171 \pm 128 \times 10^{9} / \mathrm{L}$ post procedure $)$ and was statistically significant $(p<0.001)$. Also, there was significant change in post platelet count $(p<0.001)$ and mean post hemoglobin ( $p=0.001)$. All patients improved symptomatically post procedure. As per our records, 7 patients found to be dead in follow up and remaining were on various treatment protocol.

Discussion \& Conclusion: Leukapheresis provides an immediate symptomatic relief and is an efficient useful emergency lifesaving procedure in cases of leukastasis in leukemia patients.

Disclosure of Interest: None Declared.

Keywords: Hemato-oncology, Leukapheresis, symptomatic.

\section{Abstract 10. Renal Transplantation in HLA Sensitized Patients: Bridling the Immunologic Barrier}

\section{DivyaSetya $^{* 1}$, Prashant Pandey ${ }^{1}$, Amit Pande ${ }^{1}$ \\ ${ }^{1}$ Transfusion Medicine, Jaypee Hospital, Noida, Noida, India}

Aims \& Objectives: Successful renal transplantation across HLA barrier in sensitized individuals have been on the rise during the past decade, primarily due to improved desensitization regimes. Aim of this study was to share outcome of desensitization in renal transplant recipients with Donor-Specific Anti-HLA Antibodies (DSA). Successful renal transplantation across HLA barrierin sensitized individuals have been on the rise during the past decade, primarily due to improved desensitization regimes. Aim of this study wasto share outcome of desensitization in renal transplant recipients withDonor-Specific Anti-HLA Antibodies (DSA).

Patients/Materials \& Methods: This was a retrospective analysis of all HLA immunized individuals who were prospective renal transplant recipients. All such patients underwent preconditioning as per the institutional desensitization protocol. Complement Dependent Cytoxicity based cross match (CDC-XM), Luminex based cross match (LM-XM) and Flowcytometry based cross match (FC-XM) were done in all cases. If any of these tests turned out positive, Single Antigen Bead assay (SAB) was performed. Desensitization for DSA was performed in 55 patients and all patients were followed-up for one year to assess graft function and patient outcome.

This was a retrospective analysis of all HLAimmunized individuals who were prospective renal transplant recipients.All such patients underwent preconditioning as per the institutionaldesensitization protocol. Complement Dependent Cytoxicity based crossmatch (CDC-XM), Luminex based cross match (LM-XM) andFlowcytometry based cross match (FC-XM) were done in all cases. If anyof these tests turned out positive, Single Antigen Bead assay (SAB) wasperformed. Desensitization for DSA was performed in 55 patients and allpatients were followed-up for one year to assess graft function andpatient outcome.

Results: CDC-XM being a less sensitive assay, could not detect incompatibility in $29(52.73 \%)$ cases. After desensitization, even though SAB and LM-XM results revealed an MFI within acceptable range, FC-XM being an extremely sensitive assay, continued to give a positive result in $8(14.55 \%)$ cases. The mean \pm SD number of pre-transplant TPE were $3.44 \pm 0.98(2-11)$. Out of 55, there were 10 patients who were lost to follow up. Patient and graft survival of 45 patients at one year was found to be $100 \%$.

CDC-XM being a less sensitive assay, could not detectincompatibility in $29(52.73 \%)$ cases. After desensitization, even thoughSAB and LM-XM results revealed an MFI within acceptable range, FC- 
XMbeing an extremely sensitive assay, continued to give a positive result in8 $(14.55 \%)$ cases. The mean \pm SD number of pre-transplant TPE were $3.44 \pm 0.98(2-11)$. Out of 55 , there were 10 patients who were lost tofollow up. Patient and graft survival of 45 patients at one year was foundto be $100 \%$.

Discussion \& Conclusion: Preconditioning for renal transplants in the form of immunosuppression with TPE is an extremely useful auxiliary for transplantation in HLA sensitized renal transplant recipients. Preconditioning for renal transplants in the form ofimmunosuppression with TPE is an extremely useful auxiliary fortransplantation in HLA sensitized renal transplant recipients.

Disclosure of Interest: None Declared.

Keywords: HLA, renal transplant patient, Therapeutic Plasma Exchange.

\section{Abstract 11. High Prevalence of HBB: C.9T $>$ C Polymorphism at CODON 2 of Beta Globin Gene in West Bengal Population}

\section{Ankita Biswas $^{* 1}$, SunisthaBhattacharjee ${ }^{1}$, Rudra Ray ${ }^{1,1}$, MaitreyeeBhattacharyya ${ }^{1}$}

${ }^{1}$ Transfusion Medicine, Ihtm, Medical College, Kolkata, Kolkata, India

Aims \& Objectives: The aim of the study was to investigate the prevalence and spectrum of beta globin gene mutation among the normal population (HPLC \& RBC indices normal).

Patients/Materials \& Methods: Individuals attending Thalassaemia Carrier Screening outreach camp conducted by IHTM was included in this study. Informed consent was taken from each individual. Their clinical history was recorded. $2 \mathrm{ml}$ of blood samples in EDTA vial were collected from each individual. Samples were initially subjected to Hematologicalanalyzer\& HPLC analysis. Individuals with normal RBC indices and HPLC values were subjected to Beta globin gene sequencing (ABI Variant 3500 Genetic Analyzer).

Results: The average $\mathrm{Hb}, \mathrm{MCV}, \& \mathrm{MCH}$ value of 74 individual included in this study was $12.3 \mathrm{~g} / \mathrm{dl}, 89 \mathrm{fl}$ and $28.2 \mathrm{pg}$ respectively. $\mathrm{HbA} 2(2.2 \%-3.5 \%)$ and $\mathrm{HbF}(<1 \%)$ was in the normal range (Table 1). DNA sequencing analysis revealed that among the 74 individuals, $57(77 \%)$ were found to carry a mutation (CAT $>$ CAC) at codon 2 of HBB gene [HBB: c.9T $>$ C mutation in Position- 70,603nt of refseq: NG_000007.3 Located at Exon 1 in codon 2].

Out of 57 individuals, $38(66.6 \%)$ were found to have heterozygous form and $19(33.3 \%)$ were found to have homozygous mutation for this position (Table 2). The average $\mathrm{Hb}, \mathrm{MCV}, \& \mathrm{MCH}$ value of 57 individual carrying the above said mutation was $12.3 \mathrm{~g} / \mathrm{dl}, 88.3 \mathrm{fl}$ and $28.2 \mathrm{pg}$ respectively. $\mathrm{HbA} 2$ and $\mathrm{HbF}$ was in the normal range (2.8 and 0.5 respectively) (Table 3 ). No significant difference was found in RBC indices and HPLC parameters in between the individuals carrying homozygous and heterozygous form of HBB: c.9T $>$ C mutation (Table 3 ).

Other than the HBB: c.9T $>$ C mutation, there was only one other mutation $\mathrm{Hb}$ Hamilton (GTT $>$ ATT) [HBB:c.34G $>$ A Position70,628 of ref seq: NG_000007.3, Located at Exon 1 Codon 11] found in one individual among selected population.

Discussion \& Conclusion: This study suggested that the mutation (name- HBB: c.9T $>$ C) is insignificant (His $>$ His) for beta globin expression. It has been reported to have no effect or mild effect on beta globin expression and this is kind of missense mutation (S. S. Sahoo, 2013).

Supporting Document: 080885f8-962d-4929-8531-92c78eecb7ea.
Table 1: RBC Indices and HPLC parameter of the total study population

\begin{tabular}{|c|c|c|c|c|c|c|}
\hline \multirow{2}{*}{ Indices } & \multicolumn{6}{|c|}{$\mathrm{n=74}$} \\
\cline { 2 - 7 } & $\begin{array}{c}\text { Hemoglobin } \\
(\mathbf{g} / \mathrm{dl})\end{array}$ & $\begin{array}{c}\mathbf{R B C} \\
\mathbf{1 2} \\
(\mathbf{1 0} / \mathbf{L})\end{array}$ & $\begin{array}{c}\text { MCV } \\
(\mathbf{f 1})\end{array}$ & $\begin{array}{c}\mathbf{M C H} \\
\mathbf{( p g )}\end{array}$ & $\begin{array}{c}\mathbf{H b A 2} \\
(\%)\end{array}$ & $\begin{array}{c}\mathbf{H b F} \\
(\%)\end{array}$ \\
\hline $\begin{array}{c}\text { Average } \\
\text { Value }\end{array}$ & 12.3 & 4.4 & 89 & 28.2 & 2.8 & 0.5 \\
\hline
\end{tabular}

TABLE 2: Distribution of homozygous and heterozygous state of mutation (HBB: c.9T>C) among the study Population

\begin{tabular}{|c|c|c|c|}
\hline \multirow{2}{*}{$\begin{array}{c}\text { Prevalence of } \\
\text { HBB: c.9T }>\text { C mutation }\end{array}$} & \multicolumn{3}{|c|}{ Total Study Population= 74 } \\
\cline { 2 - 4 }$(\mathrm{n}=74)$ & Heterozygous & Homozygous & Total \\
\cline { 2 - 4 } & $\mathrm{n}=38$ & $\mathrm{n}=19$ & $\mathrm{n}=57$ \\
& $(66.6 \%)$ & $(33.3 \%)$ & $(77 \%)$ \\
\hline
\end{tabular}

Table 3: RBC Indices and HPLC parameter of the population $(n=57)$ carrying HBB; c.9T>C mutation

\begin{tabular}{|c|c|c|c|c|c|c|}
\hline $\begin{array}{c}\text { Mutation } \\
\text { Type }\end{array}$ & $\begin{array}{c}\text { Hemoglobin } \\
(\mathbf{g} / \mathbf{d})\end{array}$ & $\begin{array}{c}\mathbf{R B C} \\
(\mathbf{1 0} / \mathbf{L})\end{array}$ & $\begin{array}{c}\text { MCV } \\
(\mathbf{f l})\end{array}$ & $\begin{array}{c}\text { MCH } \\
(\mathbf{p g})\end{array}$ & $\begin{array}{c}\mathbf{H b A 2} \\
(\boldsymbol{\%})\end{array}$ & $\begin{array}{c}\mathbf{H b F} \\
(\mathbf{\%})\end{array}$ \\
\hline $\begin{array}{c}\text { Total } \\
(\mathrm{n}=57)\end{array}$ & 12.3 & 4.3 & 88.3 & 28.2 & 2.8 & 0.5 \\
\hline $\begin{array}{c}\text { Heterozygous } \\
(\mathrm{n}=38)\end{array}$ & 12.4 & 4.5 & 86.4 & 27.5 & 2.7 & 0.6 \\
\hline $\begin{array}{c}\text { Homozygous } \\
(\mathrm{n}=19)\end{array}$ & 12.1 & 4.2 & 91.9 & 29.5 & 3.0 & 0.33 \\
\hline
\end{tabular}

Disclosure of Interest: None Declared.

Keywords: HBB: c.9T $>$ C polymorphism (CAT $>$ CAC), Heterozygous Mutation, Homozygous Mutation.

\section{Abstract 12. Clinical Presentation of a Rare Mutation [-90 $(C>$ T)BETA+]: A Case Study}

SunisthaBhattacharjee ${ }^{* 1}$, Rudra Ray ${ }^{1,1}$, Ankita Biswas ${ }^{1}$, MaitreyeeBhattacharyya ${ }^{1}$

${ }^{1}$ Hematology, Ihtm, Medical College, Kolkata, Kolkata, India

Aims \& Objectives: The aim of this study was to investigate the molecular defect of a thalassaemia child patient whose clinical phenotypes remain unexplained by routine hematological investigations. Patients/Materials \& Methods: A child patient attending thalassaemia OPD was recruited for this study. Informed consent was taken from his family. Clinical history of the patient and family (parents) were recorded. $2 \mathrm{ml}$ of blood samples in EDTA vial was collected from the child and both parents. Samples were initially subjected to Complete Hemogram analysis (Sysmex KX- 21), HPLC analysis (Bio-Rad Variant II, Beta Thal Short Program), and Serum Ferritin estimation by chemiluminescence immunoassay (Beckman Coulter). ARMS PCR was done to investigate for common beta mutations. GAP PCR was done to investigate the status of alpha globin gene mutation (triplication and deletion). Further DNA sequencing was done to determine the presence of any rare/uncommon beta globin gene mutation.

Results: The three year old patient recruited in this study presented with- $\mathrm{Hb} 7.10 \mathrm{~g} / \mathrm{dl}, \mathrm{MCV} 68.30 \mathrm{fl}, \mathrm{MCH} 23.00 \mathrm{pg}$. HPLC analysis showed- $-\mathrm{HbA}_{2} 4.30 \%$ and $\mathrm{HbF} 4.80 \%$. Transfusion requirement is lunit per month. His father showed $\mathrm{HbE}$ beta thalassaemia and his 
mother was beta thalassaemia carrier in HPLC (Table 1). The patient was found to carry IVS 1-5 mutation in heterozygous state as common mutation by ARMS PCR and his father was carrying both HbE and IVS-1-5 mutations in heterozygous state. There is no common mutation found in his mother (Table 1). Further, DNA sequencing analysis showed presence of a rare mutation $-90(\mathrm{C}>\mathrm{T})$ beta + in heterozygous state [HbVer, NG_000007.3] in the patient and also in his mother. His father was confirmed with $\mathrm{HbE}$ and IVS-1-5 mutations in heterozygous state (Table 1). This family screening explains the inheritance of the mutations to this patient.

Discussion \& Conclusion: $-90(\mathrm{C}>\mathrm{T})$ have been reported in West Bengal previously (Edison and Shaji et al. 2008). This mutation is located at enhancer, before the promoter site upstream to beta globin gene. It is a tandem repeat of CACCC box thus mutation in this region will assumed to affect transcription of beta globin gene.

Supporting Document: 96571332-071e-4d32-b3f5-24269eb180cb.

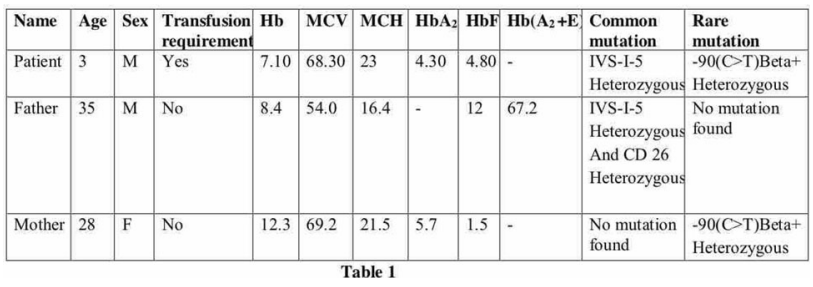

Disclosure of Interest: None Declared.

Keywords: DNA sequencing, Rare mutation, Thalassaemia.

\section{Abstract 13. An Inter-Instrument Comparison for Hemoglobin Estimation: Hemocue Vs. Automated Hematology Analyser}

\section{DhartiPadharia $^{\text {*1 }}$, KeyuriPatel ${ }^{1}$, Mustafa Ranapurwala ${ }^{1}$, Rohit Kumar Singh ${ }^{1}$, NeelofarMansuri ${ }^{1}$}

\section{${ }^{1}$ Pathology, Pramukhswami Medical College, Anand, India}

Aims \& Objectives: 1. To undertake an inter-instrument comparison for hemogobin estimation between a point of care testing device (Hemocue) against the current god standard (automated hematology analyser).

2. To validate the utilization of Hemocue for estimation of hemoglobin for blood donors.

Patients/Materials \& Methods: A prospective observational study was undertaken at the A D Gorwala Blood Bank associated with Shree Krishna Hospital, Karamsad with 200 EDTA samples. 100 samples with hemoglobin levels (run on automate hematology analyser) $>12.5 \mathrm{~g} / \mathrm{dL}$ and 100 samples with hemoglobin levels (run on automate hematology analyser) $<12.5 \mathrm{~g} / \mathrm{dL}$ were taken to be run in the hemocue machine. Regression analysis was done to validate the hemoglobin levels estimated by the hemocue by taken the hematology analyser as the gold standard.

Results: Mean $\mathrm{Hb}$ values for sysmex and hemocue runs for samples with $>12.5 \mathrm{~g} / \mathrm{dl}$ were $13.54 \pm 0.72$ and $13.7 \pm 0.75$ respectively. Mean $\mathrm{Hb}$ values for sysmex and hemocue runs for samples with $<12.5 \mathrm{~g} / \mathrm{dl}$ were $11.13 \pm 0.96$ and $11.37 \pm 0.97$ respectively. The $\mathrm{r}$ - squared value for the regression analysis was within limits.

Discussion \& Conclusion: Despite minor incongruities, hemocue showed a good agreemet with automated hematology analyser and can hence be used for screening blood donors, albiet, if done by trained staff that follow Standard Operating Procedures.

Disclosure of Interest: None Declared.

Keywords: Automated hematology analyser, Hemocue, Hemoglobin.

\section{Abstract 14. First Report of Patient with HB M Saskatoon mutation in W.B}

\section{Ankita Biswas ${ }^{* 1}$, SunisthaBhattacharjee ${ }^{1}$, Rudra Ray ${ }^{1}$, MaitreyeeBhattacharyya ${ }^{1}$}

\section{${ }^{1}$ Transfusion Medicine, Ihtm, Medical College, Kolkata, Kolkata, India}

Aims \& Objectives: The aim of this study was to investigate the molecular defect of a child patient who presented with fatigue and cyanosis/grey-blue discoloration of the distal extremities. Physical examination showed cyanosis.

Patients/Materials \& Methods: A 2 years old female child patient and her family were included in this study. The patient was recruited from Thalassaemia OPD who was referred from Pediatrics department as the patient showed symptoms of cyanosis. Informed consent was taken from them. The clinical history of the patient and family (parents) were recorded. $2 \mathrm{ml}$ of blood samples in EDTA vial were collected from each individual. Samples were initially subjected to Hematologicalanalyzer and HPLC analysis followed by ARMS PCR. DNA sequencing was done to identify the presence of beta globin gene mutation.

Results: The 2 years old patient recruited in the study presented with $\mathrm{Hb}$ value of $9.5 \mathrm{~g} / \mathrm{dl}$. Her MCV and $\mathrm{MCH}$ value was in the normal range (91.5 $\mathrm{fl}$ and $27.8 \mathrm{pg}$ respectively). HPLC analysis showed that $\mathrm{Hb} \mathrm{A} 2$ value (3.4\%) was in the border line and $\mathrm{Hb} \mathrm{F}$ value was $1.8 \%$. Symptoms of cyanosis and fatigue were reported/observed by physical examination of the patient. ARMS PCR analysis revealed absence of the common beta mutations. DNA sequencing analysis revealed that the patient was found to carry a rare mutation in the heterozygous state at Codon 63 (CAT>TAT) in the Exon 2 causing a change in amino acid sequence from $\overline{\mathrm{H}}$ is $>$ Tyr. The found mutation is reported in the globin gene server ( http://globin.cse.psu.edu/cgi-bin/hbvar/coorSeqCheck) and known as Hb-M- Saskatoon mutation [HBB:c.190C $>$ T mutation in the Position$70,914 \mathrm{nt}$ of ref seq: NG 000007.3 ]. The parents of the patient was also subjected to sequencing analysis to conform the presence/origin of $\mathrm{Hb} \mathrm{M}$ Saskatoon heterozygous mutation and the mother was found to carry the $\mathrm{Hb} \mathrm{M}$ Saskatoon heterozygous mutation. The clinical features, haematological indices and other phenotypes are given in Table 1 .

Discussion \& Conclusion: To the best of our knowledge this is the $1^{\text {st }}$ report of $\mathrm{Hb} \mathrm{M}$ Saskatoon mutation in West Bengal.

Supporting Document: 802ba236-01b1-4504-8d17-bd8dccec60d3.

Table 1: RBC Indices and HPLC parameter of the patient and her family

\begin{tabular}{|c|c|c|c|c|c|c|c|c|}
\hline Individuals & $\begin{array}{c}\text { Sex } \\
(\text { Male/ } \\
\text { Female })\end{array}$ & $\begin{array}{c}\text { Hemoglobin } \\
(\mathrm{g} / \mathrm{dl})\end{array}$ & $\begin{array}{c}\mathrm{RBC} \\
\mathbf{1 2} \\
(\mathbf{1 0} / \mathrm{L})\end{array}$ & $\begin{array}{c}\text { MCV } \\
(\mathbf{f l})\end{array}$ & $\begin{array}{c}\text { MCH } \\
(\mathbf{p g})\end{array}$ & $\begin{array}{c}\text { HbA2 } \\
(\%)\end{array}$ & $\begin{array}{c}\text { HbA0 } \\
(\%)\end{array}$ & $\begin{array}{c}\text { HbF } \\
(\%)\end{array}$ \\
\hline Patient & $\mathrm{F}$ & 9.5 & 3.42 & 91.5 & 27.8 & 3.4 & 75.8 & 1.8 \\
\hline Mother & $\mathrm{F}$ & 14.7 & 4.46 & 104.5 & 33.0 & 3.1 & 96.5 & 0.2 \\
\hline Father & $\mathrm{M}$ & 9.0 & 4.06 & 94.8 & 22.2 & 2.7 & 87.8 & 0.6 \\
\hline
\end{tabular}

Disclosure of Interest: None Declared.

Keywords: cyanosis, $\mathrm{Hb} \mathrm{M}$ Saskatoon Mutation, Heterozygous Mutation. 


\section{Abstract 15. High Dose IVIG \& Steroid for Incompatible Transfusion in a Case with Multiple Alloantibody}

\section{Chippy C S ${ }^{* 1}$, Hem chandraPandey ${ }^{1}$, TulikaSeth ${ }^{2}$, Poonam Coshic $^{1}$, Rakesh Mohan Thapliyal ${ }^{1}$, Vineet Sharma ${ }^{1}$ \\ ${ }^{1}$ Transfusion Medicine, ${ }^{2}$ Hematology, All India Institute Of Medical Sciences, NEW DELHI, India}

Aims \& Objectives: Transfusion management of case of multiple alloantibodies using non-ABO incompatible PRBC under therapeutic cover of high dose IVIG and steroid.

Patients/Materials \& Methods: Mrs. X reported to emergency department with twin pregnancy (12 weeks gestation) and $\mathrm{Hb}$ of $3.5 \mathrm{gm} / \mathrm{dl}$. She was advised emergent blood transfusion but was found to be incompatible for all blood units tested and both direct $\&$ indirect coombs tests were positive.

Patient was diagnosed with $\mathrm{HbE}-\beta$-Thalassemia intermedia at age 15 as per her outside records and was inadequately transfused every 2-2.5 months, due to transfusion reactions. In view of the present situation she was advised MTP along with transfusion of two least incompatible PRBCs, but she hemolysed both. Further workup revealed six different red cell alloantibodies (anti-C, anti-E, anti-K, anti-Jka, anti-S and an unspecified antibody). Despite all efforts we couldn't find a unit negative for these six antigens, so we transfused units (compatible for four of the antigens) under cover of high dose IVIG $(0.4 \mathrm{mg} / \mathrm{kg}$ on consecutive days for 5 days $)$ and steroid (40 mg).

Results: Her hemoglobin improved \& procedure (MTP) was done uneventfully without any signs of hemolysis post-transfusion. Patient was started on hydroxyurea and discharged one week later with a stable $\mathrm{Hb}$ of $5.1 \mathrm{~g} / \mathrm{dl}$.

Discussion \& Conclusion: Multiple red cell alloantibodies occur in thalassemia intermedia and pose difficulty for transfusion. Prophylactic use of high dose IVIG and steroid has been described for rare blood group antibodies against high frequency antigens with successful transfusion outcomes. Although the same strategy has been suggested in literature for cases where serological compatibility cannot be assured, the experience and evidence for the cases with multiple alloantibodies is lacking. Our case demonstrated that such strategy is a feasible option in cases of multiple alloantibodies without any evidence of acute hemolysis and maintenance of hemoglobin levels. However, this strategy should only be reserved for patients requiring life saving transfusions and all efforts to find a compatible unit have failed.

Disclosure of Interest: None Declared.

Keywords: high dose IVIg, incompatible transfusion, multiple alloantibodies.

\section{Abstract 16. Efficacy of Single Dose Vincristine in Immune Mediated Moderate Thrombocytopenic Dogs}

\author{
BaranidharanG.R ${ }^{* 1}$, NambiA.P ${ }^{2}$, TirumurugaanK.G ${ }^{3}$, \\ Dhinakar Raj $\mathbf{G}^{4}$
}

${ }^{1}$ Tanuvas Animal Blood Bank, Dept Of Clinics, ${ }^{2}$ Veterinary Clinical Medicine, ${ }^{3}$ Animal Biotechnology, Madras Veterinary College, Tamil Nadu Veterinary And Animal Sciences University, ${ }^{4}$ Director, Centre for Animal Health Studies, Tamil Nadu Veterinary And Animal Sciences University, Chennai, India
Aims \& Objectives: To evaluate the efficacy of single dose of vincristine sulphate @ dose of $0.025 \mathrm{mg} / \mathrm{kg}$ IV in dogs with clinical bleeding signs and moderate thrombocytopenia $(25,000$ to 50,000 cells/cmm).

Patients/Materials \& Methods: Dogs $(n=30)$ brought to the Madras Veterinary College Teaching Hospital with clinical signs of thrombocytopenia viz. petechiae, ecchymosis, purpura, epistaxis, gingival bleeding, hyphema, melena, haemetemesis, haematuria, haemoglobinuria or other spontaneous bleeding tendencies from the mucosal surfaces were selected and screened. Coagulation Profile including Prothrombin Time (PT), Activated Partial Thromboplastin Time (APTT), Buccal Mucosal Bleeding Time (BMBT) were performed on all the Thrombocytopenic dogs to evaluate the Platelet Function (PF). Platelet Function tests including Von Willebrands Factor Assay (VWF) and Platelet Associated Antibody (PSAb) Assay were analyzed. Flow cytometric study for Immune Mediated Thrombocytopenia (IMTP) was performed to detect and quantitate immune mediation. The selected dogs were administered a single dose of vincristine sulphate @ dose of $0.025 \mathrm{mg} / \mathrm{kg}$ IV and the Platelet count, Platelet Indices and the clinical outcome of bleeding signs were monitored after $24,72 \mathrm{~h}$ and 6 days of therapy.

Results: The CBC revealed a marked improvement in thrombocytopenia, moderate anemia, and mild leukocytosis in 28 out of $30 \mathrm{dogs}$ that received vincristine i.e., $\geq 75,000$ cells $/ \mathrm{cmm}$ on day2, $\geq 1,00,000$ cells $/ \mathrm{cmm}$ on day3. No adverse effects attributable to Vincristine were observed in any dog. The dogs were also clinically and hemodynamically stable after a single dose of Vincristine and moreover the absence of hemorrhagic signs with elevated platelet counts.

Discussion \& Conclusion: Dogs with Ehrlichiosis induced moderate thrombocytopenia responded good to a single dose of vincristine therapy where in a progressive increase in the platelet count was evident within 2 days and needed subsequent treatment protocols for secondary immune mediated condition and the primary disease. It could be opined that secondary moderate IMT cases can be started on a single dose of vincristine sulphate @ dose of $0.025 \mathrm{mg} / \mathrm{kg}$, IV on day of clinical presentation to avoid platelet transfusions.

Supporting Document: cd97d6d3-1635-43f5-bda9-c4db77ac6eae.
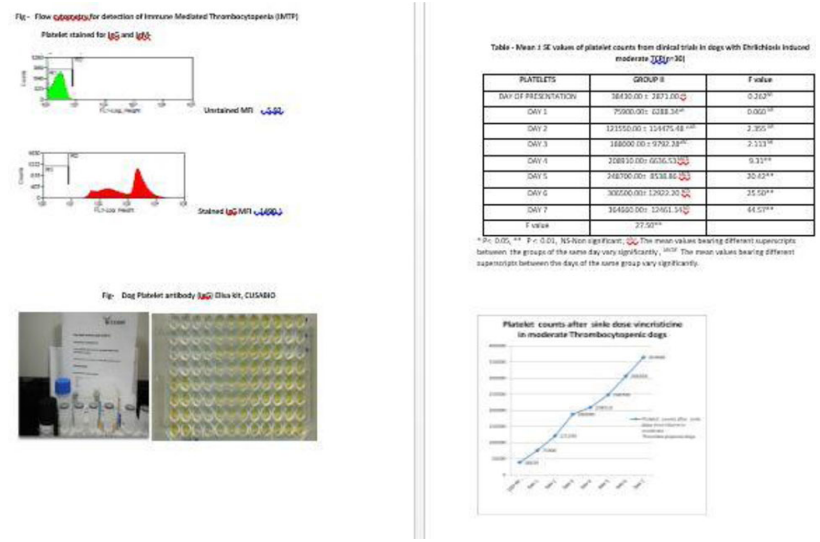

Disclosure of Interest: None Declared.

Keywords: Vincristine, Canine Ehrlichiosis, Immune Mediated Thrombocytopenia. 


\section{Abstract 17. Plasma Exchange Therapy: Effective in Case of Polyarteritis Nodosa Related to Hepatitis B Virus}

\section{ParmatmaTripathi $^{* 1}$, Rekha Hans ${ }^{1}$, Ratti Ram Sharma ${ }^{1}$ \\ ${ }^{1}$ Transfusion Medicine, PGIMER, Chandigarh, India}

Aims \& Objectives: Background: A well-known systemic necrotizing vasculitis form known as Polyarteritis nodosa (PAN) predominantly affects medium -sized muscular arteries and often involves small muscular arteries. Hepatitis B (HBV) association with PAN was described in 1970 and a rare complication of chronic Hepatitis B infection, PAN is occurring only in up to $5 \%$ of chronic $\mathrm{HBV}$ patients. Incidence of HBV related PAN is in decreasing trend due to vaccination against hepatitis $\mathrm{B}$. Role of therapeutic plasma exchange (TPE) for HBV related PAN is mentioned in literature with good prognosis in patients receiving anti-viral drugs. According to American Society For Apheresis (ASFA) 2019, HBV-PAN is category II (2C) indication for TPE and has supportive role in the therapeutic efficacy. We here report a case of PAN associated with Hepatitis B in which recovery was enhanced with plasma exchange therapy.

Patients/Materials \& Methods: Case: A twenty five year old male presented with complaints of generalized weakness, tingling numbness and weakness of extremities, joint pain, weight loss and rashes over arms and legs since two months. Hepatitis -B work up showed high viral loads of HBV (34 million IU/ml) and hepatitis e antigen positivity. Cardiac work up revealed elevated cardiac enzymes and decreased ejection fraction. CECT Chest \& abdomen with CT angiogram abdomen findings were consistent with medium vessel vasculitis. On the basis of history, examinations and investigations a diagnosis of vasculitis with probable etiology of HBV related PAN with association of mononeuritis multiplex, myocarditis was made.

Results: Twelve procedures of plasma exchange along with antiviral drugs were given. On average $2078 \mathrm{ml}$ of plasma was exchanged during each session with $4 \%$ albumin as a replacement fluid using femoral line dialysis catheter as vascular access on automated cell separator Optia ${ }^{\circledR}$ Spectra (Terumo BCT, Lakewood, Co). He was discharged with resolution of symptoms including myocarditis and regain in muscular strength and on follow up.

Discussion \& Conclusion: TPE could be an effective adjuvant therapy along with anti- viral therapy and short term corticosteroids in a rare disease like HBV related PAN.

Disclosure of Interest: None Declared.

Keywords: American Society for Apheresis ASFA, Polyarteritis nodosa PAN, therapeutic plasma exchange TPE.

Publisher's Note Springer Nature remains neutral with regard to jurisdictional claims in published maps and institutional affiliations. 